\title{
New solid state oxygen and hydrogen conducting materials. Towards their applications as high temperature electrochemical devices and gas separation membranes
}

\author{
Thesis submitted by \\ María Balaguer Ramírez \\ To apply for the Degree of Doctor \\ Supervisors: Dr. José Manuel Serra Alfaro \\ Dr. José Marcial Gozálvez Zafrilla
}

Valencia, July 2013 

A mis padres y a mi hermana

A Sergio 

"But I don't want to go among mad people," Alice remarked. "Oh, you can't help that," said the Cat: "we're all mad here. I'm mad. You're mad."

"How do you know I'm mad?" said Alice. "You must be," said the Cat, or you wouldn't have come here."

Lewis Carroll, Alice in Wonderland 



\section{Outline}

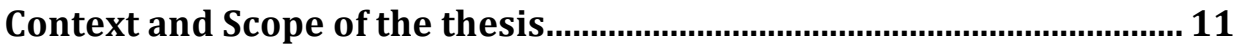

Chapter 1. Introduction ....................................................................... 15

1.1. Introduction to mixed ionic-electronic conductors (MIEC) ....... 17

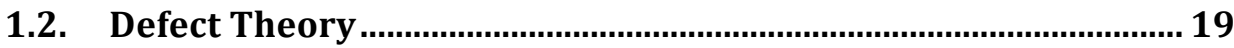

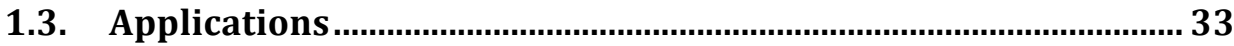

Oxygen separation membranes.................................................................. 33

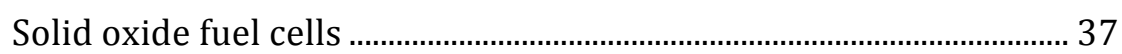

1.4. References.......................................................................................... 50

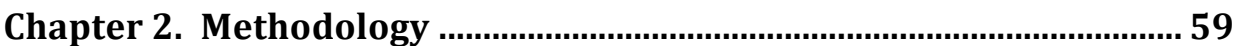

2.1. Material synthesis and sample preparation..................................61

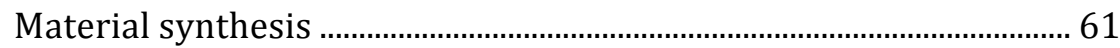

Sample preparation ................................................................................... 63

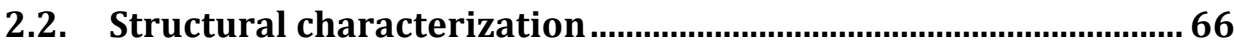

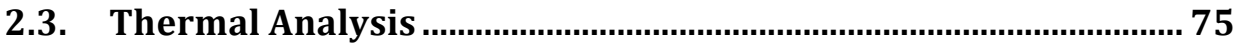

2.4. Electrochemical characterization ................................................... 78

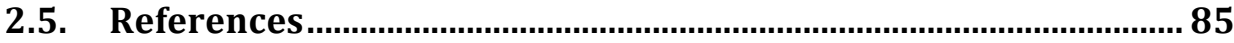

Chapter 3. Structural-transport properties relationships on

$\mathrm{Ce}_{1-\mathrm{x}} \mathrm{Ln}_{\mathrm{x}} \mathrm{O}_{2-\delta}$ system ( $\mathrm{Ln}=\mathrm{Gd}, \mathrm{La}, \mathrm{Tb}, \mathrm{Pr}, \mathrm{Eu}, \mathrm{Er}, \mathrm{Yb}, \mathrm{Nd}$ ) and effect of

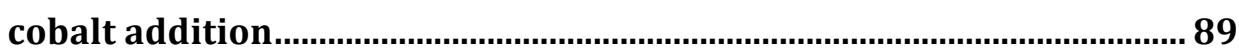

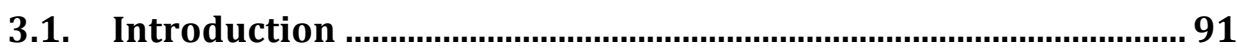

3.2. Results and discussion ................................................................ 95

3.3. Conclusions .....................................................................................114

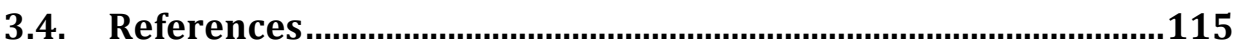

Chapter 4. Microstructure optimization of $\mathrm{Ce}_{0.9} \mathrm{Pr}_{0.1} \mathrm{O}_{2-\delta}+\mathrm{Co} 2 \mathrm{~mol} \% 121$

4.1. Introduction 
4.2. Results and discussion ........................................................124

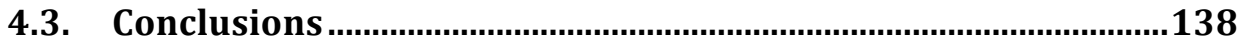

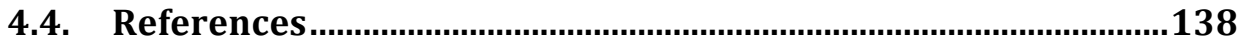

Chapter 5. Study of the bulk transport properties and oxygen surface exchange of the mixed ionic electronic conductor $C e_{1-\mathrm{x}} \mathrm{Tb}_{\mathrm{x}} \mathrm{O}_{2-\delta}$

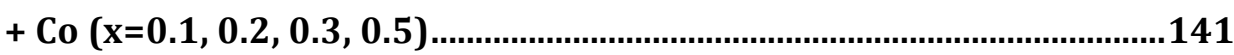

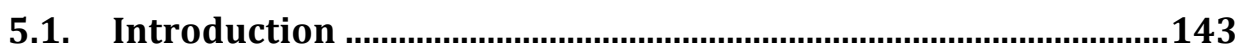

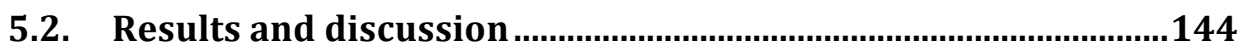

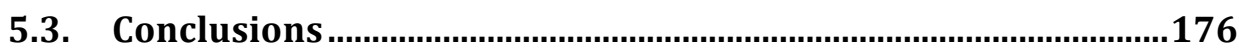

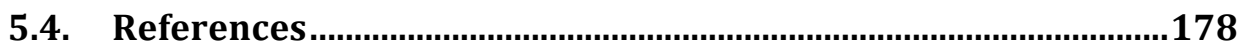

Chapter 6. LSM-ceria composite cathodes for SOFC...............................183

6.1. Introduction …….............................................................................185

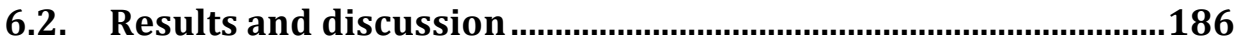

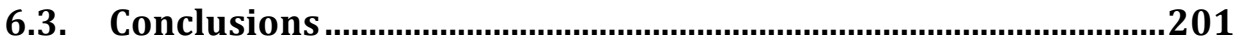

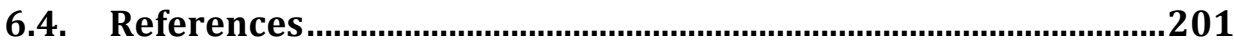

Chapter 7. Ionic transport improvement on $\mathrm{CaTiO}_{3}$ based

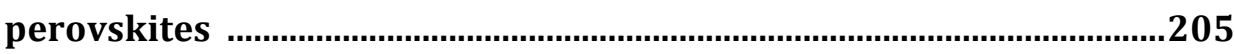

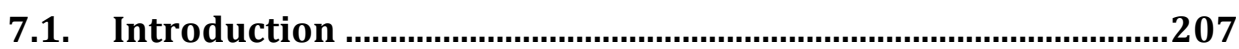

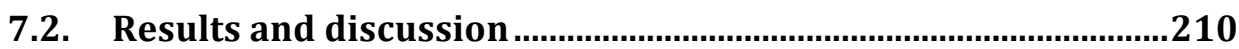

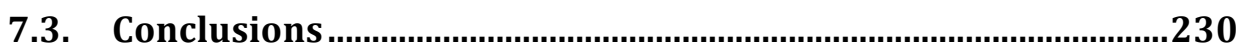

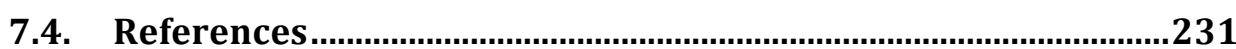

Chapter 8. Ceria-based stable dual-phase membranes for fast

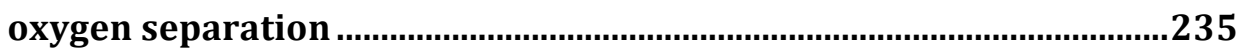

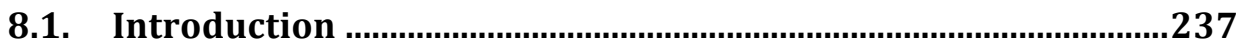

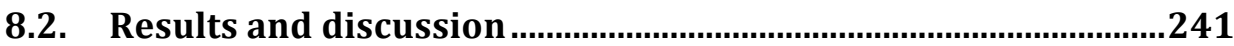

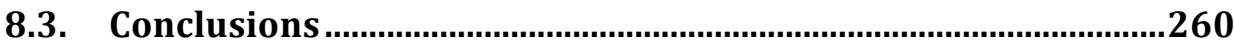

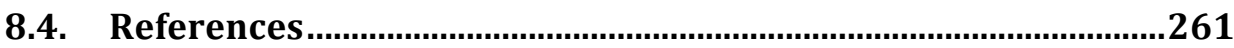


Chapter 9. Mixed protonic-electronic conductors based on

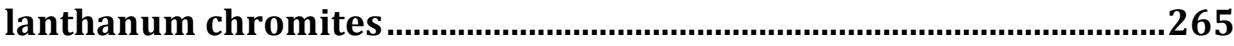

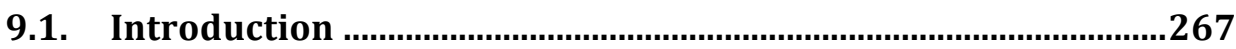

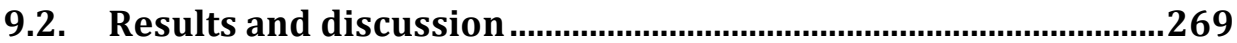

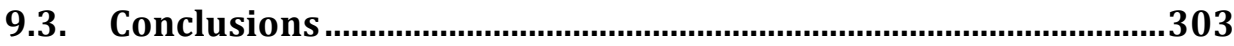

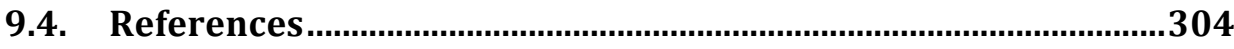

Chapter 10. Conclusions and remarks ................................................309

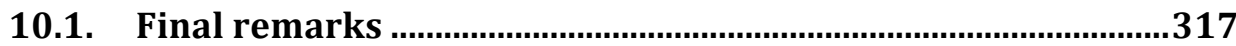

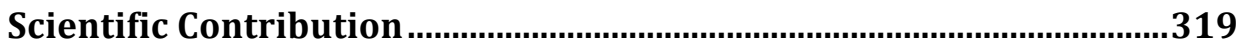

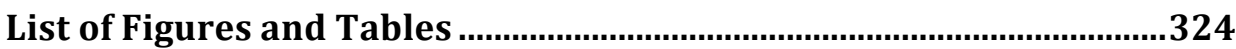

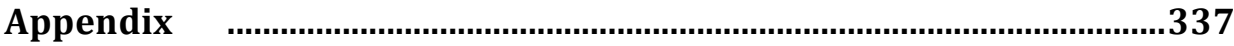

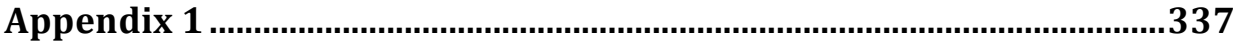

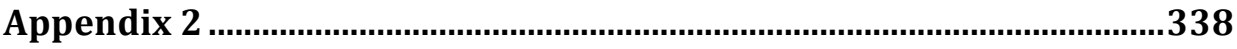

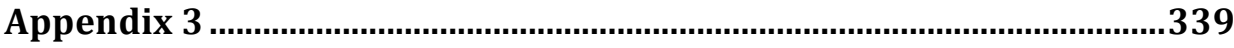

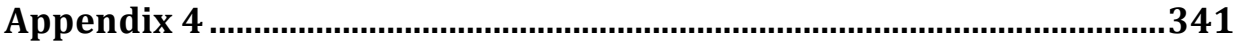

Resumen / Resum / Summary …......................................................343

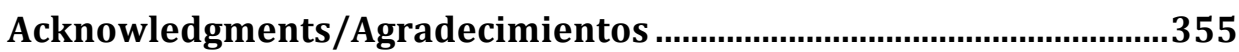





\section{Context and Scope of the thesis}

Increasing energy demand in a world where economy and sustainability are claimed to come together has given a boost to renewable sources of energy that avoid the emission of greenhouse gases. Nevertheless, under a global vision the majority of constructed power plants are still working with fossil fuels, especially coal-fired as it is cheaper and more abundant than oil and natural gas. The concentration of atmospheric $\mathrm{CO}_{2}$ has recently reached $400 \mathrm{ppm}$, far higher than the safety limit of $350 \mathrm{ppm}$ and with the last precedent 3 million years ago. Therefore, decreasing the emission of greenhouse gases and especially $\mathrm{CO}_{2}$ is a main issue that has gained great focus.

Up to date, a variety of mixed electronic and ionic (oxygen ions or protons) conducting materials are being investigated to separate oxygen or hydrogen from reforming streams at high temperature. The separation occurs through dense ceramic oxides providing selectivities close to $100 \%$. These solid oxide materials may be used in the production of electricity from fossil fuels (coal or natural gas), taking part of the separation and storage of $\mathrm{CO}_{2}$ system. Dense oxygen transport membranes (OTM) can be used in oxyfuel combustion plants or in catalytic membrane reactors (CMR), while hydrogen transport membranes (HTM) would be applied in precombustion plants. Furthermore, these materials may also be used in components for energy systems, as advanced electrodes or electrolytes for solid oxide fuel cells (SOFC) and proton conducting solid oxide fuel cells (PC-SOFC) working at high and moderate temperature. 
However, the most widely studied materials for these purposes present a series of drawbacks that make them impractical for application to gas separation. The harsh working conditions stablished by the targeted processes include high temperatures and low $\mathrm{O}_{2}$ partial pressures $\left(\mathrm{pO}_{2}\right)$, probably combined with $\mathrm{CO}_{2}$ and $\mathrm{SO}_{2}$ containing gases. Thus, the need to discover new stable inorganic materials providing high electronic and ionic conductivity is still present.

This thesis proposes a systematic search for new mixed ionic-electronic conductors with different crystalline structure and/or composition of the crystal lattice, varying the nature of the elements and the stoichiometry of the crystal. Hence, the main purpose of this thesis is to find breakthrough solid materials stable at high temperature $\left(600-1000{ }^{\circ} \mathrm{C}\right)$ which are sufficient high mixed electronic and ionic conductors, either oxygen ion or proton conducting. This main objective may break-down in several parts:

- to obtain stable crystalline phases with possible ion conduction paths through the crystal lattice. The considered crystalline phases include mixed oxides of the family of the perovskites, fluorites and spinels. Besides, physical mixtures of crystalline phases are taken into account (fluorite-spinel composites)

- to develop a technique for evaluating transport characteristics of a set of materials simultaneous and automatically

- to find and optimize new mixed ionic-electronic materials with high ionic conductivity $(\sigma>0.05 \mathrm{~S} / \mathrm{cm})$ and high chemical stability at high temperature in the presence of $\mathrm{CO}_{2}$ and $\mathrm{SO}_{2}$ and at low oxygen partial pressures

- to find and optimize new mixed protonic-electronic conductors $(\sigma>0.005 \mathrm{~S} / \mathrm{cm})$ with high temperature and chemical stability in the presence of $\mathrm{CO}_{2}$ and trace sulfur. 
The best candidates are studied in depth to understand their properties and their possible applications in gas separation, catalytic membrane reactors and SOFC components.

The obtained results are structured as follows. Chapter 1 of the thesis briefly describes the fundamental principles of solid oxides chemistry and the state of the art on the applicability of the mixed ionic-electronic conducting materials. Chapter 2 collects the procedures developed for the material synthesis and specimen fabrication. The techniques employed to characterize the samples and to determine their transport properties are outlined as well as the different setups used for the electrochemical characterization.

From this point, the following chapters may be divided in two main blocks. The first block comprises from Chapter 3 to Chapter 8 and deals with oxygen ion conducting materials. The second block, Chapter 9, is devoted to proton conducting perovskites.

In Chapter 3, a large series of lanthanide-doped ceria is characterized in order to have an overall understanding of the structural, oxygen vacancy concentration and transport properties. Besides, the addition of cobalt oxide on this system is similarly studied. The chosen lanthanides include a large range of ionic radii and different metals exhibiting variable oxidation states under the typical operating conditions encountered for these materials. Transport properties are correlated with structural features induced by the different ionic radii and the variable oxidation state of the dopants. Chapter 4 completes the study of the transport properties dependence on the microstructure. The sintering temperature of $\mathrm{Ce}_{0.9} \mathrm{Pr}_{0.1} \mathrm{O}_{2-\delta}+\mathrm{Co} 2 \mathrm{~mol} \%$ is systematically modified in order to observe the influence in particle size, $\mathrm{CoO}_{\mathrm{x}}$ location and total conductivity. In Chapter 5, the structural and bulk ionic and electronic transport properties of $\mathrm{Tb}$-doped ceria are evaluated as a function of $\mathrm{Tb}$ concentration. 
Surface properties are also reviewed. The potential use of these materials as high-temperature oxygen transport membrane is assessed. Finally, Chapter 6 includes additional metal dopants on the $\mathrm{Ce}_{0.9-\mathrm{x}} \mathrm{M}_{\mathrm{x}} \mathrm{Gd}_{0.1} \mathrm{O}_{2-\delta}$ structure with the aim of modifying the transport properties. The performance of these materials as a component of $\mathrm{La}_{1-\mathrm{x}} \mathrm{Sr}_{\mathrm{x}} \mathrm{MnO}_{3}$-based composite cathode is evaluated.

The second family of materials comprises $\mathrm{CaTiO}_{3}$-based perovskites. Chapter 7 reports the search of dopants either in A or B site that provide stable perovskites with high mixed ionic electronic conductivity. The screening drives to iron and iron-magnesium doped $\mathrm{CaTiO}_{3}$ as best candidates, which are further structural and electrochemically studied.

Chapter 8 describes the development of composite materials composed of $\mathrm{Fe}_{2} \mathrm{NiO}_{4}$ spinel material, combined with several lanthanide-doped cerias. The optimization of the synthesis method and the composition is reported as well as the electrochemical characterization and stability of the material.

Chapter 9 focuses on proton conducting perovskites based on $\mathrm{La}_{0.85} \mathrm{Sr}_{0.15} \mathrm{CrO}_{3}$. Doping strategies that aim to improve the ionic or protonic conductivity are evaluated by DC-conductivity in reducing conditions. The applicability of these materials is evaluated and the best candidates are tested as anode material for proton conducting SOFC.

Finally, general conclusions are extracted taking into account the objectives above described. 


\title{
Chapter 1. Introduction
}

\begin{abstract}
The present chapter shows a global vision about the defect chemistry of mixed ionic electronic conductors (MIEC). The main defect formation reactions are described. Secondly, the fundamentals of membrane and oxygen ion or proton conducting solid oxide fuel cells are explained, as well as their main requirements. Besides, a brief literature overview on the state of the art of the materials used for these applications is given.
\end{abstract}





\subsection{Introduction to mixed ionic-electronic conductors}

\section{(MIEC)}

Mixed ionic-electronic conductors (MIECs) are materials that conduct both ions and electronic charge carriers (electrons and/or holes). Normally, MIECs are ionic semiconductors, though the definition also holds for metals, glasses and polymers. [1] Besides, mixtures of two phases, one conducting ions the other conducting electrons/holes are considered as heterogeneous MIECs, named composites. The ionic species in solids may be oxygen anions, protons or metal cations, from which the materials presented in this work are oxygen ionic and protonic conducting oxides. The use of the term MIEC is usually reserved to those materials in which the ionic and electronic conductivities $\left(\sigma_{e}, \sigma_{i}\right)$ neither differ by more than 2 orders of magnitude nor release too low values $\left(\sigma_{\mathrm{i}}, \sigma_{\mathrm{e}}>10^{-5} \Omega^{-1} \cdot \mathrm{cm}^{-1}\right)$. [2] In fact, the conductivity of a material is proportional to the mobility and concentration of the carrier species (ions, vacancies, electrons or holes), which are examined below.

Ionic conduction in solids occurs by the movement of oxide ions through a crystal lattice, where ionic species of comparable size and opposite charge to the ions exist. In the crystal matrix, an empty site in the way of the ion is needed, which must have enough energy to overwhelm the barrier impeding the diffusion. Besides, these conditions have to be fulfilled continuously all along the sample. Thus, the movement of the ions from one to the other side of the material takes place via interstitial sites or by hopping into a vacant site or a complex combination of both interstitial and vacant sites. [3] There are three different ways to create ionic defects in a material (i) intrinsically, by thermal 
excitation, and extrinsically (ii) by changing the stoichiometry via modifications of the oxygen sublattice, or (iii) by doping with aliovalent cations.

Thermal excitation causes the smaller ion (usually the cation) to be displaced from the lattice position to an interstitial site, which create a Frenkel pair or Schottky disorder as illustrated in Figure 1.1. Frenkel pairs consist of a vacancy defect at its original site and an interstitial defect at its new location. Schottky disorder involves an equal number of cations and anions moving from the lattice to the surface. On the other hand, the change of stoichiometry modifies the composition by interaction with the environment, as introduces oxygen vacancies or interstitial oxygen in the structure, e.g. $\mathrm{CeO}_{2-\delta}$ and $\mathrm{Sr}_{4} \mathrm{Fe}_{4} \mathrm{Co}_{2} \mathrm{O}_{13+\delta}$. Finally, the strategy of doping may introduce mobile interstitial donors or mobile vacancies on the anion sublattice, which are mobile at elevated temperatures.

a)

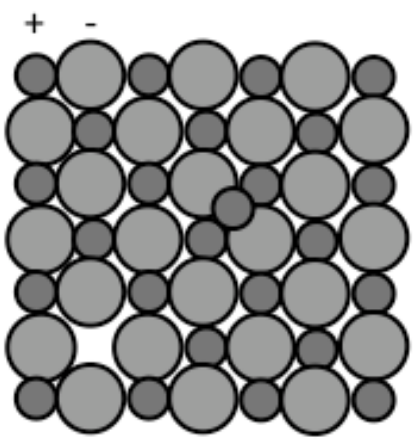

b)

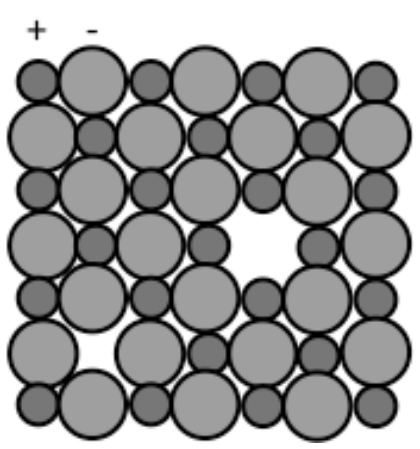

Figure 1.1. a) Frenkel defect: cation in interstitial hole, and b) Schottky disorder: missing pair of ions.

Electronic (electron/hole) conductivity occurs via delocalized states in the conduction/valence band or via localized states by a thermally assisted hopping mechanism. Similarly to the ionic conductivity, there are three ways of creating these charge carriers, (a) thermal excitation, which generates an electron-hole pair across the band gap, (b) deviation from stoichiometry that 
introduces native defects that may act as donors (oxygen vacancies) or acceptors (interstitial oxygen), and (c) doping, which also introduce donors or acceptors. [4] In some cases, unless donor level lies above the bottom of the conduction band, the electronic conduction by doping may require also thermal excitation.

It is evident that the defect concentration in an ionic or electronic conducting material is subjected to external factors such as temperature, oxygen partial pressure $\left(\mathrm{pO}_{2}\right)$ and other thermodynamic parameters. In fact, some ionic conducting materials become $p$ or $n$-type electronic conductors in certain environments. As an example, ionic conductor $\delta-\mathrm{Bi}_{2} \mathrm{O}_{3}$ becomes an $n$-type electronic conductor at low $\mathrm{pO}_{2}$. [5, 6] In the majority of applications of MIEC, they appear in the form of polycrystals; consequently, the grain boundaries (GB) are critical for the microstructure and the conductivity. The GB misorientation angles result in inter-grain blocking effect that may come from two natures (1) intrinsic charge barriers in grain-to-grain contacts, which is effectively explained by the space-charge effect where oxygen vacancies are highly depleted and (2) extrinsic segregation of impurities that block the transport of ions. [7, 8] Increasing the temperature and doping concentration minimize the 'intrinsic' blocking effect, while 'extrinsic' blocking effect may be still present up to $1000{ }^{\circ} \mathrm{C} .[7,9]$ Thus, all these factors should be taken into account. An accurate defect model could allow the systematic optimization of the electrical and transport properties of a material through cation substitution (doping), as well as the prediction of the behaviour under operating conditions. In the next section, an explanation of the defect generation is detailed.

\subsection{Defect Theory}

Key defect reactions containing the dominant electronic and ionic defects in an oxygen deficient oxide include oxidation/reduction, intrinsic lattice 
defect generation, intrinsic electron-hole generation, and ionization reactions as well as overall charge and mass balance relations. The reaction equations and their respective mass action laws are listed below. [10]

The intrinsic disorder is integrated in the equilibrium state of crystals. It is not related to impurity content or any other extrinsic origin such as deviation from stoichiometry. To achieve the equilibrium concentration of defects, the temperature needs to be sufficiently high to reach the equilibrium in reasonable times. All the movements inside a crystal that cause the lattice disorder must satisfy several conservation rules, i.e., conservation of mass, charge and structure (lattice site ratios). There are different types of intrinsic lattice disorder consistent with the conservation rules, although one type uses to be favoured compared with all others in a real case. For the compounds studied in this thesis, mainly ionic binary oxide materials, with high co-ordination number and small difference in sizes of cations and anions, the major types of intrinsic ionic disorder (schematized in Figure 1.1) are anion Frenkel (also called Anti-Frenkel) and Schottky disorder, whose defect reaction equations and their respective mass action laws are written below in Kröger-Vink notation (Eqs. 1.1-1.4). [11]

$O_{O}^{X} \rightarrow V_{O}^{\prime \prime}+O_{i}^{\prime \prime}$

$K_{F}=\left[V_{O}\right]\left[O_{i}^{\prime \prime}\right]=k_{F} \exp \left(\frac{-E_{F}}{k T}\right)$

$n u l l \rightarrow 3 V_{o}^{\prime \prime}+V_{M 1}^{\prime \prime}+V_{M 2}^{\prime \prime}$

$K_{S}=\left[V_{o}^{*}\right]^{3}\left[V_{M 1}^{\prime \prime}\right]\left[V_{M 2}^{\prime \prime}\right]=k_{S} \exp \left(\frac{-E_{S}}{k T}\right)$

where $O_{O}^{X}$ is an occupied oxygen site, $V_{O}^{\prime \prime}$ is an oxygen vacancy, $O_{i}^{\prime \prime}$ is an oxygen interstitial, $V_{M 1}^{\prime \prime}$ and $V_{M 2}^{\prime \prime}$ are the vacancies in the metals M1 and M2. Notation in brackets denotes the concentration of corresponding species and 
$K_{F}(T)$ and $K_{S}(T)$ are the equilibrium constants defining the anion Frenkel and Schottky reactions, respectively.

The intrinsic electronic disorder involves thermal excitation of electrons from chemical bonds to higher energy states. In a stoichiometric solid oxide, the ground state is a nominally filled valence band and a nominally empty conduction band. The excitation of an electron across the bandgap not only allows it to move freely through the empty states of the conduction band, but also leaves an empty electronic state at the top of the valence band. This hole can also transport charge as the electrons can move laterally into its empty state, in the opposite direction of electrons moving in the conduction band. Hence, the equilibrium reaction for the electronic excitation process that leaves behind an empty state must complete the conservation rules, and will be written as Eq. 1.5.

null $\rightarrow h^{\cdot}+e^{\prime}$

where $e^{\prime}$ is an electron in the conduction band and $h^{\prime}$ represents a hole in the valence band. So the mass action equation for this process is written as follows:

$K_{E}=n p=k_{E} \exp \left(\frac{-E_{g}}{k T}\right)$

where $n=\left[e^{\prime}\right]$ and $p=\left[h^{\prime}\right]$ are the widely used shorter notation representing the concentration of negative and positive carriers, respectively. $E_{g}$ is the energy of the bandgap that needs to be overwhelmed to create the disorder. 
a)

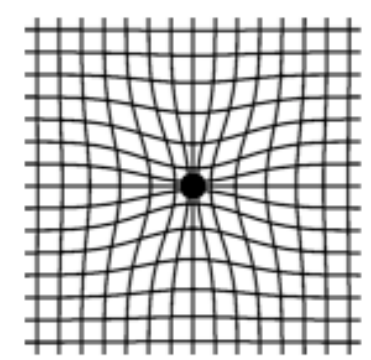

b)

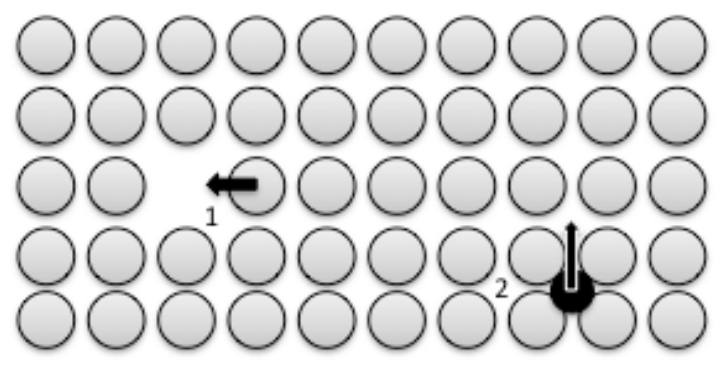

Figure 1.2. a) Polaron particle: a charge inside its self-created lattice distortion [12] and b) ionic diffusion mechanisms (1) via vacancy, (2) via interstitial site.

In the case of solid oxides where the motion of electrons is more restricted, the electronic transport occurs by discrete jumps between localized electrons via the so called hopping. Electronic defects in an ionic crystal induce a distortion in the lattice, which becomes polarized and traps the charge carrier inside the electrostatic field (self-trapping) as depicted in Figure 1.2a. The electron or hole trapped in a particular atom, ion or vacancy is considered as a particle named 'polaron', which can propagate throughout the crystal by hopping from one atom to another. Two kind of polarons can be distinguished depending on their binding energy. If the interaction with the lattice is strong and the polaron is localized, it is called 'small polaron' and the lattice distortion reaches distances close to the cell parameter. The small polaron hopping occurs between sites of optic phonon frequency, causing the low mobility (less than $\left.0.1-1 \mathrm{~cm}^{2} \mathrm{~V}^{-1} \mathrm{~s}^{-1}\right)$. By increasing temperature, the random hopping is activated so the small polaron mobilities rise. On the other hand, a weak interaction with the lattice produces longer distance distortions, which is known as 'large polaron', typical of transition metal oxides where metals may be in different oxidation states, e.g. in olivine $\left[\left(\mathrm{Mg}_{1-\mathrm{x}} \mathrm{Fe}_{\mathrm{x}}\right)_{2} \mathrm{SiO}_{4}\right]$, charge carriers are electrons transferred from $\mathrm{Fe}^{2+}$ to $\mathrm{Fe}^{3+}$ ions. $[13,14]$ Large polaron mobilities are high at low temperatures $\left(1.75 \times 10^{5} \mathrm{~cm}^{2} \mathrm{~V}^{-1} \mathrm{~s}^{-1}\right)$, which decrease with rising temperature 
through phonon scattering vibrations. [15] Focusing on carrier mobilities, the distinction between small and large polarons is shown in Figure 1.3.

The temperature dependence of the mobility is often of the form

$\mu=\mu_{0} T^{-3 / 2} e^{-E_{h} / k T}$

The localized centers hosting the electrons or holes may be lattice cations or anions or impurity centers if they are close enough to each other.

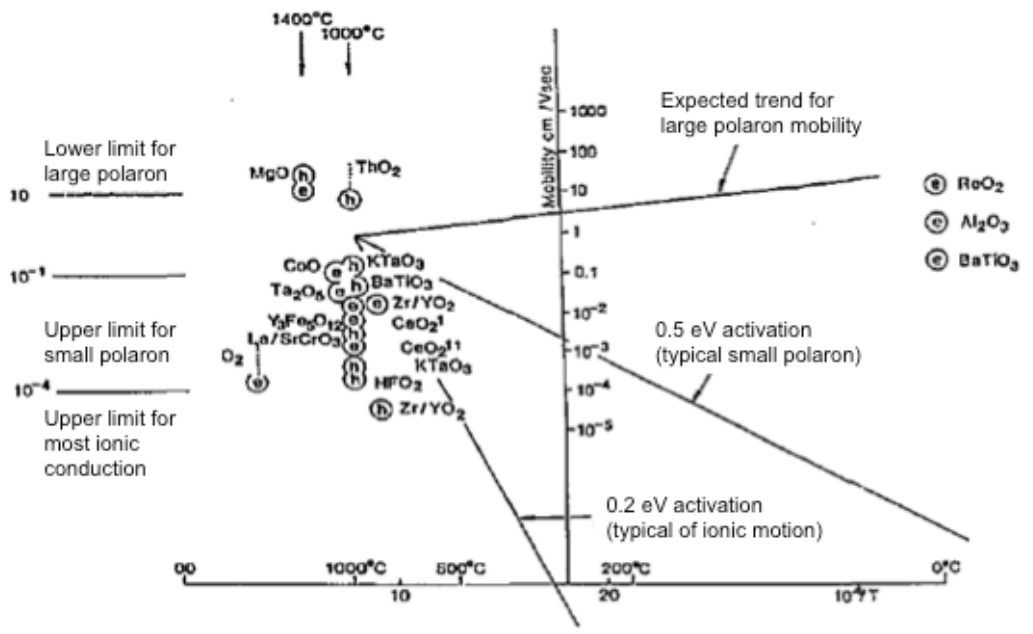

Figure 1.3. Electron (e), and hole (h), mobilities in oxides. The lower limit for large polarons corresponds to a mean-free path equal to the interatomic distance. The upper limit for small polarons corresponds to one hop every lattice vibration period. Note the opposite trends with temperature: for small polarons and ions, thermal activation is needed, so higher temperatures raise the rate; for large polarons, phonon scattering reduces the mobility at higher temperatures. [13]

Extrinsic ionic disorder involves the ionic defects that result from the presence of impurity ions dissolved in the lattice, forming a substitutional solid solution. If an isovalent ion replaces the host element, there is not need of creating other compensating defect and the properties of the solid solution are similar to the origin. Aliovalent ions carry a charge with respect to the perfect 
crystal, so that other oppositely charged defect must be present to keep the neutrality, i.e., an ionic defect. There are two possible compensating ionic defects, named vacancies or interstitials, which are different depending on the type of impurity. Donor impurities (D, positively charged) promote either cation vacancies or anion intersitials, while acceptor impurities (A, negatively charged) forms either cation interstitials or anion vacancies. Only one of these ionic defects results predominant from the impurity content. Features as electrostatic environment, space availability or formal charge on the defect will determine the most probable compensating defect.

In a material that exhibits different sort of defects, oppositely charged, the possibility of electrostatic attraction gives rise to the defect complexes and associates formation. These defects break under increasing temperature, so that their enthalpy of formation is negative. The defect complexes bind an impurity center with an oppositely charged ionic defect (sited on nearest-neighbour cation sites) turning it into a neutral inmobile complex. The subsequent drop of mobile charges produces and accelerated decrease in ionic conductivity with decreasing temperature.

In a more real scenario, it is necessary to consider that the crystal is not isolated and the thermodynamic equilibrium with the surrounding needs to be reached. This is achieved by means of electronic compensation, so that extrinsic electronic disorder appears. The interaction of doped crystals with the gaseous ambient leads to gain or loss non-metallic constituent until the ionic defects introduced by an impurity or a dopant have been eliminated and replaced by electrons or holes of equivalent charge. As a consequence, the material becomes nonstoichiometric. When doping with donor impurities the compensation occurs via the electrons left after the loss of the oxygen surplus relative to the host lattice. Acceptor dopants posses less oxygen per cation than host oxide, so that 
neutral external oxygen will enter in the lattice by taking two electrons from the valence band and leaving two compensating holes as overall result. [10]

Summarizing the effects of these mentioned cases in which an impurity or doping element is added, an ionization equation must be taken into account, different whether the dopant is an acceptor or donor:

\section{Acceptor dopant}

$A \rightarrow A_{c}^{\prime}+h^{\cdot}$

$K_{A c}=\left[A_{c}^{\prime}\right] p=k_{A c} \exp \left(\frac{-E_{A}}{k T}\right)$

\section{Donor dopant}

$D \rightarrow D_{c}^{\cdot}+e^{\prime}$

$K_{D n}=\left[D_{c}^{\cdot}\right] n=k_{D n} \exp \left(\frac{-E_{D}}{k T}\right)$

Each system will show those defects corresponding to the most favourable energetic situation. These charge-balancing defects may be used to tailor the transport properties of the material.

On the other hand, the reaction of a semiconductor compound with the surrounding gas phase components gives rise to intrinsic nonstoichiometry. The nonstoichiometry denotes a variable composition of the same crystal structure that must be adjusted with the environment by a combination of ionic and electronic defects.

\section{Reduction reaction}

In reducing atmospheres, the oxide material reacts with the atmosphere by loosing oxygen. 
$O_{O}^{X} \rightarrow V_{O}^{\ddot{\prime}}+2 e^{\prime}+\frac{1}{2} O_{2}$

The atomic defects generated in an occupied oxygen site, $O_{O}^{X}$, are oxygen vacancies that retain two electrons from the doubly ionized oxygen, $V_{o}$. The mass action law is written in Eq. 1.6

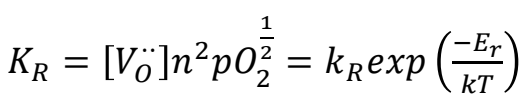

where $\left[V_{o}^{\prime \prime}\right]$ and $n$ are the oxygen vacancy and electron concentration, $p O_{2}$ is the oxygen partial pressure and $K_{r}(T)$ is the equilibrium constant defining the reaction.

\section{Oxidation reaction}

In oxidizing atmospheres, the oxide material reacts with the atmosphere by incorporating oxygen in the lattice.

$\frac{1}{2} O_{2}+V_{O}^{\ddot{H}} \rightarrow O_{O}^{X}+2 h$

$K_{O}=\left[V_{o}\right]^{-1} p^{2} p O_{2}^{-\frac{1}{2}}=k_{O} \exp \left(\frac{-E_{o}}{k T}\right)$

Finally, the electroneutrality condition inside the crystal has to be maintained, which is represented by a general equation incorporating all the charged defect species mentioned above. It can be written as Eq. 1.16 and Eq. 1.17 for Frenkel and Schottky dominion:

$$
\begin{aligned}
& n+2\left[O^{\prime \prime}\right]+\left[A^{\prime}\right] \rightarrow p+2\left[V_{O}^{*}\right]+\left[D^{\cdot}\right] \\
& n+\left[V_{M}^{\prime}\right]+\left[A^{\prime}\right] \rightarrow p+2\left[V_{\dot{X}}^{\cdot}\right]+\left[D^{\cdot}\right]
\end{aligned}
$$


If the aliovalent impurity or dopant content exceeds the concentration of defects resulting from intrinsic disorder, it gives rise to extrinsic non-stoichometry. Combination of the equations written above describes a way to evaluate the predominant type of carriers, i.e., the concentration of electrical species present at a given temperature, $\mathrm{pO}_{2}$ and dopant level. As not all the defects occur simultaneously it is possible to simplify the equations including only one defect species on either side of the equality (Eqs. 1.18-1.21). Hence, restricted ranges of $\mathrm{pO}_{2}$ and temperature usually define four defect regions dependent on the external conditions for acceptor and donor doped materials, i.e., reduction, ionic compensation, electronic compensation and oxidation regions, summarized in Table 1.1.

\begin{tabular}{llcccc}
\hline \multicolumn{3}{c}{ Region } & Acceptor & \multicolumn{2}{c}{ Donor } \\
\hline I & Reduction & \multicolumn{3}{c}{$n \approx 2\left[V_{o}^{\prime \prime}\right]$} & (Eq. 18) \\
II & Ionic compensation & $2\left[V_{o}^{\prime \prime}\right] \approx\left[A^{\prime}\right]$ & (Eq. 19) & $2\left[O_{i}^{\prime \prime}\right] \approx\left[D^{\cdot}\right]$ & (Eq. 20) \\
III & Electronic compensation & $p \approx\left[A^{\prime}\right]$ & (Eq. 21) & $n \approx\left[D^{\prime}\right]$ & (Eq. 22) \\
IV & Oxidation & & $p \approx 2\left[O_{i}^{\prime \prime}\right]$ & & (Eq. 23) \\
\hline
\end{tabular}

Table 1.1. Summary of electroneutrality equations in different regions.

Substituting these approaches solves the mentioned equation system, so that the temperature and $\mathrm{pO}_{2}$ dependence of the electrical species may be obtained. These dependencies are used in the analysis of the defect chemistry from the raw electrical data of the different systems studied in this thesis.

For an acceptor doped material, the approximation for the reduction reaction dominant region (I) indicates that the electron generation is compensated by doubly ionized oxygen vacancies. When it is substituted in Eq. 1.13 gives the following relation:

$$
n=\left(2 K_{R}\right)^{\frac{1}{3}} p O_{2}^{\frac{-1}{6}}
$$


Hence, the electrical conductivity at the reduction dominant region will have a $\mathrm{pO}_{2}^{-1 / 6}$ dependence due to electron conduction (i.e., electronic conductivity). By substituting Eq. 1.24 on the electron-hole formation equation, Eq. 1.6, it is possible to obtain p carriers dependence on $\mathrm{pO}_{2}$ :

$p=2^{-\frac{1}{3}} K_{R}^{-\frac{1}{3}} K_{e} p O_{2}^{\frac{1}{6}}$

Similarly, the equation for oxygen vacancies is obtained by substituting Eq 1.18 into Eq. 1.13:

$\left[V_{O}\right]=2^{-\frac{2}{3}} K_{R}^{\frac{1}{3}} p O_{2}^{-\frac{1}{6}}$

Finally, the oxygen partial pressure dependence for oxygen interstitials comes from the substitution of Eq. 1.26 in Eq. 1.2:

$\left[O_{X}^{\prime \prime}\right]=2^{\frac{2}{3}} K_{R}^{-\frac{1}{3}} K_{F} p O_{2}^{\frac{1}{6}}$

In region II, where less reduction conditions appear, the electrons left from the production of $\mathrm{O}_{2}$ are invested in cation reduction. Thus, by substituting Eq. 1.19 in Eq. 1.13 the electronic density will be determined by:

$n=\left(2 K_{R}\right)^{\frac{1}{2}}\left[A_{C}^{\prime}\right]^{\frac{-1}{2}} p O_{2}^{\frac{-1}{4}}$

Analogous analyses can be done for the rest of $\mathrm{pO}_{2}$ dependence of the electrical species in other regions, and the results are summarized in Table 1.2 for acceptor and Table 1.3 for donor doped materials. 


\begin{tabular}{|c|c|c|c|c|}
\hline Defect & Region I & Region II & Region III & Region IV \\
\hline $\boldsymbol{n}$ & $\left(2 K_{R}\right)^{\frac{1}{3}} p O_{2}^{-\frac{1}{6}}$ & $\left(2 K_{R}\right)^{\frac{1}{2}}\left[A_{c}^{\prime}\right]^{-\frac{1}{2}} p O_{2}^{-\frac{1}{4}}$ & {$\left[A_{c}^{\prime}\right]^{-1} K_{E}$} & $\left(2 K_{F} K_{O}\right)^{-\frac{1}{3}} K_{E} p O_{2}^{-\frac{1}{6}}$ \\
\hline $\boldsymbol{p}$ & $\left(2 K_{R}\right)^{-\frac{1}{3}} K_{E} p O_{2}^{\frac{1}{6}}$ & $\left(2 K_{R}\right)^{-\frac{1}{2}}\left[A_{c}^{\prime}\right]^{\frac{1}{2}} p O_{2}^{\frac{1}{4}}$ & {$\left[A_{c}^{\prime}\right]$} & $\left(2 K_{F} K_{O}\right)^{\frac{1}{3}} p O_{2}^{\frac{1}{6}}$ \\
\hline$\left[\boldsymbol{V}_{\boldsymbol{o}}\right]$ & $2^{-\frac{2}{3}} K_{R}^{\frac{1}{3}} p O_{2}^{-\frac{1}{6}}$ & $\frac{1}{2}\left[A_{c}^{\prime}\right]$ & $\left(2 K_{R}\right)^{\frac{1}{2}}\left[A_{c}^{\prime}\right]^{-\frac{1}{2}} p O_{2}^{-\frac{1}{4}}$ & $\left(2 K_{F}\right)^{\frac{2}{3}} K_{O}^{-\frac{1}{3}} p O_{2}^{-\frac{1}{6}}$ \\
\hline$\left[\boldsymbol{O}_{\boldsymbol{i}}^{\prime \prime}\right]$ & $2^{\frac{2}{3}} K_{R}^{-\frac{1}{3}} K_{F} p O_{2}^{-\frac{1}{6}}$ & $2\left[A_{c}^{\prime}\right]^{-1} K_{F}$ & {$\left[A_{c}^{\prime}\right]^{-2} K_{O} K_{F} p O_{2}^{\frac{1}{2}}$} & $2^{-\frac{2}{3}}\left(K_{O} K_{F}\right)^{\frac{1}{3}} p O_{2}^{\frac{1}{6}}$ \\
\hline
\end{tabular}

Table 1.2. Region dependent equation for defect species concentration in acceptor doped material.

\begin{tabular}{|c|c|c|c|c|}
\hline Defect & Region I & Region II & Region III & Region IV \\
\hline $\boldsymbol{n}$ & $\left(2 K_{R}\right)^{\frac{1}{3}} p O_{2}^{-\frac{1}{6}}$ & {$\left[D_{c}^{\cdot}\right]$} & $\left(2 K_{R} K_{F}\right)^{\frac{1}{2}}\left[D_{c}^{*}\right]^{-\frac{1}{2}} p O_{2}^{-\frac{1}{4}}$ & $2^{\frac{1}{3}} K_{O}^{-\frac{1}{3}} K_{E} p O_{2}^{-\frac{1}{6}}$ \\
\hline $\boldsymbol{p}$ & $\left(2 K_{R}\right)^{-\frac{1}{3}} K_{E} p O_{2}^{\frac{1}{6}}$ & {$\left[D_{c}^{\cdot}\right]^{-1} K_{E}$} & $\left(2 K_{R} K_{F}\right)^{-\frac{1}{2}} K_{E}\left[D_{c}\right]^{\frac{1}{2}} p O_{2}^{\frac{1}{4}}$ & $2^{-\frac{1}{3}} K_{O}^{\frac{1}{3}} p O_{2}^{\frac{1}{6}}$ \\
\hline$\left[\boldsymbol{V}_{\boldsymbol{o}}^{\prime \prime}\right]$ & $2^{-\frac{2}{3}} K_{R}^{\frac{1}{3}} p O_{2}^{-\frac{1}{6}}$ & {$\left[D_{c}^{\cdot}\right]^{-2} K_{R} p O_{2}^{-\frac{1}{2}}$} & $2\left[D_{c}^{\cdot}\right]^{-1} K_{F}$ & $\left(2 K_{F}\right)^{\frac{2}{3}} K_{O}^{-\frac{1}{3}} p O_{2}^{-\frac{1}{6}}$ \\
\hline$\left[\boldsymbol{O}_{\boldsymbol{i}}^{\prime \prime}\right]$ & $2^{\frac{2}{3}} K_{R}^{-\frac{1}{3}} K_{F} p O_{2}^{\frac{1}{6}}$ & {$\left[D_{c}^{*}\right]^{2} K_{R}^{-1} K_{F} p O_{2}^{\frac{1}{2}}$} & $\frac{1}{2}\left[D_{c}^{\prime}\right]$ & $2^{-\frac{2}{3}}\left(K_{O} K_{F}\right)^{\frac{1}{3}} p O_{2}^{\frac{1}{6}}$ \\
\hline
\end{tabular}

Table 1.3. Region dependent equation for defect species concentration in donor doped material.

The Kröger-Vink diagram (also called Brouwer diagram) is a useful representation of the variation in defect concentrations as a function of changes in the activity of a component of the system, usually $\mathrm{pO}_{2}$. For an acceptor doped material Kröger-Vink diagram is plotted in Figure 1.4, where the acceptor concentration merely represents the ionized acceptor concentration. It can be observed that electrons and oxygen vacancies are the dominant defects in Region I. However, pure electronic conduction is expected since the mobility of electrons is usually orders of magnitude higher than that of oxygen vacancies. 
Otherwise, in Region II, oxygen vacancies are maintained while the electron density decreases with increasing $\mathrm{pO}_{2}$, thus becoming dominating defects. In Region III, holes are the dominant charge carriers. In Region IV, both holes and oxygen interstitials dominate, although conduction is likely by holes since their mobility is generally much higher than that of interstitials.

A similar Kröger-Vink diagram is depicted in Figure 1.5 for donor doped material (simply the ionized donor concentration is to be considered). In Region I, the electron is expected to be the dominant electrical carrier with a $\mathrm{pO}_{2}^{-1 / 6}$ dependence. In Region II the concentration of ionized donors sets the electron density, so that it would be $\mathrm{pO}_{2}$ independent. In Region III, where higher $\mathrm{pO}_{2}$ is found, the electrical conductivity may switch to ionic towards the middle of this region if the intrinsic electron-hole product becomes low enough. Progressing to sufficiently high $\mathrm{pO}_{2}$, the higher amount of holes will again shift the balance to electronic conduction. Therefore, in Region IV, the material is likely to be dominated by hole conduction, $\mathrm{pO}_{2}{ }^{1 / 6}$ dependent. 


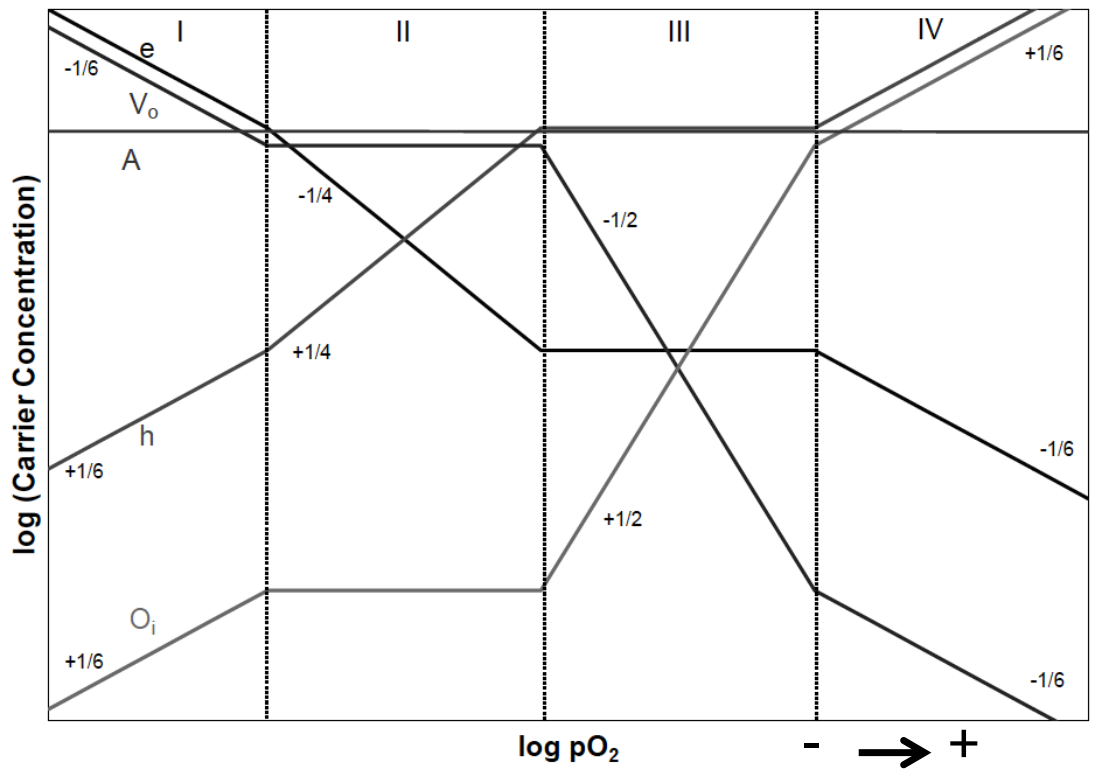

Figure 1.4. Kroger-Vink diagram for acceptor doped material. The acceptor concentration represents the ionized acceptor concentration.

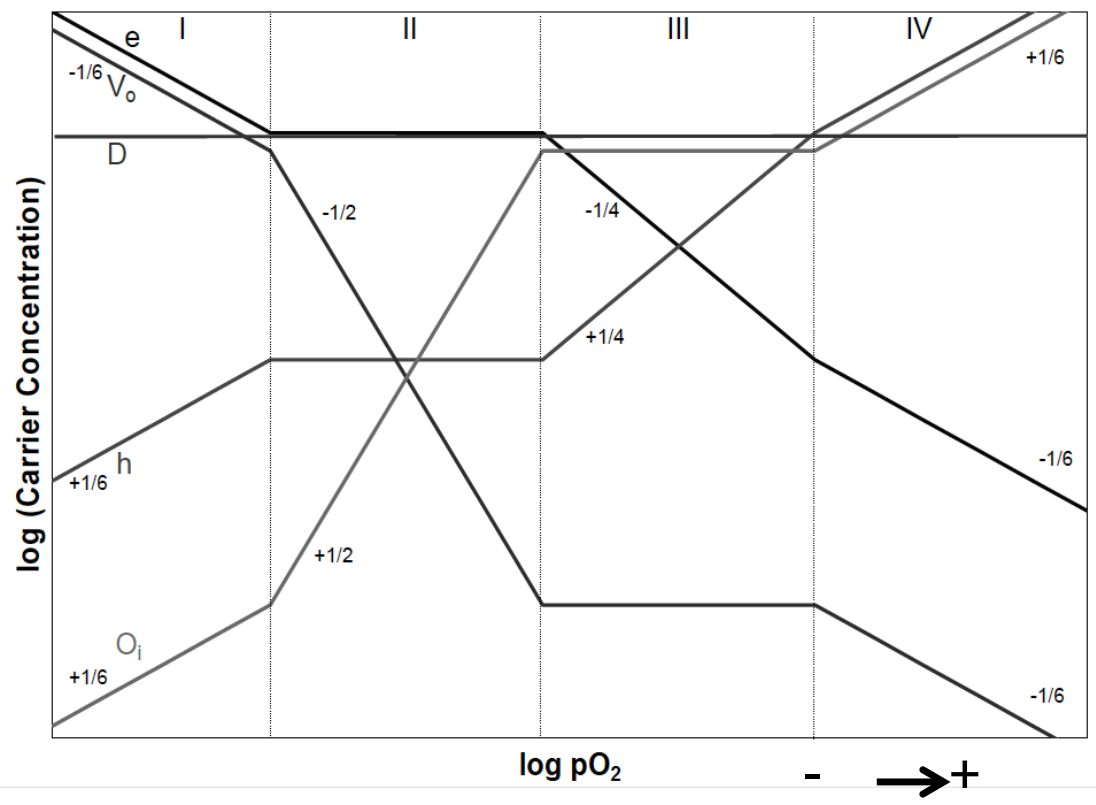

Figure 1.5. Kröger-Vink diagram for donor doped material. The donor concentration represents the ionized donor concentration. 
From this point, the conductivity obtained from these general systems will be the sum of the contribution of all charge carriers: $t$, total; $i$, ionic; $n$, electronic and $p$, holes (Eq. 1.29). For each defect, $x$, that contribution is proportional to (i) the charge carriers, $c$ (carriers $/ \mathrm{cm}^{3}$ ), (ii) to the charge that they carry, $z_{x} e(\mathrm{C} /$ carrier $)$, being $z_{x}$ the number of unit charges on the carrier and $e$ the unit electron charge $\left(1.6 \times 10^{-19} \mathrm{C}\right)$ and (iii) to the mobility $\mu_{x}$, defined as the ability to move in an electric field (Eq. 1.30). Therefore, having into account Eq. 7, a general conductivity expression in an intermediate $\mathrm{pO}_{2}$ range is given in Eq. 1.31.

$$
\begin{aligned}
& \sigma_{T}=\sigma_{i}+\sigma_{n}+\sigma_{p} \\
& \sigma_{T}=2 e\left[V_{o}^{\cdot \cdot}\right] \mu_{V_{o}^{*}}+c_{n} z_{n} e \mu_{n}+c_{p} z_{p} e \mu_{p} \\
& \left.\sigma_{T}=\sigma_{i}^{o} e^{\left(-E_{i} / k T\right)}+\sigma_{n}^{o}\left(p O_{2}\right)^{-1 / 4} e^{\left(-\Delta H_{r e d} / 2 k T\right.}\right)+\sigma_{p}^{o}\left(p O_{2}\right)^{+1 / 4} e^{\left(-\Delta H_{o x / 2 k T}\right)}
\end{aligned}
$$

In a MIEC material, the conductivity data analysis permits to distinguish electronic an ionic components by their $\mathrm{pO}_{2}$ dependency. As mentioned, the mobility of ions is far lower than the mobility of electronic carriers, so that ionic conductivity will be only observable if electron and hole concentration is minimum, that is, the $\mathrm{pO}_{2}$ is about providing $p=n$. If ionic conductivity in this range is higher than electronic, the experimental observation would be a conductivity independent on $\mathrm{pO}_{2}$. Otherwise, $n$ or $p$ electronic conductivities would be evident by $\sigma \propto p O_{2}^{-1 / 4}$ y $\mathrm{pO}_{2}^{+1 / 4}$ dependencies, respectively.

In conclusion, solid oxide materials are able to behave differently depending on their surrounding. This opens the possibility of tailoring both the structure and atmosphere to achieve desired properties for a number of 
applications, i.e., solid oxide fuel cells (SOFC), solid oxide electrolyser cells (SOEC) oxygen or hydrogen separation membranes, catalyst and gas sensors. The work presented in this thesis was focused in obtaining MIEC materials suitable for applications in SOFC components and oxygen separation membranes.

\subsection{Applications}

\section{Oxygen separation membranes}

Oxygen production at high temperature through ceramic membranes represents a current challenge that would enable the introduction of more efficient and environmental-friendly power generation and chemical production processes. In fact, reliable mass-scale $\mathrm{O}_{2}$ separation in membrane modules would allow the cost-effective operation of Oxyfuel power plants, and integrated gasification combined cycle (IGCC) plants, which can integrate $\mathrm{CO}_{2}$ capture and sequestration strategies in order to minimize $\mathrm{SO}_{2}$ particulates and $\mathrm{CO}_{2}$ emissions. [16, 17] Oxyfuel combustion principle is the removal of nitrogen from the oxidizer to carry out the combustion process in an oxygen-enriched gas stream, thus reducing gas volume and recycling the flue gas to lower the flame temperature. Hence, an air separation unit (ASU) has to be added to the power plant. The current state of technology development for ASU applies high-energy consuming cryogenic distillation units, while alternative oxygen separation technologies such as ion transport membranes would enable thermal integration and energy savings. [18] Furthermore, the development of catalytic membrane reactors (CMR) allows increasing the reaction conversion by shifting the equilibrium of the reaction. Several chemical processes as partial oxidation of 
methane (POM) to synthesis gas, oxidative coupling of methane (OCM), water splitting, selective ammonia oxidation or $\mathrm{NO}_{\mathrm{x}}$ to $\mathrm{N}_{2}$ conversion would benefit from the development of highly-permeable and $\mathrm{CO}_{2}$ stable ceramic membranes. [19-25] The thermal integration of the membrane in the process permits its intensification, which involves several advantages with respect to conventional processes, i.e., the improvement of product selectivity and conversion, energy conservation, safer operation, reduced reactor/combustor volume and higher process performance.

For these reasons, many researches are devoted to find membrane materials that can work in the processes mentioned above. Membranes based on MIEC materials enable in situ separation of oxygen in a membrane reactor fed by air. [26, 27] In MIEC ceramic membranes only oxygen ions are mobile within the ceramic lattice and all other gases, such as nitrogen, carbon monoxide, argon or methane are excluded to pass through the membrane to the permeate side. Thus, MIEC oxygen transport membranes are able to separate oxygen from air at high temperature in a process $100 \%$ selective to oxygen being competitive with conventional cryogenic separation methods. In the opposite direction to the oxygen a continuous flux of electrons keeps the electroneutrality without any external circuit. The oxygen partial pressure difference across the membrane is the driving force for the membrane to work. 


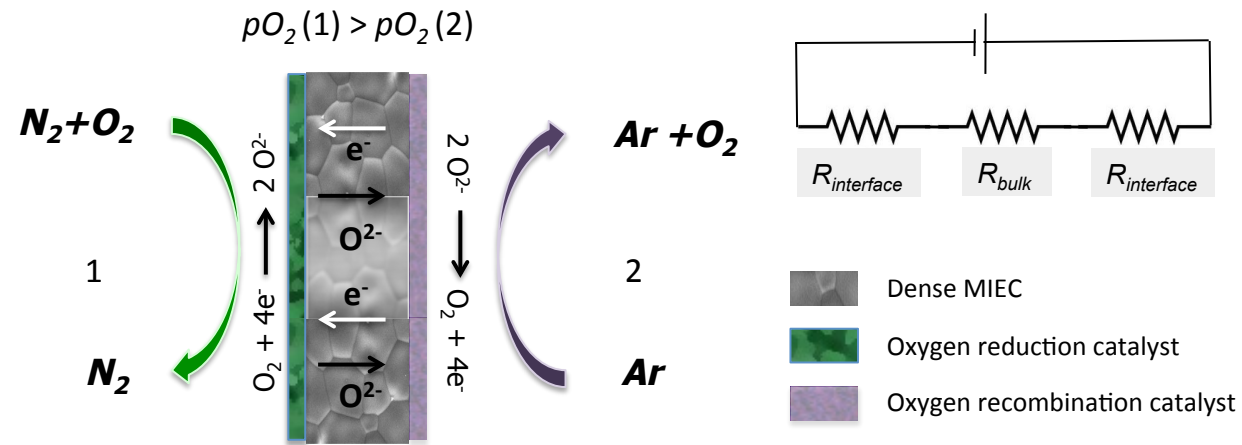

Figure 1.6. Oxygen permeation scheme for a dense membrane with catalytic layers for oxygen dissociation and recombination.

The mechanism of permeation in a ceramic membrane is depicted in Figure 1.6. In the feed side, oxygen molecules in air are dissociated and reduced to oxygen ions at the membrane reducing surface; the oxygen ions are then transported across the ceramic membrane to the oxidizing surface in the permeate side, where is combined back to oxygen gas. These three processes can be seen as a sum of series resistance $\left(R=R_{\text {interface, feed }}+R_{\text {bulk }}+R_{\text {interface, permeate }}\right)$. When the kinetics of the membrane is dominated by the bulk diffusion $\left(\mathrm{R}_{\text {bulk }}\right)$ the oxygen transport across the membrane is given by the modified Wagner's equation (Eq. 32). It predicts that the oxygen flux is proportional to (i) the temperature, (ii) the oxygen potential gradient, which is related to the $\mathrm{pO}_{2}$ difference between feed and permeate sides of the membrane, (iii) the ionic and electronic conductivities, $\sigma_{i}$ and $\sigma_{e l}\left(\sigma_{h^{\prime}}+\sigma_{e^{\prime}}\right)$, respectively, and inversely proportional to membrane thickness, $L$.

$j_{O_{2}}=\frac{R T}{16 F^{2} L} \int_{p O_{2}, f e e d}^{p O_{2}, p e r m} \frac{\sigma_{i} \cdot \sigma_{e l}}{\sigma_{i}+\sigma_{e l}} d \nabla \operatorname{lnp} O_{2}$

Thus, decreasing the membrane thickness can increase the oxygen flux through the membrane. However, there is a certain thickness in which the diffusion process is sufficiently fast and the kinetics of the membrane becomes governed by the oxygen surface exchange rate at the gas/solid interfaces 
( $\left.\mathrm{R}_{\text {interface}}\right)$. This value is known as the characteristic thickness $L_{c}$, which is defined by the ratio of oxygen diffusion constant $D\left(\mathrm{~m}^{2} \mathrm{~s}^{-1}\right)$ and oxygen surface exchange coefficient $k\left(\mathrm{~m} \mathrm{~s}^{-1}\right)$, distinctive for each membrane material, as expressed in Eq 1.33.

$L_{c}=\frac{D}{k}$

In order to decrease the resistances in the interfaces, oxygen reduction and oxidation catalyst may be attached in, respectively, feed and permeate sides of the membrane, as depicted in Figure 1.6.

Up to date, the most common type of material that exhibits both ion and electron conducting properties are perovskites e.g. $\mathrm{Ba}_{1-\mathrm{x}} \mathrm{Sr}_{\mathrm{x}} \mathrm{Co}_{0.8} \mathrm{Fe}_{0.2} \mathrm{O}_{3-\delta}$ (BSCF) and $\mathrm{La}_{1-\mathrm{x}} \mathrm{Sr}_{\mathrm{x}} \mathrm{Co}_{0.8} \mathrm{Fe}_{0.2} \mathrm{O}_{3-\delta}$ (LSCF), which enable oxygen fluxes up to $12.2 \mathrm{ml} \cdot \mathrm{min}^{-1} \cdot \mathrm{cm}^{-2}$ (BSFC) and $0.5 \mathrm{ml} \cdot \mathrm{min}^{-1} \cdot \mathrm{cm}^{-2}$ (LSFC) in $1 \mathrm{~mm}$ thick membranes measured at $900{ }^{\circ} \mathrm{C}$ using argon as sweep gas. [28-33] However, the main disadvantage of these materials is the limited chemical stability under a large oxygen concentration gradient, with one side of the membrane exposed to air oxidizing atmosphere and the other side to reducing or $\mathrm{CO}_{2}$ containing atmosphere. [34] Even at $300 \mathrm{ppm}$ [35], $\mathrm{CO}_{2}$ presence is a major issue for perovskite-based membranes (e.g. BSCF) due to the carbonation reaction of the earth alkali metals included in their structure. Improved stability against oxygen partial pressure gradients and carbonation is expected when avoiding Co alkaline-earth elements and doping by only transition metals and lanthanides instead.

On the other hand, fluorite materials such as stabilized bismuth oxide (SBO) or $\mathrm{Ce}_{1-\mathrm{x}} \mathrm{Ln}_{\mathrm{x}} \mathrm{O}_{2-\delta}$ ( $\mathrm{Ln}=$ lanthanide) have been attempted as oxygen separation membranes, as they are more stable in operational gases and against large oxygen concentration gradients. However, they are mainly ionic 
conductors and the scarcity of electronic conductivity limits the oxygen permeation performance. By doping with multivalent cations, as terbium and praseodymium, mixed conduction in ceria may be further extended to higher oxygen partial pressure range $\left(10^{-5}-10^{-1} \mathrm{~atm}\right)$, which enables its application as oxygen permeable membranes. As an example, Fagg et al. developed cobalt-doped $\mathrm{Ce}_{0.8} \mathrm{Pr}_{0.2} \mathrm{O}_{2-\delta}$ membranes that offer low but competitive levels of oxygen permeation flux at temperatures below $850{ }^{\circ} \mathrm{C}$ when compared with perovskite materials, since they are thermally and $\mathrm{CO}_{2}$ stable. [36]

As it is difficult to meet all the requirements in one single-phase material, dual phase composite membranes made from an oxygen-ion conducting oxide and a primarily electronic material (MIEC or metal) have been investigated, e.g. $\mathrm{Ce}_{0.8} \mathrm{Gd}_{0.2} \mathrm{O}_{2-\delta}$ (CGO) combined with $\mathrm{La}_{0.7} \mathrm{Sr}_{0.3} \mathrm{MnO}_{3-\delta}$ (LSM) or $\mathrm{La}_{0.8} \mathrm{Sr}_{0.2} \mathrm{Fe}_{0.8} \mathrm{Co}_{0.2} \mathrm{O}_{3-\delta}$ (LSFC), [37] or $\mathrm{Er}_{0.25} \mathrm{Bi}_{0.75} \mathrm{O}_{3-\delta}$ mixed with $\mathrm{Ag}$, Pt or Au. Nevertheless, the interaction of properties was found to be detrimental for the total conductivity and oxygen permeability.

\section{Solid oxide fuel cells}

Fuel cells are the most efficient devices for power generation as they convert the chemical energy of fuel directly to electrical energy. Since the combustion process is avoided, fuel cells produce power with minimal pollutant exhausts. Fuel cells are mainly composed of a pure ion conducting electrolyte and two electrodes: an anode and a cathode. There exist different types of fuel cells, whose classification depends on the constituent electrolyte, i.e., polymer electrolyte membrane fuel cell (PEMFC), alkaline fuel cell (AFC), phosphoric acid fuel cell (PAFC), molten carbonate fuel cell (MCFC) and solid oxide fuel cell (SOFC). 
In SOFC the electrolyte is a solid, nonporous metal oxide, which is a pure ionic $\left(\mathrm{O}^{2-}\right.$ or $\left.\mathrm{H}^{+}\right)$conductor at high temperature. It allows the fast conduction of ions between the two electrodes while the conductivity of electrons must be negligible. [38-40] The electrolyte is enclosed between two porous electrodes, the cathode, and the anode. Figure 1.7 shows the working scheme of a SOFC made by an oxygen ion conductor electrolyte that is fed by $\mathrm{H}_{2}$ in the anode side and air or $\mathrm{O}_{2}$ in the cathode. Oxygen atoms are reduced on the porous cathode surface by electrons, so that oxide ions flow through the ceramic electrolyte. In the porous anode, the oxide ions react catalytically with fuel (hydrogen) and release electrons, giving off water and heat. An external circuit conducts the electrons from the anode to the cathode and provides the electrical energy. [41]

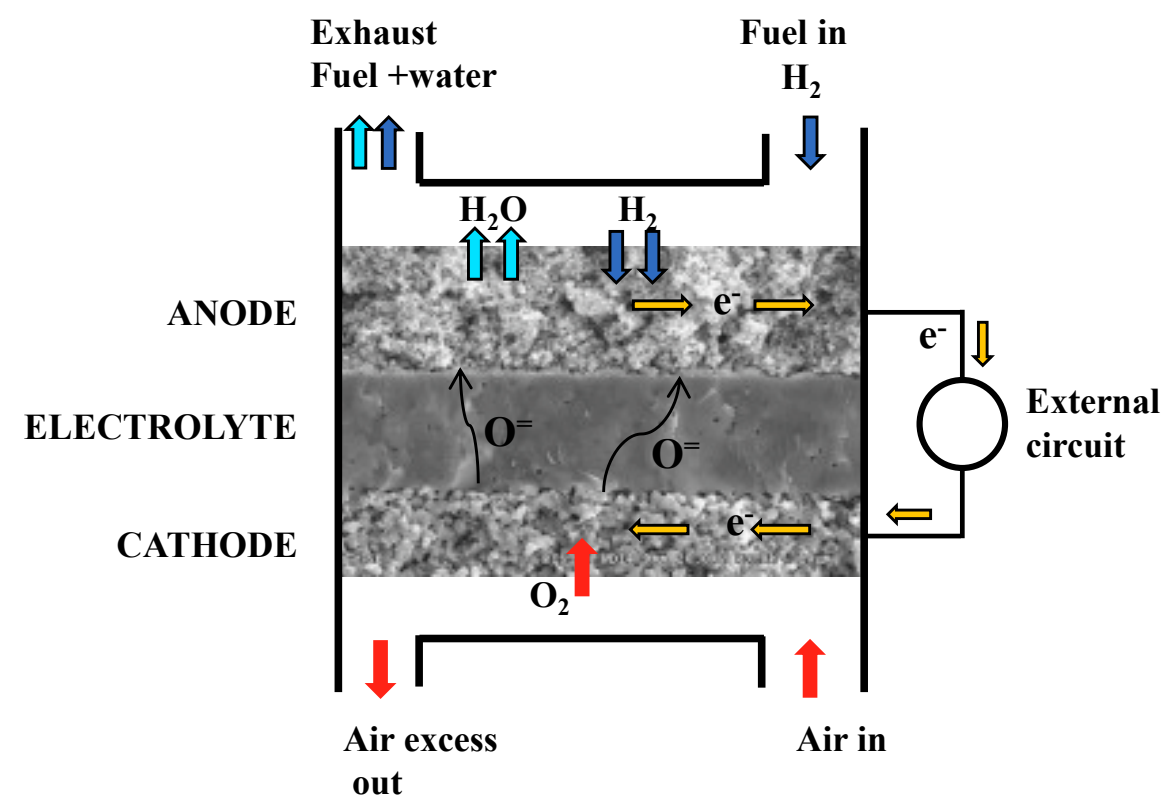

Figure 1.7. Schematic of an individual SOFC based on oxygen-ion conductors fed by $\mathrm{H}_{2}$. 
The overall reaction for the process when $\mathrm{H}_{2}$ is used as fuel can be written as

$\mathrm{H}_{2}+\frac{1}{2} \mathrm{O}_{2} \rightarrow \mathrm{H}_{2} \mathrm{O}$

The chemical energy from this reaction is directly converted into electrical energy, so that the maximum electrical work $\left(W_{e l}\right)$ obtainable in a fuel cell operating at constant temperature and pressure is given by the change in Gibbs free energy $(\Delta G)$ of the electrochemical reaction:

$W_{e l}=\Delta G=-z F E$

where $z$ is the number of electrons participating in the reaction, $F$ is Faraday's constant $\left(96487 \mathrm{C} \mathrm{mol}^{-1}\right)$, and $E$ is the ideal potential of the cell.

The Gibbs free energy change is also given by the state function of the enthalpy change, $\Delta \mathrm{H}$ and the entropy change, $\Delta \mathrm{S}$ :

$\Delta G=\Delta H-T \Delta S$

The total thermal energy available is $\Delta \mathrm{H}$. The available free energy is equal to the enthalpy change less the quantity $\mathrm{T} \Delta \mathrm{S}$, which is the energy consumed in the entropy change within the system. Reactions in fuel cells that have negative entropy change generate heat (such as hydrogen oxidation), while those with positive entropy change (such as direct solid carbon oxidation) may need an extra heat contribution if the irreversible generation of heat is smaller than the reversible absorption of heat. Thus, a fuel cell operating reversibly, i.e., the cell is in equilibrium without external current, will produce a heat equal to $\mathrm{T} \Delta \mathrm{S}$. At this point, where all the Gibbs free energy is converted to electrical energy, it is possible to obtain the ideal equilibrium potential or open circuit voltage (OCV) of the fuel cell, $(E)$. 
By substituting Eq 1.35 in Eq 1.36 under conditions different than standard, it is possible to obtain Nernst equation, which relates the ideal standard potential $\left(E^{\circ}\right)$ for the cell reaction and the ideal equilibrium potential at other partial pressures of reactants and products.

$E=E^{o}+\frac{R T}{z F} \ln \frac{\Pi(\text { reactant fugacity })}{\Pi(\text { product fugacity })}$

In the particular system of Eq. $1.34, E$ will be rewritten by taking into account the partial pressures of the gases involved.

$E=E^{o}+\frac{R T}{2 F} \ln \frac{p O_{2}^{1 / 2} p H_{2}}{p H_{2} O}$

For the overall cell reaction, the cell potential increases with an increase in the partial pressure (concentration) of reactants and a decrease in the partial pressure of products. For example, for the hydrogen reaction, operating at higher reactant pressures can increase the ideal cell potential at a given temperature. [41]

When no current is being drawn from the fuel cell, the cell voltage is at a maximum, the open circuit voltage $(E)$. As current is extracted from the cell, additional irreversible losses result in a decrease in the cell voltage $\left(E_{\text {cell }}\right)$, according to

$E_{c e l l}=E-i R-\eta_{C}-\eta_{A}$

where iR involves the ohmic losses within electrodes, interconnectors, and current take-offs due to the finite resistance of the materials used, $\eta_{A}$ refers to the overpotential at the anode, reflecting losses due to both electrode kinetics and mass transport limitations, and $\eta_{C}$ refers to the equivalent overpotential at 
the cathode. Thus, materials selection and microstructure are fundamental in minimizing these losses and, therefore, in maximizing fuel cell performance.

High temperature (ca. $1000{ }^{\circ} \mathrm{C}$ ) SOFC technology (HT-SOFCs) is based on the oxide-conducting yttria-stabilised zirconia (YSZ) electrolyte. This working regime provides highly efficient conversion to power, internal reforming, rapid electrocatalysis with non-precious metals and high quality heat by-product, which can be used for co-generation, or for use in combined cycle applications. SOFC systems have demonstrated one of the highest efficiencies (ranging from 40 to $70 \%$ ) of any power generation system, combined with very low environmental impact (virtually no acid gas or solid emissions). [42-44] The modular and solid state construction of SOFC devices allows flexibility to build stationary power generation plants in the $2 \mathrm{~kW}$ to hundreds of MW capacity range. [42] SOFCs are able to work not only with $\mathrm{H}_{2}$ fuel, but also several hydrocarbons as $\mathrm{CH}_{4}$, alcohols and $\mathrm{CO}$, giving rise to $\mathrm{CO}_{2}$ and $\mathrm{H}_{2} \mathrm{O}$ as residue.

However, SOFCs present various drawbacks, usually derived from the high operation temperature. Thermal expansion mismatches between component materials may lead to failure of solid electrolyte and sealing difficulties between cells in the flat plate configurations. The aggressive operation conditions also cause corrosion of metal components (such as the interconnects) and the modification of the materials, which may develop insulating phases. All these issues need to be taken into account for thermal cycling and stack life. [45] Hence, the material selection is very restricted and results in difficult and high cost fabrication processes.

Intermediate temperatures SOFCs (IT-SOFCs) working at ca. $500-700^{\circ} \mathrm{C}$ have been developed to face the mentioned disadvantages and to improve the durability. Combined with the ability of SOFC to use fossil fuels, the new operation conditions reduce the cost of the fuel cell since less-expensive materials of construction may be used. 
More recently, new proton conducting SOFCs (PC-SOFCs) have been developed, whose electrochemical reactions are based on the transport of protons or protonated species. The main difference regarding an oxygen ion electrolyte is the side in the fuel cell in which the water is produced. In the case of pure proton conductivity, hydrogen is oxidized in the anode to form protons, which are incorporated into the electrolyte. These protons are consumed at the cathode in the reduction of oxygen to produce water, which can easily be removed by the oxygen (air) flow. PC-SOFCs allow lower operating temperatures and achieve very high fuel utilization efficiencies, since water is formed at the air electrode and the fuel is not diluted. [46] However, there is still a need to further develop protonic electrolytes and electrode materials to achieve an optimum combination of proton transport properties, compatibility with other cell components, and thermochemical stability.

\section{General requirements for SOFC}

Target power densities of fuel cells are typically of the order of $1 \mathrm{~kW} \mathrm{~kg}^{-1}$, which means that the area specific resistivity (ASR) of the cell (electrolyte plus both electrodes) should not exceed $0.5 \Omega \mathrm{cm}^{2}$. This involves general requirements for the principal components, as low individual resistivity, chemical compatibility between materials and similar thermal expansion, high temperature stability of all the components and high strength and toughness. Traditionally, the electrolyte is made from a ceramic such as YSZ, the anode is $\mathrm{Co}-\mathrm{ZrO}_{2} \mathrm{Ni}-\mathrm{ZrO}_{2}$ or Ni-YSZ cermets, and the cathode is $\mathrm{Sr}$-doped $\mathrm{LaMnO}_{3}$ (LSM) or LSM-YSZ cercers. New strategies and materials for each cell component have been studied and developed in an attempt to reduce the ASR of the cell, which are described below. 


\section{Electrolyte}

Solid electrolytes consist of a dense non-porous microstructure that avoids the permeation of neutral gases. The electrolyte material must possess high oxygen ion or proton conductivity $\left(>0.05 \mathrm{~S} \cdot \mathrm{cm}^{-1}\right)$ at the operating temperature while electronic conductivity should be negligible both in oxidizing and reducing atmospheres to prevent losses from leakage currents. The most common oxygen conductor electrolytes are doped zirconia-based materials, especially the mentioned YSZ and scandia-doped zirconia (ScSZ). Other stabilizing dopants have been used, as $\mathrm{CaO}, \mathrm{MgO}$ and certain rare earth oxides such as $\mathrm{Nd}_{2} \mathrm{O}_{3}, \mathrm{Sm}_{2} \mathrm{O}_{3}, \mathrm{Yb}_{2} \mathrm{O}_{3}$. Also $\mathrm{Sm}, \mathrm{Gd}, \mathrm{Y}$ or Ca doped ceria (SDC, CGO and YDC, respectively), which are ionic conductors, have been tested as electrolyte materials. The main problem with the cerias is that they react with the electrodes during the sintering at high temperatures and develop electronic conductivity both at temperature above $600{ }^{\circ} \mathrm{C}$ and in the reducing atmospheres of the anode side due to the $\mathrm{Ce}^{4+}$ reaction. Other works focused on perovskitestructured electrolytes. Ishihara et al. developed Sr-Mg doped lanthanum gallate ceramics (LSGM), which demonstrates higher ionic conductivities than $\mathrm{ZrO}_{2}$ and $\mathrm{CeO}_{2}$ based oxides at intermediate operating temperatures, while keeping pure ionic behaviour in a wide $p O_{2}$ range $\left(1-10^{-20} \mathrm{~atm}\right)$. [47, 48] However, in an overall electrochemical cell LSGM reacts with almost every electrode materials and the performance is quickly degraded due to the phase change and formation of minor phases. [49]

Bismuth oxide is the suggested electrolyte material showing the highest ionic conductivity. However, it is not stable in reducing atmospheres and undergoes a phase transition from the monoclinic to the high temperature cubic phase, at approximately $730{ }^{\circ} \mathrm{C}$ where develops electronic conductivity. Many metal oxides ( $\mathrm{MO}, \mathrm{M}_{2} \mathrm{O}_{3}, \mathrm{M}_{2} \mathrm{O}_{5}$ ) dopants have been tried in order to stabilize bismuth oxide, although lanthanides (especially $\mathrm{Er}_{2} \mathrm{O}_{3}$ ) or $\mathrm{Y}_{2} \mathrm{O}_{3}$ have been 
studied in more detail. $\mathrm{Y}_{2} \mathrm{O}_{3}-\mathrm{Bi}_{2} \mathrm{O}_{3}$ structure was stable over a wide range of temperatures and shows an ionic conductivity about $0.2 \mathrm{~S} \mathrm{~cm}^{-1}$ at $700{ }^{\circ} \mathrm{C}$, but it still develops $n$-type electronic conductivity at low $p O_{2}$. [50] $\mathrm{Er}_{2} \mathrm{O}_{3}-\mathrm{Bi}_{2} \mathrm{O}_{3}$ was stable to oxygen partial pressures lower than $10^{-20} \mathrm{~atm}$ while being one of the highest ion conductors in air within this system. Finally, BIMEVOX and Aurivillius phases are families based on $\mathrm{Bi}_{4} \mathrm{~V}_{2} \mathrm{O}_{11}$ in which $\mathrm{V}$ is replaced by a metal ion (as $\mathrm{Cu}$ or $\mathrm{Ni}$ ). They are even lower temperature ionic conductors that allow high oxygen diffusion rates, but reveal surprisingly slow surface exchange kinetics. [51]

To overcome the material hitches, electrolyte performance enhancement is addressed on the cell configuration optimization. The construction of thin electrolyte films supported on porous electrode substrates allows decreasing the operation temperature. Also double layered electrolytes have been proposed, where a zirconia-coated side is exposed to the fuel gas to block the electrons, and the material that may be reduced is in contact with the cathode. Some examples are the thin coat of $\mathrm{ZrO}_{2}$ /stabilised bismuth oxide or $\mathrm{YSZ}$ deposited by sputtering over CGO; However, possible interaction and interdiffusion between phases still appear as a consequence of the electrolyte sintering temperature and influences on the performance of the composite electrolyte. This brings about the concept of protecting layers to avoid the rapid degradation of the fuel cells, as cathode-interlayer-electrolyte or sandwiching YSZ between two CGO layers. $[52-55]$

Proton conducting solid electrolytes might eventually overcome some of the limitations of oxygen ion conductors cells, since the lower activation energy of proton mobility regarding oxygen vacancies enables operation at lower temperatures. Nevertheless, they are still in an early stage of development. The first compounds with high proton conductivities were based on $\mathrm{SrCeO}_{3}$ and 
$\mathrm{BaCeO}_{3}$, which reached power densities up to $0.2 \mathrm{~W} \mathrm{~cm}^{-1}$ at $800{ }^{\circ} \mathrm{C}$. However, these materials react to carbonates even with low levels of $\mathrm{CO}_{2}$ to form alkaline earth hydroxides at high water activities, which excludes any hydrocarbon fuel. [56] Among these materials, $\mathrm{BaZrO}_{3}$ is an exception in stability, but requires high sintering temperatures that lead to high grain boundary resistances. A trade-off between stability and lower grain boundary resistance may be obtained by partial substitution of $\mathrm{Zr}$ by $\mathrm{Ce}$ in the perovskite structure. Others like yttrium-doped barium zirconate $\left(\mathrm{BaZr}_{1-x-y} \mathrm{Y}_{x} \mathrm{M}_{y} \mathrm{O}_{3-\delta}\right)$ have been investigated, using $\mathrm{M}=\mathrm{Pr}, \mathrm{Fe}$ and $\mathrm{Mn}$ as dopants. Among these, the highest total and protonic conductivity at elevated temperatures under reducing atmospheres was found for $\mathrm{BaZr}_{0.8} \mathrm{Y}_{0.15} \mathrm{Mn}_{0.05} \mathrm{O}_{3-\delta}$. [57] More recently, new classes of proton conducting oxides are derived from acceptor-doped rare earth ortho-niobates, $\mathrm{LnNbO}_{4}$ $\left(\mathrm{Ln}_{1-\mathrm{x}} \mathrm{M}_{\mathrm{x}} \mathrm{NbO}_{4-\mathrm{x} / 2}\right.$, where $\mathrm{Ln}=\mathrm{Y}, \mathrm{La}, \mathrm{Nd}, \mathrm{Gd}, \mathrm{Tb}, \mathrm{Er} ; \mathrm{M}=\mathrm{Ca}, \mathrm{Sr}, \mathrm{Ba}$ and $\mathrm{x} \leq 0.1$ ). They showed protonic conduction and a general stability against $\mathrm{CO}_{2}$. $\mathrm{LaNbO}_{4}$ based materials exhibit the highest proton conductivity, which is close to $0.001 \mathrm{~S} \mathrm{~cm}^{-1}$ for $\mathrm{La}_{0.99} \mathrm{Ca}_{0.01} \mathrm{NbO}_{4}$ under $2.5 \% \mathrm{H}_{2} \mathrm{O}$ at $\sim 950{ }^{\circ} \mathrm{C}$. [58] $\mathrm{La}_{6-\mathrm{x}} \mathrm{WO}_{\mathrm{y}}$ with $0.4<\mathrm{x}<0.7$ (LWO) is a mixed protonic-electronic conductor (mainly protonic below $800{ }^{\circ} \mathrm{C}$ ) highly stable in $\mathrm{CO}_{2}$ containing atmospheres. The applicability of LWO as a protonic electrolyte has been proved by recording the $\mathrm{OCV}$ in a $\mathrm{Pt} / \mathrm{LWO} / \mathrm{Pt}$ cell as a function of the temperature. [59-62] Proton conductivities of $0.005 \mathrm{~S} \mathrm{~cm}^{-1}$ have been achieved for $\mathrm{La}_{5.8} \mathrm{WO}_{11.7}$ at $800{ }^{\circ} \mathrm{C}$ in wet air. [63] Latest works have shown that compounds of $\mathrm{Ln}_{6} \mathrm{WO}_{12}(\mathrm{Ln}=\mathrm{La}$, $\mathrm{Nd}, \mathrm{Gd}, \mathrm{Er}, \mathrm{Eu})$ series exhibit mixed proton-electron conductivity at high temperature in humid reducing or oxidizing atmospheres. [61, 62]

\section{Cathodes}

The electrochemical reduction of oxygen takes place in the cathode side. The reaction described in Eq. 1.40 occurs when the oxygen ion conductor, the 
electronic conductor and the gas phase come in contact, sites that are known as triple-phase boundaries (TPBs).

$\frac{1}{2} O_{2}($ gas $)+2 e^{-}($cathode $) \rightarrow O^{2-}($ electrolyte $)$

Therefore, high electronic conductivity $\left(>100 \mathrm{~S} \mathrm{~cm}^{-1}\right)$ for the electron to reach the oxygen and high catalytic activity for the oxygen reduction reaction are required. Ionic conductivity is also desired in order to increase the TPBs area all along the cathode. Besides, the cathode material should not react neither with the electrolyte nor the interconnectors (or the protecting layers used to protect them, typically spinel-structured materials) to avoid changes of phase or blocking impurities. This compatibility must be extended to the thermal expansion coefficient (TEC), since great differences with the materials in contact may lead to cracks and failures in the components. The porosity of the electrode must be adequate to enhance the diffusion of oxygen gas to the cathode/electrolyte interface. $[64,65]$

Typical HT-SOFC cathode material is a composite made of LSM mixed with YSZ, to provide electronic and ionic conductivity, respectively, and to improve TEC compatibility with the YSZ electrolyte. In this situation, the electrochemically active reaction sites (TPB) increase orders of magnitude over that of porous cathodes exhibiting only electronic conductivity.

However, the new trends of reducing the operating temperature also involve the degradation of the electrode kinetics and results in large interfacial polarization resistances. At the reduced temperatures of IT-SOFC, the standard cathode material LSM cannot be used because of its low activity for the oxygen reduction reaction (due to its low oxygen diffusivity). The approach in this case is to use single-phase MIEC cathodes permitting both oxide ion and electron mobility within the cathode material, e.g., $\mathrm{La}_{1-\mathrm{x}} \mathrm{Sr}_{\mathrm{x}} \mathrm{CoO}_{3-\delta}$ (LSC), 
$\mathrm{La}_{-\mathrm{x}} \mathrm{Sr}_{\mathrm{x}} \mathrm{Co}_{1-\mathrm{y}} \mathrm{Fe}_{\mathrm{y}} \mathrm{O}_{3-\delta}$ (LSCF), $\mathrm{Pr}_{1-\mathrm{x}} \mathrm{Sr}_{\mathrm{x}} \mathrm{FeO}_{3-\delta}$ (PSF), $\mathrm{Pr}_{1-\mathrm{x}} \mathrm{Sr}_{\mathrm{x}} \mathrm{Co}_{1-\mathrm{y}} \mathrm{Fe}_{\mathrm{y}} \mathrm{O}_{3-\delta}$ (PSCF), $\mathrm{Pr}_{1-\mathrm{x}} \mathrm{Sr}_{\mathrm{x}} \mathrm{Co}_{1-\mathrm{y}} \mathrm{Mn}_{\mathrm{y}} \mathrm{O}_{3-\delta}(\mathrm{PSCM})$ or $\mathrm{Ba}_{0.5} \mathrm{Sr}_{0.5} \mathrm{Co}_{0.8} \mathrm{Fe}_{0.2} \mathrm{O}_{3-\delta}$ (BSCF), but they still lack in ionic conductivity or phase stability. [66-68] Cobalt free cathodes, both single phase or forming a composite with a ceria based fluorite have been also tested with the aim of improving TEC compatibility, but a drop in electrocatalytic properties is experienced. [69-71]

Recently, Co containing materials based on $\mathrm{YBaCo}_{4} \mathrm{O}_{7}$ swedenborgites, with CGO and YSZ compatible TEC have been developed, which demonstrated low polarization resistances compared to LSCF reference material. [72,73] Layered.perovskite oxides $\mathrm{LnBaCo}_{2} \mathrm{O}_{5}$ (LBCO) ( $\mathrm{Ln}=$ Lanthanide) with A-site $\mathrm{Ln}^{3+}$ and $\mathrm{Ba}^{2+}$ cation-ordered structures are new potential cathode materials. Particularly, the Pr based family performs the best electrochemical results due to the fast oxygen transport kinetics. [74] On the other hand, single phase fluorites, as $\mathrm{Ce}_{1-\mathrm{x}} \mathrm{Pr}_{\mathrm{x}} \mathrm{O}_{2-\delta}$ (CPO) have been proposed as cathode since they present high levels of oxygen non-stoichiometry and significant electronic contribution from electron hopping between adjacent $\mathrm{Pr}$ ions, even at high $\mathrm{pO}_{2}$. [75] By using these strategies, the cathode reaction occurs over the full electrode surface area, rather than being limited to the TPB. Thus, the overall cathode performance depends essentially on the rate of defect transport through the solid MIECs, gas transport through the pores to promote surface coverage in the MIECs, and the catalytic activity of interfaces. [76]

On the other hand, the use of PC-SOFC requires the development of tailor-made suitable electrodes for these particular electrolytes, since the main issue is the chemical compatibility between the cathode and the electrolyte materials. For barium-zirconate based electrolytes cobalt-free composites as $\mathrm{Sm}_{0.5} \mathrm{Sr}_{0.5} \mathrm{Fe}_{0.8} \mathrm{Cu}_{0.2} \mathrm{O}_{3-\delta}-\mathrm{Ce}_{0.8} \mathrm{Sm}_{0.2} \mathrm{O}_{2-\delta} \quad(\mathrm{SSFCu}-\mathrm{SDC}) \quad$ or $\quad \mathrm{Sm}_{0.5} \mathrm{Sr}_{0.5} \mathrm{FeO}_{3-\delta}-$ $\mathrm{BaZr}_{0.1} \mathrm{Ce}_{0.7} \mathrm{Y}_{0.2} \mathrm{O}_{3-\delta}$ (SSF-BZCY) have been reported as suitable composite cathodes. [77, 78] $\mathrm{La}_{0.995} \mathrm{Ca}_{0.005} \mathrm{NbO}_{4}$ (LCNO) proton conductor electrolyte is 
chemically and mechanically compatible with typical cathode material $\mathrm{La}_{0.75} \mathrm{Sr}_{0.25} \mathrm{Cr}_{0.5} \mathrm{Mn}_{0.5} \mathrm{O}_{3}$ (LSCM), while reacts with BSCF cathode and with LSM $\left(\mathrm{La}_{0.7} \mathrm{Sr}_{0.3} \mathrm{MnO}_{3}\right)$ causing $\mathrm{La}$ and $\mathrm{Nb}$ electrolyte cations to diffuse in the LSM phase. [38,79] The incorporation of the proton conducting material in a composite cathode as LCNO-LSM cer-cer increase in the TPB length providing reaction sites along the whole thickness of the electrode. [59] On the same line, specific cathodes for LWO-based cells have been developed performing a composite of LSM ( $\left.\mathrm{La}_{0.8} \mathrm{Sr}_{0.2} \mathrm{MnO}_{3}\right)$ and LWO. [80] Analogously to oxygen ion SOFC, the integration of the electrolyte material in the cathode structure has some advantages as the improvement of chemical and mechanical stability and decrease in processing cost, so that many researches are addressed in this trend. [81-83]

Anodes

The anode side hosts the fuel oxidation reaction written in Eq. 1.41. Similarly to cathode side, the reactions take place as long as an oxygen ion conductor, electronic conductor, and the gas phase converge.

$\mathrm{H}_{2}($ fuel $)+\mathrm{O}^{2-}($ electrolyte $) \rightarrow \mathrm{H}_{2} \mathrm{O}+2 e^{-}($anode $)$

Thus, the anode requires high ionic conductivity to ensure electrical contact with the electrolyte, and high electronic conductivity that provides good electrical contact with the interconnector. As for cathodes, the use of a MIEC material instead of a pure electronic conductor allows extending the active sites from the TPBs to the whole anode surface area. An anode material with high catalytic activity is necessary to promote chemisorption and dissociation of fuel at the surface and the product reaction with the oxygen ions provided by the electrolyte. Similar TEC for the anode and electrolyte prevents from cracks and problems during sintering and sealing. Also chemical stability with the materials 
in contact is needed to avoid ion or electron blocking phases. Finally, the porosity must be adequate and physically stable to allow gas transport to and from the electrolyte.

Two-phase ceramic-metal composites (cermets) may combine conduction and catalytic properties. Actually, the most commonly used anode materials are Ni/YSZ cermets, which display excellent catalytic properties for fuel oxidation and good current collection, but do exhibit disadvantages, such as low tolerance to sulphur and carbon deposition when using hydrocarbon fuels, or the fatal mechanical failure upon re-oxidation or highly moistured environments. Nickel-free SOFC anodes try to overwhelm these problems. For example, ceria based materials, which become electronic conductors at the anode reducing atmosphere have been used as anodes, being limited by their low total conductivity. Similar problem is observed for titanate based oxides, combined with fast anode degradation. [84] Perovskite structured chromites are stable in reducing conditions. $\mathrm{LaCrO}_{3}$ has been used as interconnect materials for SOFC, but their stability in both reducing and oxidizing atmospheres at high temperatures make them anode suitable materials. Substituting alkali earth and transition metal elements on the $\mathrm{La}$ and $\mathrm{Cr}$ sites of $\mathrm{LaCrO}_{3}$ may modify their electronic and catalytic properties. Typically, $\mathrm{Sr}$ is introduced in La site, while other transition elements into the Cr-site of $\mathrm{La}_{1-\mathrm{x}} \mathrm{Sr}_{\mathrm{x}} \mathrm{Cr}_{1-\mathrm{y}} \mathrm{M}_{\mathrm{y}} \mathrm{O}_{3}(\mathrm{M}=\mathrm{Mg}, \mathrm{Mn}, \mathrm{Fe}$, Co, Ni) may improve the catalytic properties for methane fed SOFC. [85-87] The use of chromite based perovskites has been also proposed as anode material for LWO electrolyte based PC-SOFC, since the typical anode materials for the barium zirconates proton conductors, $\mathrm{NiO}$ or nickel-based cermet combinations ( $\mathrm{NiO}$ or $\left.\mathrm{Ni} / \mathrm{Ba}\left(\mathrm{Ce}_{0.8-\mathrm{x}} \mathrm{Zr}_{\mathrm{x}}\right) \mathrm{Y}_{0.1} \mathrm{O}_{3-\delta}\right)$, react with the LWO electrolyte. [88, 89] $\mathrm{La}_{0.85} \mathrm{Sr}_{0.15} \mathrm{CrO}_{3-\delta}$ (LSC) based anodes have been tested as compatible and promising anode materials for LWO electrolytes, although surface reaction processes limit their operation. 
Interconnectors

The interconnectors must conduct the electron flow from the anode to the cathode by an external path. Thus, the materials require high electronic conductivity with negligible ionic conductivity. Metals are commonly used, which selection is determined by the operation temperature and TEC of the other components.

The work developed in this thesis aims to find new solid oxide materials that mean an improvement as any ionic, electronic or MIEC material. Regarding to the transport behaviour ascertained and stability, they will be classified and tested for their most suitable applications.

\subsection{References}

[1] I. Riess, Polymeric mixed ionic electronic conductors, Solid State Ionics, 136-137 (2000) 1119-1130.

[2] P.J.v. Gellings, The CRC Handbook of Solid State Electrochemistry, Portland 1999.

[3] H.L. Tuller, A.S. Nowick, Doped Ceria as a Solid Oxide Electrolyte J. Electrochem. Soc., 122 (1975) 5.

[4] W. Gao, N.M. Sammes, An introduction to Electronic and Ionic Materials, 1999.

[5] T. Takahashi, H. Iwahara, Oxide ion conductors based on bismuthsesquioxide, Materials Research Bulletin, 13 (1978) 1447-1453.

[6] A.J. Moulson, J.M. Herbert, Electroceramics, Chichester, England 2003.

[7] X. Guo, R. Waser, Electrical properties of the grain boundaries of oxygen ion conductors: Acceptor-doped zirconia and ceria, Progress in Materials Science, 51 (2006) 151-210. 
[8] X. Guo, W. Sigle, J. Maier, Blocking Grain Boundaries in Yttria-Doped and Undoped Ceria Ceramics of High Purity, Journal of the American Ceramic Society, 86 (2003) 77-87.

[9] B.C.H. Steele, Appraisal of $\mathrm{Ce}_{1-\mathrm{y}} \mathrm{Gd}_{\mathrm{y}} \mathrm{O}_{2-\mathrm{y} / 2}$ electrolytes for IT-SOFC operation at 500 ${ }^{\circ} \mathrm{C}$, Solid State Ionics, 129 (2000) 95-110.

[10] D.M. Smyth, The Defect Chemistry of Metal Oxides, 2000.

[11] F.A. Kröger, H.J. Vink, Relations between the concentrations of imperfections in solids, Journal of Physics and Chemistry of Solids, 5 (1958) 208-223.

[12] http://www.stallinga.org/ElectricalCharacterization/tft/index.html.

[13] A.L. Shluger, A.M. Stoneham, Small polarons in real crystals: concepts and problems, Journal of Physics: Condensed Matter, 5 (1993) 3049.

[14] T.J. Shankland, J. Peyronneau, J.P. Poirier, Electrical conductivity of the Earth's lower mantle, Nature, 366 (1993) 453-455.

[15] N. Itoh, A.M. Stoneham, Materials Modification by Electronic Excitation, 2001.

[16] S. Engels, F. Beggel, M. Modigell, H. Stadler, Simulation of a membrane unit for oxyfuel power plants under consideration of realistic BSCF membrane properties, Journal of Membrane Science, 359 (2010) 93-101.

[17] R. Anantharaman, O. Bolland, K.I. Asen, Novel cycles for power generation with $\mathrm{CO}_{2}$ capture using OMCM technology, Energy Procedia, 1 (2009) 335-342.

[18] M.B. Toftegaard, J. Brix, P.A. Jensen, P. Glarborg, A.D. Jensen, Oxy-fuel combustion of solid fuels, Progress in Energy and Combustion Science, 36 (2010) 581 625.

[19] H.J.M. Bouwmeester, Dense ceramic membranes for methane conversion, Catalysis Today, 82 (2003) 141-150.

[20] H. Jiang, Z. Cao, S. Schirrmeister, T. Schiestel, J. Caro, A Coupling Strategy to Produce Hydrogen and Ethylene in a Membrane Reactor, Angewandte Chemie International Edition, 49 (2010) 5656-5660.

[21] H. Jiang, L. Xing, O. Czuprat, H. Wang, S. Schirrmeister, T. Schiestel, J. Caro, Highly effective NO decomposition by in situ removal of inhibitor oxygen using an oxygen transporting membrane, Chemical Communications, 44 (2009) 6738-6740.

[22] S. Smart, C.X.C. Lin, L. Ding, K. Thambimuthu, J.C. Diniz da Costa, Ceramic membranes for gas processing in coal gasification, Energy \& Environmental Science, 3 (2010) 268-278. 
[23] D.A. Slade, Q. Jiang, K.J. Nordheden, S.M. Stagg-Williams, A comparison of mixed-conducting oxygen-permeable membranes for $\mathrm{CO}_{2}$ reforming, Catalysis Today, 148 (2009) 290-297.

[24] S. Bhatia, C.Y. Thien, A.R. Mohamed, Oxidative coupling of methane (OCM) in a catalytic membrane reactor and comparison of its performance with other catalytic reactors, Chemical Engineering Journal, 148 (2009) 525-532.

[25] S. Sun, M. Rebeilleau-Dassonneville, X. Zhu, W. Chu, W. Yang, Ammonia oxidation in $\mathrm{Ba}_{0.5} \mathrm{Sr}_{0.5} \mathrm{Co}_{0.8} \mathrm{Fe}_{0.2} \mathrm{O}_{3-\delta}$ membrane reactor, Catalysis Today, 149 (2010) 167171.

[26] J. Sunarso, S. Baumann, J.M. Serra, W.A. Meulenberg, S. Liu, Y.S. Lin, J.C. Diniz da Costa, Mixed ionic-electronic conducting (MIEC) ceramic-based membranes for oxygen separation, Journal of Membrane Science, 320 (2008) 13-41.

[27] M.L. Fontaine, Y. Larring, T. Norby, T. Grande, R. Bredesen, Dense ceramic membranes based on ion conducting oxides Annales de Chim. Sci. Mat., 32 (2007) $197-$ 212.

[28] A. Leo, S. Smart, S. Liu, J. Diniz da Costa, High performance perovskite hollow fibres for oxygen separation, Journal of Membrane Science, 368 (2011) 64-68.

[29] M. Arnold, H. Wang, A. Feldhoff, Influence of $\mathrm{CO} 2$ on the oxygen permeation performance and the microstructure of perovskite-type $\left(\mathrm{Ba}_{0.5} \mathrm{Sr}_{0.5}\right)\left(\mathrm{Co}_{0.8} \mathrm{Fe}_{0.2}\right) \mathrm{O}_{3-\delta}$ membranes, Journal of Membrane Science, 293 (2007) 44-52.

[30] Z. Shao, W. Yang, Y. Cong, H. Dong, J. Tong, G. Xiong, Investigation of the permeation behavior and stability of a $\mathrm{Ba}_{0.5} \mathrm{Sr}_{0.5} \mathrm{Co}_{0.8} \mathrm{Fe}_{0.2} \mathrm{O}_{3-\delta}$ oxygen membrane, Journal of Membrane Science, 172 (2000) 177-188.

[31] H. Kusaba, Y. Shibata, K. Sasaki, Y. Teraoka, Surface effect on oxygen permeation through dense membrane of mixed-conductive LSCF perovskite-type oxide, Solid State Ionics, 177 (2006) 2249-2253.

[32] J.M. Serra, V.B. Vert, O. Büchler, W.A. Meulenberg, H.P. Buchkremer, IT-SOFC supported on Mixed Oxygen Ionic-Electronic Conducting Composites, Chemistry of Materials, 20 (2008) 3867-3875.

[33] S. Baumann, J.M. Serra, M.P. Lobera, S. Escolastico, F. Schulze-Kuppers, W.A. Meulenberg, Ultrahigh oxygen permeation flux through supported $\mathrm{Ba}_{0.5} \mathrm{Sr}_{0.5} \mathrm{Co}_{0.8} \mathrm{Fe}_{0.2} \mathrm{O}_{3-}$ ${ }_{\delta}$, membranes, Journal of Membrane Science, 377 (2011) 198-205.

[34] A. Leo, S. Liu, J.C.D.d. Costa, Development of mixed conducting membranes for clean coal energy delivery, International Journal of Greenhouse Gas Control, 3 (2009) 357-367. 
[35] J. Hoon Park, J. Pyo Kim, S. Hwan Son, Oxygen permeation and stability of $\mathrm{Ba}_{0.5} \mathrm{Sr}_{0.5} \mathrm{Co}_{0.8} \mathrm{Fe}_{0.2} \mathrm{O}_{3-\delta}$ membrane according to trace elements and oxygen partial pressure in synthetic air, Energy Procedia, 1 (2009) 369-374.

[36] D.P. Fagg, A.L. Shaula, V.V. Kharton, J.R. Frade, High oxygen permeability in fluorite-type $\mathrm{Ce}_{0.8} \mathrm{Pr}_{0.2} \mathrm{O}_{2-\delta}$ via the use of sintering aids, Journal of Membrane Science, 299 (2007) 1-7.

[37] A.L. Shaula, V.V. Kharton, F.M.B. Marques, A.V. Kovalevsky, A.P. Viskup, E.N. Naumovich, Oxygen permeability of mixed-conducting composite membranes: effects of phase interaction, J Solid State Electrochem, 10 (2006) 28-40.

[38] K.V. Kravchyk, E. Quarez, C. Solís, J.M. Serra, O. Joubert, Cathode materials for $\mathrm{La}_{0.995} \mathrm{Ca}_{0.005} \mathrm{NbO}_{4}$ proton ceramic electrolyte, International Journal of Hydrogen Energy, 36 (2011) 13059-13066.

[39] B.C.H. Steele, Materials for IT-SOFC stacks 35 years R\&D: the inevitability of gradualness?, Solid State Ionics, 134 (2000) 3-20.

[40] A. Chroneos, B. Yildiz, A. Tarancon, D. Parfitt, J.A. Kilner, Oxygen diffusion in solid oxide fuel cell cathode and electrolyte materials: mechanistic insights from atomistic simulations, Energy Environ. Sci., 4 (2011) 2774-2789.

[41] J.H. Hirschenhofer, Stauffer, D.B., Engleman, R.R., Klett, M.G., Fuel Cell Handbook, 7 th ed., EG\&G Technical Services, Inc., Morgantown, West Virginia, 2004.

[42] A.S. Nesaraj, Recent developments in solid oxide fuel cell technology - a review, J. Sci. Ind. Res., 69 (2010) 169-176.

[43] A.B. Stambouli, E. Traversa, Solid oxide fuel cells (SOFCs): a review of an environmentally clean and efficient source of energy, Renewable and Sustainable Energy Reviews, 6 (2002) 433-455.

[44] Y. Kobayashi, Y. Ando, T. Kabata, M. Nishiura, N. Matake, Extremely Highefficiency Thermal Power System-Solid Oxide Fuel Cell (SOFC) Triple Combined-cycle System Mitsubishi Heavy Industries Technical Review 48 (2011) 9-15.

[45] A. Nakajo, J. Kuebler, A. Faes, U.F. Vogt, H.J. Schindler, L.-K. Chiang, S. Modena, J. Van herle, T. Hocker, Compilation of mechanical properties for the structural analysis of solid oxide fuel cell stacks. Constitutive materials of anode-supported cells, Ceramics International, 38 (2012) 3907-3927.

[46] H. Iwahara, Technological challenges in the application of proton conducting ceramics, Solid State Ionics, 77 (1995) 289-298.

[47] T. Ishihara, H. Matsuda, Y. Takita, Doped $\mathrm{LaGaO}_{3}$ Perovskite Type Oxide as a New Oxide Ionic Conductor, Journal of the American Chemical Society, 116 (1994) 3801-3803. 
[48] T. Ishihara, J.A. Kilner, M. Honda, Y. Takita, Oxygen Surface Exchange and Diffusion in the New Perovskite Oxide Ion Conductor $\mathrm{LaGaO}_{3}$, Journal of the American Chemical Society, 119 (1997) 2747-2748.

[49] F. Zheng, Y. Chen, High temperature electrode reactions of $\mathrm{Sr}$ and $\mathrm{Mg}$ doped $\mathrm{LaGaO}_{3}$ perovskite, Journal of Materials Science, 43 (2008) 2058-2065.

[50] N. Jiang, E.D. Wachsman, S.-H. Jung, A higher conductivity $\mathrm{Bi}_{2} \mathrm{O}_{3}$-based electrolyte, Solid State Ionics, 150 (2002) 347-353.

[51] R.N. Vannier, S.J. Skinner, R.J. Chater, J.A. Kilner, G. Mairesse, Oxygen transfer in BIMEVOX materials, Solid State Ionics, 160 (2003) 85-92.

[52] S. Uhlenbruck, N. Jordan, J.M. Serra, H.P. Buchkremer, D. Stöver, Application of electrolyte layers for solid oxide fuel cells by electron beam evaporation, Solid State Ionics, 181 (2010) 447-452.

[53] A. Tsoga, A. Gupta, A. Naoumidis, D. Skarmoutsos, P. Nikolopoulos, Performance of a double-layer CGO/YSZ electrolyte for solid oxide fuel cells, Ionics, 4 (1998) 234240 .

[54] S. Uhlenbruck, T. Moskalewicz, N. Jordan, H.J. Penkalla, H.P. Buchkremer, Element interdiffusion at electrolyte-cathode interfaces in ceramic high-temperature fuel cells, Solid State Ionics, 180 (2009) 418-423.

[55] N. Jordan, W. Assenmacher, S. Uhlenbruck, V.A.C. Haanappel, H.P. Buchkremer, D. Stöver, W. Mader, $\mathrm{Ce}_{0.8} \mathrm{Gd}_{0.2} \mathrm{O}_{2-\delta}$ protecting layers manufactured by physical vapor deposition for IT-SOFC, Solid State Ionics, 179 (2008) 919-923.

[56] E. Fabbri, L. Bi, D. Pergolesi, E. Traversa, Towards the Next Generation of Solid Oxide Fuel Cells Operating Below $600{ }^{\circ} \mathrm{C}$ with Chemically Stable Proton-Conducting Electrolytes, Advanced Materials, 24 (2012) 195-208.

[57] S. Escolastico, M. Ivanova, C. Solis, S. Roitsch, W.A. Meulenberg, J.M. Serra, Improvement of transport properties and hydrogen permeation of chemically-stable proton-conducting oxides based on the system $\mathrm{BaZr}_{1-\mathrm{x}-\mathrm{y}} \mathrm{Y}_{\mathrm{x}} \mathrm{M}_{\mathrm{y}} \mathrm{O}_{3-\delta}$, RSC Advances, 2 (2012) 4932-4943.

[58] A. Magrasó, M.L. Fontaine, Y. Larring, R. Bredesen, G.E. Syvertsen, H.L. Lein, T. Grande, M. Huse, R. Strandbakke, R. Haugsrud, T. Norby, Development of Proton Conducting SOFCs Based on $\mathrm{LaNbO}_{4}$ Electrolyte - Status in Norway, Fuel Cells, 11 (2011) 17-25.

[59] C. Solis, L. Navarrete, S. Roitsch, J.M. Serra, Electrochemical properties of composite fuel cell cathodes for $\mathrm{La}_{5.5} \mathrm{WO}_{12-\delta}$ proton conducting electrolytes, Journal of Materials Chemistry, 22 (2012) 16051-16059. 
[60] S. Escolástico, C. Solís, T. Scherb, G. Schumacher, J.M. Serra, Hydrogen separation in $\mathrm{La}_{5.5} \mathrm{WO}_{11.25-\delta}$ membranes, Journal of Membrane Science, 444 (2013) 276-284.

[61] S. Escolastico, V.B. Vert, J.M. Serra, Preparation and Characterization of Nanocrystalline Mixed Proton-Electronic Conducting Materials Based on the System $\mathrm{Ln}_{6} \mathrm{WO}_{12}$, Chemistry of Materials, 21 (2009) 3079-3089.

[62] R. Haugsrud, Defects and transport properties in $\mathrm{Ln}_{6} \mathrm{WO}_{12}(\mathrm{Ln}=\mathrm{La}, \mathrm{Nd}, \mathrm{Gd}, \mathrm{Er})$, Solid State Ionics, 178 (2007) 555-560.

[63] T. Shimura, S. Fujimoto, H. Iwahara, Proton conduction in non-perovskite-type oxides at elevated temperatures, Solid State Ionics, 143 (2001) 117-123.

[64] C. Sun, R. Hui, J. Roller, Cathode materials for solid oxide fuel cells: a review, J Solid State Electrochem, 14 (2010) 1125-1144.

[65] J.M. Serra, H.-P. Buchkremer, On the nanostructuring and catalytic promotion of intermediate temperature solid oxide fuel cell (IT-SOFC) cathodes, Journal of Power Sources, 172 (2007) 768-774.

[66] J. Piao, K. Sun, N. Zhang, X. Chen, S. Xu, D. Zhou, Preparation and characterization of $\mathrm{Pr}_{1-\mathrm{x}} \mathrm{Sr}_{\mathrm{x}} \mathrm{FeO}_{3-\delta}$ cathode material for intermediate temperature solid oxide fuel cells, Journal of Power Sources, 172 (2007) 633-640.

[67] G.C. Kostogloudis, C. Ftikos, A. Ahmad-Khanlou, A. Naoumidis, D. Stöver, Chemical compatibility of alternative perovskite oxide SOFC cathodes with doped lanthanum gallate solid electrolyte, Solid State Ionics, 134 (2000) 127-138.

[68] A. Egger, E. Bucher, M. Yang, W. Sitte, Comparison of oxygen exchange kinetics of the IT-SOFC cathode materials $\mathrm{La}_{0.5} \mathrm{Sr}_{0.5} \mathrm{CoO}_{3-\delta}$ and $\mathrm{La}_{0.6} \mathrm{Sr}_{0.4} \mathrm{CoO}_{3-\delta}$, Solid State Ionics, 225 (2012) 55-60.

[69] X. Chu, F. Liu, W. Zhu, Y. Dong, M. Ye, W. Sun, Cobalt-free composite $\mathrm{Ba}_{0.5} \mathrm{Sr}_{0.5} \mathrm{Fe}_{0.9} \mathrm{Ni}_{0.1} \mathrm{O}_{3-\delta}-\mathrm{Ce}_{0.8} \mathrm{Sm}_{0.2} \mathrm{O}_{2-\delta}$ as cathode for intermediate-temperature solid oxide fuel cell, J. Mater. Sci. Technol. (Shenyang, China), 28 (2012) 828-832.

[70] S. Huang, G. Wang, X. Sun, C. Lei, T. Li, C. Wang, Cobalt-free perovskite $\mathrm{Ba}_{0.5} \mathrm{Sr}_{0.5} \mathrm{Fe}_{0.9} \mathrm{Nb}_{0.1} \mathrm{O}_{3-\delta}$ as a cathode material for intermediate temperature solid oxide fuel cells, J. Alloys Compd., 543 (2012) 26-30.

[71] S.J. Lee, S.-M. Yong, D.S. Kim, D.K. Kim, Cobalt-free composite cathode for SOFCs: Brownmillerite-type calcium ferrite and gadolinium-doped ceria, Int. J. Hydrogen Energy, 37 (2012) 17217-17224.

[72] V.B. Vert, J.M. Serra, J.L. Jorda, Electrochemical characterisation of $\mathrm{MBaCo}_{3} \mathrm{ZnO}_{7+\delta}(\mathrm{M}=\mathrm{Y}, \mathrm{Er}, \mathrm{Tb})$ as $\mathrm{SOFC}$ cathode material with low thermal expansion coefficient, Electrochemistry Communications, 12 (2010) 278-281. 
[73] V.B. Vert, J.M. Serra, Study of $\mathrm{CO}_{2}$ stability and electrochemical oxygen activation of mixed conductors with low thermal expansion coefficient based on the $\mathrm{TbBaCo}_{3} \mathrm{ZnO}_{7+\delta}$ system, Journal of Power Sources, 196 (2011) 4270-4276.

[74] S. Pang, X. Jiang, X. Li, Q. Wang, Z. Su, Q. Zhang, Highly enhanced electrochemical performance of $\mathrm{PrBa}_{0.92} \mathrm{Co}_{2} \mathrm{O}_{5+\delta}$ cathode by introducing $\mathrm{Ba}$ cationicdeficiency, Int. J. Hydrogen Energy, 37 (2012) 3998-4001.

[75] D. Chen, S. Bishop, H. Tuller, Praseodymium-cerium oxide thin film cathodes: Study of oxygen reduction reaction kinetics, J Electroceram, 28 (2012) 62-69.

[76] B. Fan, J. Yan, W. Shi, A high performance solid oxide fuel cells operating at intermediate temperature with a modified interface between cathode and electrolyte, Journal of the European Ceramic Society, 30 (2010) 1803-1808.

[77] X. Lu, Y. Chen, Y. Ding, B. Lin, A cobalt-free $\mathrm{Sm}_{0.5} \mathrm{Sr}_{0.5} \mathrm{FeO}_{3-\delta}-\mathrm{BaZr}_{0.1} \mathrm{Ce}_{0.7} \mathrm{Y}_{0.2} \mathrm{O}_{3-\delta}$ composite cathode for proton-conducting solid oxide fuel cells, International Journal of Hydrogen Energy, 37 (2012) 8630-8634.

[78] Y. Ling, J. Yu, B. lin, X. Zhang, L. Zhao, X. Liu, A cobalt-free $\mathrm{Sm}_{0.5} \mathrm{Sr}_{0.5} \mathrm{Fe}_{0.8} \mathrm{Cu}_{0.2} \mathrm{O}_{3-\delta}-\mathrm{Ce}_{0.8} \mathrm{Sm}_{0.2} \mathrm{O}_{2-\delta}$ composite cathode for proton-conducting solid oxide fuel cells, Journal of Power Sources, 196 (2011) 2631-2634.

[79] E. Quarez, K.V. Kravchyk, O. Joubert, Compatibility of proton conducting $\mathrm{La}_{6} \mathrm{WO}_{12}$ electrolyte with standard cathode materials, Solid State Ionics, 216 (2012) 1924.

[80] C. Solis, L. Navarrete, S. Roitsch, J.M. Serra, Electrochemical properties of composite fuel cell cathodes for $\mathrm{La}_{5.5} \mathrm{WO}_{12-\delta}$ proton conducting electrolytes, Journal of Materials Chemistry, 22 (2012) 16051-16059.

[81] C. Solís, V.B. Vert, M. Fabuel, J.M. Serra, Electrochemical properties of composite cathodes for $\mathrm{La}_{0.995} \mathrm{Ca}_{0.005} \mathrm{NbO}_{4-\delta}$-based proton conducting fuel cells, Journal of Power Sources, 196 (2011) 9220-9227.

[82] M.L. Fontaine, Y. Larring, R. Haugsrud, T. Norby, K. Wiik, R. Bredesen, Novel high temperature proton conducting fuel cells: Production of $\mathrm{La}_{0.995} \mathrm{Sr}_{0.005} \mathrm{NbO}_{4-\delta}$ electrolyte thin films and compatible cathode architectures, Journal of Power Sources, 188 (2009) 106-113.

[83] F. He, T. Wu, R. Peng, C. Xia, Cathode reaction models and performance analysis of $\mathrm{Sm}_{0.5} \mathrm{Sr}_{0.5} \mathrm{CoO}_{3-\delta}-\mathrm{BaCe}_{0.8} \mathrm{Sm}_{0.2} \mathrm{O}_{3-\delta}$ composite cathode for solid oxide fuel cells with proton conducting electrolyte, Journal of Power Sources, 194 (2009) 263-268.

[84] S. Jiang, S. Chan, A review of anode materials development in solid oxide fuel cells, Journal of Materials Science, 39 (2004) 4405-4439. 
[85] S. Tao, J.T.S. Irvine, A redox-stable efficient anode for solid-oxide fuel cells, Nat Mater, 2 (2003) 320-323.

[86] J. Sfeir, P.A. Buffat, P. Möckli, N. Xanthopoulos, R. Vasquez, H. Joerg Mathieu, J. Van herle, K. Ravindranathan Thampi, Lanthanum Chromite Based Catalysts for Oxidation of Methane Directly on SOFC Anodes, Journal of Catalysis, 202 (2001) 229244.

[87] E. Lay, G. Gauthier, L. Dessemond, Preliminary studies of the new Ce-doped La/Sr chromo-manganite series as potential SOFC anode or SOEC cathode materials, Solid State Ionics, 189 (2011) 91-99.

[88] J. Seeger, M. Ivanova, W. Meulenberg, D. Stöver, S. Escolástico, J. M. Serra, S. Roitsch, Development of Mixed Proton- Electron Conducting Materials based on $\mathrm{La}_{6} \mathrm{WO}_{12}$ for Hydrogen Separation, in: ECerS XII-12th Conference of the European Ceramic Society, Stockholm, Sweden., 2011.

[89] L. Bi, E. Fabbri, E. Traversa, Effect of anode functional layer on the performance of proton-conducting solid oxide fuel cells (SOFCs), Electrochemistry Communications, 16 (2012) 37-40 



\title{
Chapter 2. Methodology
}

\begin{abstract}
This chapter describes the procedures developed for the material synthesis and specimen fabrication. The techniques employed to characterize the samples and determine their transport properties are also explained. The different assemblies used for the electrochemical characterization are outlined as well.
\end{abstract}





\subsection{Material synthesis and sample preparation}

\section{Material synthesis}

Different methods have been applied to prepare the materials depending on the desirable characteristics for the powders and on the metal precursor nature. Ceria doped compounds have been fabricated by co-precipitation method. $\mathrm{CaTiO}_{3}$ perovskites have been formed and doped by solid state reaction, commonly known as traditional ceramic method. Doped $(\mathrm{LaSr}) \mathrm{CrO}_{3}$ perovskites have been synthesized by Pechini sol-gel route, [1] and the same method has been employed for dual-phase nanocomposites. The details of the fabrication are described below.

\section{Co-precipitation}

The co-precipitation method allows the synthesis of nanometric size powders. In this work this method has been employed on the fabrication of lanthanide doped ceria materials, as it is shown in the scheme of Figure 2.1. A clear solution of commercial lanthanide nitrates mixture is prepared in deionized water at $50{ }^{\circ} \mathrm{C}$. A $\left(\mathrm{NH}_{4}\right)_{2} \mathrm{CO}_{3}$ solution is dropped into the previous solution to achieve total precipitation and the final $\mathrm{NO}_{3}^{-1} / \mathrm{CO}_{3}{ }^{-2}$ molar ratio is 0.75 . The resulting precursor powders are dried at $100-150{ }^{\circ} \mathrm{C}$ after filtration and rinsing with water. In some cases, the cerias have been codoped with cobalt. The cobalt addition is done over the dried precursor powder by incipient wetness impregnation, i.e., calculated $2 \%$ molar of $\mathrm{Co}\left(\mathrm{NO}_{3}\right)_{2} \cdot 6 \mathrm{H}_{2} \mathrm{O}$ is dissolved in deionized water (volume according with the pore volume) and mixed with 
the intermediate dried powder. Finally, each powder is calcined during 5 hours in air atmosphere at $800{ }^{\circ} \mathrm{C}$ to decompose the residual nitrates and carbonates and to favour the formation of the fluorite phase.

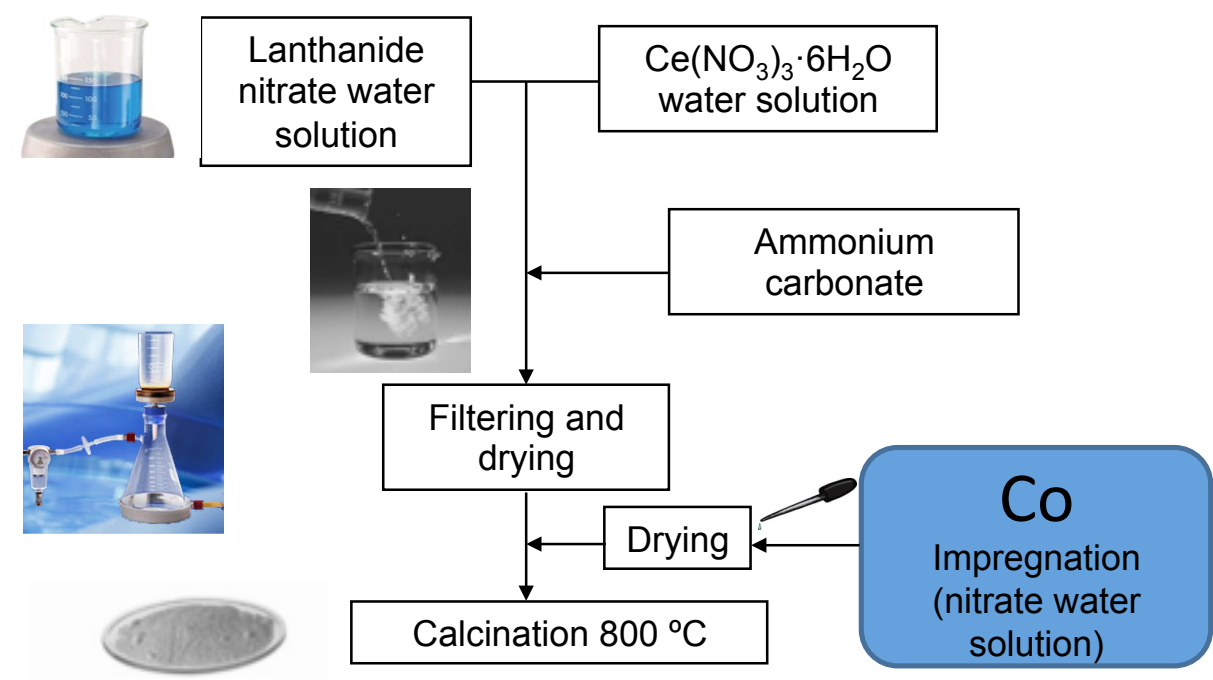

Figure 2.1. Scheme of the fabrication of the lanthanide doped ceria. The addition of Co by impregnation is indicated. The calcination temperature ensures the decomposition of the nitrates and carbonates from the precursors.

\section{Solid state reaction}

The solid state reaction is the most common synthesis process to produce conventional ceramic powders. The method consists of mixing the precursor oxides, carbonates, hydroxides or salts dispersed in acetone or other solvent in a ball mill, which reduces and homogenizes the particle size. The ball mill uses $3 \mathrm{~mm}$ diameter zirconia balls and turn at $50 \mathrm{rpm}$. After the milling, the powder is separated from the zirconia balls and dried. Then, the powder is fired at temperatures of at least two thirds of the melting point for periods up to $10 \mathrm{~h}$. [2] This method presents several drawbacks. One the one hand, it delivers low homogeneity and purity powders with broad particle size distribution. [3] On the other hand, the high-energy consumption to reach the required high 
temperatures and the issues concerning to the toxicity and evaporation of some elements have to be taken into account.

Pechini or citrate complexation route.

The Pechini route presents some advantages such as obtaining carbonate-free, chemically homogeneous final oxide compounds with a high relative density and small size. It is a sol-gel technique that involves the production of an amorphous-like gel containing a metal complex. Citric acid, ethylenediaminetetraacetic acid (EDTA) or glycine can be used as chelating agent that prevents partial segregation of metal components, which could occur in the case of different stabilities with the metal ions in solution. [4] This complexation of metal ions in citric acid is followed by dehydration at low temperature. In some cases, as in this work, a polyol (e.g. ethylene glycol) is added to the reaction batch to promote the polymerization with chelating agent and produce an organometallic polymer. Finally, thermal decomposition of the complex to form the structural phase, e.g. fluorite, perovskite or spinel, is carried out at $600{ }^{\circ} \mathrm{C}$ or $750{ }^{\circ} \mathrm{C}$.

\section{Sample preparation}

\section{Rectangular probes}

This sample configuration is used in electrochemical experiments as DC-conductivity. The as prepared powder is mixed and dispersed in acetone and ball milled with zirconia balls during 12-15 h. After rinsing and drying, rectangular probes $\left(4 \times 0.4 \times 0.2 \mathrm{~cm}^{3}\right)$ of the initially fired nanopowder were uniaxially pressed at $125 \mathrm{MPa}$ during 1 minute and subsequently sintered 5 hours at high temperature $\left(1000-1450{ }^{\circ} \mathrm{C}\right)$ in air atmosphere. Final dimensions 
will depend on the shrinkage undergone during the sintering process, as observed in the examples on Figure 2.2.

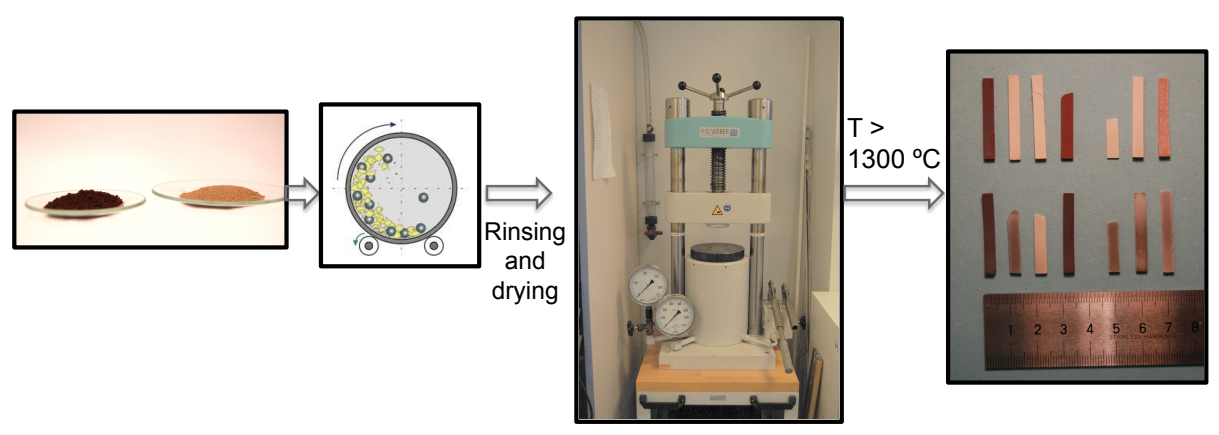

Figure 2.2. Main steps for rectangular probes fabrication. From left to rigth: initial powder, ball milling step, rinsing of the powder, pressing, high temperature calcination and final configuration of sintered samples.

\section{Membranes and electrolytes}

Membranes and electrolytes are obtained from the sintered powders by the same process, which is schematized in Figure 2.3. In this work, membranes consist of flat dense discs. The homogeneity is very important for the sintering process, which is achieved by ball milling the powder following the same process explained for solid state reaction precursors. The typical particle size of the milled powder is selected and homogenize by separating them through steel sieves of a particular mesh size $(200-400 \mu \mathrm{m})$. A $26 \mathrm{~mm}$ diameter steel dye is used to uniaxially press the material at $125 \mathrm{MPa}$ during 3 minutes. Afterwards, the green disk is exposed to a high temperature sintering process in order to densify the specimen. The temperature and time of the process depend on the material. Permeation measurements are performed on $15 \mathrm{~mm}$ diameter and 500 900 $\mu \mathrm{m}$ thick disks, which are grinded by sandpaper to reach that dimensions (Figure 2.3). In some cases both disk sides were screen printed by 20-30 $\mu \mathrm{m}$ of a catalytic layer (metals, MIECs or composite materials) in order to improve the surface catalytic behaviour. 

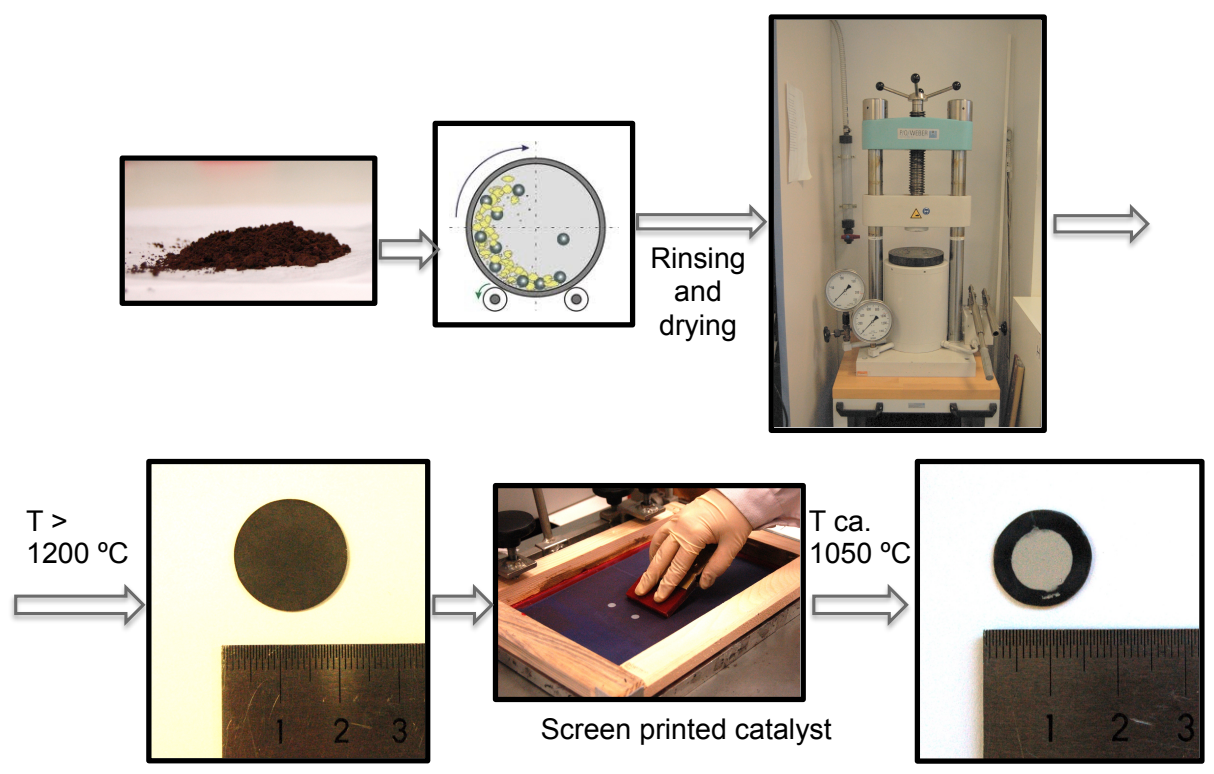

Figure 2.3. Main steps for membrane fabrication. From left to rigth: initial powder, ball milling step, rinsing and drying of the powder, uniaxial pressing, high temperature calcination and final configuration of sintered samples. Afterwards, a catalytic layer may be screen printed on the membrane surface.

\section{Electrodes}

Thick film SOFC anode and cathode electrodes have been attached to the electrolyte by screen-printing technique. This is the most economical, simple and widely used technique to produce films with a thickness in the range of $10-100 \mu \mathrm{m}$. [5] It consists of applying highly viscous ink made of the ceramic powder, an organic binder (ethyl cellulose) and a plasticizer (terpineol). A three-roll mill refines the powder and the other ingredients to form an uniform slurry. This slurry is forced to pass through a $9 \mathrm{~mm}$ diameter mesh to develop the electrode. The layer deposition is then dried and sintered at high temperature. After the drying step, more than one layer may be deposited by this method, depending on the targeted thickness. The thickness for the porous layers is $\sim 15 \mu \mathrm{m}$ and the area of the electrode is $\sim 0.6 \mathrm{~cm}^{2}$, as depicted in Figure 
2.4. In addition a gold mesh is screen printed on the electrode surface to contact the current collector.
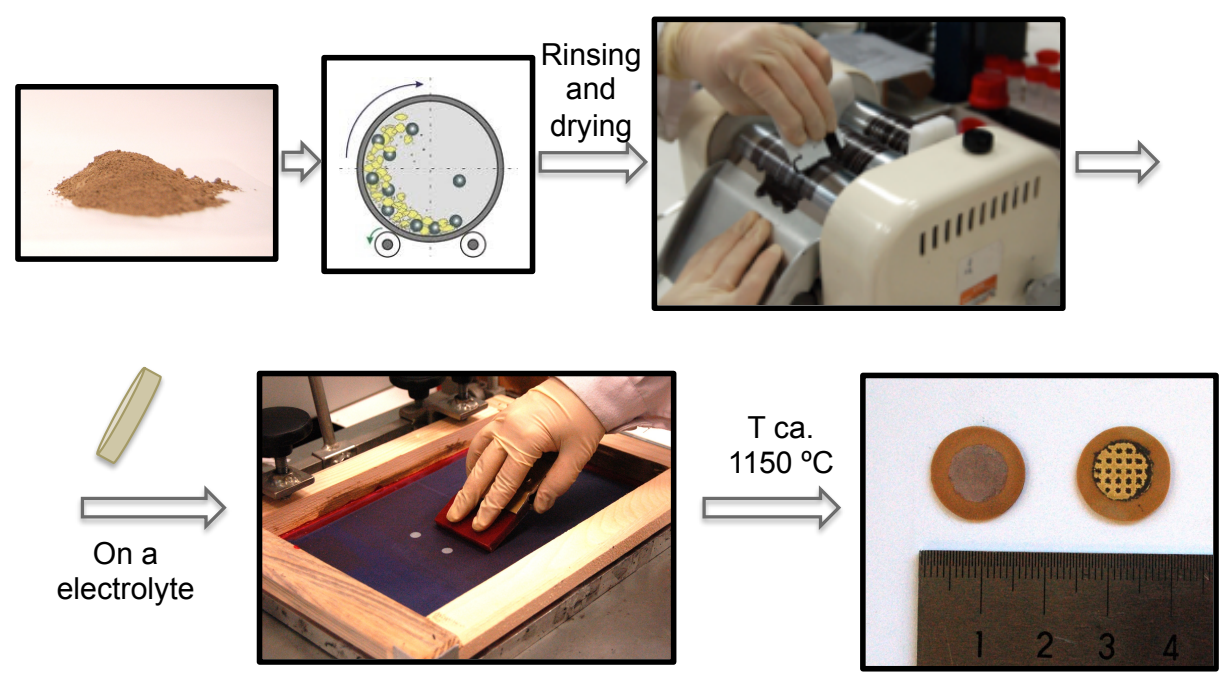

Figure 2.4. Main steps for electrode fabrication. From left to rigth: initial powder, ball milling step, rinsing and drying of the powder, ceramic ink fabrication, screen printing of the electrode on the electrolyte surface and final electrode configuration after high temperature calcination. In addition, the electrode on the right is screen printed with a gold mesh for current collection.

In this thesis, the electrodes have been tested as symmetrical cells. They consist of two porous electrodes of the same material attached to both sides of the dense electrolyte. The same atmosphere is present in both sides of the symmetrical cell for the analysis.

\subsection{Structural characterization}

Scanning Electron Microscopy

Scanning electron microscopy (SEM) allows for high-resolution imaging of surfaces by using high-energy electrons $(1.5-20 \mathrm{KeV})$ generated by 
a heated tungsten filament. The SEM has a magnification up to $10^{6} \times$ and great depth of field. For that purpose, an incident beam of monochromatic electrons causes a secondary emission across the sample surface, which is collected to form an image of the surface. The quality and contrast obtained in the image depends mainly on the conductivity and surface topography of the sample. By detecting different emissions of the sample, it is possible to obtain diverse contrast images, i.e., backscattered electrons (BSE), Auger electrons (AES) and Energy dispersive x-ray spectroscopy (EDS). BSE causes different contrast subjected to the atomic number, $\mathrm{Z}$, of the elements, so changes in composition can be distinguished; EDS is a qualitative and quantitative chemical microanalysis technique to characterize the elemental composition of the volume analysed. It is performed in conjunction with a SEM, but uses the $\mathrm{X}$-rays that are emitted from the sample due to the electron beam. The combination of SEM with EDS allows the analysis of the sample morphology and the composition of the different phases that are present. The minimum detection limits are about 0.1 weight percent, depending on the element and matrix.

Conducting materials that allow the transport of the incident beam electrons do not need any handling of the sample. However, for poor conductors or insulators a conducting layer that does not modify the topography needs to be added. This is achieved by coating the sample in vacuum with Au or graphite (for EDS) using a sputter coater.

Two SEM devices were used on the characterizations, namely the Jeol JSM 6300 and Jeol JSM 5410, both with an acceleration voltage of $20 \mathrm{kV}$. The EDS microanalyses were carried out with a detector from Oxford Instruments. The Software INCAEnergy/Wave served for the interpretation of the X-ray patterns obtained. More recently, a ZEISS Ultra55 field emission SEM (FE-SEM) has been also used. 


\section{Transmission Electron Microscopy}

Transmission Electron Microscopy (TEM) utilizes energetic electrons to provide morphologic, compositional and crystallographic information on samples. The beam of electrons is transmitted through an ultra-thin specimen $(<200 \mathrm{~nm})$, interacting with it as the beam passes through. The electrons that are not absorbed by the sample are detected forming a two dimensions image, which is magnified and focused onto an imaging device.

The TEM device employed for the experiments is a Philips CM10, which has allowed obtaining particle sizes and morphologies of the nanoparticles. In addition, a transmission electron microscope FEI Tecnai F20 (with acceleration voltage of $200 \mathrm{kV}$ ) has been used. TEM lamellas were prepared by means of a FIB (Focused-Ion Beam, FEI Helios Nanolab 400s) with the subsequent thinning performed in a BAL-TEC RES-120.

\section{$X$-ray diffraction}

$\mathrm{X}$-ray diffraction $(\mathrm{XRD})$ is a non-destructive technique that allows the identification of the crystalline phases and orientation of crystalline materials, their lattice parameters (10-4 $\AA$ ), strain, crystallite size and thermal expansion, which define the fingerprint of the substance. In a crystalline material, the atoms are arranged in a regular pattern. The unit cell is the smallest volume element that by repetition in three dimensions describes the crystal. Three axes describe the dimensions of the unit cell: a, b, c and so the angles between them $\alpha, \beta, \gamma$. Different combinations of these parameters release fourteen kinds of cells covering all possible point lattices or all crystals, which were classified by August Bravais. Since some of the Bravais lattices can be expressed by other simpler lattices, they can be represented by seven primitive lattices, summarized in Table 2.1. [6] 


\begin{tabular}{llll}
\hline System & Axial lengths and angles & Bravais lattice & Lattice symbol \\
\hline Cubic & Three equal axes at right angles & Simple & $P$ \\
& $a=b=c, \alpha=\beta=\gamma=90^{\circ}$ & Body-centered & $I$ \\
Tetragonal & Three axes at right angles, two equals & Simple & $F$ \\
& $a=b \neq c, \alpha=\beta=\gamma=90^{\circ}$ & Body-centered & $P$ \\
Orthorhombic & Three unequal axes at right angles & Simple & $I$ \\
& $a \neq b \neq c, \alpha=\beta=\gamma=90^{\circ}$ & Body-centered & $P$ \\
& & Base-centered & $I$ \\
Trigonal* & Three equal axes, equally inclined & Face-centered & $F$ \\
Hexagonal & $a=b=c, \alpha=\beta=\gamma \neq 90^{\circ}$ & Simple & $R$ \\
& Two equal coplanar axes at, & & \\
& $120^{\circ}$ third axis at right angles & Simple & \\
Monoclinic & $a=b \neq c, \alpha=\beta=90^{\circ}, \gamma=120^{\circ}$ & & \\
& Three unequal axes, one pair not & Simple & \\
& at right angles $a \neq b \neq c$, & Base-centered & \\
Triclinic & $\alpha \neq \gamma=90^{\circ} \neq \beta$ & & \\
& Three unequal axes, unequally & Simple & \\
& inclined and none at right angles & & \\
*Also called rhom & $a \neq b \neq c, \alpha \neq \beta \neq \gamma \neq 90^{\circ}$ & &
\end{tabular}

Table 2.1. Summary of the seven crystal systems and Bravais lattices. [6] The axial length and angles, and lattice symbols are also indicated.

XRD measurements are based on the physical principle that a monochromatic X-ray beam with a wavelength $\lambda$ comes into contact with the crystalline material at a particular angle. The diffraction is produced only when the distance traveled by the rays reflected from successive planes differs by a complete number of wavelengths. As shown in Figure 2.5, the diffracted angle from the family of crystallographic planes ( $h k l$ ) has a value of $2 \theta$, which is employed to determine the distance between layers of atoms in a sample using the Bragg's law:

$$
\mathrm{n} \lambda=2 \mathrm{~d}_{\mathrm{hkl}} \sin \theta
$$


where $\mathrm{d}$ is the $h k l$ interplanar spacing $(\mathrm{nm}), \theta$ is the angle of incidence of $\mathrm{X}$-rays (rad), $\lambda$ is the wavelength of X-rays (m); and $n$ is an integer representing the reflection order.

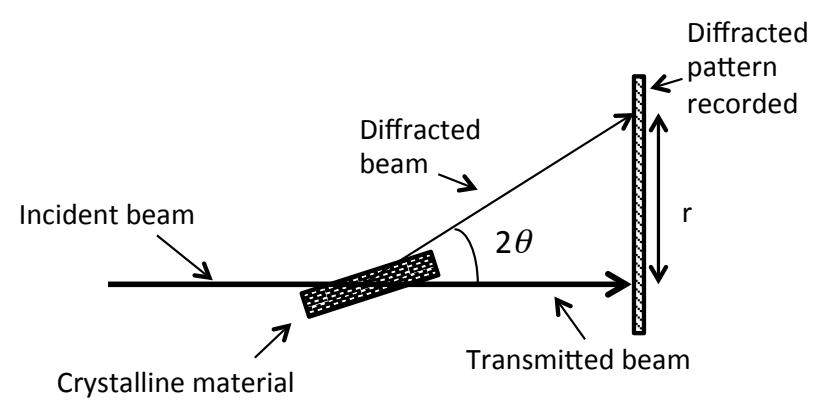

Figure 2.5. Incident beam on a crystalline material and diffracted angle $(2 \theta)$, which is recorded.

By combining the Bragg condition with the plane spacing for a system, it is feasible to directly obtain the positions of atoms in the unit cell. For example, for a cubic system the diffraction peaks satisfy the following equation [7]:

$\frac{\sin ^{2} \theta}{\mathrm{h}^{2}+\mathrm{k}^{2}+\mathrm{l}^{2}}=\frac{\lambda^{2}}{4 \mathrm{a}^{2}}$

where $a$ is the cell parameter and $\mathrm{h}, \mathrm{k}$, and $\mathrm{l}$, the Miller indices defining the plane. The plane spacing for solving other systems is provided in table of the Appendix 2.

On the other hand, XRD peak profile is also employed to estimate the particle size of the crystallites in a powder sample. It is worthy here to emphasize the difference between the grain size of a crystalline powder sample and the size of crystallites. The term 'particle size' or 'grain size' is used when the size of individual crystals is less than about $100 \mathrm{~nm}$, because a particle of a real crystalline powder sample generally consists of many fine units called 
'crystallites', which can be considered as single crystals, as drawn in Figure 2.6. Although the size of crystallites may be the same as that of grain size in some cases, they are essentially different physical quantities. The size of crystallites is related with the peak width, broader as smaller the crystallite. Crystallites in a crystalline powder smaller than $5 \mathrm{~nm}$ will show a diffraction peak profile clearly different from that of the same sample with the standard size of 0.5-10 $\mu \mathrm{m}$ in diameter, which will present sharper peaks. [6]

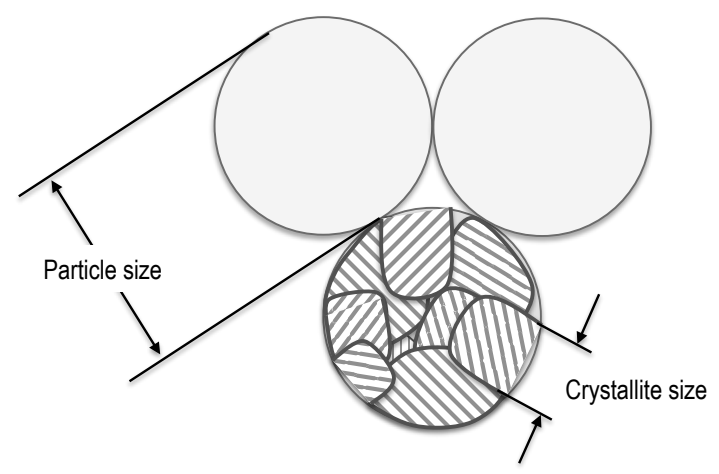

Figure 2.6. Schematic diagram for grain size and crystallite of a crystalline powder sample. A particle or grain of a real crystalline powder generally consists of many crystallites, which can be considered as single crystals.

Calculations of crystallite size commonly employ the Scherrer's equation (Eq. 2.3) for estimating the size of crystallites from a measured diffraction peak profile.

$\mathrm{t}=\frac{0.9 \cdot \lambda}{\beta \cdot \cos \theta}$

where $t$ is diameter of crystallites and $\beta$ represents the full width at half the maximum peak intensity. As order of magnitude crystallites down to about $50 \mathrm{~nm}$, may show peaks of ca. 0.2 degrees broad. [6]

The measurements developed during the present thesis were carried out by a Cubix fast diffractometer, using $\mathrm{CuK} \alpha_{1,2}$ radiation $\left(\lambda_{1}=1.5406 \AA\right)$ and an 
X'Celerator detector in Bragg-Brentano geometry. XRD patterns were recorded in the $2 \theta$ range from $2^{\circ}$ to $90^{\circ}$ and analyzed using X'Pert Highscore Plus software. Some studies required obtaining the XRD patterns at high temperature (HT-XRD), which were performed by an Anton Paar XRK-900 reaction chamber attached to the diffractometer, either in dry air atmosphere or in $\mathrm{N}_{2}$ up to $900{ }^{\circ} \mathrm{C}$.

\section{Density measurements}

Density features of the materials were calculated from mass and volume values, obtained by a helium pycnometer, and comparing them with the theoretical values. This method measures the volume of gas (He) displaced by a known mass of powder, and gives the real density of the material by (Eq. 2.4). The sample must be completely dried. The density measurements were carried out at least three times to average out the variation in values measured.

$\rho_{\mathrm{e}}=\frac{\text { Sample mass }}{\text { Displaced volume }}$

The theoretical density $\left(\rho_{\mathrm{T}}\right)$ is determined from the molecular weigth and the cell parameters obtained by XRD. As an example, for a cubic fluorite structure, which has 4 lanthanide and 8 oxygen atoms, $\rho_{T}$ is calculated as follows:

$\rho_{\mathrm{T}}=\frac{\sum_{\mathrm{n}} \mathrm{X}_{\mathrm{n}} \cdot\left[4 \cdot \mathrm{AW}_{\mathrm{n}}+8 \cdot \mathrm{AW}_{\mathrm{O}}\right]}{[\mathrm{CV}] \cdot\left[\mathrm{N}_{\mathrm{A}}\right]}$

Where $n$ represents the number of different elements forming the material; $x$ is the molar fraction of the element in the material; $A W$ is the atomic weight of the n-lanthanide and the oxygen; $C V$ is the volume of the unit cell and $N_{A}$ is the Avogadro's number. 


\section{X-Ray photoelectron Spectroscopy}

The X-ray photoelectron spectroscopy (XPS) technique analyzes the surface chemistry of a material. XPS method uses X-rays of energy $h v$ to eject electrons from inner-shell orbitals. Typically, the monochromatic sources of radiation are $\mathrm{Al} \mathrm{K} \alpha(1486.6 \mathrm{eV})$ or $\mathrm{Mg} \mathrm{K \alpha}(1253.6 \mathrm{eV})$. The photon is absorbed by an atom in a molecule or solid, which leads to ionization and emission of a core (inner shell) electron. The energy of electrons emitted by the material can be measured by using any appropriate electron energy analyser and thus, a photoelectron spectrum can be recorded, given by:

$E_{K}=h v+E_{b}+E_{w}$

Being $E_{k}$ the kinetic energy of the electrons, $h v$ the energy of the photons, $E_{b}$ is the binding energy characteristic of each element, and $E_{w}$ is the work function of the spectrometer. The measurement yields a spectrum with a series of photoelectron peaks whose peak areas determine the composition of the materials surface. The shape of each peak and the binding energy can be slightly altered by the chemical state of the emitting atom. Thus, these abnormalities can be used to extract chemical bonding information. As a limitation, XPS is not sensitive to hydrogen or helium, and must be carried out in ultra high vacuum conditions. [8]

Sintered pellets of the materials under study were quenched from high temperature in the desired atmosphere to air saturated liquid $\mathrm{N}_{2}$ to imitate the surface in working experimental conditions. Measurements were carried out on a SPECS spectrometer with a MCD-9 detector and using a non-monochromatic Al K X-ray source. Spectra were recorded using analyzer pass energy of $30 \mathrm{~V}$, an X-ray power of $100 \mathrm{~W}$ and under an operating pressure of $10^{-9}$ mbar. CASA software has been used to perform the spectra treatment. Binding energies (BE) 
were referenced to the $\mathrm{C} 1 \mathrm{~s}$ peak at $284.5 \mathrm{eV}$. The energy regions of $\mathrm{O} 1 \mathrm{~s}$ and $\mathrm{C} 1 \mathrm{~s}$ transitions and the specific ones of the materials were recorded.

\section{Raman spectroscopy}

Raman spectroscopy is a useful tool for obtaining information about the structure and properties of molecules from their vibrational transitions. Raman vibrational bands are characterized by their frequency (energy), intensity, and band shape (environment of bonds). The frequencies of these molecular vibrations depend on the masses of the atoms, their geometric arrangement, and the strength of their chemical bonds. [9, 10] Thus, it is possible to detect structural and compositional changes by the anomalies in position, intensity and shape of the bands.

Raman spectra were measured with a Renishaw inVia Raman spectrometer equipped with a Leica DMLM microscope and a 514-nm $\mathrm{Ar}^{+}$ion laser as an excitation source. A $\times 50$ objective of 8 -mm optical length was used to focus the depolarized laser beam on a spot of about $3 \mu \mathrm{m}$ in diameter. The Raman scattering was collected with a charged coupled device (CCD) array detector.

\section{$U V$-vis spectrophotometry}

The electronic properties of a semiconductor may be determined from the analysis of its energy band diagram. The separation between the energy of the lowest conduction band and that of the highest valence band is called the band-gap $\left(E_{g}\right)$. UV-vis diffuse reflectance spectroscopy is a commonly employed optical method to evaluate the $E_{g}$. It describes the electronic behavior present in the structure of the solid giving information about the electronic transitions of the different orbitals of a solid. 
The UV-vis technique measures the increase in the absorbance at a given wavelength (band gap energy) due to the optical excitation of the electrons from the valence band to the conduction band. Graphical Kubelka-Munk ( $K-M)$ transformation (Eq. 2.7) is commonly employed to obtain the optical $E_{g}$.

$\mathrm{F}(\mathrm{R})=\frac{(1-\mathrm{R})^{2}}{2 \mathrm{R}}$

where $R$ is the reflectance; $F(R)$ is proportional to the extinction coefficient $(\alpha)$. Any electronic transition has been considered here, so the plot directly represents $F(R)$ versus the energy in eV. The optical gap has been inferred by linear extrapolation of the absorbance from the high slope region obtained from the spectra. [11]

By doping the materials under study, it is possible to introduce electronic levels that will make the band gap narrower. These changes are followed by UV-vis spectra of the compounds recorded on a Varian 5000 UV-Vi-NIR spectrophotometer. The wavelength range is $200-800 \mathrm{~nm}$ (with a lamp change at $350 \mathrm{~nm}$ ) using $\mathrm{BaSO}_{4}$ as reference material.

\subsection{Thermal Analysis}

Thermal analysis techniques detect the transformations occurring in a sample under a temperature programme in a controlled atmosphere.

Thermogravimetry/Differential Thermal Analysis (TG/DTA)

Chemical and thermal stability of materials can be studied by TG technique, which is commonly used to investigate decomposition, dehydration, 
oxidation/reduction reactions, reaction pathways, kinetics of reaction and the quantity of a specific component that occurs accompanied by a mass change. The TG device registers the mass changes undergone by the sample as a function of temperature, time and atmosphere. The TG is combined with DTA, which records exothermic or endothermic processes that enclose transformations in the material when compared with an inert reference material. DTA is mainly used for studying transitions, chemical reactions, adsorption, crystallisation, melting and sublimation. In addition it is possible to measure the heat of reaction and to determine kinetic parameters. [12]

In the current study, the thermobalance was used to monitor changes in the oxygen non-stoichiometry, to ensure the complete decomposition of precursors (carbonates, nitrates and polymers) and to test mass changes of the material in the reaction against $\mathrm{CO}_{2}$. The TG/DTA analysis was performed on Mettler-Toledo StarE equipment, which has different gases available, such as synthetic Air (21 vol\% $\mathrm{O}_{2}$ and 79 vol $\% \mathrm{~N}_{2}$ ), $\mathrm{N}_{2}$ and $5 \% \mathrm{CO}_{2} /$ air. The powders were analysed in these atmospheres in the temperature range from room temperature to $1000^{\circ} \mathrm{C}$, with heating and cooling rates of $10{ }^{\circ} \mathrm{C} / \mathrm{min}$.

\section{Temperature programmed desorption}

Temperature programmed desorption (TPD) techniques are important for the determination of the kinetic and thermodynamic parameters of desorption processes or decomposition reactions. The method consists of heating a sample and simultaneously detecting certain absorbed species that have enough energy to escape, by means of a mass analyser. The mass spectrometer measures the mass of gas phase ions produced from the ionisation of the molecules and atoms of a specimen and allows qualitative and quantitative determination of its elemental composition. These ions are separated according to their mass to charge ratio $(\mathrm{m} / \mathrm{z})$ and then detected. [13] 
TPD measurements were performed in order to observe the oxygen release with temperature. Around $100 \mathrm{mg}$ of powdery material sintered at high temperature are placed in a quartz reactor and heated up and cooled down in air up to it $1000{ }^{\circ} \mathrm{C}$. Then the sample is heated up at $10 / \mathrm{min}$ up to $1000^{\circ} \mathrm{C}$ in the desired atmosphere, i.e. $\mathrm{He}, \mathrm{N}_{2}$, $\mathrm{Ar}$ or $\mathrm{CO}_{2}$. The ions and molecules delivered are monitored and recorded with a mass spectrometer Omnistar (Balzers), by following the corresponding ionic masses: $m / e=32$ and 16 amu for oxygen, or $m / e=44 \mathrm{amu}$ for $\mathrm{CO}_{2}$.

\section{Temperature programmed reduction}

Temperature programmed reduction (TPR) method is a widely used tool for the characterization of metal oxides and mixed metal oxides. Quantitative information of the reducibility of the oxide's surface, as well as the heterogeneity of the reducible surface can be obtained by TPR. A reducing gas mixture (typically $3 \%$ to $17 \%$ hydrogen diluted in argon or nitrogen) flows over the sample, while a thermal conductivity detector (TCD) measures the changes in the thermal conductivity of the gas stream. The TCD signal is then converted to concentration of active gas using a level calibration. Integrating the area under the concentration vs. time (or temperature) yields the total gas consumed.

In the present work, a 2910 Micromeritics system was used to carry out the measurements. Thus, $100 \mathrm{mg}$ of sample was degassed under Ar flow for $1 \mathrm{~h}$ and then was subjected to reduction under $\mathrm{H}_{2} / \mathrm{Ar}$ (1/9) flow and heating rate of $10{ }^{\circ} \mathrm{C} /$ min till $1000^{\circ} \mathrm{C}$. The $\mathrm{H}_{2}$ consumption was measured by a TCD. 


\subsection{Electrochemical characterization}

DC total electrical conductivity

Electrical conductivity measurements are conducted by standard four-point DC technique. Compared to two-point measurements, this configuration eliminates the influence of the contact resistance and wiring.

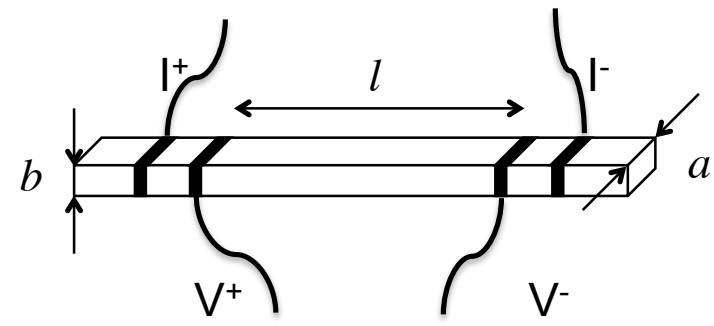

Figure 2.7. Four point configuration for DC conductivity measurements. The contacts are made with silver paste and wires.

Samples consisting of sintered rectangular bars are contacted using silver wire and paste, as shown in Figure 2.7. The measurements are carried out in a temperature range from 400 to $800{ }^{\circ} \mathrm{C}$ by cooling down $\left(1{ }^{\circ} \mathrm{C} / \mathrm{min}\right)$ in constant different $\mathrm{O}_{2}$ contained atmospheres (Linde calibrated gas mixtures checked by an YSZ oxygen sensor). Exceptionally, the temperature range may be extended to $400-1000{ }^{\circ} \mathrm{C}$. A new combinatorial setup for measuring simultaneously the DC conductivity of ten samples has been developed (Figure $2.8 \mathrm{a}$ and $\mathrm{b}$ ). It consists of a constant $\mathrm{pO}_{2}$ chamber that is inserted in a tubular oven. The samples and the YSZ sensor are radially attached around the gas inlet. A programmable current source (Keithley 2601) supplies the constant current and a sixteen channel multimeter (Keithley 3706) detects the voltage drop (Figure 2.8c). The I-V slope provides the resistance imposed by the 
sample, from which the electrical conductivity $\left(\sigma_{\text {total }}\right)$ is extracted by applying the Ohm's law:

$\sigma_{\text {total }}=\frac{\mathrm{l}}{\mathrm{a} \cdot \mathrm{b}} \cdot \frac{1}{\mathrm{R}}$

where $l$ is the distance between contacts, $a$ is the width and $b$ is the thickness of the sample and $R$, the resistance of the material. The conductivity measurements are thermally activated and were analysed on the basis of Arrhenius behaviour

$\sigma(T)=(A / T) \exp \left(-E_{a} / k T\right)$

The activation energy $\left(E_{a}\right)$ is extracted from the slope of the graphs. Once the highest temperature $\left(800^{\circ} \mathrm{C}\right)$ was reached, the samples were stabilized for two hours in order to warrantee the high-temperature reduction state corresponding to the specific $p \mathrm{O}_{2}$. 


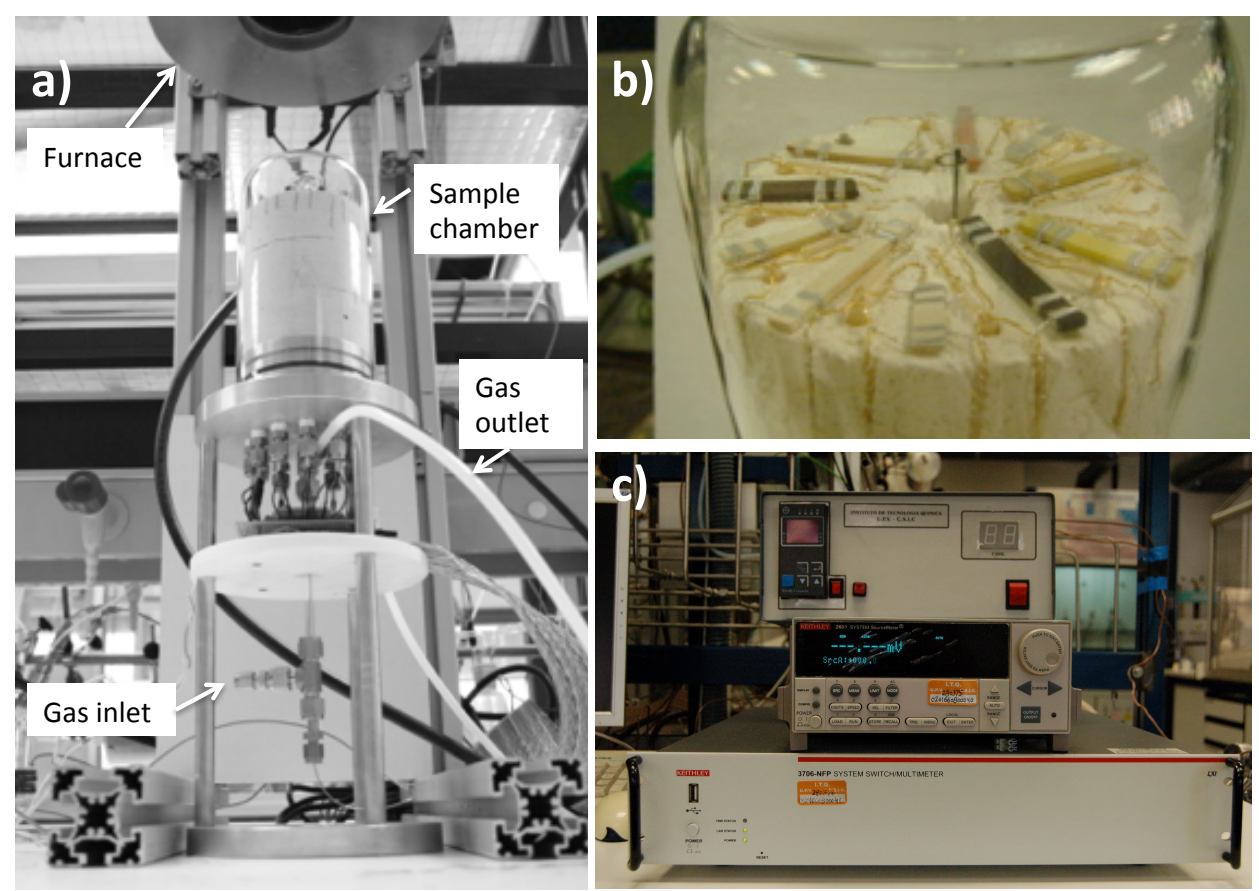

Figure 2.8. a) Combinatorial DC conductivity setup, b) detail of the sample chamber containing the ten samples and c) from top to bottom, furnace controller, programmable current source (Keithley 2601) that and sixteen channel multimeter (Keithley 3706).

\section{Pulse Isotopic Exchange (PIE)}

The fast surface exchange of neutral oxygen and reduction/dissociation to $\mathrm{O}^{2-}$ is a basic requirement for conduction in dense materials. It is characterized by the surface exchange coefficient $k$. Pulse isotopic exchange (PIE) [16] technique allows working in a surface exchange dominated regime since it uses powders of a size smaller than the characteristic thickness $\left(L_{c}=D / k\right)$. Therefore, it provides a more reliable method to measure $k$ than other techniques as the electrical conductivity relaxation (ECR). [17]

For PIE experiments, the powder was uniaxially pressed at $400 \mathrm{MPa}$. The disk was sintered at $1450{ }^{\circ} \mathrm{C}$ for $10 \mathrm{~h}$ in air, and crushed into a coarse powder. The fraction passing through $125 \mu \mathrm{m}$ stainless steel sieve was pre- 
annealed at $1200{ }^{\circ} \mathrm{C}$ for $10 \mathrm{~h}$ in air, and sieved again to remove agglomerates. For the measurement, approximately $80-100 \mathrm{mg}$ of sample powder were loaded between two quartz wool plugs in a quartz tubular microreactor with inner diameter of $2 \mathrm{~mm}$. The length of the packed-bed was about $10 \mathrm{~mm}$. A ${ }^{16} \mathrm{O}_{2} / \mathrm{Ar}$ gas mixture was used as carrier gas with a flow rate $50 \mathrm{ml} / \mathrm{min}$ (STP). The response to ${ }^{18} \mathrm{O}_{2} / \mathrm{N}_{2}$ pulse $(500 \mu \mathrm{l})$, with the same $\mathrm{pO}_{2}$ than the carrier gas was analyzed by online mass spectrometry at the exit of the reactor. A six-port valve with sample loop was used for injection of the ${ }^{18} \mathrm{O}_{2} / \mathrm{N}_{2}$ pulse into the ${ }^{16} \mathrm{O}_{2} / \mathrm{Ar}$ carrier gas flow stream. Oxygen isotope gas was greater than 97 atom $\%{ }^{18} \mathrm{O}_{2}$ (Cambridge Isotope Laboratory). Nitrogen used as diluent for the ${ }^{18} \mathrm{O}_{2}$ gas was also used for internal calibration of the mass spectrometer. Prior to measurements, the powder packed-bed was pre-treated at $800^{\circ} \mathrm{C}$ for $0.5 \mathrm{~h}$ under flowing synthetic air in order to remove possibly adsorbed water and $\mathrm{CO}_{2}$, and subsequently cooled to room temperature at a rate of $5{ }^{\circ} \mathrm{C} / \mathrm{min}$. The pulse-response isotope exchange measurements were performed in the temperature and $\mathrm{pO}_{2}$ ranges $450-900{ }^{\circ} \mathrm{C}$ and $0.21 \mathrm{~atm}$, respectively. The reactor was equilibrated $0.5 \mathrm{~h}$ at fixed temperature and $\mathrm{pO}_{2}$ before data collection. The packed-bed microreactor was designed to approximate ideal plug flow behaviour. [18] The isotopic exchange measurements details are explained by Yoo et al. [19]

\section{Permeation tests}

Permeation measurements were performed on a cylindrical two chambers quartz reactor schematized in Figure 2.9. The inner chamber is $16 \mathrm{~mm}$ diameter with a flat holed bottom that supports the membrane. Sealing was done using gold rings heated at temperature $>1000{ }^{\circ} \mathrm{C}$ and pressed by a spring sited in the lid. Permeation measurements were performed on the $15 \mathrm{~mm}$ diameter disks mentioned in section 2.1.2. Both disk sides may be coated by micrometric layer of catalyst e.g. Pt ink (Mateck, Germany). 


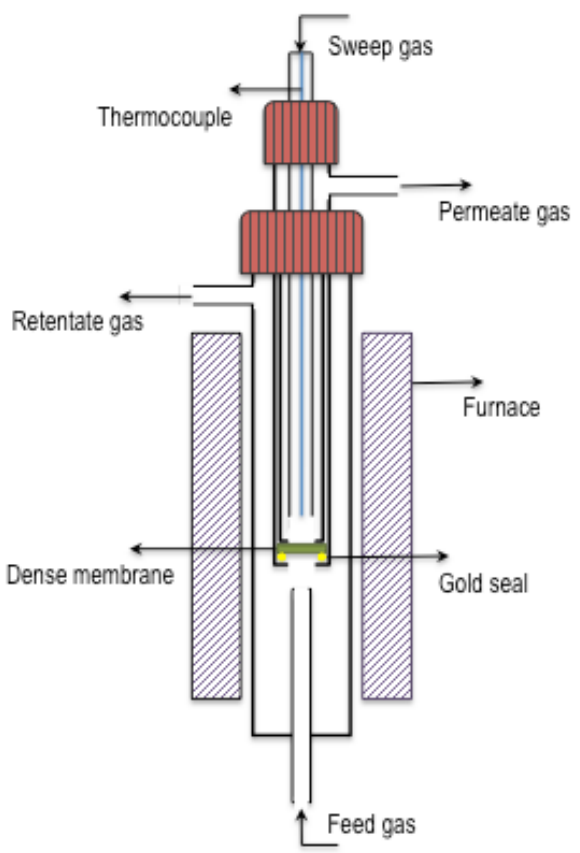

Figure 2.9. Experimental setup permeation scheme. The gas inlet and outlet may be reversed. The membrane is pressed by a spring sited in the lid.

Oxygen was separated from fed syntethic air using argon as sweep gas. The flow rate of all gas feed streams was controlled using mass flow controllers (MFCs). The standard flow rates used were $100 \mathrm{~mL} \mathrm{~min}^{-1}$ for feed and $150 \mathrm{~mL}$ $\min ^{-1}$ for sweep, although they are modify in the different experiments in order to determine their influences on the oxygen separation. In fact, the oxygen permeation has been followed under different parameters, i.e., temperature (500-1000 $\left.{ }^{\circ} \mathrm{C}\right)$, sweep gas flow or fuel gas.

From the oxygen content measured in the argon side (permeate side) and the argon flow rate, the total oxygen permeation rate was calculated, assuming the ideal gas law. The oxygen content in the permeate side was analyzed using micro-GC Varian CP-4900 equipped with Molsieve5A, PoraPlot-Q glass capillary and CP-Sil modules. The Molsieve5A detects $\mathrm{O}_{2}, \mathrm{~N}_{2}$, 
$\mathrm{He}$ and $\mathrm{H}_{2}$. Qualitative analysis of water concentration in the permeate is done in the PoraPlot-Q channel and the CP-Sil measures heavier compounds. Appropriate sealing was confirmed by measuring a tracer concentration in the permeate stream. An acceptable sealing was achieved when the $\mathrm{N}_{2}$ concentration was lower than $5 \%$ of the permeated $\mathrm{O}_{2}$. Data reported are achieved at steady state after $30 \mathrm{~min}$ of stabilization and each test was repeated three times to minimize analysis relative error, which was found to have a standard deviation of $10^{-4}$.

Oxygen concentration is calculated from the $\mathrm{O}_{2}$ signal area and its response factor, previously calibrated. Total permeation is calculated as the product of $\mathrm{O}_{2}$ concentration and Ar flux. Permeation flux $\left(\mathrm{mL} \cdot \mathrm{min}^{-1} \cdot \mathrm{cm}^{-2}\right)$ is extracted from the oxygen flux and the effective area ( $\left.A_{\text {effective }}\right)$ of the membrane by the following equation:

$\mathrm{J}\left(\mathrm{O}_{2}\right)=\frac{\% \mathrm{O}_{2, \text { permeate }}}{100 \%-\% \mathrm{O}_{2, \text { permeate }}} \cdot \frac{\cdot \mathrm{Q}_{\text {Ar }}}{\mathrm{A}_{\text {effective }}}\left(\mathrm{mL} \cdot \mathrm{min}^{-1} \cdot \mathrm{cm}^{-2}\right)$

Electrochemical impedance spectroscopy (EIS) and Alterning Current (AC) electrode testing

EIS is based on the response of an electrochemical cell material to the AC passing through the cell. The different processes occurring inside the fuel cell, i.e., gas transfer to the reaction sites, the electrochemical reaction at those sites, the transfer of ions and electrons in the bulk and in the grain boundary and their combination at the cathode, have different rates. They can be disclosed by measuring in a range of frequencies, whose impedance response is in the complex plane. Thus, it covers circuit elements that exhibit much more complex behavior than that observed by simplified DC conductivity, which is considered 
as an ideal resistor. [20] Experimentally, it is carried out by passing a small sinusoidal current (or voltage) through the interface and measuring the phase shift and amplitude, or real and imaginary parts, of the resulting voltage/current signal:

$\mathrm{v}(\mathrm{t})=\mathrm{V}_{\mathrm{m}} \sin \omega \mathrm{t}$

$i(t)=I_{m} \sin (\omega t+\theta)$

$Z(\omega)=\frac{v(t)}{i(t)}=\operatorname{Re}(Z)+i \operatorname{Im}(Z)$

where $Z$ is the system impedance, $\omega$ the frequency and $\theta$ the phase shift between voltage and current intensity.
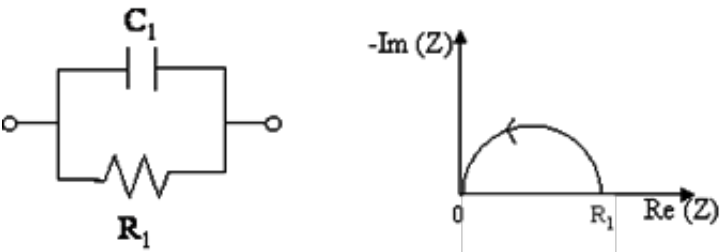

a)
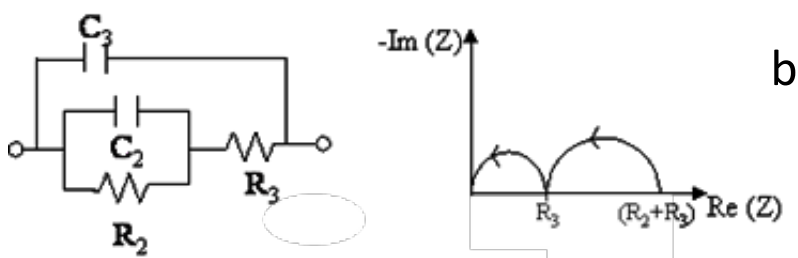

Figure 2.10. Equivalent circuits a) simple parallel resistance-capacitor (RC) circuit and its corresponding perfect semicircle response and b) double RC circuit, represented as a double arc.

EIS data analysis is typically made by graphical representation and modelling to an electrical equivalent circuit that allows the physical interpretation of the single characteristics. Nyquist-plot represents real (Z') and imaginary (Z”) part of impedance values, whose spectra have a semicircle 
shape. The high frequency intercept (for $\omega \rightarrow \infty$ ) with the real axis represents the purely ohmic resistance, $\mathrm{R}_{0}$ of the cell, whereas the intercept at the lower frequency (for $\omega \rightarrow 0$ ) represents the differential resistance, which can be obtained from the corresponding I-U characteristic at the given operating point. The difference between the low and high frequency intercept is the so-called polarisation resistance $(R p)$ of the cell that is the sum of each single polarisation resistance caused by the loss mechanisms inside the cell. Figure 2.10 shows two examples of typical equivalent circuits and the corresponding representation in the complex plane. A simple parallel resistance-capacitor (RC) circuit will deliver a perfect semicircle response as shown in Figure 2.10a, whose extremes are in the origin and in $\mathrm{R}_{0}$, and the maximum is found at $\omega_{M}=1 /(R C)$. Figure $2.10 \mathrm{~b}$ is a double RC circuit, represented as a double arc if $R_{3} C_{3}<<R_{2} C_{2}$.

Electrodes in symmetrical cells were tested by EIS in two-point configuration. Input signal was $0 \mathrm{~V} \mathrm{DC}-20 \mathrm{mV}$ AC amplitude signal in the $0.01-3 \cdot 10^{5} \mathrm{~Hz}$ frequency range. This signal was generated by a Solartron $1470 \mathrm{E}$ and a 1455A FRA module equipment. For anodes, EIS measurements were performed in the $650-900{ }^{\circ} \mathrm{C}$ range, under moistened atmospheres $(2.5 \%$ vol. $\left.\mathrm{H}_{2} \mathrm{O}\right)$ at different $\mathrm{pH}_{2}\left(100 \%, 50 \%\right.$ and $\left.5 \% \mathrm{H}_{2}\right)$. On the other hand, cathodes in symmetrical cells were measured under air in the $700-900{ }^{\circ} \mathrm{C}$ temperature ranges. In all the cases, the total flow remained constant $\left(100 \mathrm{~mL} \cdot \mathrm{min}^{-1}\right)$. Zview $^{\mathrm{TM}} 2$ fitting programme was employed to analyze the impedance spectra.

\subsection{References}

[1] M.P. Pechini, US Patent no 3330 697, in, 1967.

[2] A.S. Bhalla, R. Guo, R. Roy, The perovskite structure: a review of its role in ceramic science and technology, Mat Res Innovat, 4 (2000) 3-26. 
[3] P. Cousin, R.A. Ross, Preparation of mixed oxides: a review, Materials Science and Engineering: A, 130 (1990) 119-125.

[4] S. Liu, X. Tan, K. Li, R. Hughes, Synthesis of strontium cerates-based perovskite ceramics via water-soluble complex precursor routes, Ceramics International, 28 (2002) 327-335.

[5] J. Will, A. Mitterdorfer, C. Kleinlogel, D. Perednis, L.J. Gauckler, Fabrication of thin electrolytes for second-generation solid oxide fuel cells, Solid State Ionics, 131 (2000) 79-96.

[6] Y. Waseda, E. Matsubara, K. Shinoda, X-Ray Diffraction Crystalography, Berlin, Heidelberg, 2011.

[7] E. Lifshin, X-ray Characterization of Materials, Wiley-VCH, Weinheim, Germany, 1999.

[8] H.R. Verma, Atomic and nuclear analytical methods: XRF, Mössbauer, XPS, NAA and B63ion-beam Spectroscopic Techniques, Springer-Verlag Berlin Heidelberg, Berlin; Heidelberg, 2006.

[9] P. Larkin, Infrared and Raman Spectroscopy, Elsevier, cop., Boston, 2011.

[10] M. Baia, S. Astilean, T. Iliescu, Raman and SERS investigation of Pharmaceuticals, Berlin; Heidelberg, 2008.

[11] R. Lopez, R. Gomez, Band-gap energy estimation from diffuse reflectance measurements on sol-gel and commercial $\mathrm{TiO}_{2}$ : a comparative study, J Sol-Gel Sci Technol, 61 (2012) 1-7.

[12] C.J. Keattch, D. Dollimore, An introduction to thermogravimetry, 2nd ed., Heyden, London, 1975.

[13] http://www.uksaf.org/tech/tpd.html.

[14] C.-R. Song, H.-I. Yoo, Chemical diffusivity of $\mathrm{BaTiO}_{3-\delta}$ : III. Conductivitynonstoichiometry (d) correlation in a mixed n/p regime, Solid State Ionics, 124 (1999) 289-299.

[15] M. Sogaard, P. Vang Hendriksen, M. Mogensen, Oxygen nonstoichiometry and transport properties of strontium substituted lanthanum ferrite, Journal of Solid State Chemistry, 180 (2007) 1489-1503.

[16] H.J.M. Bouwmeester, C. Song, J. Zhu, J. Yi, M. van Sint Annaland, B.A. Boukamp, A novel pulse isotopic exchange technique for rapid determination of the oxygen surface exchange rate of oxide ion conductors, Physical Chemistry Chemical Physics, 11 (2009) 9640-9643. 
[17] E.N. Armstrong, K.L. Duncan, D.J. Oh, J.F. Weaver, E.D. Wachsman, Determination of Surface Exchange Coefficients of LSM, LSCF, YSZ, GDC Constituent Materials in Composite SOFC Cathodes, Journal of The Electrochemical Society, 158 (2011) B492-B499.

[18] H.J.M. Bouwmeester, H. Kruidhof, A.J. Burggraaf, Importance of the surface exchange kinetics as rate limiting step in oxygen permeation through mixed-conducting oxides, Solid State Ionics, 72, Part 2 (1994) 185-194.

[19] C.-Y. Yoo, B. Boukamp, H.M. Bouwmeester, Oxygen surface exchange kinetics of erbia-stabilized bismuth oxide, J Solid State Electrochem, 15 (2011) 231-236.

[20] R. Macdonald, Impedance Spectroscopy, J John Wiley \& Sons, 1987. 



\title{
Chapter 3. Structural-transport properties relationships on $\mathrm{Ce}_{1-x} \mathrm{Ln}_{x} \mathrm{O}_{2-\delta}$ system $(L n=G d, L a, T b, P r, E u, E r, Y b$, Nd) and effect of cobalt addition
}

\begin{abstract}
A large series of doped ceria has been prepared by co-precipitation method and completely characterized in order to have an overall understanding of the structural, oxygen vacancy concentration and transport properties. Several lanthanides have been disolved in the $\mathrm{CeO}_{2}$ fluorite structure and the effect of the final sintering temperature and the addition of cobalt oxide on the structural properties have been studied. The chosen lanthanides ( $\mathrm{Gd}, \mathrm{La}, \mathrm{Tb}, \mathrm{Pr}, \mathrm{Eu}, \mathrm{Er}$, $\mathrm{Yb}$ and $\mathrm{Nd}$ ) include a large range of ionic radii and different metals exhibiting variable oxidation states. The materials have been characterized by powder $\mathrm{XRD}$, HT XRD, $\mu$-Raman spectroscopy, helium pycnometry and DC conductivity. Transport properties have been correlated with the structural features induced by the different ionic radii and variable oxidation state of the dopants. The highest ionic conductivity has been obtained for the less distorted cells ( $\mathrm{Gd}$ and $\mathrm{Nd}$ doped ceria), which represent the optimum balance between Coulomb interactions, steric effects and vacancy distribution. The lowest $E a$ value has been found for materials with long cell parameters.
\end{abstract}





\subsection{Introduction}

Pure $\mathrm{CeO}_{2}$ is a fluorite structured oxide in which the cations have eightfold coordination. At low oxygen partial pressures, $\mathrm{Ce}^{4+}$ cations are easily reduced to the $\mathrm{Ce}^{3+}$ valence state in order to charge compensate the oxygen vacancy formation while the fluorite structure is retained. This material exhibits $n$-type electronic conductivity in reducing environments due to the formation of small polarons, which migrate by a thermally activated hopping. [1] The ceria fluorite phase is very tolerant to such reduction and to the dissolution of other oxides that introduce oxide vacancies and thus extend the electrolytic region (area of predominant ionic conductivity) over that of ceria. Nevertheless, both acceptor doping and reduced cation formation result in a strain termed chemical expansion, which produces stresses or constrictions in the structure. [1] Such strains affect the conductivity and might result in mechanical failure of the solid state device. [2, 3] Actually, the highest ionic conductivities are reported for cations causing the lowest size mismatch with respect to $\mathrm{Ce}^{4+}$, i.e., gadolinium and samarium. $[4,5]$ 


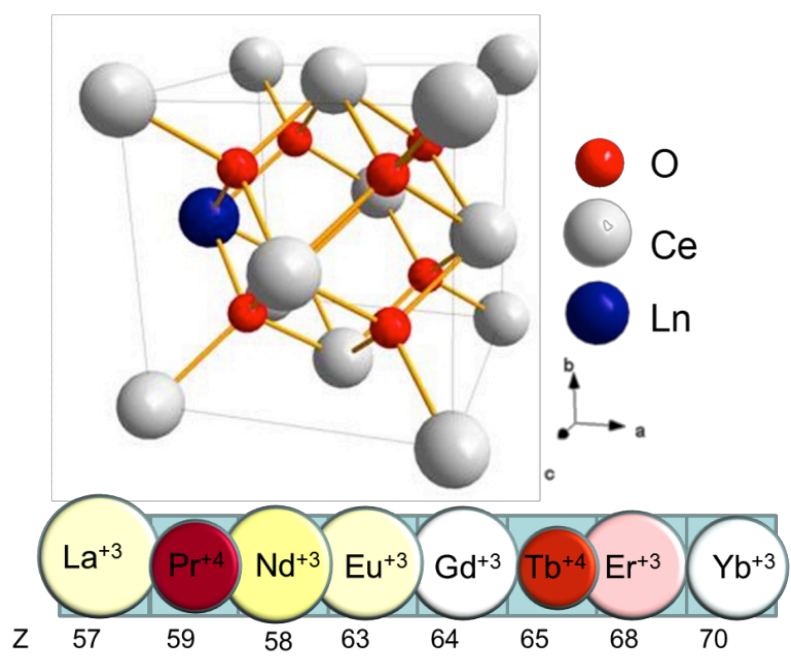

Figure 3.1. Fluorite cubic structure of $\mathrm{CeO}_{2}$. Doping occurs by the $\mathrm{Ln}$ substitution of a Ce cation.

By the mentioned modifications, doped cerias are able to combine high oxygen-ion mobility, redox catalytic properties, chemical compatibility with water and carbon dioxide at high temperatures and sufficient resistance to reduction under relatively low oxygen partial pressures. Therefore, depending on the main characteristics introduced by the dopant, these materials have been suggested and studied for a number of applications, e.g. electrolyte $[6,7]$ and anode $[8,9]$ in solid oxide fuel cells (SOFCs), mixed-conductive membranes for oxygen separation $[10,11]$ and partial oxidation of hydrocarbons, $[12,13]$ oxygen sensors, [14] oxygen storage materials and catalysts for industrial relevant processes. [15-18]

Special attention has been paid to doping with lanthanide $(L n)$ oxides as depicted in Figure 3.1, which are soluble in ceria up to values around $40 \%$. $[19,20]$ The addition of trivalent lanthanides promotes the ionic conductivity since an oxygen vacancy, $\mathrm{V}_{\mathrm{O}}$, is formed for every two substituting ions as indicated by the equation 
$\mathrm{Ln}_{2} \mathrm{O}_{3} \stackrel{2 \mathrm{CeO}_{2}}{\longrightarrow} 2 \mathrm{Ln}_{\mathrm{Ce}}^{\prime}+\mathrm{V}_{\mathrm{O}}^{*}+3 \mathrm{O}_{\mathrm{O}}^{\times}$

where following the Kröger-Vink notation employed in the Chapter $1, \operatorname{Ln}_{\mathrm{Ce}}^{\prime}$ means an acceptor dopant substition in a Ce site. The oxygen vacancies are mostly free at low dopant content, however, as the dopant level increases defect associations due to Coulombic attractions might appear [4]. It has been widely reported that $\mathrm{Sm}$ provides high ionic conductivity combined with high resistance to reduction, followed by Gd. [5] That is why these two dopants are the most commonly used in ceria-based electrolytes for SOFC. [2, 4, 11] Doping with $\mathrm{La}$ increases the lattice constant of the ceria phase favouring the mobility of the ions, but the material becomes quite reducible instead. [5, 15, 21] Dy has been proposed on ceria systems as yttrium co-dopant and Er has been used as dopant in the catalytic ceria-zirconia system. [22, 23] The addition of elements with two valence states has been less studied. They increase the ionic conductivity, but some of these materials develop mixed ionic electronic conductivity, i.e., ceria doped with $\mathrm{Tb}$ and $\mathrm{Pr}$. The rise in electronic conductivity and the lattice expansion undergone at low partial pressures, persuade the use of these materials as SOFC electrolyte. However, at high oxygen partial pressures ionic and $p$-type electronic conductivity are available in some materials and make them suitable for $\mathrm{O}_{2}$ permeable membranes and SOFC cathodes. [24, 25] Specifically, Pr has been employed as dopant for gravimetric gas sensors because it increases the level of nonstoichiometry in ceria under oxidizing conditions. [26] However, it has a doping limit close to $30 \%$ due to the high and non linear thermal expansion behaviour and large chemical expansion upon reduction, which may lead to the device failure. [27] Thus, initial dopant content of $10 \%$ has been chosen. Trivalent lanthanides as Gd, La, Nd and Er have been added. Pr, Tb, Eu and $\mathrm{Yb}$ are elements with two possible valence states. 
On the other hand, doping with lanthanide elements does not generally affect the sintering properties, with the exception of $\mathrm{Yb}, \mathrm{Nd}$ and La. Their pellets or probes remain fragile and crumble on the edges despite they may give densities greater than $85-90 \%$ with regard to theoretical density. [28] Many metal oxides have been proposed to improve the density such as $\mathrm{MnO}_{2}, \mathrm{Mn}_{2} \mathrm{O}_{3}$, $\mathrm{Ga}_{2} \mathrm{O}_{3}, \mathrm{Co}_{3} \mathrm{O}_{4}, \mathrm{CoO}$ and $\mathrm{Fe}_{2} \mathrm{O}_{3}$. [29, 30] They act as sintering aid because of their melting point, which is lower than that of ceria-lanthanide and thus promotes the atomic diffusion throughout the particle surface under high temperatures causing the sintering at lower temperatures. Cobalt oxide has been demonstrated to be an attractive sintering aid since it is able to reduce the sintering temperature more effectively than other metal oxides. [31] Cobalt oxide has been added to the same series of doped ceria in order to check whether it increases the mixed conductivity of these materials as previously reported for Gd and Pr doped ceria. [32, 33]

Previous reported works about series of $L n$ doped ceria are based on theoretical studies or on the collection of literature conductivity values, which are usually either quite scattered or does not take multivalent dopants into account. [34-36] The screening presented in this chapter deals with a systematic and exhaustive study of both structural and transport properties of several $L n$ substituted ceria compounds and the effect of $\mathrm{Co}$ as a sintering aid. First of all, the materials have been systematically prepared and characterized by powder $\mathrm{XRD}, \mu$-Raman spectroscopy, helium pycnometry and DC conductivity. Specifically, Raman spectroscopy is a widely used technique for structural analysis of ceria and lanthanide doped ceria due to its sensitivity to any structural changes (cell parameter and particle size) as well as to the oxygen vacancy concentration. $[37,38]$ 


\subsection{Results and discussion}

Lanthanide doped ceria compounds have been prepared by the coprecipitation method explained in Chapter 2.1, in order to synthesize powders of nanometric size. Thermogravimetric analysis of $\mathrm{Ce}_{0.9} \mathrm{~Tb}_{0.1} \mathrm{O}_{2-\delta}$ and $\mathrm{Ce}_{0.9} \mathrm{Er}_{0.1} \mathrm{O}_{2-\delta}$ precipitate powder dried at $150{ }^{\circ} \mathrm{C}$ are shown in Figure 3.2. The decomposition of the precursor proceeds through two stages. The first weight loss may be attributed to the evaporation of the absorbed moisture and the release of ammonia and water. The second stage may correspond to the decomposition of the nitrates and (hydroxy)-carbonates. That proves that the mass is completely stabilized at $600{ }^{\circ} \mathrm{C}$ and calcination at $800{ }^{\circ} \mathrm{C}$ ensures that there will not remain any phase related to the precursor salts. Afterwards, the samples are sintered at $1300{ }^{\circ} \mathrm{C}$ to perform the electrochemical experiments where dense specimens are required, so that characterization at this temperature was also carried out.

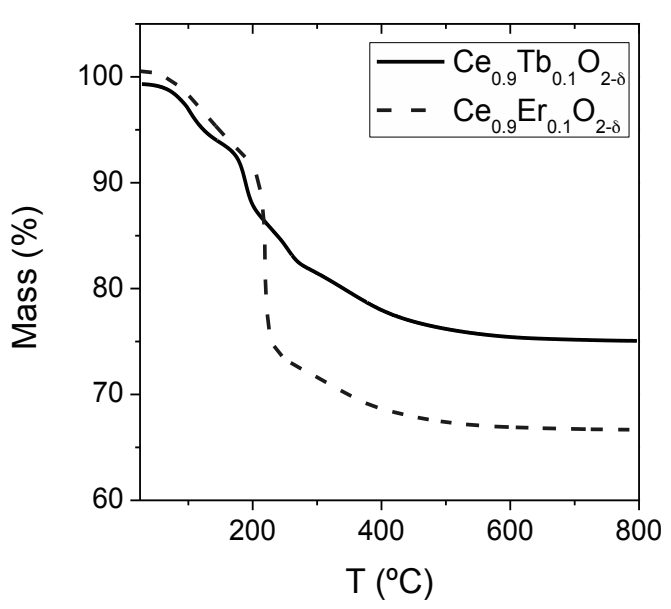

Figure 3.2 Thermogravimetry analysis of $\mathrm{Ce}_{0.9} \mathrm{~Tb}_{0.1} \mathrm{O}_{2-\delta}$ and $\mathrm{Ce}_{0.9} \mathrm{Er}_{0.1} \mathrm{O}_{2-\delta}$, in air. The TG was performed up to $800{ }^{\circ} \mathrm{C}$, the calcination temperature of the as prepared materials. 
XRD patterns of $\mathrm{Ce}_{0.9} \mathrm{Ln}_{0.1} \mathrm{O}_{2-\delta}$ and $\mathrm{Ce}_{0.9} \mathrm{Ln}_{0.1} \mathrm{O}_{2-\delta}+\mathrm{Co} 2 \mathrm{~mol} \%$ series $(\mathrm{Ln}=\mathrm{La}, \mathrm{Gd}, \mathrm{Eu}, \mathrm{Pr}, \mathrm{Tb}, \mathrm{Yb}, \mathrm{Er})$ are depicted in Figure 3.3. After calcination at $800{ }^{\circ} \mathrm{C}$ all the compounds show the expected cubic fluorite structure for $\mathrm{CeO}_{2}$ (ICDD 00-043-1002). Diffraction peaks corresponding to any other lanthanide oxide or precursor are not observed, which means that the dopants are fully incorporated to the lattice of ceria. Likewise, the addition of Co does not cause the formation of any impurity phase. The stability is maintained at $1300{ }^{\circ} \mathrm{C}$. In this case, XRD patterns are sharper due to the crystallite size growing induced by the increasing temperature. XRD peak positions of doped specimens are shifted regarding to the pure $\mathrm{CeO}_{2}$, which indicates deviations from its cell dimensions. The shift to shorter angle implies the enlargement of the unit cell, which happens for all dopants with ionic radius $\left(r_{d}\right)$ bigger that of $\mathrm{Ce}^{4+}$ $\left(r_{c}=1.024 \AA\right)$, while deviations to higher $2 \theta$ denote the shrinkage of the unit cell (dopants with $r_{d}<r_{c}$ ). The unit cell of the present cubic system is characterized by the lattice or cell parameter $(a)$. It was extracted by fitting (4 2 2) reflection peak with a Gaussian function. [39] The lattice parameters for the ( $h \mathrm{kl}$ ) plane were calculated using the equation 2.2 (Chapter 2 ) for a cubic system. 

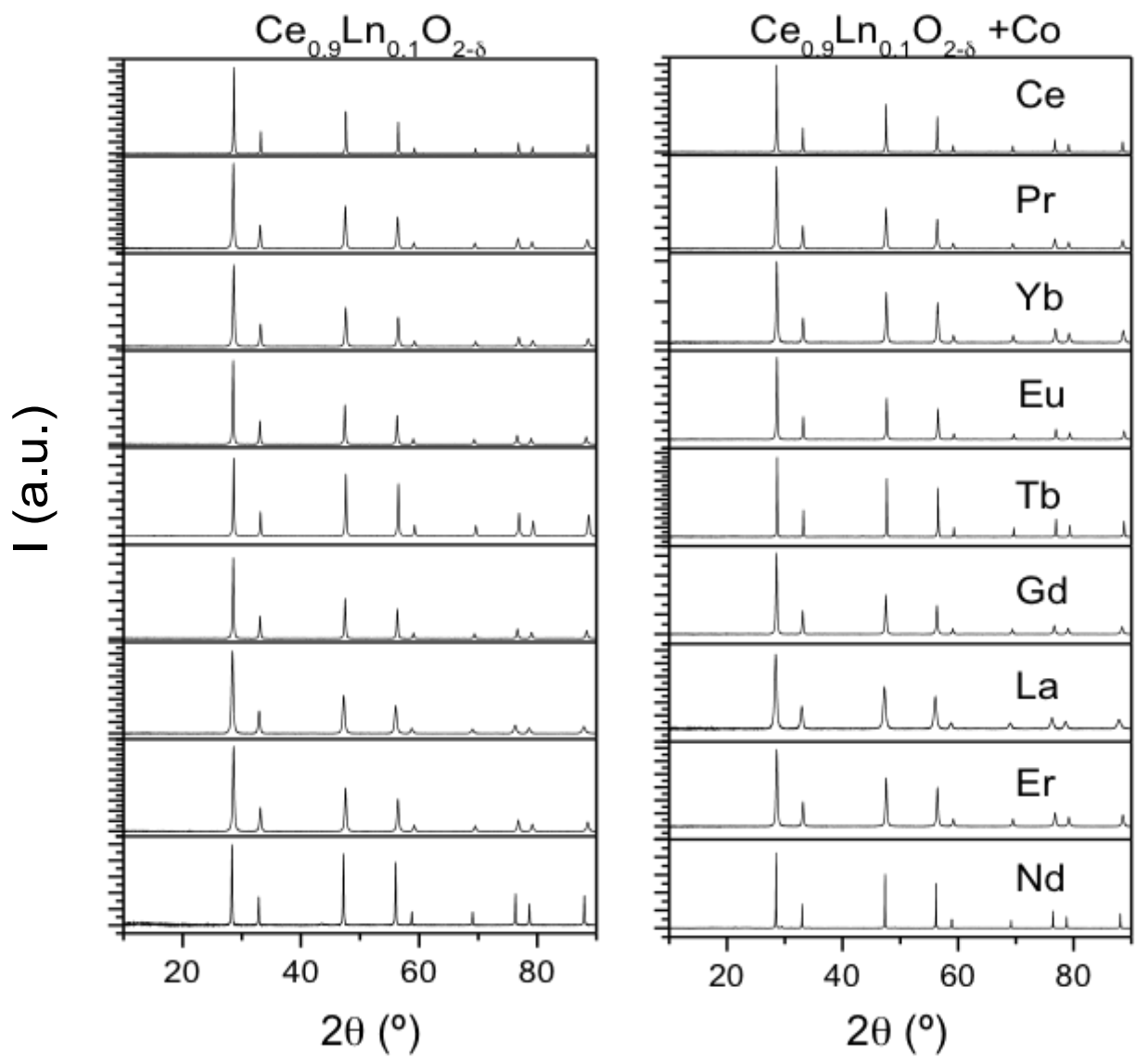

Figure 3.3. XRD pattern of $\mathrm{Ce}_{0.9} \mathrm{Ln}_{0.1} \mathrm{O}_{2-\delta}$ and $\mathrm{Ce}_{0.9} \mathrm{Ln}_{0.1} \mathrm{O}_{2-\delta}+\mathrm{Co} 2 \mathrm{~mol} \%$ calcined at $800{ }^{\circ} \mathrm{C}$.

Figure 3.4 depicts the cell parameter of all the materials as a function of the trivalent ionic radii in 8 -fold coordination given by Shannon for the two different annealing temperatures, 800 and $1300{ }^{\circ} \mathrm{C}$. [40] The cell parameter follows a linear correlation except for $\mathrm{Pr}$ and $\mathrm{Tb}$. Despite the two possible oxidation states $(+2 /+3)$ of $\mathrm{Eu}$ and $\mathrm{Yb}$, they remain trivalent in the fluorite structure. On the other hand, multivalent $\mathrm{Pr}$ and $\mathrm{Tb}(+3 /+4)$ would need an apparent ionic radii between those corresponding to +3 and +4 oxidation states in order to preserve the general trend. By applying the lever rule, it is possible 
to estimate, at room temperature, the amount of trivalent and tetravalent cations, which gives rise to $40 \%$ and $62 \%$ of trivalent cations for $\operatorname{Pr}$ and $\mathrm{Tb}$ respectively.

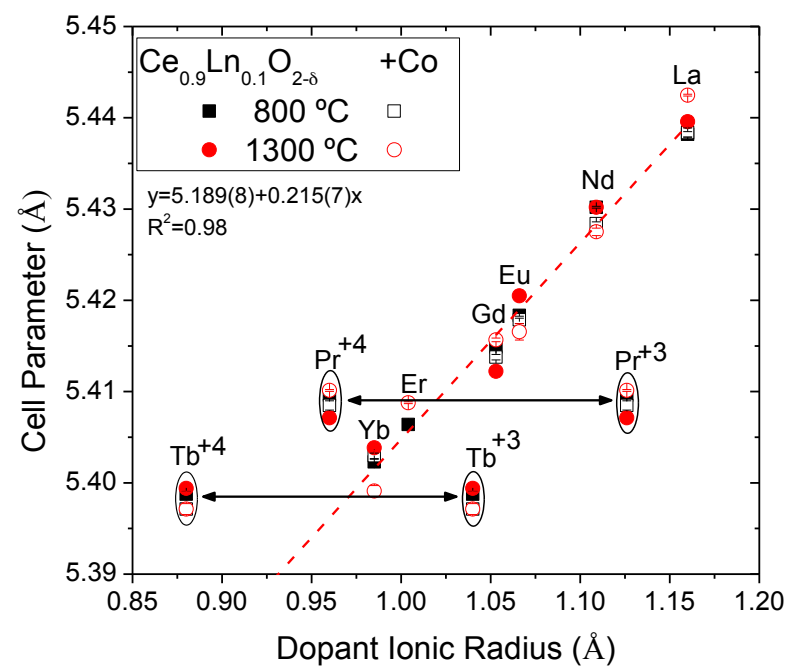

Figure 3.4. Lattice parameter of doped ceria as a function of the trivalent ionic radius of the dopant cation (and tetravalent for Pr and $\mathrm{Tb}$ ) in eight-fold coordination.

Within the range of accuracy of the XRD device, Co addition does not cause any common cell parameter change. Several studies about Co addition in cerias attempted to elucidate the location of Co atoms. Chen et al. [41] studied the solubility of $\mathrm{Co}$ in $\mathrm{CeO}_{2}$ and found a value of $3 \mathrm{~mol} \%$ after sintering at $1580{ }^{\circ} \mathrm{C}$. Also Co has been reported to be soluble less than $0.5 \mathrm{~mol} \%$ in CGO, at $900{ }^{\circ} \mathrm{C}$ and diffuse into the grain boundary to form a film of roughly $0.5 \mathrm{~nm}$ thickness together with Gd or clustering particles. [42] For Pr doped ceria, the addition of $\mathrm{Co}$ at $2 \mathrm{~mol} \%$ results in the apparent dissolution of Co while the addition of $5 \mathrm{~mol} \%$ shows traces of Co oxides, observed by XRD, which are segregated along the grain boundaries. [33] On the contrary, Co causes shrinkage in the cell parameter of $\mathrm{Tb}$ doped ceria indicating that it might be soluble in the structure to some extent. Regardless the location, Co enhances the 
sinterability and the grain size growth, as inferred from the sharpening of the XRD peaks of samples calcined at the same temperature with respect to the Co-free samples (Figure 3.3). [27]

SEM images for Tb, Pr, Eu and La doped ceria and analogous pictures of these materials with Co are shown in Figure 3.5. All the specimens were calcined at the same temperature $\left(1300{ }^{\circ} \mathrm{C}\right)$. The mentioned differences in grain size when Co is added are observed, i.e., an average size of $0.3 \pm 0.1 \mu \mathrm{m}$ is observed for samples without Co, while the average size increases up to $1.3 \pm 0.3 \mu \mathrm{m}$ for Co containing compounds. This suggests that Co doping accelerates the densification rate and the grain growth remarkably. Works of Zhang et al. on the sintering behavior of several dopants included Co oxide. They reported that Co doping reduces the activation energy for grain growth of $\mathrm{CeO}_{2}$, which would explain why Co doping leads to a rapid grain enlargement. $[43,44]$ The SEM pictures do not show difference of phases that could appear from $\mathrm{CoO}_{\mathrm{x}}$ or from its reaction with the doped ceria, although this is not concluding due to the limitations of the device and will be studied in more detail in Chapter 4. On the other hand, all samples show porosity, which should be taken into account depending on the application of the material, i.e., dense membranes or SOFC electrolyte, or porous electrodes. 


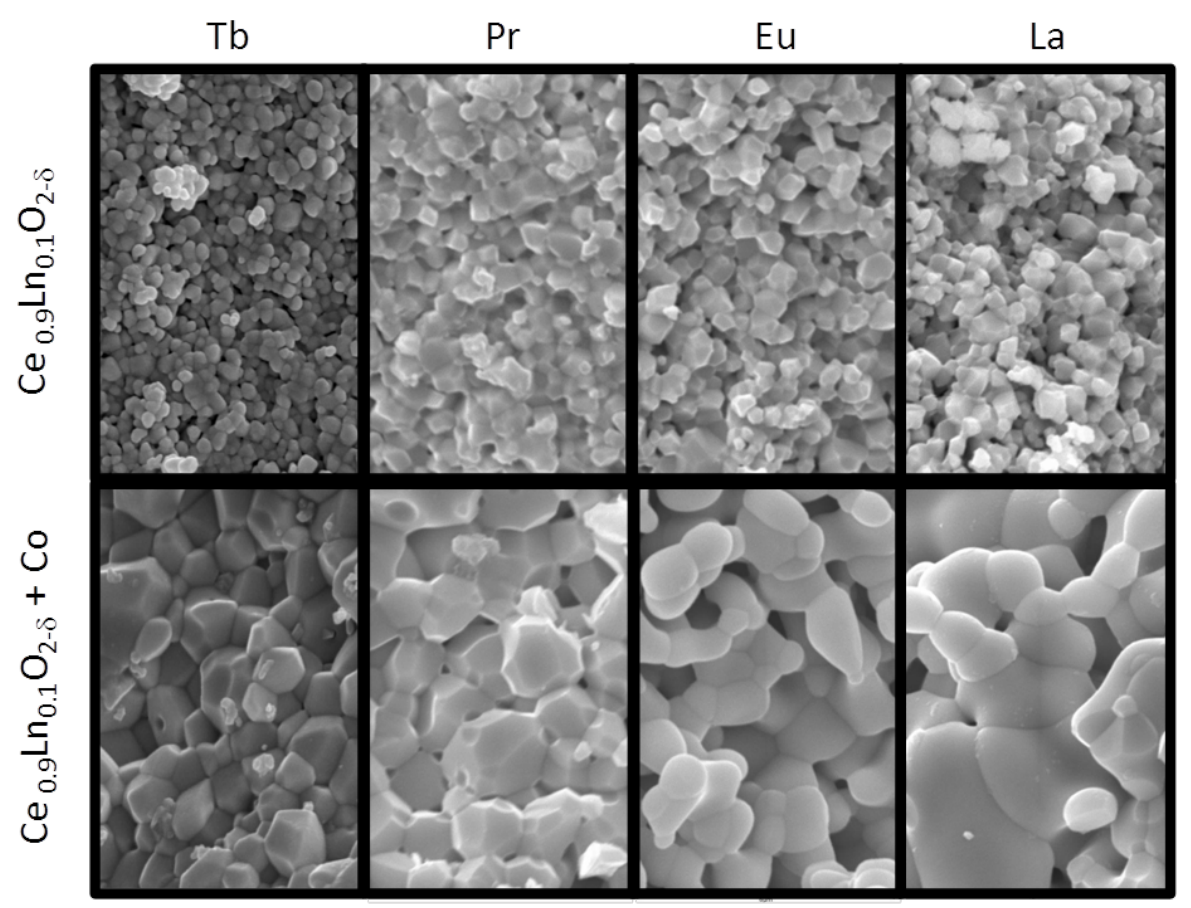

$5 \mu \mathrm{m}$

Figure 3.5. SEM images of $\mathrm{Ce}_{0.9} \mathrm{Ln}_{0.1} \mathrm{O}_{2-\delta}$ and $\mathrm{Ce}_{0.9} \mathrm{Ln}_{0.1} \mathrm{O}_{2-\delta}+\mathrm{Co} 2 \mathrm{~mol} \%$ calcined at $1300{ }^{\circ} \mathrm{C}(\mathrm{Ln}=\mathrm{Tb}, \mathrm{Pr}, \mathrm{Eu}$ and $\mathrm{La})$.

The experimental density values have been obtained from mass and volume values acquired by a helium pycnometer and compared with the theoretical values calculated from the unit cell volume obtained by XRD. The results summarized in Table 3.1 indicate that almost all the materials achieve $>97 \%$ of the estimated density. Pr-doped ceria shows the highest deviation with a $92 \%$ of the expected value, which is ascribed principally to closed porosity. However we should take into account the experimental error obtained in these measurements $(\approx 3 \%$ ), which also explains densities over $100 \%$ for Gd doped ceria. 


\begin{tabular}{cccc}
\hline Materials & $\boldsymbol{\rho}_{\text {theor }}( \pm \mathbf{0 . 0 0 1}) \mathbf{g} / \mathbf{c m}^{\mathbf{3}}$ & $\boldsymbol{\rho}_{\text {exp }}( \pm \mathbf{0 . 2 5}) \mathbf{g} / \mathbf{c m}^{\mathbf{3}}$ & $\mathbf{\%}$ \\
\hline $\mathrm{CeO}_{2-\mathrm{d}}$ & 7.209 & 7.11 & 98.6 \\
$\mathrm{Ce}_{0.9} \mathrm{Pr}_{0.1} \mathrm{O}_{2-\mathrm{d}}$ & 7.197 & 6.63 & 92.1 \\
$\mathrm{Ce}_{0.9} \mathrm{Yb}_{0.1} \mathrm{O}_{2-\mathrm{d}}$ & 7.345 & 7.29 & 99.3 \\
$\mathrm{Ce}_{0.9} \mathrm{Eu}_{0.1} \mathrm{O}_{2-\mathrm{d}}$ & 7.190 & 6.76 & 94.0 \\
$\mathrm{Ce}_{0.9} \mathrm{~Tb}_{0.1} \mathrm{O}_{2-\mathrm{d}}$ & 7.304 & 7.25 & 99.3 \\
$\mathrm{Ce}_{0.9} \mathrm{Gd}_{0.1} \mathrm{O}_{2-\mathrm{d}}$ & 7.245 & 7.45 & 102.8 \\
$\mathrm{Ce}_{0.9} \mathrm{La}_{0.1} \mathrm{O}_{2-\mathrm{d}}$ & 7.061 & 7.05 & 99.8 \\
$\mathrm{Ce}_{0.9} \mathrm{Er}_{0.1} \mathrm{O}_{2-\mathrm{d}}$ & 7.314 & 7.12 & 97.4 \\
\hline
\end{tabular}

Table 3.1. Theoretical and experimental density of doped cerias.

The real operating conditions in which the object materials of this thesis should work involve high temperatures and gradients of $\mathrm{pO}_{2}$. In this context, different redox processes dependent on temperature and $\mathrm{pO}_{2}$ may occur. Therefore, the evolution of cell parameters associated to thermal expansion and oxygen vacancy formation is studied by means of high temperature XRD (HT-XRD). The measurements are done by heating the specimens in air up to $900{ }^{\circ} \mathrm{C}$ and cooling them down in nitrogen. The fluorite structure is maintained in the whole temperature range and corresponding cell parameters are plotted in Figure 3.6 for some selected compositions. $\mathrm{Ln}=\mathrm{La}, \mathrm{Gd}$ and $\mathrm{Eu}$ show a linear dependency of cell parameter with temperature, which relates to thermal expansion coefficients (TEC) in air and in He respectively. On the other hand, $\mathrm{Tb}$ - and Pr-doped cerias show an increase in the slope at ca. 480 and $550{ }^{\circ} \mathrm{C}$, respectively, when they are heated up in air. Accordingly, an additional chemical expansion coefficient (CEC) is calculated, which is assigned to the reduction of $\mathrm{Ln}^{4+}$ to $\mathrm{Ln}^{3+}$ and the associated release of molecular oxygen. CEC is not observed for Eu doped ceria, which confirms that there is not reduction of the cation either with the temperature or with lower $\mathrm{pO}_{2}\left(\mathrm{~N}_{2}\right)$. When $\operatorname{Pr}$ and $\mathrm{Tb}$ 
doped samples are cooled down in $\mathrm{N}_{2}$ the material cannot be re-oxidized due to the lack of molecular oxygen necessary for re-oxidation and only thermal expansion can be observed for the material in the achieved reduced state. TEC values in both atmospheres and CEC values in air are summarized in Table 3.2.

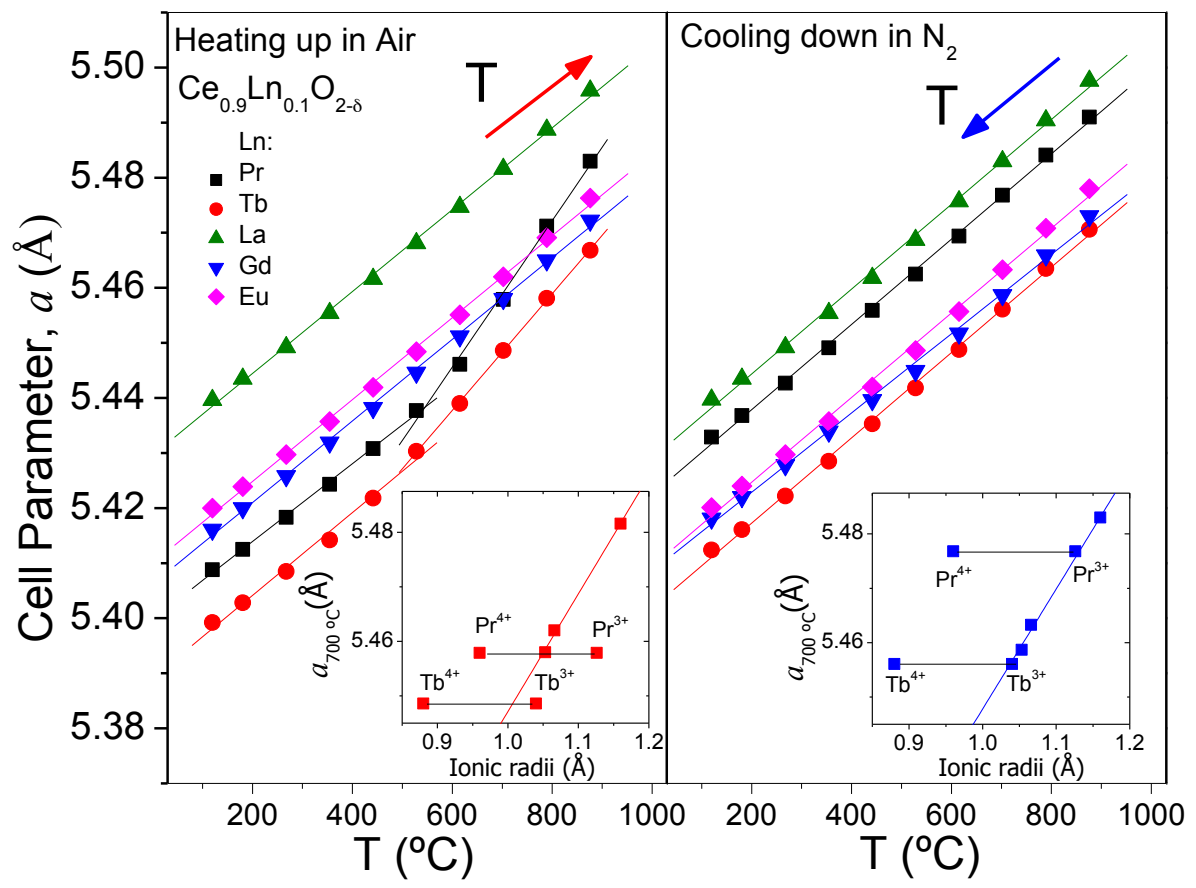

Figure 3.6. HT-XRD heating up in air (left side) and cooling down in $\mathrm{N}_{2}$ (right side) of $\mathrm{Ce}_{0.9} \mathrm{Ln}_{0.1} \mathrm{O}_{2-\delta}$. In the insets, the calculated cell parameters $\left(a_{700}{ }^{\circ} \mathrm{C}\right)$ at $700{ }^{\circ} \mathrm{C}$ in air (left side) and in $\mathrm{N}_{2}$ (right side) are plotted as a function of the trivalent ionic radii (and tetravalent for $\mathrm{Pr}$ and $\mathrm{Tb}$ ).

Based on the HT-XRD cell parameters and the ionic radii for the different oxidation states, it is possible to estimate the amount of trivalent cation for $\mathrm{Pr}$ and $\mathrm{Tb}$ by comparing with those theoretical cell parameters of totally reduced and oxidized samples. The insets of plot the cell parameters in air (left) and $\mathrm{N}_{2}$ (right) at $700{ }^{\circ} \mathrm{C}$ as a function of the ionic radii (trivalent and tetravalent 
when required). From room temperature (RT) to $700{ }^{\circ} \mathrm{C}$ in air the estimated amount of trivalent cation is increased from $40 \%$ to $53 \%$ for Pr and from $62 \%$ to $78 \%$ for $\mathrm{Tb}$. After heating the materials up to $900{ }^{\circ} \mathrm{C}$ in air only $9.4 \%$ of $\mathrm{Tb}^{4+}$ remains and $16 \%$ of $\operatorname{Pr}^{4+}$ is still present. By changing the atmosphere to $\mathrm{N}_{2}$ both materials are completely reduced and the concentration of trivalent cations remains fixed during the cooling down in $\mathrm{N}_{2}$ to room temperature. The relative amount of $\mathrm{Pr}$ and $\mathrm{Tb}$ cations in each of their oxidation states proved by HT-XRD is related to changes in the oxygen vacancy concentration whose importance will be discussed latter in the transport measurements.

\begin{tabular}{cccc}
\hline Ln & TEC in Air $\left(\mathrm{K}^{-1}\right)$ & TEC in $\mathrm{N}_{2}\left(\mathrm{~K}^{-1}\right)$ & CEC in Air $\left(\mathrm{K}^{-1}\right)$ \\
\hline $\mathrm{La}$ & $11.9 \times 10^{-6}$ & $12.2 \times 10^{-6}$ & \\
$\mathrm{Eu}$ & $11.9 \times 10^{-6}$ & $12.2 \times 10^{-6}$ & \\
$\mathrm{Gd}$ & $11.9 \times 10^{-6}$ & $11.4 \times 10^{-6}$ & \\
$\mathrm{Pr}$ & $11.3 \times 10^{-6}$ & $12.3 \times 10^{-6}$ & $11.51 \times 10^{-6}$ \\
$\mathrm{~Tb}$ & $12.1 \times 10^{-6}$ & $12.3 \times 10^{-6}$ & $5.77 \times 10^{-6}$ \\
\hline
\end{tabular}

Table 3.2. Thermal expansion coefficients calculated in Air and in $\mathrm{N}_{2}$ for different La, $\mathrm{Eu} \mathrm{Gd,} \mathrm{Pr}$ and $\mathrm{Tb}$ doped ceria.

The creation of oxygen vacancies by the dopants causes changes in the lattice bond that may be detected by Raman spectroscopy. $\mathrm{CeO}_{2}$ has a cubic fluorite structure that belongs to the $\mathrm{O}_{\mathrm{h}}^{5}(\mathrm{Fm} \overline{3} \mathrm{~m})$ space group. The $\mathrm{F}_{2}$ Raman-active mode frequency (at $465 \mathrm{~cm}^{-1}$ ) can be understood as a symmetric breathing mode of the $\mathrm{O}$ atoms around each cation (Ce-O8 unit). Therefore, this unit should be very sensitive to any disorder in the oxygen sublattice due to induced non-stoichiometry. [45] The normalized Raman spectra obtained for $\mathrm{Ce}_{0.9} \mathrm{Ln}_{0.1} \mathrm{O}_{2-\delta}$ series ( $\mathrm{Ln}=\mathrm{Pr}, \mathrm{Yb}, \mathrm{Eu}, \mathrm{Tb}, \mathrm{Gd}, \mathrm{La}$ ) are plotted in Figure 3.7a, where they are compared with pure ceria. There are also 
reported additional peaks close to $F_{2 \mathrm{~g}}$ mode, mainly a shoulder at $\sim 570 \mathrm{~cm}^{-1}$ attributed to the formation of oxygen vacancies (see inset of Figure 3.7a). The higher relative intensity observed for $\operatorname{Pr}$ and $\mathrm{Tb}$ of the $\sim 570 \mathrm{~cm}^{-1}$ shoulder is ascribed to the higher optical adsorption for these coloured compounds (dark red) and not only to the oxygen vacancy existence. [37] There is also a broad band at $600 \mathrm{~cm}^{-1}$ consequence of either an ordering effect in the oxygen vacancies to produce confinement of the phonons within an inhomogeneous distribution of domains or structural distortions of the O sublattice. [46]
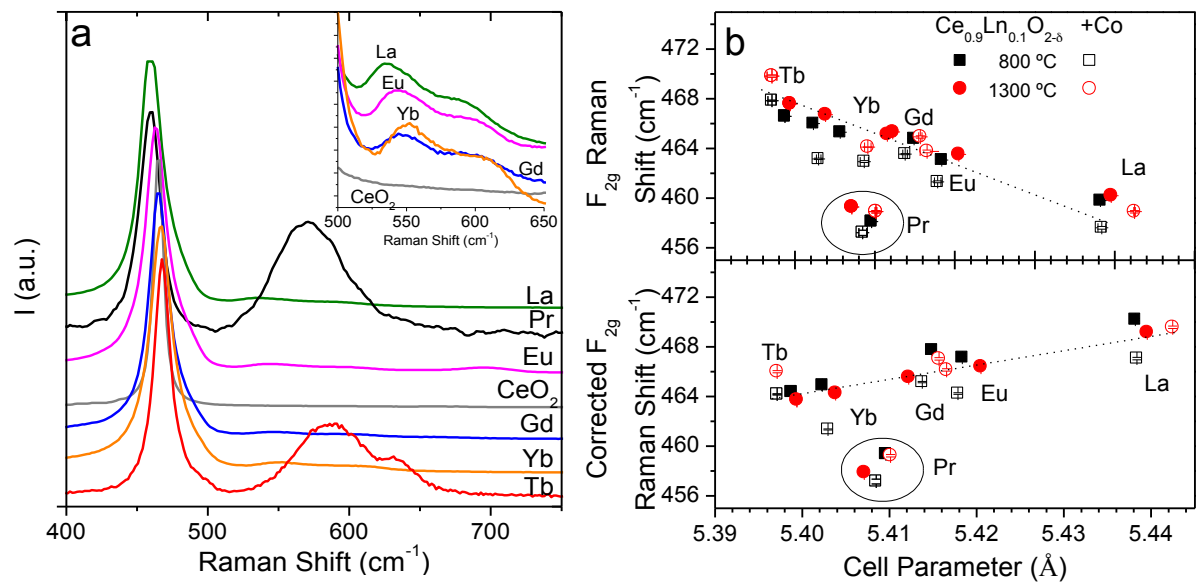

Figure 3.7. a) Raman spectra of the $\mathrm{Ce}_{0.9} \mathrm{Ln}_{0.1} \mathrm{O}_{2-\delta}$ ( $\mathrm{Ln}=\mathrm{La}, \mathrm{Gd}, \mathrm{Eu}, \mathrm{Pr}, \mathrm{Tb}, \mathrm{Yb}, \mathrm{Er}$ ) (sintered at $1300{ }^{\circ} \mathrm{C}$ ) from $400-750 \mathrm{~cm}^{-1}$ and b) $\mathrm{F} 2 \mathrm{~g}$ Raman shift as a function of the cell parameter $\mathrm{Ce}_{0.9} \mathrm{Ln}_{0.1} \mathrm{O}_{2-\delta}$ and $\mathrm{Ce}_{0.9} \mathrm{Ln}_{0.1} \mathrm{O}_{2-\delta}+\mathrm{Co}$ calcined at $1300{ }^{\circ} \mathrm{C}$ and $800{ }^{\circ} \mathrm{C}$ (top) and after subtracting Grüneisen shifts (bottom). The inset shows a magnification of the band related to the oxygen vacancy formation.

The frequency shift $(\Delta \omega)$ produced by a change in the lattice parameter $(\Delta a)$ can be written in terms of the Grüneisen parameter:

$\Delta \omega=-3 \gamma \omega_{0} \Delta \mathrm{a} / \mathrm{a}_{0}$ 
where $\omega_{0}$ and $a_{0}$ are the Raman frequency and the lattice parameter for $\mathrm{CeO}_{2}$ respectively, and $\gamma \equiv\left(\frac{B}{\omega}\right) \mathrm{d} \omega / \mathrm{dP}$ is the Grüneisen constant $\left(1.24\right.$ for $\mathrm{CeO}_{2}$ [37]). Therefore, the influence of the Ln doping on the structure can be detected by Raman spectroscopy. Figure $3.7 \mathrm{~b}$ (top) plots the Raman shift of the $F_{2 \mathrm{~g}}$ mode as a function of the cell parameter. Raman mode shifts down as the cell parameter increases and only $\operatorname{Pr}$ doped $\mathrm{CeO}_{2}$ does not follow the linear tendency. The general trend agrees with expected Raman shift due to cell volume changes.

Other contributions, apart from volume, can be analyzed if the Grüneisen shifts are taken into account. Figure 3.7b (bottom) shows corrected Raman frequencies after subtracting Grüneisen shifts, as a function of the cell parameter. Now, the Raman shift increases with the cell parameter except for Pr, again out of the general trend. This shift up in frequency in the $F_{2 g}$ mode can be directly assigned to the increasing disorder produced by the oxygen vacancies. [37] It can be observed that La-doped material present the highest shift, while the behaviour of the Pr-doped compound remains unexplained in this work.

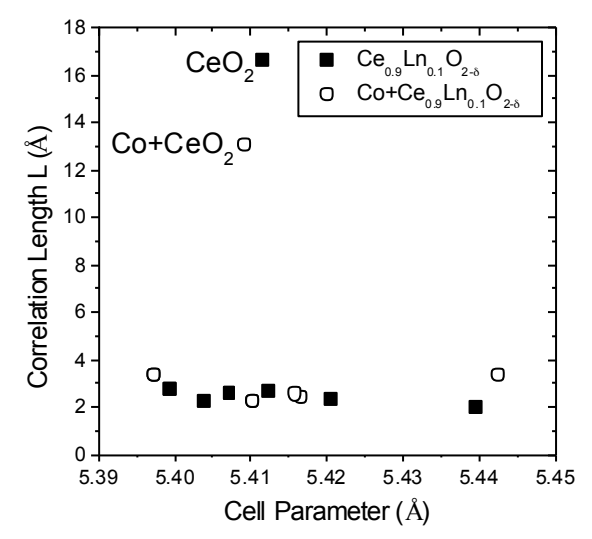

Figure 3.8. Correlation length obtained from Raman line-shape fitting as a function of the cell parameter. 
The defect concentration may be estimated from Raman intensity by using the spatial correlation length, [47] which considers them as spheres with diameters equal to the distance between two next oxygen vacancies. According to the spatial correlation model, Raman intensity $I(\omega)$ at a frequency $\omega$ can be written as [47]:

$$
I=\int_{0}^{1} \exp \left(\frac{-\mathrm{q}^{2} \mathrm{~L}^{2}}{4}\right) \frac{\mathrm{d}^{3} \mathrm{q}}{[\mathrm{w}-\mathrm{w}(\mathrm{q})]^{2}+(\Gamma / 2)^{2}}
$$

where the wave vector $q$ is expressed in $2 \pi / a$ units (a is the lattice parameter), $L$ is the correlation length, and $\Gamma$ is the linewidth of the Raman mode (FWHM). $\exp \left(\frac{-\mathrm{q}^{2} \mathrm{~L}^{2}}{4}\right)$ is the Gaussian spatial correlation function and $\omega(q)$ is the phonon dispersion for selected mode, represented by parabolic fits to the $\mathrm{CeO}_{2}$ phonon dispersion curves determined by Weber et al. [45] Figure 3.8 shows the correlation length obtained from Raman line-shape fitting as a function of the cell parameter. Correlation length is 1.7 and $1.3 \mathrm{~nm}$ for $\mathrm{CeO}_{2}$ (grain size $85 \mathrm{~nm}$ ) and $\mathrm{Co}$ doped $\mathrm{CeO}_{2}$ (grain size $87.7 \mathrm{~nm}$ ) respectively, while for the rare earth doped samples (with and without $\mathrm{Co}$ ) the correlation length decreases and it is shorter than the unit cell. It has been previously reported that when the oxygen vacancy concentration reaches $10^{21} \mathrm{~cm}^{-3}$, practically each unit cell is influenced by defects and then the correlation length, which is related to the short-range order parameter, should be attributed to the average oxygen-oxygen distance. When $10 \%$ of Ln dopant is introduced in ceria samples there is an induced oxygen defect concentration of around $4 \times 10^{21} \mathrm{~cm}^{-3}$ so, in this case, the correlation length corresponds to the oxygen - oxygen averaged distances, which is in all cases close to $2.71 \AA$. [48, 49] For pure and Co added ceria the defect concentration is three orders of magnitude smaller and the correlation length can be assigned to the distance between two next oxygen vacancies. This 
defect concentration can be seen as the volume between two next oxygen vacancies, and assuming they are spherical, it is possible to calculate the defect concentration $N$ as a function of the correlation length by $\mathrm{N}=\frac{3}{4 \pi \mathrm{L}^{3}}$. Then $5 \times 10^{19} \mathrm{~cm}^{-3}$ is obtained for $\mathrm{CeO}_{2}$ and $1 \times 10^{20} \mathrm{~cm}^{-3}$ for Co doped $\mathrm{CeO}_{2}$, which is in agreement with other reported values. [48]

Raman spectroscopy results demonstrate that the introduction of $\mathrm{Ln}^{3+}$ dopants produces a disorder due to the oxygen vacancies that increases with the cell parameter. The generated disorder is reflected in transport properties, whose features are analysed in Figure 3.9 and Figure 3.10. For the sake of simplicity dopants are displayed in two different plots and compared with $\mathrm{CeO}_{2}$ as a reference.

Figure 3.9a shows a representative sample of each kind of dopant: $\mathrm{CeO}_{2}$ as a reference, $\mathrm{Eu}(+2 /+3), \mathrm{Tb}$ and $\operatorname{Pr}(+3 /+4)$ and $\mathrm{Er}(+3)$. The Arrhenius plot for the conductivity in air of the selected materials shows that any dopant improves the total conductivity of pure ceria due to the generation of extrinsic vacancies. Trivalent dopants may enhance the ionic conductivity better than those in a mixed valence as $\mathrm{Tb}$ or $\mathrm{Pr}$, as they produce a fixed number of oxygen vacancies at this $\mathrm{pO}_{2}$. The oxygen release and consequent reduction of the dopant caused by the rising temperature results in a change of the Arrhenius slope (apparent activation energy) for $\mathrm{Tb}$ and $\mathrm{Pr}$ doped ceria in air. Actually, the temperature of change in the slope matches that observed by HT-XRD analysis. On the other hand, the conductivity results for the rest of samples available in Figure 3.10a ( $\mathrm{Yb}, \mathrm{Gd}, \mathrm{La}$ and $\mathrm{Nd})$ do not show such transition. Indeed, it is expected for fixed valence dopants and indicates that $\mathrm{Yb}$ and $\mathrm{Eu}$ remain in their +3 oxidation state. 

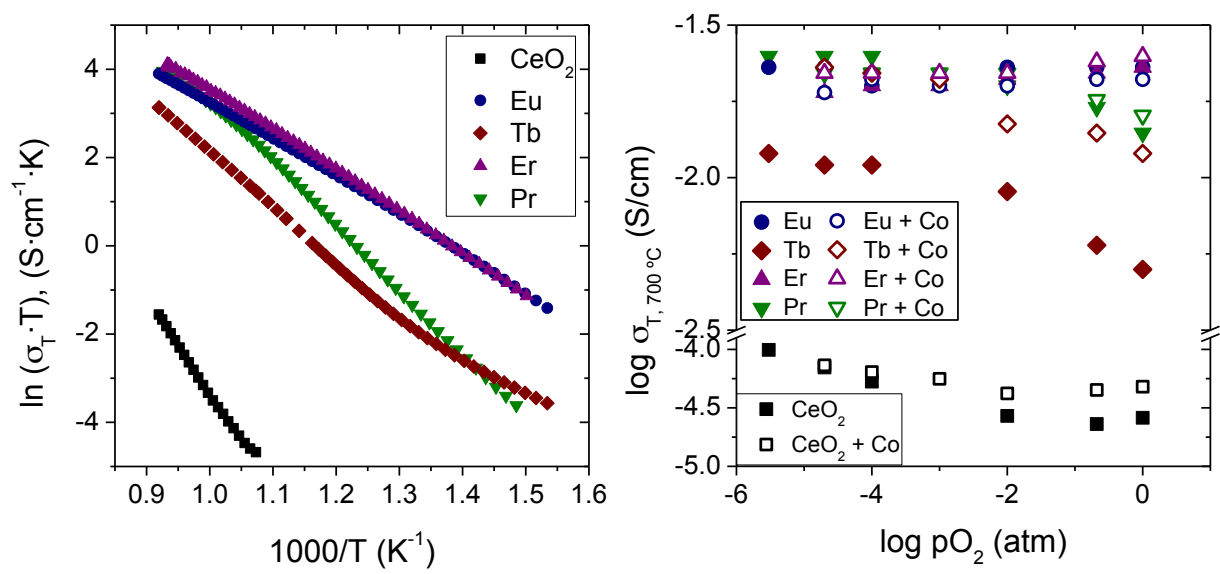

Figure 3.9. a) Arrhenius plot of the total conductivity in air of $\mathrm{CeO}_{2}$ and $\mathrm{Ce}_{0.9} \mathrm{Ln}_{0.1} \mathrm{O}_{2-\delta}$ $(\mathrm{Ln}=\mathrm{Tb}, \mathrm{Pr}, \mathrm{Eu}$ and $\mathrm{Er})$, and b) $\log -\log$ plot of total conductivity at $700{ }^{\circ} \mathrm{C}$ of the Cofree and Co-containing $\mathrm{CeO}_{2}$ and $\mathrm{Ce}_{0.9} \mathrm{Ln}_{0.1} \mathrm{O}_{2-\delta}(\mathrm{Ln}=\mathrm{Tb}, \mathrm{Pr}, \mathrm{Eu}$ and $\mathrm{Er})$ as a function of $\mathrm{pO}_{2}$.
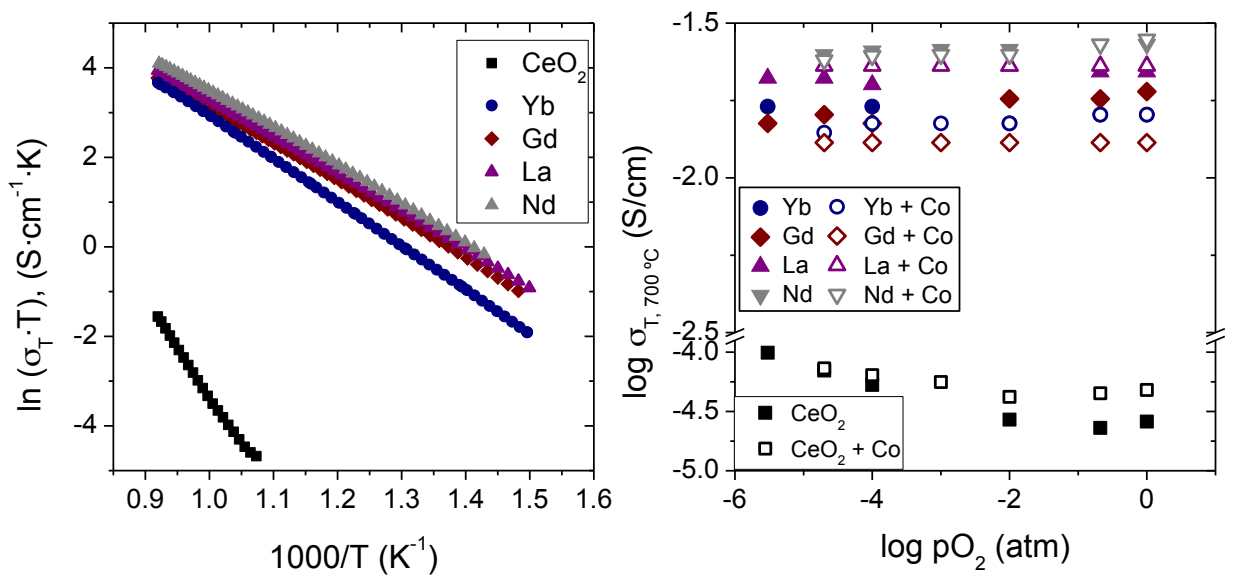

Figure 3.10. a) Arrhenius plot of the total conductivity in air of Co-free $\mathrm{Ce}_{0.9} \operatorname{Ln}_{0.1} \mathrm{O}_{2-\delta}$ $\mathrm{Ce}_{0.9} \mathrm{Ln}_{0.1} \mathrm{O}_{2-\delta}(\mathrm{Ln}=\mathrm{Yb}, \mathrm{Gd}, \mathrm{La}$ and $\mathrm{Nd})$ and b) $\log$-log plot of the total conductivity versus $\mathrm{pO}_{2}$ at $700{ }^{\circ} \mathrm{C}$ of the $\mathrm{Co}$-free and Co-containing $\mathrm{CeO}_{2}$ and $\mathrm{Ce}_{0.9} \mathrm{Ln}_{0.1} \mathrm{O}_{2-\delta}$ $\mathrm{Ce}_{0.9} \mathrm{Ln}_{0.1} \mathrm{O}_{2-\delta}(\mathrm{Ln}=\mathrm{Yb}, \mathrm{Gd}$, La and $\mathrm{Nd})$. 
Figure $3.9 \mathrm{~b}$ shows the conductivity dependence on the $\mathrm{pO}_{2}$ for the selected materials with and without Co taken at $700{ }^{\circ} \mathrm{C}$. The studied range of $\mathrm{pO}_{2}$ includes from oxidant (0.21-1 atm) to moderately reducing atmospheres $\left(10^{-5}-0.21 \mathrm{~atm}\right)$, where the $\mathrm{Ce}^{4+}$ does not reduce to $\mathrm{Ce}^{3+}$, since it has been demonstrated that this reaction initiates at oxygen partial pressures lower than $10^{-10} \mathrm{~atm}$. [27, 50-52] Except for $\mathrm{Tb}$ and $\mathrm{Pr}$, all the materials show a mainly ionic conductivity behaviour, which is independent on $\mathrm{pO}_{2}$. Tb and $\operatorname{Pr}$ doped ceria present a dependency on $\mathrm{pO}_{2}^{-1 / 6}$ at the most oxidizing atmospheres attributed to the dopant reduction as $\mathrm{pO}_{2}$ decreases, and which turns into a $\mathrm{pO}_{2}$ independent plateau. [53] Taking into account the defect chemistry explained in the Chapter 1 (see Table 1.2), the conductivity is identified as mainly ionic in these oxidizing conditions and the multiple oxidation state of some dopants makes the ionic conductivity $\mathrm{pO}_{2}$ dependent (region not observed in singleoxidation-state acceptor doped systems as $\mathrm{Er}$ and $\mathrm{Eu}$ ). In fact, this behaviour is well in agreement with the defect chemistry of an acceptor doped fluorite working in the boundary between region III and region IV developed in Figure 1.4. It is worthy to mention here that the defect concentration is also determined by temperature, so that there will be a different diagram at each temperature for any given compound. That means the $\mathrm{pO}_{2}$ region edges will shift as a function of temperature and related to the enthalpy of the diverse defect reactions.

In general the addition of cobalt to doped ceria improves the total conductivity being especially evident for $\mathrm{Tb}$ and $\mathrm{Pr}$. The conductivity enhancement is ascribed to the creation of percolating electronic paths through the grain boundary, where a nanosized Co-rich phase has been reported to be preferentially located. [27] Moreover, $\mathrm{Pr}$ and $\mathrm{Tb}$ with cobalt addition show enhanced total conductivity due to (a) the reducibility of the material at lower temperature with respect to the Co-free samples; (b) the electronic conductivity promotion; and (c) the higher sample density and grain packing due to the high 
sintering activity of Co-containing ceria observed by SEM (Figure 3.5). [27, 31, 42]

\section{Relationship between structural and transport properties}

From the conductivity features, it may be stated that the dopants in fixed trivalent state contribute only ionic charge carriers to the total conductivity. In that case, for the same dopant concentration, differences in conductivity should be related not only to the defects, but also to the mobility of ions over the oxygen vacancies formed in the fluorite lattice. In the following section the correlation between structural and transport properties of these series of lanthanide doped ceria is discussed. It has been widely reported that a dopant minimizing the internal strain will maximize the ionic conductivity as it has been traditionally observed for Gd or Sm doped ceria. [54] Some computational studies are devoted to the understanding of the factors that affect the ionic conductivity in doped ceria, as dopant ionic radius or clustering phenomena and how they are reflected in the mobility of ions. [35, 55, 56] They allow identifying and evaluating the energy barriers for diffusion inside the ceria lattice, which are related to the total activation energy $\left(E_{a}\right)$.

Fundamental physics behind the ionic conductivity determine that $E_{a}$ is equal to the sum of the vacancy formation energy $\left(E_{f}\right)$ and the migration barrier $\left(E_{m}\right)$ for pure ceria. The introduction of aliovalent dopants produces extrinsic oxygen vacancies whose effective charges are opposite so that they may attract each other forming a $\mathrm{Ln}_{\mathrm{Ce}}^{\prime}-\mathrm{V}_{\mathrm{O}}^{*}$ (vacancy-dopant) associate. Vacancy-dopant association energy $\left(E_{a s s}\right)$ also influences the vacancy mobility. Hence, $E_{a}$ can be understood as the sum of $E_{m}$ and $E_{\text {ass }}$ for bulk diffusion in doped ceria. Quantum mechanical calculations on Sm-doped ceria have revealed $E_{m}$ values around $0.46 \mathrm{eV}$, when there are no trivalent ions around a vacancy, while $E_{m}$ values 
increase up to $\sim 0.5 \mathrm{eV}$ when neighboring dopant cations are present. This is due to the oxygen atom movement through a point close to large trivalent ions is less favorable than to move to smaller $\mathrm{Ce}^{4+}$ ions. $[56,57]$ On the other hand, $E_{\text {ass }}$ depends on the distribution and concentration of the trivalent ions. In the present work the concentration of trivalent ions is the same $(10 \%)$ for all dopants except for $\mathrm{Tb}$ and $\mathrm{Pr}$ that have a variable concentration depending on temperature and $\mathrm{pO}_{2}$. Thus, the vacancy-dopant distribution will be essential in the $E_{\text {ass }}$ performance.

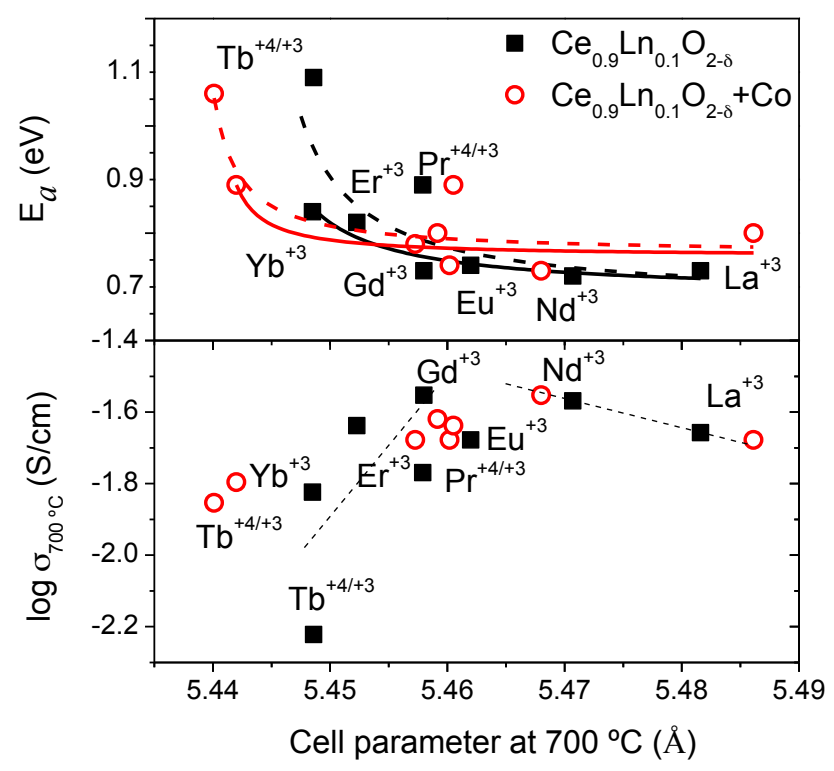

Figure 3.11: $E_{a}$ (top) and total conductivity at $700{ }^{\circ} \mathrm{C}$ in air (bottom) of the different doped materials versus cell parameter measured at $700{ }^{\circ} \mathrm{C}$. Cell parameters at $700{ }^{\circ} \mathrm{C}$ in air have been calculated from HT-XRD or by using TEC values for similar dopants. $E_{a}$ solid lines have been fitted to Eq. 4 only taken into account samples with trivalent dopants while dashed fits include $\operatorname{Pr}$ and $\mathrm{Tb}$.

The experimental $E_{a}$ values derived from the conductivity measurements corresponding to the different samples are represented in Figure 
3.11 (top) as a function of the cell parameter at $700{ }^{\circ} \mathrm{C}$ in air. $E_{a}$ has been calculated over the full temperature range for samples with trivalent dopants. For $\mathrm{Tb}$ and $\operatorname{Pr}$ doped ceria, the plotted $E_{a}$ was the one in the $800-650{ }^{\circ} \mathrm{C}$ range that includes the temperature at which the cell parameter was measured.

$E_{a}$ decreases with the increasing cell parameter of the materials following a trend proportional to $1 / a^{2}$ and tend to reach a fix value of $0.73 \mathrm{eV}$. The observed tendency can be easily related to coulombic interactions and steric effects due to the strong dependency of the displacement distance upon dopant type and relative dopant-vacancy positions. [58] The lowest $E_{a}$, obtained for samples with the largest cell parameters, are related to materials with an optimal balance between elastic (lattice distortions) and electronic defect interactions (redistribution of the electronic structure). When the cell parameter is shorter than that of pure ceria (which is approximately equal to Gd doped material) the structure is shrunk and $E_{a}$ follows Coulombic tendency. For longer cell parameters, from the point where the structure is not changed (Gd doped) up to the largest cell parameter (La doped) the Coulombic limit is overpassed and $E_{a}$ reaches a steady value defined by dopant concentration.

Experimental data has been fitted following Eq. 3.4

$$
E_{a}=E_{0}+\frac{A}{a^{2}-B^{2}}
$$

where $a$ is the calculated cell parameter at $700{ }^{\circ} \mathrm{C} ; E_{0}$ is the limit where Coulomb interactions do not affect the total energy for sufficient large cell parameters; $A$ is a constant that principally includes charges and electric constants; and $B$ is the minimum cell parameter that allows oxygen diffusivity. [58] 
Solid lines have been fitted to Eq. 3.4 including only samples with trivalent dopants and dashed fits also include $\operatorname{Pr}$ and $\mathrm{Tb}$. The deviation from general trend of these dopants, especially $\operatorname{Pr}$, is due to the remaining and variable tetravalent cations. This fact confirms the importance of the interatomic distances that govern $E_{a}$ behavior, as they present a cell parameter according to their mixed valence state (as discussed in Figure 3.4 and 3.6) and almost fall in the same trend as all the other trivalent dopants. In fact $E_{a}$ decreases for $\operatorname{Pr}$ and $\mathrm{Tb}$ doped samples at lower $\mathrm{pO}_{2}$ (the more trivalent dopant the higher vacancy density, and thus longer cell parameter) while trivalent dopants maintain their $E_{a}$ because there are not new structural changes. Raman measurements also confirm the $E_{a}$ behavior, since they predicted the highest oxygen vacancy disorder, and thus the lowest $E_{\text {ass }}$, for the longest cell parameters (Figure 3.7b) and the exceptional behavior of Pr doped samples.

By fitting to Eq. 3.4, a steady $E_{0}$ value of $0.70 \pm 0.08 \mathrm{eV}(0.68 \pm 0.16 \mathrm{eV}$ considering $\mathrm{Tb}$ and $\mathrm{Pr}$ ) for Co-free series and $0.77 \pm 0.08 \mathrm{eV}(0.76 \pm 0.13 \mathrm{eV}$ considering $\mathrm{Tb}$ and $\mathrm{Pr}$ ) for Co-containing samples have been obtained. The higher activation energy of Co-doped samples may result from the location of Co along the grain boundary as mentioned above. [42] It is worthy to note the opposite behavior of the $\mathrm{Tb}$ doped sample, whose $E_{a}$ decreases when Co is added. It may be attributed to the partial incorporation of Co in the ceria solid phase and increasing in oxygen vacancy concentration as will be studied in detail in Chapter 5. The minimum cell parameter that allows oxygen diffusivity, $B$, was found to be close to $5.4 \pm 0.9 \AA$. As the accuracy of $B$ parameter is not high enough, it can be found in the literature a minimum cell parameter of $5.3987 \AA$ corresponding to $\mathrm{Lu}$, the smallest rare earth dopant, which presents the minimum ionic conductivity. [59] Finally, $E_{a} \approx E_{0}$ is found for non-distorted cell materials and longer cell parameters i.e., Gd. 
The influence of the structural parameters on the transport properties is also illustrated in Figure 3.11 (bottom), which represents the total conductivity of all the samples (with and without Co) measured in air $700{ }^{\circ} \mathrm{C}$ as a function of the cell parameter at $700{ }^{\circ} \mathrm{C}$ in air. Predominant ionic conductivity is expected and, confirming reported studies, there is a maximum conductivity for Gd and $\mathrm{Nd}$ doped ceria due to the low association energy between the oxygen vacancies and the trivalent dopants and the lowest migration energy, minimized to those materials with cell parameters close to pure ceria, which represents the less distorted cell as well. [1, 59] Furthermore, it should be pointed out that electronic transport begins to contribute to the total transport for the $\mathrm{Tb}$ and $\mathrm{Pr}$ doped materials with Co addition, which could explain the dispersion of the data for these materials.

\subsection{Conclusions}

A large series of partially-substituted cerias has been prepared and characterized by XRD, $\mu$-Raman spectroscopy and conductivity measurements in order to have an overall understanding on the relationship of structural and transport properties. Several lanthanides ( $\mathrm{Gd}, \mathrm{La}, \mathrm{Tb}, \mathrm{Pr}, \mathrm{Eu}, \mathrm{Er}, \mathrm{Yb}$ and $\mathrm{Nd}$ ) have been disolved in the fluorite structure. XRD and HT-XRD shows that the doping produces modifications in the cell parameter of the pure ceria, which follows a linear dependency on the trivalent ionic radii of the dopants. $\mathrm{Tb}$ and $\operatorname{Pr}$ are out of this tendency due to their mixed $3+/ 4+$ oxidation state. The amount of each dopant in each oxidation state has been estimated finding that $\mathrm{Eu}$ and $\mathrm{Yb}$ remain always trivalent but the amount of trivalent $\mathrm{Pr}$ and $\mathrm{Tb}$ depends on temperature and atmosphere. Raman spectroscopy allowed studying the 
substitution effect and Co-doping on the final fluorite structure and estimating the vacancy disorder.

The lowest $E_{a}$ value was found for materials with long cell parameters, which is reached by the non distorted cell (Gd) and by cells with longer lattice parameters. This is ascribed to the decreasing Coulombic interactions combined with the lowest $E_{\text {ass }}$ due to the higher vacancy disorder, which was predicted by Raman. Once the cell parameter of the non distorted cell is overwhelmed, the increase in ionic radii is not joined by the ionic conductivity improvement. Vacancy-dopant concentration and distribution affect the total conductivity, giving rise to the highest ionic conductivity for the non distorted cells (Gd-doped ceria), which represent the optimum balance between Coulomb interactions, steric effects and vacancy distribution.

This first screening permits the reorientation of the next steps on the research of new electronic and/or ionic materials. Questions about macrostructure optimization of the sintering aids, the possibility of the electronic promotion in multivalent doped ceria and potential in their redox reactions or the possibility to enhance or introduce new properties by co-doping the present materials will be formulated and discussed in the upcoming chapters.

\subsection{References}

[1] M. Mogensen, N.M. Sammes, G.A. Tompsett, Physical, chemical and electrochemical properties of pure and doped ceria, Solid State Ionics, 129 (2000) 63-94.

[2] A. Atkinson, Chemically-induced stresses in gadolinium-doped ceria solid oxide fuel cell electrolytes, Solid State Ionics, 95 (1997) 249-258. 
[3] R. Krishnamurthy, B.W. Sheldon, Stresses due to oxygen potential gradients in non-stoichiometric oxides, Acta Materialia, 52 (2004) 1807-1822.

[4] B.C.H. Steele, Appraisal of $\mathrm{Ce}_{1-\mathrm{y}} \mathrm{Gd}_{\mathrm{y}} \mathrm{O}_{2-\mathrm{y} / 2}$ electrolytes for IT-SOFC operation at $500{ }^{\circ} \mathrm{C}$, Solid State Ionics, 129 (2000) 95-110.

[5] H. Yahiro, K. Eguchi, H. Arai, Electrical properties and reducibilities of ceria-rare earth oxide systems and their application to solid oxide fuel cell, Solid State Ionics, 36 (1989) $71-75$.

[6] J.M. Serra, V.B. Vert, M. Betz, V.A.C. Haanappel, W.A. Meulenberg, F. Tietz, Screening of A-Substitution in the System $\mathrm{A}_{0.68} \mathrm{Sr}_{0.3} \mathrm{Fe}_{0.8} \mathrm{Co}_{0.2} \mathrm{O}_{3-\delta}$ for SOFC Cathodes, Journal of The Electrochemical Society, 155 (2008) B207-B214.

[7] M. Sahibzada, B.C.H. Steele, K. Zheng, R.A. Rudkin, I.S. Metcalfe, Development of solid oxide fuel cells based on a $\mathrm{Ce}(\mathrm{Gd}) \mathrm{O}_{2-\delta}$ electrolyte film for intermediate temperature operation, Catalysis Today, 38 (1997) 459-466.

[8] C. Sun, U. Stimming, Recent anode advances in solid oxide fuel cells, Journal of Power Sources, 171 (2007) 247-260.

[9] J.W. Fergus, Oxide anode materials for solid oxide fuel cells, Solid State Ionics, 177 (2006) 1529-1541.

[10] H.J. Park, G.M. Choi, Oxygen permeability of gadolinium-doped ceria at high temperature, Journal of the European Ceramic Society, 24 (2004) 1313-1317.

[11] J. Sunarso, S. Baumann, J.M. Serra, W.A. Meulenberg, S. Liu, Y.S. Lin, J.C. Diniz da Costa, Mixed ionic-electronic conducting (MIEC) ceramic-based membranes for oxygen separation, Journal of Membrane Science, 320 (2008) 13-41.

[12] D.P. Fagg, V.V. Kharton, A. Shaula, I.P. Marozau, J.R. Frade, Mixed conductivity, thermal expansion, and oxygen permeability of $\mathrm{Ce}(\mathrm{Pr}, \mathrm{Zr}) \mathrm{O}_{2-\delta}$, Solid State Ionics, 176 (2005) 1723-1730.

[13] P. Shuk, M. Greenblatt, Hydrothermal synthesis and properties of mixed conductors based on $\mathrm{Ce}_{1-\mathrm{x}} \mathrm{Pr}_{\mathrm{x}} \mathrm{O}_{2-\delta}$ solid solutions, Solid State Ionics, 116 (1999) 217-223.

[14] P. Jasinski, T. Suzuki, H.U. Anderson, Nanocrystalline undoped ceria oxygen sensor, Sensors and Actuators B: Chemical, 95 (2003) 73-77.

[15] M. Sugiura, Oxygen Storage Materials for Automotive Catalysts: Ceria-Zirconia Solid Solutions, Catalysis Surveys from Asia, 7 (2003) 77-87. 
[16] W.C. Chueh, C. Falter, M. Abbott, D. Scipio, P. Furler, S. M. Haile, A. Steinfeld, , High-Flux Solar-Driven Thermochemical Dissociation of $\mathrm{CO}_{2}$ and $\mathrm{H}_{2} \mathrm{O}$ Using Nonstoichiometric Ceria Science, 330 (2010) 1797-1801.

[17] A. Grirrane, A. Corma, H. García, Gold-Catalyzed Synthesis of Aromatic Azo Compounds from Anilines and Nitroaromatics, Science, 322 (2008) 1661-1664.

[18] A. Trovarelli, Catalytic Properties of Ceria and $\mathrm{CeO}_{2}-$ Containing Materials, Catalysis Reviews, 38 (1996) 439-520.

[19] T.H. Etsell, S.N. Flengas, Electrical properties of solid oxide electrolytes, Chemical Reviews, 70 (1970) 339-376.

[20] V.V. Kharton, F.M. Figueiredo, L. Navarro, E.N. Naumovich, A.V. Kovalevsky, A.A. Yaremchenko, A.P. Viskup, A. Carneiro, F.M.B. Marques, J.R. Frade, Ceria-based materials for solid oxide fuel cells, Journal of Materials Science, 36 (2001) 1105-1117.

[21] T. Mori, J. Drennan, J.-H. Lee, J.-G. Li, T. Ikegami, Oxide ionic conductivity and microstructures of Sm- or La-doped $\mathrm{CeO}_{2}$-based systems, Solid State Ionics, 154-155 (2002) 461-466.

[22] S.K. Tadokoro, E.N.S. Muccillo, Effect of Y and Dy co-doping on electrical conductivity of ceria ceramics, Journal of the European Ceramic Society, 27 (2007) 4261-4264.

[23] S. Maschio, E. Aneggi, A. Trovarelli, V. Sergo, Influence of erbia or europia doping on crystal structure and microstructure of ceria-zirconia (CZ) solid solutions, Ceramics International, 34 (2008) 1327-1333.

[24] H. Luo, K. Efimov, H. Jiang, A. Feldhoff, H. Wang, J. Caro, $\mathrm{CO}_{2}-$ Stable and Cobalt-Free Dual-Phase Membrane for Oxygen Separation, Angewandte Chemie International Edition, 50 (2011) 759-763.

[25] M.P. Lobera, J.M. Serra, S.P. Foghmoes, M. Sögaard, A. Kaiser, On the use of supported ceria membranes for oxyfuel process/syngas production, Journal of Membrane Science, 385-386 (2011) 154-161.

[26] T.S. Stefanik, H.L. Tuller, Ceria-based gas sensors, Journal of the European Ceramic Society, 21 (2001) 1967-1970.

[27] D.P. Fagg, S. García-Martin, V.V. Kharton, J.R. Frade, Transport Properties of Fluorite-Type $\mathrm{Ce}_{0.8} \mathrm{Pr}_{0.2} \mathrm{O}_{2-\delta}$ : Optimization via the Use of Cobalt Oxide Sintering Aid, Chemistry of Materials, 21 (2009) 381-391.

[28] G.B. Balazs, R.S. Glass, ac impedance studies of rare earth oxide doped ceria, Solid State Ionics, 76 (1995) 155-162. 
[29] E.Y. Pikalova, A.N. Demina, A.K. Demin, A.A. Murashkina, V.E. Sopernikov, N.O. Esina, Effect of doping with $\mathrm{Co}_{2} \mathrm{O}_{3}, \mathrm{TiO}_{2}, \mathrm{Fe}_{2} \mathrm{O}_{3}$, and $\mathrm{Mn}_{2} \mathrm{O}_{3}$ on the properties of $\mathrm{Ce}_{0.8} \mathrm{Gd}_{0.2} \mathrm{O}_{2-\delta}$, Inorg Mater, 43 (2007) 735-742.

[30] J.D. Nicholas, L.C. De Jonghe, Prediction and evaluation of sintering aids for Cerium Gadolinium Oxide, Solid State Ionics, 178 (2007) 1187-1194.

[31] E. Jud, L. Gauckler, The Effect of Cobalt Oxide Addition on the Conductivity of Ce0.9Gd0.1O1.95, J Electroceram, 15 (2005) 159-166.

[32] D. Perez-Coll, J.C. Ruiz-Morales, D. Marrero-Lopez, P. Nuñez, J.R. Frade, Effect of sintering additive and low temperature on the electrode polarization of CGO, Journal of Alloys and Compounds, 467 (2009) 533-538.

[33] D.P. Fagg, D. Perez-Coll, P. Nuñez, J.R. Frade, A.L. Shaula, A.A. Yaremchenko, V.V. Kharton, Ceria based mixed conductors with adjusted electronic conductivity in the bulk and/or along grain boundaries, Solid State Ionics, 180 (2009) 896-899.

[34] E.Y. Pikalova, A.A. Murashkina, V.I. Maragou, A.K. Demin, V.N. Strekalovsky, P.E. Tsiakaras, $\mathrm{CeO}_{2}$ based materials doped with lanthanides for applications in intermediate temperature electrochemical devices, International Journal of Hydrogen Energy, 36 (2011) 6175-6183.

[35] M. Yashima, T. Takizawa, Atomic Displacement Parameters of Ceria Doped with Rare-Earth Oxide $\mathrm{Ce}_{0.8} \mathrm{R}_{0.2} \mathrm{O}_{1.9}(\mathrm{R}=\mathrm{La}, \mathrm{Nd}, \mathrm{Sm}, \mathrm{Gd}, \mathrm{Y}$, and $\mathrm{Yb})$ and Correlation with Oxide-Ion Conductivity, The Journal of Physical Chemistry C, 114 (2010) 2385-2392.

[36] A. Nakamura, New defect-crystal-chemical approach to non-Vegardianity and complex defect structure of fluorite-based $\mathrm{MO}_{2}-\mathrm{LnO}_{1.5}$ solid solutions $\left(\mathrm{M}^{4+}=\mathrm{Ce}\right.$, Th; $\mathrm{Ln}^{3+}=$ lanthanide): Part II: Detailed local-structure and ionic-conductivity analysis, Solid State Ionics, 181 (2010) 1631-1653.

[37] J.R. McBride, K.C. Hass, B.D. Poindexter, W.H. Weber, Raman and X-ray studies of $\mathrm{Ce}_{1-\mathrm{x}} \mathrm{RE}_{\mathrm{x}} \mathrm{O}_{2-\mathrm{y}}$, where $\mathrm{RE}=\mathrm{La}, \mathrm{Pr}, \mathrm{Nd}, \mathrm{Eu}, \mathrm{Gd}$, and Tb, Journal of Applied Physics, 76 (1994) 2435-2441.

[38] F. Zhang, S.-W. Chan, J.E. Spanier, E. Apak, Q. Jin, R.D. Robinson, I.P. Herman, Cerium oxide nanoparticles: Size-selective formation and structure analysis, Applied Physics Letters, 80 (2002) 127-129.

[39] A. Kumar, S. Babu, A.S. Karakoti, A. Schulte, S. Seal, Luminescence Properties of Europium-Doped Cerium Oxide Nanoparticles: Role of Vacancy and Oxidation States, Langmuir, 25 (2009) 10998-11007. 
[40] R. Shannon, Revised effective ionic radii and systematic studies of interatomic distances in halides and chalcogenides, Acta Crystallographica Section A, 32 (1976) 751-767.

[41] M. Chen, B. Hallstedt, A.N. Grundy, L.J. Gauckler, $\mathrm{CeO}_{2}-\mathrm{CoO}$ Phase Diagram, Journal of the American Ceramic Society, 86 (2003) 1567-1570.

[42] E. Jud, Z. Zhang, W. Sigle, L. Gauckler, Microstructure of cobalt oxide doped sintered ceria solid solutions, J Electroceram, 16 (2006) 191-197.

[43] T. Zhang, P. Hing, H. Huang, J. Kilner, Sintering and grain growth of CoO-doped $\mathrm{CeO}_{2}$ ceramics, Journal of the European Ceramic Society, 22 (2002) 27-34.

[44] R. Yan, F. Chu, Q. Ma, X. Liu, G. Meng, Sintering kinetics of samarium doped ceria with addition of cobalt oxide, Mater. Lett., 60 (2006) 3605-3609.

[45] W.H. Weber, K.C. Hass, J.R. McBride, Raman study of $\mathrm{CeO}_{2}$ : Second-order scattering, lattice dynamics, and particle-size effects, Physical Review B, 48 (1993) 178-185.

[46] X. Wang, J.C. Hanson, G. Liu, J.A. Rodriguez, A. Iglesias-Juez, M. FernandezGarcia, The behavior of mixed-metal oxides: Physical and chemical properties of bulk $\mathrm{Ce}_{1-\mathrm{x}} \mathrm{Tb}_{\mathrm{x}} \mathrm{O}_{2}$ and nanoparticles of $\mathrm{Ce}_{1-\mathrm{x}} \mathrm{Tb}_{\mathrm{x}} \mathrm{O}_{\mathrm{y}}$, The Journal of Chemical Physics, 121 (2004) 5434-5444.

[47] S. Patil, S. Seal, Y. Guo, A. Schulte, J. Norwood, Role of trivalent La and Nd dopants in lattice distortion and oxygen vacancy generation in cerium oxide nanoparticles, Applied Physics Letters, 88 (2006) 243110-243113.

[48] I. Kosacki, T. Suzuki, H.U. Anderson, P. Colomban, Raman scattering and lattice defects in nanocrystalline $\mathrm{CeO}_{2}$ thin films, Solid State Ionics, 149 (2002) 99-105.

[49] G.n.1. Gouadec, P. Colomban, Raman Spectroscopy of nanomaterials: How spectra relate to disorder, particle size and mechanical properties, Progress in Crystal Growth and Characterization of Materials, 53 (2007) 1-56.

[50] D.P. Fagg, I.P. Marozau, A.L. Shaula, V.V. Kharton, J.R. Frade, Oxygen permeability, thermal expansion and mixed conductivity of $\mathrm{Gd}_{\mathrm{x}} \mathrm{Ce}_{0.8-\mathrm{x}} \mathrm{Pr}_{0.2} \mathrm{O}_{2-\delta}, \mathrm{x}=0$, 0.15, 0.2, Journal of Solid State Chemistry, 179 (2006) 3347-3356.

[51] K.L. Duncan, Y. Wang, S.R. Bishop, F. Ebrahimi, E.D. Wachsman, The role of point defects in the physical properties of nonstoichiometric ceria, Journal of Applied Physics, 101 (2007) 044906-044906. 
[52] C. Chatzichristodoulou, P.V. Hendriksen, A. Hagen, Defect Chemistry and Thermomechanical Properties of $\mathrm{Ce}_{0.8} \mathrm{Pr}_{\mathrm{x}} \mathrm{Tb}_{0.2-\mathrm{x}} \mathrm{O}_{2-\delta}$, Journal of The Electrochemical Society, 157 (2010) B299-B307.

[53] S.R. Bishop, T.S. Stefanik, H.L. Tuller, Electrical conductivity and defect equilibria of $\operatorname{Pr}_{0.1} \mathrm{Ce}_{0.9} \mathrm{O}_{2-\delta}$, Physical Chemistry Chemical Physics, 13 (2011) 10165-10173.

[54] M. Mogensen, D. Lybye, N. Bonanos, P.V. Hendriksen, F.W. Poulsen, Factors controlling the oxide ion conductivity of fluorite and perovskite structured oxides, Solid State Ionics, 174 (2004) 279-286.

[55] J. Hooper, A. Ismail, J.B. Giorgi, T.K. Woo, Computational insights into the nature of increased ionic conductivity in concentrated samarium-doped ceria: a genetic algorithm study, Physical Chemistry Chemical Physics, 12 (2010) 12969-12972.

[56] A. Ismail, J. Hooper, J.B. Giorgi, T.K. Woo, A DFT+U study of defect association and oxygen migration in samarium-doped ceria, Physical Chemistry Chemical Physics, 13 (2011) 6116-6124.

[57] D.A. Andersson, S.I. Simak, N.V. Skorodumova, I.A. Abrikosov, B.r. Johansson, Optimization of ionic conductivity in doped ceria, Proceedings of the National Academy of Sciences of the United States of America, 103 (2006) 3518-3521.

[58] C. Frayret, A. Villesuzanne, M. Pouchard, F. Mauvy, J.-M. Bassat, J.-C. Grenier, Identifying Doping Strategies To Optimize the Oxide Ion Conductivity in Ceria-Based Materials, The Journal of Physical Chemistry C, 114 (2010) 19062-19076.

[59] S. Omar, E.D. Wachsman, J.L. Jones, J.C. Nino, Crystal Structure-Ionic Conductivity Relationships in Doped Ceria Systems, Journal of the American Ceramic Society, 92 (2009) 2674-2681. 


\title{
Chapter 4. Microstructure \\ optimization of $\mathrm{Ce}_{0.9} \mathrm{Pr}_{0.1} \mathrm{O}_{2-\delta}+\mathrm{Co} 2 \mathrm{~mol} \%$
}

\begin{abstract}
Praseodymium doped ceria shows a combination of mixed ionic and electronic conductivity, redox catalytic properties and chemical compatibility with water and carbon dioxide at high temperatures. Minor additions of cobalt oxide have been demonstrated to be a sintering aid and an electronic conduction promoter. However, an excess of sintering temperature causes cobalt aggregation into the grain boundaries, which is demonstrated by scanning electron microscopy (SEM) and temperature programmed desorption (TPD). In this chapter, the evolution of the microstructure has been investigated in order to understand the role of Co and to optimize both electronic and oxygen ion transport properties.
\end{abstract}





\subsection{Introduction}

By doping with multivalent cations, mixed conduction in ceria may be further extended to higher oxygen partial pressure range $\left(10^{-5}-10^{-1}\right.$ atm). In the previous chapter, terbium and praseodymium doped ceria have shown MIEC properties arising from their possible $3+/ 4+$ valences at these $\mathrm{pO}_{2}$ thus being suitable for the development of oxygen permeation membranes. [1-3] However, the low electronic conductivity is still the bottleneck for the oxygen permeation. The addition of small amounts of cobalt oxide is a strategy to improve the electronic conductivity and the material sintering. Since this addition modifies the microstructure, the sintering conditions need to be optimized.

For that purpose, a systematic study of $\mathrm{Ce}_{0.9} \mathrm{Pr}_{0.1} \mathrm{O}_{2-\delta}+\mathrm{Co} 2 \mathrm{~mol} \%$ synthesized by co-precipitation and impregnation and sintered at different temperatures was carried out. XRD, FE-SEM and TEM were employed on the structural characterization. The electrochemical properties were studied by means of DC-conductivity, which results allow finding a correlation between the grain boundary length, composition and the conduction properties. The aim is to engineer the grain boundary contribution to the total conductivity by promoting the electronic conductivity and to optimize the compound micro and macrostructe for oxygen membrane applications. 


\subsection{Results and discussion}

The initial powders of $\mathrm{Ce}_{0.9} \mathrm{Pr}_{0.1} \mathrm{O}_{2-\delta}(\mathrm{CP})$ and $\mathrm{Ce}_{0.9} \mathrm{Pr}_{0.1} \mathrm{O}_{2-\delta}+\mathrm{Co} 2 \mathrm{~mol} \%$ (CPCo) after co-precipitation were calcined at $800{ }^{\circ} \mathrm{C}$, since this temperature is sufficient to ensure the elimination of the precursor nitrates and carbonates (Chapter 3). Subsequently, rectangular probes $\left(4 \times 0.4 \times 0.2 \mathrm{~cm}^{3}\right)$ of the powders fired at $800{ }^{\circ} \mathrm{C}$ were uniaxially pressed at $125 \mathrm{MPa}$ during 1 minute and subsequently sintered 5 hours at $900,1000,1100,1200,1300$ and $1400{ }^{\circ} \mathrm{C}$ in air atmosphere. Besides, one probe of $\mathrm{CP}$ was calcined at $1300^{\circ} \mathrm{C}$ for comparison with CPCo.

In first place, the effect of the Co addition on CP is considered. XRD patterns of $\mathrm{CP}$ and $\mathrm{CPCo}$ calcined at $1300{ }^{\circ} \mathrm{C}$ show a cubic fluorite structure, which is depicted in Figure 4.1a. [4] The obtained patterns indicate that praseodymium forms a solid solution and incorporates in the lattice of ceria. Diffraction peaks corresponding to any precursor or Co oxides were not observed on the initial powder to the limit of the apparatus. As previously reported, $\mathrm{CoO}_{\mathrm{x}}$ is a sintering aid that enables lowering the densification temperature with respect to the Co-free specimen. [5] Thus, CPCo sample shows sharper and more intense diffraction peaks for the same calcination temperature as $\mathrm{CP}$. This is a consequence of the grain size enlargement, which suggests that $\mathrm{CoO}_{\mathrm{x}}$ presence accelerates the densification rate and grain growth. As it has been reported and mentioned in Chapter 3, the addition of Co oxides may reduce the activation energy of grain growth of $\mathrm{CeO}_{2}$, causing larger grains for CPCo with respect to CP. [6, 7] SEM images in Figure 4.1a illustrate this effect for $\mathrm{CP}$ and $\mathrm{CPC}$ materials sintered at $1300^{\circ} \mathrm{C}$. 
a)

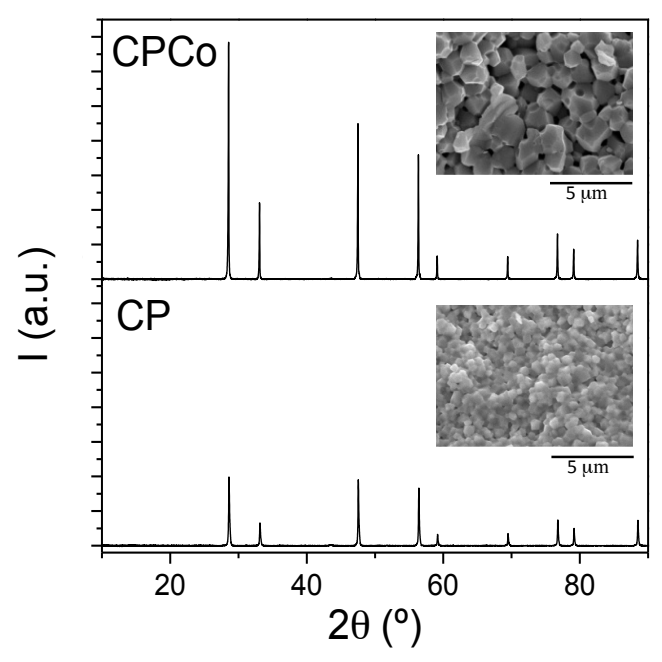

b)

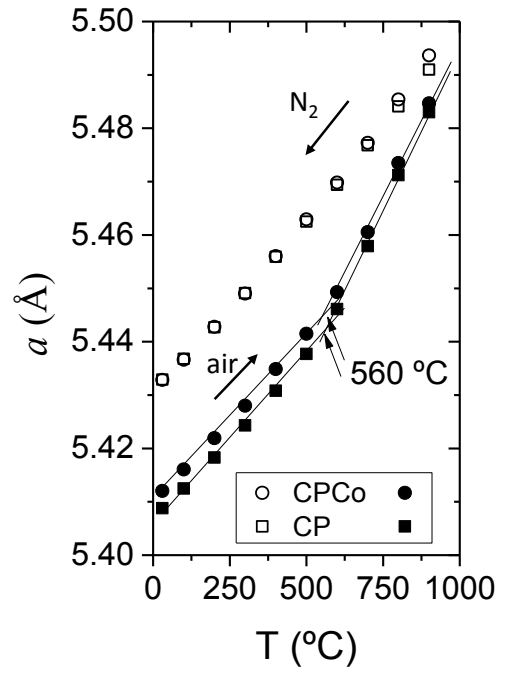

Figure 4.1. a) XRD patterns and SEM images and b) temperature dependence of cell parameter of $\mathrm{CP}$ and $\mathrm{CPCo}$ sintered at $1300^{\circ} \mathrm{C}$.

CPCo cell parameter, calculated from the XRD measured in air at room temperature, was $5.412 \pm 0.001 \AA$, slightly higher than that obtained for $\mathrm{CP}(5.407 \pm 0.001 \AA)$. This fact points out that (i) the Co is not incorporated in the ceria lattice, as it would result in the shrinkage of the cell parameter (the ionic radius of $\mathrm{Co}^{2+}$ in 8-fold coordination is $0.9 \AA$, smaller than that of $\operatorname{Pr}^{3+}$ and $\mathrm{Ce}^{4+}$ cations, 1.126 and $0.97 \AA$, respectively) and (ii) the catalytic effect of Co on the reduction of part of $\mathrm{Pr}^{4+}$ to $\mathrm{Pr}^{3+}$, with higher ionic radius and thus increasing the cell parameter. [8]

On the other hand, different redox processes dependent on temperature and $\mathrm{pO}_{2}$ occur at the most interesting operating conditions for this kind of materials as oxygen separation membranes and SOFC cathodes. In order to check the evolution of the cell parameter and associated vacancy concentration at high temperature, XRD measurements were performed on $\mathrm{CP}$ and $\mathrm{CPCo}$ sintered at $1300{ }^{\circ} \mathrm{C}$ by heating the specimens in air up to $900{ }^{\circ} \mathrm{C}$ and cooling 
them down in $\mathrm{N}_{2}$. The results plotted in Figure 4.1b show a linear dependence of cell parameter with temperature and an increase of the slope at $560{ }^{\circ} \mathrm{C}$. The first slope corresponds to the thermal expansion coefficient in air. Above $560{ }^{\circ} \mathrm{C}$, the increase in slope is caused by the additional contribution of chemical expansion due to the reduction of $\operatorname{Pr}^{4+}$ to $\operatorname{Pr}^{3+}$, the consequent expansion of the ionic radius and the associated oxygen release. From these results, a linear chemical expansion coefficient can be estimated from 830 to $900{ }^{\circ} \mathrm{C}$. After stabilization at the highest temperature the atmosphere is switched to $\mathrm{N}_{2}$ and the sample is cooled down. Both samples in $\mathrm{N}_{2}$ have similar cell parameters and expansion coefficients due to the achievement of totally reduced $\mathrm{Pr}^{3+}$ concentration at this $\mathrm{pO}_{2}$. [9] The material is not re-oxidized upon cooling due to the lack of molecular oxygen, and only thermal expansion/shrinkage can be observed. All the coefficients are summarized in Table 4.1 considering an experimental error of $1 \%$. It should be noted that the linear thermal expansion coefficient coincides for both oxidized and reduced material.

\begin{tabular}{cccc}
\hline Material & $\boldsymbol{\alpha}_{\mathbf{L}, \text { AIR1 }}(\mathbf{x 1 0}), \mathbf{K}^{-1}$ & $\boldsymbol{\alpha}_{\mathbf{L}, \mathbf{A I R 2}}\left(\mathbf{x 1 0}^{\mathbf{6}}\right), \mathbf{K}^{-1}$ & $\boldsymbol{\alpha}_{\mathbf{L}, \mathbf{N} 2}(\mathbf{x 1 0}), \mathbf{K}^{-1}$ \\
\hline CP & 11.4 & 11.4 & 12.3 \\
CPCo & 11.6 & 10.4 & 12.7 \\
\hline
\end{tabular}

Table 4.1 Thermal expansion coefficient of $\mathrm{Ce}_{0.9} \mathrm{Pr}_{0.1} \mathrm{O}_{2-\text { - }}$ and $\mathrm{CPCo} 2 \%$ sintered at $1300{ }^{\circ} \mathrm{C}$.

Temperature programmed reduction in $\mathrm{H}_{2}\left(\mathrm{H}_{2}-\mathrm{TPR}\right)$ measurements were done in order to analyze the redox properties of pure $\mathrm{CeO}_{2}$ and $\mathrm{CP}$. The effect of Co addition is studied as well for the two bulk compositions. The profiles depicted in Figure 4.2 demonstrate the higher reducibility of the Co containing samples in terms of lower temperature reduction and $\mathrm{H}_{2}$ consumption. $\mathrm{H}_{2}$-TPR Figure 4.2a shows the $\mathrm{H}_{2}$-TPR plot for the pure $\mathrm{CeO}_{2}$ and $\mathrm{CP}$ samples calcined in air at $1300{ }^{\circ} \mathrm{C}$. The $\mathrm{CeO}_{2}$ sample show peaks at 440 and 
$860{ }^{\circ} \mathrm{C}$, which are ascribed to the easily reducible surface oxygen and that in the bulk ceria, respectively. Doping with Pr, however, shows multiple reduction peaks. The first one around $400{ }^{\circ} \mathrm{C}$ overlaps several peaks attributed principally to $\operatorname{Pr}^{4+}$ reduction $\left(380{ }^{\circ} \mathrm{C}\right)$ and in a minor degree to $\mathrm{Ce}^{4+}$ surface reduction (shoulder at $440{ }^{\circ} \mathrm{C}$ as observed in pure ceria). The hydrogen consumption at this low temperature range corresponds to an oxygen substoichiometry of 0.05 . Consequently, a large percentage of Pr cations in ceria are originally expected in $\operatorname{Pr}^{4+}$ oxidized state. The second peak is attributed to the bulk $\mathrm{Ce}^{4+}$ reduction temperature, which is substantially diminished up to $720{ }^{\circ} \mathrm{C}$. This shift in temperature has been attributed in other works to the oxygen vacancies provided by the Pr, which increases the oxygen mobility in the lattice. [10]

a)

b)

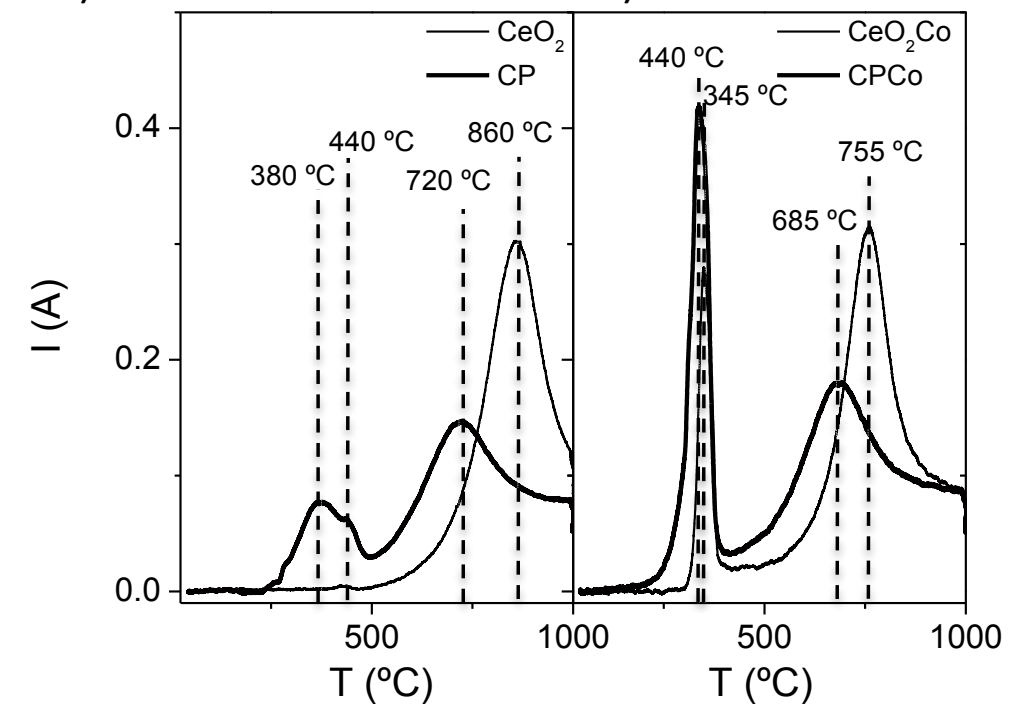

Figure 4.2. a) TPR profile of $\mathrm{CeO}_{2}$ and $\mathrm{CP}$ and b) TPR profile of $\mathrm{CeO}_{2}+2 \mathrm{~mol} \% \mathrm{Co}$ and CPCo. All the samples are samples sintered at $1300{ }^{\circ} \mathrm{C}$. The flow gas is $10 \% \mathrm{H}_{2}$ in Ar; heating ramp was $5^{\circ} \mathrm{C} / \mathrm{min}$. 
Figure $4.2 \mathrm{~b}$ represents the effect of Co on the reduction temperature. The spectrum of $\mathrm{CeO}_{2}$ presents a new reduction peak centered at $347{ }^{\circ} \mathrm{C}$ related to reducible species, $\mathrm{Co}^{3+2+}$ (which masks the surface $\mathrm{Ce}^{4+}$ peak). With respect to the bulk ceria, the reducibility temperature decreases around $100{ }^{\circ} \mathrm{C}$. The system becomes more complicated when the cobalt is added to Pr doped ceria. Up to four overlapped peaks can be found CPCo reduction in the range from 300 to $350{ }^{\circ} \mathrm{C}$. These peaks are related to the reduction of $\mathrm{Co}^{3+/ 2+}, \mathrm{Pr}^{4+/ 3+}$ and in a minor extent of surface $\mathrm{Ce}^{4+}$. Bulk ceria reduction occurs also at lower temperature, i.e., the maximum reduction peak is observed at $675^{\circ} \mathrm{C}$. Cobalt has a catalytic effect on the reduction of ceria which shifts bulk ceria reduction temperature in all cases. Moreover, the cobalt addition allows increasing the total amount of ceria reduced, i.e., the final oxygen substoichiometry. Specifically, the final vacancy concentration is increased as follows: $15 \%$ $\left(\mathrm{CeO}_{2-\mathrm{x}}\right)$, and $30 \%(\mathrm{CP})$. From the point of view of the material application (as oxygen membranes or SOFC electrodes), the improvement in the reducibility would bring the possibility of reducing the operation temperatures.

The following section focuses on the optimization of the CPCo sintering conditions. The variation of the sintering temperature may induce substantial changes in the microstructure and consequently in the redox and transport properties. [11] The SEM pictures of CPCo exposed in Figure 4.3 show how the grain size evolves as a function of the sintering temperature (from 900 to $1450{ }^{\circ} \mathrm{C}$ ). The grain size grows from the $60 \mathrm{~nm}$ of sample sintered at $900{ }^{\circ} \mathrm{C}$ up to $7 \mu \mathrm{m}$ at $1450{ }^{\circ} \mathrm{C}$. The density is improved as sintering temperature rises. 


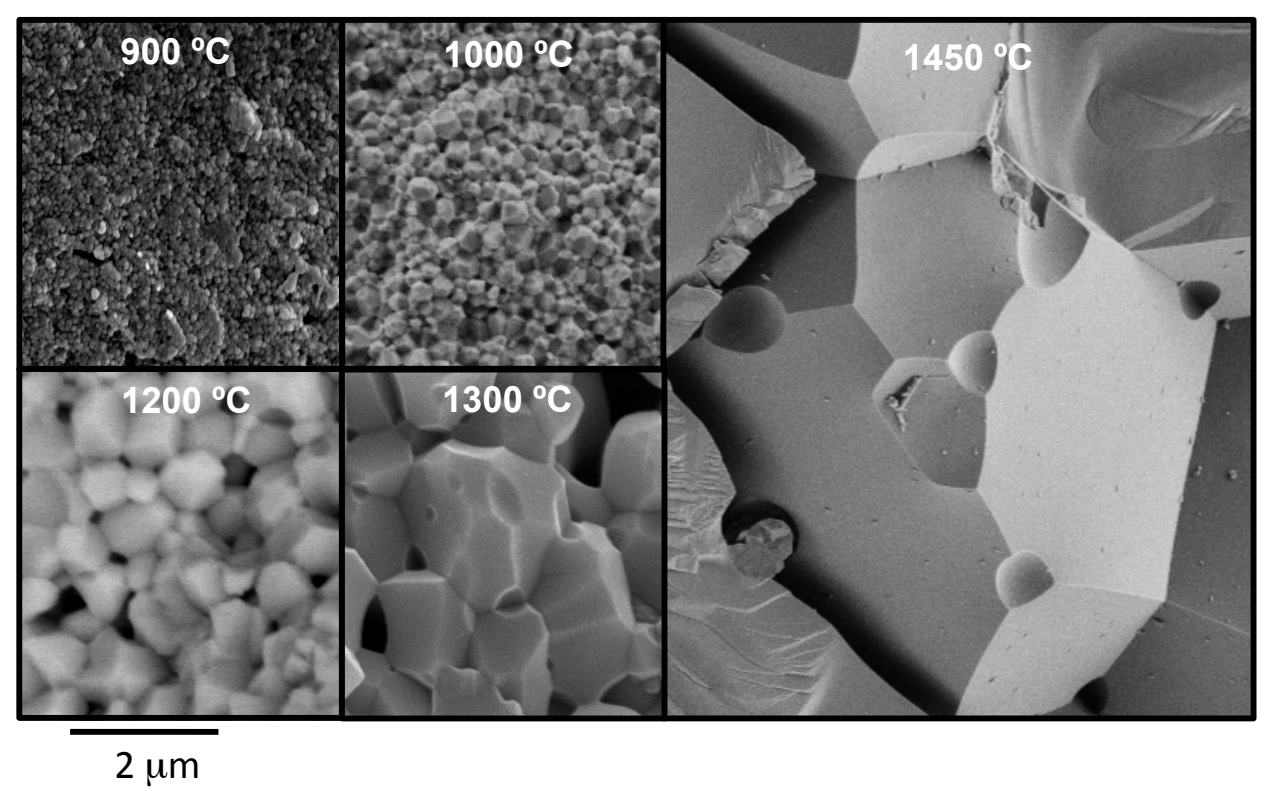

Figure 4.3 SEM pictures of CPCo for different sintering temperature: 900, 1000, 1200, 1300 and $1450{ }^{\circ} \mathrm{C}$. The grain size grows with the temperature from $60 \mathrm{~nm}$ at $900{ }^{\circ} \mathrm{C}$ to 7 $\mu \mathrm{m}$ at $1450^{\circ} \mathrm{C}$.

Changes in composition of the different grains and element distribution can be distinguished by backscattered electron (BS) FE-SEM. The pictures in Figure $4.4 \mathrm{a}$ and $\mathrm{b}$ show two different phases for both CPCo samples sintered at 1000 and $1450{ }^{\circ} \mathrm{C}$, respectively. An EDX mapping analysis (Figure 4.4d) over the BS image of Figure 4.4c indicates that the dark particles correspond to $\mathrm{CoO}_{\mathrm{x}}$ and spots its distribution along the grain boundary (GB). [14] Further, the sintering temperature influences the size of these $\mathrm{CoO}_{x}$ particles. The sample sintered at $1000{ }^{\circ} \mathrm{C}$ shows $\mathrm{CoO}_{\mathrm{x}}$ nanoparticles (below $50 \mathrm{~nm}$ ). However, at $1450{ }^{\circ} \mathrm{C}$ these nanoparticles appear spread over the grain boundary surface next to aggregates of 1.5-2 $\mu \mathrm{m}$ in a non-homogeneous distribution. 


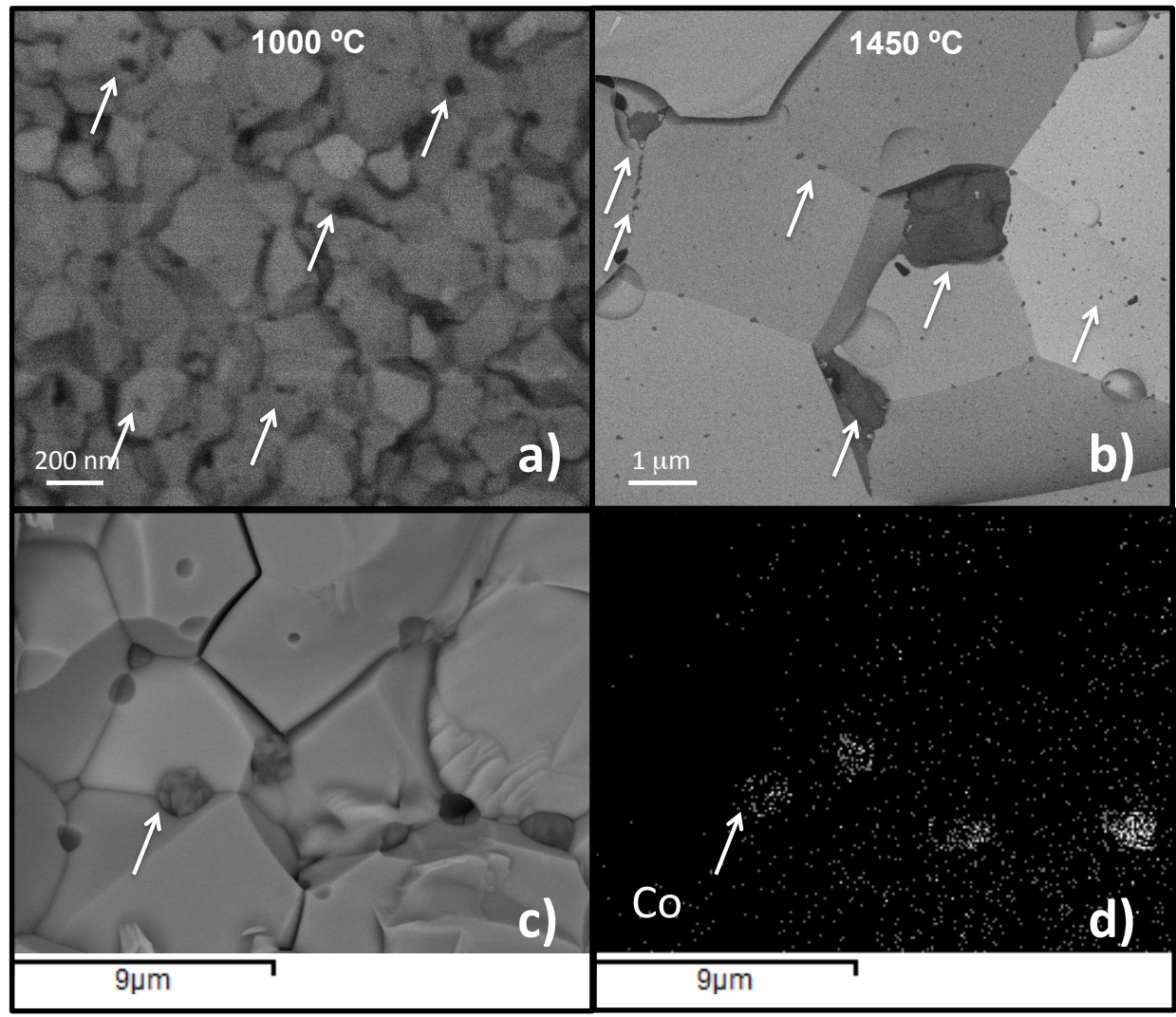

Figure 4.4. Back scattering SEM of CPCo sintered at a) $1000^{\circ} \mathrm{C}$ and b) $1450{ }^{\circ} \mathrm{C}$. c) Less magnified image of CPCo at $1450^{\circ} \mathrm{C}$ and d) EDS Co mapping indicating Co rich zones. Arrows indicate the presence of the $\mathrm{CoO}_{x}$ particles.

Indeed, the TEM images of a focused ion beam (FIB) lamella of Figure 4.5 show clean grain boundaries, with no reacting layers. The Co appears well distributed in the sample at $1000{ }^{\circ} \mathrm{C}$, with fine grain interface and it may be detected by EDS (Figure 4.6). On the other hand, the interface between grains of PCCo at $1450{ }^{\circ} \mathrm{C}$ is very fine and no traces of Co have been found by EDS, even in the fringes (Figure 4.7). This proves that the $\mathrm{CoO}_{\mathrm{x}}$ particles are out from the lattice, and they agglomerate as a secondary phase that was not possible to find in such a small lamella. 


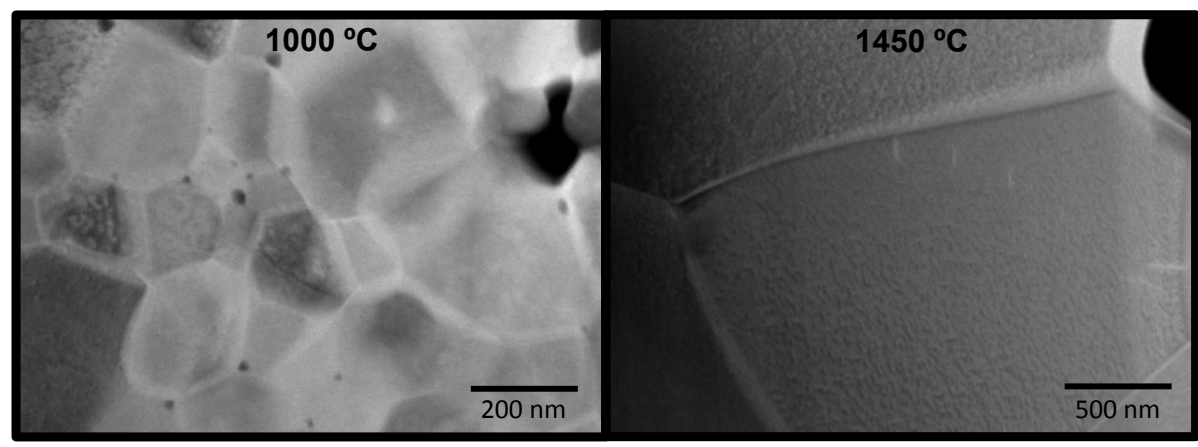

Figure 4.5. FIB-lamella TEM of CPCo sintered at $1000^{\circ} \mathrm{C}$ (left) and $1450^{\circ} \mathrm{C}$ (right).

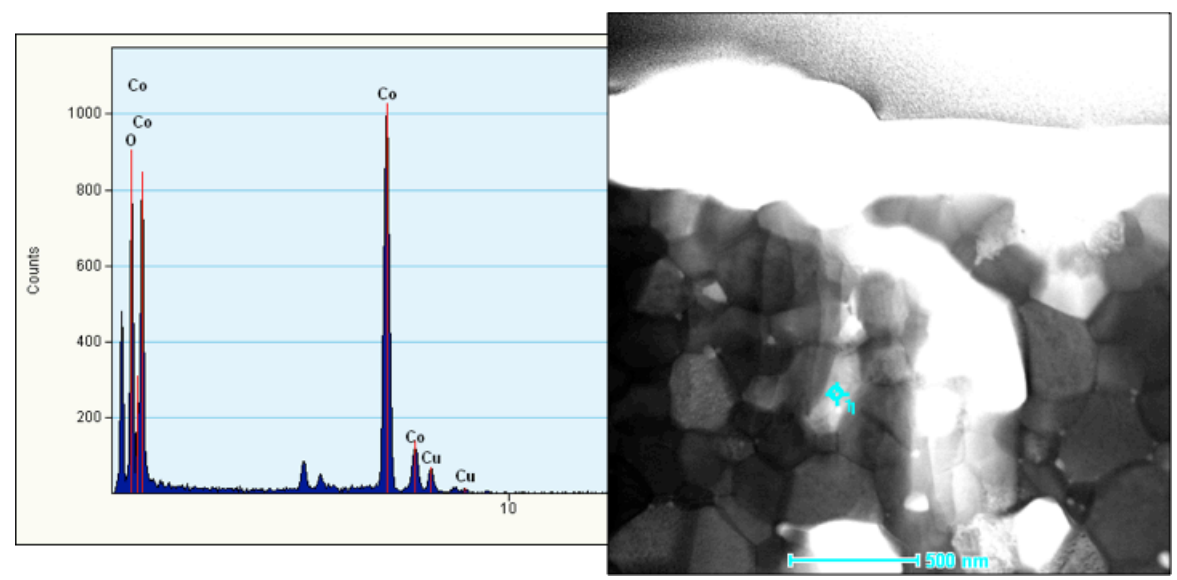

Figure 4.6. TEM-EDS on a $1000^{\circ} \mathrm{C} \mathrm{CPCo} \mathrm{grain.} \mathrm{Co} \mathrm{appears} \mathrm{dispersed} \mathrm{along} \mathrm{the} \mathrm{grain}$ surface, while fine $\mathrm{CP}-\mathrm{CP}$ grain interface is shown. 


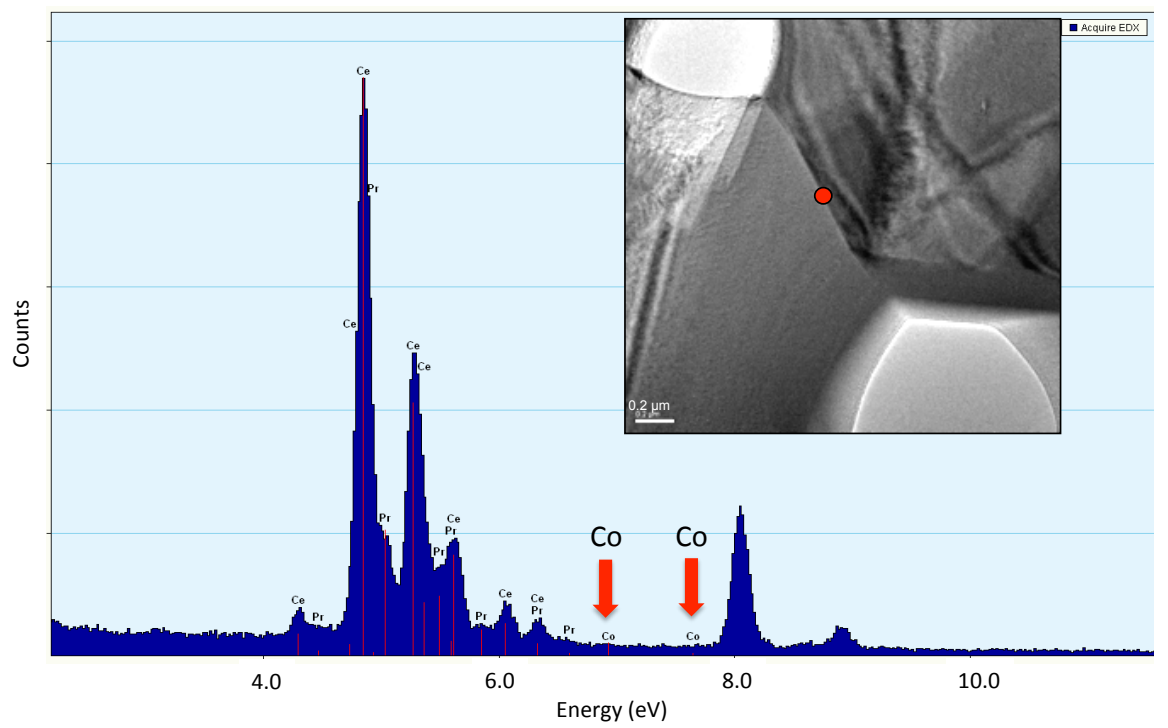

Figure 4.7. TEM-EDS on a $1450{ }^{\circ} \mathrm{C}$ CPCo grain boundary. A fine CP-CP grain interface is shown. Co was not detected either in the grain boundaries or in fringes and surfaces.

XRD patterns of CPCo samples sintered at 1000,1200 and $1450{ }^{\circ} \mathrm{C}$ are plotted in Figure 4.8a. All compositions demonstrate the cubic defect fluorite structure. More accurate XRD measurements of CPCo at 1000, 1200 and $1450{ }^{\circ} \mathrm{C}$ have revealed some low intensity peaks when they are represented in logarithmic scale. These peaks are due to the steel sample holder and not to the $\mathrm{CoO}_{\mathrm{x}}$ related species observed by FE-SEM, which indicates than the $2 \mathrm{~mol} \%$ of cobalt is below the resolution of the difractometer.

On the other hand, although all samples are fluorite structured and they have the same nominal composition, they may show different redox properties related to the microstructure. In order to check the oxygen release and simultaneous cation reduction and oxygen vacancy formation of the material, temperature programmed desorption (TPD) of CPCo sintered at 1000, 1200 and $1450{ }^{\circ} \mathrm{C}$ have been carried out in Ar as plotted in Figure 4.8b. For comparison, 
TPD of CP was also measured and plotted in Figure 4.8b (bottom). All compounds have a similar oxygen desorption peak at around $600{ }^{\circ} \mathrm{C}$. As $\mathrm{Ce}^{4+}$ reduction initiates at oxygen partial pressures lower than $10^{-10}$ atm $[1,8,12,13]$ and keeps nearly constant at higher oxygen contents (in the present study $p O_{2}>10^{-5} \mathrm{~atm}$ ), the observed reduction is most likely ascribed to the reduction of $\mathrm{Pr}^{4+} \rightarrow \operatorname{Pr}^{3+}$ and would cause the chemical expansion of the lattice previously observed by HT-XRD (Figure 4.1b). A second peak appears at around $790{ }^{\circ} \mathrm{C}$ only for the three Co containing samples, which indicates a different reduction process corresponding to $\left[\mathrm{Co}_{3} \mathrm{O}_{4}\right] \mathrm{Co}^{3+} \rightarrow \mathrm{Co}^{2+}[\mathrm{CoO}]$. [14] The relative intensity of this peak decreases as sintering temperature rises. It can be ascribed to the better Co distribution at lower sintering temperatures, as showed by SEM analysis.

a)

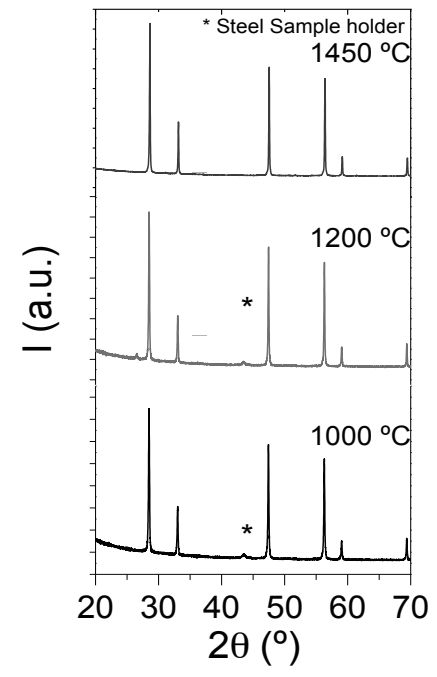

b)

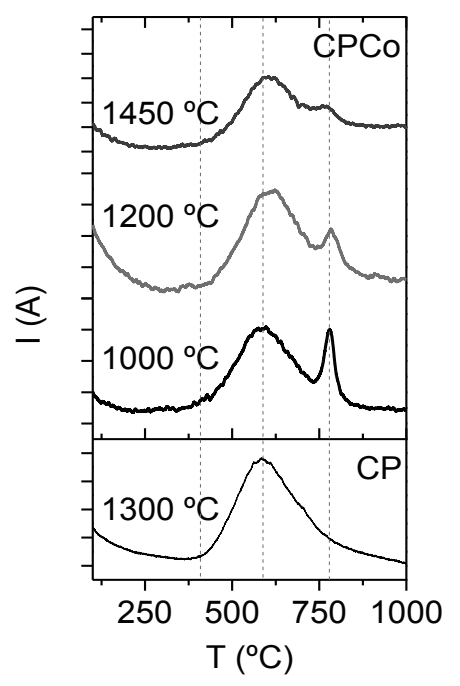

Figure 4.8. a) XRD patterns and b) TPD of oxygen in Ar for CPCo sintered at 1000, 1200 and $1450{ }^{\circ} \mathrm{C}$. TPD of CP is also depicted for comparison.

The oxygen-release thermal behavior, i.e., the specific oxygen nonstoichiometry, is directly related to the solid-state transport properties (both 
ionic and electronic transport). In this regard, the total conductivity in air at $800{ }^{\circ} \mathrm{C}$ and $400{ }^{\circ} \mathrm{C}$ is represented as a function of the sintering temperature in the Figure 4.9a. The highest total conductivity value observed at $400{ }^{\circ} \mathrm{C}$ is found for the sample sintered at the lowest temperature (i.e., $900{ }^{\circ} \mathrm{C}$ ). At $800^{\circ} \mathrm{C}$, the sample sintered at $1000{ }^{\circ} \mathrm{C}$ shows the highest total conductivity. The different behaviour observed at different operating temperatures suggests that the dominating transport mechanisms strongly depends on the operating temperature and sintering temperature of the sample.

a)

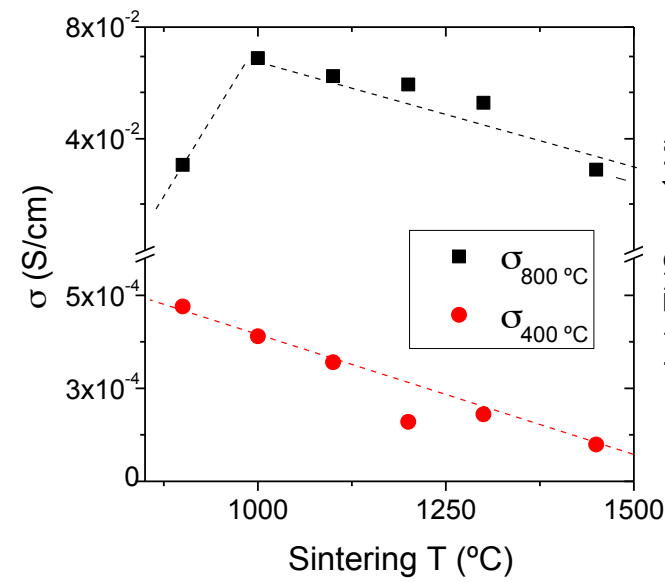

b)

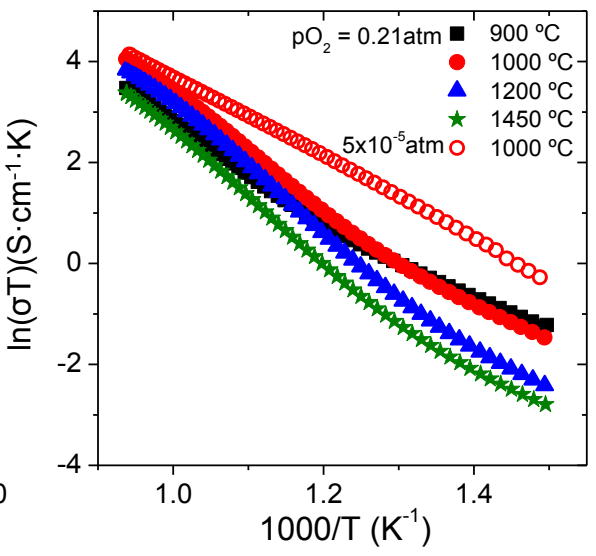

Figure 4.9. a) Total conductivity in air at $800{ }^{\circ} \mathrm{C}$ and $400{ }^{\circ} \mathrm{C}$ as a function of the sintering temperature and b) total conductivity of $\mathrm{CPCo}$ sintered at five different temperatures in air. For comparison, conductivity at $5 \times 10^{-5}$ atm for CPCo sintered at $1000{ }^{\circ} \mathrm{C}$ is plotted.

Figure $4.9 \mathrm{~b}$ shows the conductivity $\ln (\sigma \mathrm{T})$ as a function of the reverse temperature for the CPCo samples sintered at five different temperatures and measured in air. Changes in activation energy $\left(E_{a}\right)$ are observed at high $\mathrm{pO}_{2}$ as temperature drops due to oxygen re-incorporation. This process has been observed in Chapter 3 as a consequence of the thermal reduction of dopants that 
may be present in more than one oxidation state, as the case of Pr. Actually, $E_{a}$ change of CPCo in air is produced around $560^{\circ} \mathrm{C}$, which matches temperature range of the oxygen desorption/incorporation shown by HT-XRD Figure $4.1 \mathrm{~b}$ and by TPD (Figure 4.8). For comparison, the graph also plots the conductivity at $5 \times 10^{-5} \mathrm{~atm}$ for $\mathrm{CPCo}$ sintered at $1000^{\circ} \mathrm{C}$. At this $\mathrm{pO}_{2}$ (lower than $10^{-3} \mathrm{~atm}$ ), the oxygen incorporation in cooling down does not take place and only one $E_{a}$ is observed. The similar behavior of all the samples suggests that differences in conductivity are not caused by disparities in the composition.

In order to shed further light on the specific effect of the cobalt addition and minimize the influence of the microstructure change, the following comparison is done. Figure 4.10a plots the conductivity in air of (i) CPCo and Co-free CP sintered at $1300{ }^{\circ} \mathrm{C}$ and (ii) CPCo sintered at $1000^{\circ} \mathrm{C}$, which shows same grain size as $\mathrm{CP}$ sintered at $1300{ }^{\circ} \mathrm{C}$. As aforementioned (Figure 4.1), the addition of Co to $\mathrm{CP}$, causes the grain size growth. The conductivity at high temperatures is lower for $\mathrm{CPCo}$ with respect to $\mathrm{CP}$, possibly due to the grain size. However, at low temperatures, $\mathrm{CPCo}$ sintered at $1300{ }^{\circ} \mathrm{C}$ presents higher total conductivity, which is attributed to electronic contribution. When comparing samples with similar grain size, i.e., CPCo sintered at $1000{ }^{\circ} \mathrm{C}$ and $\mathrm{CP}$ sintered at $1300{ }^{\circ} \mathrm{C}$, the conductivity of the former is higher over the whole temperature range. This is ascribed to the homogeneous spreading of Co-enriched nanoparticles over the grains (observed in the BS/FE-SEM image Figure 4.4), which promotes the preferential percolation of electronic carriers through the grain boundaries. 
a)

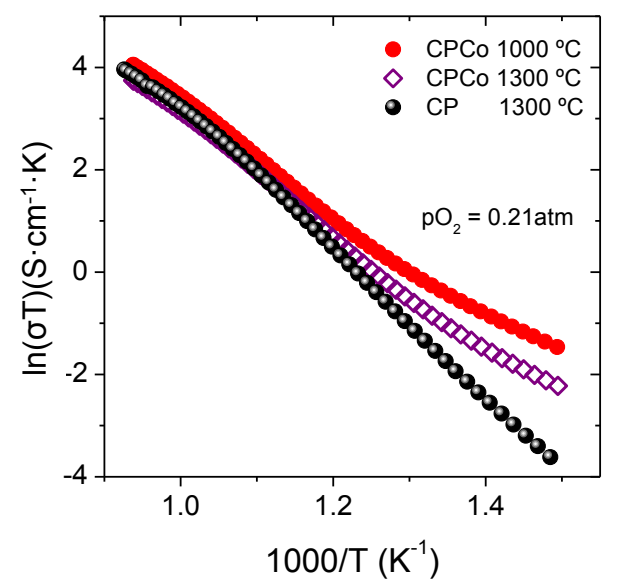

b)

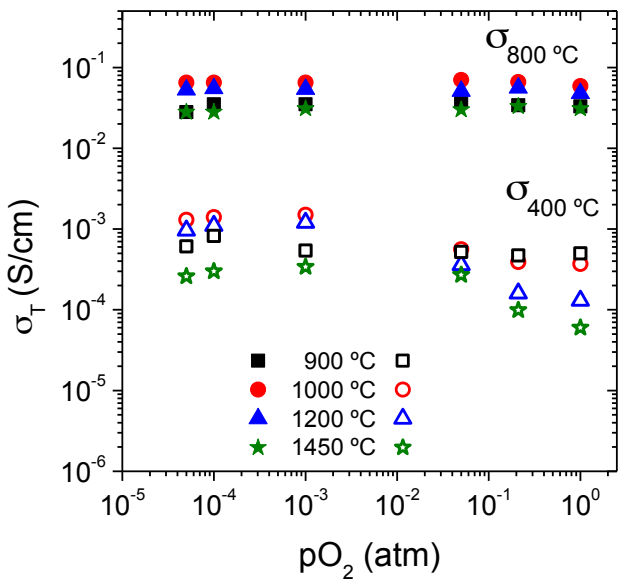

Figure 4.10. a) Arrhenius plot of the total conductivity of CPCo sintered at 1000 and $1300{ }^{\circ} \mathrm{C}$ and $\mathrm{CP}$ sintered at $1300{ }^{\circ} \mathrm{C}$, in air. b) $\mathrm{pO}_{2}$ dependence of the total conductivity of CPCo sintered at different temperatures, $400{ }^{\circ} \mathrm{C}$ (open symbols) and $800{ }^{\circ} \mathrm{C}$ (solid symbols).

The results described Chapter 3 show that different electronic and ionic charge carriers contribute to the total conductivity in multivalent doped ceria. The specific contribution depends on the dopant content, operating temperature range and $\mathrm{pO}_{2}$. Pr reduction (from $\mathrm{Pr}^{+4}$ to $\mathrm{Pr}^{+3}$ ) results in a $p O_{2}$ dependent ionic conductivity (-1/6 dependency) as well as in the introduction of electronic levels within the energy gap of ceria. $[15,16]$ On the other hand, the electronic conductivity improvement by the addition of Co has been reported. [17] Figure $4.10 \mathrm{~b}$ depicts the $\mathrm{pO}_{2}$ dependency of the total conductivity measured at 400 and $800{ }^{\circ} \mathrm{C}$, in a $\log$ - $\log$ representation. The conductivity at $400{ }^{\circ} \mathrm{C}$ of the CPCo specimen sintered $1450{ }^{\circ} \mathrm{C}$ follows a $\mathrm{pO}_{2}^{-1 / 6}$ dependence up to $p O_{2}=10^{-2} \mathrm{~atm}$, when it reaches a plateau. This dependence is stepwise attenuated as sintering temperatures are lower, up to the nearly independent-like behavior of CPCo sintered at $900{ }^{\circ} \mathrm{C}$. Such dependence mitigation may be due to the $p$-type electronic contribution, which follows a counter 1/6 slope. 
Since all the samples have the same nominal compositions, these behaviour differences should be most likely caused by microstructural issues. The conductivity enhancement observed for the low temperature sintered CPCo is related to their smaller grain size. Small grain size distribution produces the homogenous spreading of the Co additive, observed along the grain boundary (GB) area by BS FE-SEM (Figure 4.4a). As the sintering temperature rises, not only the fluorite grain size grows but also the Co-enriched particles aggregate and grow substantially in isolated positions among three or more fluorite grains (see Figure 4.4). Under this scenario, the electronic percolating network provided by the $\mathrm{CoO}_{\mathrm{x}}$ nanoparticles is not continuous and the contribution to the electronic conductivity along GB becomes negligible. In fact, the lowest conductivity values are obtained for the sample sintered at $1450{ }^{\circ} \mathrm{C}$, for which mainly ionic bulk transport through large grains. At $800{ }^{\circ} \mathrm{C}$ the conductivity for this sample is apparently $\mathrm{pO}_{2}$ independent, due to the prevailing contribution of ionic carriers, particularly considering that at this temperature most of the $\mathrm{Pr}$ cations are reduced to $\operatorname{Pr}^{3+}$ and the oxygen vacancies concentration is the highest. Under these conditions very large grains result in a very low electronic conductivity and the negligible effect of grain boundaries. Additionally, the transport in CPCo samples with very small grains will be more influenced by the grain boundary mechanisms, which in this case turns to be favorable for the mixed conductivity thanks to the well-distributed $\mathrm{CoO}_{\mathrm{x}}$ electronic percolating network. As a result of this microstructure, the most performing material is that sintered at $1000{ }^{\circ} \mathrm{C}$. This conductivity results suggest that by tailoring grain sizes and Co distribution along the GB, it is possible to modify not only total conductivity but also the nature of the main transport mechanism. 


\subsection{Conclusions}

This chapter summarizes the effect of the Co addition in praseodymiun doped cerias: (1) as sintering aid that increases the grain size; (2) as reduction promoter, increasing the proportion $\operatorname{Pr}^{3+}$ versus $\operatorname{Pr}^{4+}$ at high $p O_{2}$ and (3) as promoter of the total conductivity, most likely by enhancing the electronic transport.

The variation of the sintering temperature allowed the structural and transport properties of Co-doped $\mathrm{Ce}_{0.9} \mathrm{Pr}_{0.1} \mathrm{O}_{2-\delta}$ to be optimized. Specifically, important differences in fluorite grain size, grain boundary nature and composition as well as the oxygen release capacity have been found depending on the sintering temperature. The sample sintered at $1000{ }^{\circ} \mathrm{C}$ showed the highest oxygen release upon thermal treatment in argon and this is ascribed to the very high dispersion on the grain boundary and the nanoscale of cobalt-rich particles. Furthermore, the sample sintered at $1000{ }^{\circ} \mathrm{C}$ exhibited the highest total conductivity at high temperatures, which is directly related to the enhancement of the electronic conductivity. In summary, the mixed ionic electronic conductivity of the nanocomposite Co-doped $\mathrm{Ce}_{0.9} \mathrm{Pr}_{0.1} \mathrm{O}_{2-\delta}$ could be optimized by engineering the microstructure and grain boundary.

\subsection{References}

[1] C. Chatzichristodoulou, P.V. Hendriksen, A. Hagen, Defect Chemistry and Thermomechanical Properties of $\mathrm{Ce}_{0.8} \mathrm{Pr}_{\mathrm{x}} \mathrm{Tb}_{0.2-\mathrm{x}} \mathrm{O}_{2-\delta}$, Journal of The Electrochemical Society, 157 (2010) B299-B307.

[2] D.P. Fagg, A.L. Shaula, V.V. Kharton, J.R. Frade, High oxygen permeability in fluorite-type $\mathrm{Ce}_{0.8} \mathrm{Pr}_{0.2} \mathrm{O}_{2-\delta}$ via the use of sintering aids, Journal of Membrane Science, 299 (2007) 1-7. 
[3] C. Chatzichristodoulou, P.V. Hendriksen, A. Hagen, J.-C. Grivel, Oxygen Nonstoichiometry and Defect Chemistry Modelling of $\mathrm{Ce}_{0.8} \operatorname{Pr}_{\mathrm{x}} \mathrm{Tb}_{0.2-\mathrm{x}} \mathrm{O}_{2-\delta}, \mathrm{ECS}$ Transactions, 13 (2008) 347-359.

[4] S. Hull, S.T. Norberg, I. Ahmed, S.G. Eriksson, D. Marrocchelli, P.A. Madden, Oxygen vacancy ordering within anion-deficient Ceria, Journal of Solid State Chemistry, 182 (2009) 2815-2821.

[5] J.D. Nicholas, L.C. De Jonghe, Prediction and evaluation of sintering aids for Cerium Gadolinium Oxide, Solid State Ionics, 178 (2007) 1187-1194.

[6] T. Zhang, P. Hing, H. Huang, J. Kilner, Sintering and grain growth of CoO-doped $\mathrm{CeO}_{2}$ ceramics, Journal of the European Ceramic Society, 22 (2002) 27-34.

[7] R. Yan, F. Chu, Q. Ma, X. Liu, G. Meng, Sintering kinetics of samarium doped ceria with addition of cobalt oxide, Mater. Lett., 60 (2006) 3605-3609.

[8] D.P. Fagg, I.P. Marozau, A.L. Shaula, V.V. Kharton, J.R. Frade, Oxygen permeability, thermal expansion and mixed conductivity of $\mathrm{Gd}_{\mathrm{x}} \mathrm{Ce}_{0.8-\mathrm{x}} \mathrm{Pr}_{0.2} \mathrm{O}_{2-\delta}, \mathrm{x}=0$, 0.15, 0.2, Journal of Solid State Chemistry, 179 (2006) 3347-3356.

[9] C. Chatzichristodoulou, P.V. Hendriksen, Oxygen Nonstoichiometry and Defect Chemistry Modeling of $\mathrm{Ce}_{0.8} \mathrm{Pr}_{0.2} \mathrm{O}_{2-\delta}$, Journal of The Electrochemical Society, 157 (2010) B481-B489.

[10] B.M. Reddy, P. Saikia, P. Bharali, S.-E. Park, M. Muhler, W. Grünert, Physicochemical Characteristics and Catalytic Activity of Alumina-Supported Nanosized Ceria-Terbia Solid Solutions, The Journal of Physical Chemistry C, 113 (2009) 2452-2462.

[11] J. Ayawanna, D. Wattanasiriwech, S. Wattanasiriwech, P. Aungkavattana, Effects of cobalt metal addition on sintering and ionic conductivity of Sm(Y)-doped ceria solid electrolyte for SOFC, Solid State Ionics, 180 (2009) 1388-1394.

[12] D.P. Fagg, S. García-Martin, V.V. Kharton, J.R. Frade, Transport Properties of Fluorite-Type $\mathrm{Ce}_{0.8} \mathrm{Pr}_{0.2} \mathrm{O}_{2-\delta}$ : Optimization via the Use of Cobalt Oxide Sintering Aid, Chemistry of Materials, 21 (2009) 381-391.

[13] K.L. Duncan, Y. Wang, S.R. Bishop, F. Ebrahimi, E.D. Wachsman, The role of point defects in the physical properties of nonstoichiometric ceria, Journal of Applied Physics, 101 (2007) 044906-044906.

[14] C.-W. Tang, C.-B. Wang, S.-H. Chien, Characterization of cobalt oxides studied by FT-IR, Raman, TPR and TG-MS, Thermochimica Acta, 473 (2008) 68-73. 
[15] T.S. Stefanik, H.L. Tuller, Ceria-based gas sensors, Journal of the European Ceramic Society, 21 (2001) 1967-1970.

[16] S.R. Bishop, T.S. Stefanik, H.L. Tuller, Electrical conductivity and defect equilibria of $\operatorname{Pr}_{0.1} \mathrm{Ce}_{0.9} \mathrm{O}_{2-\delta}$, Physical Chemistry Chemical Physics, 13 (2011) 1016510173.

[17] D. Perez-Coll, J.C. Ruiz-Morales, D. Marrero-Lopez, P. Nuñez, J.R. Frade, Effect of sintering additive and low temperature on the electrode polarization of CGO, Journal of Alloys and Compounds, 467 (2009) 533-538. 


\title{
Chapter 5. Study of the bulk transport
}

\section{properties and oxygen surface exchange of \\ the mixed ionic electronic conductor \\ $C e_{1-x} T b_{x} O_{2-\delta}+C o(x=0.1,0.2,0.3,0.5)$}

\begin{abstract}
Structural, bulk ionic and electronic transport properties and surface operational of $\mathrm{Tb}$-doped ceria have been evaluated as a function of $\mathrm{Tb}$ concentration, aiming to assess potential use of the materials as high-temperature oxygentransport membrane. The materials were synthesized by the co-precipitation method. Cobalt oxide ( $2 \mathrm{~mol} \%$ ) was added in order to improve sinterability and conductivity. XRD measurements suggest that a part of the cobalt is incorporated in the ceria lattice. $\mathrm{Ce}_{1-\mathrm{x}} \mathrm{Tb}_{\mathrm{x}} \mathrm{O}_{2-\delta}$ materials showed predominantly ionic conductivity, but the mixed ionic electronic conductivity could be tuned by modifying $\mathrm{Tb}$ (and Co-doping) concentration and temperature. Low $\mathrm{Tb}$ content materials $(\mathrm{x}=0.1$ and 0.2$)$ are predominant ionic conductors, but the materials with $50 \mathrm{~mol} \% \mathrm{~Tb}$ show both $p$-type electronic and ionic conductivity. The enhanced electronic conduction in $\mathrm{Ce}_{0.5} \mathrm{~Tb}_{0.5} \mathrm{O}_{2-\delta}$ is associated with narrowing of the band gap upon doping ceria with $\mathrm{Tb}$. The ambipolar conductivity was determined by galvanic method coupled with gas permeation. These results support the applicability of these materials as oxygen transport
\end{abstract}


membranes at high temperature. $\mathrm{Ce}_{1-\mathrm{x}} \mathrm{Tb}_{\mathrm{x}} \mathrm{O}_{2-\delta}+\mathrm{Co} 2 \mathrm{~mol} \%$ membranes are $\mathrm{CO}_{2}$ stable and yield oxygen fluxes that can compete with reported perovskite materials.

In addition, the surface chemistry of the samples was investigated by means of X-ray photoelectron spectroscopy (XPS) and pulse isotopic exchange (PIE). The surface exchange rate was found to increase by increasing the level of $\mathrm{Tb}$ doping. The highest surface exchange rates in this study were found for materials doped with $50 \mathrm{~mol} \% \mathrm{~Tb}$. 


\subsection{Introduction}

As it was studied in chapter 3 the redox properties of $\mathrm{Pr}$ and $\mathrm{Tb}$ change depending on the temperature and oxygen partial pressures. The possibility of electron-hole hopping between dopant cations allows extending the mixed ionic-electronic conduction to higher oxygen partial pressures $\left(10^{-5}-10^{-1} \mathrm{~atm}\right)$, which enables its application as oxygen permeable membrane. $[1,2]$

Basic requirements for oxygen permeation are fast kinetics for bulk oxygen transport, e.g., via oxygen defect sites, and electron and/or electron hole transport, as well as fast surface oxygen exchange kinetics, involving dissociation of molecular $\mathrm{O}_{2}$, its reduction to oxygen adatoms, and successive incorporation in the oxide lattice as $\mathrm{O}^{2-}$ anions. These features have been exhaustively studied in the present chapter, where different compositions based on undoped and cobalt-doped $\mathrm{Ce}_{1-\mathrm{x}} \mathrm{Tb}_{\mathrm{x}} \mathrm{O}_{2-\delta}(\mathrm{x}=0.1,0.2,0.3,0.5)$ have been prepared and characterized to assess its applicability as an oxygen separation membrane. The influence of the dopant concentration is also examined.

Several bulk-related properties were investigated to gain insight into the transport properties of terbium-doped ceria. These were investigated by means of X-ray diffraction (XRD), temperature programmed desorption and reduction (TPD and TPR), thermogravimetry (TG), DC-conductivity in high $\mathrm{pO}_{2}$ range and UV-vis spectrophotometry, which enabled to gain insight into the conduction mechanisms dependent on $\mathrm{pO}_{2}$ and temperature. The surface chemistry of the samples was studied by X-ray photoelectron spectroscopy (XPS). Pulse isotopic exchange (PIE) experiments were conducted to measure 
the rate of oxygen surface exchange. Subsequently, $\mathrm{CO}_{2}$ stability, oxygen permeation and ion pumping experiments were done.

\subsection{Results and discussion}

\section{Structural characterization}

$\mathrm{XRD}$ patterns of $\mathrm{Ce}_{1-\mathrm{x}} \mathrm{Tb}_{\mathrm{x}} \mathrm{O}_{2-\delta}(\mathrm{CTx}, \mathrm{x}$ in percentage $)$ and $\mathrm{Ce}_{1-\mathrm{x}} \mathrm{Tb}_{\mathrm{x}} \mathrm{O}_{2-\delta}+\mathrm{Co} 2 \mathrm{~mol} \%$ (CTxCo) sintered at $1450^{\circ} \mathrm{C}$ are shown in Figure 5.1. All the powders are single phase with cubic fluorite structure. Within experimental error, diffraction peaks corresponding to any precursor or secondary phase related to $\mathrm{Tb}$ or Co oxides could not be detected. The obtained patterns indicate that terbium concentrations remain below the solubility limit of $\mathrm{Tb}$ in ceria compounds forming a solid solution within the lattice of ceria. [3, 4] However, reported studies on the microstructure of (Co free) Tb-doped ceria showed the presence of nano-sized precipitates in the fluorite-structured matrix, which are $\mathrm{Tb}^{3+}$ and $\mathrm{Ce}^{3+}$ rich. [5] As previously reported [6] and observed in Chapters 3 and 4, cobalt enables lowering the densification temperature and enhances the sintering. SEM pictures of CTx and CTxCo membranes calcined at $1200{ }^{\circ} \mathrm{C}$ showed in Figure 5.2 show the grain size growth difference caused by the addition of Co. [7] Besides, dark $\mathrm{CoO}_{\mathrm{x}}$ aggregates are observed, similar to that observed for Pr-doped ceria and other reported doped cerias, as Gd-doped. [8-10] 


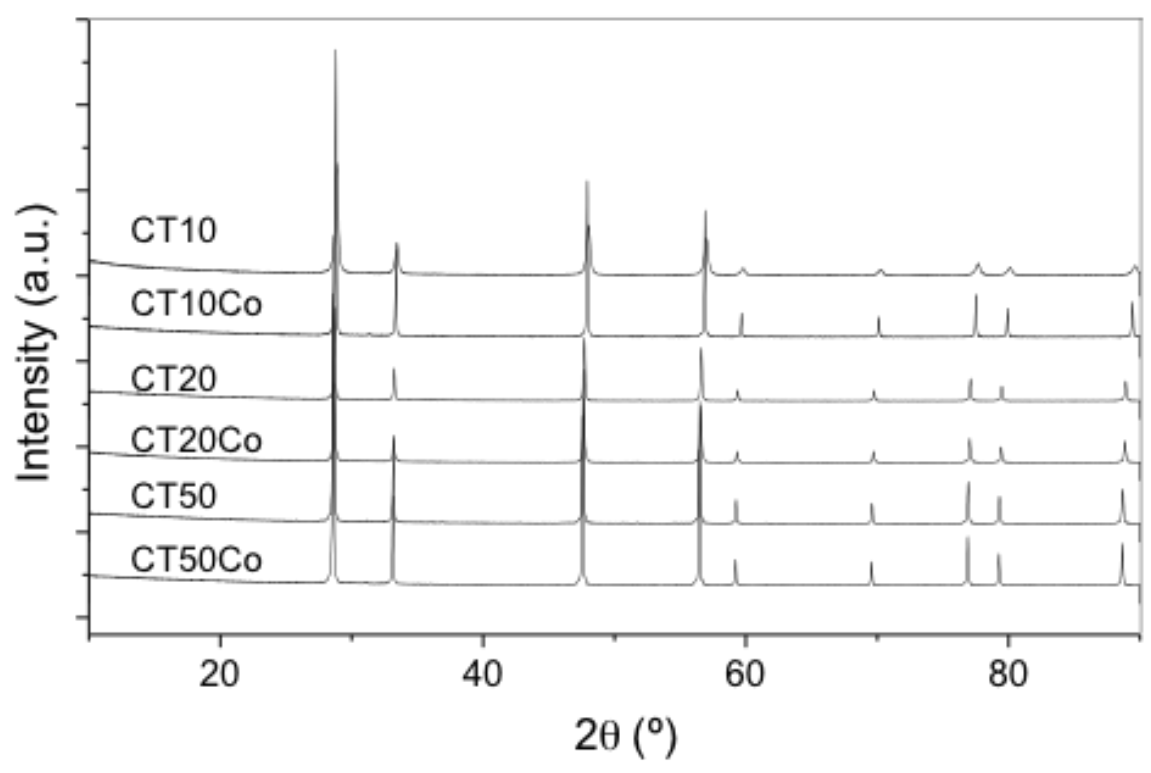

Figure 5.1. XRD patterns of CTx and CTxCo sintered at $1450{ }^{\circ} \mathrm{C}$ recorded at room temperature.

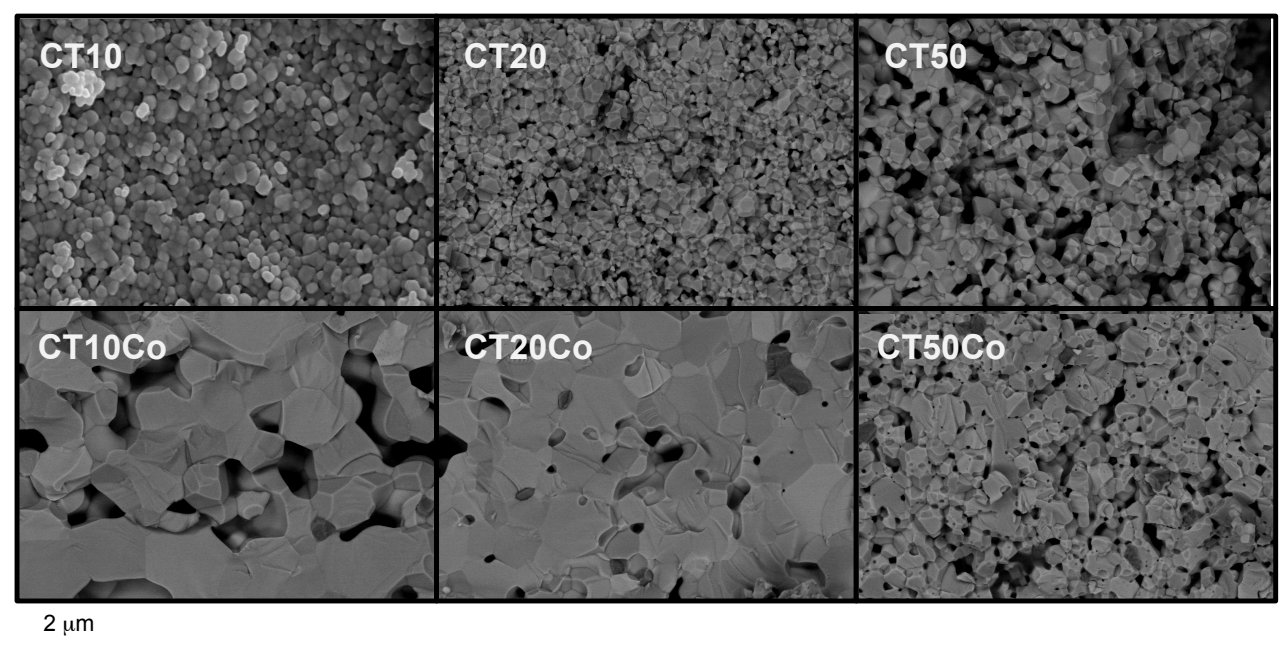

Figure 5.2. Scanning microscope pictures taken by backscattered electrons of CTx and CTxCo membrane cross sections, at $1200{ }^{\circ} \mathrm{C}$. 
Inside the solubility limits of a solid solution the lattice parameter of a fluorite crystal follows the Vegard's rule, i.e., a linear relationship exists at constant temperature between crystal lattice parameter and the concentration of the solute or dopant. $[4,11]$ For the ceria particular case the general relation is:

$a(\AA)=5.4113+\frac{4}{\sqrt{3}}\left[r_{M}-1.024\right] \cdot x$

where $r_{M}$ is the ionic radius of the dopant cation, $x$ is the dopant amount in $\mathrm{Ce}_{1-\mathrm{x}} \mathrm{Tb}_{x} \mathrm{O}_{2-\delta}$, and the constant $a$-intercept belongs to the non doped ceria cell parameter.

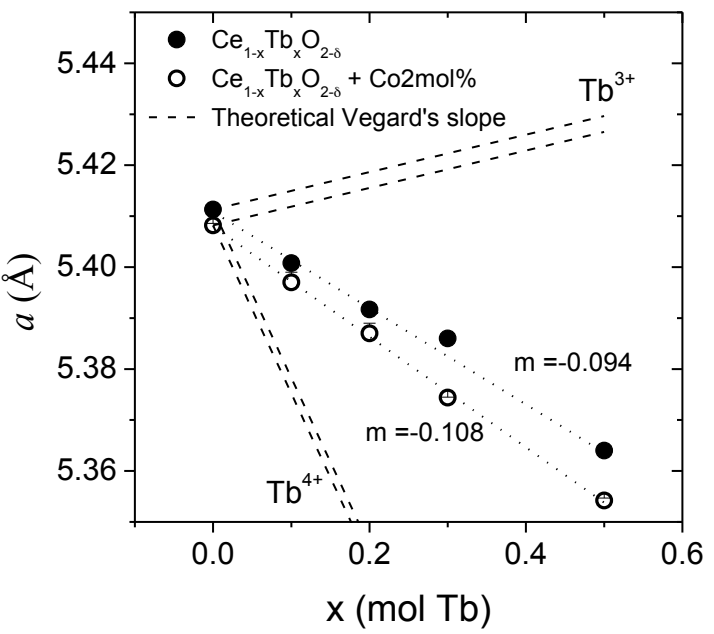

Figure 5.3 Cell parameter dependence on the dopant amount for CTx and CTxCo and theoretical Vegard's slope for $\mathrm{Tb}^{3+}$ and $\mathrm{Tb}^{4+}$ total substitution.

The cell parameters of CTx and CTxCo depicted in Figure 5.3 as a function of the concentration of $\mathrm{Tb}(x=0,0.1,0.2,0.3,0.5)$ are calculated from the XRD patterns measured at room temperature of samples sintered at $1450{ }^{\circ} \mathrm{C}$. The slope of this line is known as Vegard's slope. Also the theoretical Vegard's slope for $\mathrm{Tb}^{3+}$ and $\mathrm{Tb}^{4+}$ total substitution is drawn following Eq. 5.1. The mentioned general equation is recalculated taking into account the experimental 
cell parameters for $\mathrm{Tb}$ undoped ceria $\left(a_{\mathrm{CeO}_{2}}=5.411 \AA\right.$ and $\left.a_{\mathrm{CeO} 2 \mathrm{Co}}=5.408 \AA\right)$, which underwent the same treatment and came from the same raw precursors than doped specimens. They give a corrector factor from the perfect fluorite $(a=5.427 \AA$ ) of 0.9971 and 0.9965 for CTx and CTxCo, respectively. [3, 11]

$a\left(x_{T b}, r_{M}\right)=5.411+2.293 \cdot\left(r_{M}-1.024\right) \cdot x_{T b}$

$a\left(x_{T b}, r_{M}\right)=5.408+2.292 \cdot\left(r_{M}-1.024\right) \cdot x_{T b}$

Where the ionic radii are $r_{M}\left(T b^{4+}\right)=0.88 \AA$ and $r_{M}\left(T b^{3+}\right)=1.04 \AA$ for the cations in eight-fold coordination according to Shannon. [12] The reduction of Ce cations has been ruled out in the calculations since the non-stoichiometry of ceria and doped ceria materials has been exhaustively studied demonstrating that $\mathrm{Ce}^{4+}$ reduction initiates at oxygen partial pressures lower than $10^{-10} \mathrm{~atm}$ and keeps nearly constant at higher oxygen contents. [13-16] Consequently, in the present study where $\mathrm{pO}_{2}>10^{-5}$ atm, the changes observed in oxygen non-stoichiometry are most likely related to the reduction of $\mathrm{Tb}^{4+} / \mathrm{Tb}^{3+}$ rather than $\mathrm{Ce}^{4+}$.

For each series, Figure 5.3 shows the experimental cell parameters obtained in oxidizing atmospheres and at room temperature. They follow a nearly linear trend with the $\mathrm{Tb}$ concentration that lay inside the defined theoretical limits. Deviations from the Vegard's slope frequently indicate non-solubility of the dopant. $[3,11,17]$ However, single phase fluorite without impurities was observed for all the samples denoting that the experimentally observed deviations rather indicate that $\mathrm{Tb}$ is in a mixed valence. Considering the theoretical and experimental slopes for Co-free (solid circles) and Co-containing compounds (open circles), a rough estimation of the $\mathrm{Tb}^{4+} / \mathrm{Tb}$ proportion at room temperature can be done, i.e., 38\% (Co-free) and $48 \%$ (Co-containing). The first value agrees with that obtained by 
Chatzichristodoulou et al. for CT20 measured by XANES. [16] The lattice parameter decreases as $\mathrm{Tb}$ concentration rises and the calculated ratio $\mathrm{Tb}^{4+} / \mathrm{Tb}$ remains constant within the studied $\mathrm{Tb}$ concentration range.

On the other hand, the cell parameters of Co-containing compounds are slightly shorter than that of Co-free samples. This can be attributed to the partial incorporation of Co cations into the ceria lattice while the rest should be in the grain boundary. Specifically, incorporation of $1.05 \mathrm{~mol} \%$ of $\mathrm{Co}^{2+}$ would give rise to the cell parameter change observed in $\mathrm{CeO}_{2}$. Moreover, the Co addition causes the increase in the Vegard's slope, i.e., it produces a slight decrease in the cell parameter, when compared to Co-free samples. This is an indication of the higher average oxidation state of $\mathrm{Tb}$ cations in the Co-containing compounds previously calculated. It should be pointed out that such a shrinkage in cell parameter cannot be explained by a higher amount of incorporated Co in the lattice, since almost twice the Co nominally available $\mathrm{Co}^{2+}$ in eight-fold coordination is $0.90 \AA$ ) would be needed. Otherwise, it is well-known that cobalt cations/species have high redox activity and this enables to increase the reduction and re-oxidation rate of bulk material. [18] The improved reduction activity of cobalt on the overall material has been studied by temperature programmed oxygen desorption (TPD) in argon and temperature programmed reduction (TPR) in hydrogen. TPR measurements in Figure 5.4 show that introduction of the $\mathrm{Tb}$ dopant decreases the reduction temperature of $\mathrm{Ce}^{4+}$ to $\mathrm{Ce}^{3+}$. The addition of cobalt does not cause any change in the Ce reduction peak but it lowers the reduction temperature of $\mathrm{Tb}^{4+}$ to $\mathrm{Tb}^{3+}$, and causes higher overall reducibility of the material, i.e., a greater consumption of $\mathrm{H}_{2}$ due to the Co extra reduction peak. 
a)

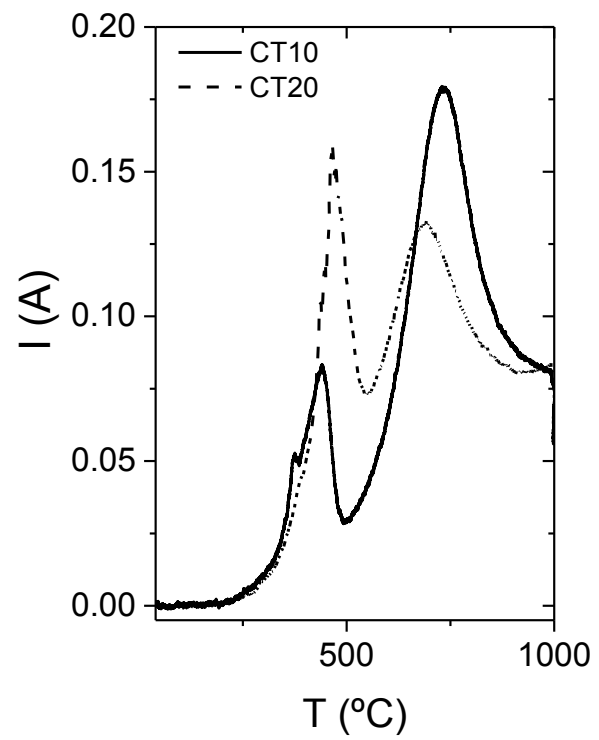

b)

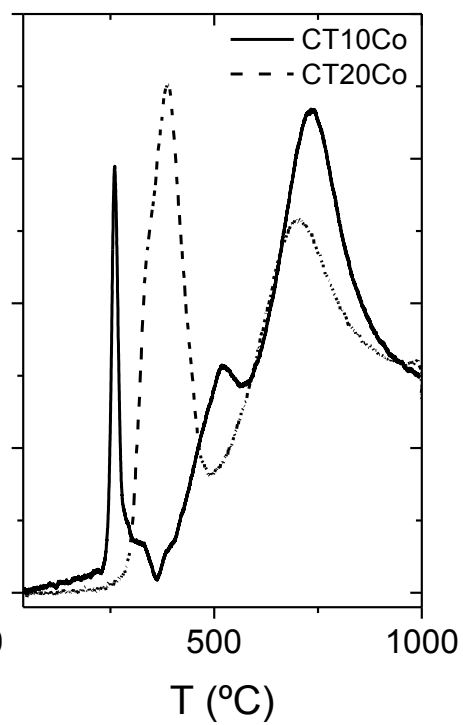

Figure 5.4. a) $\mathrm{H}_{2}$ TPR signal for $\mathrm{CT} 10$ and b) $\mathrm{H}_{2}$ TPR for $\mathrm{CTxCo}$ ( $\mathrm{x}=10$ and 20).

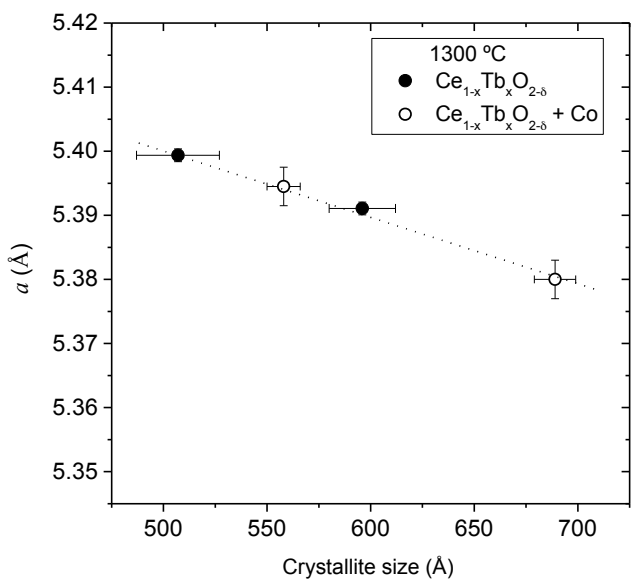

Figure 5.5. Crystallite size dependence on the cell parameter for CT10, CT20, CT10Co and $\mathrm{CT} 20 \mathrm{Co}$ after sintering at $1300^{\circ} \mathrm{C}$. 
The evolution of the cell parameter with different compositions also agrees with the evolution of crystallite size of the samples calculated by Scherrer equation (Eq. 2.3, Chapter 2). Figure 5.5 shows that $10 \% \mathrm{~Tb}$ doped samples have a larger cell parameter and smaller crystallite sizes. This lattice expansion with decreasing grain size at room temperature is due to the larger concentration of oxygen vacancies associated to the presence of intrinsic defects of $\mathrm{Ce}^{3+}$ and $/$ or $\mathrm{Tb}^{3+}$ as previously reported for other cerias. [19-21]

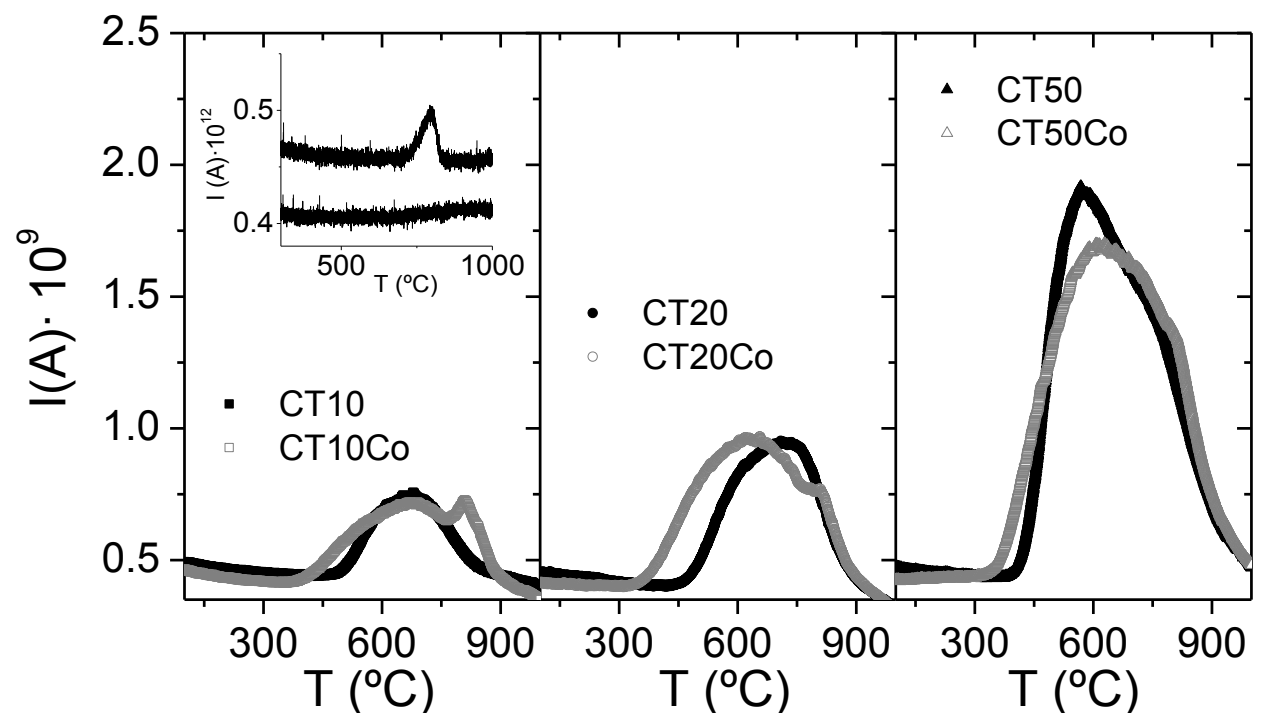

Figure 5.6. Normalized TPD measured in Ar of CTx and CTxCo. Inset: $\mathrm{CeO}_{2}$ and $\mathrm{CeO}_{2}+\mathrm{Co}$. The intensity (ion current) corresponds to $m / z=32$.

The evolution of cell parameters and associated vacancy concentration may predict the different redox processes occurring in a material dependent on temperature and $\mathrm{pO}_{2}$. In the Figure 3.6 of Chapter 3, HT-XRD measurements performed on CT10 showed a linear dependency of cell parameter on the temperature and an increase of the slope at $480{ }^{\circ} \mathrm{C}$. The first slope corresponds to the thermal expansion coefficient (ca. $12.1 \times 10^{-6} \mathrm{~K}^{-1}$ ) in air while the increase in slope beyond $430{ }^{\circ} \mathrm{C}$ is the additional contribution of chemical expansion due 
to the reduction of $\mathrm{Tb}^{4+}$ to $\mathrm{Tb}^{3+}$ and the associated oxygen release (ca. $5.77 \times 10^{-6} \mathrm{~K}^{-1}$ ).

The mentioned oxygen release can be observed in the TPD measurements depicted in Figure 5.6, where the ion current corresponding to the released oxygen from the solid oxide powder (as determined by mass spectroscopy) is plotted as a function of temperature. CT10 oxygen desorption starts at $\sim 480{ }^{\circ} \mathrm{C}$, approximately the same temperature at which the chemical expansion becomes tangible by HT-XRD. It reaches a maximum at $670{ }^{\circ} \mathrm{C}$ and decreases hereafter. This oxygen release implies the reduction of the material and the oxygen vacancies formation that, taking into account that the $p O_{2}>10^{-5}$ atm, are related to the reduction of $\mathrm{Tb}^{4+} / \mathrm{Tb}^{3+}$. Higher dopant content is found to shift the oxygen release to lower temperatures (CT20 and CT50), i.e., it increases the reducibility of the material.

Co-containing samples present two distinguished redox processes: (1) the oxygen release associated with $\mathrm{Tb}^{4+}$ reduction, which starts at lower temperatures than their analogous Co-free samples; and (2) a new important well-defined reduction process that takes place at temperatures ranging from 730 to $880{ }^{\circ} \mathrm{C}$ and that reaches a maximum at $780{ }^{\circ} \mathrm{C}, 800{ }^{\circ} \mathrm{C}$ and $810{ }^{\circ} \mathrm{C}$ for CT10Co and CT20Co, CT50Co, respectively. This high-temperature reduction process can be ascribed to two different processes:

- reduction of Co itself $\left(\mathrm{Co}^{3+} \rightarrow \mathrm{Co}^{2+}\right)$, which is confirmed by the existence of this reduction in in $\mathrm{CeO}_{2-\delta}+\mathrm{Co}$ sample, while it is not present in $\mathrm{CeO}_{2-\delta}$ as shown in the inset of Figure 5.6. [18] Note that the intensity of the Co-related peak changes due to the $\mathrm{Tb}$ background.

- further reduction of bulk $\mathrm{Tb}^{4+}$ to $\mathrm{Tb}^{3+}$ is confirmed by the existence of a broadened peak at higher temperatures. 
In summary, TPD measurements reveal that addition of either Co or a higher amount of $\mathrm{Tb}$ increases the oxygen release i.e., the material reducibility.

a)

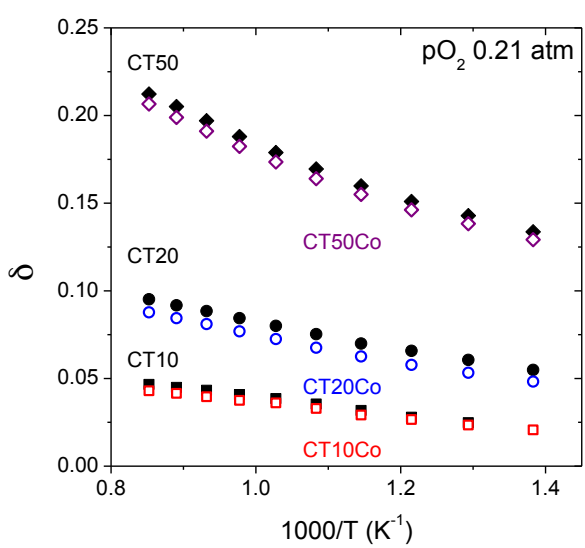

b)

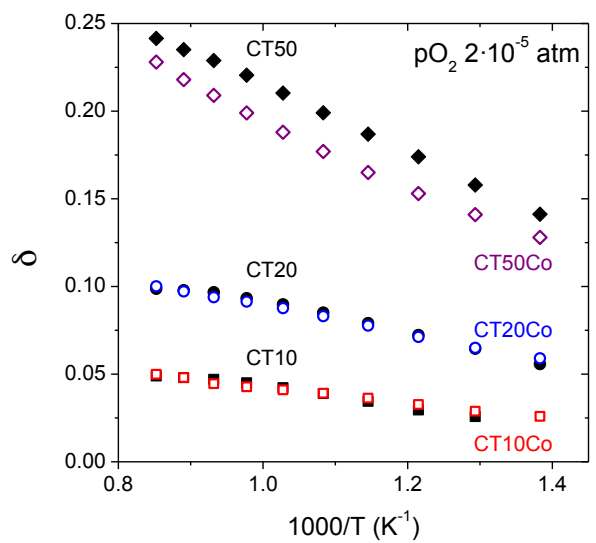

Figure 5.7. (a) Oxygen non-stoichiometry $(\delta$ ) determined by TG in air and (b) $\delta$ determined by TG in $\mathrm{N}_{2}$, for CTx and CTxCo. Temperature range include from 430 to $1000{ }^{\circ} \mathrm{C}$.

Figure 5.7 shows the evolution of the oxygen non-stoichiometry parameter $\delta$ in air (a) and in $\mathrm{N}_{2}$ (b) with temperature for the different samples, as determined by thermogravimetry. The estimations were done by taking into account that the mass loss corresponds to oxygen release (after a drying step). To obtain the final non-stoichiometry, the total $\mathrm{O}_{2}$ mol per mol of fluorite has been added to the oxygen of the totally reduced fluorite in $\mathrm{N}_{2}$, following the equation:

$2-\delta=\frac{n_{\mathrm{O}_{2}}}{n_{\text {fluorite }}}+\left(2-\frac{x}{2}\right)$

As expected, at high temperatures $\delta$ is not affected by the Co addition while only minor changes in $\delta$ are observed at low temperatures. On the other hand, a substantial change in $\delta$ arising from the Co addition is observed for the 
sample with $50 \% \mathrm{~Tb}$ (CT50Co). In fact, the lower oxygen release observed in CT50Co with respect to CT50 seems to be related to the higher concentration of $\mathrm{Tb}^{3+}$ at lower temperatures, which also emerged from the cell parameter data of $\mathrm{XRD}$ at room temperature (Figure 5.3).

\section{Electrochemical characterization}

In order to analyze the complete conductivity mechanism in the studied range of temperatures and $\mathrm{pO}_{2}$, it would be interesting to explore the defect chemistry of these compounds. The dominant defect equilibrium at high $\mathrm{pO}_{2}$ in the system CTx is thereby suggested to be that of Tb reduction. Stefanik and Tuller [22] and Fagg et al [17] described a defect model including a variable valency acceptor dopant. The four regions plotted in Figure 5.8 delimite the main key defect reactions for a general fluorite. In this study, the considered limits are regions II and IV, which are listed below for the particular case of $\mathrm{Tb}$ doped ceria, starting with the consideration of pure $\mathrm{CeO}_{2}$.

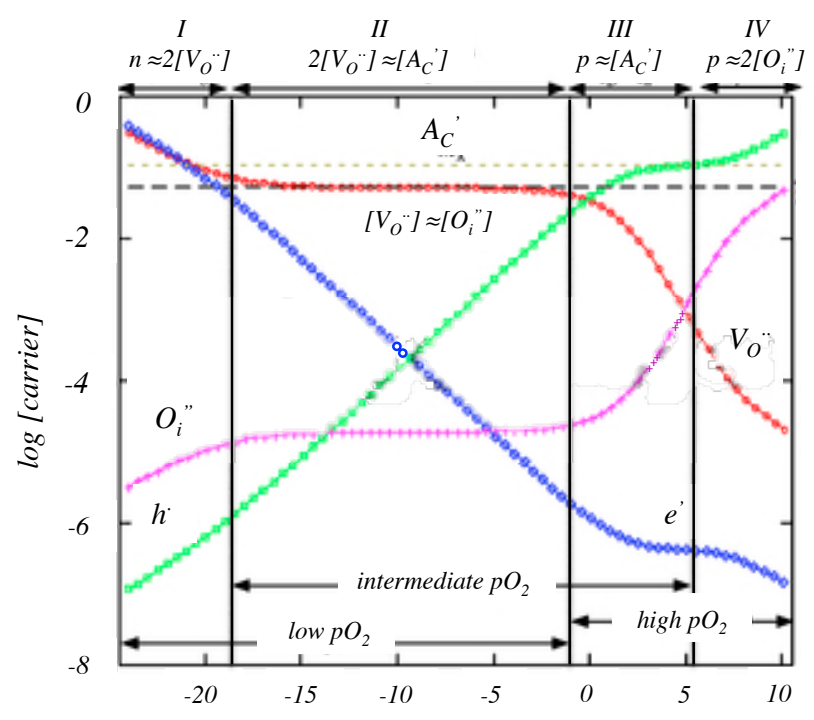

Figure 5.8. Kröger-Vink diagram for acceptor doped fluorite. Four $\mathrm{pO}_{2}$ regions delimit the main key defect reactions for a general fluorite. 
First, the formation of oxygen vacancies and interstitials is dominated by the Frenkel defect mechanism for pure $\mathrm{CeO}_{2}$ as described in Eq. 5.5, while intrinsic electron-hole pair generation produced by thermal excitation over the band gap is governed by Eq. 5.6. On the other hand, molecular oxygen release causes oxygen vacancy generation (charge compensated by the formation of free electrons localized on $\mathrm{Ce}$ ions) and hopping conduction will occur over the $\mathrm{Ce}^{3+} / \mathrm{Ce}^{4+}$ from intrinsic defects of pure ceria sublattice following Eq. 5.7.

$$
\begin{aligned}
& O_{O}^{\times} \rightarrow V_{O}^{\prime \prime}+O_{i}^{\prime \prime} ; \quad\left[V_{O}^{\prime \prime}\right]\left[O_{i}^{\prime \prime}\right]=K_{F}(T) \\
& n i l=e^{\prime}+h^{\prime} ; \quad n p=K_{e}(T) \\
& O_{O}^{\times} \rightarrow \frac{1}{2} O_{2}(g)+V_{o}^{\prime \prime}+2 e^{\prime} ; \quad\left[V_{O}^{\prime \prime}\right] n^{2} p O_{2}^{1 / 2}=K_{R}(T)
\end{aligned}
$$

In a $\mathrm{Tb}$ doped system it has to be taken into account the mass conservation law Eq. 5.8, the ionization of Tb cations Eq. 5.9, and the added charge defect $T b_{C e}^{\prime}$ in order to keep the electroneutrality condition Eq. 5.10.

$$
\begin{aligned}
& {\left[T b_{C e}^{\prime}\right]+\left[T b_{C e}^{\times}\right]=T b_{\text {total }}} \\
& T b_{C e}^{\prime} \rightarrow T b_{C e}^{\times}+e^{\prime} ; \quad \frac{\left[T b_{C e}^{\times}\right] n}{\left[T b_{C e}^{\prime}\right]}=K_{T b}(T) \\
& 2\left[O_{i}^{\prime \prime}\right]+n+\left[T b_{C e}^{\prime}\right]=2\left[V_{O}^{*}\right]+p
\end{aligned}
$$

Considering the region IV boundary, since $\mathrm{Tb}$ introduces extrinsic oxygen vacancies, the concentration of interstitial oxygen will be suppressed $\left[O_{i}^{\prime \prime}\right] \rightarrow 0$, improving the ionic conductivity in the high $p O_{2}$ range. Besides, in oxidizing conditions and low temperatures (below $480{ }^{\circ} \mathrm{C}$ ) most terbium exists as $\mathrm{Tb}^{4+}$ and then $T b_{C e}^{\prime}<<T b_{C e}^{\times}\left(\mathrm{n}<<\mathrm{K}_{\mathrm{Tb}}\right)$. Therefore: 
$n \propto p O_{2}^{-1 / 6}$

$\left[V_{O}^{*}\right] \propto p O_{2}^{-1 / 6}$

$p \propto p O_{2}^{1 / 6}$

In the case of region II, when $T b_{C e}^{\prime}>>T b_{C e}^{\times}\left(\mathrm{n}>>\mathrm{K}_{\mathrm{Tb}}\right)$ but $T \mathrm{~b}_{\text {total }}>>\mathrm{n}$; consequently:

$n \propto p O_{2}^{-1 / 4}$

$\left[V_{O}^{*}\right] \propto p O_{2}^{0}$

$p \propto p O_{2}^{-1 / 4}$

Thus, vacancy concentration is independent on $\mathrm{pO}_{2}$ and proportional to the total terbium concentration.

On the other hand, XRD analysis suggests that the Co added in CTXCo samples is partially incorporated in the fluorite lattice as $\mathrm{Co}^{2+}$, giving rise to one oxygen vacancy per substituted Ce. [13] Therefore, the electroneutrality balance (Eq. 5.10) must be modified with the addition of $\left[\mathrm{Co}_{\mathrm{Ce}}^{\prime \prime}\right]$ in the left term. The rest of Co is likely placed along the grain boundaries and does not affect bulk defect chemistry in equilibrium.

Hence, equations above describe a way to make an evaluation of the predominant type of carriers from the conductivity behaviour with temperature and $\mathrm{pO}_{2}$. Primarily, the influence of the temperature is considered.

Figure 5.9 shows the conductivity $\ln (\sigma \mathrm{T})$ as a function of the reverse temperature for $\mathrm{CTx}$ (a) and $\mathrm{CTxCo}$ (b) measured in $\mathrm{pO}_{2}$ of 0.21 and 
$5 \times 10^{-5} \mathrm{~atm}$. Given a sample composition, differences in conductivity at different $\mathrm{pO}_{2}$ indicate a change in the level of reduction. Moreover, oxygen can be more easily incorporated at high $\mathrm{pO}_{2}$ as temperature drops while it cannot be incorporated (reoxidization) at low $\mathrm{pO}_{2}$. This causes an apparent change in activation energy $\left(E_{a}\right)$ under oxidizing atmospheres $\left(\mathrm{pO}_{2}=1\right.$ and $\left.0.21 \mathrm{~atm}\right)$, in CT10, CT10Co, CT20 and CT20Co, while reoxidization cannot be observed at $\mathrm{pO}_{2}$ of $5 \times 10^{-5} \mathrm{~atm}$ in the measured temperature range $\left(800-400{ }^{\circ} \mathrm{C}\right)$. The variations in $E_{a}$ at given conditions indicate a temperature dependent change in the main conduction process contributing to the total conductivity. Therefore, changes in the $E_{a}$ in this kind of mixed conducting materials may denote variations in the proportion between ionic and electronic contributions. Taking into account that at these high $p O_{2}$ doped ceria materials have been reported as pure ionic conductors, the change in apparent $E_{a}$ could be analyzed in terms of vacancy concentration increase due to the release of oxygen ( $\mathrm{Tb}$ reduction). Actually, $E_{a}$ change of CT10 in air is produced at $470-480{ }^{\circ} \mathrm{C}$, which matches the temperature range observed by XRD for oxygen desorption/incorporation and TPD (Figure 5.6).

On the other hand, the conductivity of CT50Co and specially CT50 is higher than for the cerias with lower Tb concentration. In this case, the change in $E_{a}$ occurs in the low oxygen content environment and at higher temperature (ca. $680{ }^{\circ} \mathrm{C}$ ). At this temperature, TPD in Ar shows that the majority of $\mathrm{Tb}$ is already reduced. Accordingly, the increase in the ionic conduction that causes the $E_{a}$ change may be attributed to oxygen vacancies that form associates at lower temperatures. 
a)

b)
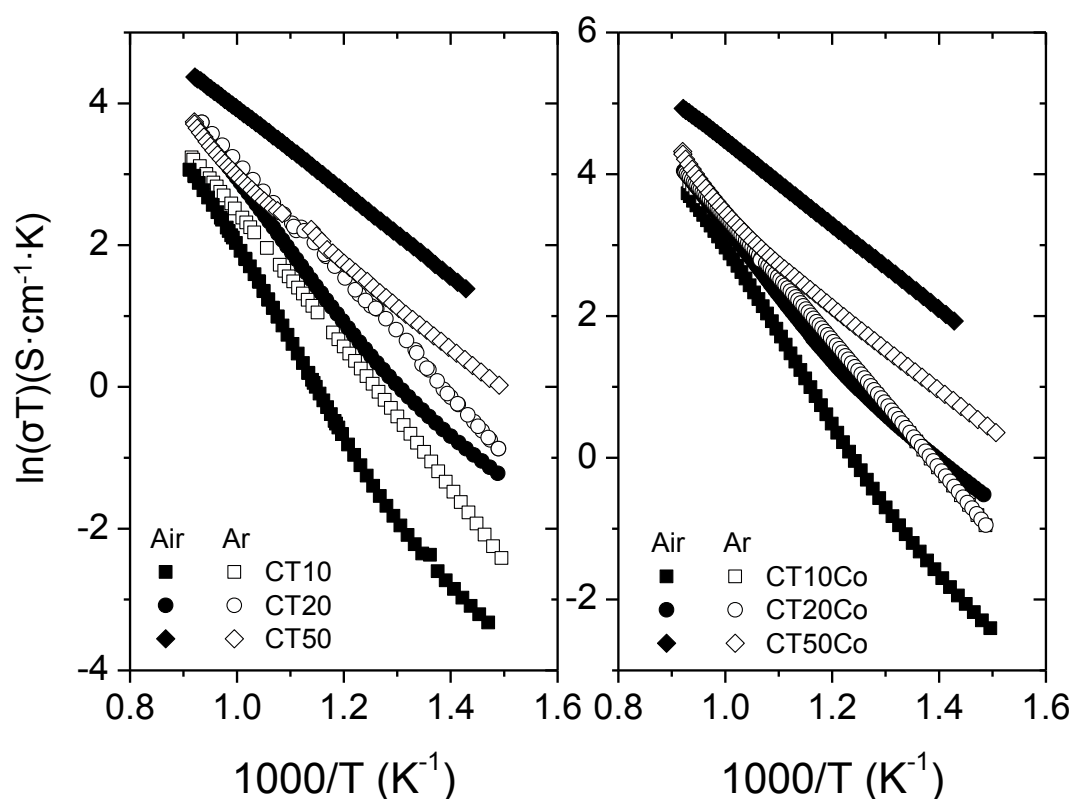

Figure 5.9. Arrhenius plot of the total conductivity in air and in $\operatorname{Ar}\left(0.21\right.$ and $5 \cdot 10^{-5} \mathrm{~atm}$, respectively) for a) CTx and b) CTxCo.

Table 5.1 summarizes apparent $E_{a}$ in the temperature ranges of $800-480{ }^{\circ} \mathrm{C}$ (high) and $480-400{ }^{\circ} \mathrm{C}$ (low) for 10 and $20 \% \mathrm{~Tb}$ doped ceria and $800-700{ }^{\circ} \mathrm{C}$ (high) and $700-400{ }^{\circ} \mathrm{C}$ (low) for $50 \% \mathrm{~Tb}$. Experimental $E_{a}$ matches the typical values found for ionic conductors. As mentioned, at $\mathrm{pO}_{2}<10^{-3} \mathrm{~atm}$, there exists only one $E_{a}$ for CT10, CT10Co, CT20 and CT20Co, i.e., terbium is mainly reduced to $\mathrm{Tb}^{3+}$, over the whole studied temperature range, thus behaving as a fixed valence dopant, which enhances principally the ionic conductivity. CT50 and CT50Co constant $E_{a}$ appears at $p O_{2}>10^{-3}$. 


\begin{tabular}{ccccccc}
\hline \multicolumn{9}{c}{$\boldsymbol{E}_{\boldsymbol{a}}(\mathbf{e V})$} & \multicolumn{2}{c}{$\boldsymbol{\sigma}(\mathbf{S} / \mathbf{c m}) \mathbf{8 0 0}{ }^{\circ} \mathbf{C}$} \\
\hline & High & Low T & High T & Low T & & \\
& T & & & & & \\
& & & & & & \\
CT10 & 1.08 & 0.67 & 0.80 & 0.80 & 0.018 & 0.025 \\
CT10Co & 1.06 & 0.76 & 0.75 & 0.75 & 0.038 & 0.045 \\
CT20 & 0.87 & 0.59 & 0.71 & 0.71 & 0.035 & 0.040 \\
CT20Co & 0.82 & 0.55 & 0.77 & 0.77 & 0.048 & 0.051 \\
CT50 & 0.51 & 0.51 & 0.78 & 0.52 & 0.071 & 0.034 \\
CT50Co & 0.51 & 0.51 & 0.83 & 0.51 & 0.122 & 0.040 \\
\hline
\end{tabular}

Table 5.1. Apparent activation energies in the temperature ranges of $800-480{ }^{\circ} \mathrm{C}$ (high T) and $480-400{ }^{\circ} \mathrm{C}$ (low T) for CT10, CT10Co, CT20 and CT20Co and in the temperature range of $800-700{ }^{\circ} \mathrm{C}$ (high T) and $700-400{ }^{\circ} \mathrm{C}$ (low T) for CT50 and CT50Co. The conductivities at $800{ }^{\circ} \mathrm{C}$ under air and $5 \times 10^{-5}$ atm is indicated.

Total conductivity at $800{ }^{\circ} \mathrm{C}$ under 0.21 and $5 \times 10^{-5}$ atm $p O_{2}$ is also specified in Table 5.1. The increase of $\mathrm{Tb}$ content and the addition of cobalt enables increasing substantially the total conductivity and the best conductivity values have been obtained for $50 \% \mathrm{~Tb}$ content. Figure 5.10a depicts the dependency of total conductivity on $\mathrm{Tb}$ dopant concentration under air. The total conductivity in air increases with the $\mathrm{Tb}$ dopant concentration, which is interpreted as a superior concentration of oxygen vacancies at higher $\mathrm{Tb}$ doping levels having into account that only the dopant is reduced $\left(\mathrm{Tb}^{+4} \rightarrow \mathrm{Tb}^{3+}\right)$. [14] When Co is added to both compositions, the reduction of the sample is enhanced as shown by TPD (Figure 5.6) and TPR measurements (Figure 5.4). This allows increasing the oxygen vacancy concentration at the same temperature with respect to the Co-free samples. Thus, the ionic conductivity increases and electronic conductivity is promoted as confirmed later on by oxygen permeation results. 
a)

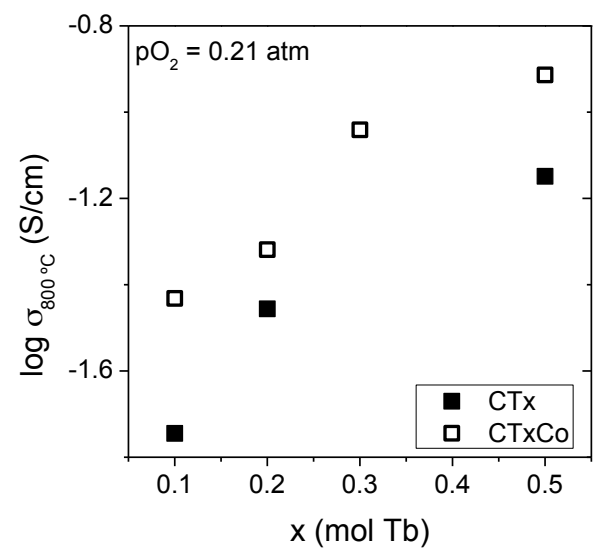

b)

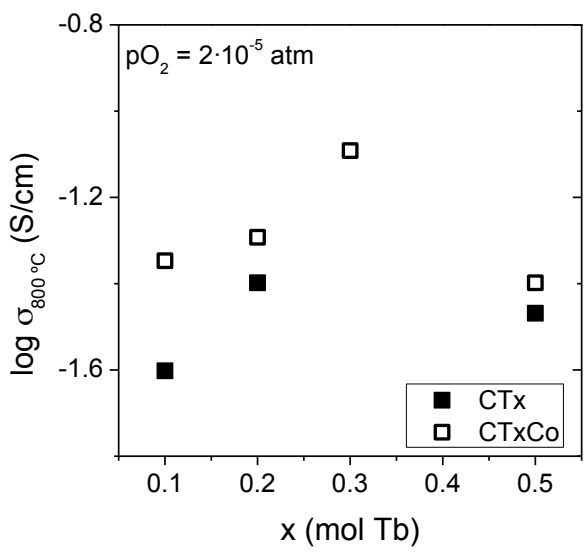

Figure 5.10. Total conductivity dependency on $\mathrm{Tb}$ dopant concentration in (a) air, $p \mathrm{O}_{2}=0.21 \mathrm{~atm}$ and (b) Ar, $p O_{2}=2 \cdot 10^{-5} \mathrm{~atm}$.

Figure $5.10 \mathrm{~b}$ shows the dependency of the total conductivity on $\mathrm{Tb}$ dopant concentration under $\operatorname{Ar}\left(\mathrm{pO}_{2}=2 \cdot 10^{-5}\right.$ atm $)$. The total conductivity in $\operatorname{Ar}$ reaches a maximum at about $\mathrm{x}=30 \mathrm{~mol} \% \mathrm{~Tb}$. For CT50 and CT50Co, the total conductivity is found to decline with decreasing $\mathrm{pO}_{2}$. This observation can be attributed to a mainly $p$-type electronic conductor behavior. In this case, the conductivity diminishes with the decrease in $\mathrm{pO}_{2}$ since the formation of oxygen vacancies diminishes the electron-hole density. Besides, defect association between dopant $\mathrm{Tb}^{3+}$ cations and oxygen vacancies can occur, according to Eq. 5.15,

$T b_{C e}^{\prime}+V_{o}^{*} \leftrightarrow\left(T b_{C e}^{\prime} V_{o}^{*}\right)$

This new type of defect occurs at high concentrations of oxygen vacancies due to the electrostatic interaction among dopant $\mathrm{Tb}^{3+}$ and the oppositely charged oxygen vacancy. These associated defects prevent oxygen vacancies to move and, consequently, the ionic conductivity diminishes. [15, 23] 
a)

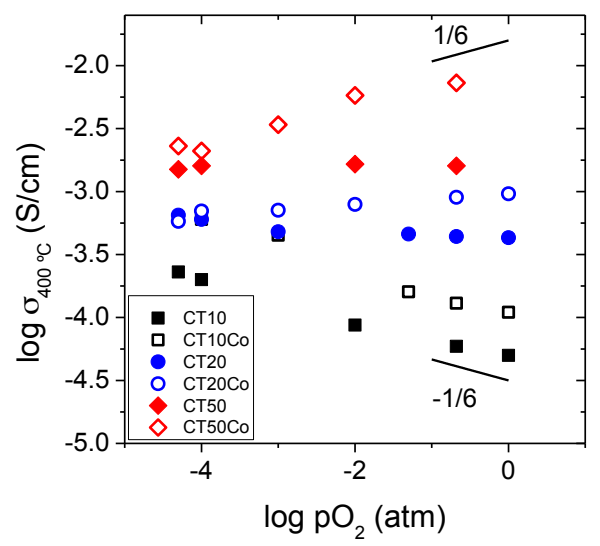

b)

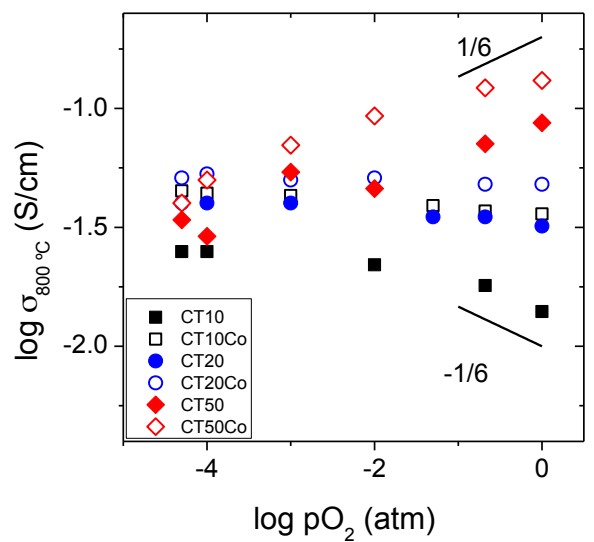

Figure 5.11. Conductivity of CTx and CTxCo systems as a function of $\mathrm{pO}_{2}$ measured at (a) $400{ }^{\circ} \mathrm{C}$ and (b) $800{ }^{\circ} \mathrm{C}$.

Figure 5.11 plots the conductivity logarithm at $400{ }^{\circ} \mathrm{C}$ (a) and $800{ }^{\circ} \mathrm{C}$ (b) versus $\mathrm{pO}_{2}$ logarithm for the different compositions. The plots show that (1) in general, the total conductivity is enhanced by cobalt addition, and (2) the conductivity- $\mathrm{pO}_{2}$ behavior depends on the Tb content.

At $400{ }^{\circ} \mathrm{C}$, over the whole range of $p O_{2}$, CT10 shows $p O_{2}^{-1 / 6}$ dependency, which could be characteristic of both $n$-type electronic conductivity and ionic conductivity via oxygen vacancies for this material and conditions, as described by Eq. 5.11 and Eq. 5.12, respectively. At $800{ }^{\circ} \mathrm{C}$ the $\mathrm{pO}_{2}{ }^{-1 / 6}$ dependency exists but there is a conductivity plateau at $\mathrm{pO}_{2}<10^{-3} \mathrm{~atm}$, characteristic of ionic transport as illustrated in Eq. 5.15. This plateau is related to a maximum reduction of $\mathrm{Tb}$ reachable at these temperatures in this $\mathrm{pO}_{2}$ range that causes the material to achieve a steady vacancy concentration independently on the $\mathrm{pO}_{2}$ and proportional to the oxygen vacancy concentration $\left(\sigma \propto\left[V_{o}^{*}\right]\right)$. The absence of a continuous slope of $-1 / 6$ indicates that, even at $p O_{2}$ higher than $10^{-3} \mathrm{~atm}$, the total conductivity should be predominantly ionic because $n$-type electronic conductivity would result in a continuous increase 
when $\mathrm{pO}_{2}$ decreases, which is not observed in the experimental results. Hence, conductivity is mainly ionic in these oxidizing conditions and the multiple oxidation state of the dopant makes the ionic conductivity $\mathrm{pO}_{2}$ dependent (region not observed in single-oxidation-state acceptor doped systems as $\mathrm{Gd}$ ).

The previous behaviour can be extended to CT10Co even though the increase in conductivity is not only due to the oxygen vacancies but also to enhanced densification of the sample. It has been reported that the use of Co as a sintering aid may result in the decrease of grain boundary resistance. [13, 24] By XRD the maximum solubility of the Co into the ceria lattice was determined to be $1.05 \mathrm{~mol} \%$, which increases the $p$-type contribution shifting the transition to the ionic plateau to higher $p O_{2}$. The remaining Co appears as a cobalt-rich phase at the grain boundary (observed by SEM but below the detection limit of the XRD device). This cobalt-rich phase might act as a percolating network, increasing the electronic conductivity as observed for other doped cerias. [24] A beneficial effect is that the catalytic activity for the oxygen reduction reaction at the surface may change by the presence of Co, which will be discussed below. As a result, the $\mathrm{pO}_{2}$ slope is smoothed and an extended plateau range is observed.

As aforementioned, CT20 introduces more oxygen vacancies than the $10 \%$ doped material broadening the electrolytic region over that of CT10. The $\mathrm{pO}_{2}$ dependency of the conductivity for CT20 is lower than $-1 / 6$ at both temperatures, and reaches the ionic plateau at higher $\mathrm{pO}_{2}$ than for CT10. $\mathrm{pO}_{2}$ dependency smaller than $-1 / 6$ sets the behaviour of the material in the diffuse region between the vacancy concentration $\mathrm{pO}_{2}$ independent and the $-1 / 4$ dependence (boundary between regions II and III). This shift to region III with respect to CT10 may be the consequence of the introduction of energetic levels of $\mathrm{Tb}$ into the ceria band gap, which permits electron-holes to hop from $\mathrm{Tb}^{3+}$ to 
$\mathrm{Tb}^{4+}$ and the concurrent enhancement of the electronic conductivity. The small addition of Co oxide allows further increasing of the p-type electronic conductivity. The slight increase in conductivity observed for these samples with decreasing $\mathrm{pO}_{2}$ is still attributed to $\mathrm{Tb}^{4+}$ reduction with concomitant formation of oxygen vacancies.

The total conductivity of CT50 is found proportional to $\mathrm{pO}_{2}{ }^{1 / 6}$, which is ascribed to predominant $p$-type conductivity. For highly Tb-doped ceria, the impurity band contributing to the electron-hole hopping and causing additional electronic transport is more evident. This small polaron hopping requires the mixed valent $\mathrm{Tb}$, since electrons on $\mathrm{Tb}^{3+}$ sites can only hop to an adjacent $\mathrm{Tb}$ site if it is empty, e.g. in the $\mathrm{Tb}^{4+}$ state. The maximum contribution to the electronic conductivity is, therefore, $\mathrm{pO}_{2}$ dependent, and it will be reached when the concentration of the two valence states are equal. [22] These results confirm that transport of electron-holes is the dominant conduction mechanism at high $\mathrm{pO}_{2}$ for CT50. Finally, Co addition to CT50 provides the highest conductivity values obtained in this study. However, the slightly positive slope observed at $400{ }^{\circ} \mathrm{C}$ suggests that the enhancement of vacancy formation by $\mathrm{Co}$ is such that causes defect interaction typical for highly doped specimens, $\left(T b_{C e}^{\prime} V_{O}^{\prime \prime}\right)^{\prime}$. [15, 22] This effect is more appreciable at low temperatures since there is not enough energy to exceed the defect dissociation barrier and oxygen vacancies are not free and able to account on the ionic transport process.

On the other hand, UV-vis absorption experiments were carried out in order to study the influence of the dopant on the band gap and thus, the $p$-type electronic conductivity. The band gap measures the energy difference between the top of the valence band occupied with electrons and the bottom of the conduction band unfilled of electrons, thus being related to the extent of electronic conductivity of the materials. In fact, the optical band gap energy is 
comparable to the thermal band gap related to the formation of electron-hole pairs. Kubelka-Munk (K-M) theory has been used to estimate the forbidden energy gap $E_{g}$ of the material. [25, 26] This consists of plotting the obtained reflectance $h v F(R \infty)^{2}$ as a function of the photon energy, $h v$. The band gap $E_{g}$ can be obtained by extrapolating to zero a tangent line drawn in the point of inflection of the curve, i.e., the point of intersection with the $\mathrm{h} v$ horizontal axis.

a)

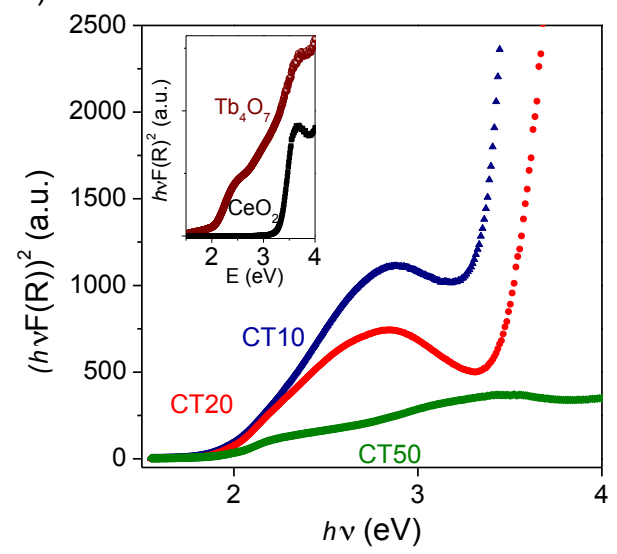

b)

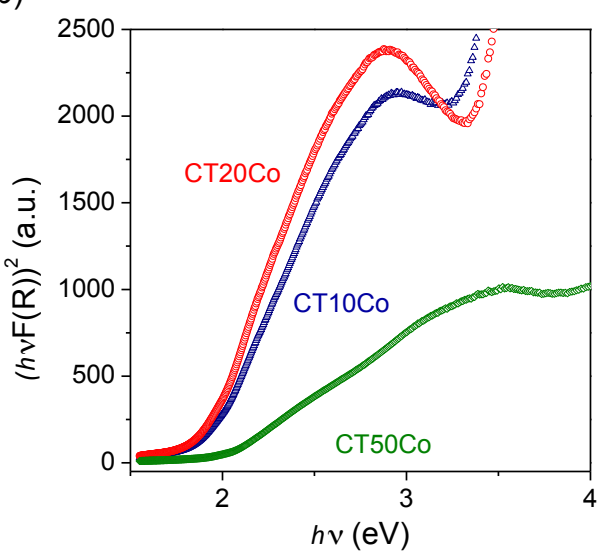

Figure 5.12. UV-vis absorption spectra $(h v F(R))^{2}$ versus photon energy of (a) CTx and (b) CTxCo.

Figure 5.12 shows shows the room temperature UV-vis absorption spectra of the Tb-doped series. $\mathrm{CeO}_{2}$. and $\mathrm{Tb}_{4} \mathrm{O}_{7}$ were taken as reference (see inset in Figure 5.12a). The energy gaps for $\mathrm{CeO}_{2}$ and $\mathrm{Tb}_{4} \mathrm{O}_{7}$ are found to be $3.3 \mathrm{eV}$ and $1.94 \mathrm{eV}$, respectively. The band gap energy of all doped samples decreases from the pure ceria to be close to that of $\mathrm{Tb}_{4} \mathrm{O}_{7}$. This fact suggests that $\mathrm{Tb}$ doping makes it easier electron hop and increases electronic-hole conductivity. For low $\mathrm{Tb}$ content materials $(\mathrm{x}=0.1,0.2)$ the addition of Co results in even smaller values for $\mathrm{E}_{\mathrm{g}}$. 


\section{Surface characterization}

The overall oxygen transport through a dense oxide is governed not only by the electron (or hole) and oxygen ion in the bulk, but also by the surface exchange reaction at the device interfaces. Near-surface defect chemistry plays an important role in determining the chemical surface chemistry and gas exchange activity. Therefore, X-ray photoelectron spectroscopy (XPS) has been performed to gain insight into both (1) valence bands and (2) core levels of the atoms present in the first layer of the material surface. Samples of CT20 and CT20Co were quenched from $800{ }^{\circ} \mathrm{C}$ under air stream to liquid nitrogen in order to have the similar material state as for the conductivity and PIE measurements.
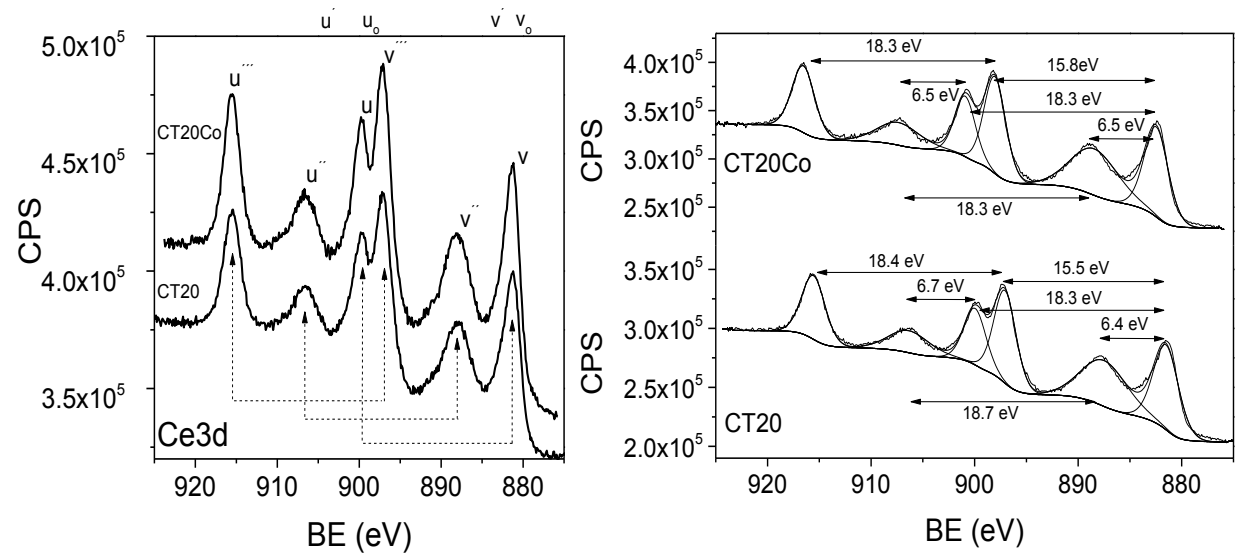

Figure 5.13. a) Ce $3 d$ XPS spectra of CT20 and CT20Co quenched in air from $800{ }^{\circ} \mathrm{C}$ and b) Ce3d XPS separation of peaks of CT20 and CT20Co spectra. Pass energy was 30 $\mathrm{eV}$.

The Ce $3 d$ XPS spectra of CT20 and CT20Co quenched in air from $800{ }^{\circ} \mathrm{C}$ are plotted in Figure 5.13. Both of them show three peaks, separated by several $\mathrm{eV}$ for each of the spin-orbit split 5/2;3/2 components (Figure 5.13b). Thus, the $3 d_{3 / 2}$ and $3 d_{5 / 2}$ contributions are represented as $\mathrm{u}$ and $\mathrm{v}$, respectively. The $\mathrm{u} "$ 
component at $915.5 \mathrm{eV}$, which is a fingerprint of the $\mathrm{Ce}^{4+}$ state, presents a considerable intensity and does not overlap with other peaks. This peak is coupled with $\mathrm{v}^{\prime \prime \prime}(897.2 \mathrm{eV})$ and they both come up from an almost pure $4 \mathrm{f}^{0}$ electron configuration. [27] Besides, good separation between $\mathrm{v}(881.3 \mathrm{eV})$ and $\mathrm{v}^{\prime \prime}(888.1 \mathrm{eV})$ peaks can be observed, which is a characteristic of only $\mathrm{Ce}^{4+}$ state. [28] The overall spectrum appears around $1 \mathrm{eV}$ lower than positions found in the literature for $\mathrm{Ce} 3 \mathrm{~d}$, ascribed to charge shifting of the device. There are slight differences between peak separation from CT20 to CT20Co, which are attributed to accumulated experimental errors rather than a mixture of oxidation states. $\mathrm{Ce}^{3+}$ peaks represented as $\mathrm{v}_{0}, \mathrm{u}_{0}$ should be expected in these spectra at around 879.6 and $898.3 \mathrm{eV}$, respectively (marked by dotted vertical lines). [29] Here, it can be seen that the latter peaks do not appear, which confirms that there is a negligible amount of $\mathrm{Ce}^{3+}$ in the samples. Furthermore, no meaningful changes in the cerium oxidation state have been found due to the influence of cobalt for the studied conditions.

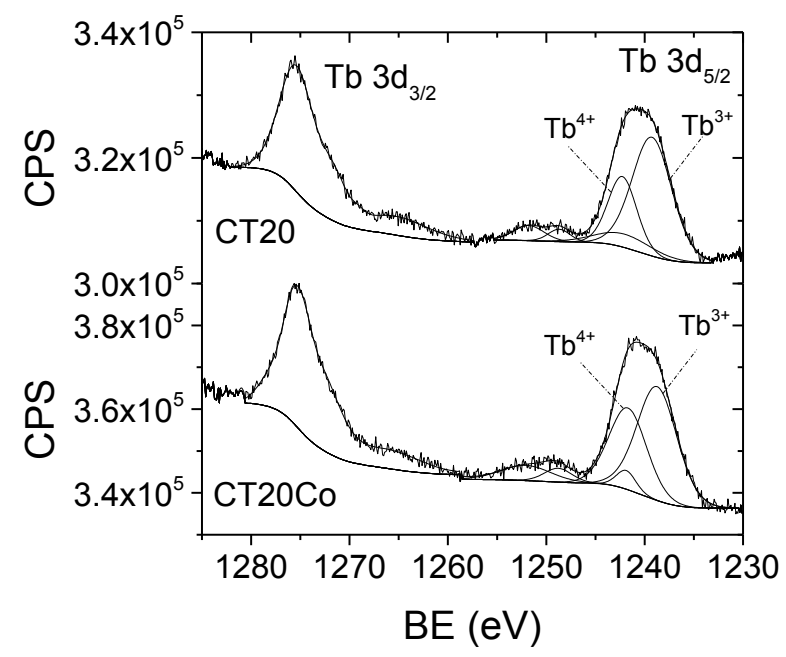

Figure 5.14. XPS of the Tb $3 d$ region of a) CT20 and b) CT20Co, quenched in air from $800{ }^{\circ} \mathrm{C}$, acquired with a pass energy of $30 \mathrm{eV}$. 
The most intense core level for terbium is $\mathrm{Tb} 3 \mathrm{~d}$ at the high binding energy between 1230 and $1290 \mathrm{eV}$. However, it is difficult to distinguish accurately the chemical states of $\mathrm{Tb} 3 \mathrm{~d}$ line as binding energies of the different oxidation states are very similar. Figure 5.14 shows the $\mathrm{Tb} 3 d$ XPS patterns of quenched samples. Two principal components are commonly distinguished related to $\mathrm{Tb} 3 \mathrm{~d}_{3 / 2}$ and the $\mathrm{Tb} 3 \mathrm{~d}_{5 / 2}$ binding energy peak position. The binding energy peak position is shifted from that earlier published for pure $\mathrm{TbO}_{1.5}\left(\mathrm{~Tb}^{3+}\right)$ where the $\mathrm{Tb} 3 \mathrm{~d}_{5 / 2}$ and $\mathrm{Tb} 3 \mathrm{~d}_{3 / 2}$ peak are reported at about 1239.1 and $1274 \mathrm{eV}$. $\mathrm{Tb}^{4+}$ related peaks should appear at 1244 and $1278 \mathrm{eV}$. [30] In addition to the main peaks, terbium shows relatively intense satellites in the $\mathrm{Tb} 3 \mathrm{~d}_{5 / 2}$ component at $1251.5 \mathrm{eV}$ for the tetravalent state and $1250.6 \mathrm{eV}$ for the trivalent state, and at $1264 \mathrm{eV}$ for the $\mathrm{Tb} 3 \mathrm{~d}_{3 / 2}$ component of $\mathrm{Tb}^{4+}$. [31] The spectra in Figure 5.14 show two peaks at 1241.6 and $1276.3 \mathrm{eV}$, and 1240.9 and $1275.7 \mathrm{eV}$ for CT20Co and CT20, respectively. Also two satellites appear between the main peaks. These results suggest that the $\mathrm{Tb}$ introduced in the position of $\mathrm{Ce}$ in the $\mathrm{CeO}_{2}$ lattice as an extrinsic dopant appears in more than one oxidation state i.e., $3+$ and 4+. [32] The CT20Co spectrum is more shifted to higher energies, which indicates a higher oxidation state, i.e. higher amount of $\mathrm{Tb}^{4+}$. From the deconvolution of the spectra it is possible to estimate $60.5 \%$ and $54 \%$ of $\mathrm{Tb}^{3+}$ in the surface of CT20 and CT20Co respectively. The surface oxidation state of $\mathrm{Tb}$ differs from the bulk oxidation state estimated by $\mathrm{TG}$ measurements in air $\left(800{ }^{\circ} \mathrm{C}\right)$, ca. $88.5 \%$ for CT20 and $81 \%$ for CT20Co. Nevertheless, XPS results support the presence of $\mathrm{Tb}$ in both $3+$ and $4+$ oxidation states inferred from lattice parameter extracted from XRD of CT20 and CT20Co. 


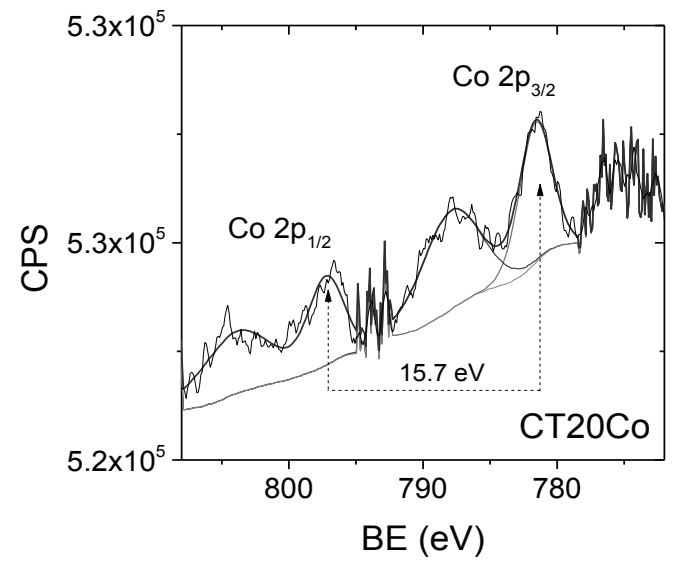

Figure 5.15. XPS of the Co $2 d$ region of CT20 (a) and CT20Co (b), quenched in air from $800^{\circ} \mathrm{C}$, acquired with a pass energy of $30 \mathrm{eV}$. The Co $2 \mathrm{~d}$ peak is shifted to higher binding energy than found in $\mathrm{CoO}(778 \mathrm{eV})$, suggesting Co lattice substitution.

The presence of Co atoms on the grain surface is confirmed by the XPS. The binding energy (BE) values of the Co $2 \mathrm{p}_{3 / 2}$ core-level are close to $780 \mathrm{eV}$ for $\mathrm{Co}^{2+}$ on $\mathrm{CoO}_{\mathrm{x}}$. The main peak is accompanied by a relatively intense satellite peak at $787.5 \mathrm{eV}$ characteristics of $\mathrm{Co}^{2+}$ species and its interaction with other metal. [33] Spin-orbital splitting upon ionization between $2 p_{3 / 2}$ and $2 p_{1 / 2}$ is $16 \mathrm{eV}$. XPS spectra of the synthesized CT20Co material in the Co $2 \mathrm{p}$ region are shown in Figure 5.15. The low intensity of the Co $2 p$ region is due to the very low Co content $(2 \mathrm{~mol} \%)$. The BE of Co $2 \mathrm{p}_{3 / 2}$ peak is ca. $780 \mathrm{eV}$ and the satellite peak at $787.5 \mathrm{eV}$. These binding energies correspond to cobalt oxide species highly dispersed with a strong interaction with the structure, which may indicate the partial incorporation of $\mathrm{Co}$ in the lattice of $\mathrm{CeO}_{2}$. Then, the $\mathrm{BE}$ is shifted to lower values (about $0.2 \mathrm{eV}$ ) compared to the $\mathrm{BE}$ of Co $2 \mathrm{p}_{3 / 2}$ on $\mathrm{Co}_{3} \mathrm{O}_{4}$ or $\mathrm{CoO}(780.2 \mathrm{eV})$. [34] Nevertheless, the proximity of BE for both oxide states, i.e., $\mathrm{Co}_{3} \mathrm{O}_{4}$ or $\mathrm{CoO}$, makes it very difficult to indentify the Co state at the surface of these materials. 
The oxygen exchange kinetics in the CTx and CTxCo surface has been studied by pulse response ${ }^{18} \mathrm{O}-{ }^{16} \mathrm{O}$ isotope exchange (PIE) measurements. Figure 5.16 displays Arrhenius plots of the overall exchange rate $\left(\Re_{0}\right)$ for all materials investigated in this study as evaluated from data of PIE measurements. $\Re_{0}$ is found to increase profoundly with increasing $\mathrm{Tb}$ concentration. The data listed in Table 5.2 support a relationship between the surface exchange rate, at $700{ }^{\circ} \mathrm{C}$, and the corresponding concentration of oxygen vacancies in the Tb-doped ceria samples.

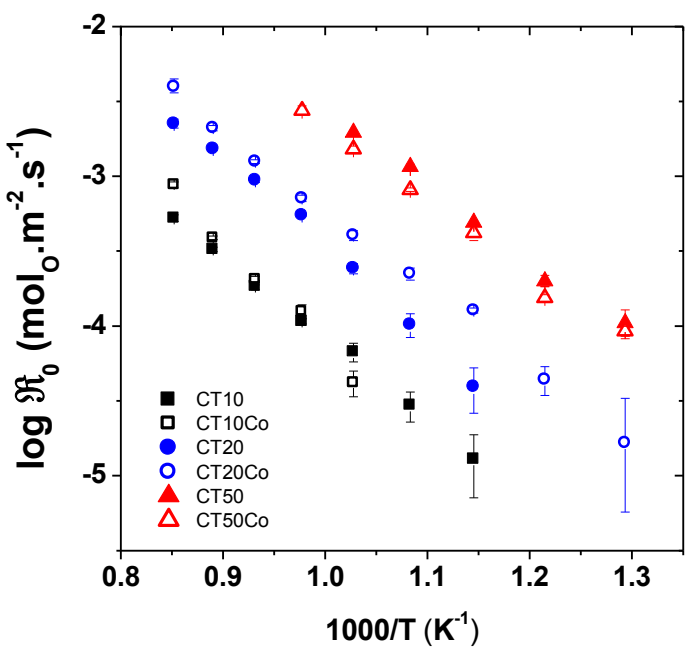

Figure 5.16. Arrhenius plot of the exchange rates of CT10, CT10Co, CT20. CT20Co, CT50, CT50Co.

Lane et al reported that the rate of oxygen surface exchange might be influenced by the surface concentration of oxygen vacancies and by the supply of electrons, which are necessary for charge transfer. [35] Note from Figure 5.16 that Co addition to either CT10 or CT50 does not significantly improve $\Re_{0}$. However, the surface exchange rate exceeds that of the CT10 at temperatures above $750{ }^{\circ} \mathrm{C}$. Above this temperature, the $\left[\mathrm{V}_{\mathrm{O}}{ }^{\cdots}\right]$ reaches a maximum, i.e. $\mathrm{Tb}^{3+}$ is the prevailing $\mathrm{Tb}$ cation, as inferred from XRD, DC conductivity and TG 
experiments. Below $700{ }^{\circ} \mathrm{C}$ surface exchange appears to be limited by the $\left[\mathrm{V}_{\mathrm{O}} \cdot\right]$, partly due to the annihilation of oxygen vacancy associated with the partial oxidation of $\mathrm{Tb}$ from $3+$ to $4+$. However, when the $\mathrm{Tb}^{4+}$ starts to reduce at higher temperatures, $\Re_{0}$ is improved through Co addition, which slightly boosts $p$-type conductivity.

CT20Co increases the surface exchange rate of oxygen with respect to the Co-free counterpart at any tested temperature. This behavior suggests that for $\mathrm{CT} 20,\left[\mathrm{~V}_{\mathrm{O}}{ }^{\prime \prime}\right]$ was enough to ensure the reaction and that the rate limiting step is the electronic contribution. As a consequence, the increase in $p$-type conductivity by Co-addition alleviates the electronic limitation and raises the overall surface exchange rate. Table 5.2 shows the apparent $\mathfrak{R}_{0}$ activation energy obtained for all six compounds. The $E_{a}$ observed for CT20Co decreases with respect to CT20, suggesting a possible change in the rate limiting step, presumably from limitation by insufficient electronic supply (CT20, $118 \mathrm{~kJ} / \mathrm{mol})$ to limitation by deficient $\left[\mathrm{V}_{\mathrm{O}}{ }^{\prime \prime}\right](\mathrm{CT} 20 \mathrm{Co}, 101 \mathrm{~kJ} / \mathrm{mol})$. Indeed, the $E_{a}$, seemingly ascribed to $\left[\mathrm{V}_{\mathrm{O}}{ }^{\prime \prime}\right]$ limitation coincides with the $E_{a}$ observed for CT10 and CT10Co.

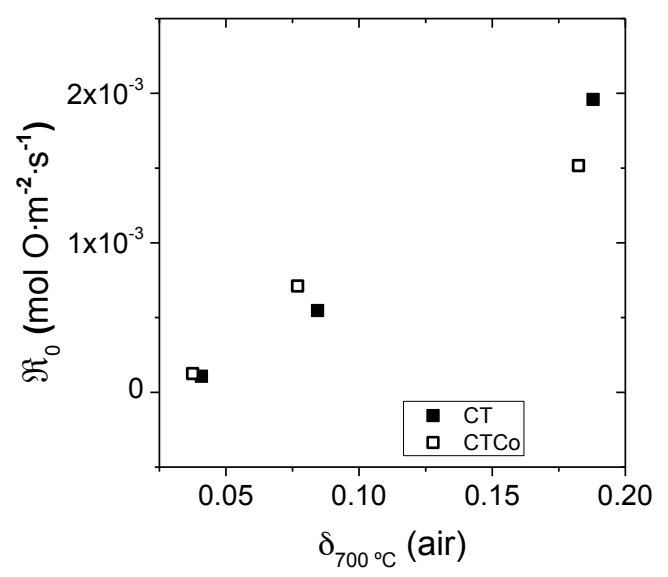

Figure 5.17. Surface exchange rate as a function of the oxygen nonstoichiometry at $700{ }^{\circ} \mathrm{C}$. 


\begin{tabular}{|c|c|c|c|}
\hline Compound & $\delta_{700{ }^{\circ} \mathrm{C}}$ & $\Re_{0}\left(\mathrm{~mol} \mathrm{O} \cdot \mathrm{m}^{-2} \cdot \mathrm{s}^{-1}\right)_{700{ }^{\circ} \mathrm{C}}$ & $\mathrm{E}_{\mathrm{a}}(\mathrm{kJ} / \mathrm{mol})$ [temperature range] \\
\hline CT10 & 0.042 & $6.71 \cdot 10^{-5}$ & $105\left[600-900{ }^{\circ} \mathrm{C}\right]$ \\
\hline CT10Co & 0.036 & $4.18 \cdot 10^{-5}$ & $136\left[700-900{ }^{\circ} \mathrm{C}\right]$ \\
\hline $\mathrm{CT} 20$ & 0.08 & $2.42 \cdot 10^{-4}$ & $118\left[600-900^{\circ} \mathrm{C}\right]$ \\
\hline CT20Co & 0.073 & $4.02 \cdot 10^{-4}$ & $101\left[500-900^{\circ} \mathrm{C}\right]$ \\
\hline CT50 & 0.179 & $1.96 \cdot 10^{-3}$ & $96\left[600-700^{\circ} \mathrm{C}\right]$ \\
\hline CT50Co & 0.174 & $1.52 \cdot 10^{-3}$ & $97 \quad\left[500-750^{\circ} \mathrm{C}\right]$ \\
\hline
\end{tabular}

Table 5.2: Oxygen non-stoichiometry and surface exchange rate, at $700{ }^{\circ} \mathrm{C}$, and apparent activation energies for the surface exchange rate of the different compounds.

Similar $\Re_{0}$ increase through Co addition is not observed for the case of CT50Co. $E_{a}$ is not altered, which may indicate that there is no change in the rate limiting step when cobalt is added. Both CT50 and CT50Co showed $p$-type electronic conductivity (Figure 5.11). Additionally, the $E_{a}$ is similar to that observed for the samples limited by a process related to oxygen vacancies. Therefore, 50\%-doped samples seem not to be limited by electronic supply but by a process involving oxygen vacancies. Accordingly, slightly higher exchange rates are observed for CT50, which can be explained by the CT50 higher nonstoichiometry than that for CT50Co (Figure 5.17). These observations support the conclusion that the rate of exchange in these materials is determined by $i$ ) the concentration of oxygen vacancies, which is related to the ionic conductivity, ii) the $p$-type electronic conductivity, and iii) the balance between these two parameters. 

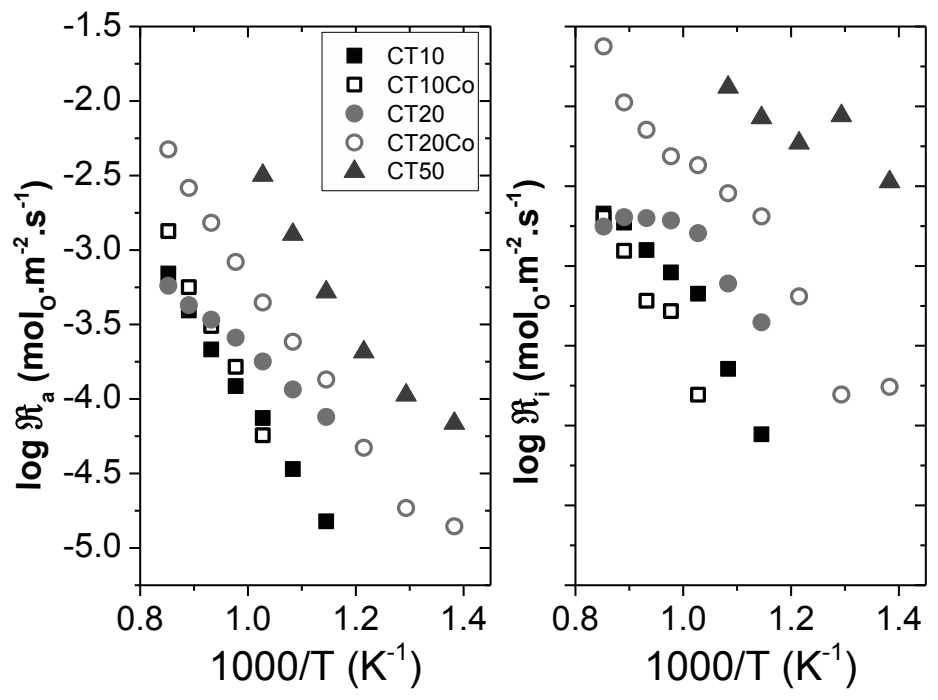

Figure 5.18. Inverse temperature dependence of the rates of dissociative adsorption $(\mathfrak{R}$ a), oxygen incorporation $(\mathfrak{R} i)$ of the terbium doped studied series.

PIE experiments also provide information about the surface exchange mechanism. [36] The distribution of oxygen isotopomers $\left({ }^{18} \mathrm{O}_{2},{ }^{16} \mathrm{O}^{18} \mathrm{O},{ }^{16} \mathrm{O}_{2}\right)$ in the effluent pulse can be modeled by adopting a two-step exchange mechanism, in which isotopic scrambling is determined by the rate of dissociative adsorption of $\mathrm{O}_{2}$ molecules at the surface $\left(\Re_{\mathrm{a}}\right)$ and that of successive incorporation of adsorbed $\mathrm{O}$ adatoms in the oxide lattice $\left(\Re_{\mathrm{i}}\right)$. Thus, overall exchange rate is considered to occur as a series process ${ }^{\mathrm{i}}$. Figure 5.18 plots the rates of dissociative adsorption, $\Re_{\mathrm{a}}$, and oxygen incorporation, $\mathfrak{R}_{\mathrm{i}}$, as a function of the inverse temperature. The overall rate of surface exchange on both CTx and CTxCo samples appears to be controlled by the dissociative adsorption reaction,

$$
\text { i } \frac{1}{\mathfrak{K}_{o}}=\frac{1}{\mathfrak{R}_{a}}+\frac{1}{\mathfrak{R}_{i}}
$$


as was found for, e.g., $25 \mathrm{~mol} \%$ erbia-stabilized bismuth oxide (BE25) [37] and $\mathrm{Ba}_{1-\mathrm{x}} \mathrm{Sr}_{\mathrm{x}} \mathrm{Co}_{0.8} \mathrm{Fe}_{0.2} \mathrm{O}_{3-\delta}$ (BSCF). [36] For all compositions studied, the rate of oxygen incorporation was found between one or two orders of magnitude higher (Figure 5.18). The observations made in this study can be reconciled by assuming that $\mathrm{O}_{2}$ dissociative adsorption and, hence, overall oxygen exchange is rate determined by electron transfer to intermediate superoxide ions $\left(\mathrm{O}_{2}^{-}\right)$at the surface:

$$
\mathrm{O}_{2}^{-}+\mathrm{e}^{-} \rightarrow 2 \mathrm{O}^{-}
$$

with the notion that surface oxygen vacancies are assumed to be the sites onto which adsorption of $\mathrm{O}_{2}$ molecules takes place.

Permeation measurements and stability tests

In this section, the oxygen permeation through different samples has been determined under certain $\mathrm{pO}_{2}$ gradients, which may enable to assess the presence of mixed conductivity and the applicability as $\mathrm{O}_{2}$ separating membranes. Oxygen permeation through CT10, CT10Co, and CT20Co membranes sintered at $1200{ }^{\circ} \mathrm{C}$ has been measured. Membranes made of CT20, CT50 and CT50Co were not measured due to manufacturability issues, i.e. no dense membrane specimens were achieved by the current sintering techniques. Figure 5.19 shows the oxygen flux as a function of temperature for two sweep gases: argon and diluted methane. 


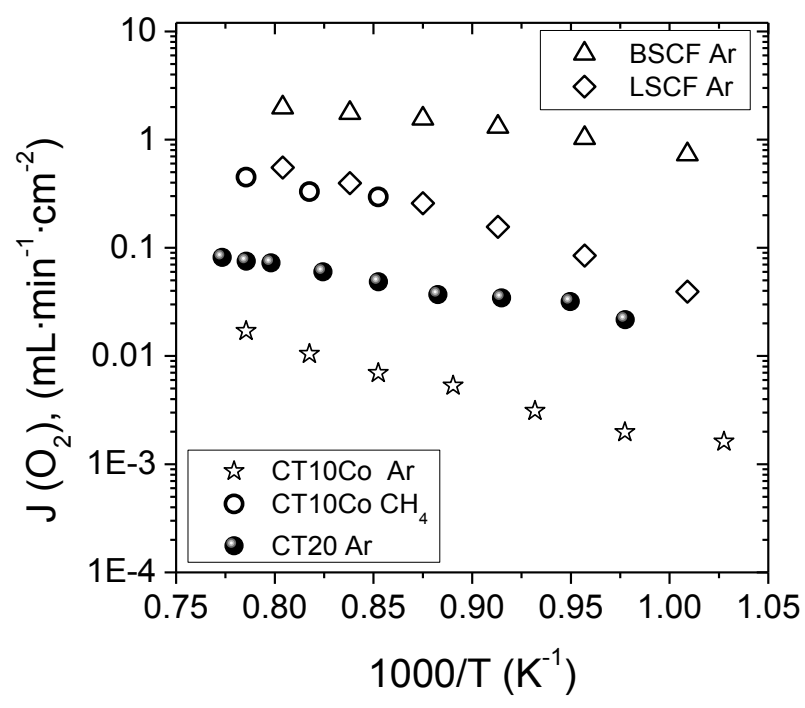

Figure 5.19. Oxygen permeation flux as a function of inverse temperature for CT10Co and CT20Co. The oxygen flux for similarly configuration BSCF and LSCF membranes is shown for comparison.

Figure 5.19 illustrates also the flux obtained with benchmark perovskite-based membranes as BSCF and LSCF in a similar configuration (thickness and sample history). CT10 membrane shows nearly negligible permeability (not shown) and this confirms the main ionic conductivity of this composition. However, 0.9 and $1.2 \mathrm{~mm}$-thick membranes made of CT10Co and $\mathrm{CT} 20 \mathrm{Co}$ present permeation values at $1000{ }^{\circ} \mathrm{C}$ around 0.02 and $0.08 \mathrm{~mL} \cdot \mathrm{min}^{-1} \cdot \mathrm{cm}^{-2}$ for argon sweeping, respectively.

The combination of conductivity and permeation measurements using imposed current allowed determining the ambipolar conductivity at high temperature for CT20Co. CT20Co transference numbers were obtained across a $1.2 \mathrm{~mm}$ thick membrane under a $\mathrm{pO}_{2}$ gradient $0.21 / 5 \times 10^{-5} \mathrm{~atm}$ (air/Ar). The transference numbers showed in Figure 5.20a indicate that CT20Co performs electronic conductivity although ionic conduction prevails. This study is performed in the temperature range from 750 to $1020{ }^{\circ} \mathrm{C}$, which is suitable for 
application as oxygen transport membranes. Ionic conductivity is predominant in the whole temperature range but electronic contribution becomes more important as the temperature drops. Figure $5.20 \mathrm{~b}$ presents the ionic, electronic and total conductivity values obtained by using the transference number. The $\mathrm{O}_{2}$ flux obtained agrees perfectly with the expected value calculated considering the ambipolar conductivity, $\sigma_{a m b}=\frac{\sigma_{i} \sigma_{e}}{\left(\sigma_{i}+\sigma_{e}\right)}$, and assuming a constant mean value in the experimental $p O_{2}$ gradient, e.g. at $860{ }^{\circ} \mathrm{C}$, the obtained $J\left(O_{2}\right)$ is $2.8 \cdot 10^{-8} \mathrm{~mol} \cdot \mathrm{s}^{-1} \cdot \mathrm{cm}^{-2}$, and the $\sigma_{\text {amb }}$ is $0.0085 \mathrm{~S} \cdot \mathrm{cm}^{-1}$. By using Wagner's equation (Eq. 1. 26), the $J\left(O_{2}\right)$ is given by Eq. 5.19

$$
J(O 2)_{(\text {calculated })}=\frac{R T}{16 F^{2}} 0.0085 \ln \frac{0.21}{0.00019}
$$

which finally gives $\mathrm{j}_{\mathrm{O} 2}=3.1 \cdot 10^{-8} \mathrm{~mol} \cdot \mathrm{s}^{-1} \cdot \mathrm{cm}^{-2}$.

a)

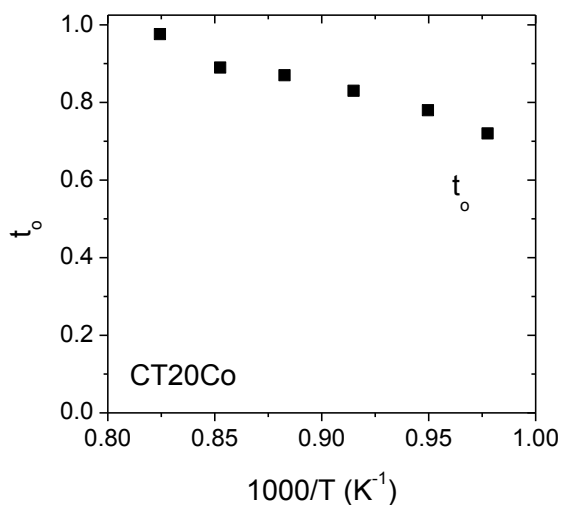

b)

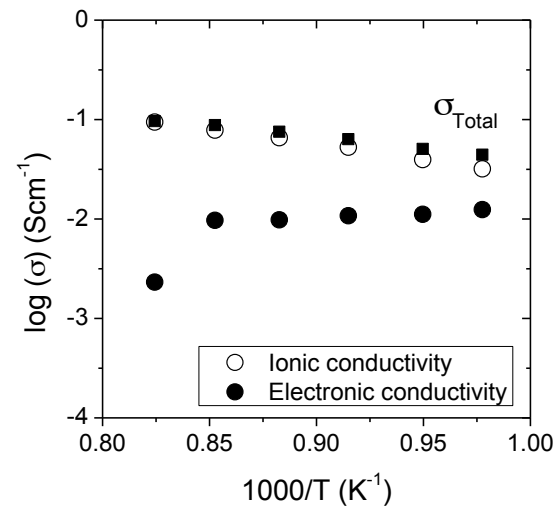

Figure 5.20. a) Ionic transport number and b) total, ionic and electronic conductivity of CT20Co measured across a $1.2 \mathrm{~mm}$ thick membrane under a nominal gradient of $\mathrm{pO}_{2}$ $0.21 \| 5 \times 10^{-5} \mathrm{~atm}$ (air/Ar). $J\left(\mathrm{O}_{2}\right)$ measured using chromatography analytical data.

In more reducing conditions the permeation can be enhanced since the driving force is augmented. For methane sweeping CT10Co sample is able to achieve $0.5 \mathrm{~mL} \cdot \mathrm{min}^{-1} \cdot \mathrm{cm}^{-2}$. This flux increase is additionally attributed to the 
substantial rise of $n$-type conductivity achieved preferentially through $\mathrm{Ce}^{4+}$ reduction. The permeation values are also interesting when compared to some perovskite type permeation membranes, especially due to the high stability under reducing and $\mathrm{CO}_{2}$ containing atmospheres at high temperatures, where broadly applied perovskite materials are prone to decompose. Specifically, these harsh operation conditions are encountered in the targeted applications in oxyfuel processes using flue gas as sweep and high-temperature catalytic membrane reactors for hydrocarbon conversion into syngas and added-value products. The stability of CT10 and CT10Co has been studied by thermogravimetry under continuous flow of air with $5 \% \mathrm{CO}_{2}$. Figure 5.21 shows the mass evolution of these samples and the evolution of a highly permeable perovskite, i.e., BSCF. Carbonates are formed (mass increase) in the range from 700 to $900{ }^{\circ} \mathrm{C}$ and decompose (mass decrease) at higher temperatures, as it can be observed for the BSCF measurement. Ceria sample presents a negligible formation of carbonates while it is also detected the mass loss due to oxygen release at temperatures above $480{ }^{\circ} \mathrm{C}$.

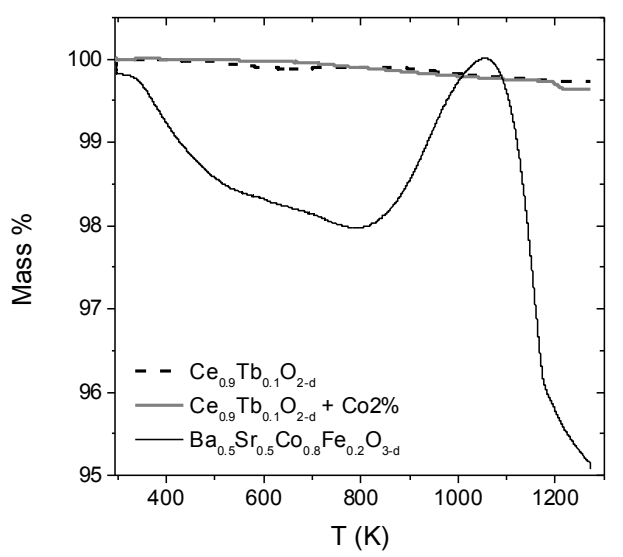

Figure 5.21. Thermogravimetric analysis under $\mathrm{CO}_{2}$ flow of CT10 and CT10Co in $\mathrm{CO}_{2}$, and BSCF. 


\subsection{Conclusions}

$\mathrm{Tb}$ doped ceria shows predominant ionic conductivity or mixed ionicelectronic conductivity depending on the $\mathrm{Tb}$ and $\mathrm{Co}$ doping level and temperature range, which proves MIEC properties of these compounds. The extent of mixed ionic-electronic conductivity is a function of temperature and can be tuned by modifying the $\mathrm{Tb}$ - (and Co-doping) concentration. Low $\mathrm{Tb}$ content materials $(\mathrm{x}=0.1)$ show predominantly ionic conductivity. Higher doping levels $(>20 \%)$ introduce new $\mathrm{Tb}$ electronic levels in the ceria bandgap that allow the small polaron hopping between $\mathrm{Tb}$ ions of different oxidation state (since $\mathrm{Ce}$ does not reduce under the studied $\mathrm{pO}_{2}$ range). As a consequence, the electronic conductivity of these compositions in oxidizing conditions is not negligible and will be limited by the number of available sites for electron hops. CT50 and CT50Co materials show $p$-type electronic conductivity. XRD results showed that $\mathrm{Co}$ addition does affect the oxidation state of $\mathrm{Tb}$, increasing the ratio $\mathrm{Tb}^{4+} / \mathrm{Tb}^{3+}$. However, it is not entirely incorporated into the ceria lattice, but in the grain boundary. Consequently, the addition of cobalt oxide enables the improvement of the sintering and densification of specimens and the significant enhancement in total conductivity due to (i) the improved material reducibility at high temperatures and the associated rise in electronic conductivity and (ii) the increased concentration of oxygen vacancies.

XPS measurements proved the existence of $\mathrm{Tb}^{3+}$ on the CT20 and CT20Co surfaces and thus the availability of oxygen vacancies. The oxygen exchange rate of cobalt-free and cobalt-containing CTx materials was measured using PIE. The highest surface exchange rates were obtained for CT50 and CT50Co samples. On the whole, the results indicate that the exchange rate exhibited by CTx is limited by the dissociative adsorption of molecular oxygen 
at the oxide surface, and its magnitude is bounded by the relative magnitudes of the ionic and electronic conductivities in the materials.

Oxygen permeation was negligible for cobalt-free samples while CT10Co and CT20Co showed an oxygen flux at $1000{ }^{\circ} \mathrm{C}$ and $1 \mathrm{~mm}$ thickness of ca. 0.02 and $0.08 \mathrm{~mL} \cdot \mathrm{min}^{-1} \cdot \mathrm{cm}^{-2}$ for argon sweeping, respectively. CT10Co oxygen permeation flux was increased until reaching $0.5 \mathrm{~mL} \cdot \mathrm{min}^{-1} \cdot \mathrm{cm}^{-2}$ by using methane as sweep gas at $1000{ }^{\circ} \mathrm{C}$. Permeability results confirm the higher ambipolar conductivity of the CT20Co composition and the possibility to achieve suitable mixed conductivity values by the appropriate dopants and doping level.

Finally, thermogravimetric tests carried out in $5 \% \mathrm{CO}_{2}$-containing air confirmed the stability of ceria material in contrast with the instability observed for $\mathrm{Ba}-$ and $\mathrm{Sr}$-containing perovskites. The combined oxygen permeation and stability under reducing and carbonated atmospheres suggests the application of $\mathrm{Ce}_{1-\mathrm{x}} \mathrm{Tb}_{\mathrm{x}} \mathrm{O}_{2-\delta}+\mathrm{Co} 2 \%$ as membrane materials in catalytic membrane reactors for hydrocarbon and syngas conversion and oxygen separation using flue gas sweeping.

From the conductivity and surface results, a good membrane would be expected from CT20, CT50 and CT50C0 materials. However, new manufacturing methods need to be developed to obtain dense monoliths. New sintering strategies that allow obtaining dense specimens may be intended in the future, as spark plasma sintering or the combination of the material in dual-phase membranes. 


\subsection{References}

[1] D.P. Fagg, A.L. Shaula, V.V. Kharton, J.R. Frade, High oxygen permeability in fluorite-type $\mathrm{Ce}_{0.8} \mathrm{Pr}_{0.2} \mathrm{O}_{2-\delta}$ via the use of sintering aids, Journal of Membrane Science, 299 (2007) 1-7.

[2] C. Chatzichristodoulou, P.V. Hendriksen, A. Hagen, J.-C. Grivel, Oxygen Nonstoichiometry and Defect Chemistry Modelling of $\mathrm{Ce}_{0.8} \operatorname{Pr}_{\mathrm{x}} \mathrm{Tb}_{0.2-\mathrm{x}} \mathrm{O}_{2-\delta}, \quad \mathrm{ECS}$ Transactions, 13 (2008) 347-359.

[3] D.-J. Kim, Lattice Parameters, Ionic Conductivities, and Solubility Limits in Fluorite-Structure MO2 Oxide $\left[\mathrm{M}=\mathrm{Hf}^{4+}, \mathrm{Zr}^{4+}, \mathrm{Ce}^{4+}, \mathrm{Th}^{4+}, \mathrm{U}^{4+}\right]$ Solid Solutions, Journal of the American Ceramic Society, 72 (1989) 1415-1421.

[4] M. Mogensen, N.M. Sammes, G.A. Tompsett, Physical, chemical and electrochemical properties of pure and doped ceria, Solid State Ionics, 129 (2000) 6394.

[5] F. Ye, T. Mori, D.R. Ou, J. Zou, J. Drennan, Microstructural characterization of terbium-doped ceria, Materials Research Bulletin, 42 (2007) 943-949.

[6] J.D. Nicholas, L.C. De Jonghe, Prediction and evaluation of sintering aids for Cerium Gadolinium Oxide, Solid State Ionics, 178 (2007) 1187-1194.

[7] J. Ayawanna, D. Wattanasiriwech, S. Wattanasiriwech, P. Aungkavattana, Effects of cobalt metal addition on sintering and ionic conductivity of $\mathrm{Sm}(\mathrm{Y})$-doped ceria solid electrolyte for SOFC, Solid State Ionics, 180 (2009) 1388-1394.

[8] D. Pérez-Coll, P. Núñez, J.C.C. Abrantes, D.P. Fagg, V.V. Kharton, J.R. Frade, Effects of firing conditions and addition of Co on bulk and grain boundary properties of CGO, Solid State Ionics, 176 (2005) 2799-2805.

[9] Z. Zhang, W. Sigle, M. Rühle, E. Jud, L.J. Gauckler, Microstructure characterization of a cobalt-oxide-doped cerium-gadolinium-oxide by analytical and high-resolution TEM, Acta Materialia, 55 (2007) 2907-2917.

[10] D.P. Fagg, D. Perez-Coll, P. Nuñez, J.R. Frade, A.L. Shaula, A.A. Yaremchenko, V.V. Kharton, Ceria based mixed conductors with adjusted electronic conductivity in the bulk and/or along grain boundaries, Solid State Ionics, 180 (2009) 896-899.

[11] S.J. Hong, A.V. Virkar, Lattice Parameters and Densities of Rare-Earth Oxide Doped Ceria Electrolytes, Journal of the American Ceramic Society, 78 (1995) 433-439. 
[12] R. Shannon, Revised effective ionic radii and systematic studies of interatomic distances in halides and chalcogenides, Acta Crystallographica Section A, 32 (1976) 751-767.

[13] D.P. Fagg, S. García-Martin, V.V. Kharton, J.R. Frade, Transport Properties of Fluorite-Type $\mathrm{Ce}_{0.8} \mathrm{Pr}_{0.2} \mathrm{O}_{2-\delta}$ : Optimization via the Use of Cobalt Oxide Sintering Aid, Chemistry of Materials, 21 (2009) 381-391.

[14] D.P. Fagg, I.P. Marozau, A.L. Shaula, V.V. Kharton, J.R. Frade, Oxygen permeability, thermal expansion and mixed conductivity of $\mathrm{Gd}_{\mathrm{x}} \mathrm{Ce}_{0.8-\mathrm{x}} \mathrm{Pr}_{0.2} \mathrm{O}_{2-\delta}, \mathrm{x}=0$, 0.15, 0.2, Journal of Solid State Chemistry, 179 (2006) 3347-3356.

[15] K.L. Duncan, Y. Wang, S.R. Bishop, F. Ebrahimi, E.D. Wachsman, The role of point defects in the physical properties of nonstoichiometric ceria, Journal of Applied Physics, 101 (2007) 044906-044906.

[16] C. Chatzichristodoulou, P.V. Hendriksen, A. Hagen, Defect Chemistry and Thermomechanical Properties of $\mathrm{Ce}_{0.8} \mathrm{Pr}_{\mathrm{x}} \mathrm{Tb}_{0.2-\mathrm{x}} \mathrm{O}_{2-\delta}$, Journal of The Electrochemical Society, 157 (2010) B299-B307.

[17] D.P. Fagg, J.R. Frade, M. Mogensen, J.T.S. Irvine, Effects of firing schedule on solubility limits and transport properties of $\mathrm{ZrO}_{2}-\mathrm{TiO}_{2}-\mathrm{Y}_{2} \mathrm{O}_{3}$ fluorites, Journal of Solid State Chemistry, 180 (2007) 2371-2376.

[18] C.-W. Tang, C.-B. Wang, S.-H. Chien, Characterization of cobalt oxides studied by FT-IR, Raman, TPR and TG-MS, Thermochimica Acta, 473 (2008) 68-73.

[19] S. Tsunekawa, K. Ishikawa, Z.Q. Li, Y. Kawazoe, A. Kasuya, Origin of Anomalous Lattice Expansion in Oxide Nanoparticles, Physical Review Letters, 85 (2000) 3440-3443.

[20] X.D. Zhou, W. Huebner, Size-induced lattice relaxation in $\mathrm{CeO}_{2}$ nanoparticles, Applied Physics Letters, 79 (2001) 3512-3514.

[21] I. Kosacki, T. Suzuki, H.U. Anderson, P. Colomban, Raman scattering and lattice defects in nanocrystalline $\mathrm{CeO}_{2}$ thin films, Solid State Ionics, 149 (2002) 99-105.

[22] T.S. Stefanik, H.L. Tuller, Ceria-based gas sensors, Journal of the European Ceramic Society, 21 (2001) 1967-1970.

[23] C. Solís, W. Jung, H.L. Tuller, J. Santiso, Defect Structure, Charge Transport Mechanisms, and Strain Effects in $\mathrm{Sr}_{4} \mathrm{Fe}_{6} \mathrm{O}_{2+\delta}$ Epitaxial Thin Films, Chemistry of Materials, 22 (2010) 1452-1461. 
[24] K. Schmale, M. Grünebaum, M. Janssen, S. Baumann, F. Schulze-Küppers, H.-D. Wiemhöfer, Electronic conductivity of $\mathrm{Ce}_{0.8} \mathrm{Gd}_{0.2-\mathrm{x}} \mathrm{Pr}_{\mathrm{x}} \mathrm{O}_{2-\delta}$ and influence of added $\mathrm{CoO}$, physica status solidi (b), 248 (2011) 314-322.

[25] R. López, R. Gómez, Band-gap energy estimation from diffuse reflectance measurements on sol-gel and commercial $\mathrm{TiO}_{2}$ : a comparative study, J Sol-Gel Sci Technol, 61 (2012) 1-7.

[26] A.B. Murphy, Band-gap determination from diffuse reflectance measurements of semiconductor films, and application to photoelectrochemical water-splitting, Solar Energy Materials and Solar Cells, 91 (2007) 1326-1337.

[27] E. Paparazzo, On the curve-fitting of XPS Ce(3d) spectra of cerium oxides, Materials Research Bulletin, 46 (2011) 323-326.

[28] M.B. Reddy, G. Thrimurthulu, K. Lakshmi, Nanosized Unsupported and AluminaSupported Ceria-Zirconia and Ceria-Terbia Solid Solutions for CO Oxidation, Chinese Journal of Catalysis, 32 (2011) 800-806.

[29] F. Larachi, J. Pierre, A. Adnot, A. Bernis, Ce 3d XPS study of composite $\mathrm{Ce}_{\mathrm{x}} \mathrm{Mn}_{1-}$ ${ }_{x} \mathrm{O}_{2-y}$ wet oxidation catalysts, Applied Surface Science, 195 (2002) 236-250.

[30] I.M. Nagpure, S.S. Pitale, E. Coetsee, O.M. Ntwaeaborwa, J.J. Terblans, H.C. Swart, Low voltage electron induced cathodoluminescence degradation and surface characterization of $\mathrm{Sr}_{3}\left(\mathrm{PO}_{4}\right)_{2}$ : $\mathrm{Tb}$ phosphor, Applied Surface Science, 257 (2011) $10147-$ 10155.

[31] G. Blanco, J.M. Pintado, S. Bernal, M.A. Cauqui, M.P. Corchado, A. Galtayries, J. Ghijsen, R. Sporken, T. Eickhoff, W. Drube, Influence of the nature of the noble metal $(\mathrm{Rh}, \mathrm{Pt})$ on the low-temperature reducibility of a $\mathrm{Ce} / \mathrm{Tb}$ mixed oxide with application as TWC component, Surface and Interface Analysis, 34 (2002) 120-124.

[32] D.D. Sarma, C.N.R. Rao, XPES studies of oxides of second- and third-row transition metals including rare earths, Journal of Electron Spectroscopy and Related Phenomena, 20 (1980) 25-45.

[33] Z. Zsoldos, L. Guczi, Structure and catalytic activity of alumina supported platinum-cobalt bimetallic catalysts. 3. Effect of treatment on the interface layer, The Journal of Physical Chemistry, 96 (1992) 9393-9400.

[34] S.G. Aspromonte, Á. Sastre, A.V. Boix, M.J. Cocero, E. Alonso, Cobalt oxide nanoparticles on mesoporous MCM-41 and Al-MCM-41 by supercritical $\mathrm{CO}_{2}$ deposition, Microporous and Mesoporous Materials, 148 (2012) 53-61.

[35] J.A. Lane, J.A. Kilner, Oxygen surface exchange on gadolinia doped ceria, Solid State Ionics, 136-137 (2000) 927-932. 
[36] H.J.M. Bouwmeester, C. Song, J. Zhu, J. Yi, M. van Sint Annaland, B.A. Boukamp, A novel pulse isotopic exchange technique for rapid determination of the oxygen surface exchange rate of oxide ion conductors, Physical Chemistry Chemical Physics, 11 (2009) 9640-9643.

[37] C.-Y. Yoo, B.A. Boukamp, H.M. Bouwmeester, Oxygen surface exchange kinetics of erbia-stabilized bismuth oxide, J Solid State Electrochem, 15 (2011) 231-236. 



\title{
Chapter 6. LSM-ceria composite cathodes for SOFC
}

\begin{abstract}
Ceria-based materials have been incorporated into LSM conventional cathodes in order to improve electrochemical performance. Gadolinium and other elements $(\mathrm{Mg}, \mathrm{Cr}$ and $\mathrm{Bi})$ have been used as ceria co-dopants for enhancing conductivity and electrocatalytic properties of several LSM-ceria composites. Among different tested LSM-ceria cathodes, $\mathrm{Ce}_{0.8} \mathrm{Gd}_{0.1} \mathrm{Mg}_{0.1} \mathrm{O}_{2-\delta}$-based composite offers the lowest electrode polarization resistance in the $700-900{ }^{\circ} \mathrm{C}$ temperature range. However, at the lowest temperatures these results are similar to those for the $\mathrm{Ce}_{0.8} \mathrm{Gd}_{0.2} \mathrm{O}_{2-\delta}$ binary system, thus the selection of $\mathrm{Gd}$-co-doping elements should principally address economic issues. Since LSM-based cathodes are pointed for high temperature applications $\left(>800{ }^{\circ} \mathrm{C}\right)$ this magnesium-co-doped ceria-gadolinia compound could be an alternative to widely used $\mathrm{Ce}_{1-\mathrm{x}} \mathrm{Gd}_{\mathrm{x}} \mathrm{O}_{2-\delta}$ binary systems for composite SOFC cathodes.
\end{abstract}





\subsection{Introduction}

Strontium doped lanthanum manganite $\left(\mathrm{La}_{1-\mathrm{x}} \mathrm{Sr}_{\mathrm{x}} \mathrm{MnO}_{3}-\mathrm{LSM}\right)$ perovskites are the principal component of the state-of-the-art materials for SOFC cathodes. LSM-based compositions show interesting electrochemical properties for oxygen activation at high temperatures while they are thermochemically compatible with the widely used YSZ electrolyte materials. However, the lack of ionic conductivity and the decreasing performance of LSM cathodes at temperatures below $800{ }^{\circ} \mathrm{C}$ require mixing LSM cathodes with electrolyte materials. These LSM-electrolyte composites allow increasing the global ionic conductivity of the cathode while augments the three phase boundary (TPB) region thus enhancing the cathode performance. In order to increase the electrochemical performance of LSM-based composite cathodes, it is desirable that the electrolyte-like component possesses high oxygen ion conductivity, some electronic paths and/or enhanced catalytic activity for the oxygen reduction reaction at the operating temperature.

Rare earth-doped cerias studied in the previous chapters have demonstrated mainly ionic conductivity. Among them, gadolinia doped cerium oxide (CGO) shows the highest ionic conductivity, which is also higher than that of the YSZ electrolyte. Hence, this material seems to be an interesting electrolyte material, specifically at low temperatures. [1, 2] In addition, thermochemical properties of CGO material fit much closer with LSM than YSZ electrolytes. [3] The presence of gadolinium favors the oxygen ion conductivity while the addition of other doping elements may promote 
electronic conductivity. Moreover, the use of doped ceria materials in composite cathodes allows incorporating additional redox electrocatalytic activity. [4, 5]

In order to increase the ionic conductivity and improve other related properties of the materials, co-doping approach has been proved to be effective. Co-doping ceria with alkaline earth and rare earth ion shows significantly higher conductivity in air than singly doped materials with the same dopant concentration. [6-8] The possibility of adding mixed ionic-electronic conductivity is a plus for the use as a cathode, since the electronic conduction is not blocked by the electrolyte particles, thus boosting the TPB area.

With the premises exposed above, this chapter shows the electrochemical performance of composite SOFC cathodes, fabricated with LSM and different co-doped gadolinium-cerium oxides based on the $\mathrm{Ce}_{0.8} \mathrm{Gd}_{0.1} \mathrm{X}_{0.1} \mathrm{O}_{2-\delta}(\mathrm{X}=\mathrm{Gd}, \mathrm{Cr}, \mathrm{Mg}, \mathrm{Bi}, \mathrm{Ce})$ nominal formula.

\subsection{Results and discussion}

\section{Structural characterization}

The $\mathrm{La}_{0.8} \mathrm{Sr}_{0.2} \mathrm{MnO}_{3}$ (LSM) commercial powders are obtained from Fuel Cell Materials (USA). Pure fluorite compounds with the nominal formula $\mathrm{Ce}_{0.8} \mathrm{Gd}_{0.1} \mathrm{X}_{0.1} \mathrm{O}_{2-\delta}(\mathrm{X}=\mathrm{Gd}, \mathrm{Cr}, \mathrm{Mg}, \mathrm{Bi}, \mathrm{Ce})$ were synthesized by means of co-precipitation method after an $800{ }^{\circ} \mathrm{C}$ firing step under air. Table 6.1 summarizes the short nomenclature of the compounds that is used along the text. The compositions comprise both CG82 and CG91 parent materials and also the corresponding magnesium-, bismuth- and chromium-co-doped materials. 


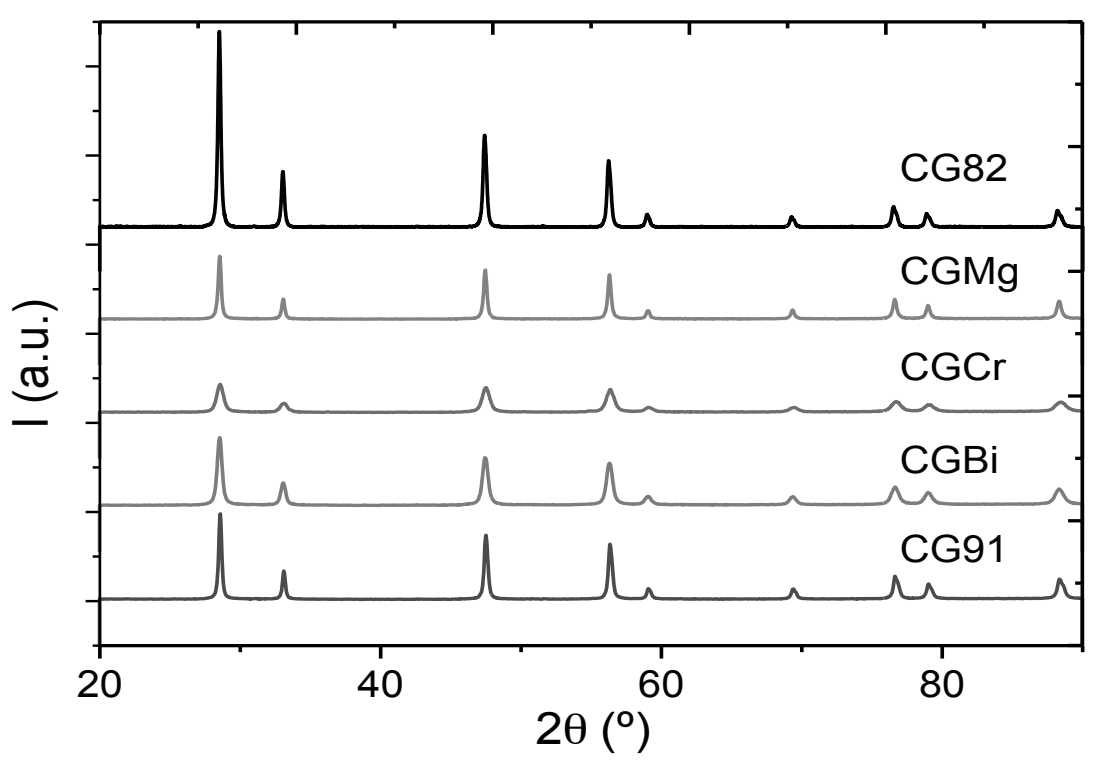

Figure 6.1. Room temperature XRD patterns of the as-sintered doped cerias CG82, $\mathrm{CGMg}, \mathrm{CGCr}, \mathrm{CGBi}$ and CG91) at $800^{\circ} \mathrm{C}$.

Figure 6.1 plots the XRD patterns of the as-sintered powders showing the expected cubic fluorite structure. Diffraction peaks corresponding to any other oxide or any precursor are not observed, which means that the different dopants are fully dissolved into the ceria lattice. Crystallographic lattice parameters $(a)$ for the $(h k l)$ planes were calculated using the corresponding equation for a cubic system and the results are summarized in Table 6.1. Crystallite sizes were extracted by fitting the $\left(\begin{array}{lll}4 & 2 & 2\end{array}\right)$ plane reflection to a Gaussian function. [9] The small amount of the co-dopant has small influence on the lattice size, calculated from the XRD patterns (Table 6.1), but it seems to have some effect on the ceria crystallite size as compared from Table 6.1. Indeed, by using the same synthesis method and sample processing, it is possible to obtain a range of crystallite sizes depending on the co-doping added element. Further investigations should be focused on this fact, studying the 
nucleation and crystal growth during precipitation and later sintering process, and its influence on particle size.

\begin{tabular}{lccc}
\hline Composition & Nomenclature & Lattice parameter $(\AA)$ & Crystallite size ( $\AA$ ) \\
\hline $\mathrm{Ce}_{0.8} \mathrm{Gd}_{0.2} \mathrm{O}_{2-\delta}$ & $\mathrm{CG} 82$ & $5.4224(1)$ & 529 \\
$\mathrm{Ce}_{0.9} \mathrm{Gd}_{0.1} \mathrm{O}_{2-\delta}$ & $\mathrm{CG} 91$ & $5.4152(1)$ & 535 \\
$\mathrm{Ce}_{0.8} \mathrm{Gd}_{0.1} \mathrm{Bi}_{0.1} \mathrm{O}_{2-\delta}$ & $\mathrm{CGBi}$ & $5.4153(3)$ & 255 \\
$\mathrm{Ce}_{0.8} \mathrm{Gd}_{0.1} \mathrm{Cr}_{0.1} \mathrm{O}_{2-\delta}$ & $\mathrm{CGCr}$ & $5.4143(9)$ & 169 \\
$\mathrm{Ce}_{0.8} \mathrm{Gd}_{0.1} \mathrm{Mg}_{0.1} \mathrm{O}_{2-\delta}$ & $\mathrm{CGMg}$ & $5.4159(6)$ & 477 \\
\hline
\end{tabular}

Table 6.1 Cerium-based compositions synthesized as cubic fluorite structure with the nomenclature used along the text, and the corresponding lattice parameter and crystallite size extracted from XRD patterns

Irrespective of differences in crystallite size among co-doping elements (Table 6.1), co-precipitation synthesis method permits obtaining powders nanometric particles, as observed in Figure 6.2a. Comparing, for instance, the particle size of the ceria-based CG82 material with the as-received LSM powder $\left(\mathrm{d}_{50}=0.6-0.3 \mu \mathrm{m}\right)$ it can be observed by SEM imaging (Figure $6.2 \mathrm{a}$ and $\mathrm{b}$ ) that particle size is much smaller for the ceria-based co-precipitated powders. 


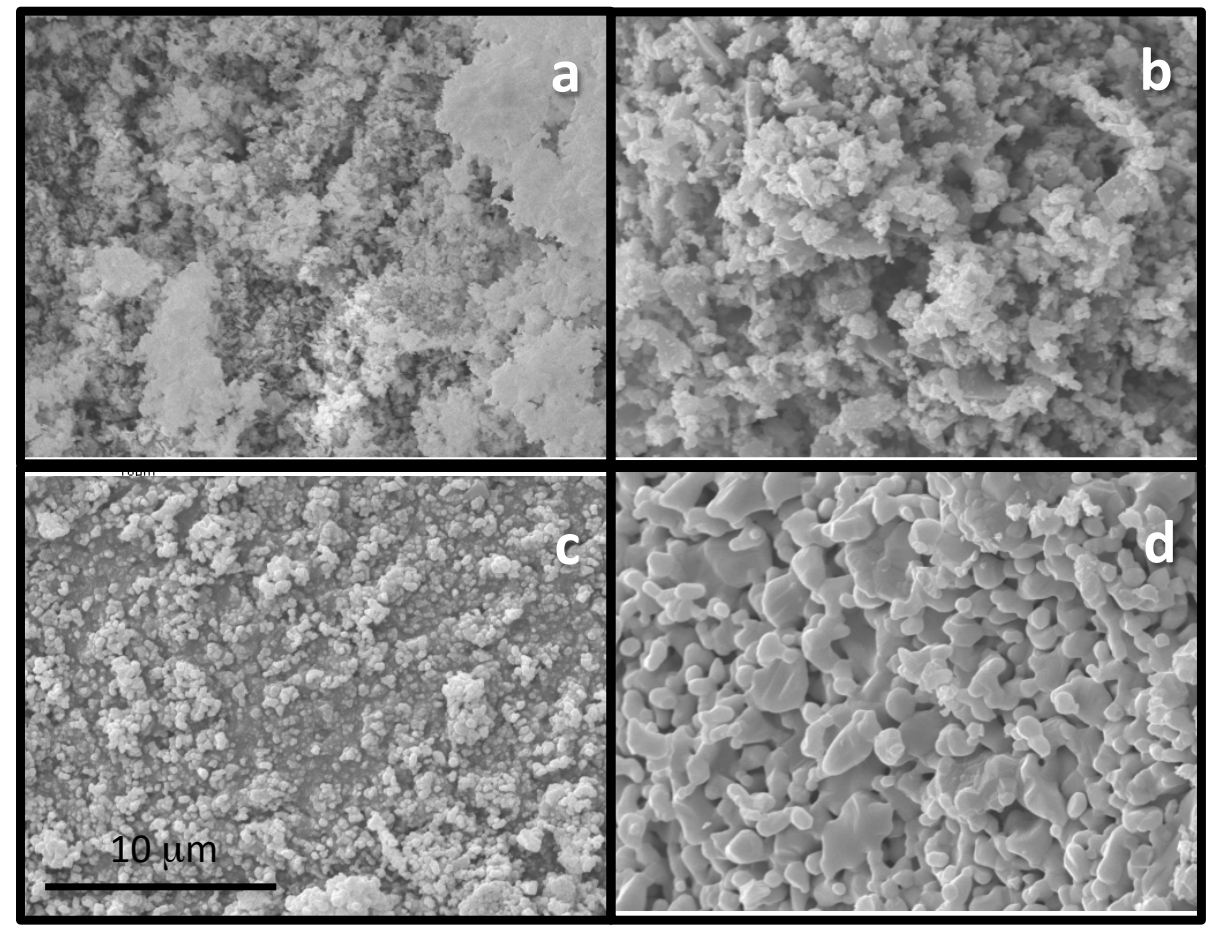

Figure 6.2. Comparison of the initial particle size of a) CG82 (as-sintered) and b) LSM (as-received) and the resulting powders after calcination at $1150{ }^{\circ} \mathrm{C}$ : c) $\mathrm{CG} 82$ and $\mathrm{d}$ ) LSM.

In addition and despite the fact that initial sintering temperature for the ceria-based compounds is lower than the commercial LSM $\left(1000{ }^{\circ} \mathrm{C}\right)$, after a $1150{ }^{\circ} \mathrm{C}$ calcination step, LSM powder has bigger and more sintered grains than ceria-based CG82 powder (Figure 6.2c and d) indicating the higher sintering activity of LSM material compared to the co-precipitated ceria compound. On the contrary, ceria-based CG82 material seems to produce smaller grains, with respect to LSM, after treatment at high temperature even when the initial synthesis temperature is low $\left(800^{\circ} \mathrm{C}\right)$.

Regarding this particle size evolution it should be assumed that the bigger LSM particles will become covered by smaller ceria-based ones in a 
non-well distributed composite. However, SEM images of several LSM-ceria composite cathodes (Figure 6.3) revealed that particle size is similar for both materials (LSM and ceria-based) after sintering together at $1150{ }^{\circ} \mathrm{C}$. Therefore, the sintering evolution of composite components that have been together calcined is not as different as when materials are sintered separately. This fact enables that mixtures of these materials have well distributed and connected particles and the fabricated composite cathodes can maintain proper conducting paths and enough porosity for SOFC cathode purposes. Spatial constraints favored by the good mixing of LSM and doped-ceria phases in the composite cathode allow preventing particle agglomeration and coarsening of the electrode materials. Therefore, both materials achieve similar particle size when calcined together. In addition, the presumably presence of small nanoparticles of ceria compounds (Figure 6.2c) on the surface of bigger LSM grains might be beneficial from the point of view of the cathode performance, as reported for both LSM-YSZ [10] and GDC-impregnated LSM. [11] This could be a further optimization step, which would entail a final infiltration step of the previously sintered cathode.

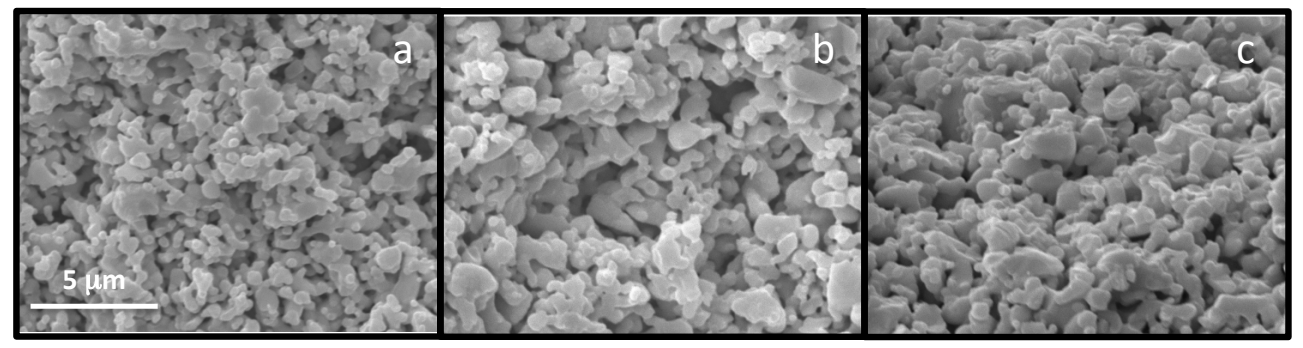

Figure 6.3. SEM images of several composite cathodes sintered at $1150{ }^{\circ} \mathrm{C}$ : a) CG82-LSM, b) CG91-LSM and c) CGBi-LSM. 


\section{DC-conductivity characterization}

As an extension of the Chapter 3, metallic dopants are introduced in combination with the Gd in order to improve the transport properties. Electrical conductivity of ceria-based materials under air is shown in Figure 6.4a at several temperatures. As described for several experimental works on $\mathrm{Ce}_{1-\mathrm{x}} \mathrm{Gd}_{\mathrm{x}} \mathrm{O}_{2-\delta}$ system, it can be observed that the total conductivity for CG91 is lower than for CG82 (Figure 6.4a). [12, 13] Conductivity enhancement on the ceria fluorite lattice obtained by $\mathrm{Gd}^{3+}$ addition arises from the ionic conductivity introduced by extrinsic vacancies formed to maintain the electroneutrality in the structure. Nevertheless, the best ratio for $\mathrm{CeO}_{2}-\mathrm{Gd}_{2} \mathrm{O}_{3}$ solid solution is still not clearly defined for applications as solid oxide fuel cells, since it depends on the proposed limiting conductivity mechanism. [14-17] Co-doped materials with the general formula $\mathrm{Ce}_{0.8} \mathrm{Gd}_{0.1} \mathrm{X}_{0.1} \mathrm{O}_{2-\delta}$ have a cerium and gadolinium molar content in between CG82 and CG91. Conductivity of these co-doped ceria materials (Figure 6.4a) lies in between the values observed for $20 \%$ and $10 \%$ Gd-doped ceria, i.e., CG82 and CG91. The total conductivity of these co-doped materials is high enough for SOFC cathode applications since CG82 and CG91 conductivity fits with the SOFC application requirements. 
a)

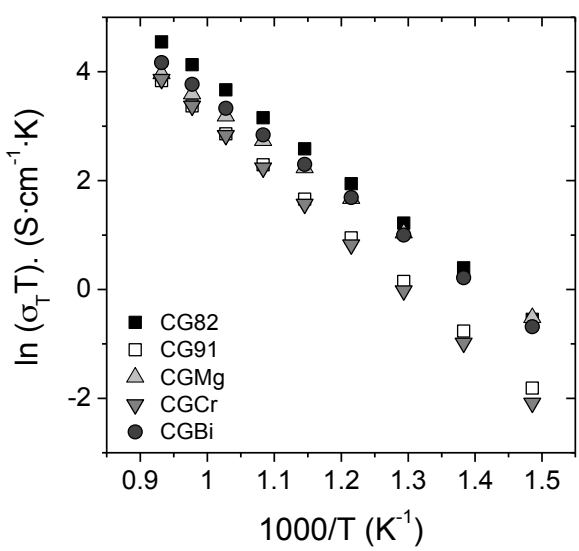

b)

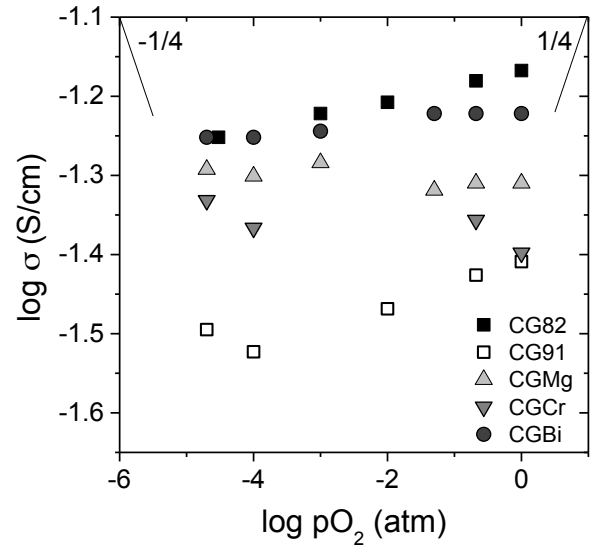

Figure 6.4. a) Arrhenius plot in air and b) $\mathrm{pO}_{2}$ dependence, of the total conductivity of ceria-based materials

Among the different co-doped materials, those based on bismuth (CGBi) and magnesium (CGMg) have similar conductivities. These conductivity values are close to those obtained for CG82 material at low temperatures (Figure 6.4a). On the other hand, CGCr composition has lower conductivity than the other two co-doped materials and it is even lower than CG91 compound when temperature decreases. Therefore, by using $\mathrm{Mg}$ or $\mathrm{Bi}$, it is possible to reduce gadolinium and/or cerium content in ceria-gadolinia materials without significantly decreasing the total electrical conductivity. This partial replacement of the gadolinium or cerium by other elements would make possible to reduce production costs whereas both electrochemical and catalytic activity are not significantly affected.

In addition to the conductivity measurements performed under air, different oxygen partial pressure atmospheres were used for measuring the total conductivity of these materials (Figure $6.4 \mathrm{~b}$ ) at $800{ }^{\circ} \mathrm{C}$. The studied range of $\mathrm{pO}_{2}$ includes both oxidant $(0.21-1 \mathrm{~atm})$ and moderately reducing atmospheres $\left(10^{-5}-0.21 \mathrm{~atm}\right)$, in where $\mathrm{Ce}^{4+}$ does not reduce to $\mathrm{Ce}^{3+}$. [18-21] Conductivity 
values in this oxygen partial pressure range (Figure 6.4b) seem to be independent on $\mathrm{pO}_{2}$ for all the materials, and consequently $p$-type conductivity is negligible. Therefore, conductivity of these ceria-based materials is mainly ionic in these oxidizing conditions. It seems not possible to promote the electronic conductivity either in the bulk or in the grain boundary through the chosen doping strategy on the ceria-based fluorite structure.

\section{Cathode testing on symmetric cells}

The enhancement of electrocatalytic properties of LSM cathode after mixing with ceria-based materials is shown in Figure 6.5. Electrode polarization resistance (Rp) values were obtained from electrochemical impedance spectroscopy (EIS) measurements on symmetrical cells, fabricated with the different LSM-ceria-based composite cathodes and using CG82 cathode that used Co as a sintering aid (CG82-Co). The chosen LSM to ceria ratio (in weight) is $50 / 50$ because it has been reported as the optimum for SOFC LSM-based cathode purpose. Irrespective to the ceria-based composition, the corresponding $\mathrm{Rp}$ of the resulting composite electrode is lower than single LSM electrode at any tested temperature. This confirms that the addition of ion conducting paths (ceria-based percolating network) allows extending the TPB area into a certain thickness of the electrode. Indeed, the presence of ionic conducting phase on LSM-YSZ state-of-the-art composite cathode also introduces ionic paths and increases TPB sites thus reducing the electrode polarization resistance compared to single LSM cathode. As previously explained, the use of ceria-compounds instead of YSZ materials could also increase the composite cathode electroactivity. [4] As a consequence, obtained electrode polarization resistance for ceria-based composites is lower than for LSM-YSZ cathodes as observed in Figure 6.5. 


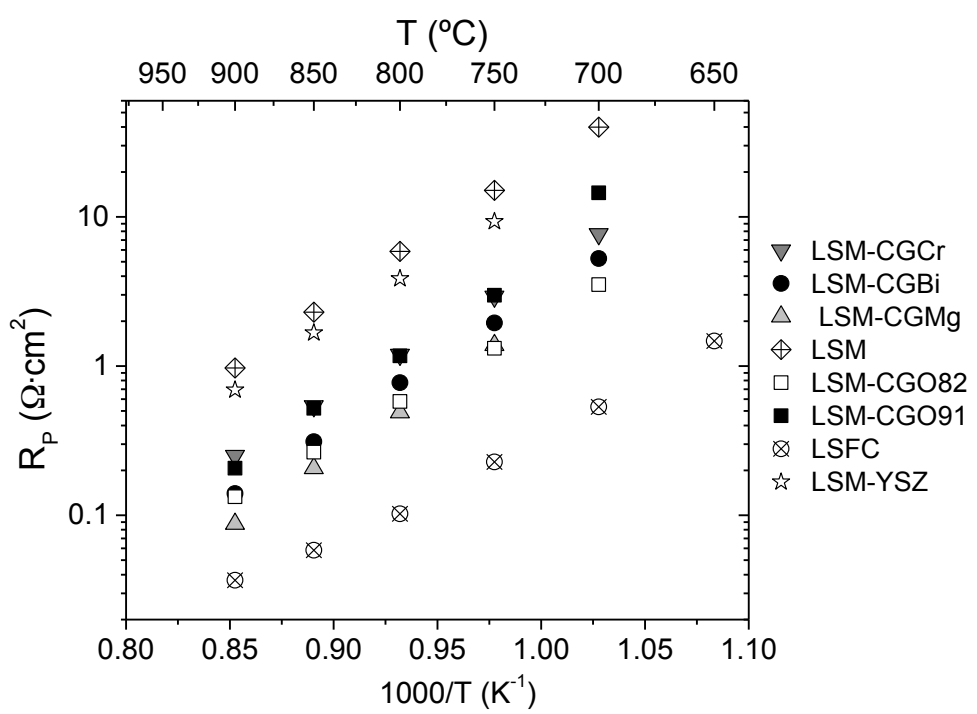

Figure 6.5. Arrhenius plot of the electrode polarization resistance of LSM-ceria-based composites and the single LSM electrodes. LSFC and LSM-YSZ are shown for comparison.

Figure 6.5 shows that the electrode polarization resistance values for the LSM-co-doped-ceria composites lie in between LSM-CG82 and LSM-CG91 electrodes. This is in agreement with the previously observed electrical conductivity measurements of single ceria-doped materials (Figure 6.4a). The best composite electrode regarding Rp values (Figure 6.5) is the LSM-CGMg cathode at any tested temperature. It also presents lower electrode polarization resistances than the LSM-CG82 cathode at higher temperatures $\left(800-900^{\circ} \mathrm{C}\right)$. Nonetheless, electrode resistance of LSM-CGMg increases with decreasing operating temperatures, i.e., this cathode presents high activation energy. This performance worsening may be ascribed to the less mobile oxygen vacancies at lower temperatures. Chromium- or bismuth-co-doping do not improve the electrochemical reaction for oxygen reduction with respect to the LSM-CG82 composite. 
Moreover, it can be inferred from Figure 6.5 that all ceria-based material composites exhibit higher polarization resistances than the well-established high-performing LSFC (tested on same symmetrical cell support) cathode. Blending ionic materials (CGMg, CGBi, CGCr and Gd-doped ceria) with LSM allows increasing the concentration of TPB sites. However, the extension of the active zone for the oxygen reduction reaction in these composites is still restricted to TPB points and not to the whole electrode surface area, as for LSFC mixed ionic-electronic conducting (MIEC) electrode. Despite that fact, composite microstructure of the present ceria-based electrodes could be further optimized in order to approach the corresponding performance of MIEC cathodes. [11, 12, 22]

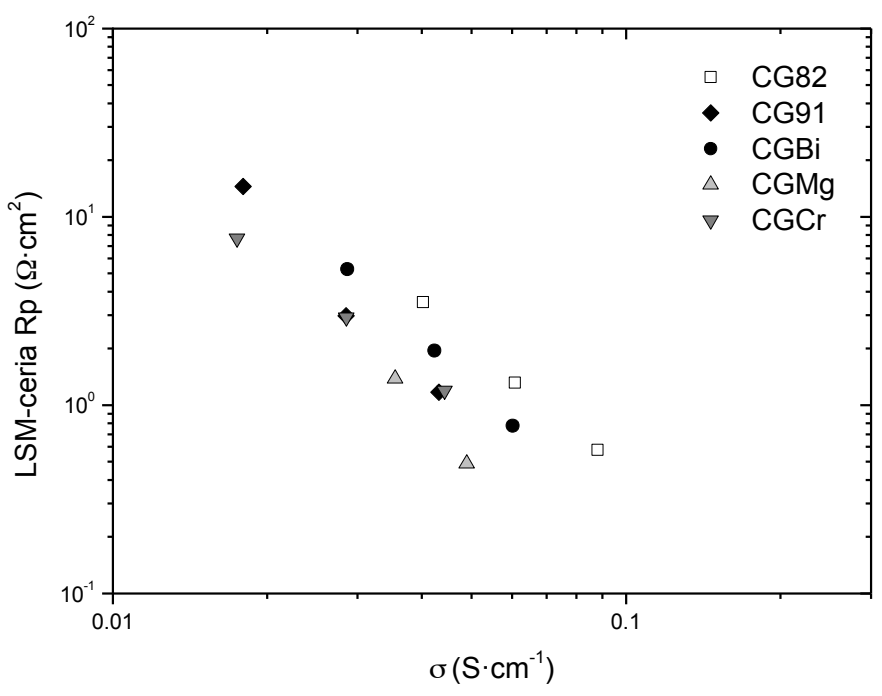

Figure 6.6. Polarization resistance as a function of conductivity at three different temperatures $\left(700,750\right.$ and $\left.800{ }^{\circ} \mathrm{C}\right)$

Figure 6.6 represents the polarization resistance of LSM-ceria-based composites as a function of ceria-based material conductivity at three temperatures $\left(700,750\right.$ and $\left.800{ }^{\circ} \mathrm{C}\right)$ for all tested electrodes. In general, there 
exists a trend suggesting that the resistance decreases with increasing conductivities for selected cathode composites. However, all data points do not fall in the same (straight) trend line, which suggests that other factors are affecting the polarization resistance such as surface reaction, interfacial resistance, electrode microstructure, etc.

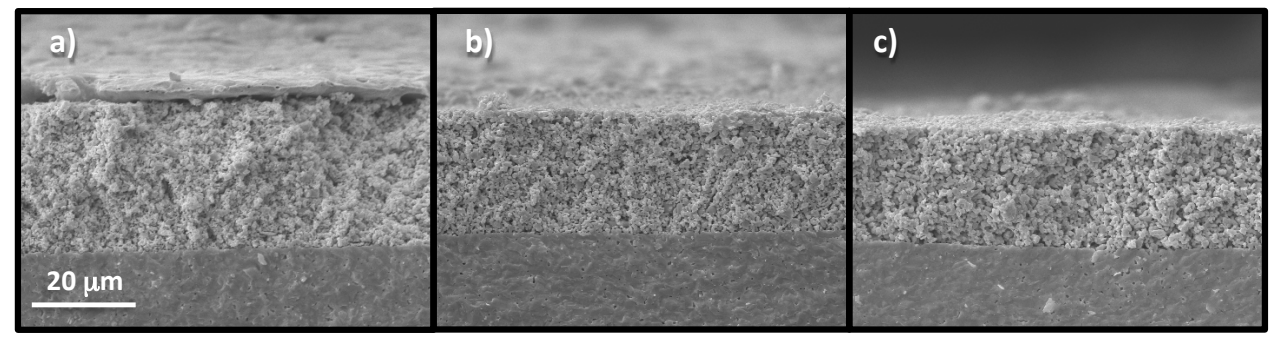

Figure 6.7. Cross section SEM images of a) CGCr-LSM, b) CG82-LSM and c) CG91LSM electrodes on a dense CG82-Co electrolyte. The upper layer seen in a) corresponds to the gold current collecting mesh.

The high density of CG82-Co electrolyte and the thickness of all porous electrode layers $(\sim 35 \mu \mathrm{m})$ can be inferred from the SEM images recorded on symmetrical cells fracture cross-section in Figure 6.7. [9] Electrodes are apparently very well attached to the electrolyte upon sintering in air at $1150{ }^{\circ} \mathrm{C}$. Moreover, the grains of the two materials of the composite are well connected and distributed. Despite the initial particle size of the different doped-cerias varies significantly (Table 6.1 and Figure 6.2a), a similar particle size distribution is observed for all electrodes, i.e., when they are mixed and sintered together with LSM (Figure 6.3 and Figure 6.7).

\section{Impedance spectroscopy modeling}

Previous results were analyzed in terms of the electrode polarization resistance obtained from EIS measurements. As an example of such EIS 
obtained data, Figure 6.8a represents the Nyquist plot for the different LSM-ceria-based composites while Figure $6.8 \mathrm{~b}$ shows the corresponding imaginary Bode plot, both at $800{ }^{\circ} \mathrm{C}$ under air. Spectra in Figure 6.8a correspond to the sum of both identical electrode contributions from symmetrical cells and from it the electrode polarization resistance can be calculated. In addition, data presented in Figure 6.8 could also be fitted to an equivalent circuit comprising three ZARC elements, as named by J. R. McDonald, [23] in series with an inductance (from wiring) and a pure resistance, which comes mainly from current collection and electrolyte resistance. Indeed, in Figure 6.8a the values corresponding to the pure resistance (R) vary among samples because of current collecting and contact resistances.
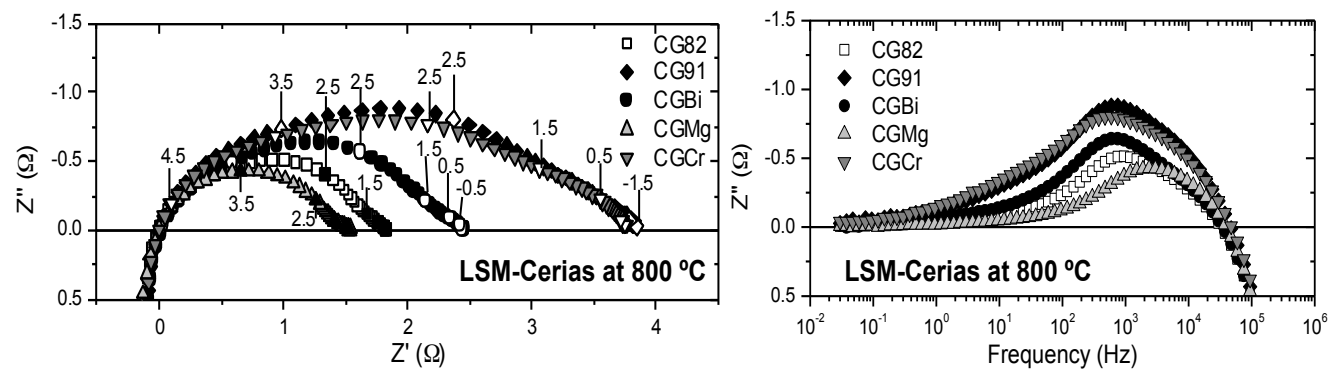

Figure 6.8. Electrochemical impedance spectra of symmetrical cells with different LSM-doped-ceria electrodes at $800{ }^{\circ} \mathrm{C}$ under air: a) Nyquist and b) imaginary impedance Bode plots. Logarithm of selected frequencies is provided on the Nyquist plot.

Fitting values of spectra in Figure 6.8, regarding to resistance, constant phase element parameters $\left(\mathrm{Q}_{0}\right.$ and $\left.n\right)$ and corresponding summit frequency of ZARC elements, are presented in Table 6.2, in which the area-corrected resistance values for single electrode contribution are presented. Taking into account the calculated summit frequency (as the inverse product of the resistance times the equivalent capacitance of the constant phase element [24]), 
two processes occur at similar relaxation times at high frequencies (A and $\mathrm{B})$ and the other one at relatively low frequencies (C).

\begin{tabular}{lcccccc}
\hline Fit & Units & LSM-CG91 & LSM-CG82 & LSM-CGMg LSM-CGCr LSM-CGBi \\
\hline $\mathbf{R}_{\mathbf{A}}$ & $\Omega \cdot \mathrm{cm}^{2}$ & 0.21 & 0.10 & 0.09 & 0.21 & 0.12 \\
$\mathbf{Q}_{\mathbf{0 A}}$ & $\mathrm{S} \cdot \mathrm{s}^{-\mathrm{n}}$ & $5.26 \cdot 10^{-5}$ & $4.99 \cdot 10^{-5}$ & $1.08 \cdot 10^{-5}$ & $6.34 \cdot 10^{-5}$ & $1.21 \cdot 10^{-4}$ \\
$\boldsymbol{n}_{\mathrm{A}}$ & -- & 0.91 & 0.96 & 1.00 & 0.90 & 0.86 \\
$\mathbf{\omega}_{\mathrm{A}}$ & $\mathrm{Hz}$ & $8.10 \cdot 10^{4}$ & $1.08 \cdot 10^{5}$ & $1.74 \cdot 10^{5}$ & $8.00 \cdot 10^{4}$ & $1.10 \cdot 10^{5}$ \\
$\mathbf{R}_{\mathbf{B}}$ & $\Omega \cdot \mathrm{cm}^{2}$ & 0.77 & 0.48 & 0.41 & 0.69 & 0.64 \\
$\mathbf{Q}_{\mathbf{0 B}}$ & $\mathrm{S} \cdot \mathrm{s}^{-\mathrm{n}}$ & $9.62 \cdot 10^{-4}$ & $1.01 \cdot 10^{-3}$ & $5.80 \cdot 10^{-4}$ & $1.30 \cdot 10^{-3}$ & $1.28 \cdot 10^{-3}$ \\
$\boldsymbol{n}_{\mathrm{B}}$ & -- & 0.72 & 0.75 & 0.75 & 0.70 & 0.71 \\
$\mathbf{\omega}_{\mathbf{B}}$ & $\mathrm{Hz}$ & $4.65 \cdot 10^{3}$ & $6.10 \cdot 10^{3}$ & $1.47 \cdot 10^{4}$ & $4.47 \cdot 10^{3}$ & $4.49 \cdot 10^{3}$ \\
$\mathbf{R}_{\mathrm{C}}$ & $\Omega \cdot \mathrm{cm}$ & 0.29 & 0.06 & 0.05 & 0.36 & 0.08 \\
$\mathbf{Q}_{\mathbf{0 C}}$ & $\mathrm{S} \cdot \mathrm{s}^{-\mathrm{n}}$ & $8.23 \cdot 10^{-2}$ & $6.45 \cdot 10^{-1}$ & $4.26 \cdot 10^{-1}$ & $5.11 \cdot 10^{-2}$ & $4.52 \cdot 10^{-1}$ \\
$\boldsymbol{n}_{\mathrm{C}}$ & -- & 0.54 & 0.54 & 0.58 & 0.56 & 0.61 \\
$\boldsymbol{\omega}_{\mathrm{C}}$ & $\mathrm{Hz}$ & $1.21 \cdot 10^{2}$ & $5.12 \cdot 10^{1}$ & $9.82 \cdot 10^{1}$ & $1.63 \cdot 10^{2}$ & $3.47 \cdot 10^{1}$
\end{tabular}

Table 6.2. Fitting parameters obtained for each LSM-ceria-based composite at $800{ }^{\circ} \mathrm{C}$ under air. $\mathrm{R}$ is the resistance, $\mathrm{Q}_{0}$ and $n$ are the constant phase element parameters and $\omega$ the summit frequency of each ZARC element, respectively. Capital letters (A, B, C) indicate the process according to the proposed electrical equivalent circuit

Electrochemical impedance response of electrodes based on LSM-YSZ composites has been described to possess up to five contributions. [25] Nonetheless, three different electrode processes named A, B and C seem to be more predominant in Figure 8 spectra. [26] According to the possible processes occurring in ceramic-ceramic composite cathodes for oxygen reduction reaction and the proposed model in Figure 6.9 and results (Table 6.2), [25-27] the two high frequency processes (named A and B) can be related to (1) transference of the oxygen intermediates/oxide ions or electrons [28-30] between ceria and LSM particles; and (2) the transport through the ceria-based particles of the composite towards the electrolyte. On the other hand, the low frequency 
contribution (named C) is related to the dissociative adsorption, dissociation and transfer of species at TPB coupled with the surface diffusion, similar to the processes occurring in MIEC cathodes. The last assignment is supported on the exponent values close to 0.5 of the constant phase elements $\left(n_{C}\right)$ (Table 6.2). Therefore the ZARC configuration could also have been modeled by a diffusion element [31] like a Gerischer or a Warburg. [32, 33] However, in order to directly analyze the contribution of the resistance of each process to the total electrode resistance, it was decided to model this contribution using a ZARC element.

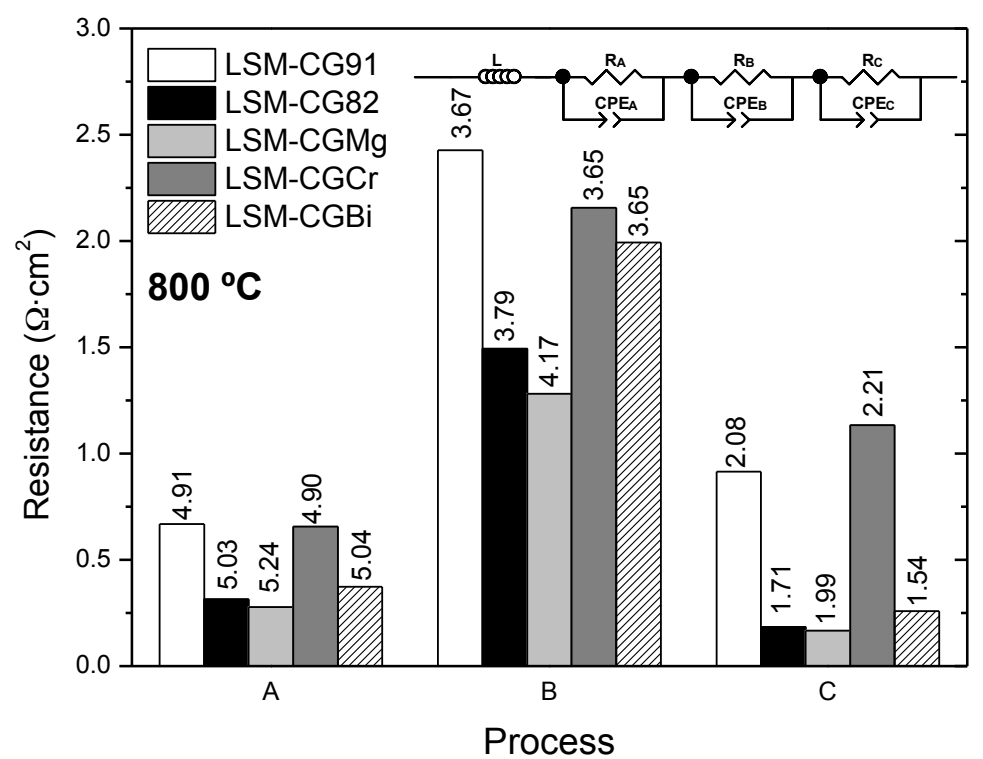

Figure 6.9. Resistances corresponding to the different processes obtained by fitting the spectra of symmetrical cells at $800{ }^{\circ} \mathrm{C}$ under air according to the equivalent circuit $\mathrm{LR}\left(\mathrm{R}_{\mathrm{A}} \mathrm{Q}_{\mathrm{A}}\right)\left(\mathrm{R}_{\mathrm{B}} \mathrm{Q}_{\mathrm{B}}\right)\left(\mathrm{R}_{\mathrm{C}} \mathrm{Q}_{\mathrm{C}}\right)$ shown in the inset. The corresponding logarithm of summit frequency is indicated on top of each bar.

In Figure 6.9 the resistance values for each fitted process are compared. Composite cathodes based on CG82 and CGMg showed resistance values lower than CG91- and CGCr-based composites for all three (A, B, C) processes. For 
LSM-CGBi cathode, the resistance assigned to process B seems to limit the whole cathode performance (Figure 6.9) even when the other two contributions (A and C) have relatively low values. CGMg-based cathode presents the lowest LF resistance of all and this improvement of the surface processes should be related to both (1) the higher surface concentration of oxygen vacancies/active (adsorption) sites in the oxygen reduction mechanism; and (2) the alkali character of the $\mathrm{Mg}$ cation, which improves the molecular oxygen adsorption. On the contrary, the highest LF contribution is observed for CG91 and CGCr. This could be caused by the lower surface concentration of oxygen vacancies and, in the particular case of CGCr, some detrimental effect of $\mathrm{Cr}$ species on the ceria surface and TPB sites.

The nature of limiting processes at the highest frequency range (A and B) cannot be unambiguously established. However, assuming that total conductivity at $800{ }^{\circ} \mathrm{C}$ for all doped cerias is similar (Figure 6.4a), high frequency limitations should be originated principally from cathode microstructure, i.e., effective paths for oxygen ions/intermediates or electrons transfer are limited. [26] Additionally, the potential role of grain boundary oxygen resistances of these ceria-based compounds cannot be excluded, especially at lower temperatures. [18]

On the whole, high amount of trivalent/divalent dopants (e.g. $\mathrm{Gd}^{3+}$ and $\left.\mathrm{Mg}^{2+}\right)$, in the ceria lattice benefits the oxygen vacancy formation and the resulting processes, at different frequency ranges, are enhanced due to the promotion of adsorption/dissociation steps, but also by better surface and bulk diffusion processes. On the contrary, multivalent chromium or gadoliniumisovalent bismuth have a detrimental effect on the global polarization resistance of the electrode. As a consequence, all three limiting processes analyzed and modeled here appear to be highly related one to each other. 


\subsection{Conclusions}

Several ceria-based materials have been properly synthesized by co-precipitation method. Total electrical conductivity of $\mathrm{Ce}_{0.8} \mathrm{Gd}_{0.1} \mathrm{X}_{0.1} \mathrm{O}_{2-\delta}$ ( $\mathrm{X}=\mathrm{Gd}, \mathrm{Cr}, \mathrm{Mg}, \mathrm{Bi}, \mathrm{Ce}$ ) materials has been proved to be sufficient for SOFC applications. Specifically, the electrochemical properties of several LSM-Ce ${ }_{0.8} \mathrm{Gd}_{0.1} \mathrm{X}_{0.1} \mathrm{O}_{2-\delta}$ composite cathodes have been analyzed in symmetrical cells. In general, polarization resistance decreases with increasing ceria-based materials total conductivities. Among different co-dopants, $\mathrm{Mg}$ seems to enhance both the oxygen ion transferring and the surface processes for oxygen reduction. Addition of chromium and bismuth to the ceria-gadolinia-based materials seems not to improve the LSM-composite cathode performance. Nevertheless, performance and conductivity of these new co-doped composite materials as SOFC cathodes is not significantly higher than $\mathrm{Ce}_{0.8} \mathrm{Gd}_{0.2} \mathrm{O}_{2-\delta}$ material. The use of the Mg-Gd-co-doped ceria may be viable due to economic reasons, regarding the price of cerium and gadolinium raw materials. Microstructural issues should be focused in order to further improve cathode performance.

\subsection{References}

[1] A.S. Nesaraj, Recent developments in solid oxide fuel cell technology - a review, J. Sci. Ind. Res., 69 (2010) 169-176.

[2] A.B. Stambouli, E. Traversa, Solid oxide fuel cells (SOFCs): a review of an environmentally clean and efficient source of energy, Renewable and Sustainable Energy Reviews, 6 (2002) 433-455.

[3] F. Tietz, Thermal expansion of SOFC materials, Ionics, 5 (1999) 129-139. 
[4] T.Z. Sholklapper, H. Kurokawa, C.P. Jacobson, S.J. Visco, L.C. De Jonghe, Nanostructured Solid Oxide Fuel Cell Electrodes, Nano Letters, 7 (2007) 2136-2141.

[5] N. Imanishi, R. Ohno, K. Murata, A. Hirano, Y. Takeda, O. Yamamoto, K. Yamahara, LSM-YSZ Cathode with Infiltrated Cobalt Oxide and Cerium Oxide Nanoparticles, Fuel Cells, 9 (2009) 215-221.

[6] Y. Zheng, Y. Shi, H. Gu, L. Gao, H. Chen, L. Guo, La and Ca co-doped ceria-based electrolyte materials for IT-SOFCs, Materials Research Bulletin, 44 (2009) 1717-1721.

[7] J. van Herle, D. Seneviratne, A.J. McEvoy, Lanthanide co-doping of solid electrolytes: AC conductivity behaviour, Journal of the European Ceramic Society, 19 (1999) 837-841.

[8] Y. Zheng, H. Gu, H. Chen, L. Gao, X. Zhu, L. Guo, Effect of Sm and Mg co-doping on the properties of ceria-based electrolyte materials for IT-SOFCs, Materials Research Bulletin, 44 (2009) 775-779.

[9] A. Kumar, S. Babu, A.S. Karakoti, A. Schulte, S. Seal, Luminescence Properties of Europium-Doped Cerium Oxide Nanoparticles: Role of Vacancy and Oxidation States, Langmuir, 25 (2009) 10998-11007.

[10] E. Perry Murray, S.A. Barnett, (La,Sr) $\mathrm{MnO}_{3}-(\mathrm{Ce}, \mathrm{Gd}) \mathrm{O}_{2-\mathrm{x}}$ composite cathodes for solid oxide fuel cells, Solid State Ionics, 143 (2001) 265-273.

[11] S.P. Jiang, W. Wang, Novel structured mixed ionic and electronic conducting cathodes of solid oxide fuel cells, Solid State Ionics, 176 (2005) 1351-1357.

[12] Z. Tianshu, P. Hing, H. Huang, J. Kilner, Ionic conductivity in the $\mathrm{CeO}_{2}-\mathrm{Gd}_{2} \mathrm{O}_{3}$ system $(0.05 \leq \mathrm{Gd} / \mathrm{Ce} \leq 0.4)$ prepared by oxalate coprecipitation, Solid State Ionics, 148 (2002) $567-573$.

[13] N.P. Brandon, D. Thompsett, Fuel cells compendium, Oxford, 2005.

[14] V.V. Kharton, F.M. Figueiredo, L. Navarro, E.N. Naumovich, A.V. Kovalevsky, A.A. Yaremchenko, A.P. Viskup, A. Carneiro, F.M.B. Marques, J.R. Frade, Ceria-based materials for solid oxide fuel cells, Journal of Materials Science, 36 (2001) 1105-1117.

[15] B.C.H. Steele, Appraisal of $\mathrm{Ce}_{1-\mathrm{y}} \mathrm{Gd}_{\mathrm{y}} \mathrm{O}_{2-\mathrm{y} / 2}$ electrolytes for IT-SOFC operation at $500{ }^{\circ} \mathrm{C}$, Solid State Ionics, 129 (2000) 95-110.

[16] D.S. Jung, S.H. Lee, J.M. Han, H.J. Hwang, J.-H. Lee, Y.C. Kang, Microstructure and electrical properties of nano-sized $\mathrm{Ce}_{1-\mathrm{x}} \mathrm{Gd}_{\mathrm{x}} \mathrm{O}_{2}(0 \leq \mathrm{x} \leq 0.2)$ particles prepared by spray pyrolysis, Journal of The Ceramic Society of Japan, 116 (2008) 969-974. 
[17] V.V. Kharton, Solid state electrochemistry: Fundamentals, materials and their applications, Weinheim, 2009.

[18] D.P. Fagg, S. García-Martin, V.V. Kharton, J.R. Frade, Transport Properties of Fluorite-Type $\mathrm{Ce}_{0.8} \mathrm{Pr}_{0.2} \mathrm{O}_{2-\delta}$ : Optimization via the Use of Cobalt Oxide Sintering Aid, Chemistry of Materials, 21 (2009) 381-391.

[19] D.P. Fagg, I.P. Marozau, A.L. Shaula, V.V. Kharton, J.R. Frade, Oxygen permeability, thermal expansion and mixed conductivity of $\mathrm{Gd}_{\mathrm{x}} \mathrm{Ce}_{0.8-\mathrm{x}} \mathrm{Pr}_{0.2} \mathrm{O}_{2-\delta}, \mathrm{x}=0$, 0.15, 0.2, Journal of Solid State Chemistry, 179 (2006) 3347-3356.

[20] K.L. Duncan, Y. Wang, S.R. Bishop, F. Ebrahimi, E.D. Wachsman, The role of point defects in the physical properties of nonstoichiometric ceria, Journal of Applied Physics, 101 (2007) 044906-044906.

[21] C. Chatzichristodoulou, P.V. Hendriksen, A. Hagen, Defect Chemistry and Thermomechanical Properties of $\mathrm{Ce}_{0.8} \mathrm{Pr}_{\mathrm{x}} \mathrm{Tb}_{0.2-\mathrm{x}} \mathrm{O}_{2-\delta}$, Journal of The Electrochemical Society, 157 (2010) B299-B307.

[22] V.A.C. Haanappel, J. Mertens, D. Rutenbeck, C. Tropartz, W. Herzhof, D. Sebold, F. Tietz, Optimisation of processing and microstructural parameters of LSM cathodes to improve the electrochemical performance of anode-supported SOFCs, Journal of Power Sources, 141 (2005) 216-226.

[23] J.R. Macdonald, Impedance Spectroscopy: Emphasizing Solid Materials and Systems, New York, 1987.

[24] J. Fleig, The grain boundary impedance of random microstructures: numerical simulations and implications for the analysis of experimental data, Solid State Ionics, 150 (2002) 181-193.

[25] M.J. Jørgensen, M. Mogensen, Impedance of Solid Oxide Fuel Cell LSM/YSZ Composite Cathodes, Journal of The Electrochemical Society, 148 (2001) A433-A442.

[26] M.J.L. Østergård, M. Mogensen, ac Impedance study of the oxygen reduction mechanism on $\mathrm{La}_{1-\mathrm{x}} \mathrm{Sr}_{\mathrm{x}} \mathrm{MnO}_{3}$ in solid oxide fuel cells, Electrochimica Acta, 38 (1993) 2015-2020.

[27] E. Barsoukov, J.R. Macdonald, Impedance spectroscopy: theory, experiment and applications, New Jersey, 2005.

[28] V.B. Vert, J.M. Serra, Influence of Barium Incorporation on the Electrochemical Performance of $\mathrm{Ln}_{0.58} \mathrm{Sr}_{0.4} \mathrm{Fe}_{0.8} \mathrm{Co}_{0.2} \mathrm{O}_{3-\delta}$ ( $\left.\mathrm{Ln}=\mathrm{La}, \mathrm{Pr}, \mathrm{Sm}\right)$ Perovskites for Oxygen Activation at Intermediate Temperatures, Fuel Cells, 9 (2009) 663-678. 
[29] V.B. Vert, J.M. Serra, Improvement of the Electrochemical Performance of $\mathrm{Ln}_{0.58} \mathrm{Sr}_{0.4} \mathrm{Fe}_{0.8} \mathrm{Co}_{0.2} \mathrm{O}_{3-\delta}$ IT-SOFC Cathodes by Ternary Lanthanide Combinations (LaPr-Sm), Fuel Cells, 10 (2010) 693-702.

[30] J.M. Serra, V.B. Vert, Optimization of Oxygen Activation Fuel-Cell Electrocatalysts by Combinatorial Designs, ChemSusChem, 2 (2009) 957-961.

[31] http://consultrsr.com/resources/eis/diffusion.htm.

[32] M.E. Orazem, P. Shukla, M.A. Membrino, Extension of the measurement model approach for deconvolution of underlying distributions for impedance measurements, Electrochimica Acta, 47 (2002) 2027-2034.

[33] M. González-Cuenca, W. Zipprich, B.A. Boukamp, G. Pudmich, F. Tietz, Impedance Studies on Chromite-Titanate Porous Electrodes under Reducing Conditions, Fuel Cells, 1 (2001) 256-264. 


\title{
Chapter 7. Ionic transport improvement on $\mathrm{CaTiO}_{3}$ based perovskites
}

\begin{abstract}
The second family of materials under research comprises $\mathrm{CaTiO}_{3}$-based perovskites. $\mathrm{CaTiO}_{3}$ perovskite is a $p$-type electronic conductor whose ionic conductivity may be increased by doping with lower valent cations. However, the stability of the structure may be drastically affected by the guest cations turming temperature or atmosphere dependent or developing a different non-perovskite structure. The series $\mathrm{Ca}_{1-\mathrm{z}} \mathrm{A}_{\mathrm{z}} \mathrm{Ti}_{1-\mathrm{x}}\left(\mathrm{M} 1_{1-\mathrm{y}} \mathrm{M} 2_{\mathrm{y}}\right)_{\mathrm{x}} \mathrm{O}_{3-\delta}$ has been prepared by solid state reaction to evaluate the structural stability and ionic conductivity improvement of $\mathrm{CaTiO}_{3}$ perovskites. The first screening shows that even the structural predictions are contrary, single phase perovskite structure is not achieved for all the compositions. DC conductivity indicates that all the dopants allow increasing the conductivity, especially $\mathrm{CaTi}_{0.8} \mathrm{Fe}_{0.2} \mathrm{O}_{3-\delta}$ and $\mathrm{CaTi}_{0.73} \mathrm{Fe}_{0.18} \mathrm{Mg}_{0.09} \mathrm{O}_{3-\delta}$. The former has been well reported in literature and compared with the second one in the present chapter.

Bulk transport and surface properties have been characterized by means of DC conductivity up to $1000{ }^{\circ} \mathrm{C}$, thermogravimetry (TG) and temperature programmed desorption (TPD), X-ray photoelectron spectroscopy (XPS) and pulse isotope exchange (PIE). The present study presents the preparation of a membrane made of $\mathrm{CaTi}_{0.73} \mathrm{Fe}_{0.18} \mathrm{Mg}_{0.09} \mathrm{O}_{3-\delta}$. The systematic study of the
\end{abstract}


membrane operation variables on the oxygen flux is shown, i.e. inlet gas flow rate at sweep membrane compartment, temperature and oxygen feed gas nature. Moreover, membrane stability is proved under $\mathrm{CO}_{2}$ as sweep gas. 


\subsection{Introduction}

Perovskite-structured compounds with the formula $\mathrm{ABO}_{3}$ take their name from the mineral $\mathrm{CaTiO}_{3}$. [1] The ideal perovskite possesses the cubic symmetry in which A is a large cation sited on the corners in 12-fold oxygen coordination(as a lanthanide, alkali or alkali earth), while the body center $\mathrm{B}$ is a small cation in 6-fold oxygen coordination, as transition metals or small lanthanides. The oxygen anions occupy the faces center. Similar to the fluorites, the structure is able to host cations with different valence and ionic radii to achieve high concentrations of oxygen vacancies and ionic conductivity. This affects the crystal stability, which is predicted by the tolerance factor established by Goldschmidt

$t=\frac{\left(r_{A}+r_{0}\right)}{\sqrt{2}\left(r_{B}+r_{0}\right)}$

where $r_{A}, r_{B}$ and $r_{O}$ are the ionic radii of the cations in $A$ and $B$ site and the oxygen anion, respectively. The A cation in an ideal perovskite has the same size as the oxygen anion (1.4 $\AA$ ) forming a well packed cube that hosts the B-cation. Cubic perovskites will be obtained for values close to $t=1$, while other distorted perovskites are obtained provided $0.8<t<1$. Goldschmidt tolerance condition is necessary but not sufficient for the stability of the perovskite. Another governing parameter for the formability of perovskites is known as octahedral factor (o.f.), the ratio of radii of the small cation B over the radii of anion $\mathrm{O}$, which is related to the stability of $\mathrm{BO}_{6}$ basic octahedron. The lowest limit for the o.f. to deliver a stable perovskite is 0.425 , which means that the minimum $r_{B}$ is $0.53 \AA$. [2] 
Perovskites as $\mathrm{LaGaO}_{3}$ are mainly ionic conductors, while in perovskites containing $\mathrm{Fe}, \mathrm{Co}, \mathrm{Ni}$ or $\mathrm{Mn}$ the electronic conductivity is usually orders of magnitude larger than the ionic conductivity. [3, 4] The ionic conductivity takes place by the hopping of oxygen ions from one oxygen vacant site to neighbouring vacant sites, so that the oxygen deficiency $\mathrm{ABO}_{3-\delta}$ is needed. The A site is commonly used to tune the oxygen vacancy concentration while the B site is basically responsible for overall electronic conductivity through $\mathrm{B}^{\mathrm{n}+}-\mathrm{O}-\mathrm{B}^{(\mathrm{n}+1)+}$ conduction pairs. [5] For example, the ionic conductivity can be enhanced by the substitution of A-site cations with lower valent cations. On the contrary, higher valent ions may increase the phase stability, although the annihilation of oxygen vacancies is expected to decrease the ionic conductivity. Partially substituting the A site by larger isovalent cations may improve the chemical and structural stability in reducing environments while keeping the oxygen flux levels. [4] On the other hand, the decrease of average A cations radii may reduce the oxygen flux. [4]

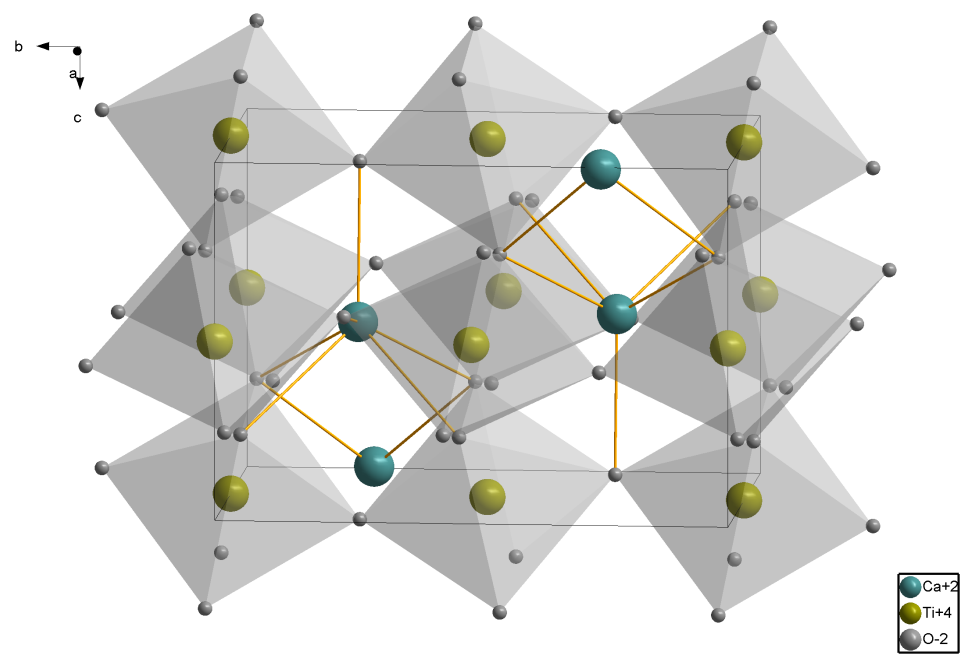

Figure 7.1. $\mathrm{CaTiO}_{3}$ orthorhombic perovskite from the family $\mathrm{A}^{\mathrm{II}} \mathrm{B}^{\mathrm{IV}} \mathrm{O}_{3}$. 
As mentioned in Chapter 1, iron and cobalt-based perovskites typically possess high electron conductivity as well as good ionic conductivity, so that intensive research devoted to the preparation and characterization of MIEC perovskite membranes has been developed in the last decades. [6-9] The most extensively used perovskite materials are large A-site BSCF and LSCF, which despite their high oxygen fluxes still present fundamental disadvantages for a proper use, as temperature dependent phase transitions. [4, 10-12] On the other hand, $\mathrm{CaTiO}_{3}$ based materials are stable perovskite in a vast range of temperatures and $p O_{2}$. [13]

The perovskite $\mathrm{CaTiO}_{3}$ represented in Figure 7.1 is slightly distorted from cubic structure and belong to orthorhombic symmetry with Pnma spatial group. $[1,13] \mathrm{CaTiO}_{3}$ is a MIEC at low $p O_{2}$, but the electrolytic range of $\mathrm{pO}_{2}$ may be extended to higher oxygen content atmospheres by doping, since $\mathrm{CaTiO}_{3}$ is able to keep the perovskite structure also at high defect concentrations. When acceptor dopants are substituted in the Ti site, both ionic and electronic conductivities may be enhanced. The ionic conductivity can be also enhanced by the substitution of Ca cations with lower valent cations, while higher valent dopants create A-site deficiencies. $[14,15]$ In this study, different dopants have been introduced in the mainly electronic $\mathrm{CaTiO}_{3}$, attending to criteria as ionic radii affinity and aliovalence or multivalence of the cations with the aim of improving the ionic and the electronic conductivity of the material. A first approach has been done to obtain the perovskite structure discarding such compositions that were not single phase or perovskite at all. The compounds showing the perovskite structure were tested by DC conductivity with the aim of selecting MIEC materials suitable for oxygen transport. 


\subsection{Results and discussion}

The series $\mathrm{Ca}_{1-\mathrm{z}} \mathrm{A}_{\mathrm{z}} \mathrm{Ti}_{1-\mathrm{x}}\left(\mathrm{M} 1_{1-\mathrm{y}} \mathrm{M} 2_{\mathrm{y}}\right)_{\mathrm{x}} \mathrm{O}_{3-\delta}$ has been prepared by solid state reaction. The synthesis has been done at $1200{ }^{\circ} \mathrm{C}$ during 14 hours to favour the formation of the perovskite phase. Several dopants have been selected on their ionic radii and aliovalences of the substituted element. Table 7.1 lists the combination of dopants and the ionic radii of $\mathrm{A}$ and $\mathrm{B}$ positions, as well as an identification number. A preliminary prediction of the formation of stable perovskite phase has been tried on the basis of the Goldschmidt and octahedral factors, and the experimental results are also exposed. The crystalline phase(s) of the materials were determined by XRD. Even though all the compounds exhibit $0.8<t<1$ and o.f. $<0.425$ (except $\mathrm{CaTi}_{0.8} \mathrm{Fe}_{0.2} \mathrm{O}_{3-\delta}$ and $\mathrm{CaTi}_{0.6} \mathrm{Fe}_{0.4} \mathrm{O}_{3-\delta}$ ), several of them did not deliver pure perovskite structure. Therefore, they were not further investigated. Several reasons may contribute to the instability, as that the dopant does not substitute in the expected site or with the expected oxidation state (so that expected ionic radii) and thus the predictions are not valid. 


\begin{tabular}{|c|c|c|c|c|c|c|}
\hline Material & $\#$ & $r_{A}(\AA)$ & $r_{B}(\AA)$ & $\mathbf{t}$ & o.f. & Symmetry \\
\hline $\mathrm{CaTiO}_{3}$ & 0 & 1.34 & 0.605 & 0.966 & 0.432 & $\mathrm{O}$ \\
\hline $\mathrm{CaTi}_{0.8} \mathrm{Fe}_{0.2} \mathrm{O}_{3-\delta}$ & 1 & 1.34 & 0.594 & 0.972 & 0.424 & $\mathrm{O}$ \\
\hline $\mathrm{CaTi}_{0.9} \mathrm{Co}_{0.1} \mathrm{O}_{3-\delta}$ & 2 & 1.34 & 0.6 & 0.969 & 0.428 & $\mathrm{O}$ \\
\hline $\mathrm{CaTi}_{0.9} \mathrm{Cu}_{0.1} \mathrm{O}_{3-\delta}$ & 3 & 1.34 & 0.618 & 0.960 & 0.441 & $\mathrm{O}$ \\
\hline $\mathrm{CaTi}_{0.8} \mathrm{Ga}_{0.2} \mathrm{O}_{3-\delta}$ & 4 & 1.34 & 0.608 & 0.965 & 0.434 & $\mathrm{X}$ \\
\hline $\mathrm{CaTi}_{0.9} \mathrm{Mg}_{0.1} \mathrm{O}_{3-\delta}$ & 5 & 1.34 & 0.617 & 0.961 & 0.440 & $\mathrm{O}$ \\
\hline $\mathrm{CaTi}_{0.73} \mathrm{Fe}_{0.18} \mathrm{Mg}_{0.09} \mathrm{O}_{3-\delta}$ & 6 & 1.34 & 0.605 & 0.966 & 0.432 & $\mathrm{O}$ \\
\hline $\mathrm{CaTi}_{0.7} \mathrm{Fe}_{0.2} \mathrm{Mg}_{0.1} \mathrm{O}_{3-\delta}$ & 6 bis & 1.34 & 0.606 & 0.966 & 0.432 & $\mathrm{X}$ \\
\hline $\mathrm{CaTi}_{0.8} \mathrm{~V}_{0.1} \mathrm{Mg}_{0.1} \mathrm{O}_{3-\delta}$ & 7 & 1.34 & 0.610 & 0.964 & 0.436 & $\mathrm{X}$ \\
\hline $\mathrm{CaTi}_{0.85} \mathrm{~V}_{0.05} \mathrm{Mg}_{0.1} \mathrm{O}_{3}$ & 7 bis & 1.34 & 0.613 & 0.963 & 0.438 & $\mathrm{X}$ \\
\hline $\mathrm{CaTi}_{0.8} \mathrm{Cr}_{0.1} \mathrm{Mg}_{0.1} \mathrm{O}_{3-\delta}$ & 8 & 1.34 & 0.618 & 0.960 & 0.441 & $\mathrm{O}$ \\
\hline $\mathrm{CaTi}_{0.8} \mathrm{Nb}_{0.1} \mathrm{Mg}_{0.1} \mathrm{O}_{3-\delta}$ & 9 & 1.34 & 0.62 & 0.959 & 0.443 & $\mathrm{O}$ \\
\hline $\mathrm{CaTi}_{0.85} \mathrm{Mo}_{0.05} \mathrm{Mg}_{0.1} \mathrm{O}_{3-\delta}$ & 10 & 1.34 & 0.616 & 0.961 & 0.440 & $\mathrm{O}$ \\
\hline $\mathrm{CaTi}_{0.9} \mathrm{Al}_{0.1} \mathrm{O}_{3-\delta}$ & 11 & 1.34 & 0.598 & 0.970 & 0.427 & $\mathrm{O}$ \\
\hline $\mathrm{CaTi}_{0.9} \mathrm{Y}_{0.1} \mathrm{O}_{3-\delta}$ & 12 & 1.34 & 0.635 & 0.952 & 0.453 & $\mathrm{X}$ \\
\hline $\mathrm{CaTi}_{0.9} \mathrm{Mn}_{0.1} \mathrm{O}_{3-\delta}$ & 13 & 1.34 & 0.612 & 0.963 & 0.437 & $\mathrm{X}$ \\
\hline $\mathrm{CaTi}_{0.8} \mathrm{Co}_{0.1} \mathrm{Mg}_{0.1} \mathrm{O}_{3-\delta}$ & 14 & 1.34 & 0.611 & 0.963 & 0.436 & $\mathrm{X}$ \\
\hline $\mathrm{CaTi}_{0.8} \mathrm{Mg}_{0.2} \mathrm{O}_{3-\delta}$ & 15 & 1.34 & 0.628 & 0.956 & 0.449 & $\mathrm{X}$ \\
\hline $\mathrm{CaTi}_{0.8} \mathrm{Ni}_{0.1} \mathrm{Mg}_{0.1} \mathrm{O}_{3-\delta}$ & 16 & 1.34 & 0.612 & 0.963 & 0.437 & $\mathrm{X}$ \\
\hline $\mathrm{CaTi}_{0.8} \mathrm{Mn}_{0.1} \mathrm{Mg}_{0.1} \mathrm{O}_{3-\delta}$ & 17 & 1.34 & 0.618 & 0.960 & 0.441 & $\mathrm{X}$ \\
\hline $\mathrm{Ca}_{0.9} \mathrm{Eu}_{0.1} \mathrm{Ti}_{0.8} \mathrm{Fe}_{0.1} \mathrm{Mg}_{0.1} \mathrm{O}_{3-\delta}$ & 18 & 1.303 & 0.611 & 0.950 & 0.436 & $\mathrm{O}$ \\
\hline $\mathrm{Ca}_{0.9} \mathrm{Pr}_{0.1} \mathrm{Ti}_{0.8} \mathrm{Fe}_{0.1} \mathrm{Mg}_{0.1} \mathrm{O}_{3-\delta}$ & 19 & 1.303 & 0.611 & 0.916 & 0.436 & $\mathrm{O}$ \\
\hline $\mathrm{Ca}_{0.9} \mathrm{Ag}_{0.1} \mathrm{Ti}_{0.8} \mathrm{Fe}_{0.1} \mathrm{Mg}_{0.1} \mathrm{O}_{3-\delta}$ & 20 & 1.321 & 0.615 & 0.957 & 0.436 & $\mathrm{O}$ \\
\hline $\mathrm{CaTi}_{0.9} \mathrm{Fe}_{0.1} \mathrm{O}_{3-\delta}$ & 21 & 1.34 & 0.6 & 0.969 & 0.428 & $\mathrm{O}$ \\
\hline $\mathrm{CaTi}_{0.9} \mathrm{Ni}_{0.1} \mathrm{O}_{3-\delta}$ & 22 & 1.34 & 0.6 & 0.960 & 0.429 & $\mathrm{X}$ \\
\hline $\mathrm{CaTi}_{0.8} \mathrm{Al}_{0.1} \mathrm{Fe}_{0.1} \mathrm{O}_{3-\delta}$ & 23 & 1.34 & 0.593 & 0.973 & 0.423 & $\mathrm{O}$ \\
\hline $\mathrm{CaTi}_{0.8} \mathrm{Mn}_{0.1} \mathrm{Fe}_{0.1} \mathrm{O}_{3-\delta}$ & 24 & 1.34 & 0.606 & 0.966 & 0.433 & $\mathrm{O}$ \\
\hline $\mathrm{CaTi}_{0.8} \mathrm{Ga}_{0.1} \mathrm{Fe}_{0.1} \mathrm{O}_{3-\delta}$ & 25 & 1.34 & 0.601 & 0.968 & 0.429 & $\mathrm{X}$ \\
\hline $\mathrm{Ca}_{0.9} \mathrm{Bi}_{0.1} \mathrm{Ti}_{0.9} \mathrm{Fe}_{0.1} \mathrm{O}_{3-\delta}$ & 26 & 1.282 & 0.6 & 0.949 & 0.428 & $\mathrm{O}$ \\
\hline $\mathrm{CaTi}_{0.9} \mathrm{Sb}_{0.1} \mathrm{O}_{3-\delta}$ & 27 & 1.34 & 0.605 & 0.969 & 0.432 & $\mathrm{X}$ \\
\hline $\mathrm{CaTi}_{0.85} \mathrm{Pd}_{0.05} \mathrm{Fe}_{0.1} \mathrm{O}_{3-\delta}$ & 28 & 1.34 & 0.607 & 0.965 & 0.434 & $\mathrm{X}$ \\
\hline $\mathrm{CaTi}_{0.8} \mathrm{Ge}_{0.1} \mathrm{Fe}_{0.1} \mathrm{O}_{3-\delta}$ & 29 & 1.34 & 0.612 & 0.963 & 0.437 & $\mathrm{X}$ \\
\hline
\end{tabular}

Table 7.1. Ionic radii of constituent ions $A$ and $B$, tolerance factor, octahedral factor $\left(r_{B} / r_{O}\right)$ and symmetry of different compounds $(\mathrm{O}=$ orthorrombic, $\mathrm{X}=$ not single phase $)$ 
The lattice parameters of the succeeded perovskites, with pure perovskite crystalline phase, have been determined from XRD and summarized in Table 7.2 .

\begin{tabular}{llllll}
\hline \multicolumn{1}{c}{ Material } & $\#$ & Cell volume $\left(\AA^{3}\right)$ & a $(\AA)$ & $\mathbf{b}(\AA)$ & $\mathbf{c}(\AA)$ \\
\hline $\mathrm{CaTiO}_{3}$ & $\mathrm{~T} 0$ & 223.60 & $7.640(2)$ & $5.440(1)$ & $5.382(1)$ \\
$\mathrm{CaTi}_{0.8} \mathrm{Fe}_{0.2} \mathrm{O}_{3-\delta}$ & $\mathrm{T} 1$ & 223.35 & $7.637(4)$ & $5.425(3)$ & $5.391(3)$ \\
$\mathrm{CaTi}_{0.9} \mathrm{Co}_{0.1} \mathrm{O}_{3-\delta}$ & $\mathrm{T} 2$ & 223.36 & $7.65(1)$ & $5.435(7)$ & $5.372(5)$ \\
$\mathrm{CaTi}_{0.9} \mathrm{Cu}_{0.1} \mathrm{O}_{3-\delta}$ & $\mathrm{T} 3$ & 222.24 & $7.640(2)$ & $5.440(1)$ & $5.382(1)$ \\
$\mathrm{CaTi}_{0.9} \mathrm{Mg}_{0.1} \mathrm{O}_{3-\delta}$ & $\mathrm{T} 5$ & 223.16 & $7.65(1)$ & $5.441(4)$ & $5.36(4)$ \\
$\mathrm{CaTi}_{0.73} \mathrm{Fe}_{0.18} \mathrm{Mg}_{0.09} \mathrm{O}_{3-\delta}$ & $\mathrm{T} 6$ & 219.88 & $7.65(1)$ & $5.419(6)$ & $5.30(6)$ \\
$\mathrm{CaTi}_{0.8} \mathrm{Nb}_{0.1} \mathrm{Mg}_{0.1} \mathrm{O}_{3-\delta}$ & $\mathrm{T} 9$ & 224.30 & $7.65(2)$ & $5.460(9)$ & $5.37(1)$ \\
$\mathrm{CaTi}_{0.85} \mathrm{Mo}_{0.05} \mathrm{Mg}_{0.1} \mathrm{O}_{3-\delta}$ & $\mathrm{T} 10$ & 225.40 & $7.653(3)$ & $5.463(1)$ & $5.392(1)$ \\
$\mathrm{CaTi}_{0.9} \mathrm{Al}_{0.1} \mathrm{O}_{3-\delta}$ & $\mathrm{T} 11$ & 225.48 & $7.83(2)$ & $5.40(1)$ & $5.33(1)$ \\
$\mathrm{Ca}_{0.9} \mathrm{Eu}_{0.1} \mathrm{Ti}_{0.8} \mathrm{Fe}_{0.1} \mathrm{Mg}_{0.1} \mathrm{O}_{3-\delta}$ & $\mathrm{T} 18$ & 224.18 & $7.650(7)$ & $5.447(5)$ & $5.379(5)$ \\
$\mathrm{Ca}_{0.9} \mathrm{Pr}_{0.1} \mathrm{Ti}_{0.8} \mathrm{Fe}_{0.1} \mathrm{Mg}_{0.1} \mathrm{O}_{3-\delta}$ & $\mathrm{T} 19$ & 225.32 & $7.52(2)$ & $5.52(2)$ & $5.42(1)$ \\
$\mathrm{Ca}_{0.9} \mathrm{Ag}_{0.1} \mathrm{Ti}_{0.8} \mathrm{Fe}_{0.1} \mathrm{Mg}_{0.1} \mathrm{O}_{3-\delta}$ & $\mathrm{T} 20$ & 447.54 & $7.648(3)$ & $5.431(2)$ & $10.774(6)$ \\
$\mathrm{CaTi}_{0.9} \mathrm{Fe}_{0.1} \mathrm{O}_{3-\delta}$ & $\mathrm{T} 21$ & 223.45 & $7.65(1)$ & $5.432(8)$ & $5.378(7)$ \\
$\mathrm{CaTi}_{0.8} \mathrm{Al}_{0.1} \mathrm{Fe}_{0.1} \mathrm{O}_{3-\delta}$ & $\mathrm{T} 23$ & 262.66 & $7.62(1)$ & $7.82(4)$ & $4.407(8)$ \\
$\mathrm{CaTi}_{0.8} \mathrm{Mn}_{0.1} \mathrm{Fe}_{0.1} \mathrm{O}_{3-\delta}$ & $\mathrm{T} 24$ & 223.26 & $7.63(8)$ & $5.442(1)$ & $5.380(7)$ \\
$\mathrm{Ca}_{0.9} \mathrm{Bi}_{0.1} \mathrm{Ti}_{0.9} \mathrm{Fe}_{0.1} \mathrm{O}_{3-\delta}$ & $\mathrm{T} 26$ & 223.82 & $7.70(2)$ & $5.436(9)$ & $5.349(2)$ \\
\hline
\end{tabular}

Table 7.2. Cell volume and cell parameters of formed perovskite compounds (fitting errors in brackets).

DC electrical conductivity measurements have been conducted on sintered rectangular bars, both as a function of temperature and in different oxygen containing atmospheres. Figure 7.2 shows the total conductivity dependence on the $\mathrm{pO}_{2}$ of doped calcium titanates that present pure perovskite structure. Samples T3, T5 were not measured since they reacted with the holder upon calcination. T26 is not dense even uprising the sintering temperature, which explains the low conductivity. 


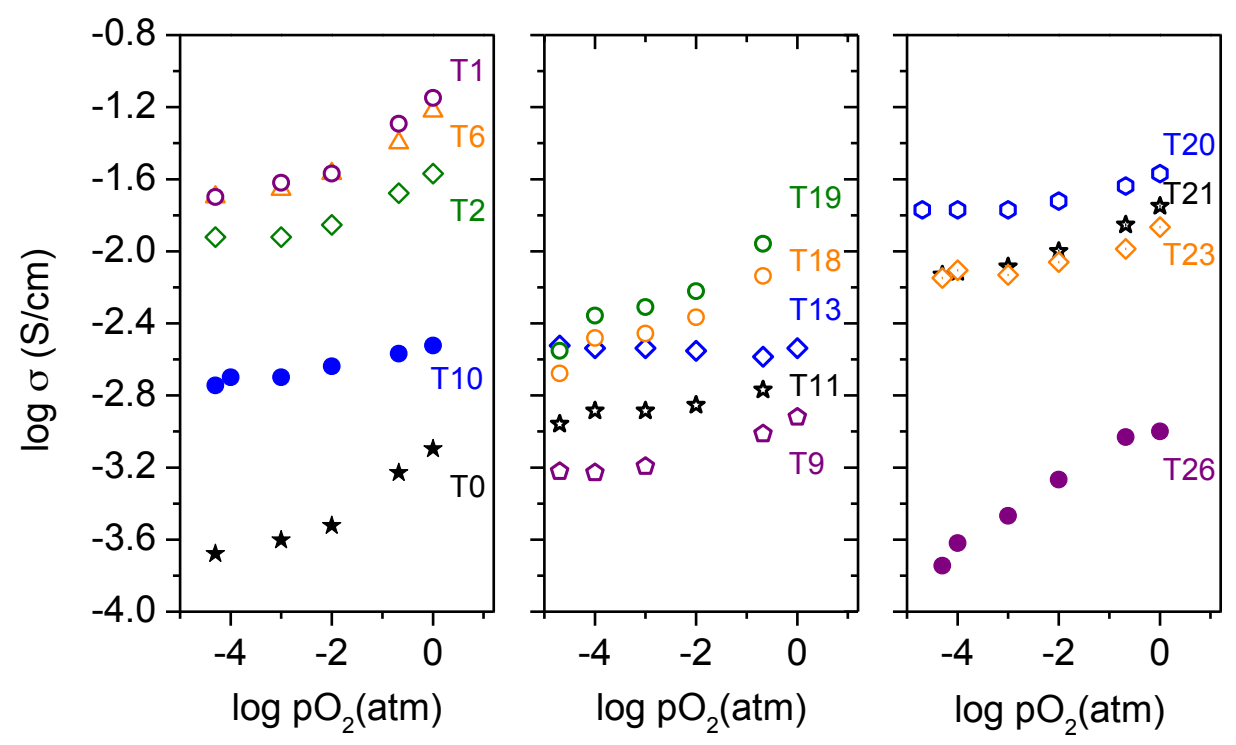

Figure 7.2. Comparative $\mathrm{pO}_{2}$ dependence of total electrical conductivity for pure and doped $\mathrm{CaTiO}_{3}$ perovskites.

All the introduced dopants are able to increase total conductivity with respect to pure $\mathrm{CaTiO}_{3}$. The majority of these materials diminish conductivity as the $\mathrm{pO}_{2}$ decreases indicating that they are mainly $p$-type electronic conductors, even though doping with lower valence ions produces oxygen vacancies and ionic conductivity was also expected. On the other hand, $\mathrm{CaTi}_{0.85} \mathrm{Mo}_{0.05} \mathrm{Mg}_{0.1} \mathrm{O}_{3-\delta}$ (T10), $\mathrm{CaTi}_{0.9} \mathrm{Al}_{0.1} \mathrm{O}_{3-\delta}$ (T11) and $\mathrm{CaTi}_{0.9} \mathrm{Mn}_{0.1} \mathrm{O}_{3-\delta}$ (T13) are not $\mathrm{pO}_{2}$ dependent, which suggests mainly ionic conduction.

Among all of them, the best conductivity is found when doping the B-site with $20 \% \mathrm{Fe}$, which has been widely studied in literature and used here as a reference. $[13,16-19]$ Iron oxide is an acceptor dopant that enhances the total conductivity in oxidising conditions by simultaneously increasing the $p$-type and the ionic conductivity. In a similar composition $\mathrm{Mg}^{2+}$ is added with the aim of further increase the oxygen vacancy concentration, i.e. 
$\mathrm{CaTi}_{0.73} \mathrm{Fe}_{0.18} \mathrm{Mg}_{0.09} \mathrm{O}_{3-\delta}$ (T6). The preliminary conductivity screening shows that this is the only composition with a comparable electric behaviour with respect to the reference $\mathrm{CaTi}_{0.8} \mathrm{Fe}_{0.2} \mathrm{O}_{3-\delta}$ (T1), although the total conductivity is not improved. Therefore, further studies on this composition have been carried out in order to determine the role of the ionic carriers and possible effects of the $\mathrm{Mg}$ on the overall properties of $\mathrm{CaTi}_{0.73} \mathrm{Fe}_{0.18} \mathrm{Mg}_{0.09} \mathrm{O}_{3-\delta}$.

Characterization of $\mathrm{CaTi}_{0.73} \mathrm{Fe}_{0.18} \mathrm{Mg}_{0.09} \mathrm{O}_{3-\delta}(\mathrm{CTFM})$

XRD patterns of initial $\mathrm{CaTi}_{0.73} \mathrm{Fe}_{0.18} \mathrm{Mg}_{0.09} \mathrm{O}_{3-\delta}$ (CTFM) calcined at $1200{ }^{\circ} \mathrm{C}$ show perovskite structure with only orthorhombic distorted symmetry depicted in Figure 7.3 ( $a=7.65$ (1) $\AA, b=5.419$ (6) $\AA, c=5.30$ (6) $\AA$ ). The obtained patterns indicate that a solid solution is formed and the dopant elements are mainly dissolved in the lattice of $\mathrm{CaTiO}_{3}$, since no additional phases were observed. Goldschmidt tolerance factor, and octahedral factor were found to be 0.96 and 0.432 respectively, optimum values to form a perovskite. Figure $7.3 \mathrm{~b}$ depicts the SEM picture of the membrane, where a closed packing is observed. The grain size is estimated to be in the range of 3 to $6 \mu \mathrm{m}$. Based on the literature optimization of the microstructure of $\mathrm{CaTi}_{0.8} \mathrm{Fe}_{0.2} \mathrm{O}_{3-\delta}$ (CTF), these large grains may favor the ionic conductivity as the grain boundary becomes negligible. [17] Besides the large grains, small grains with a cubic shape are glimpsed in the SEM picture despite XRD does not clearly indicate a secondary phase. They could be related to the segregation of any of the elements on a minor separated phase, although the XRD or EDS do not give any concluding proof on their nature. 
a)

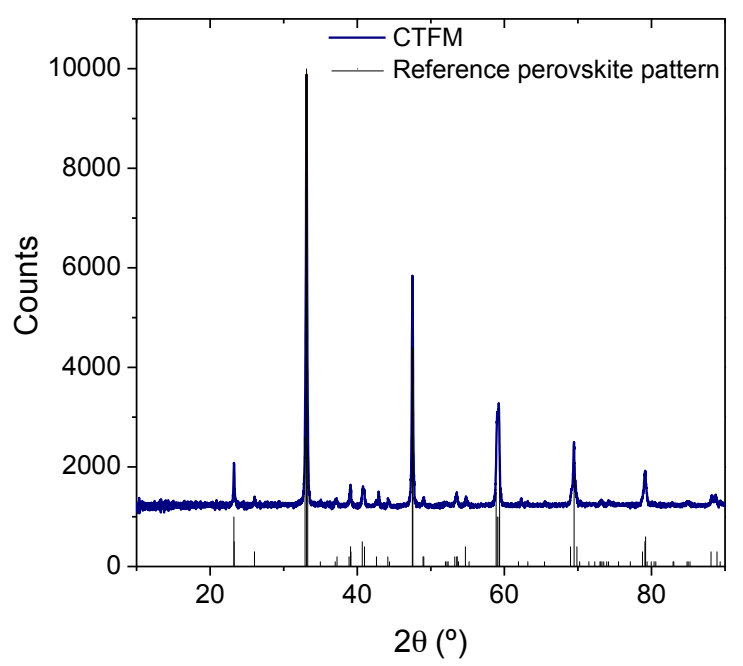

b)

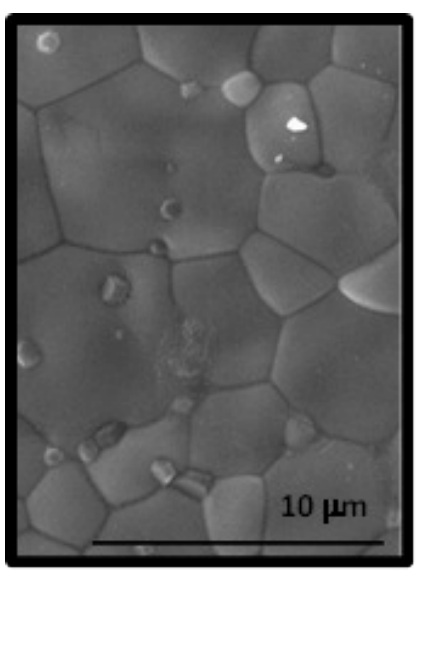

Figure 7.3. a) Room temperature XRD pattern of orthorhombic CTFM calcined at $1200{ }^{\circ} \mathrm{C}$ and b) SEM picture of the formed membrane.

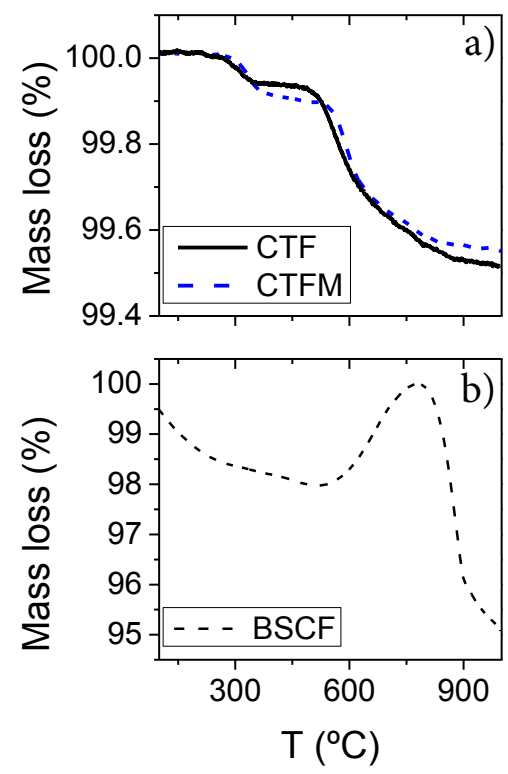

Figure 7.4. TG analysis under $5 \% \mathrm{CO}_{2} /$ air of a) CTFM and CTF and b) BSCF. Heating rate was $10^{\circ} \mathrm{C} \cdot \mathrm{min}^{-1}$. 
Figure 7.4a shows the TG analysis of CTFM and CTF materials in $\mathrm{CO}_{2}$ containing atmosphere. The analysis of the BSCF is also depicted in Figure 7.4b for comparison. The BSCF gain of mass around $580^{\circ} \mathrm{C}$ evidences the formation of carbonates and the later decomposition when temperature keeps increasing. $[20,21]$ On the other hand, CTFM and CTF do not undergo any visible $\mathrm{CO}_{2}$ uptake under $\mathrm{CO}_{2}$ containing atmospheres to the limits of this technique. The observed weight loss is attributed to the sample dehydration up to $200{ }^{\circ} \mathrm{C}$ and latter oxygen release from the perovskite lattice and subsequent oxygen vacancy formation.

a)

c)
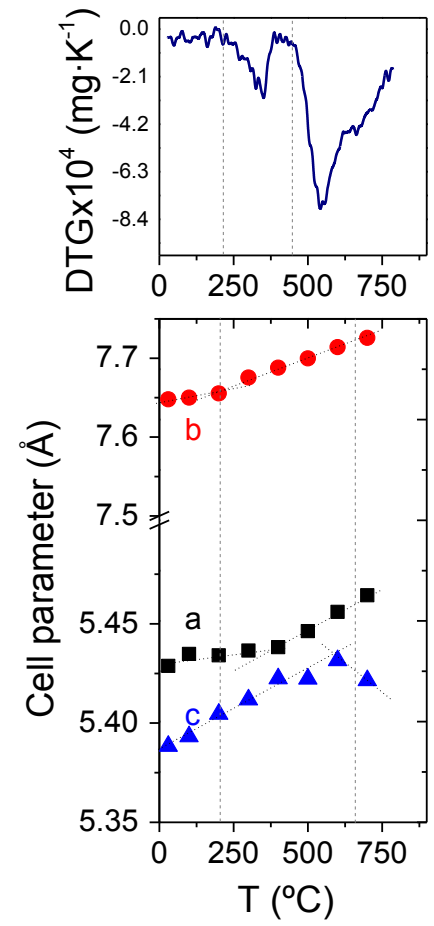

b)

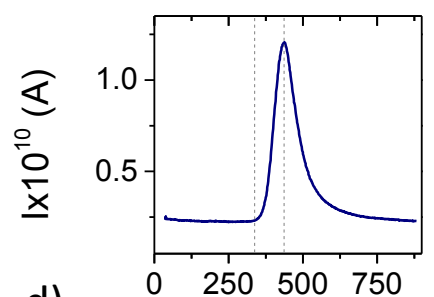

d)

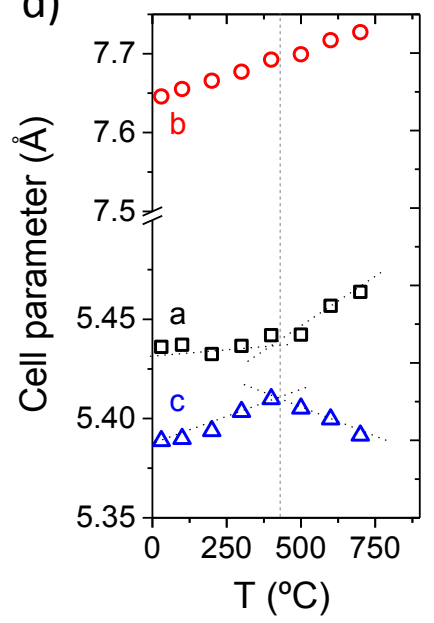

Figure 7.5. a) DTG in air, b) TPD of oxygen, $\mathrm{m} / \mathrm{z}=32$, in $\mathrm{He}\left(\mathrm{pO}_{2}=10^{-5} \mathrm{~atm}\right)$, c) cell parameters extracted from HT-XRD in air and d) cell parameters extracted from HT-XRD in $\mathrm{N}_{2}$, for CTFM. Heating rate was $10^{\circ} \mathrm{C} \cdot \mathrm{min}^{-1}$. 
Figure 7.5 confirms the oxygen release both in high (air) and low $\left(10^{-5} \mathrm{~atm}\right)$ oxygen partial pressures. Figure $7.5 \mathrm{a}$ plots the differential thermogravimetry (DTG) in air of CTFM. Two maxima in oxygen desorption are observed at 361 and $557{ }^{\circ} \mathrm{C}$. The starting desorption temperatures (220 and $453^{\circ} \mathrm{C}$ ) correspond to changes in slope observed in Figure 7.5c, which depicts the evolution of the cell parameters with increasing temperature extracted from HT-XRD. CTFM undergoes chemical expansion as oxygen desorbs and the cations are reduced (larger ionic radius), which occurs at different temperatures for each lattice constant. Figure $7.5 \mathrm{~b}$ plots mass spectrometry results on TPD of CTFM performed in He, which settles that oxygen is the released specie (both 16 and 32 a.m.u.). Under low $\mathrm{pO}_{2}$ (e.g. He or $\mathrm{N}_{2}$ ), lattice parameters evolve differently than in air, as lattice parameter, $\mathrm{c}$, is shortened.

Figure 7.6 shows the conductivity, $\ln (\sigma \mathrm{T})$ of CTFM as a function of the reverse temperature measured at $0.21 \mathrm{~atm}$. In the preliminary screening, a change in behaviour is glimpsed at temperatures close to $800{ }^{\circ} \mathrm{C}$. Thus, the temperature range is extended from 400 to $1000{ }^{\circ} \mathrm{C}$ and compared with the reference CTF. The addition of dopants to the perovskite results in an increase of the total conductivity with respect to the non-doped $\mathrm{CaTiO}_{3}$. The equilibrium of this oxide in air favours the loss of oxygen and subsequent formation of oxygen vacancies under increasing temperature, which explains the $E_{a}$ changes. Below $800{ }^{\circ} \mathrm{C}$, total conductivity of CTFM is slightly lower than the corresponding to $\mathrm{CTF}$, which has been reported to have maximum conductivity on the $20 \% \mathrm{Fe}$ dopant concentration as a consequence of the balance between moderate oxygen vacancy concentration and a relatively disordered $\mathrm{Fe}^{3+}$ structure. [13]

On the contrary, above $800{ }^{\circ} \mathrm{C}$, CTFM exceeds the conductivity observed for CTF. This behaviour can be explained by the trapping of the 
oxygen vacancies into ordered microdomains that avoid the mobility in CTFM. [13] By increasing the temperature, the large energy required to break down the pairs of these associated defects is achieved and the conductivity of CTFM increases over that of CTF.

a)

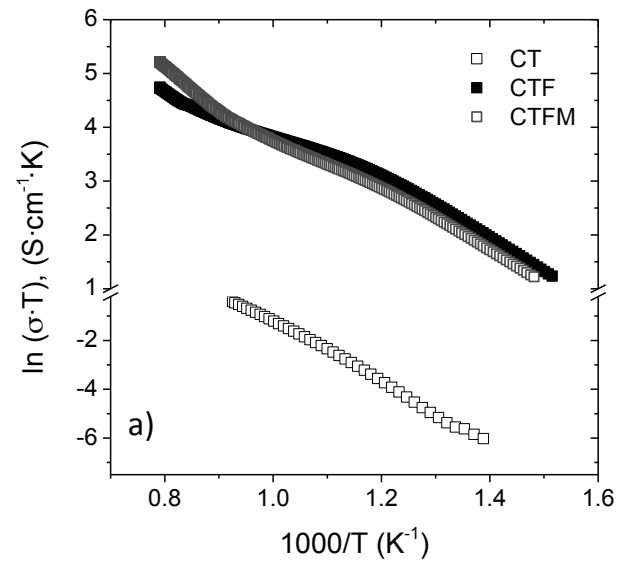

b)

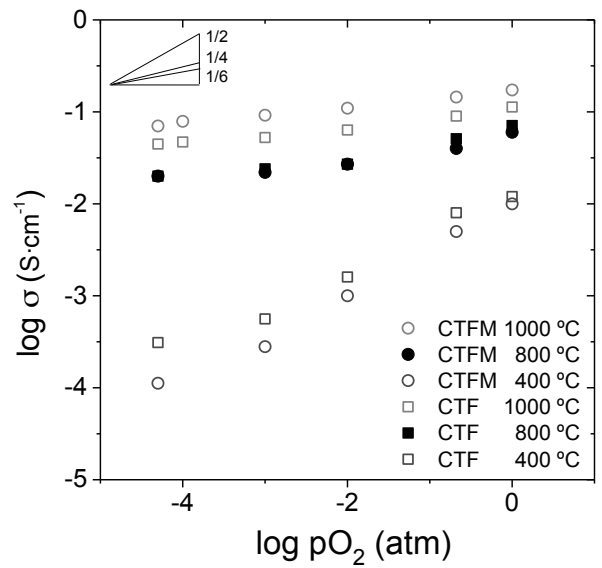

Figure 7.6. a) Arrhenius plot of the conductivity of CTFM, CTF and $\mathrm{CaTiO}_{3}$ and b) logarithm of the total conductivity versus $\mathrm{pO}_{2}$ of CTFM and CTF at 1000, 800 and $400{ }^{\circ} \mathrm{C}$.

The dependence of conductivity on the oxygen partial pressure for CTFM and CTF is presented in Figure 7.6b. The drop in conductivity as $\mathrm{pO}_{2}$ decreases indicates $p$-type electronic conductor behaviour for both CTFM and CTF. The ionic character is enhanced as temperature increases, as can be observed from the weaker dependence on the $\mathrm{pO}_{2}$. The results confirm the mixed ionic-electronic conductivity of these compositions. The high temperature bulk transport properties comprise a major ionic conductivity while electronic contribution becomes more important as the temperature drops. Particularly, at $400{ }^{\circ} \mathrm{C}$, over the whole range of $p O_{2}$, CTFM shows $1 / 2$ dependency. This unexpected behaviour is attributed to the coupling of 
reduction processes (as inferred from TPD results). At this temperature the formed oxygen vacancies have low mobility and may be trapped as associates $\left(\mathrm{V}_{\mathrm{O}} \cdot \mathrm{Mg}_{\mathrm{Ti}}^{\prime \prime}\right)$. In such a case both the $p$-type electronic and ionic conductivities will be decreased providing the observed dependence. At $1000{ }^{\circ} \mathrm{C}$ the $\mathrm{pO}_{2}$ dependency is $1 / 6$ and a plateau starts to appear at $p O_{2}$ lower that $10^{-3} \mathrm{~atm}$, characteristic of ionic transport. This plateau should be related with the steady vacancy concentration independent on the $\mathrm{pO}_{2}$ and $\sigma \propto\left[\mathrm{V}_{\mathrm{o}}\right]$, and indicates the higher ionic conductivity of the CTFM over the CTF.

On the other hand, reported studies on the oxygen permeability of $\mathrm{CaTi}_{1-\mathrm{x}} \mathrm{Fe}_{\mathrm{x}} \mathrm{O}_{3-\delta}$ dense ceramic membranes with different thicknesses demonstrate significant oxygen surface exchange limitations. [22] The study of the surface properties of CTFM is of interest to evaluate the suitability as oxygen permeation membrane and catalyst. First, the composition of the surface has been studied by XPS. Also the oxygen exchange rate has been measured by PIE and compared with that of CTF.

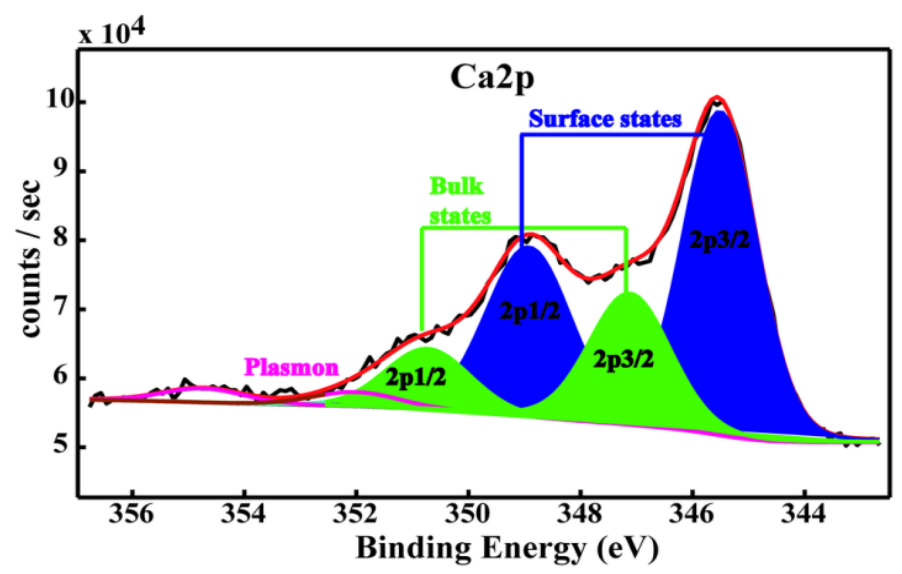

Figure 7.7. Ca $2 p$ spectrum of CTFM quenched from $800{ }^{\circ} \mathrm{C}$ in Ar. Pass energy was 30 $\mathrm{eV}$. 
Figure 7.7 shows the deconvoluted Ca 2p XPS spectrum of CTFM quenched from $800{ }^{\circ} \mathrm{C}$ in Ar. The $\mathrm{Ca} 2 \mathrm{p}$ band-like spectrum exhibits two features: the 'bulk' one as $\mathrm{Ca}^{2+}$ oxidation state in oxide at $347.1 \mathrm{eV}\left(\mathrm{Ca} 2 \mathrm{p}_{1 / 2}\right)$ and $350.7 \mathrm{eV}\left(\mathrm{Ca} 2 \mathrm{p}_{3 / 2}\right)$ binding energies and a 'surface' feature for $\mathrm{Ca}^{2+}$ bonded in the perovkite structure $(345.5 \mathrm{eV}+348.1 \mathrm{eV})$. The last two small peaks are the characteristic plasmon features in the Ca 2p XPS spectrum. [23]

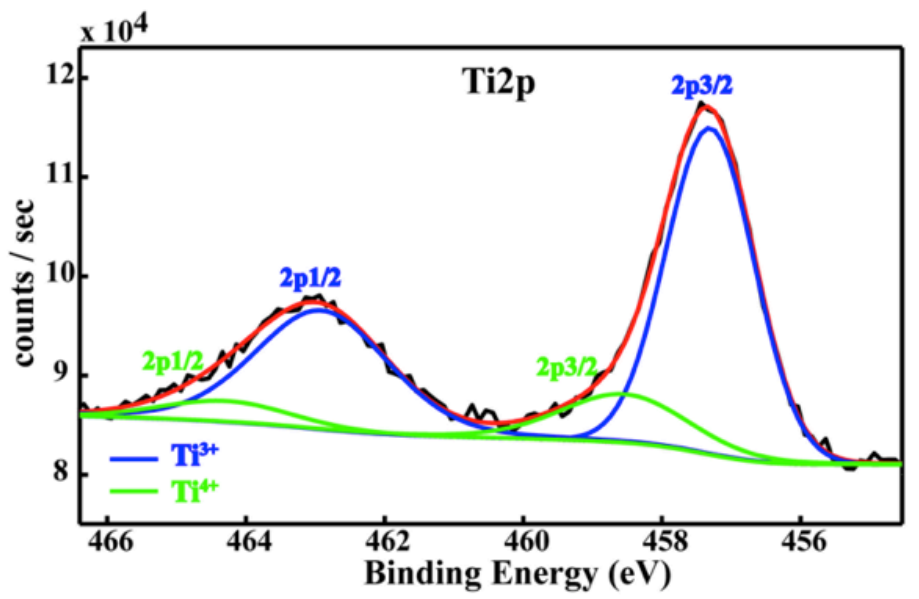

Figure 7.8. Ti $2 \mathrm{p}$ spectrum of CTFM quenched from $800{ }^{\circ} \mathrm{C}$ in $\mathrm{Ar}$ and deconvolution of the spectrum. $\mathrm{Ti}^{4+}$ and $\mathrm{Ti}^{3+}$ components are highlighted. Pass energy was $30 \mathrm{eV}$.

Figure 7.8 shows the fitting results of the XPS spectrum of Ti $2 p$ for CTFM. According to characteristic BEs and the split-orbit parameter $(\Delta \sim 6 \mathrm{eV})$, the Ti $2 p$ main oxidation state is $\mathrm{Ti}^{3+}$. A percentage of surface $\mathrm{Ti}^{4+}$ was highlighted after deconvolution $(\sim 17 \%)$, whose peaks appear at binding energies of $458.4 \mathrm{eV}\left(\mathrm{Ti} 2 \mathrm{p}_{1 / 2}\right)$ and $464.1 \mathrm{eV}\left(\mathrm{Ti} 2 \mathrm{p}_{3 / 2}\right)$. [23, 24] 
a)

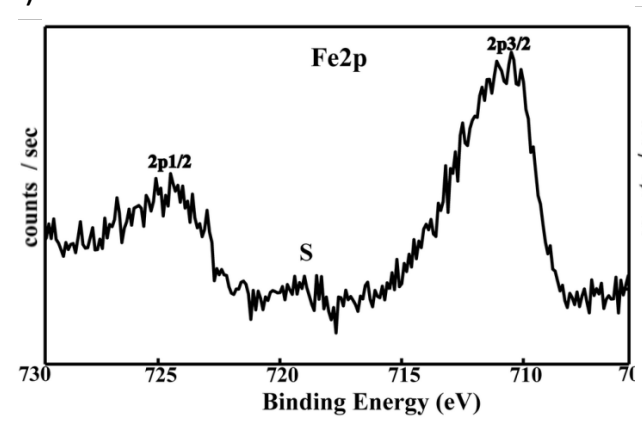

b)

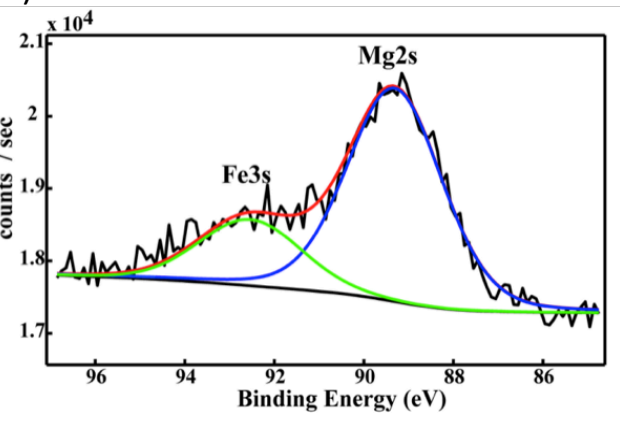

Figure 7.9. a) Fe 2p XPS spectrum and b) $\mathrm{Mg} 2 \mathrm{~s}$ and Fe3s XPS spectra of CTFM quenched from $800{ }^{\circ} \mathrm{C}$ in Ar. Pass energy was $30 \mathrm{eV}$.

Figure 7.9a shows the XPS spectrum of Fe 2p. According to the BEs and to the presence of the characteristic satellite at $719.2 \mathrm{eV}$, the oxidation state is identified as $\mathrm{Fe}^{3+}$. However, the presence of $\mathrm{FeOOH}$ confined on the top of the surface cannot be completely ruled out. $[25,26]$ The XPS spectrum of Mg $2 \mathrm{~s}$ for CTFM is shown in Figure 7.9b. The peak observed at $89.3 \mathrm{eV}$ belongs to $\mathrm{Mg}^{2+}$ in a non-stoichiometric environment. A second peak in the $\mathrm{Mg} 2 \mathrm{~s}$ deconvoluted spectrum is observed at $92.5 \mathrm{eV}$. It corresponds to $\mathrm{Fe} 3 \mathrm{~s}$ component and confirms the $\mathrm{Fe}^{3+}$ oxidation state. $[27,28]$

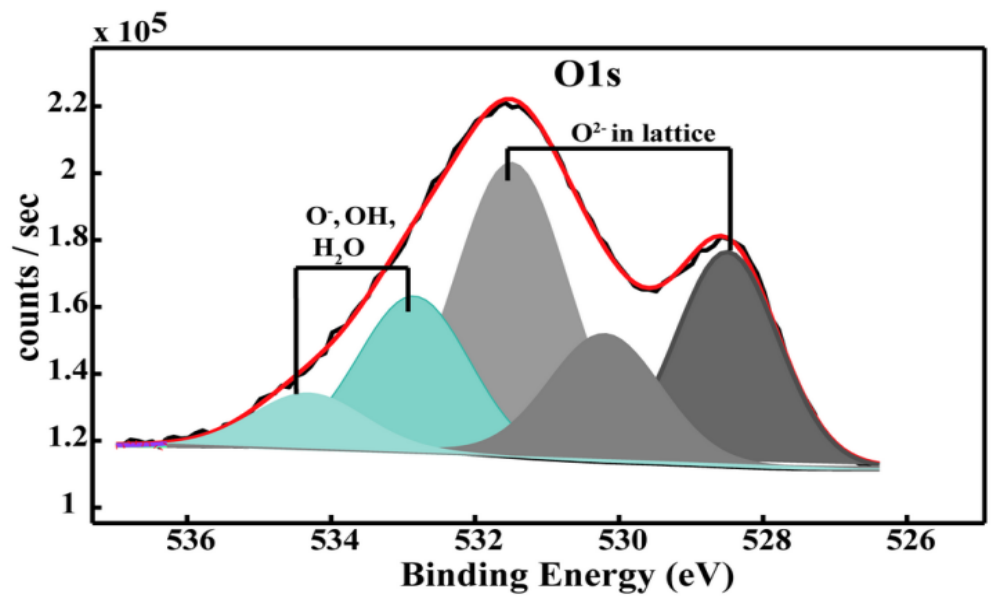

Figure 7.10. O1s XPS spectra of CTFM quenched from $800^{\circ} \mathrm{C}$. Pass energy was $30 \mathrm{eV}$. 
The XPS spectrum of Figure 7.10 shows O1s peaks at binding energies of $528.5 \mathrm{eV}, 530.2 \mathrm{eV}$ and $531.5 \mathrm{eV}$, which are attributed to the chemical state $\mathrm{O}^{2-}$ bonded in the lattice. The last two components at $532.9 \mathrm{eV}$ and $534.3 \mathrm{eV}$ can be assigned to strongly adsorbed oxygen as $\mathrm{O}^{-},{ }^{-} \mathrm{OH}$, or $_{2} \mathrm{O}$ (the surface is hygroscopic).

Thus, the XPS demonstrates the presence of oxygen vacancies in the CTFM surface, from the tri- and divalent oxidation states of $\mathrm{Ti}, \mathrm{Fe}$ and $\mathrm{Mg}$, respectively. These surface oxygen vacancies are expected to provide adsorption sites for the oxygen exchange.

The interaction between gaseous $\mathrm{O}_{2}$ and the oxide surface may be evaluated by pulse isotope exchange (PIE). The overall oxygen exchange reaction in the surface can be expressed as,

$\mathrm{O}_{2}+2 \mathrm{~V}_{\mathrm{O}}^{\ddot{*}}+4 \mathrm{e}^{\prime} \rightarrow 2 \mathrm{O}_{\mathrm{O}}^{\mathrm{X}}$

which indicates that both lattice oxygen vacancies and electronic carriers at the oxide surface participate in the rate of surface exchange. Figure 7.11a plots the PIE results of oxygen surface exchange rate, $\Re_{0}$, of CTF and CTFM obtained by PIE at $p O_{2}=0.21 \mathrm{~atm}$. Up to $650{ }^{\circ} \mathrm{C} \Re_{\mathrm{o}}$ is the same for both materials, so that a similar surface limitations are expected on the oxygen permeation. Below $550{ }^{\circ} \mathrm{C}$ an abrupt drop in CTFM $\Re_{0}$ is observed, which is attributed to the decrease of free oxygen vacancies as temperature decreases, also observed by conductivity and TPD. The range of temperature at which PIE measurements could be performed limits the direct comparison, as the measurement is enabled as long as the effective time constant associated with the isotopic exchange is of similar order as the time scale of the experiment, i.e. the residence time of the packed bed reactor. Thus, higher temperature experiments were not possible. 
Figure $7.11 \mathrm{~b}$ shows the temperature dependence of the rates of dissociative adsorption $\Re_{\mathrm{a}}$, and oxygen incorporation $\Re_{\mathrm{i}}$, of CTFM. The overall rate of surface exchange appears to be controlled by the dissociative adsorption reaction, as the rate of oxygen incorporation is found between one or two orders of magnitude higher than the adsorption one, similar to reported studies of $\mathrm{BSCF}$ and $\mathrm{Bi}_{0.75} \mathrm{Er}_{0.25} \mathrm{O}_{2-\delta}$. [29, 30]
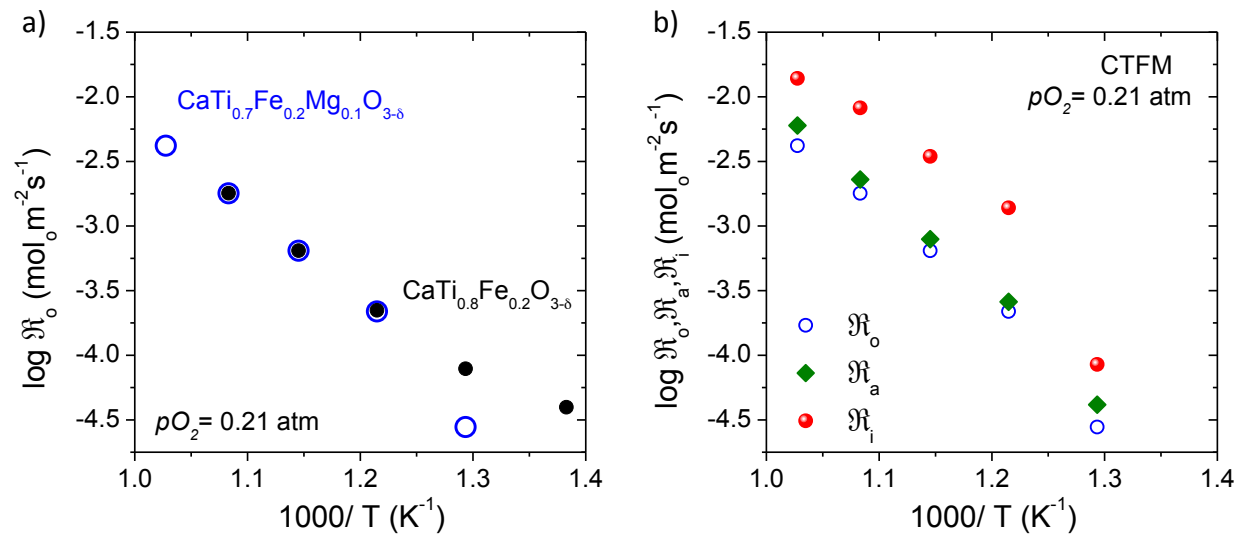

Figure 7.11. Arrhenius plot of CTF and CTFM (a) the overall surface exchange rate $\mathfrak{R}_{0}$ and (b) partial $\mathfrak{R}_{\mathrm{a}}, \mathfrak{R}_{\mathrm{i}}$ and $\mathfrak{R}_{0}$.

Figure 7.12 collects the Arrhenius plots of the overall oxygen surface exchange rate, $\Re_{0}$ of some reference oxide materials measured by PIE (YSZ, CGO10, CGO20, CT50) or isotopic exchange followed by deep profiling (IEDP) as $\mathrm{La}_{0.8} \mathrm{Sr}_{0.2} \mathrm{CoO}_{2-\delta}$ (LSC20) and BSCF. CTF and CTFM show a comparatively high surface exchange rate with regard to YSZ and CGO10, known as solid pure ionic conductors, and LSC20 perovskite. $\Re_{\mathrm{o}}$ is comparable to that of CGO20, CT50. On the other hand CTF and CTFM $\Re_{\mathrm{o}}$ are lower than BSCF. [30-32] The limits in oxygen surface rate may be less significant by applying a catalytic layer over the membrane surface. 


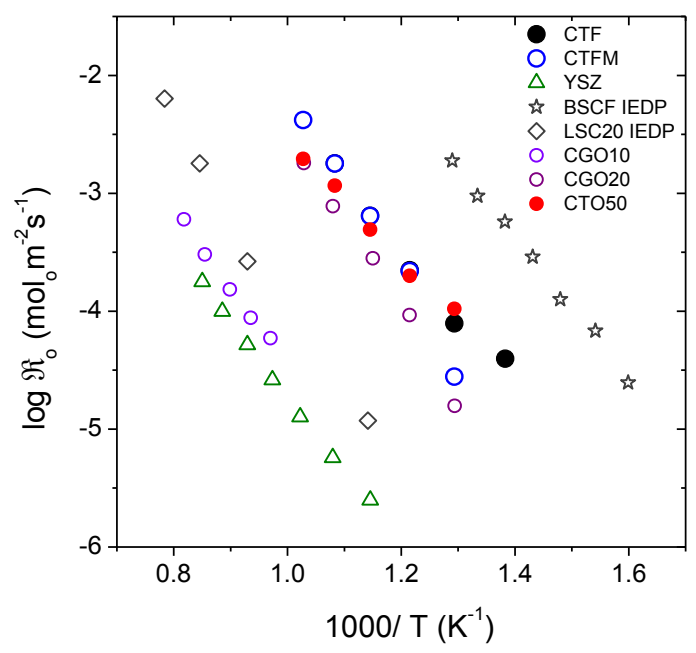

Figure 7.12. Comparative Arrhenius plot of the CTFM and CTF $\mathfrak{R}_{0}$ with literature data of widely used oxides measured by PIE as YSZ, CGO10, CGO20, CT50 or IEDP as LSC20 and BSCF. [30-32]

\section{Oxygen permeation test}

Permeation measurements have been performed on a CTFM membrane $900 \mu \mathrm{m}$ thick. Both disc sides are coated by a Pt ink $20 \mu \mathrm{m}$ layer aiming to improve surface catalytic behaviour. Oxygen is separated from synthetic air flow using argon as sweep gas. The permeation fluxes $\left(\mathrm{mL} \cdot \mathrm{min}^{-1} \cdot \mathrm{cm}^{-2}\right)$ are calculated by dividing the permeation rates by the effective surface area of the membranes.

First, oxygen permeation flux is represented in Figure 7.13 as a function of reciprocal temperature using $100 \mathrm{~mL} \cdot \mathrm{min}^{-1}$ of synthetic air and pure oxygen as feed and $400 \mathrm{~mL} \cdot \mathrm{min}^{-1} \mathrm{Ar}$ as sweep flow. It can be observed that in both feeding conditions, oxygen flux increases with temperature following Arrhenius behaviour. The oxygen permeation flux increases 2 -fold by using oxygen as 
feed gas, and reaches values up to $2 \mathrm{~mL} \cdot \mathrm{min}^{-1} \cdot \mathrm{cm}^{-2}$ at $1000{ }^{\circ} \mathrm{C}$. This increase is a consequence of the higher driving force, which corresponds to the oxygen chemical potential gradient along the thickness of the membrane. Based on the magnitude of the membrane thickness $(0.9 \mathrm{~mm})$, which is over the characteristic length expected for perovskites and on the catalytic activation of the surface achieved by depositing a Pt porous layer, the bulk diffusion is expected to limit the oxygen permeation rate. [4, 33] In such a case, the relationship between the oxygen potential gradient and the oxygen flux is postulated by the Wagner equation:

$\mathrm{J}\left(\mathrm{O}_{2}\right)=\frac{\mathrm{RT}}{16 \mathrm{~F}^{2} \mathrm{~L}} \int_{\mathrm{pO}_{2}^{\mathrm{II}}}^{\mathrm{pO} \mathrm{I}} \sigma_{\mathrm{amb}}\left(\mathrm{pO}_{2}\right) \mathrm{dlnpO}_{2}$

where $\mathrm{J}\left(\mathrm{O}_{2}\right)$ is the oxygen permeation rate, $R$ and $F$ are gas and Faraday constants, $L$ is the membrane thickness, $\sigma_{\mathrm{amb}}$ is the ambipolar conductivity, and $p O_{2}{ }^{I}$ and $\mathrm{pO}_{2}{ }^{I I}$ are the oxygen partial pressure at the high pressure side and the low pressure side, respectively.

Constant apparent activation energies for oxygen transport are observed in the whole temperature range $\left(1000-400{ }^{\circ} \mathrm{C}\right)$, regardless of $\mathrm{pO}_{2}$ in the feed stream. This suggests that the ion diffusion through the bulk and grain boundary is the rate limiting step. Values of the apparent $E_{a}$ are $1.05 \mathrm{eV}(57.58 \mathrm{~kJ} / \mathrm{mol})$ and $0.96 \mathrm{eV}(43.89 \mathrm{~kJ} / \mathrm{mol})$ for air and pure oxygen feed, respectively. Obtained $E_{a}$ values are in the order of those obtained for thick BSCF membranes at high temperatures, where oxygen permeation process is limited by the bulk oxygen ions diffusion. [34] Otherwise, the $E_{a}$ for oxygen surface exchange is expected to be higher than that for the oxygen ion diffusion. $[4,7]$ The porous Pt layer on the surface enhances the catalytic activity and the oxygen uptake due to the increase of the available area for the surface exchange reactions. Thus, this 
surface modification avoids the surface limitation leading to an improvement of surface exchange rate and the subsequent increase in the $J\left(0_{2}\right) .[11,35]$

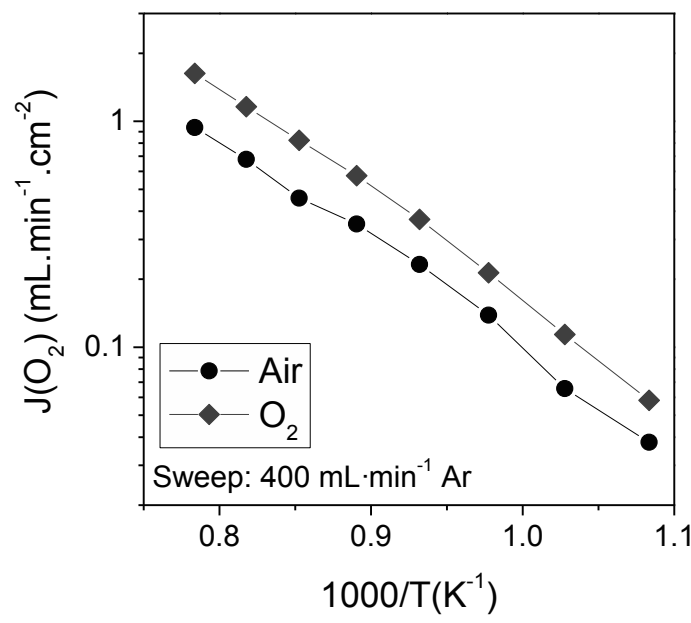

Figure 7.13. Oxygen permeation flow for CTFM membrane as a function of the temperature, with a sweep flow of $400 \mathrm{~mL} \cdot \mathrm{min}^{-1}$ of Ar. Synthetic air and pure oxygen at a flow rate of $100 \mathrm{~mL} \cdot \mathrm{min}^{-1}$ are used as feed gas.

Figure $7.14 \mathrm{a}$ shows the oxygen permeation fluxes in the temperature range of $700-1000{ }^{\circ} \mathrm{C}$ for different flow rates of sweep gas and synthetic air used as feed gas. The increase of the gas flow rate causes an increase of the driving force by the reduction of $\mathrm{pO}_{2}$ in the sweep stream, as the permeated oxygen is diluted. As predicted by the Wagner's equation, it leads to an improvement in the oxygen permeation flux. On the other hand, the enhancement can be further produced by the improvement in the fluid dynamics in the permeate chamber that avoids the accumulation of species on the permate side surface. This allows reducing concentration polarization resistance and decreasing substantially the local $\mathrm{pO}_{2}$ at the membrane surface. [8] The influence of the gas flow rates is more pronounced in the high temperature range. It may be the consequence of the improvement in ionic conduction for 
CTFM achieved above $800{ }^{\circ} \mathrm{C}$ (increasing the ambipolar conductivity) together with the influence of the higher $\mathrm{pO}_{2}$ gradient.

Figure 7.14b depicts the permeation results for pure $\mathrm{O}_{2}$ and air as feed gas. An important increase in the $\mathrm{J}\left(\mathrm{O}_{2}\right)$ is observed by the growth of the chemical potential across the membrane (provided by the pure $\mathrm{O}_{2}$ ). Specifically, an oxygen flux of ca. $1.6 \mathrm{~mL} \cdot \mathrm{min}^{-1} \cdot \mathrm{cm}^{2}$ is achieved at $1000{ }^{\circ} \mathrm{C}$ by the highest studied $\mathrm{pO}_{2}$ gradient.

a)

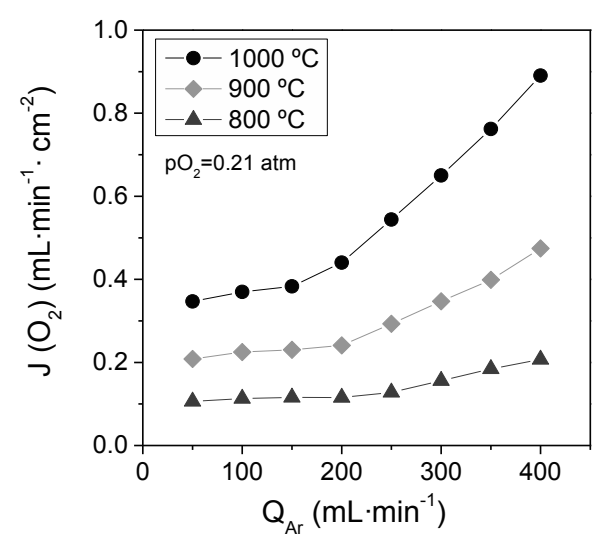

b)

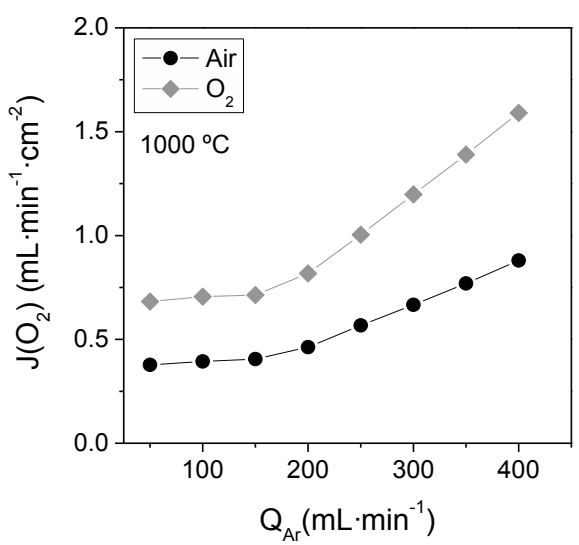

Figure 7.14. a) Oxygen permeation flux for CTFM membrane as a function of the $\mathrm{Ar}$ sweep flow at three different temperatures using synthetic air as feed and b) using air and $\mathrm{O}_{2}$ as feed gas at $1000^{\circ} \mathrm{C}$.

The oxygen flux obtained for the reference CTF $1 \mathrm{~mm}$ membrane is reported to achieve $7.08 \cdot 10^{-9} \mathrm{~mol} \mathrm{~s}^{-1} \mathrm{~cm}^{-2}$ at $950{ }^{\circ} \mathrm{C}$, which uses a $\mathrm{CTF}$ porous layer as catalyst on the both sides, and is fed by air. [22] This value is more than one order of magnitude lower than that obtained for CTFM, which was $4.6 \cdot 10^{-7} \mathrm{~mol} \mathrm{~s}^{-1} \mathrm{~cm}^{-2}$ at $950{ }^{\circ} \mathrm{C}$ using air as feed gas and $400 \mathrm{~mL} \cdot \mathrm{min}^{-1}$ of $\mathrm{Ar}$ as sweep gas. The improvement is ascribed to the enhanced ionic conductivity observed above $800{ }^{\circ} \mathrm{C}$. 
Paying attention to the most probable operating conditions of an oxygen transport membrane, the stability of CTFM against $\mathrm{CO}_{2}$ has been checked. The sample has been exposed to $15 \% \mathrm{CO}_{2} / \mathrm{Ar}$ atmosphere during 24 hours at $750{ }^{\circ} \mathrm{C}$, since the carbonates formation, if any, would occur at this temperature. After this treatment, it has been cooled down to $650{ }^{\circ} \mathrm{C}$, and the oxygen permeation flow is measured from $650{ }^{\circ} \mathrm{C}$ to $1000{ }^{\circ} \mathrm{C}$ in the same atmosphere. In Figure $7.15 \mathrm{a}$, it can be observed that the permeation values of heating up and cooling down measurements are slightly different. Since TG of CTFM in $5 \% \mathrm{CO}_{2}$ containing Ar does not show any carbonation reaction, the flux gap among both processes in $\mathrm{CO}_{2} / \mathrm{Ar}$ sweep side can be ascribed to the $\mathrm{CO}_{2} / \mathrm{O}_{2}$ competitive adsorption on the membrane surface. Actually, oxygen flux values obtained after $\mathrm{CO}_{2}$ treatment using $\mathrm{Ar}$ as sweep gas are very similar to those measured before $\mathrm{CO}_{2}$ treatment (Figure 7.15b). The oxygen flux drop when the membrane is exposed to $\mathrm{CO}_{2}$ may be produced by a change in the Pt porous layer.
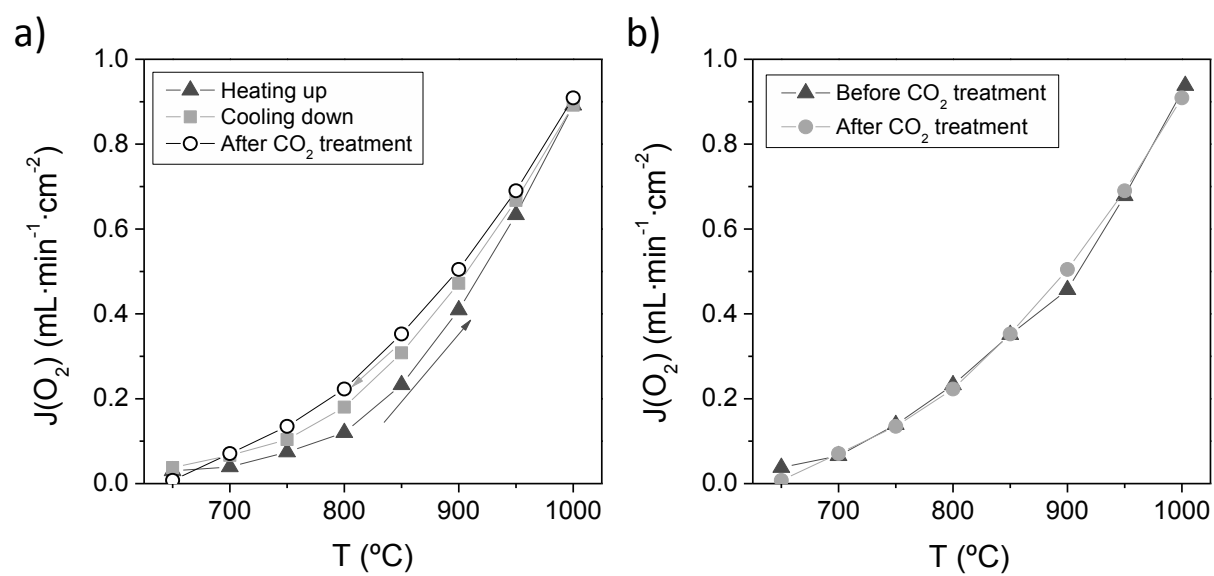

Figure 7.15. a) Oxygen permeation flow after 24 hours in $\mathrm{CO}_{2} / \mathrm{Ar}$ atmosphere. Oxygen flux using Ar as sweep gas is represented using open symbols, b) Oxygen permeation flux in Ar before and after of $\mathrm{CO}_{2}$ treatment. $\mathrm{Q}_{\mathrm{Ar}}=400 \mathrm{~mL} \cdot \mathrm{min}^{-1}$. 


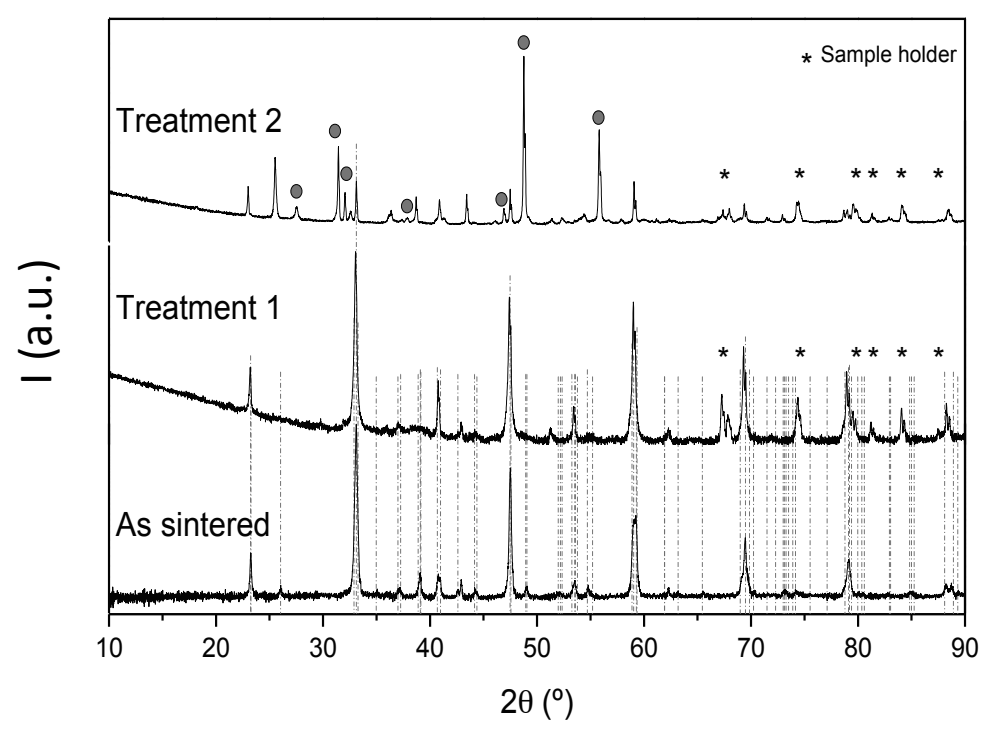

Figure 7.16. XRD patterns of as sintered CTFM powder and membranes after the stability treatments. Reference pattern and XRD of sample holder has been plotted for comparison.

Finally, CTFM membrane material was tested in harsh conditions. On one hand, the membrane was exposed to a continuous gas flow composed of 1000 ppm COS, 100 ppm $\mathrm{HCN}, 4 \% \mathrm{CO}_{2}, 46 \% \mathrm{CO}, 46 \% \mathrm{H}_{2}$ and $4 \% \mathrm{H}_{2} \mathrm{O}$ at 35 bar and a thermal treatment up to $400{ }^{\circ} \mathrm{C}$ (treatment 1). On the other hand, a treatment of two weeks on a continuous stream of $\mathrm{CO}_{2}$ containing $5 \% \mathrm{O}_{2}, 1 \%$ of $\mathrm{SO}_{2}$ and $2.5 \%$ of $\mathrm{H}_{2} \mathrm{O}$, was carried out during 14 days at $800{ }^{\circ} \mathrm{C}$ (treatment 2). From the XRD patterns of Figure 7.16, it can be inferred that materials do not suffer any degradation by the treatment 1 , and the formation of carbonates, sulfides or sulfates phases is negligible up to the limit of XRD analysis. However, the $\mathrm{SO}_{2}$ containing treatment 2 causes the reaction of the material and the loss of the perovskite structure. $\mathrm{SO}_{2}$ preferentially reacted with $\mathrm{Ca}$ and $\mathrm{Ti}$. The impurities founded are indexed as $\mathrm{CaFeO}_{2}$ (ICSD 00-021-0917), $\mathrm{CaSO}_{4}$ 
(ICSD 00-006-0226) and $\mathrm{Ti}_{2} \mathrm{~S}$ (00-011-0664). Note that diffraction peaks corresponding to the sample holder material are also observed.

\subsection{Conclusions}

The series $\mathrm{Ca}_{1-\mathrm{z}} \mathrm{A}_{\mathrm{z}} \mathrm{Ti}_{1-\mathrm{x}}\left(\mathrm{M} 1_{1-\mathrm{y}} \mathrm{M} 2_{\mathrm{y}}\right)_{\mathrm{x}} \mathrm{O}_{3-\delta}$ has been prepared by solid state reaction to evaluate the formability and ionic conductivity improvement of $\mathrm{CaTiO}_{3}$ perovskites. Although the Goldschmidt and the octahedral factors anticipated the perovskite structure for the majority of the compounds, they are not experimentally achieved for all the compositions. It has been attributed to different sitting of the dopant or to different oxidation states than expected (so that expected ionic radii), but no more research has been developed yet on this regard.

DC conductivity indicates that all the dopants allow increasing the conductivity and particularly CTF and CTFM, which have been further characterized. DC conductivity up to $1000{ }^{\circ} \mathrm{C}$ demonstrates an increase of conductivity of CTFM over that of CTF upon $800{ }^{\circ} \mathrm{C}$, which has been ascribed to the mobility of associated oxygen vacancies released at high temperature.

XPS shows the presence of $\mathrm{Fe}^{2+}$ and $\mathrm{Fe}^{3+}$, and $\mathrm{Mg}^{2+}$ in CTFM surface, while PIE results do not show any surface exchange rate improvement with respect to CTF up to $750{ }^{\circ} \mathrm{C}$, so that the CTFM membrane is covered with $\mathrm{Pt}$ ink layer to avoid surface restrictions. The systematic study of the CTFM membrane variables on the oxygen flux and temperature delivers an oxygen permeation flux one order of magnitude higher than referenced CTF membranes, which is attributed to the temperature dependent increase of oxygen vacancies concentration and mobility. Moreover, membrane stability is 
proved under $\mathrm{CO}_{2}$ as sweep gas, and after being submitted to harsh $1000 \mathrm{ppm}$ COS, $100 \mathrm{ppm} \mathrm{HCN}, 4 \% \mathrm{CO}_{2}, 46 \% \mathrm{CO}, 46 \% \mathrm{H}_{2}$ and $4 \% \mathrm{H}_{2} \mathrm{O}$ at 35 bar environment up to $400{ }^{\circ} \mathrm{C}$. However, the material is not stable against wet $\mathrm{SO}_{2}$.

\subsection{References}

[1] H.F. Kay, P.C. Bailey, Structure and properties of $\mathrm{CaTiO}_{3}$, Acta Crystallographica, 10 (1957) 219-226.

[2] C. Li, K.C.K. Soh, P. Wu, Formability of $\mathrm{ABO}_{3}$ perovskites, Journal of Alloys and Compounds, 372 (2004) 40-48.

[3] V.V. Kharton, E.N. Naumovich, F.M.B. Marques, Ionic and electronic transport in perovskite-type $\mathrm{La}(\mathrm{Ga}, \mathrm{M}) \mathrm{O}_{3-\delta}(\mathrm{M}=\mathrm{Mg}, \mathrm{Cr}, \mathrm{Fe}, \mathrm{Co}, \mathrm{Ni}, \mathrm{Nb}$ ), Ionics, 5 (1999) 183-193.

[4] J. Sunarso, S. Baumann, J.M. Serra, W.A. Meulenberg, S. Liu, Y.S. Lin, J.C. Diniz da Costa, Mixed ionic-electronic conducting (MIEC) ceramic-based membranes for oxygen separation, Journal of Membrane Science, 320 (2008) 13-41.

[5] V.V. Kharton, A.A. Yaremchenko, A.V. Kovalevsky, A.P. Viskup, E.N. Naumovich, P.F. Kerko, Perovskite-type oxides for high-temperature oxygen separation membranes, Journal of Membrane Science, 163 (1999) 307-317.

[6] Y. Teraoka, H. Shimokawa, C.Y. Kang, H. Kusaba, K. Sasaki, Fe-based perovskitetype oxides as excellent oxygen-permeable and reduction-tolerant materials, Solid State Ionics, 177 (2006) 2245-2248.

[7] H. Wang, S. Werth, T. Schiestel, J. Caro, Perovskite Hollow-Fiber Membranes for the Production of Oxygen-Enriched Air, Angewandte Chemie International Edition, 44 (2005) 6906-6909.

[8] Y. Teraoka, T. Nobunaga, N. Yamazoe, Effect of Cation Substitution on the Oxygen Semipermeability of Perovskite-type Oxides, Chemistry Letters, 17 (1988) 503-506.

[9] Y. Teraoka, H.-M. Zhang, S. Furukawa, N. Yamazoe, Oxygen Permeation Through Perovskite-Tipe Oxides, Chemistry Letters, 14 (1985) 1743-1746.

[10] M. Arnold, H. Wang, A. Feldhoff, Influence of $\mathrm{CO} 2$ on the oxygen permeation performance and the microstructure of perovskite-type $\left(\mathrm{Ba}_{0.5} \mathrm{Sr}_{0.5}\right)\left(\mathrm{Co}_{0.8} \mathrm{Fe}_{0.2}\right) \mathrm{O}_{3-\delta}$ membranes, Journal of Membrane Science, 293 (2007) 44-52. 
[11] A. Leo, S. Smart, S. Liu, J.C. Diniz da Costa, High performance perovskite hollow fibres for oxygen separation, Journal of Membrane Science, 368 (2011) 64-68.

[12] J. Hoon Park, J. Pyo Kim, S. Hwan Son, Oxygen permeation and stability of $\mathrm{Ba}_{0.5} \mathrm{Sr}_{0.5} \mathrm{Co}_{0.8} \mathrm{Fe}_{0.2} \mathrm{O}_{3-\delta}$ membrane according to trace elements and oxygen partial pressure in synthetic air, Energy Procedia, 1 (2009) 369-374.

[13] F.M. Figueiredo, J. Waerenborgh, V.V. Kharton, H. Näfe, J.R. Frade, On the relationships between structure, oxygen stoichiometry and ionic conductivity of $\mathrm{CaTi}_{1}$. ${ }_{\mathrm{x}} \mathrm{Fe}_{\mathrm{x}} \mathrm{O}_{3-\delta}(\mathrm{x}=0.05,0.20,0.40,0.60)$, Solid State Ionics, 156 (2003) 371-381.

[14] V. Vashook, L. Vasylechko, M. Knapp, H. Ullmann, U. Guth, Lanthanum doped calcium titanates: synthesis, crystal structure, thermal expansion and transport properties, Journal of Alloys and Compounds, 354 (2003) 13-23.

[15] J. Cheng, W. Bao, D. Zhu, M. Ding, Preparation and electrical properties of $\mathrm{La}_{0.1} \mathrm{Ca}_{0.9} \mathrm{Ti}_{0.9} \mathrm{Zr}_{0.1} \mathrm{O}_{3}$ as a novel electrolyte material for SOFCs, Journal of Alloys and Compounds, 486 (2009) 895-899.

[16] A.L. Shaula, R.O. Fuentes, F.M. Figueiredo, V.V. Kharton, F.M.B. Marques, J.R. Frade, Grain size effects on oxygen permeation in submicrometric $\mathrm{CaTi}_{0.8} \mathrm{Fe}_{0.2} \mathrm{O}_{3-\delta}$ ceramics obtained by mechanical activation, Journal of the European Ceramic Society, 25 (2005) 2613-2616.

[17] F.M. Figueiredo, V.V. Kharton, J.C. Waerenborgh, A.P. Viskup, E.N. Naumovich, J.R. Frade, Influence of Microstructure on the Electrical Properties of Iron-Substituted Calcium Titanate Ceramics, Journal of the American Ceramic Society, 87 (2004) 22522261.

[18] L.A. Dunyushkina, A.K. Demin, B.V. Zhuravlev, Electrical conductivity of irondoped calcium titanate, Solid State Ionics, 116 (1999) 85-88.

[19] E. Chinarro, J.R. Jurado, F.M. Figueiredo, J.R. Frade, Bulk and grain boundary conductivity of $\mathrm{Ca}_{0.97} \mathrm{Ti}_{1-\mathrm{x}} \mathrm{Fe}_{\mathrm{x}} \mathrm{O}_{3-\delta}$ materials, Solid State Ionics, 160 (2003) 161-168.

[20] K.H. Ryu, S.M. Haile, Chemical stability and proton conductivity of doped $\mathrm{BaCeO}_{3}-\mathrm{BaZrO}_{3}$ solid solutions, Solid State Ionics, 125 (1999) 355-367.

[21] M.J. Scholten, J. Schoonman, J.C. van Miltenburg, H.A.J. Oonk, Synthesis of strontium and barium cerate and their reaction with carbon dioxide, Solid State Ionics, 61 (1993) 83-91.

[22] F.M. Figueiredo, V.V. Kharton, A.P. Viskup, J.R. Frade, Surface enhanced oxygen permeation in $\mathrm{CaTi}_{1-\mathrm{x}} \mathrm{Fe}_{\mathrm{x}} \mathrm{O}_{3-\delta}$ ceramic membranes, Journal of Membrane Science, 236 (2004) 73-80. 
[23] C.-Y. Chen, K. Ozasa, K.-I. Katsumata, M. Maeda, K. Okada, N. Matsushita, $\mathrm{CaTiO}_{3}$ nanobricks prepared from anodized TiO2 nanotubes, Electrochemistry Communications, 22 (2012) 101-104.

[24] M.C. Biesinger, L.W.M. Lau, A.R. Gerson, R.S.C. Smart, Resolving surface chemical states in XPS analysis of first row transition metals, oxides and hydroxides: Sc, Ti, V, Cu and Zn, Applied Surface Science, 257 (2010) 887-898.

[25] M.C. Biesinger, B.P. Payne, A.P. Grosvenor, L.W.M. Lau, A.R. Gerson, R.S.C. Smart, Resolving surface chemical states in XPS analysis of first row transition metals, oxides and hydroxides: Cr, Mn, Fe, Co and Ni, Applied Surface Science, 257 (2011) 2717-2730.

[26] M. Hadnadjev, T. Vulic, R. Marinkovic-Neducin, Y. Suchorski, H. Weiss, The iron oxidation state in Mg-Al-Fe mixed oxides derived from layered double hydroxides: An XPS study, Applied Surface Science, 254 (2008) 4297-4302.

[27] S. Ardizzone, C.L. Bianchi, M. Fadoni, B. Vercelli, Magnesium salts and oxide: an XPS overview, Applied Surface Science, 119 (1997) 253-259.

[28] H.B. Yao, Y. Li, A.T.S. Wee, An XPS investigation of the oxidation/corrosion of melt-spun Mg, Applied Surface Science, 158 (2000) 112-119.

[29] C.-Y. Yoo, B.A. Boukamp, H.M. Bouwmeester, Oxygen surface exchange kinetics of erbia-stabilized bismuth oxide, J Solid State Electrochem, 15 (2011) 231-236.

[30] H.J.M. Bouwmeester, C. Song, J. Zhu, J. Yi, M. van Sint Annaland, B.A. Boukamp, A novel pulse isotopic exchange technique for rapid determination of the oxygen surface exchange rate of oxide ion conductors, Physical Chemistry Chemical Physics, 11 (2009) 9640-9643.

[31] J.A. Lane, J.A. Kilner, Oxygen surface exchange on gadolinia doped ceria, Solid State Ionics, 136-137 (2000) 927-932.

[32] R.A. De Souza, J.A. Kilner, Oxygen transport in $\mathrm{La}_{1-\mathrm{x}} \mathrm{Sr}_{\mathrm{x}} \mathrm{Mn}_{1-\mathrm{y}} \mathrm{Co}_{\mathrm{y}} \mathrm{O}_{3-\delta}$ perovskites: Part II. Oxygen surface exchange, Solid State Ionics, 126 (1999) 153-161.

[33] H.J.M. Bouwmeester, Dense ceramic membranes for methane conversion, Catalysis Today, 82 (2003) 141-150.

[34] M.P. Lobera, S. Escolástico, J.M. Serra, High Ethylene Production through Oxidative Dehydrogenation of Ethane Membrane Reactors Based on Fast Oxygen-Ion Conductors, ChemCatChem, 3 (2011) 1503-1508. 
[35] A. Leo, S. Liu, J.C.D.d. Costa, Z. Shao, Oxygen permeation through perovskite membranes and the improvement of oxygen flux by surface modification, Science and Technology of Advanced Materials, 7 (2006) 819. 


\title{
Chapter 8. Ceria-based stable dual-phase membranes for fast oxygen separation
}

\begin{abstract}
As another approach of the new materials research, dual phase materials composed of spinel and fluorite phases have been developed. They have been selected on the basis of their electrical conductivities and the expected contributions to the overall composite material to enhance the mixed ionic and electronic conductivity. $\mathrm{Fe}_{2} \mathrm{NiO}_{4}$ is the spinel of choice as it is prone to form dense composite without reacting with the fluorites.

60 vol\% $\mathrm{Fe}_{2} \mathrm{NiO}_{4}-40$ vol\% $\mathrm{Ce}_{0.8} \mathrm{Ln}_{0.2} \mathrm{O}_{2-\delta}$ (FNO-CLnO) composites have been successfully obtained by one-pot fabrication method showing both spinel and fluorite pure phases. Narrow grain size distribution centred around $1 \mu \mathrm{m}$ and homogeneous distribution of grains is attained in the gastight membrane, as well as percolative pathways from side to side of the dual-phase membranes. Specifically, composite membranes with enhanced oxygen permeability and unprecedented stability in oxyfuel-like gas environments are reported. The conductivity of the composite is investigated as a function of temperature and $\mathrm{pO}_{2}$. Oxygen separation in this kind of FNO-CLnO composite membranes occurs via the separate ambipolar transport through the two distinct percolating
\end{abstract}


networks. Further study of a 60 vol\% $\mathrm{Fe}_{2} \mathrm{NiO}_{4}-40$ vol\% $\mathrm{Ce}_{0.8} \mathrm{~Tb}_{0.2} \mathrm{O}_{2-\delta}$ (FNOCTO) composite membrane has been developed. Oxygen permeation flux values of $0.17 \mathrm{~mL} \cdot \mathrm{min}^{-1} \cdot \mathrm{cm}^{-2}$ and $0.20 \mathrm{~mL} \cdot \mathrm{min}^{-1} \cdot \mathrm{cm}^{-2}$ are achieved at $1000{ }^{\circ} \mathrm{C}$ when $\mathrm{Ar}$ and pure $\mathrm{CO}_{2}$ are used as sweep gas, respectively, through a $0.68 \mathrm{~mm}$ thick membrane. Long term experiments at $900{ }^{\circ} \mathrm{C}$ show that the material is stable and effective in pure $\mathrm{CO}_{2}$ atmospheres and the oxygen permeation is even improved after $76 \mathrm{~h}$ on $\mathrm{CO}_{2}$ stream. Moreover, the composite resists a stability test in $\mathrm{SO}_{2}$ and $\mathrm{CO}_{2}$ containing gas at $800{ }^{\circ} \mathrm{C}$ for $170 \mathrm{~h}$, which represents a step forward towards its potential use in oxyfuel power plants. 


\subsection{Introduction}

Materials with perovskite structure are the MIEC materials showing the highest electrical conductivities but permeabilities are often limited by the ionic conductivity. Doping strategies to increase the oxygen ion conductivity normally affect the crystal and thermo-mechanical stability and increase their vulnerability when exposed to large oxygen concentration gradients and atmospheres containing $\mathrm{CO}_{2}, \mathrm{SO}_{2}$ and $\mathrm{H}_{2} \mathrm{O}$, the most probable operation conditions in the oxyfuel process and catalytic membrane reactors in different intensified industrial processes. [1-3]

In previous chapters, the difficulty of joining all the desired characteristics in a single material has been recognized, which points out to achieve them separately. Dual phase composite membranes try to combine the best characteristics of different compounds to achieve large oxygen permeability and relatively good chemical and mechanical stability at elevated temperatures. Thus, composite materials should consist of an electron conducting material, which allows the percolation of electrons, and an ionic conductor that transports the oxygen ions through the membrane. Due to the existence of two phases, the influence of the grain boundary between them plays an important role, since it can either promote or block the transport of ions and electrons across the membrane. Furthermore, large catalytic activity towards surface oxygen exchange is required. 
The first reported dual phase membranes were ceramic-metal (cermet) composites consisting of a continuous oxygen ion-conducting oxide phase and a continuous electron-conducting noble metal phase, but the high costs arising from the use of noble metals prevent the industrial applications. [4-6] Therefore, attempts to decrease the total price of the membranes involve the substitution of the metallic phase by solid oxides with high electronic conductivity. Indeed, the use of ceramic oxide composites also brings the possibility of substituting the electronic or ionic phase by a MIEC phase that does not block the ionic or electronic transport. Ceramic-ceramic (cercer) dual-phase compounds have been successfully prepared since early 2000's decade. [7] In a cercer, other important requirements besides the transport properties have to be taken into account, i.e. the stability of both phases against reaction with each other and the compatibility of thermal expansion coefficient (TEC) of both compounds. Following this composite strategy, several crystalline structures have been combined, e.g. fluorites, perovskites, spinels and rock salts. [8] Materials with perovskite and spinel $\left(\mathrm{A}_{2} \mathrm{BO}_{4}\right)$ structure may be used as electronic phase. [9] Fluorite materials such as yttria stabilized zirconia (YSZ), stabilized bismuth oxide (SBO) or $\mathrm{Ce}_{1-\mathrm{x}} \mathrm{B}_{\mathrm{x}} \mathrm{O}_{2-\delta}(\mathrm{B}=$ lanthanide) play the oxygen conductor role in composites since they present mainly ionic conductivity. Moreover, the chemical stability of doped cerias is significantly higher than that of perovskites. [10]

On these premises, cercers composed of doped ceria fluorites and several spinels have allowed the development of $\mathrm{CO}_{2}$ stable composite membranes. First suggested membranes joined $\mathrm{Ce}_{0.8} \mathrm{Gd}_{0.2} \mathrm{O}_{1.9}$ (CGO20) with $\mathrm{CoFe}_{2} \mathrm{O}_{4}$ (CFO) and $\mathrm{MnFe}_{2} \mathrm{O}_{4}$ (MFO). [11] An optimum ratio for the percolation of both phases was found to be $30 \%$ volume of spinel phase. However, these compositions presented reaction between phases and showed a small amount of $\mathrm{GdFeO}_{3}$, which reduces the Gd content in $\mathrm{CGO} 20$ and 
consequently drops the ionic conductivity. Lower fractions of spinel (ca. 17\%) are also oxygen permeating despite percolation of the electronic phase was not achieved. [11] To further improve the oxygen permeation properties, the same authors studied the composite formed by $85 \mathrm{vol} \%$ of $\mathrm{Ce}_{0.8} \mathrm{Sm}_{0.2} \mathrm{O}_{1.9}$ (SDC20) and $15 \mathrm{vol} \%$ of $\mathrm{Mn}_{(1.5-0.5 \mathrm{y})} \mathrm{Co}_{(1+0.5 \mathrm{y})} \mathrm{Ni}_{0.5} \mathrm{O}_{4}(\mathrm{MCNO}, 0 \leq \mathrm{y} \leq 1)$ spinel oxide. The microstructural analysis found out that two spinel type oxides are formed, one consisted of Mn-Co-Ni and another of Co-Ni. The highest oxygen flux density was obtained when $y=1$, which is comparable to those for SDC-15MFO. [12]

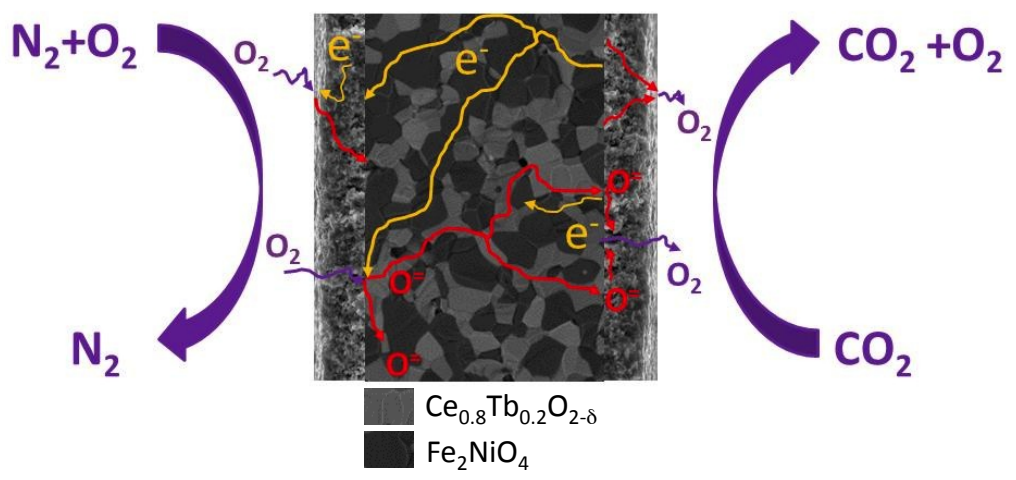

Figure 8.1. Oxygen and electron transport scheme in a dual phase composite membrane composed of a) pure electronic and mixed ionic electronic conducting phases and b) pure electronic and ionic conducting phases.

Co-free spinel $\mathrm{Fe}_{2} \mathrm{NiO}_{4}$ (FNO) $40 \mathrm{wt} \%$ was mixed with $\mathrm{Ce}_{0.9} \mathrm{Gd}_{0.1} \mathrm{O}_{1.95}$ (CGO10) both by powder mixing method and one pot. [9] No intermixing of cations between the two phases occurs while the dual phase structure at high temperatures is retained even in $50 \% \mathrm{CO}_{2}$ containing atmospheres. The enhancement of the oxygen flux was obtained by the one pot method due to the improvement in morphology and homogeneity. [9, 13] In fact, oxygen flux improves with decreasing grain sizes in composite materials while high density 
is needed to avoid leakages. Therefore, sintering temperatures should be optimized for each composite composition. 20\% Pr-doped $\mathrm{CeO}_{2}$ (CPO) fluorite possesses redox properties that bring about both ionic and electronic conductivities, which is favorable for oxygen permeable ceramics. Oxygen permeation through $\mathrm{Ce}_{1-\mathrm{x}} \mathrm{Pr}_{\mathrm{x}} \mathrm{O}_{2-\delta}$ membranes has been obtained under small $p \mathrm{O}_{2}$ gradients, but the large expansion under reducing atmospheres such as methane causes severe strains in the membrane up to the final fail. $[14,15] \mathrm{CPO} / 40 \mathrm{wt} \%$ MFO composite has demonstrated several benefits over the single fluorite. [16, 17] From the fact that $\mathrm{CPO}$ is a MIEC conductor arises that even non percolative fractions of spinel phase tolerate the oxygen permeation. Although a small amount of $\mathrm{PrFeO}_{3}$ is formed when MFO is over $10 \%$, the optimum volume fraction of MFO was determined to be from 5 to $25 \%$. [16]

The present chapter reports the research on dual phase membranes composed of the spinel and fluorite phases listed in Table 8.1. They have been selected on the bases of their electrical conductivities and the expected contributions to the overall composite material.

\begin{tabular}{ccc}
\hline Spinels & Fluorites & Properties \\
\hline $\mathrm{Fe}_{2} \mathrm{NiO}_{4}$ & $\mathrm{Ce}_{0.8} \mathrm{Gd}_{0.2} \mathrm{O}_{2-\delta}$ & Electronic + ionic \\
$\mathrm{Mn}_{2} \mathrm{CuO}_{4}$ & $\mathrm{Ce}_{0.8} \mathrm{Gd}_{0.1} \mathrm{Pr}_{0.1} \mathrm{O}_{2-\delta}$ & Electronic + mixed ionic-electronic \\
$\mathrm{Fe}_{2} \mathrm{CrO}_{4}$ & $\mathrm{Ce}_{0.8} \mathrm{~Tb}_{0.2}$ & Electronic + mixed mainly ionic \\
$\mathrm{Co}_{2} \mathrm{FeO}_{4}$ & $\mathrm{Ce}_{0.8} \mathrm{~Tb}_{0.2} \mathrm{O}_{2-\delta}+\mathrm{Co}$ & Electronic + ionic-electronic + sintering aid \\
\hline
\end{tabular}

Table 8.1. Spinel and fluorite structured compounds selected for dual-phase membranes.

An optimization of phase ratio and synthesis method is carried out, as well as a screening of several materials combinations to develop dual-phase membranes. X-Ray Diffraction (XRD) and Scanning Electron Microscopy (SEM) carry out characterization of powders and sintered membranes. The 
electrochemical study is performed by 4-point DC conductivity. The oxygen permeation of a planar disk dense membrane with both sides covered with a previously optimized catalytic composite layer has been tested by $\mathrm{Ar}$ and $\mathrm{CO}_{2}$ sweeping gases. Finally, the stability of the material for 7 days against exposure to a $1 \mathrm{~mol} \% \mathrm{SO}_{2}$-containing wet gas environment is checked.

\subsection{Results and discussion}

\section{Component and microstructural optimization}

In a first step, the synthesis of the spinels was checked separately. They were prepared by Pechini reaction method and sintered at $1300{ }^{\circ} \mathrm{C}$, which is a normal temperature to obtain dense membranes. Initial identification of the crystalline phases by XRD is shown in Figure 8.2. Only $\mathrm{Fe}_{2} \mathrm{NiO}_{4}$ and $\mathrm{Fe}_{2} \mathrm{CrO}_{4}$ show pure spinel structure. Thus $\mathrm{Mn}_{2} \mathrm{CuO}_{4}$ and $\mathrm{Co}_{2} \mathrm{FeO}_{4}$ were not further considered. $\mathrm{Mn}_{2} \mathrm{CuO}_{4}$ was modified to $\mathrm{Mn}_{1.5} \mathrm{Cu}_{1.5} \mathrm{O}_{4}$ and formed pure spinel phase. However, even though both $\mathrm{Mn}_{1.5} \mathrm{Cu}_{1.5} \mathrm{O}_{4}$ and $\mathrm{Fe}_{2} \mathrm{CrO}_{4}$ form spinel and a stable composite with fluorites, they were not able to deliver dense monoliths and were not further considered, either. 


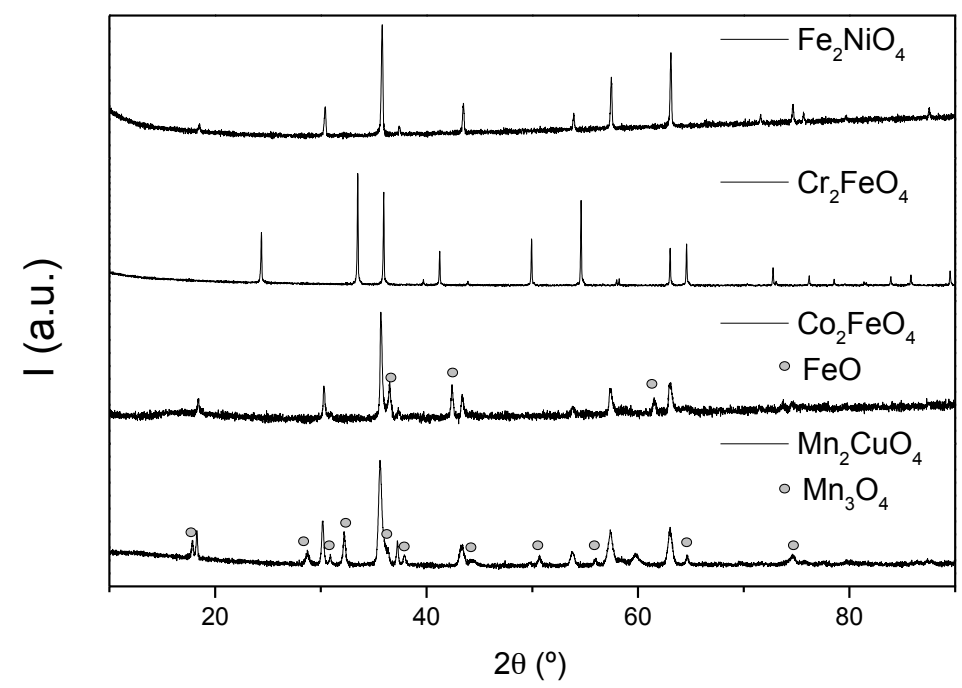

Figure 8.2. XRD patterns of tested spinels: $\mathrm{Fe}_{2} \mathrm{NiO}_{4}, \mathrm{Cr}_{2} \mathrm{FeO}_{4}, \mathrm{Co}_{2} \mathrm{FeO}_{4}, \mathrm{Mn}_{2} \mathrm{CuO}_{4}$. Grey circles indicate impurity peaks.

In order to optimize the microstructure, the composite powder has been prepared by powder mixing method (PM) and one pot method (OP). The first method blends the oxides separately, i.e., the fluorite compounds formed by co-precipitation method and incipient wetness impregnation (if Co oxide is added) and the spinels prepared by Pechini, both calcined at $1300{ }^{\circ} \mathrm{C}$. Otherwise, the OP method allows the composite to be synthesized by a single step Pechini method. [9, 18] Figure 8.3a shows the XRD pattern of $50 \% \mathrm{Fe}_{2} \mathrm{NiO}_{4}-50 \% \mathrm{Ce}_{0.8} \mathrm{Gd}_{0.2} \mathrm{O}_{1.95}$ (FNO-CGO) sintered in air at $1400{ }^{\circ} \mathrm{C}$. For comparison, separate CGO and FNO structure peaks are also plotted. Both structural phases are well identified in the composites with no evidence of impurities or reaction between them up to the limit of the XRD device, demonstrating the validity of both fabrication methods. 
a)

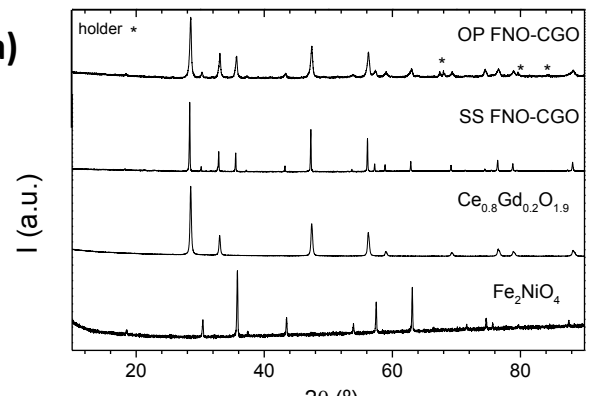

b)
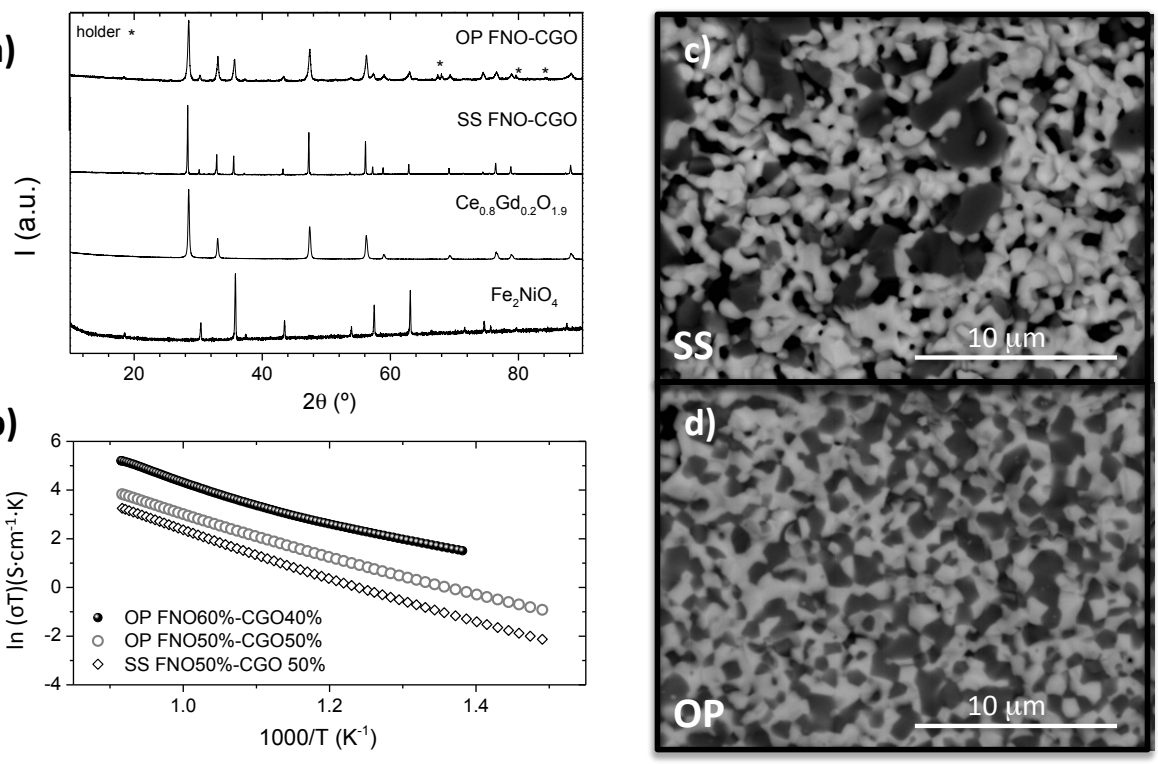

Figure 8.3. a) XRD patterns of the composites prepared by SS and OP, and the $\mathrm{Fe}_{2} \mathrm{NiO}_{4}$ and $\mathrm{Ce}_{0.8} \mathrm{Gd}_{0.2} \mathrm{O}_{1.9}$ single phases. b) Arrhenius plot of total electrical conductivity for the 50/50 phase ratio composites prepared by SS and OP and for 60/40 phase ratio OP composite. c) SEM picture of 50/50 FNO-CGO prepared by PM and d) SEM picture of 50/50 FNO-CGO prepared by OP.

Figure $8.3 \mathrm{c}$ and $\mathrm{d}$ show the backscattered SEM images of the $50 \% \mathrm{Fe}_{2} \mathrm{NiO}_{4}-50 \% \mathrm{Ce}_{0.8} \mathrm{Gd}_{0.2} \mathrm{O}_{1.9}$ composite membranes prepared by PM and OP methods, respectively. While the specimens blended by PM form agglomerates of spinel phase (dark grey), the OP method enables the formation of homogeneous-nanometric sized grains in a well-distributed mixture of the two phases forming the composite. On the other hand, the lack of continuity of spinel phase observed in SEM pictures suggests that percolative pathways for electrons may be not completely achieved. 
To confirm the importance of materials uniformity, Arrhenius plot of the total electrical conductivity in air for the differently sintered materials is shown in Figure $8.3 \mathrm{~b}$. Conductivity increases the more uniform grain size and homogeneous mixture. Besides, increasing the spinel content from 50 to $60 \%$ provides a continuous pathway for electrons across the bulk material. Therefore, 60 vol.\% of electronic FNO and 40 vol.\% of doped ceria ionic fluorite phases synthesized via OP method have been selected since this rate allows the electronic percolation and a proper microstructure.

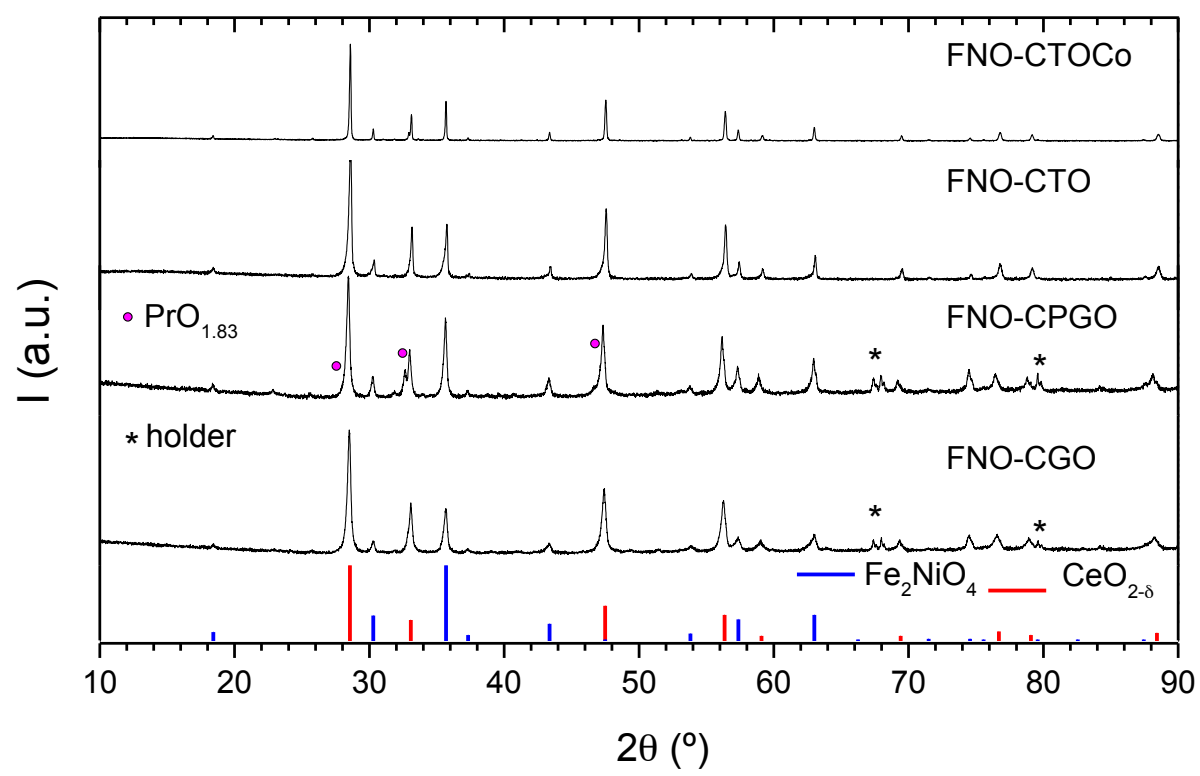

Figure 8.4. XRD patterns of FNO-CLnO synthesized by OP and sintered in air at $1400{ }^{\circ} \mathrm{C}$. Reference fluorite and spinel structure peaks are also indicated (ICDD 00-0431002 and 00-044-1485, respectively).

Figure 8.4 shows the XRD patterns of FNO-CLnO synthesized by OP and sintered in air at $1400{ }^{\circ} \mathrm{C}$. For comparison, reference fluorite and spinel structure peaks are also indicated (ICDD 00-043-1002 and 00-044-1485, respectively). Both structural phases are well identified in FNO-CGO, 
FNO-CTO and FNO-CTOCo composites with no evidence of impurities or reaction between them up to the limit of the XRD device. Otherwise, FNO-CPGO has another phase identified as $\operatorname{Pr}_{7} \mathrm{O}_{11}$. This impurity is detrimental for the conductivity as it may block the diffusion of ions and electrons. Therefore, this composition is not further considered.

The conductivity in air of stable FNO-CLnO series is compared in Figure 8.5 as a function of temperature. At high temperatures, the FNO-CGO is the compound with higher conductivity, ascribed to the ionic conductivity given by the higher extrinsic nonstoichiometry of CGO. At low temperatures FNO-CTO shows an enhanced conductivity over that of FNO-CGO, which can be attributed to the improved electrical conductivity of the fluorite phase containing multivalent dopants. CTOCo showed the lowest conductivity, probably due to the increased particle size induced by the Co.

\section{$\mathrm{Fe}_{2} \mathrm{NiO}_{4}-\mathrm{Ce}_{0.8} \mathrm{~Tb}_{0.2} \mathrm{O}_{2-\delta}$ composite characterization}

In the following, an exhaustive study on FNO-CTO composite material is carried out. It is compared in some points with with FNO-CGO. Different conduction mechanisms are expected from the CGO and CTO fluorite phases since CGO is an ionic conductor at high $\mathrm{pO}_{2}$ and the CTO may provide electronic conduction, as deduced in Chapter 5. 


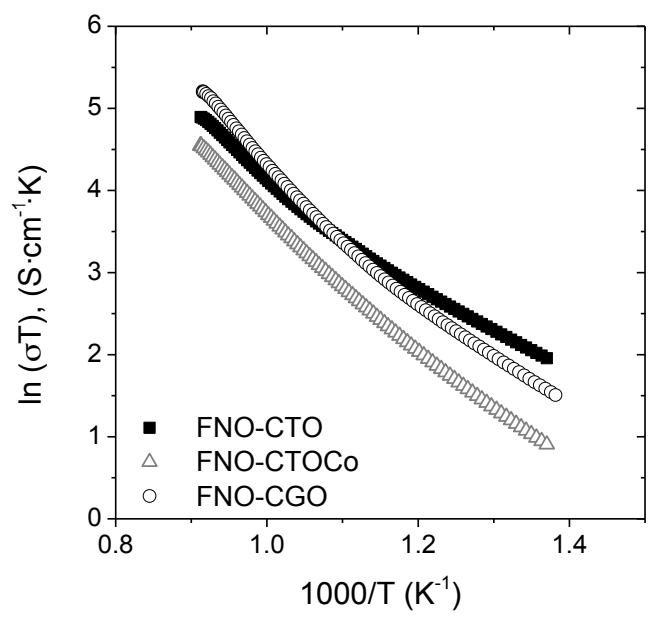

Figure 8.5. Arrhenius plot of the total electrical conductivity in air of FNO-CLnO composite series.

Dense membrane and probes of FNO-CTO have been achieved by sintering the composite at $1400{ }^{\circ} \mathrm{C}$. XRD pattern of FNO-CTO shown in Figure 8.4 indicate that pure spinel and cubic fluorite phases are obtained. The calculated cell parameters of FNO and CTO are $=8.332 \AA$ and $\mathrm{a}=5.406 \AA$. They are very similar to those obtained for single phases separately ( $\mathrm{a}=8.339 \AA$ and $\mathrm{a}=5.392 \AA$, respectively), which point out that there is not inter-diffusion of the elements. Despite these differences, diffusion of $\mathrm{Ce}$ or $\mathrm{Tb}$ inside the spinel structure (if any) should cause an expansion of the cell parameter, but the experimental data shows a slight shrinkage. On the other hand, the cell parameter of the ceria, which is longer than that observed previously for the single phase, could suggest that $\mathrm{Tb}$ dopant is not fully solved in the fluorite lattice, so that the rest should appear as $\mathrm{Tb}_{2} \mathrm{O}_{3}$ impurity. However, $\mathrm{Tb}_{2} \mathrm{O}_{3}$ is not detected by XRD, up to the limits of the device. The composite has been calcined in atmospheric air. Under such condition not all the $\mathrm{Tb}$ is introduced as $\mathrm{Tb}^{3+}$ (i.r. $1.04 \AA$ ) but as $\mathrm{Tb}^{4+}$ (i.r. $0.88 \AA$ ), as studied in chapters 3 and 5. Both the sample thermal history and the influence of the spinel on the Tb reduction 
properties may cause the slight difference in cell parameter by delivering higher ratio $\mathrm{Tb}^{3+} / \mathrm{Tb}^{4+}$. [19]
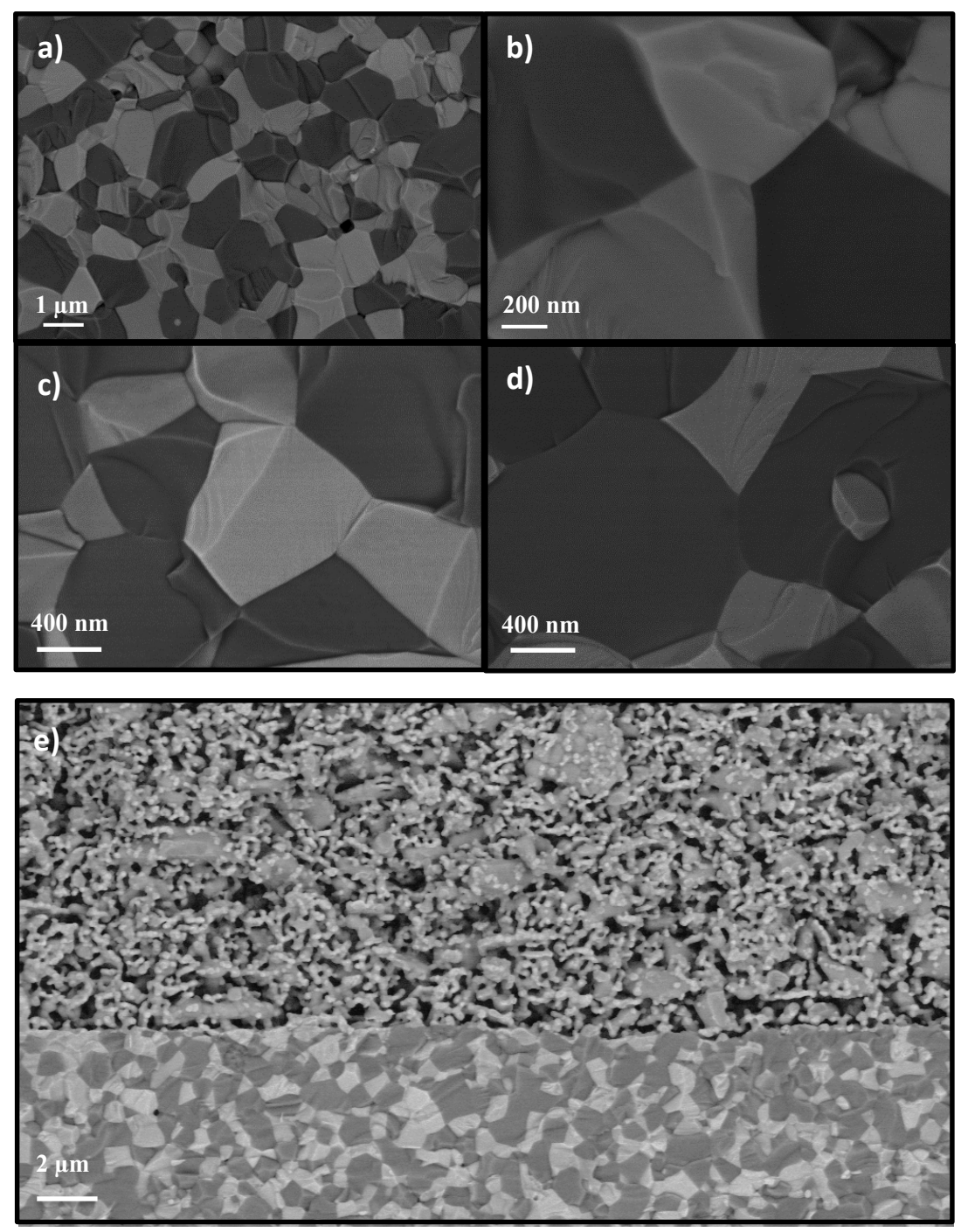

Figure 8.6. a) BS-SEM picture of the fractured cross-section of a FNO-CTO composite membrane sintered at $1400{ }^{\circ} \mathrm{C}$ in air; b) detail of the grain boundary between spinel (dark) and fluorite (light) phases; c) detail of two fluorite grains fringe; d) detail of two spinel grains fringe and; e) CGO-LSM-Pd catalytic layer on the composite. 
SEM images allow the analysis of the membrane microstructure, e.g. grain size distribution, pores, grain boundaries as well as offering information about the possible solid state reaction between the two composite phases. SEM backscattered electrons detector (BS-SEM) provide images with compositional contrast related to the averaged atomic number of the elements of each phase, and therefore changes in composition of the different grains and element distribution can be distinguished. The images in Figure 8.6 show a dark phase that corresponds to the spinel and a bright one that belongs to the fluorite. In Figure 8.6a, a narrow grain size distribution (centred around $1 \mu \mathrm{m}$ ) and a homogeneous distribution of grains can be observed. The existence of percolative paths from side to side of the dual-phase membranes necessary for the oxygen permeation is ascertained. Image analysis confirms the expected 60/40-volume phase ratio (spinel/fluorite). Figure $8.6 \mathrm{~b}$ presents a higher magnification image offering details about the grain boundaries between the two different phases, and apparently no phase reaction, impurities segregation or inter-diffusion take place during the membrane sintering at $1400{ }^{\circ} \mathrm{C}$. Further, Figure $8.6 \mathrm{~b}-\mathrm{d}$ reveal the very good bounding between grains made of the same phase (CTO-CTO or FNO-FNO) or different phases (FNO-CTO). Additionally, Figure 8.6e shows a detail of the $18 \mu \mathrm{m}$-thick CGO-LSM catalytic layer, which can be easily identified by its smaller grain size and higher porosity. The BS-SEM image (Figure 8.6e) also makes visible the two distinct phases of this catalytic layer, i.e.,, CGO corresponds to the bright phase and the LSM to the dark one. From this image, it is also visible a very clean interface between the membrane and the catalytic layer, with no reaction interfaces or cracks, which reveals the chemical compatibility of all the different materials and the good bounding between layer and membrane. 
a)

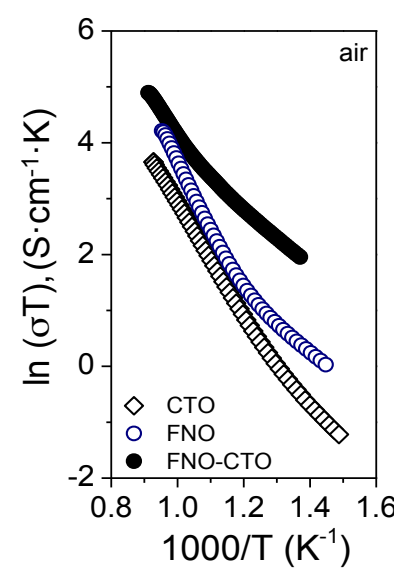

b)

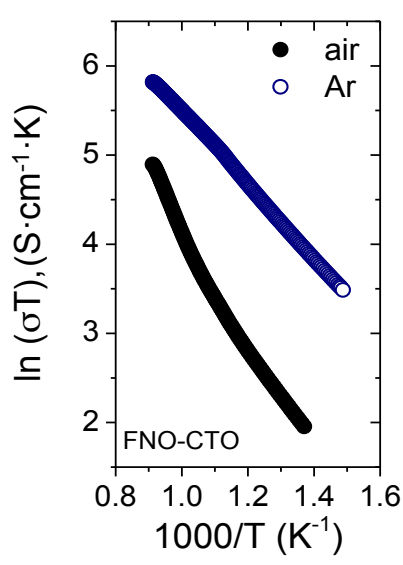

c)

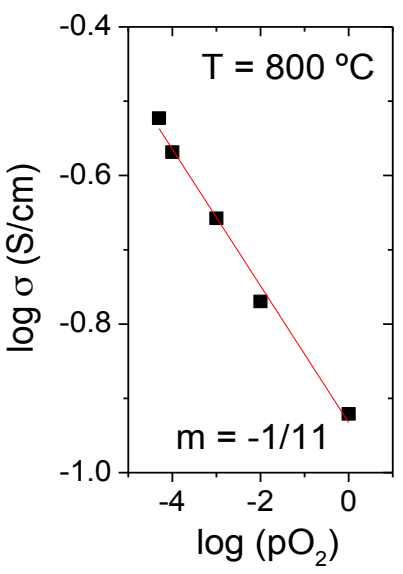

Figure 8.7. a) Total electrical conductivity recorded in air as a function of temperature for the single phase CTO, FNO and for the composite, b) Arrhenius plot of the total electrical conductivity of the composite in air and $\mathrm{Ar}$ atmospheres and c) total electrical conductivity at $800^{\circ} \mathrm{C}$ as a function of the $\mathrm{pO}_{2}$.

Once proved the compatibility between both crystalline phases and the appropriate microstructure of the sintered composite body, the transport properties are investigated. Due to the selected percentage of phases in the composite, preferential pathways for oxygen and electrons are expected, so that the total conductivity would result from the sum of CTO and FNO contributions. The plot in Figure 8.7a shows the electrical conductivity recorded in air as a function of temperature for the single phase CTO, FNO and for the composite. The composite conductivity is surprisingly higher than the equivalent sum of the single components, which is attributed to the improvement of densification for the composite in contrast to the FNO, with lower sintering activity. FNO sample displayed in Figure 8.8 is not totally dense 
(relative density $\sim 90 \%$ ) when sintered at $1400{ }^{\circ} \mathrm{C}$ for $10 \mathrm{~h}$, while CTO and the composite are dense at the same conditions.

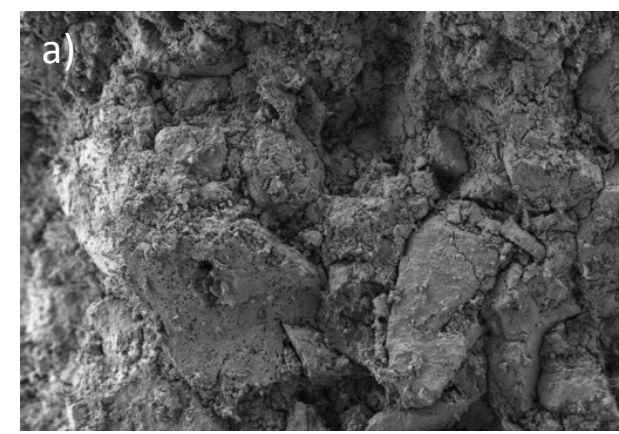

$\overline{100 \mu \mathrm{m}}$

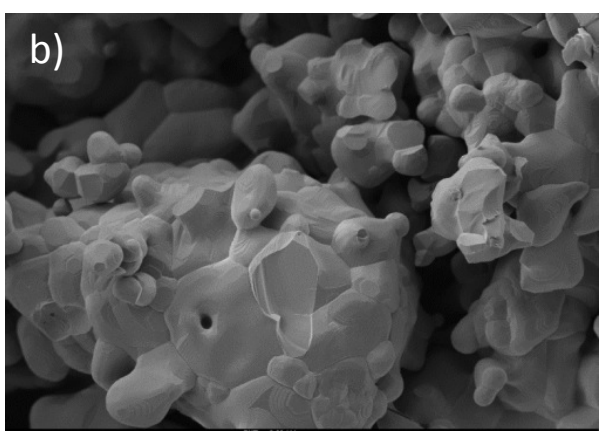

$\overline{5 \mu \mathrm{m}}$

Figure 8.8. SEM image of $\mathrm{Fe}_{2} \mathrm{NiO}_{4}$ sample sintered at $1400{ }^{\circ} \mathrm{C}$ in air dwelling $10 \mathrm{~h}$. $\sim 90 \%$ relative density was achieved at these conditions.

The Figure $8.7 \mathrm{~b}$ displays the total conductivity, $\ln (\sigma \mathrm{T})$ as a function of reciprocal temperature measured in air and argon $\left(\mathrm{pO}_{2}=0.21\right.$ and $5 \times 10^{-5} \mathrm{~atm}$, respectively) for the composite. An apparent change in activation energy $\left(E_{a}\right)$ is observed in air, which suggests a change in the dominant conduction mechanisms. $E_{a}$ values of 0.45 and $0.71 \mathrm{eV}$ have been calculated from the fitted data below and above $655{ }^{\circ} \mathrm{C}$, respectively. The low temperature $E_{a}$ may correspond to FNO phase and is characteristic for the hopping of electronic charge carriers, i.e., hopping of localized electrons between multivalent cations located in the octahedral Fe sublattice. [20]

$$
F e^{3+}+e^{\prime} \rightarrow F e^{2+}
$$

For temperatures above $655{ }^{\circ} \mathrm{C}$, oxygen is released from CTO phase (oxygen vacancy formation) and induce $\mathrm{Tb}^{4+}$ reduction to $\mathrm{Tb}^{3+}$, which is charge compensated by the formation of electrons localized on $\mathrm{Tb}$ ions, as indicated by the following equations: 


$$
\begin{aligned}
& \frac{1}{2} O_{2}(g)+V_{O}^{*} \rightarrow O_{O}^{\times}+2 h^{\prime} ;\left[V_{O}^{\prime \prime}\right]^{-1} p^{2} p O_{2}^{-1 / 2}=K_{O}(T) \\
& T b_{C e}^{\times}+e^{\prime} \rightarrow T b_{C e}^{\prime} \quad \frac{\left[T b_{C e}^{\prime}\right]}{\left[T b_{C e}^{\times}\right] n}=K_{T b}(T)
\end{aligned}
$$

The oxygen vacancies concentration in combination with the strong thermal activation of the oxide ion mobility leads to the noticeable increase in the ionic conductivity. As a result, the contribution of the ionic transport to the total conductivity of the FNO-CTO composite becomes significant at temperatures above $655{ }^{\circ} \mathrm{C}$. This thermal process in CTO is responsible for the raise in the overall composite $E_{a}$, which is related to the higher $E_{a}$ associated to oxygen vacancy diffusion with regard to the electron hopping $E_{a}$ observed at lower temperatures. [21, 22]

Figure $8.7 \mathrm{~b}$ also presents the thermal evolution of the composite conductivity in Ar atmosphere upon equilibration of the samples in argon at 800 ${ }^{\circ} \mathrm{C}$ for $2 \mathrm{~h}$. At this low $\mathrm{pO}_{2}$ terbium and iron cations are mainly reduced to $\mathrm{Tb}^{3+}$, and $\mathrm{Fe}^{2+}$ valence state along the whole studied temperature range. Hence, in $\mathrm{Ar}$, the concentration of oxygen vacancies in CTO remains constant with decreasing temperatures. Consequently a monotonic Arrhenius behaviour is observed, i.e., the $E_{a}$ remains constant. $E_{a}$ is $0.38 \mathrm{eV}$, which suggests that the electronic conductivity of the partially-reduced FNO governs the composite conductivity. Additionally, the conductivity increases substantially at higher temperatures due not only to the further activation of electron hopping rate but also to the increased probability of the localized electrons in the spinel to transfer from one ion to another due to the lattice vibrations. Thus, these trapped electronic carriers in the ferrite may contribute to the conduction process leading to an overall decrease in resistivity. 
Total electrical conductivity at $800{ }^{\circ} \mathrm{C}$ as a function of the $\mathrm{pO}_{2}$ is depicted in Figure 8.7c. Nickel ferrite is a $p$ or $n$-type semiconductor depending on the oxygen deficiency determined by the sintering temperature and atmosphere. The present sintering conditions, i.e., high temperatures and oxygen partial pressures lower than 4 atm of oxygen, favour the $\mathrm{Fe}^{2+}$ concentrations instead of $\mathrm{Ni}^{3+}$ holes. $[20,23]$ Thus, $n$-type conductivity $(-1 / 4$ dependency with $p O_{2}$ ) is expected. However, the influence of the slight increase in the CTO ionic conductivity $\left(<-1 / 6\right.$ dependency with $\left.p O_{2}\right)$ provokes the adjustment of conductivity- $\mathrm{pO}_{2}$ dependency of both phases giving rise to $-1 / 11$ $p \mathrm{O}_{2}$ dependency, as shown in Figure 8.7c. [20]

\section{Dual phase $\mathrm{Fe}_{2} \mathrm{NiO}_{4}-\mathrm{Ce}_{0.8} \mathrm{~Tb}_{0.2} \mathrm{O}_{2-\delta}$ membranes}

The preceding results provide sufficient evidences about the participation of both ionic and electronic carriers in the ambipolar conduction. Consequently this stable composite presents the appropriate characteristics to work as oxygen permeation membrane. The catalytic layer consisted of CGO-LSM porous composite (Figure 8.6e) and Pd infiltration is selected to further promote oxygen activation. In chapter 6 , this mixture demonstrated to be the optimum between LSM-doped ceria compositions with interesting electrochemical properties for oxygen activation at high temperatures, while these phases are known to be stable and effective in $\mathrm{CO}_{2}$-rich atmospheres. CGO provides the ionic conductivity that LSM lacks and thus allow increasing the global three-phase-boundary (TPB) region of the oxygen activation and incorporating additional redox electrocatalytic activity. Further, no reaction between the layer and the membrane surface is observed. 


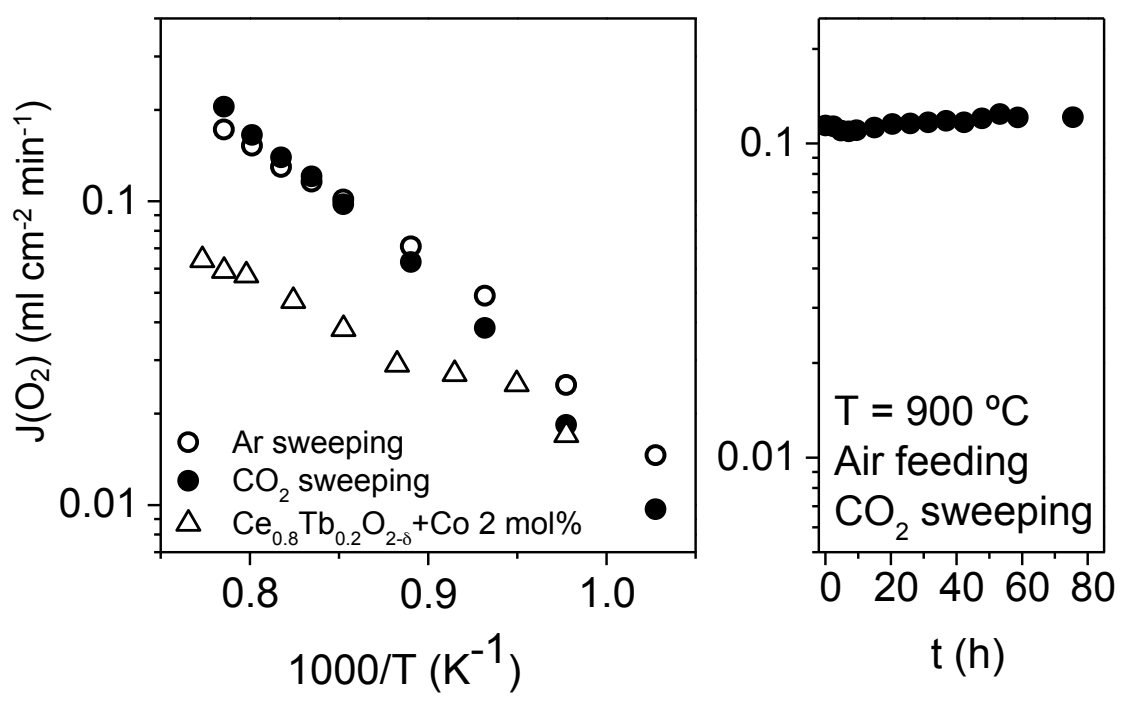

Figure 8.9. a) Oxygen permeation flux as a function of temperature and sweep gas ( $\mathrm{Ar}$ or $\mathrm{CO}_{2}$ ) for FNO-CTO membrane and in Ar for single phase CTOCo; and b) flux stability with time on stream for FNO-CTO membrane in $\mathrm{CO}_{2}$ sweeping at $900{ }^{\circ} \mathrm{C}$.

Figure $8.9 \mathrm{a}$ shows the oxygen flux as a function of temperature when two different sweep gases are used, i.e., argon and pure $\mathrm{CO}_{2}$. Using a $0.68 \mathrm{~mm}$ thick membrane, permeation fluxes of $0.17 \mathrm{~mL} \cdot \mathrm{min}^{-1} \cdot \mathrm{cm}^{-2}$ and $0.20 \mathrm{~mL} \cdot \mathrm{min}^{-1} \cdot \mathrm{cm}^{-2}$ for $\mathrm{Ar}$ and $\mathrm{CO}_{2}$ sweeping are obtained at $1000{ }^{\circ} \mathrm{C}$, respectively. These permeation values are twice the permeation values achieved in Chapter 5 using a single phase Co-containing $\mathrm{Ce}_{0.8} \mathrm{~Tb}_{0.2} \mathrm{O}_{2-\delta}$ (CTOCo) membrane. This result stresses the importance of the electronic contribution provided by the spinel percolating network.

Oxygen permeation observed in Figure 8.9 represents a two-fold Arrhenius behavior with a change in the activation energy at $800{ }^{\circ} \mathrm{C}$. This indicates that the nature of the processes limiting the oxygen permeation rate changes with the temperature. In the high temperature range $\left(1000-800^{\circ} \mathrm{C}\right)$, the 
bulk diffusion through the dense membrane controls the permeation. In the low temperature range, the limiting step is the surface exchange reaction.

By using $\mathrm{CO}_{2}$ as a sweep gas, the oxygen permeation is maintained and even improved at high temperatures with respect to Ar sweeping. As observed for broadly applied perovskite materials as BSCF or LSCF, the oxygen permeation could be affected by either carbonate formation or $\mathrm{O}_{2}$ competitive adsorption on the membrane surface. [24-26] These two phenomena would lead to a reduction in oxygen permeation rates, the first being the most dramatic. Since terbium doped ceria is not prone to interact with $\mathrm{CO}_{2}$ to form carbonates, oxygen permeation diminution observed in Figure 8.9a at temperatures below $900{ }^{\circ} \mathrm{C}$ would be related with the process involving $\mathrm{O}_{2}-\mathrm{CO}_{2}$ competitive adsorption on reaction sites. This process is more favorable at low temperatures, as previously observed on several oxygen-ion conducting surfaces. [2, 27] Figure 8.9a also indicates that above $900{ }^{\circ} \mathrm{C}, \mathrm{CO}_{2}$ utilization as sweep gas improves the oxygen permeation. This could be related with the better sweeping properties of $\mathrm{CO}_{2}$ with respect to argon at these high temperatures and the smaller $\mathrm{CO}_{2}$ adsorption constant as temperature is increased, thus neglecting the effect of competitive adsorption.

Figure $8.9 \mathrm{~b}$ represents the evolution of the oxygen permeation as a function of time in $\mathrm{CO}_{2}$ sweeping conditions, at $900{ }^{\circ} \mathrm{C}$. The flux kept stable during $76 \mathrm{~h}$ and slightly increased with time on stream. The origin of this intriguing activation with time remains unclear and may be related with surface and/or grain boundary processes. 


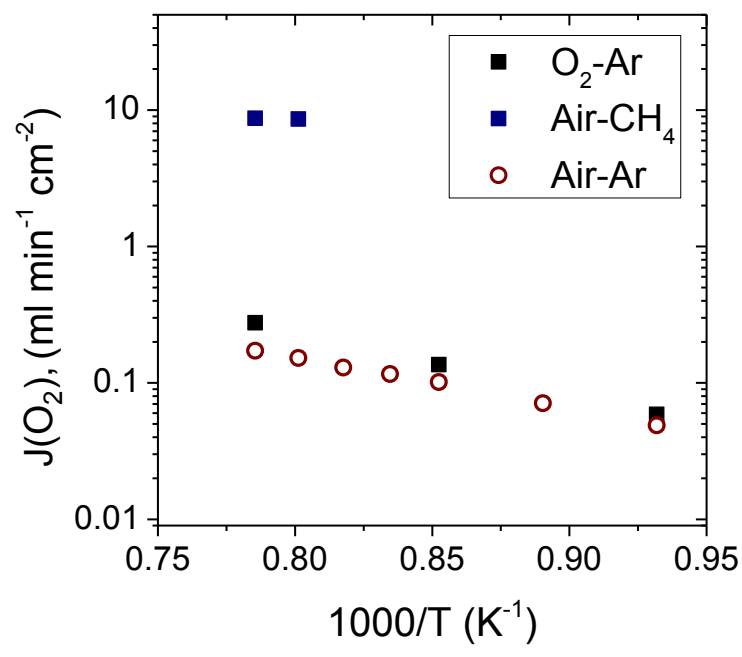

Figure 8.10. Oxygen flux as a function of reciprocal temperature for different $p \mathrm{O}_{2}$ gradients across the membrane, i.e., air- $\mathrm{Ar}, \mathrm{O}_{2}-\mathrm{Ar}$ and air- $10 \% \mathrm{CH}_{4} / \mathrm{Ar}$, on a $0.68 \mathrm{~mm}$ thick FNO-CTO membrane sintered at $1400^{\circ} \mathrm{C}$.

By increasing the separation driving force, i.e., the chemical potential across the membrane, the oxygen permeation can be enlarged depending on the $\mathrm{pO}_{2}$ gradients. Figure 8.10 presents the permeation results when pure $\mathrm{O}_{2}$ was fed instead of air and provides evidence about the important increase in the $J\left(\mathrm{O}_{2}\right)$, specifically flux values up to $0.28 \mathrm{~mL} \cdot \mathrm{min}^{-1} \cdot \mathrm{cm}^{-2}$ were achieved at $1000{ }^{\circ} \mathrm{C}$. This improvement in the flux at high temperature may be related to bulk diffusion limited oxygen permeation, whereas other processes such as surface exchange reactions are more limiting below $800{ }^{\circ} \mathrm{C}$. Alternatively, when methane is employed as sweep gas instead of Ar (and air feeding), $J\left(O_{2}\right)$ is strongly boosted, reaching flux values up to $8.71 \mathrm{~mL} \cdot \mathrm{min}^{-1} \cdot \mathrm{cm}^{-2}$ at $1000{ }^{\circ} \mathrm{C}$. This last increase in flux is not only related to the large increase in the transport driving force but also to the substantial rise in $n$-type conductivity achieved thanks to the higher reducing conditions in the permeate side. Such reduction 
induces the lattice expansion and the generation of new strains, leading to the formation of cracks through the membrane inside the quartz setup. However, after measuring the cracked membrane, XRD pattern does not reveal the presence of reacted phases or impurities (Figure 8.11). Therefore, the membrane failure is attributed only to the mechanical stresses associated with the chemical expansion.

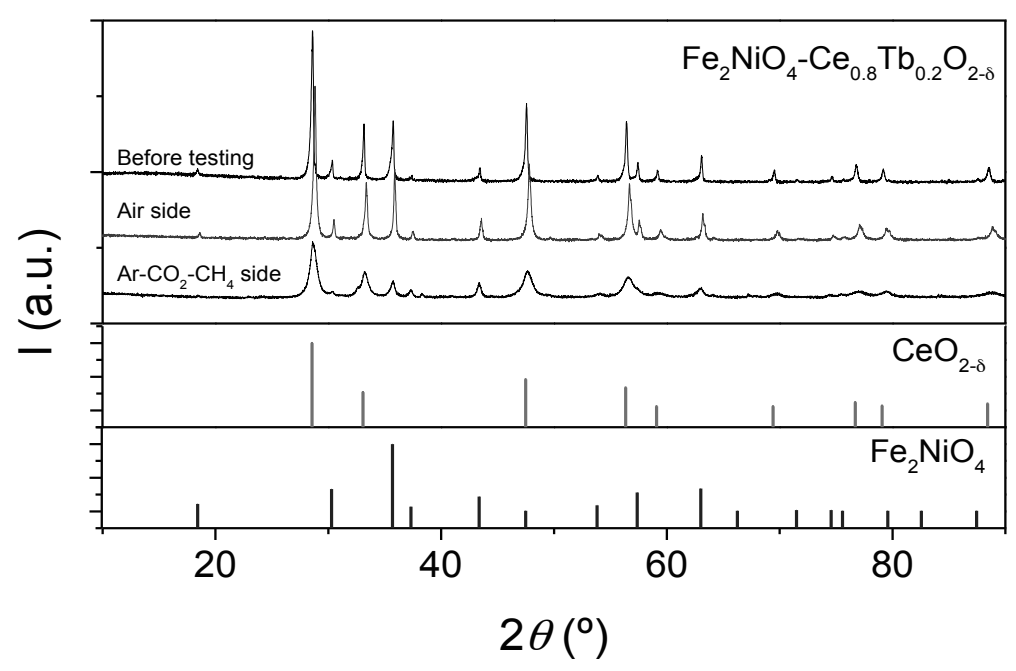

Figure 8.11. XRD pattern of both sides of the FNO-CTO membranes after 7 days of permeation experiments in $\mathrm{Ar}, \mathrm{CO}_{2}, \mathrm{CH}_{4}$ sweeping.

Finally, the membrane is compared with a reference composite made of FNO-CGO as this membrane is reported to be $\mathrm{CO}_{2}$ stable. [9] Figure 8.12 shows the permeation flux of both membranes. FNO-CTO composite membrane exhibits higher oxygen permeation and this difference becomes more important with decreasing temperatures, despite the lower ionic conductivity of CTO. The better behavior of the FNO-CTO membrane may originate from the higher p-type electronic conductivity of $\mathrm{CTO}$, which increases the overall mixed 
ionic-electronic conductivity of the composite and makes possible to enlarge the surface reaction area from single TPB points, where the two solid phases and the gas phase converge, to the overall CTO grain surface. This is in contrast with the FNO-CGO composite since CGO behaves as a purely ionic conductor in the applied $\mathrm{pO}_{2}$ environments and oxygen exchange is limited to single TPB points.

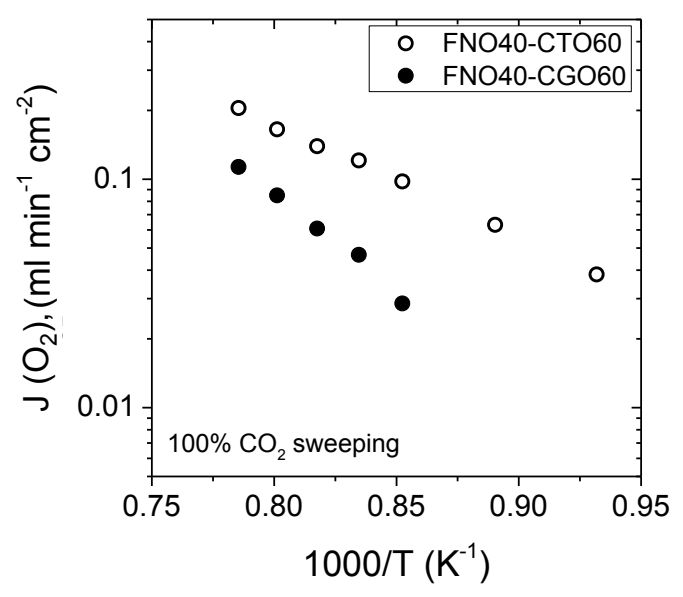

Figure 8.12. Temperature dependency of oxygen permeation of FNO-CTO and FNOCGO composites. The membranes were fed by air and the sweep gas was $100 \% \mathrm{CO}_{2}$.

In addition to the permeation in $\mathrm{CO}_{2}$, the stability of FNO-CTO composite has been studied by treating the membrane during 7 days at $800{ }^{\circ} \mathrm{C}$ under continuous flow of $\mathrm{CO}_{2}$ with $5 \% \mathrm{O}_{2}, 1 \%$ of $\mathrm{SO}_{2}$ and $2.5 \%$ of $\mathrm{H}_{2} \mathrm{O}$. The membrane surface directly exposed to this gas environment was analyzed by XRD. The pattern plotted in Figure 8.13 reveals that only spinel and fluorite phases appear and neither new oxide phases nor minor impurities are detected up to the limits of the diffractometer. On the contrary, the membrane reference material $\mathrm{La}_{0.8} \mathrm{Sr}_{0.2} \mathrm{Co}_{0.5} \mathrm{Fe}_{0.5} \mathrm{O}_{3-\delta}$ (LSCF) is completely destroyed when exposed 
at the same treatment (Figure 8.14). This is a very remarkable result since these harsh environmental conditions are likely encountered in the targeted applications in (i) oxyfuel processes using recirculated flue gas as sweep and (ii) high-temperature catalytic membrane reactors for hydrocarbon conversion.

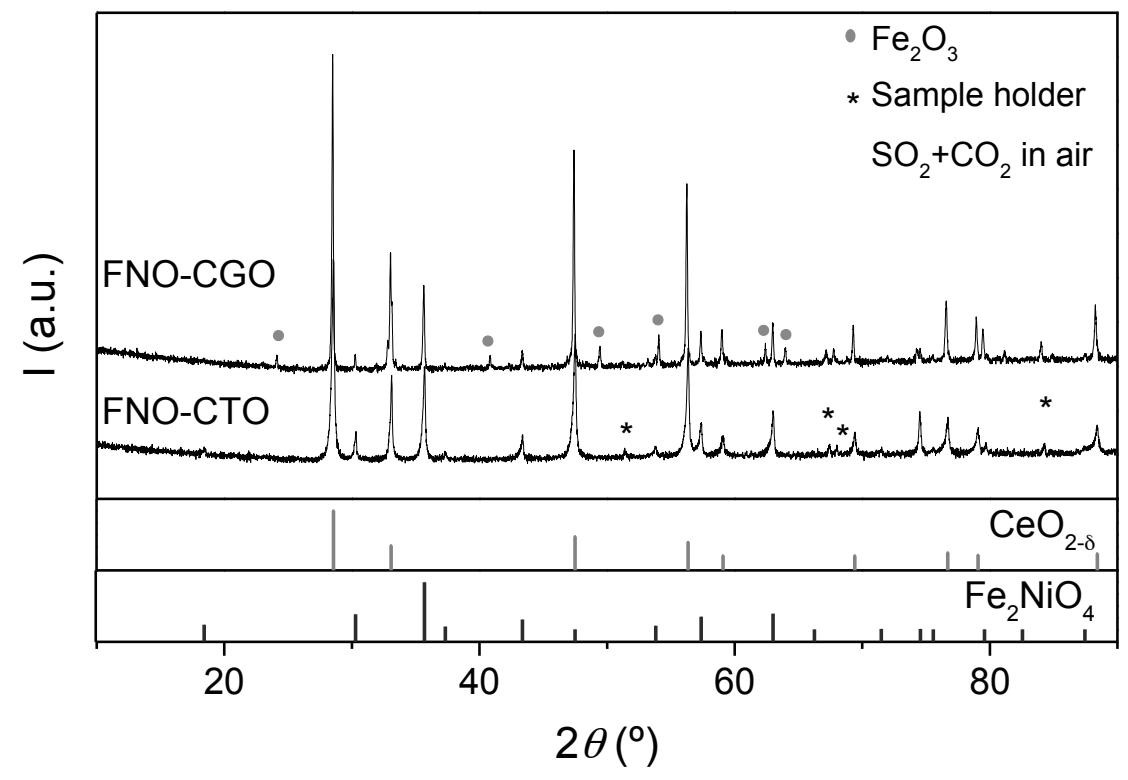

Figure 8.13. XRD pattern of FNO-CTO and FNO-CGO composites after 7 days of treatment under a continuous flux consisted of $1 \% \mathrm{SO}_{2}-5 \% \mathrm{O}_{2}$ in $\mathrm{CO}_{2}$, at $800{ }^{\circ} \mathrm{C}$. 


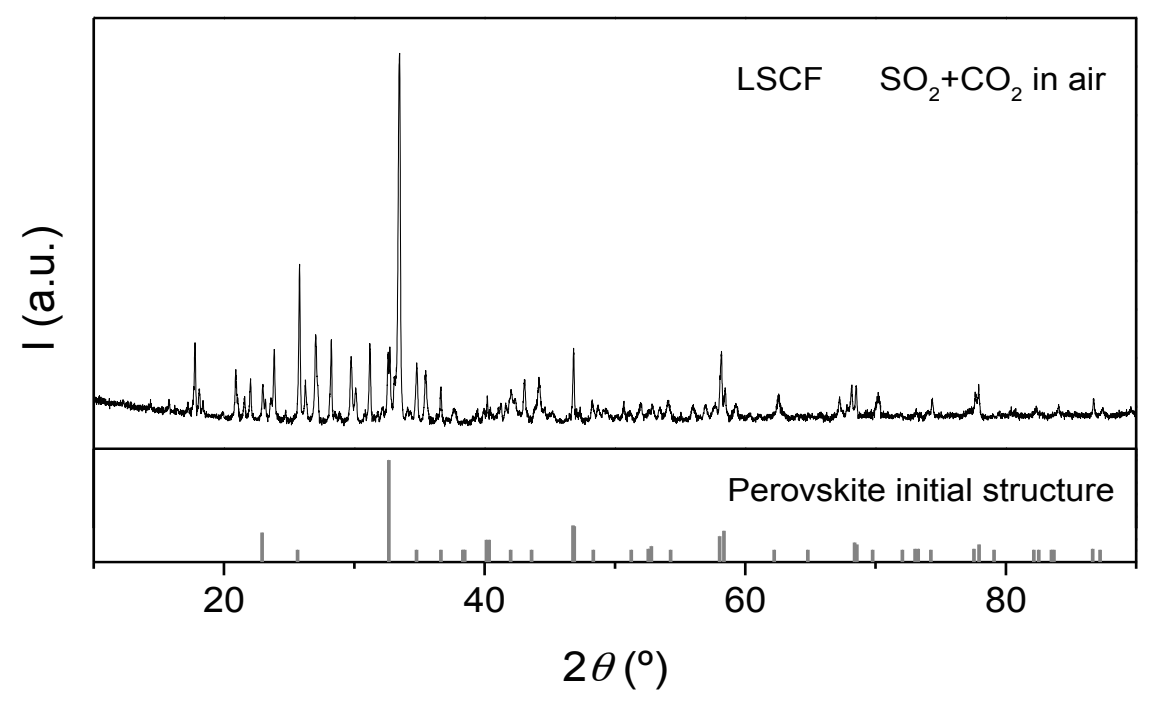

Figure 8.14. XRD pattern of LSCF after 7 days of treatment under a continuous flux consisted of $1 \% \mathrm{SO}_{2}-5 \% \mathrm{O}_{2}$ in $\mathrm{CO}_{2}$, at $800{ }^{\circ} \mathrm{C}$, and comparison with pure perovskite phase.

Summarizing, FNO-CTO composites seem appealing materials for oxygen separation devices for integration in oxyfuel processes using recirculated flue gas as sweep due to the particular combination of reasonable oxygen fluxes and unrecorded chemical stability at high temperatures. However, the oxygen fluxes are still orders of magnitude lower than the state of the art BSCF perovskites, which in addition has more economic components than the cercer composite. These issues should be taken into account. A step forward in the optimization of the membrane would imply the use of the cercer as a dense thin film, which is expected to increase the oxygen flux rate and decrease the material costs as the necessary amount of cercer would be substantially reduced. 


\subsection{Conclusions}

One-pot fabrication of $60 \% \mathrm{Fe}_{2} \mathrm{NiO}_{4}-40 \% \mathrm{Ce}_{0.8} \mathrm{Ln}_{0.2} \mathrm{O}_{2-\delta}$ (FNO-CLnO) composite has been proved to be the most suitable method to obtain both spinel and fluorite pure phase materials with a narrow grain size distribution and homogeneous distribution of grains, while the $60 / 40$ phase ratio permits the existence of percolative paths from side to side of the dual-phase membranes. From DC-conductivity screening FNO-CTO composite has been selected having a comparable conductivity with FNO-CGO composite. An exhaustive study of this material has been carried out to assess the suitability as oxygen transport membrane.

FNO-CTO composite does not show evidence of impurities or reaction between phases up to the limits of the XRD. Additionally, SEM analysis reveals a narrow grain size distribution and homogeneous allocation of grains. Furthermore, the existence of percolative paths from side to side of the dual-phase membranes necessary for the oxygen permeation can be ascertained whilst the packing and bounding among the different grains in the membrane is appropriate.

The conductivity of both FNO-CTO phases is investigated as a function of temperature and $\mathrm{pO}_{2}$. The ambipolar transport involves the concomitant (i) hopping of charge carriers, i.e., hopping of localized electrons between multivalent cations located in the octahedral $\mathrm{Fe}$ sublattice and (ii) the ionic transport in though the doped ceria lattice. Additionally, CTO presents significant $p$-type electronic conductivity although the ionic conductivity prevails. 
Oxygen permeation flux values of $0.17 \mathrm{~mL} \cdot \mathrm{min}^{-1} \cdot \mathrm{cm}^{-2}$ and $0.20 \mathrm{~mL} \cdot \mathrm{min}^{-1} \cdot \mathrm{cm}^{-2}$ when argon and pure $\mathrm{CO}_{2}$ are used as sweep gas, respectively, are measured through a $0.68 \mathrm{~mm}$-thick membrane at $1000^{\circ} \mathrm{C}$. These values are higher than those achieved using the reference composite FNO-CGO. Imposing higher $\mathrm{pO}_{2}$ gradients across the membrane leads to a substantial increase in the oxygen flux. Long term experiments at $900{ }^{\circ} \mathrm{C}$ showed that the material is $\mathrm{CO}_{2}$ stable and that oxygen permeation is even improved after $76 \mathrm{~h}$ on stream. Moreover, the composite was stable against $\mathrm{SO}_{2}$ and $\mathrm{CO}_{2}$ gas environments, as can be inferred from the stability test at $800{ }^{\circ} \mathrm{C}$. However, permeation tests on $\mathrm{SO}_{2}-\mathrm{CO}_{2}$ containing sweep gases would be necessary.

Conclusively, FNO-CTO composites seem appealing materials for oxygen separation devices for integration in oxyfuel processes since they combine reasonable oxygen fluxes and unrecorded chemical stability at high temperatures. A step forward in the optimization of the membrane would imply the use of the cercer as dense thin films.

\subsection{References}

[1] M.P. Lobera, J.M. Serra, S.P. Foghmoes, M. Sogaard, A. Kaiser, On the use of supported ceria membranes for oxyfuel process/syngas production, Journal of Membrane Science, 385 (2011) 154-161.

[2] I. Garcia-Torregrosa, M.P. Lobera, C. Solis, P. Atienzar, J.M. Serra, Development of $\mathrm{CO} 2$ Protective Layers by Spray Pyrolysis for Ceramic Oxygen Transport Membranes, Advanced Energy Materials, 1 (2011) 618-625. 
[3] H. Stadler, F. Beggel, M. Habermehl, B. Persigehl, R. Kneer, M. Modigell, P. Jeschke, Oxyfuel coal combustion by efficient integration of oxygen transport membranes, International Journal of Greenhouse Gas Control, 5 (2011) 7-15.

[4] T.J. Mazanec, T.L. Cable, J.G. Frye Jr, Electrocatalytic cells for chemical reaction, Solid State Ionics, 53-56, Part 1 (1992) 111-118.

[5] C.S. Chen, H. Kruidhof, H.J.W. Bouwmeester, H. Verweij, A.J. Burggraaf, Oxygen permeation through oxygen ion oxide-noble metal dual phase composites, Solid State Ionics, 86-88 (1996) 569-572.

[6] C.S. Chen, A.J. Burggraaf, Stabilized bismuth oxide-noble metal mixed conducting composites as high temperature oxygen separation membranes, Journal of Applied Electrochemistry, 29 (1999) 355-360.

[7] U. Nigge, H.D. Wiemhöfer, E.W.J. Römer, H.J.M. Bouwmeester, T.R. Schulte, Composites of $\mathrm{Ce}_{0.8} \mathrm{Gd}_{0.2} \mathrm{O}_{1.9}$ and $\mathrm{Gd}_{0.7} \mathrm{Ca}_{0.3} \mathrm{CoO}_{3-\delta}$ as oxygen permeable membranes for exhaust gas sensors, Solid State Ionics, 146 (2002) 163-174.

[8] A.L. Shaula, V.V. Kharton, F.M.B. Marques, A.V. Kovalevsky, A.P. Viskup, E.N. Naumovich, Oxygen permeability of mixed-conducting composite membranes: effects of phase interaction, J. Solid State Electrochem., 10 (2006) 28-40.

[9] H. Luo, K. Efimov, H. Jiang, A. Feldhoff, H. Wang, J. Caro, $\mathrm{CO}_{2}$-Stable and CobaltFree Dual-Phase Membrane for Oxygen Separation, Angewandte Chemie International Edition, 50 (2011) 759-763.

[10] J. Yi, Y. Zuo, W. Liu, L. Winnubst, C. Chen, Oxygen permeation through a $\mathrm{Ce}_{0.8} \mathrm{Sm}_{0.2} \mathrm{O}_{2-\delta}-\mathrm{La}_{0.8} \mathrm{Sr}_{0.2} \mathrm{CrO}_{3-\delta}$ dual-phase composite membrane, J. Membr. Sci., 280 (2006) 849-855.

[11] H. Takamura, K. Okumura, Y. Koshino, A. Kamegawa, M. Okada, Oxygen permeation properties of ceria-ferrite-based composites, J. Electroceram., 13 (2004) 613-618. 
[12] H. Takamura, T. Kobayashi, T. Kasahara, A. Kamegawa, M. Okada, Oxygen permeation and methane reforming properties of ceria-based composite membranes, J. Alloys Compd., 408-412 (2006) 1084-1089.

[13] H. Luo, H. Jiang, K. Efimov, J. Caro, H. Wang, Influence of the preparation methods on the microstructure and oxygen permeability of a $\mathrm{CO}_{2}$-stable dual phase membrane, AIChE J., 57 (2011) 2738-2745.

[14] T. Stefanik, H. Tuller, Nonstoichiometry and Defect Chemistry in PraseodymiumCerium Oxide, J Electroceram, 13 (2004) 799-803.

[15] D.P. Fagg, V.V. Kharton, A. Shaula, I.P. Marozau, J.R. Frade, Mixed conductivity, thermal expansion, and oxygen permeability of $\mathrm{Ce}(\mathrm{Pr}, \mathrm{Zr}) \mathrm{O}_{2-\delta}$, Solid State Ionics, 176 (2005) 1723-1730.

[16] H. Takamura, H. Sugai, M. Watanabe, T. Kasahara, A. Kamegawa, M. Okada, Oxygen permeation properties and surface modification of acceptor-doped $\mathrm{CeO}_{2} / \mathrm{MnFe}_{2} \mathrm{O}_{4}$ composites, J. Electroceram., 17 (2006) 741-748.

[17] D.P. Fagg, A.L. Shaula, V.V. Kharton, J.R. Frade, High oxygen permeability in fluorite-type $\mathrm{Ce}_{0.8} \mathrm{Pr}_{0.2} \mathrm{O}_{2-\delta}$ via the use of sintering aids, Journal of Membrane Science, 299 (2007) 1-7.

[18] J.M. Serra, V.B. Vert, M. Betz, V.A.C. Haanappel, W.A. Meulenberg, F. Tietz, Screening of A-substitution in the system $\mathrm{A}_{(0.68)} \mathrm{Sr}_{(0.3)} \mathrm{Fe}_{(0.8)} \mathrm{Co}_{(0.2)} \mathrm{O}_{(3-\delta)}$ for $\mathrm{SOFC}$ cathodes, Journal of the Electrochemical Society, 155 (2008) B207-B214.

[19] R. Shannon, Revised effective ionic radii and systematic studies of interatomic distances in halides and chalcogenides, Acta Crystallographica Section A, 32 (1976) 751-767. 
[20] B. Liu, K. Zhou, Z. Li, D. Zhang, L. Zhang, Microstructure and DC electrical conductivity of spinel nickel ferrite sintered in air and nitrogen atmospheres, Materials Research Bulletin, 45 (2010) 1668-1671.

[21] N. Ponpandian, P. Balaya, A. Narayanasamy, Electrical conductivity and dielectric behaviour of nanocrystalline $\mathrm{NiFe}_{2} \mathrm{O}_{4}$ spinel, Journal of Physics: Condensed Matter, 14 (2002) 3221.

[22] A.L. Shluger, A.M. Stoneham, Small polarons in real crystals: concepts and problems, Journal of Physics: Condensed Matter, 5 (1993) 3049.

[23] R. Parker, B.A. Griffiths, D. Elwell, The effect of cobalt substitution on electrical conduction in nickel ferrite, British Journal of Applied Physics, 17 (1966) 1269.

[24] S.M. Fang, C.Y. Yoo, H.J.M. Bouwmeester, Performance and stability of niobiumsubstituted $\mathrm{Ba}_{0.5} \mathrm{Sr}_{0.5} \mathrm{Co}_{0.8} \mathrm{Fe}_{0.2} \mathrm{O}_{3-\delta}$ membranes, Solid State Ionics, 195 (2011) 1-6.

[25] M. Schulz, R. Kriegel, A. Kaempfer, Assessment of $\mathrm{CO}_{2}$ stability and oxygen flux of oxygen permeable membranes, Journal of Membrane Science, 378 (2011) 10-17.

[26] S.J. Benson, D. Waller, J.A. Kilner, Degradation of $\mathrm{La}_{0.6} \mathrm{Sr}_{0.4} \mathrm{Fe}_{0.8} \mathrm{Co}_{0.2} \mathrm{O}_{3-\delta}$ in carbon dioxide and water atmospheres, Journal of the Electrochemical Society, 146 (1999) 1305-1309.

[27] V.B. Vert, J.M. Serra, Study of $\mathrm{CO}_{2}$ stability and electrochemical oxygen activation of mixed conductors with low thermal expansion coefficient based on the $\mathrm{TbBaCo}_{3} \mathrm{ZnO}_{7+\delta}$ system, Journal of Power Sources, 196 (2011) 4270-4276. 


\title{
Chapter 9. Mixed protonic-electronic conductors based on lanthanum chromites
}

\begin{abstract}
A second block of materials on the research of mixed protonic-electronic conductors is presented. Perovskite-structured $\mathrm{LaCrO}_{3}$ has been doped with the aim of modifying the electronic and catalytic properties in hydrogen-containing atmospheres, where these materials turn out to be proton conductors.
\end{abstract}

The results have addressed to a new generation of anodes for PC-SOFCs based on catalytically-promoted $\mathrm{La}_{0.87} \mathrm{Sr}_{0.13} \mathrm{CrO}_{3} \quad$ (LSC). $\quad \mathrm{La}_{0.85} \mathrm{Sr}_{0.15} \mathrm{Cr}_{0.9} \mathrm{Ni}_{0.1} \mathrm{O}_{3-\delta}$ (LSCN10), $\mathrm{La}_{0.85} \mathrm{Sr}_{0.15} \mathrm{Cr}_{0.8} \mathrm{Ni}_{0.2} \mathrm{O}_{3-\delta}$ (LSCN) and $\mathrm{La}_{0.75} \mathrm{Ce}_{0.1} \mathrm{Sr}_{0.15} \mathrm{CrO}_{3-\delta}$ (LSCCe) chromite based materials are a good alternative to the widely used $\mathrm{NiO}$, as they show good compatibility with LWO electrolyte in contrast to the highly reactive NiO. LSCCe is selected as electrode backbone structure, due to its superior total conductivity with respect to LSC, while LSCN10 and LSCN are selected to evaluate the role of the $\mathrm{Ni}$ in the structure. The infiltration of nickel nanoparticles in the sintered LSCCe and LSCN electrodes boosted the surface limiting reactions while preserving the compatibility. Metallic Ni nanoparticles become catalytically active centres for the $\mathrm{H}_{2}$ oxidation reaction improving the anode performance regarding to pure LSC, previously limited by low frequency surface related processes. 



\subsection{Introduction}

$\mathrm{LaCrO}_{3}$ is a stable perovskite in both reducing and oxidizing atmospheres at high temperatures. Substituting alkali earth and transition metal elements on the A and B sites may modify the electronic and catalytic properties. Typically, $\mathrm{Sr}$ is introduced in A site, while other transition elements into the B-site of $\mathrm{La}_{1-\mathrm{x}} \mathrm{Sr}_{\mathrm{x}} \mathrm{Cr}_{1-\mathrm{y}} \mathrm{M}_{\mathrm{y}} \mathrm{O}_{3}(\mathrm{M}=\mathrm{Mg}, \mathrm{Mn}, \mathrm{Fe}, \mathrm{Co}, \mathrm{Ni})$ may improve the catalytic properties for, as example, methane fed SOFC. [1-3] These materials are traditionally known for their oxide ion conductivity, however, the conductivity experiments systematically conducted in different atmospheres evidence that these materials might turn out to be proton conductors in hydrogen-containing atmospheres.

Therefore, this kind of compounds is proposed to their potential application as hydrogen-separation membranes and catalytic membrane reactions. Hydrogen separation at high temperatures participates in the integrated gasification combined cycle (IGCC) of power plants. Thus, these membranes are the step to separate the $\mathrm{H}_{2}$ and the $\mathrm{CO}_{2}$ after fuel gasification, providing a moisturized $\mathrm{CO}_{2}$ exhaust, ready to store.

On the other hand, recent interest in solid oxide fuel cells (SOFC) is focused on research and development of proton conducting SOFCs (PC-SOFC) that allow lowering the operation temperature and achieving very high fuel utilization, since it is not diluted by water that is formed in the air electrode. In this way, it emerges the possibility of simpler and cheaper cell designs. [4, 5] However, there still exists the need of developing novel protonic electrolytes 
and electrode materials, which combine excellent proton transport properties, compatibility with other cell components and thermochemical stability.

$\mathrm{La}_{6-\mathrm{x}} \mathrm{WO}_{\mathrm{y}}(\mathrm{LWO}, 0.4<\mathrm{x}<0.7)$ [6] is a promising proton conductor, with high and predominant proton conductivity, and stable in wet $\mathrm{CO}_{2}$ under typical fuel cell operating conditions. [6-10] Therefore, compatible electrodes with the LWO electrolyte are currently being investigated. [11, 12] Recently, the development of specific LSM-LWO composite cathodes for LWO-based cells has been addressed. [11] Concerning the anodes, strong requirements entail high electrical conductivity, sufficient protonic conductivity, fast gas transport and exchange through a porous scaffold and fine-grained homogeneous microstructure, besides of high catalytic activity concerning hydrogen oxidation. Several nickel-based cermet combinations (Ni/YSZ, Ni/CGO, $\left.\mathrm{Ni} / \mathrm{Ba}\left(\mathrm{Ce}_{0.8-\mathrm{x}} \mathrm{Zr}_{\mathrm{x}}\right) \mathrm{Y}_{0.1} \mathrm{O}_{3-\delta}\right)$ and mixed conductor $\mathrm{Sr}_{0.94} \mathrm{Ti}_{0.9} \mathrm{Nb}_{0.1} \mathrm{O}_{3}$ (STN) have been proposed and investigated as SOFC anode materials. [13-16] However, these systems react with the LWO electrolyte, the reaction between LWO and $\mathrm{NiO}$ typical anode being especially dramatic. [17]

The present chapter focuses on the use of chromite based perovskites as PC-SOFC anode materials. Indeed, doped $\mathrm{La}_{0.85} \mathrm{Sr}_{0.15} \mathrm{CrO}_{3}$ has already been tested as anode for conventional SOFCs, due to its particular combination of redox stability and sufficient $p$-type electronic and oxygen-ion conductivity under reducing conditions, while its electrochemical activity can be fine-tuned by incorporating catalytic doping elements, such as nickel and ruthenium. [1822] Furthermore, doped $\mathrm{LaCrO}_{3}$ compounds have been claimed as hydrogen permeation membrane materials and this particular function suggests the presence of mixed protonic-electronic conductivity in this material. [23] 
Here, the initial LSC electrode has been improved stepwise by tackling the two major rate-limiting steps, i.e., electrode total conductivity and surface processes. Firstly, the enhancement of the transport properties of the chromite has been tried by doping on $\mathrm{A}$ and $\mathrm{B}$-perovskite positions $(\mathrm{A}=\mathrm{Sm}, \mathrm{Ce}$, and $\mathrm{Nd}$ and $\mathrm{B}=\mathrm{Ni}, \mathrm{Y}, \mathrm{Al}, \mathrm{Fe}, \mathrm{Ti}, \mathrm{Ga}, \mathrm{Mn}, \mathrm{Zn}$ ) and $\mathrm{Ce}$ and Ni-doped LSC have been selected for further investigation. Secondly, nickel infiltration into the sintered $\mathrm{Ce}$ and Ni-doped electrodes has been carried out in order to promote the surface reactions, since $\mathrm{Ni}$ is a well-known, highly active $\mathrm{H}_{2}$ bond breaking catalyst. [20-22]

\footnotetext{
Symmetrical cells made by $\mathrm{La}_{0.87} \mathrm{Sr}_{0.13} \mathrm{CrO}_{3}$ (LSC), $\mathrm{La}_{0.85} \mathrm{Sr}_{0.15} \mathrm{Cr}_{0.9} \mathrm{Ni}_{0.1} \mathrm{O}_{3} \quad$ (LSCN10), $\quad \mathrm{La}_{0.85} \mathrm{Sr}_{0.15} \mathrm{Cr}_{0.8} \mathrm{Ni}_{0.2} \mathrm{O}_{3} \quad$ (LSCN) and $\mathrm{La}_{0.75} \mathrm{Sr}_{0.15} \mathrm{Ce}_{0.1} \mathrm{CrO}_{3}$ (LSCCe), both bare and infiltrated with Ni nanoparticles, are tested as anodes for LWO based PC-SOFCs aiming (1) to compare results of the new Ni and Ce doped LSC material with previously studied LSC based anodes and (2) to analyse the effect of the surface catalytic promotion due to the $\mathrm{Ni}$ infiltration.
}

\subsection{Results and discussion}

\section{Screening of doped LSC}

LSC and doped-LSC compounds have been prepared by the Pechini method explained in section 2.1. Powders of nanometric size are obtained after calcination of the dry precursors at $600{ }^{\circ} \mathrm{C}$. Table 9.1 summarizes the nominal stoichiometry and short nomenclature of the A and B-site doped perovskite 
compositions ( $\mathrm{A}=\mathrm{Sm}, \mathrm{Ce}$, and $\mathrm{Nd}$ and $\mathrm{B}=\mathrm{Ni}, \mathrm{Y}, \mathrm{Al}, \mathrm{Fe}, \mathrm{Ti})$. Initial $\mathrm{XRD}$ measurements evaluate whether the doping succeeded to form pure perovskite phase. As example, the patterns of LSC calcined at $900{ }^{\circ} \mathrm{C}, \mathrm{LSCN} 10$ and LSCN20 at $1050{ }^{\circ} \mathrm{C}$ and $\mathrm{LSCrF}$ at $1000^{\circ} \mathrm{C}$ are represented in Figure 9.1.

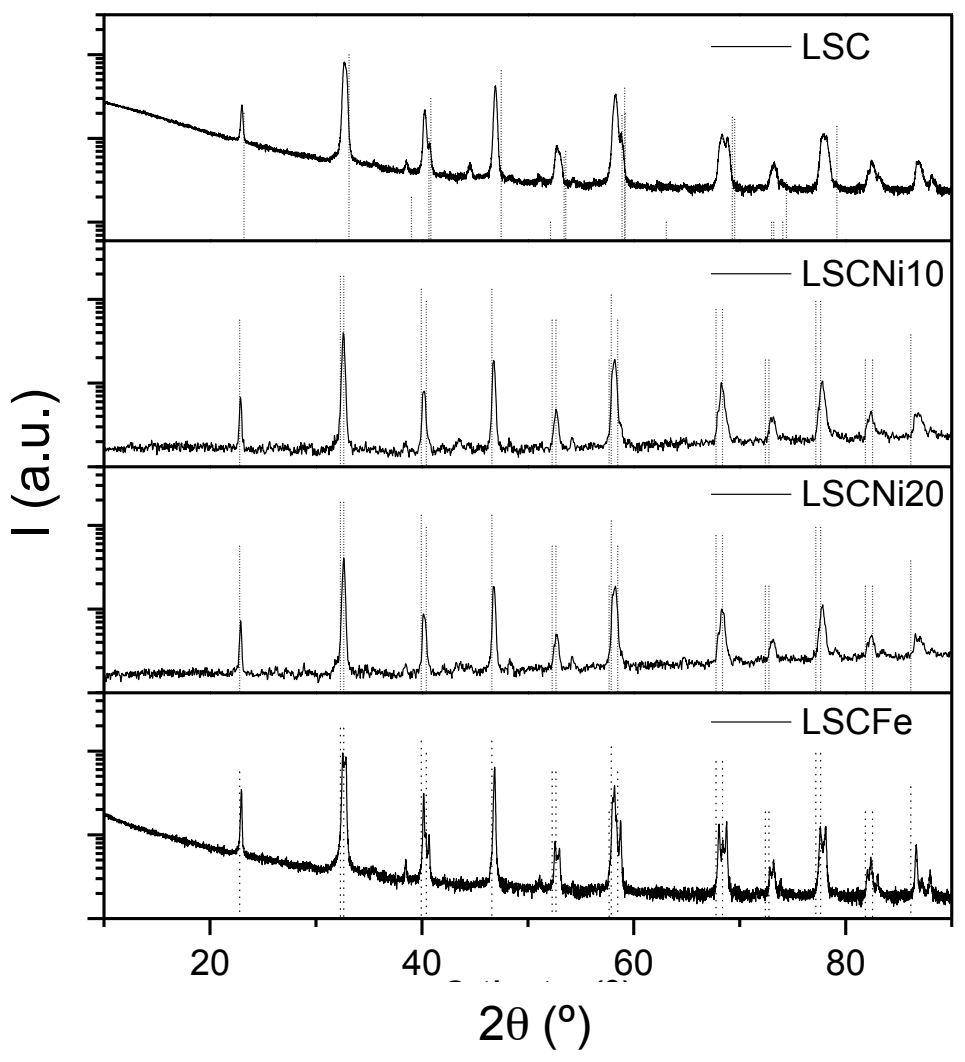

Figure 9.1. XRD patterns of LSC calcined at $900{ }^{\circ} \mathrm{C}, \mathrm{LSCN} 10$ and $\mathrm{LSCN}$ at $1050{ }^{\circ} \mathrm{C}$ and $\mathrm{LSCrF}$ at $1000^{\circ} \mathrm{C}$. The dotted lines represent the LSC reference pattern (ICSD 00032-1240).

However, the chromites need very high temperatures to obtain the dense specimens required by the transport measurements, so that the structure was checked after pressing and firing the samples at $1600{ }^{\circ} \mathrm{C}$. 
Figure 9.2 shows the XRD patterns of A-site doped LSC beside their corresponding SEM microstructure images of SmSC, NSC and LSCCe. Only SmSC and LSCCe are pure phase, while NSC presents $\mathrm{Nd}_{2} \mathrm{O}_{3}$ and $\mathrm{CrO}_{2}$ impurities. Regarding to the microstructure, the LSCCe shows the most homogeneous grain size distribution.

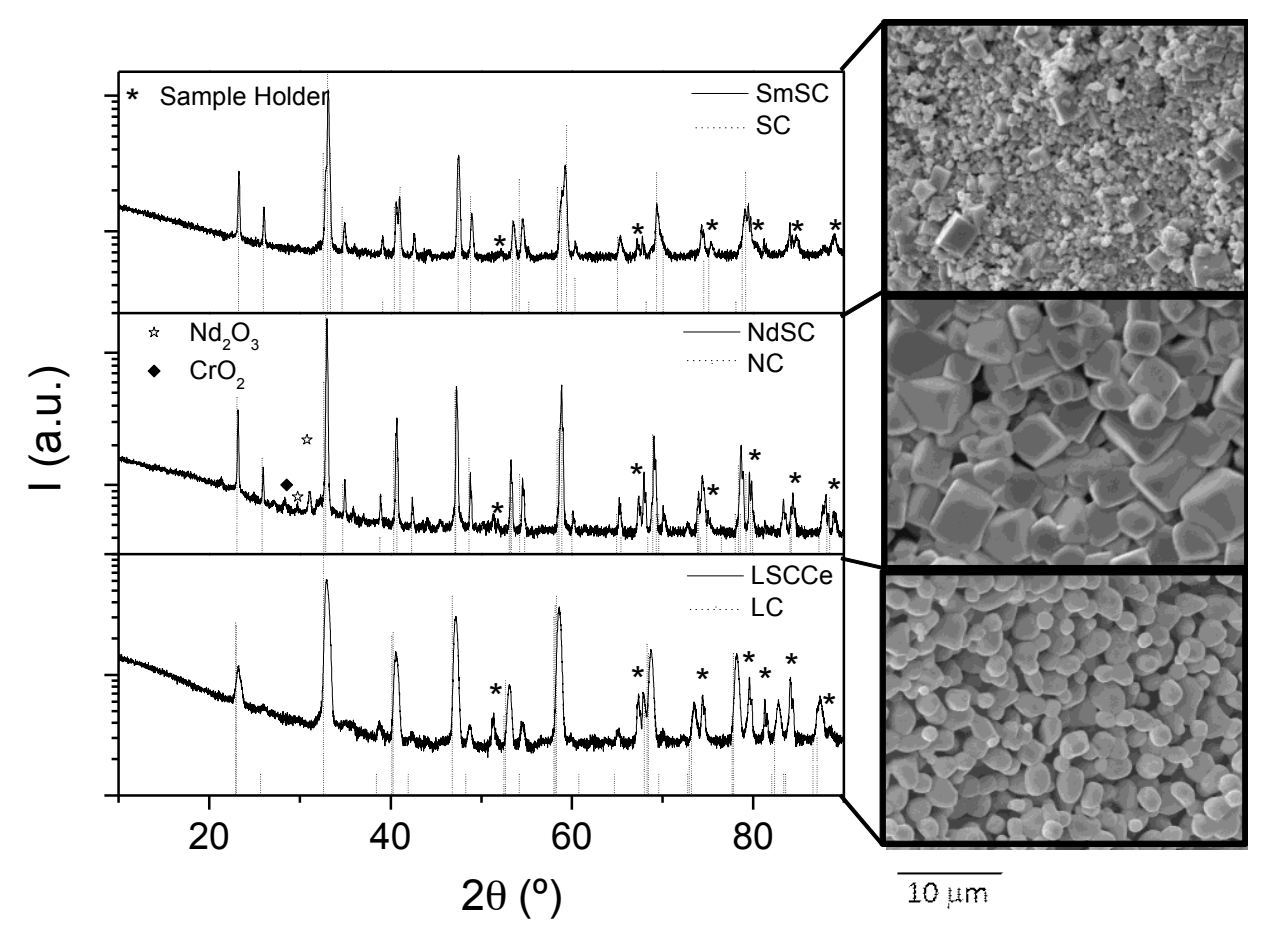

Figure 9.2. XRD patterns of A-site doped LSC calcined at $1600{ }^{\circ} \mathrm{C}$ and corresponding SEM microstructure images of (top to bottom) SmSC, NSC and LSCCe.

On the other hand, B-site doped LSC does not deliver single perovskites but a combination of hexagonal (LSC) and orthorhombic perovskite (LC) when they are submitted to $1600{ }^{\circ} \mathrm{C}$. However, dense specimens are obtained, with 
only closed porosity. In particular, LSCrF is very well packed, which is expected to be beneficial on the transport properties.

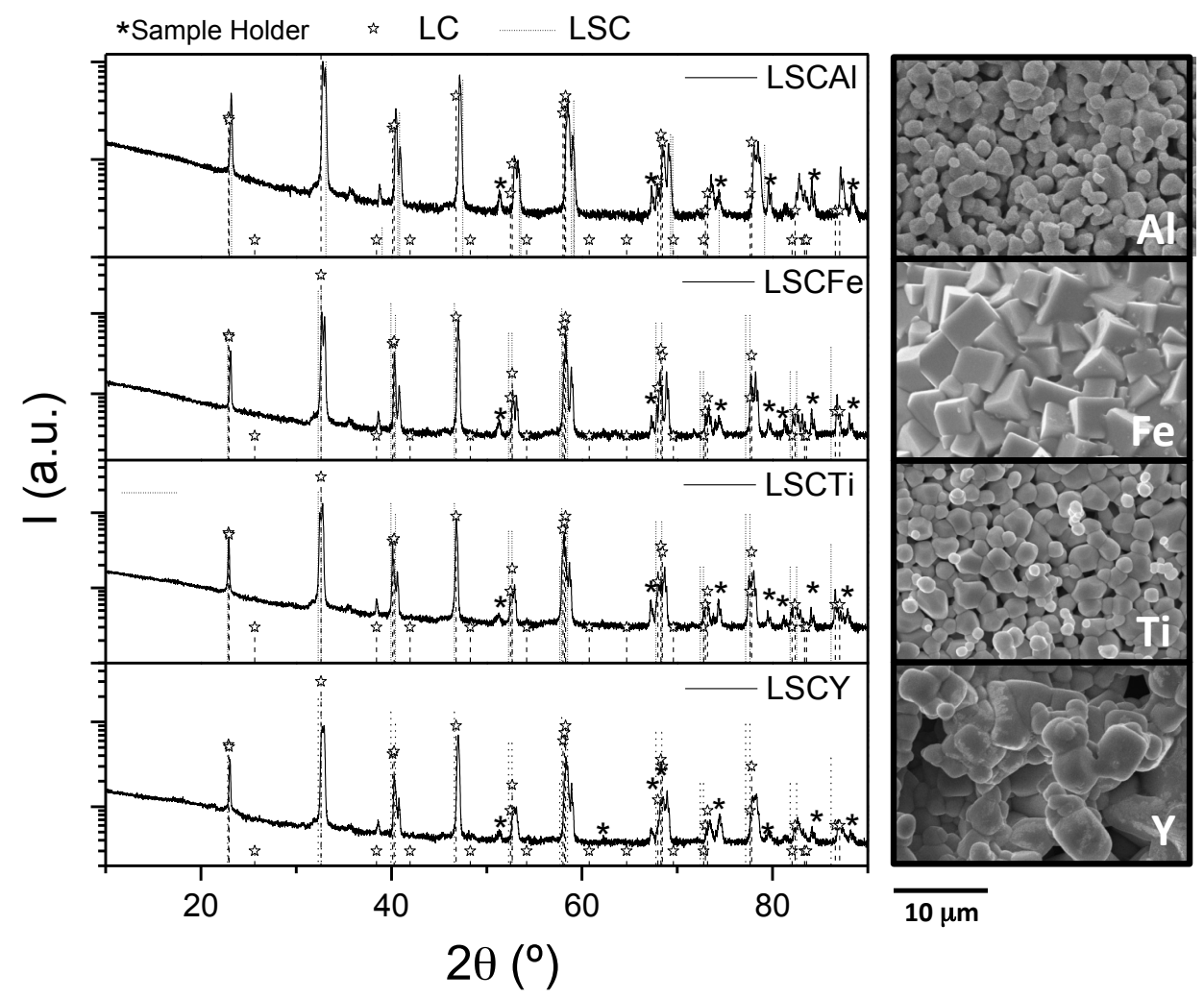

Figure 9.3. XRD patterns and corresponding SEM images of B-site doped LSC pressed and calcined at $1600{ }^{\circ} \mathrm{C}$ : LSCY, LSCT, LSCA and LSCrF. The peak positions of $\mathrm{LaCrO}_{3}$ (LC, ICSD 00-024-1016) and LSC (ICSD 00-032-1240) perovskites are represented for comparison as dotted vertical lines.

In order to test the possibility of both protonic and $p$-type electronic conductivity of the materials, the transport properties were measured by DC-conductivity in reducing atmospheres, i.e., $5 \% \mathrm{H}_{2}$ and $5 \% \mathrm{D}_{2}$ dry and wet (2.5\% atm of $\mathrm{H}_{2} \mathrm{O}$ and $\mathrm{D}_{2} \mathrm{O}$ respectively). It is observed that the conductivity in wet atmospheres is higher than in dry atmospheres, especially at low temperatures. At higher temperatures (ca. $800^{\circ} \mathrm{C}$ ), the exothermal oxide 
dehydration occurs, which drops the protonic carriers equalling the conductivity in wet and dry conditions. [28] Secondly, the conductivities in $\mathrm{H}_{2}$ are higher than in $\mathrm{D}_{2}$ in both dry and wet atmospheres, the so-called isotopic effect. The results at $700{ }^{\circ} \mathrm{C}$ indicate the mixed protonic-electronic character of doped LSC showing a clear isotopic effect. As direct comparison, the total conductivity in wet $\mathrm{H}_{2}$ atmosphere at $700{ }^{\circ} \mathrm{C}$, and the corresponding activation energy are written in the Table 9.1. The best conductivity results in wet $\mathrm{H}_{2}$ are obtained for LSCCe. For a question of clarity, only further details about the transport properties of the selected lanthanum chromites are shown and explained below.

\begin{tabular}{|c|c|c|c|c|}
\hline Composition & Nomenclature & $\begin{array}{c}\text { Structure } \\
\left(\text { at } 1600^{\circ} \mathrm{C}\right)\end{array}$ & $\begin{array}{c}\sigma_{700}{ }^{\circ} \mathrm{C} \\
\left(\mathrm{H}_{2}-\mathrm{H}_{2} \mathrm{O}\right) \\
\mathrm{S} \cdot \mathrm{cm}^{-1}\end{array}$ & $\begin{array}{l}E_{a} \\
\mathrm{eV}\end{array}$ \\
\hline $\mathrm{La}_{0.87} \mathrm{Sr}_{0.13} \mathrm{CrO}_{3}$ & LSC & Perovskite & 6 & 0.28 \\
\hline $\mathrm{La}_{0.85} \mathrm{Sr}_{0.15} \mathrm{Cr}_{0.9} \mathrm{Ni}_{0.1} \mathrm{O}_{3}$ & LSCN10 & Perovskite & 1 & 0.25 \\
\hline $\mathrm{La}_{0.85} \mathrm{Sr}_{0.15} \mathrm{Cr}_{0.8} \mathrm{Ni}_{0.2} \mathrm{O}_{3}$ & LSCN & Perovskite & - & - \\
\hline $\mathrm{La}_{0.75} \mathrm{Sr}_{0.15} \mathrm{Ce}_{0.1} \mathrm{CrO}_{3}$ & LSCCe & Perovskite & 11 & 0.28 \\
\hline $\mathrm{La}_{0.85} \mathrm{Sr}_{0.15} \mathrm{Cr}_{0.9} \mathrm{Fe}_{0.1} \mathrm{O}_{3}$ & $\mathrm{LSCrF}$ & Perovskite mixture & 6.4 & 0.38 \\
\hline $\mathrm{La}_{0.85} \mathrm{Sr}_{0.15} \mathrm{Cr}_{0.9} \mathrm{Ti}_{0.1} \mathrm{O}_{3}$ & LSCT & Perovskite mixture & 1.1 & 0.51 \\
\hline $\mathrm{La}_{0.85} \mathrm{Sr}_{0.15} \mathrm{Cr}_{0.9} \mathrm{Al}_{0.1} \mathrm{O}_{3}$ & LSCA & Perovskite mixture & 1.9 & 0.43 \\
\hline $\mathrm{La}_{0.85} \mathrm{Sr}_{0.15} \mathrm{Cr}_{0.9} \mathrm{Y}_{0.1} \mathrm{O}_{3}$ & LSCY & Perovskite mixture & 6.2 & 0.20 \\
\hline $\mathrm{Sm}_{0.85} \mathrm{Sr}_{0.15} \mathrm{CrO}_{3}$ & $\mathrm{SmSC}$ & Perovskite & 4.1 & 0.72 \\
\hline $\mathrm{Nd}_{0.85} \mathrm{Sr}_{0.15} \mathrm{CrO}_{3}$ & $\mathrm{NdSC}$ & Perovskite+impurities & 4.1 & 0.4 \\
\hline
\end{tabular}

Table 9.1. Summary of doped lanthanum strontium chromites composition and short nomenclature. The main structural phase formation is indicated as well as the total conductivity in wet $\mathrm{H}_{2}$ atmosphere at $700^{\circ} \mathrm{C}$, and the corresponding activation energy.

Although highly conducting materials have been obtained by doping LSC, the difficulty to obtain dense specimens in combination with a single phase structure limits the use of these materials for some applications, al least 
with the fabrication approaches considered in this thesis. For hydrogen membrane application, other densification methods that do not employ such high temperatures, as spark plasma, or the development of a composite might be tried. Nevertheless, these materials may be used as anode in PC-SOFC, since a proper porous microstructure is a condition necessary for the gas current through the electrode to the electrolyte and vice versa.

Thus, further studies on the materials showing a single perovskite phase and good conductivity in wet $\mathrm{H}_{2}$ are done. It is the case of LSCCe, which will be tested as anode backbone to improve the protonic transport through a proton conducting electrolyte, i.e., the LWO specified in the introduction. On the other hand, even though it does not improve the conductivity, the composition LSCN10 is tested as anode material due to the Ni structural loading, as the typical $\mathrm{NiO}$ anode material is incompatible with LWO under the oxidizing conditions necessary for the sintering and assembling of the fuel cell.

$L a_{0.85} \mathrm{Sr}_{0.15} \mathrm{Cr}_{0.9} \mathrm{Ni}_{0.1} \mathrm{O}_{3-\delta}(\mathrm{LSCN10})$ anode

In first place, the behavior of a $10 \% \mathrm{Ni}$ doped-LSC, i.e., LSCN10, as anode has been tested and compared with pure LSC. Symmetrical cells have been prepared by screen printing LSC and LSCN10 inks on LWO electrolyte and fired at $1050^{\circ} \mathrm{C}$. The chemical compatibilities of LSC and LSCN10 anodes on LWO electrolyte have been tested by XRD analysis of both phases after sintering at high temperature. Figure 9.5 shows the XRD pattern of a mixture of LSC and LWO 50/50 vol.\% fired at $1400{ }^{\circ} \mathrm{C}$. Single phase component materials are also plotted as reference. Both phases appear in the composite pattern without reaction evidences or impurities, confirming the material compatibility. 


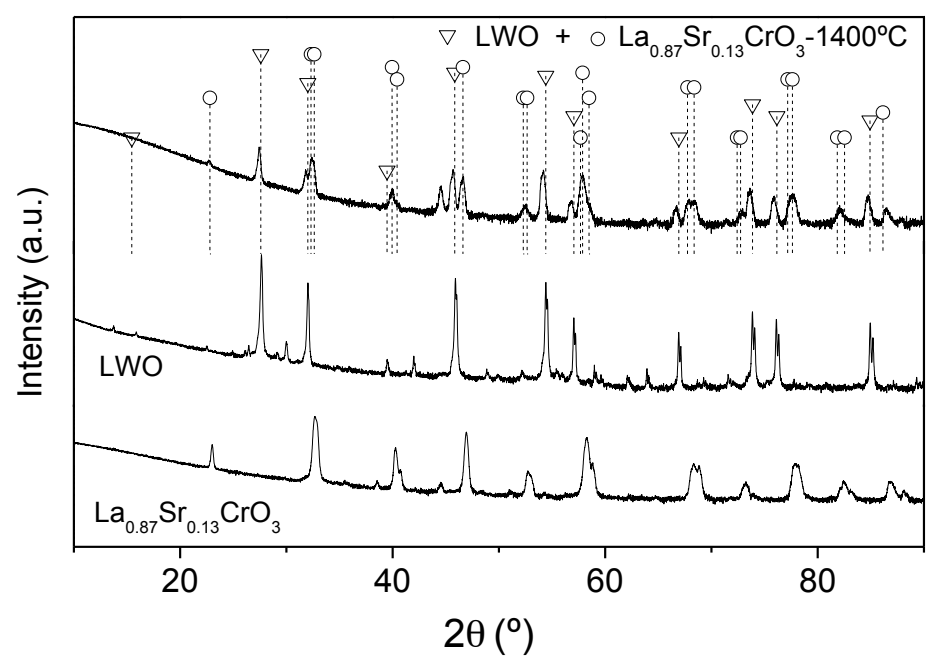

Figure 9.4. XRD patterns of (from bottom to top): LSC, LWO and their 50 vol.\% mixture at RT, treated at $1400{ }^{\circ} \mathrm{C}$ in $\mathrm{H}_{2}$ for $48 \mathrm{~h}$ and treated at $1150{ }^{\circ} \mathrm{C}$ in air for $5 \mathrm{~h}$.

On the other hand, $10 \% \mathrm{Ni}$ doping LSC aims to introduce catalytic properties on the anode without stability degradation. Figure 9.5 shows the XRD pattern of a mixture of LSCN10 and LWO 50/50 vol.\% after being treated for $5 \mathrm{~h}$ at $1150{ }^{\circ} \mathrm{C}$ in air. No new crystalline phases appear whereas all diffraction peaks can be completely assigned to LSCN10 or LWO phases. This is in contrast with the high reactivity of LWO with $\mathrm{NiO}$, which leads to the formation of several perovskite-related products (also shown in Figure 9.5). The confirmation of the lack of reactivity between LWO and metallic Ni can be inferred from the XRD pattern of the $\mathrm{LWO}+\mathrm{NiO} 50$ vol.\% after treatment at $900{ }^{\circ} \mathrm{C}$ for $48 \mathrm{~h}$ in $\mathrm{H}_{2}$, since no reaction products appear. 


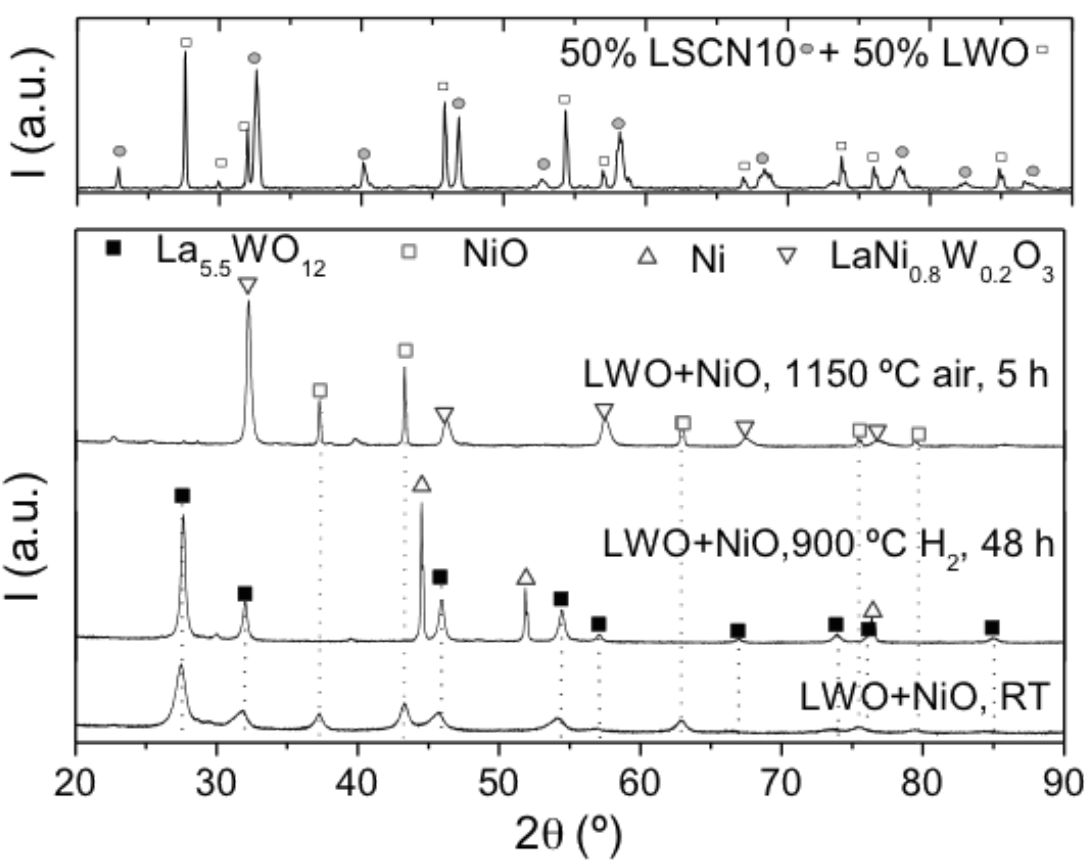

Figure 9.5: XRD patterns of (from bottom to top): LWO and NiO 50 vol.\% mixture at $\mathrm{RT}$, treated at $900{ }^{\circ} \mathrm{C}$ in $\mathrm{H}_{2}$ for $48 \mathrm{~h}$ and treated at $1150{ }^{\circ} \mathrm{C}$ in air for $5 \mathrm{~h}$; and LSCN10 and LWO 50 vol.\% mixture after calcination in air at $1150{ }^{\circ} \mathrm{C}$ for $5 \mathrm{~h}$

SEM and TEM images of the LSCN10 anode on LWO electrolyte (Figure 9.6a and b, respectively) permit ascertaining that the integrity of the interface between both materials is preserved after sintering and no reaction layers appear. This is confirmed by an EDS analysis of the different phases observed in the TEM picture. In addition, anode porosity for gas exchange is confirmed, as well as relatively high surface area available for the electrochemical reaction. The LWO electrolyte is dense and only presents closed porosity. 

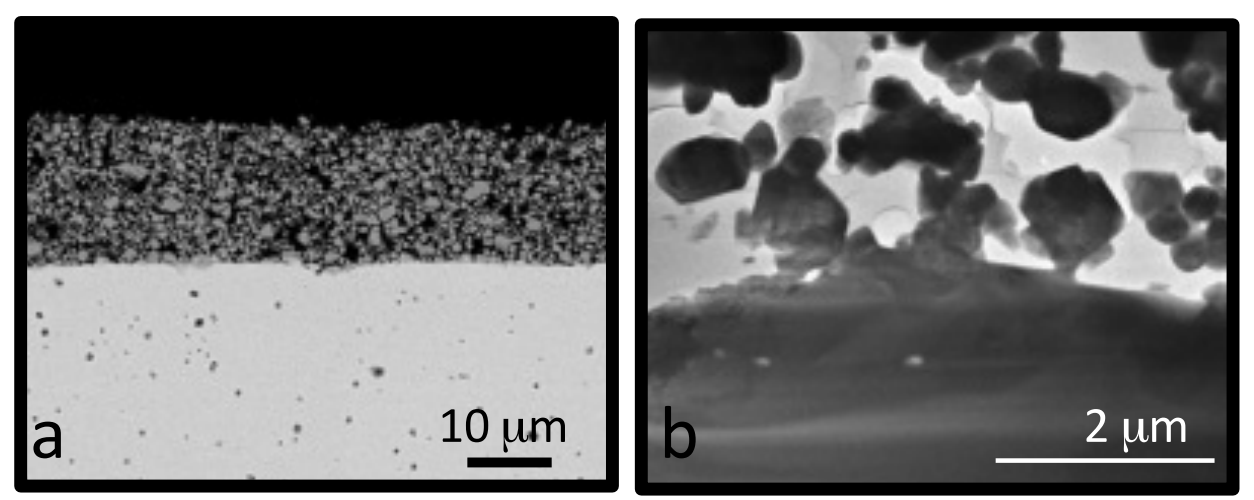

Figure 9.6: a) SEM image and b) TEM image of a FIB-lamella of a LSCN10 anode on LWO electrolyte sintered at $1150^{\circ} \mathrm{C}$

On the other hand, TEM images of the reduced electrode have been taken in order to see the effect of the operation conditions in the microstructure, i.e., high temperature and reducing conditions. It is observed that LSCN10 splits the Ni dissolved into the structure distributing metallic Ni nanoparticles over the surface. Figure $9.7 \mathrm{a}$ and $\mathrm{b}$ show the TEM images of as sintered LSCN10 anode on LWO electrolyte and reduced LSCN10 powder, where Ni nanoparticles are visible, respectively. These Ni nanoparticles $(19.7 \pm 3 \mathrm{~nm}$ when material is reduced at $900{ }^{\circ} \mathrm{C}$ ) turn into catalytic active particles boosting the anodic hydrogen oxidation reaction. [24-26] Furthermore, Ni particles are chemically compatible with LWO as concluded from Figure 9.5. Hence, the TEM analysis suggests that a higher Ni loading would increase the surface concentration of metallic particles, which will be studied below. 


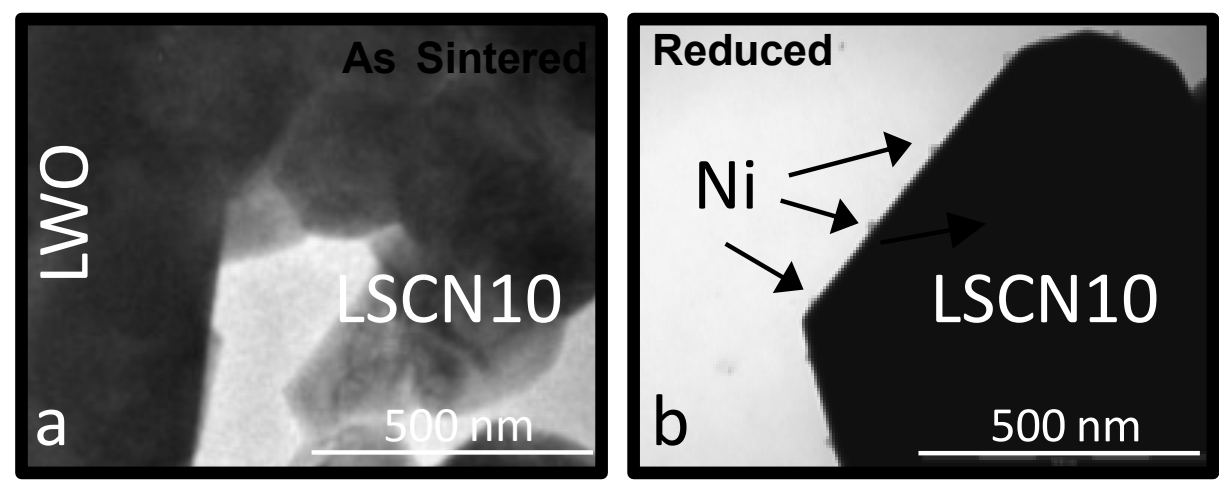

Figure 9.7: TEM images of a) as sintered LSCN10 on LWO electrolyte revealing the good bounding between electrolyte and porous electrode particles and b) LSCN10 powder after reduction at $900{ }^{\circ} \mathrm{C}$.

The formation of the metallic $\mathrm{Ni}$ nanoparticles upon reduction in hydrogen is monitored by TPR measurements, as showed in Figure 9.8a. While no reduction takes place in the undoped $\mathrm{LaCrO}_{3}$, a reduction peak appears centred at $380{ }^{\circ} \mathrm{C}$ when $\mathrm{Cr}$ is partially substituted by $\mathrm{Ni}$. This reduction is related to two reduction processes, i.e., the reduction of $\mathrm{Ni}^{2+}$ to metallic nickel, and the reduction of $\mathrm{Cr}^{4+}$ to $\mathrm{Cr}^{3+}$ and more reduced species $\left(\mathrm{Cr}^{\delta+}\right)$ as supported by reported XPS measurements, which involves the concomitant formation of new oxygen vacancies. [27] This reduction peak increases substantially when $\mathrm{Sr}^{2+}$ further substitutes $\mathrm{La}^{3+}$, since the concentration of $\mathrm{Cr}^{4+}$ increases to compensate the presence of $\mathrm{Sr}^{2+}$ and $\mathrm{Ni}^{2+}$ in oxidizing conditions. This additional reduction is attributed to (1) the formation of metallic $\mathrm{Ni}$ nanoparticles active in the anodic reactions and (2) the formation of oxygen vacancies, indispensable for the oxide hydration and protonic transport, through $\mathrm{Cr}^{4+}$ reduction.

The kinetics of Ni particle formation has been studied using a LSCN10 porous electrode on LWO. The anode polarization resistance $\left(\mathrm{R}_{\mathrm{p}}\right)$ was monitored at $900{ }^{\circ} \mathrm{C}$ upon feeding pure moist hydrogen. The total reduction of 
the LSCN10 material with the consequent formation of metallic Ni lasted for $6.6 \mathrm{~h}$, as can be inferred from Figure 9.8b, while the promotion of the electrochemical reactions through the Ni nanoparticles is confirmed.

a)

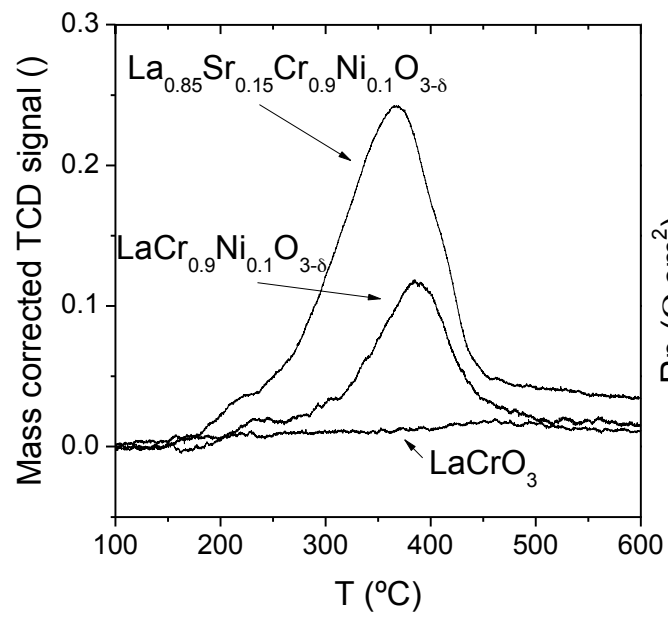

b)

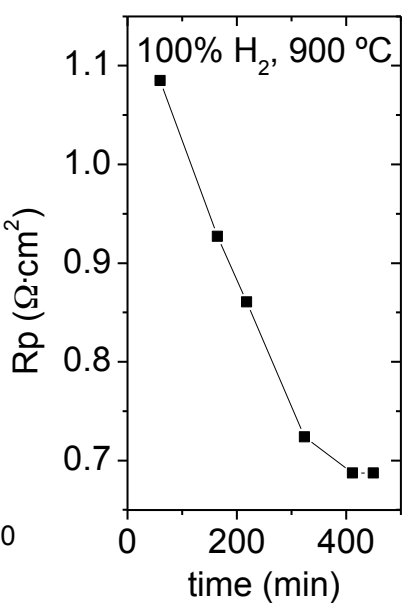

Figure 9.8: a) TPR analysis of $\mathrm{LaCrO}_{3-\delta}, \mathrm{LaCr}_{0.9} \mathrm{Ni}_{0.1} \mathrm{O}_{3-\delta}$ and LSCN10 materials and b) $\mathrm{R}_{\mathrm{p}}$ of LSCN10 as a function of time at $900{ }^{\circ} \mathrm{C}$ in $100 \% \mathrm{H}_{2}$.

The transport properties of reduced LSCN10 in $5 \% \mathrm{H}_{2}$ and $5 \% \mathrm{D}_{2}$ dry and wet $\left(2.5 \%\right.$ atm of $\mathrm{H}_{2} \mathrm{O}$ and $\mathrm{D}_{2} \mathrm{O}$ respectively) have been studied by DC-conductivity. The results plotted in Figure 9.9 show that (i) the conductivity in wet atmospheres is higher than in dry atmospheres although the exothermal oxide dehydration at high temperatures drops the protonic conductivity and equals the conductivity in wet and dry conditions, e.g. at $800^{\circ} \mathrm{C}$ in wet and dry $\mathrm{H}_{2}$; this effect is expected to occur at higher temperatures in $\mathrm{D}_{2}$ due to slightly different oxide hydration equilibrium for $\mathrm{D}_{2} \mathrm{O}$; [28] (ii) the conductivities in $\mathrm{H}_{2}$ are higher than in $\mathrm{D}_{2}$ (very close to the theoretical $\sqrt{2}$ factor) in both dry and wet atmospheres. The isotopic effect in dry atmospheres is ascribed to the presence 
of residual amounts of $\mathrm{H}_{2} \mathrm{O} / \mathrm{D}_{2} \mathrm{O}(\sim 30 \mathrm{ppm})$ in the so-called dry atmospheres; and (iii) the dependency of conductivity with $p O_{2}\left(\mathrm{D}_{2}<\mathrm{H}_{2} ; \mathrm{D}_{2}-\mathrm{D}_{2} \mathrm{O}<\mathrm{H}_{2}-\mathrm{H}_{2} \mathrm{O}\right)$ indicates that the conductivity rises with increasing $p O_{2}$, specially at temperatures below $800{ }^{\circ} \mathrm{C}$. The results suggest that LSCN presents both protonic and $p$-type electronic conductivity under typical anode operating conditions. In fact, the conductivity data follow the expected hydration and isotopic effects for a proton conductor while electron-hole is affected by the corresponding $\mathrm{pO}_{2}$ being attributed to $p$-type conduction. [11] At $600{ }^{\circ} \mathrm{C}$ the mixed protonic-electronic character of LSCN is well evidenced showing a clear isotopic effect. However, at $800{ }^{\circ} \mathrm{C}$, the $\mathrm{pO}_{2}$ dependency becomes minor and the isotopic effect is still visible, which suggests the growing contribution of oxide-ion conductivity at high temperatures.

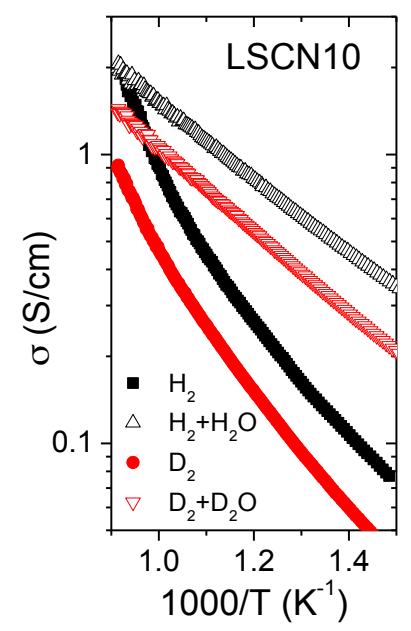

Figure 9.9: Total conductivity of $\mathrm{La}_{0.85} \mathrm{Sr}_{0.15} \mathrm{Cr}_{0.9} \mathrm{Ni}_{0.1} \mathrm{O}_{3-8}$ in $5 \% \mathrm{H}_{2}, 5 \% \mathrm{D}_{2}, 5 \% \mathrm{H}_{2}+\mathrm{H}_{2} \mathrm{O}$ and $5 \% \mathrm{D}_{2}+\mathrm{D}_{2} \mathrm{O}$.

Up to here, the compatibility of the LSCN10 with LWO, and the adequate transport properties and catalytic activity of LSCN material have been evidenced. Next, the suitability as PC-SOFC anode material is analysed by 
electrochemical impedance spectroscopy (EIS) in symmetrical cells on LWO electrolyte.

Figure $9.10 \mathrm{a}$ and $\mathrm{b}$ respectively represent Nyquist and Bode plots of the EIS analysis of LSC recorded in wet $100 \% \mathrm{H}_{2}$ at $750{ }^{\circ} \mathrm{C}$ after subtracting electrolyte contribution. Figure 9.10c and d shows similar representation for LSCN10 recorded in wet $100 \% \mathrm{H}_{2}$ at different temperatures. The impedance of LSCN10 is decreased by some orders of magnitude with respect to LSC. This effect is attributed to surface activation when $\mathrm{Ni}$ is finely dispersed on the chromite grain surface.

a)
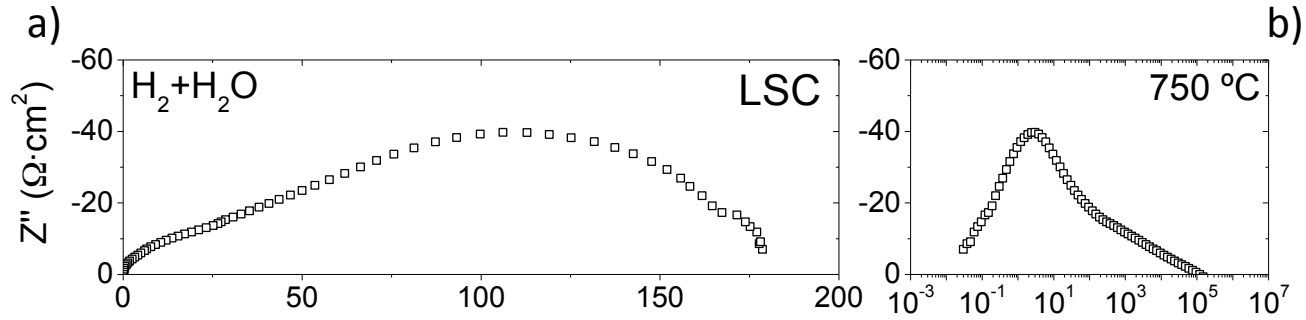

c)
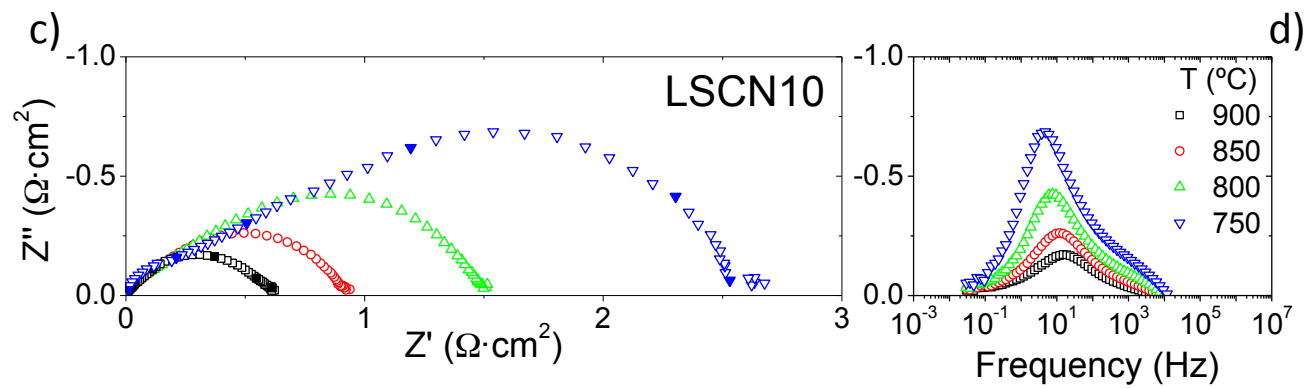

Figure 9.10: a) Nyquist and b) Bode plots of impedance spectra recorded in wet $(2.5 \%$ vol. $\left.\mathrm{H}_{2} \mathrm{O}\right) 100 \% \mathrm{H}_{2}$ for LSC at $700{ }^{\circ} \mathrm{C}$ and c) Nyquist and d) Bode plots of impedance spectra recorded in wet $\left(2.5 \%\right.$ vol. $\left.\mathrm{H}_{2} \mathrm{O}\right) 100 \% \mathrm{H}_{2}$ at different temperatures for LSCN10.

Figure 9.10 show two main contributions that are fitted to the double $\mathrm{R} \| \mathrm{CPE}$ equivalent circuit illustrated in Figure 9.11, which comprises two 
depressed arcs, one observed at low frequencies (LF, 10-100 Hz) and another at medium frequency (MF, 0.1-1 kHz), whose overlapping depends on the operating conditions. The depressing arcs need the use of these CPEs (Constant Phase Elements) instead of capacitors as corresponds to the porous structure of the electrode. The impedance of a CPE can be expressed as:

$$
Z=A^{-1}(j \omega)^{-n}
$$

where $A$ is a frequency independent constant, $\omega$ is the angular frequency and $j=\sqrt{ }-1$. Note that there are two limiting cases, first when $n=1$, the CPE operates as an ideal capacitor and second when $n=0$, the CPE acts as a pure resistor. [29] The equivalent capacitance of the CPEs can be calculated according to the following equation. [30]

$$
C_{C P E}=R^{(1-n) / n} A^{1 / n}
$$

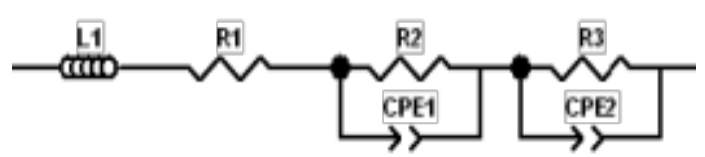

Figure 9.11. Equivalent circuit used for EIS impedance modeling. CPS are used instead of perfect capacitors due to the porous nature of the anode.

LF and MF resistances (R) of LSCN10 measured in $100 \%$ moist $\mathrm{H}_{2}$ are represented as a function of temperature in Arrhenius arrangement (Figure 9.12a). Above $850{ }^{\circ} \mathrm{C}$ both $\mathrm{LF}$ and $\mathrm{MF}$ contributions are similar but below $850{ }^{\circ} \mathrm{C}$ the rate limiting mechanism corresponds to the LF associated processes, being twice the MF contribution. The resistance increases when reducing $\mathrm{pH}_{2}$, and at $750{ }^{\circ} \mathrm{C}$, both LF and MF associated mechanisms have a $\mathrm{pH}_{2}$ dependency close to $-1 / 4$ (Figure 9.12b). The LF process (with capacitances in the range of $10^{-2} \mathrm{~F} / \mathrm{cm}^{2}$ ) may be related to surface processes: catalytic reactions between chemisorbed species, adsorption/desorption reactions from/to the gas phase and 
surface diffusion of intermediate species. [31] This important contribution to the anode impedance of the adsorption/desorption of $\mathrm{H}_{2} \mathrm{O}$ and $\mathrm{OH}^{-}$species has been previously observed in similar frequency ranges and activation energies for Ni-YSZ/Ni-CGO anodes. [32] These results anticipate that the further development of chromite-based PC-SOFC anodes should focus on the optimization of surface activity, either increasing the intrinsic activity of the catalyst or improving the catalyst dispersion, in order to diminish LF associated processes. Furthermore, MF processes (with capacitances in the range of $10^{-3}-10^{-2} \mathrm{~F} / \mathrm{cm}^{2}$ ) might be associated to charge transfer reactions, [33] i.e., (i) the charge transfer between $\mathrm{Ni}$ nanoparticles and the oxide and (ii) the diffusion charged species to the three phase boundary. [34]

a)

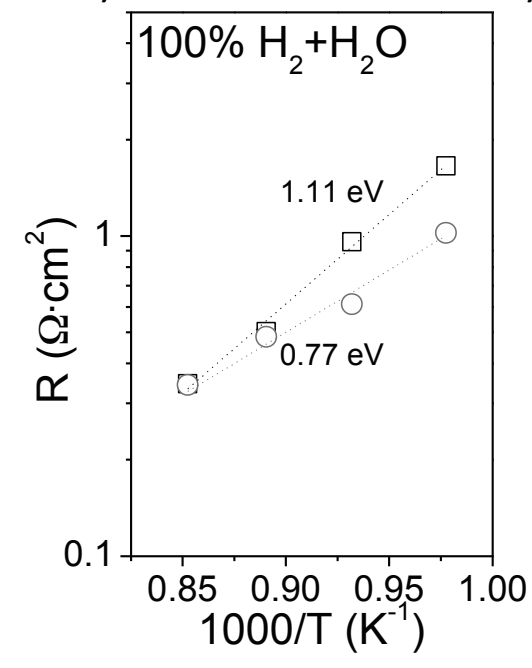

b)

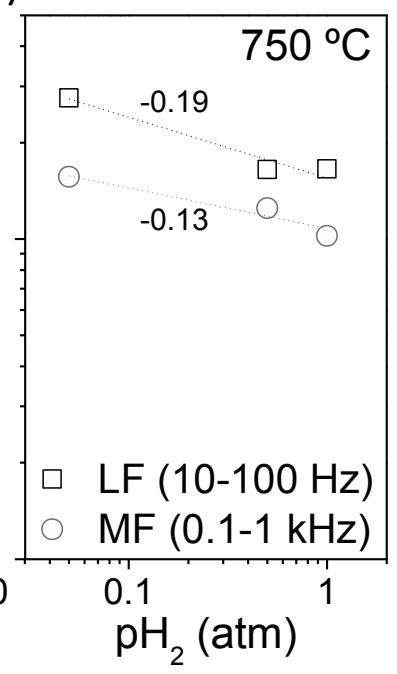

Figure 9.12. a) Modeled LF and MF resistances as a function of the temperature and b) as a function of the $\mathrm{pH}_{2}$. 
Finally, the XRD in Figure 9.13 shows the structure pattern of the reduced LSCN10 anode on LWO after electrochemical cell testing. It demonstrates the stability of the material in working conditions. The perovskite and the fluorite phases are observed without any reaction between them. Also peaks corresponding to the metallic $\mathrm{Ni}$ in the surface after the reduction and the $\mathrm{Au}$ from the current collector are distinguished.

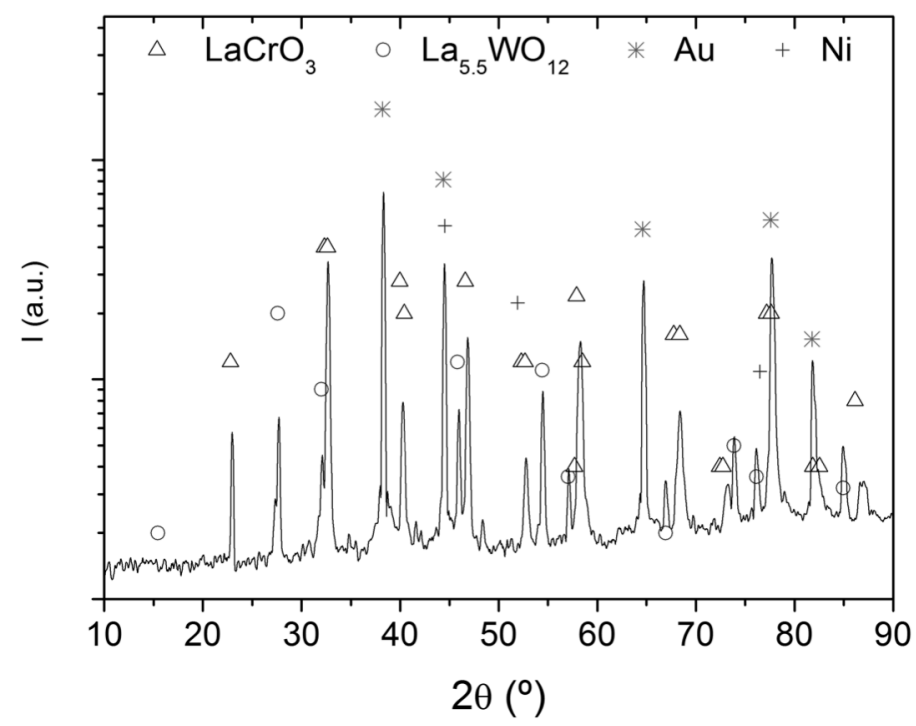

Figure 9.13. XRD pattern in logarithm scale of LSCN10 anode after electrochemical test. The materials maintains its pure perovskite structure.

Catalytic surface promotion of $\mathrm{La}_{0.85} \mathrm{Sr}_{0.15} \mathrm{Cr}_{0.8} \mathrm{Ni}_{0.2} \mathrm{O}_{3-\delta}(\mathrm{LSCN})$ anodes

The LSC and LSCN10 anodes have been shown above to be specially limited by surface reactions. Therefore, further development of chromite-based PC-SOFC anodes should focus on the optimization of surface activity. Nickel infiltration into the electrodes has been considered, since metallic $\mathrm{Ni}$ 
nanoparticles have been demonstrated to be compatible with LWO under anode operating conditions and they are active catalyst in the $\mathrm{H}_{2}$ bond breaking reaction. [20-22] Concerning the anode backbone, the first material of choice is $20 \% \mathrm{Ni}$ doped in the $\mathrm{B}$ site, which was deduced in the previous section to increase Ni nanoparticles segregation.

The structure of the LSCN powder sintered at $900{ }^{\circ} \mathrm{C}$ can be observed from XRD pattern plotted in Figure 9.14a. The main diffraction peaks can be assigned to the orthorhombic $\mathrm{LaCrO}_{3}$ perovskite phase, although some traces of $\mathrm{SrCrO}_{4}$ spinel phase can also be observed. [35] These traces of $\mathrm{SrCrO}_{4}$ were previously observed at this low sintering temperature but it was also proved that they disappear upon reducing conditions. SEM image of Figure $9.14 \mathrm{~b}$ shows the microstructure of this powder, which presents a homogenous grain size distribution.

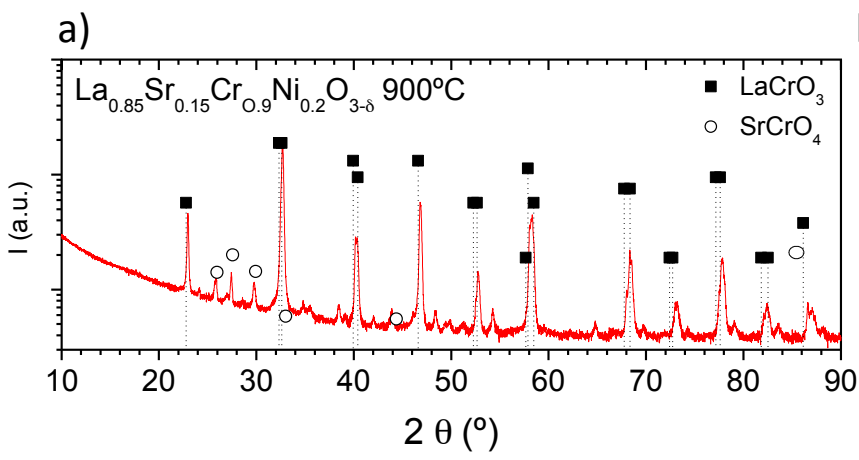

b)

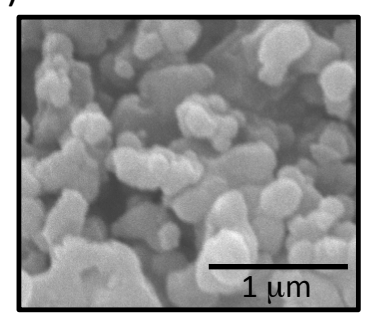

Figure 9.14. a) XRD pattern and b) SEM image of LSCN powder sintered at $900{ }^{\circ} \mathrm{C}$

Symmetrical cells were prepared on LWO electrolyte by using LSCN ink, as explained in Chapter 2.1. Once the electrolyte was fired at $1050{ }^{\circ} \mathrm{C}$, the infiltration of $\mathrm{Ni}$ in the $\mathrm{LSCN}$ electrode $(\mathrm{LSCN}+5 \mathrm{Ni})$ was done by dropping a 
$5 \mathrm{M}$ water solution of $\mathrm{Ni}$ nitrate on the LSCN electrode surface. It was allowed to penetrate into the pores for $30 \mathrm{~min}$ and calcined at $300{ }^{\circ} \mathrm{C}$ to decompose the nitrates. This process was repeated 4 times. XRD patterns of the different symmetrical cells, LSC, LSCN and LSCN $+5 \mathrm{Ni}$, are represented in Figure 9.15 (intensity in $\log$ scale) after the impedance measurements at high temperature and reducing conditions. The diffraction peaks of the LSC cell can be assigned to (1) the chromite perovskite phase, (2) the LWO electrolyte fluorite phase and (3) metallic Au, which is used as current collector in the symmetrical cell testing. As expected after the reducing conditions, no traces of $\mathrm{SrCrO}_{4}$ are observed. When the anode material is doped with Ni (LSCN symmetrical cell) after reducing conditions diffraction peaks corresponding to metallic $\mathrm{Ni}$ are also visible. This metallic Ni is segregated from the LSCN compound due to the high temperature and the reducing conditions, as it has been previously proved. [24] The intensity of these Ni peaks is increased in the case of LSCN $+5 \mathrm{Ni}$ symmetrical cell.

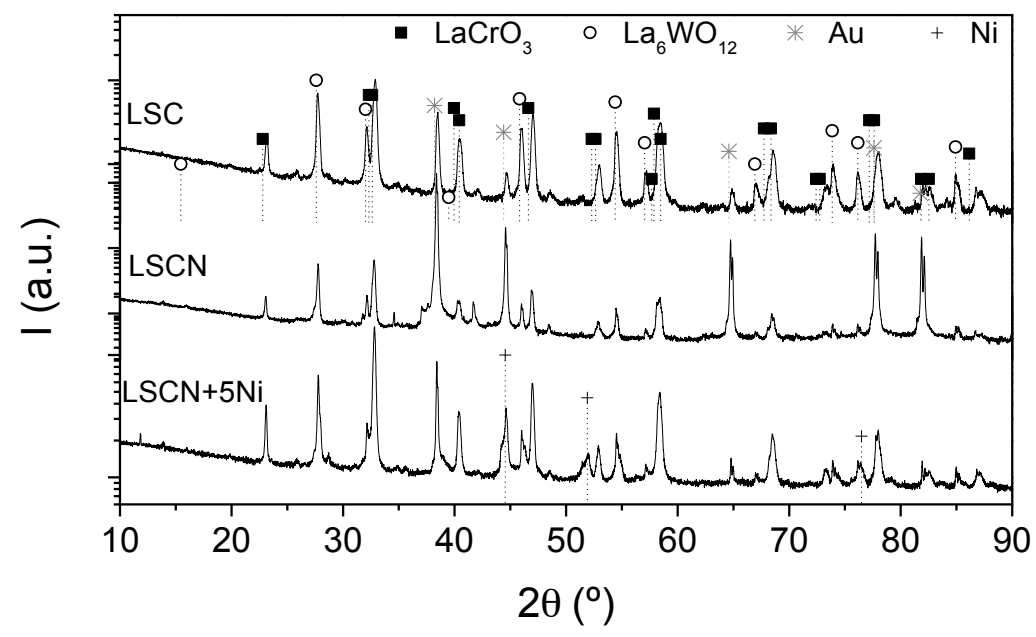

Figure 9.15. XRD patterns of LSC, LSCN and LSCN+5Ni symmetrical cells. The peaks corresponding to metallic Au from the current collector of the symmetrical cell and metallic Ni (infiltrated and structural) are indicated. 
The Ni nanoparticle segregation from the LSCN material after reduction at high temperature is represented in the scheme of Figure 9.16a. However, from its real SEM image, showed in Figure 9.16b, it is not easy to distinguish the presence of these nanoparticles, due to the very small size and low amount of Ni. For this reason, in previous reported works, TEM was used for the characterization of these segregated particles. [24] The scheme of the LSCN electrode infiltrated by using $5 \mathrm{M}$ water solution of $\mathrm{Ni}$ nitrate $(\mathrm{LSCN}+5 \mathrm{Ni})$ is showed in Figure 9.16c aside with corresponding SEM images of the cross section anodes after measurements depicted in Figure 9.16d and e (two different magnifications). These SEM images show the covering by Ni nanoparticles on the $\mathrm{LSCN}+5 \mathrm{Ni}$ electrode backbone after reduction at $800^{\circ} \mathrm{C}$. In fact, the surface of the $\mathrm{LSCN}+5 \mathrm{Ni}$ grains is fully covered with well distributed Ni nanoparticles, along the thickness of the electrode. The average Ni particle size infiltrated in the electrode is $10-20 \mathrm{~nm}$. This size matches with $\sim 10-20 \mathrm{~nm}$ Ni particles resulted from Ni nitrate calcination and subsequent reduction at $800{ }^{\circ} \mathrm{C}$ in $\mathrm{H}_{2}$, which are observed in the TEM image of Figure 9.17 (measured in powder). Electrode microstructural parameters are of great importance especially for infiltrated electrodes, since the pore should host the Ni nanoparticles and allow the mean free path of the gas molecules. Indeed, the SEM images of the anodes (Figure 9.16) after measurements in reducing conditions show that the highly-porous backbone is adequate for Ni hosting and proper gas transport. 

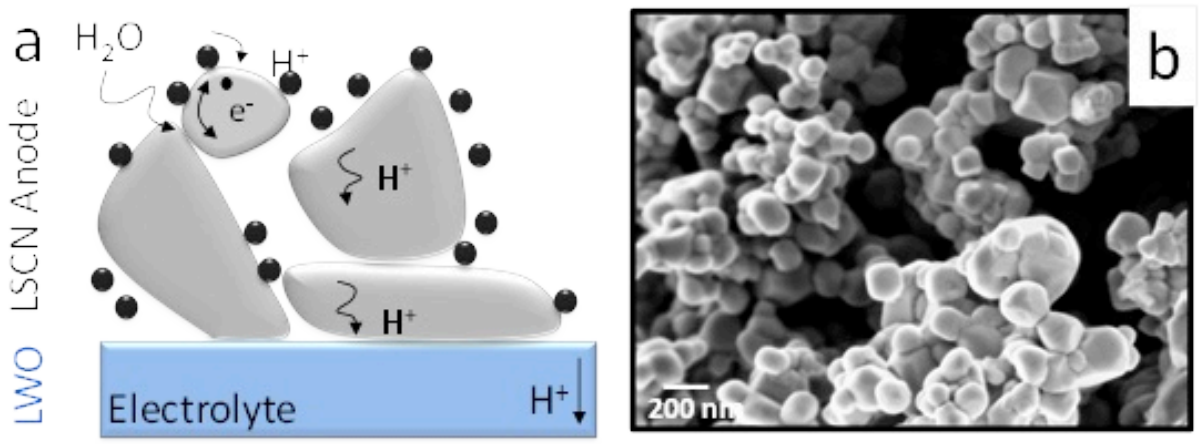

C Ni-nanoparticles $\mathrm{H}_{2, \mathrm{gas}}$
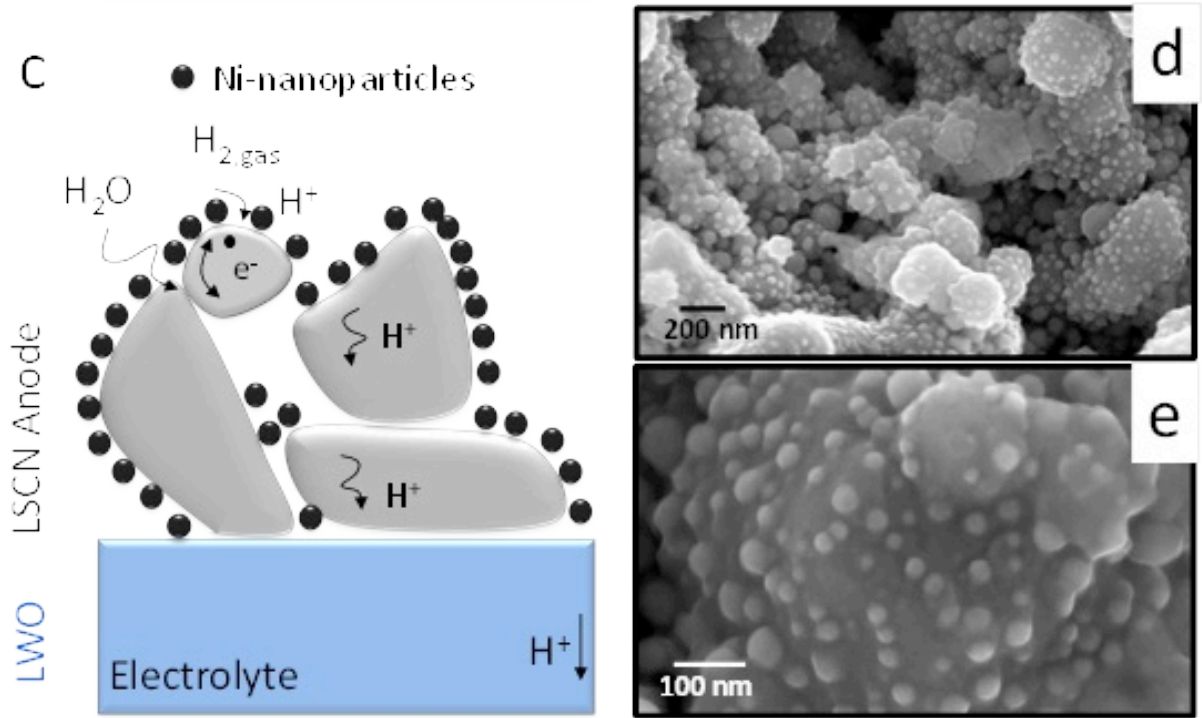

Figure 9.16. Schematic diagram of the LSCN anode a) and its corresponding SEM image (b) and scheme of the LSCN+5Ni anode c) and its real SEM images d) and e). $5 \mathrm{M}$ water solution of $\mathrm{Ni}$ Nitrate was dropped on the surface of the LSCN electrode and allowed to penetrate into the pores for $30 \mathrm{~min}$. The infiltration step was repeated 4 times. Each infiltration step was followed by calcination at $300{ }^{\circ} \mathrm{C}$ to decompose the nitrates. 


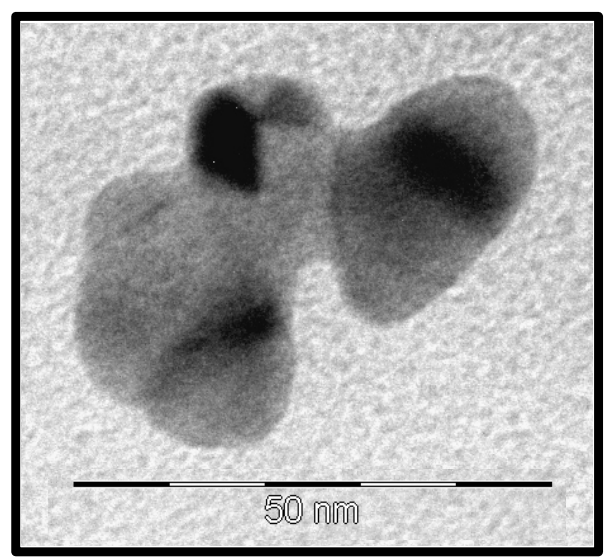

Figure 9.17. TEM image of $\mathrm{Ni}$ nanoparticles obtained by firing nitrate precursor at $800{ }^{\circ} \mathrm{C}$ in $\mathrm{H}_{2}$. Particle size is in between 10 and $20 \mathrm{~nm}$.

The electrochemical properties of the three different tested anodes, LSC, LSCN and LSCN $+5 \mathrm{Ni}$, can be evaluated to determine the polarization resistance $(\mathrm{Rp})$ values as a function of the temperature, plotted in Figure 9.18. At $700{ }^{\circ} \mathrm{C}$ in wet hydrogen LSC anode shows $\mathrm{Rp}$ of $347 \Omega \cdot \mathrm{cm}^{2}$. In the case of the LSCN anode, the segregation of the Ni nanoparticles results in two orders of magnitude $\mathrm{Rp}$ decrease, showing a $\mathrm{Rp}$ value of $3.54 \Omega \cdot \mathrm{cm}^{2}$ at $700{ }^{\circ} \mathrm{C}$. This indicates that the presence of the Ni nanoparticles actively helps the anode performance, which may be attributed to the Ni catalytic activity over the as $\mathrm{H}_{2}$ oxidation reaction. Ni infiltration on LSCN anode decreases the Rp almost another order of magnitude, reaching a value of $0.47 \Omega \cdot \mathrm{cm}^{2}$ at $700{ }^{\circ} \mathrm{C}$. This improvement can be directly related to the higher amount of active $\mathrm{Ni}$ nanoparticles in the anode. 


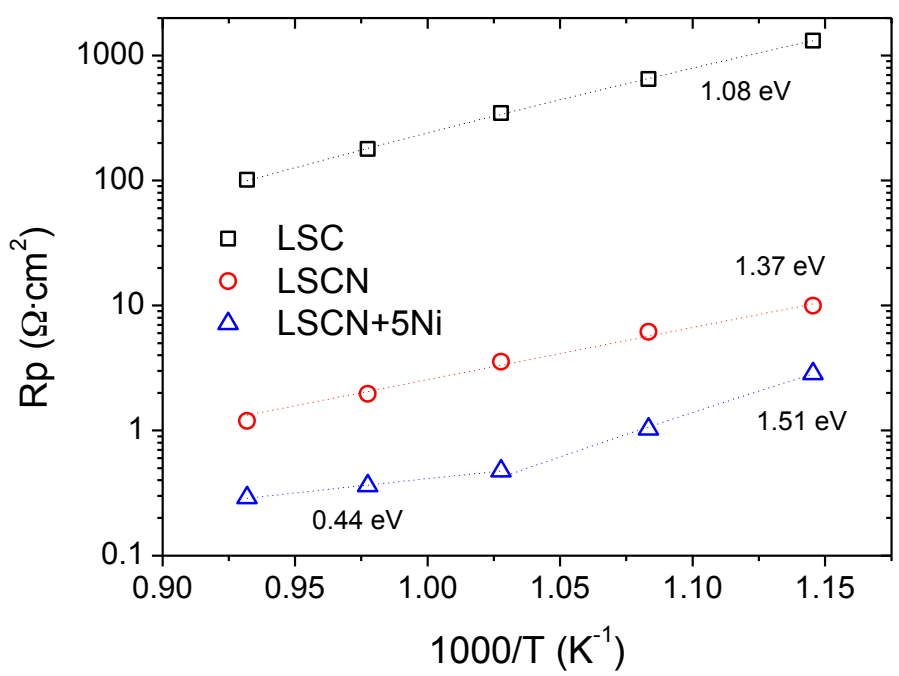

Figure 9.18. Rp as a function of inverse temperature for LSC, LSCN and $\mathrm{LSCN}+5 \mathrm{Ni}$ electrodes.

In order to discern the nature of the electrochemical improvements due to the $\mathrm{Ni}$ nanoparticles that are on one hand segregated from the $\mathrm{Ni}$ doped compound and, on the other, obtained by $\mathrm{Ni}$ infiltration, EIS spectra are analyzed for the different materials.

Figure 9.19 shows the EIS spectra at $700{ }^{\circ} \mathrm{C}$ of LSC anode (a and b, Nyquist and Bode plots, shown again for a direct comparison) and LSCN and $\mathrm{LSCN}+5 \mathrm{Ni}$ (c and d, Nyquist and Bode plots) after subtracting the electrolyte contribution. The spectra show two different contributions in both LSC and LSCN anodes, i.e., two different arcs at different frequencies. Otherwise, $\mathrm{LSCN}+5 \mathrm{Ni}$ anode only shows one main contribution, despite a small noise ascribed to the contacts appears at low frequencies and high temperatures. Figure $9.19 \mathrm{~b}$ demonstrates that the LSC anode is limited by low frequency (LF) associated processes, although high frequency (HF) associated processes remain an important contribution as well. The structural Ni doping the LSCN is able to segregate in the surface of the material under operating conditions as 
nanoclusters, so that the LF contribution is drastically decreased (almost two orders of magnitude), although it is still limiting the overall anode performance. LSCN anode also shows another contribution at higher frequencies. By $\mathrm{Ni}$ nanoparticles infiltration along the anode surface, the LF contribution disappears and high frequency (HF) associated processes limit the LSCN+5Ni anode performance.
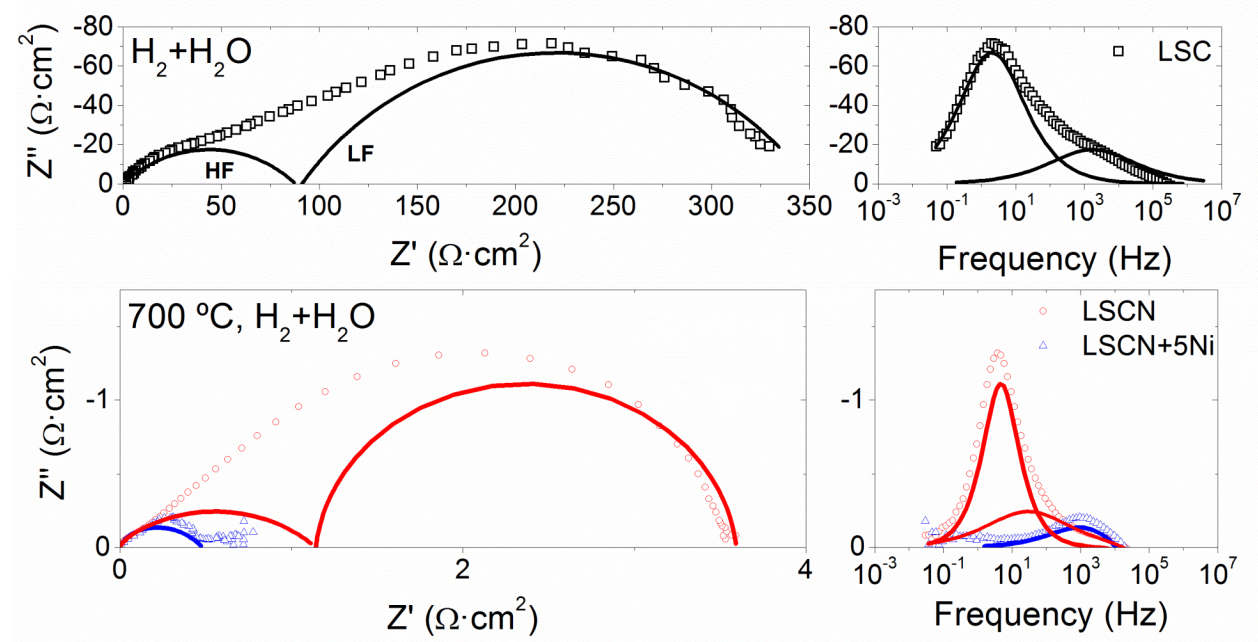

Figure 9.19. Impedance spectra recorded at $700{ }^{\circ} \mathrm{C}$ in wet hydrogen of LSC a) Nyquist and b) Bode plots and LSCN and LSCN $+5 \mathrm{Ni}$ c) Nyquist and d) Bode plots. Solid lines represent the depressed arcs fitting the different electrode resistance contributions.

These different HF and LF contributions observed for LSC and LSCN can be analyzed in terms of two depressed arcs by fitting EIS spectra to a double $\mathrm{R} \| \mathrm{CPE}$ circuit (Figure 9.11). In the case of $\mathrm{LSCN}+5 \mathrm{Ni}$ anode only one arc is needed, fitted to a single $\mathrm{R} \| \mathrm{CPE}$ circuit. Modeled resistances and capacitances (calculated from CPEs) are depicted in Figure 9.20a and b, and their characteristic frequencies are written in the graph. LSC anode shows both limiting LF $(2-25 \mathrm{~Hz})$ associated processes and also very high resistances 
associated to $\mathrm{HF}(1-10 \mathrm{kHz})$ processes. Its associated capacitance values are $10^{-3} \mathrm{~F} / \mathrm{cm}^{2}$ for LF processes and $10^{-5}-10^{-4} \mathrm{~F} / \mathrm{cm}^{2}$ for HF, which combined with their characteristic frequencies can be assigned to surface associated processes and LSC transport properties, respectively.
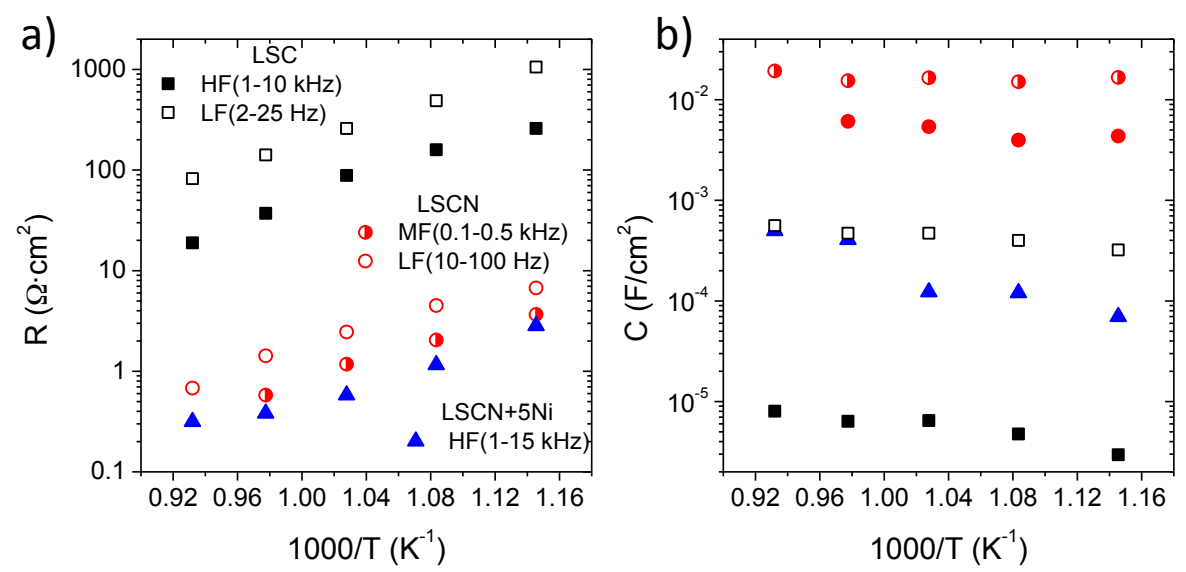

Figure 9.20. Temperature dependency of modelled resistances a) and capacitances b) of LSC, LSCN and LSCN $+5 \mathrm{~N}$ samples.

When the $\mathrm{Ni}$ is added as dopant, the LSCN anode shows LF $(10-100 \mathrm{~Hz})$ and medium frequency, MF $(0.1-0.5 \mathrm{kHz})$, contributions. Associated capacitances are $10^{-2} \mathrm{~F} / \mathrm{cm}^{2}$ and $10^{-3}-10^{-2} \mathrm{~F} / \mathrm{cm}^{2}$ for LF and MF processes, respectively. These characteristic frequencies and their corresponding associated capacitances can be related to surface processes. In particular, the MF is associated to surface exchange coupled with bulk ionic transport in mixed conducting electrodes. [36] LF resistance decreases more than one order of magnitude compared to that of LSC, while the range of characteristic frequencies shift to higher values. This decrease of the LF resistance can only be attributed to the effect of the active $\mathrm{Ni}$ nanoparticles formed on the surface of the anode after reduction, which improves the surface dissociation of hydrogen. The disappearance of this LF contribution in the 
LSCN $+5 \mathrm{Ni}$ anode demonstrates the great importance of the catalytic activity of these Ni nanoparticles.

In fact, $\mathrm{LSCN}+5 \mathrm{Ni}$ anode only shows $\mathrm{HF}(1-15 \mathrm{kHz})$ resistance with associated capacitances of $10^{-3}-10^{-4} \mathrm{~F} / \mathrm{cm}^{2}$. These HF processes may be attributed to the transport properties of the LSCN material or to the LSCN/LWO interfacial resistances, i.e., (i) the charge transfer between Ni nanoparticles and the oxide and (ii) the diffusion of charged species to the three phase boundary. $[33,34]$ The pronounced resistance improvement confirms that infiltration is a suitable method to support $\mathrm{Ni}$ catalyst nanoparticles to increase the surface contact with fuel.

Catalytic surface promotion of $\mathrm{La}_{0.75} \mathrm{Ce}_{0.1} \mathrm{Sr}_{0.15} \mathrm{CrO}_{3-\delta}(\mathrm{LSCCe}$ ) anodes

The second anode development strategy consisted of improving the total conductivity of the material, which was achieved by Ce doping on the A-site. Symmetrical LSCCe electrodes were screen printed on the LWO dense electrolyte and were infiltrated afterwards by different $\mathrm{Ni}$ loads $(0,1$ and $5 \mathrm{Ni}$ loads) to boost the surface reaction.

Figure 9.21 presents the XRD analysis of the LSCCe powder and the Ni-infiltrated LSCCe anode after being submitted to the reduction and to the electrochemical characterization. Powder sintered at $1200^{\circ} \mathrm{C}$ still present traces of $\mathrm{SrCrO}_{4}$ and $\mathrm{CeO}_{2}$, which disappear either at higher temperatures or in reducing atmospheres. $[24,30]$ In fact, LSCCe anode after testing only shows diffraction peaks corresponding to the chromite, the LWO from the electrolyte, $\mathrm{Au}$ from the current collector and Ni catalyst. The lack of any other diffraction 
peaks that could be assigned to any reaction product denotes the compatibility of the LSCCe with the LWO electrolyte, even after the Ni infiltration, and the stability in anode working conditions.

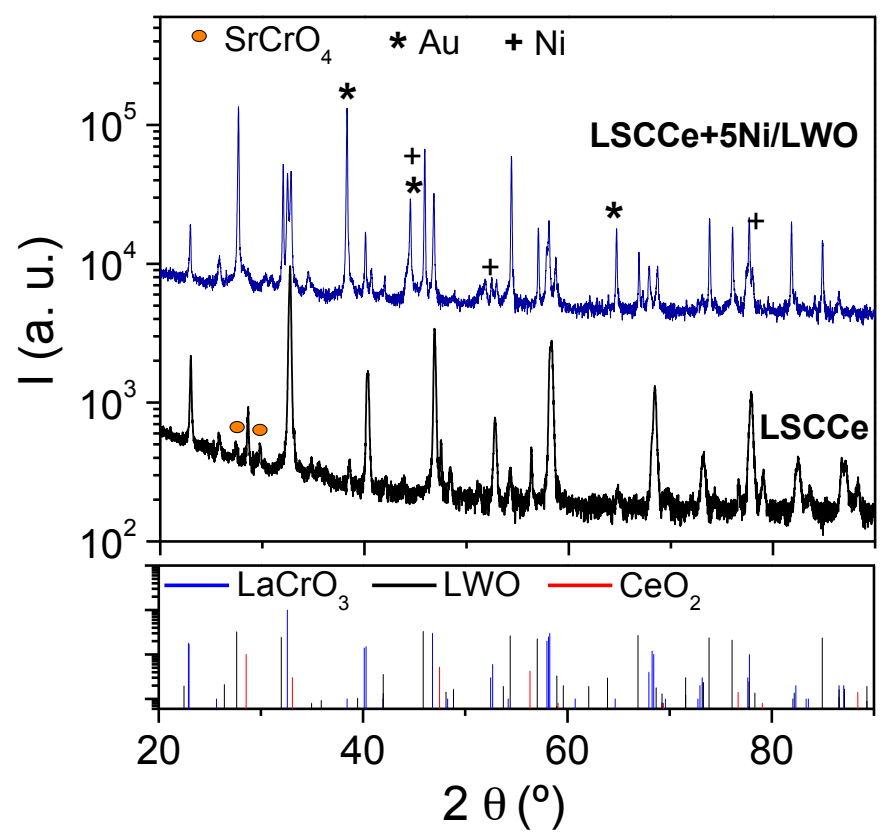

Figure 9.21. XRD patterns of $\mathrm{LSCCe}$ as prepared powder and anode after $\mathrm{Ni}$ infiltration and reduction in wet $\mathrm{H}_{2}$ up to $800{ }^{\circ} \mathrm{C}$. XRD positions of $\mathrm{LaCrO}_{3}, \mathrm{LWO}$ and $\mathrm{CeO}_{2}$ are indicated as reference.

Besides the material stability under working conditions, Figure 9.22 shows that the material is stable in $\mathrm{CO}_{2}$ containing atmospheres. The thermogravimetric curve (Figure 9.22b) shows a slight weight loss at temperatures up to $400{ }^{\circ} \mathrm{C}$, attributed to water and oxygen desorption, while at higher temperatures the mass remains practically constant. The lack of weight gain indicates that carbonation processes/reactions $\left(\mathrm{CO}_{2}\right.$ uptake) at temperatures below $800{ }^{\circ} \mathrm{C}$ are not happening. 


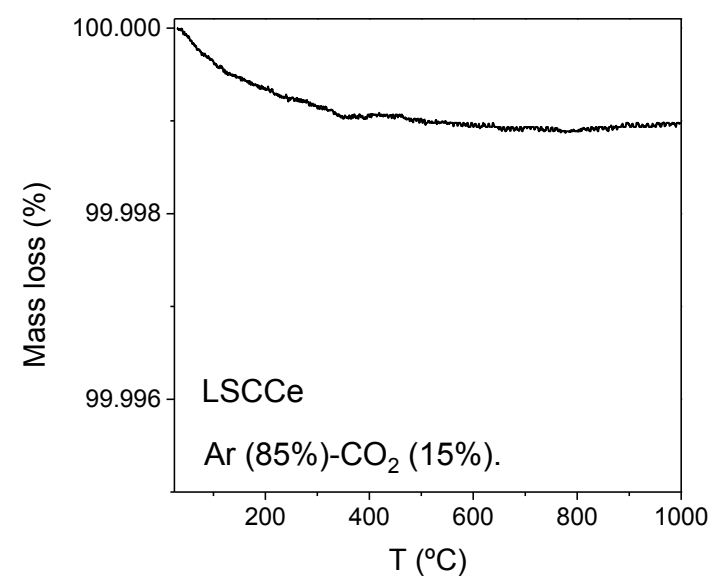

Figure 9.22. TG analysis of the LSCCe in $\mathrm{Ar}(85 \%)-\mathrm{CO}_{2}(15 \%)$. The temperature was increased at a rate of $5^{\circ} \mathrm{C} / \mathrm{min}$.

In order to investigate the transport properties of the material, DC-conductivity measurements were carried out. Figure 9.23a plots the total conductivity of LSCCe in wet $\mathrm{H}_{2}$ and $\mathrm{D}_{2}$ as a function of temperature. For comparison the LSC conductivity in $\mathrm{H}_{2}+\mathrm{H}_{2} \mathrm{O}$ (anode operating conditions) is also depicted. LSCCe exhibits higher total conductivity under reducing atmospheres than LSC. Besides, the conductivity in $\mathrm{H}_{2}+\mathrm{H}_{2} \mathrm{O}$ is higher than in $\mathrm{D}_{2}+\mathrm{D}_{2} \mathrm{O}$, which denotes an isotopic effect typical of protonic transport.

In the studied $\mathrm{pO}_{2}$ range, the conductivity of chromites usually presents a predominant $p$-type electronic character. $[18,19]$ Moreover, ionic $\left(\mathrm{O}^{2-}\right.$ and $\left.\mathrm{H}^{+}\right)$ transport represents an important contribution due to the oxygen vacancies created upon $\mathrm{Cr}$ and Ce reduction. [24, 30, 36, 37] This is confirmed for LSCCe by means of DC-conductivity analysis as a function of $\mathrm{pO}_{2}$. Figure $9.23 \mathrm{~b}$ and $9.23 \mathrm{c}$ display the conductivity power dependency on $p O_{2}\left(\sigma \propto p O_{2}{ }^{n}\right)$ at $700{ }^{\circ} \mathrm{C}$ in dry and wet conditions, respectively. Figure $9.23 \mathrm{~b}$ confirms the conductivity dependence of $\mathrm{pO}_{2}{ }^{1 / 4}$ expected for a predominant $p$-type electronic conductor in 
dry atmospheres, as it is the case of the reported chromites. [20-22] In wet atmospheres there is a decrease of the $\mathrm{pO}_{2}$ slope up to $1 / 6$, which is likely a consequence of the introduction of protonic carriers.

a)

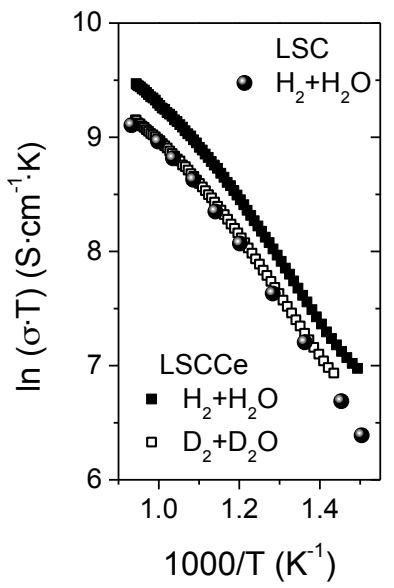

b)

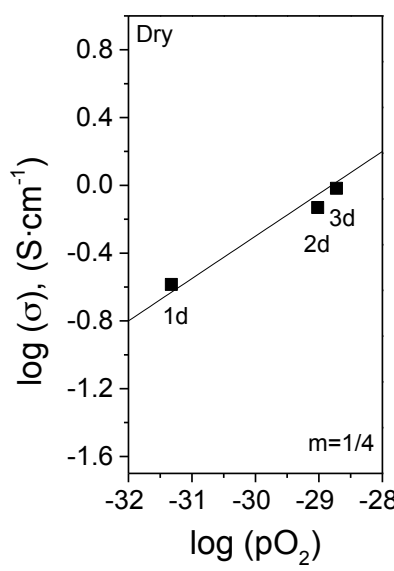

c)

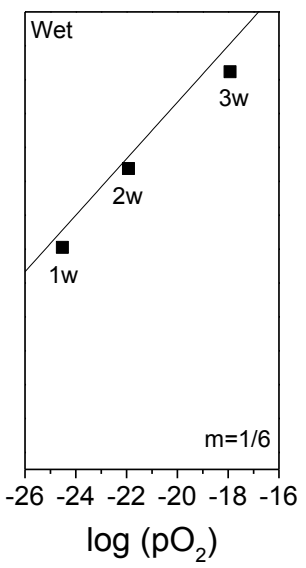

Figure 9.23. a) LSCCe total conductivity in $\mathrm{H}_{2}+\mathrm{H}_{2} \mathrm{O}$ and $\mathrm{D}_{2}+\mathrm{D}_{2} \mathrm{O}$ and LSC in $\mathrm{H}_{2}+\mathrm{H}_{2} \mathrm{O}$, b) $\mathrm{pO}_{2}$ dependence of the total conductivity at $700{ }^{\circ} \mathrm{C}$ of LSCCe in dry atmosphere and c) $\mathrm{pO}_{2}$ dependence of the total conductivity at $700{ }^{\circ} \mathrm{C}$ of LSCCe wet atmospheres.

Summarizing, the protonic conductivity is deduced from the observed isotopic effect and from the smaller $\mathrm{pO}_{2}$ dependency (1/6), and combined with the dry dependency, the mixed ionic-protonic transport of the material under these conditions is pointed out. Due to this mixed electronic-protonic behaviour one may expect the length of triple phase boundary (TPB) within the electrode to increase up to the whole electrode surface, enhancing the anode performance. 


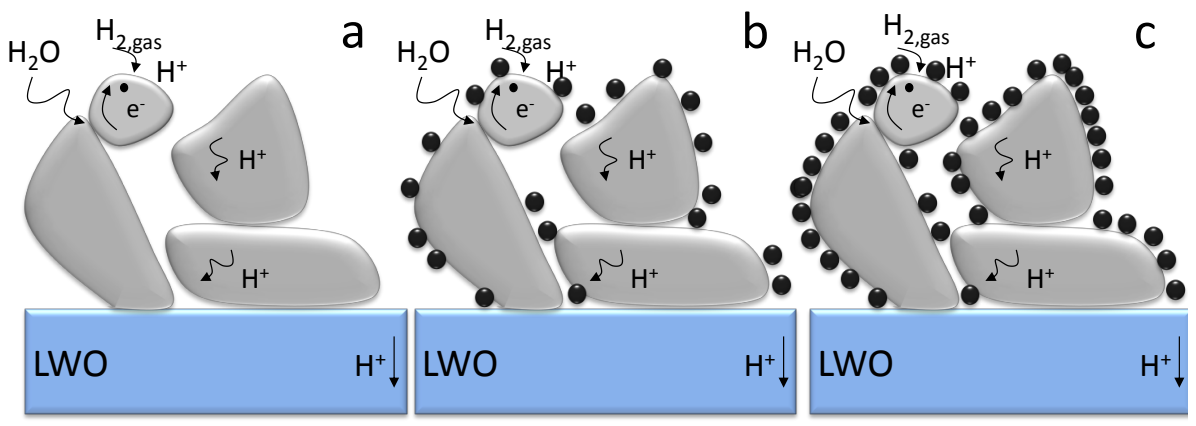

LSCCe: mixed $\mathrm{H}^{+} / \mathrm{e}^{-}$conductor LWO: protonic conductor Ni-nanoparticles
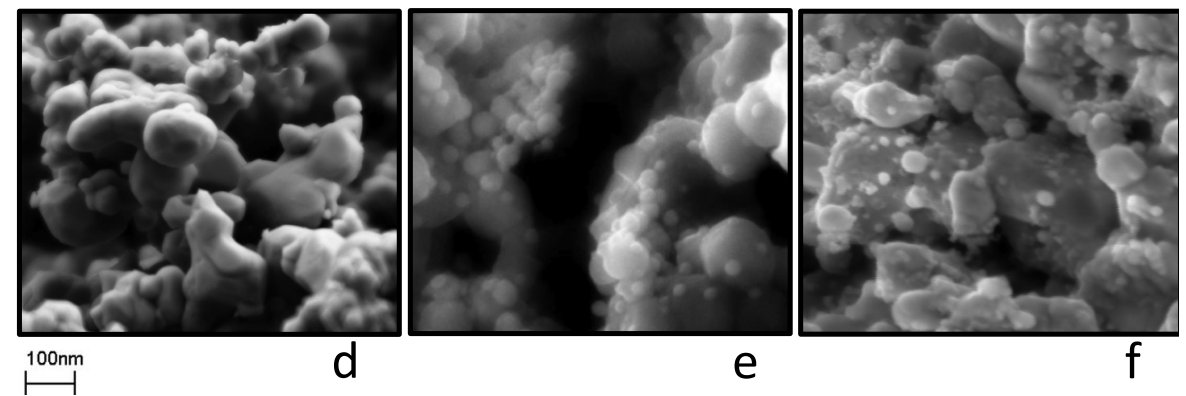

Figure 9.24. Schematic diagrams of the LSCCe a), LSCCe+1Ni b) and LSCCe+5Ni c) anodes and the corresponding SEM images d), e) and f). $1 \mathrm{M}$ and $5 \mathrm{M}$ water solution of $\mathrm{Ni}$ Nitrate (LSCe+1Ni and $\mathrm{LSCe}+5 \mathrm{Ni}$, respectively) were dropped on the surface of the electrodes and allowed to penetrate into the electrodes pores for $30 \mathrm{~min}$. The infiltration step with the $5 \mathrm{M}$ solution was repeated 4 times. Each infiltration step was followed by calcination at $300{ }^{\circ} \mathrm{C}$ to decompose the nitrates.

In order to further improve this anode, different amounts of $\mathrm{Ni}$ were infiltrated into the sintered anode aiming to study the effect of the catalytic coating on the electrochemical activity. The three different LSCCe anodes discussed are shown schematically in Figure 9.24: (a) bare LSCCe anode, (b) LSCCe infiltrated by using $1 \mathrm{M}$ water solution of $\mathrm{Ni}$ nitrate (LSCCe+1Ni) and (c) LSCCe infiltrated by using $5 \mathrm{M}$ water solution of Ni nitrate $(\mathrm{LSCCe}+5 \mathrm{Ni})$. The real SEM images of the cross section anodes after measurements are shown in Figures 9.24d, 9.24e and 9.24f, respectively. The study of these three 
different anodes aims (1) to compare results of the new Ce-doped LSC material with previously studied LSC based anodes and (2) to analyse the effect of the surface catalytic promotion due to the Ni infiltration.

SEM images (Figure 9.24e and f) provide evidence that the quantity of $\mathrm{Ni}$ nanoparticles increases with the $\mathrm{Ni}$ concentration of aqueous solution employed in the infiltration. Further, the average Ni particle size is similar $(\sim 10-20 \mathrm{~nm})$ to that shown for the Ni powder reduced from precursor nitrates in Figure 9.17.

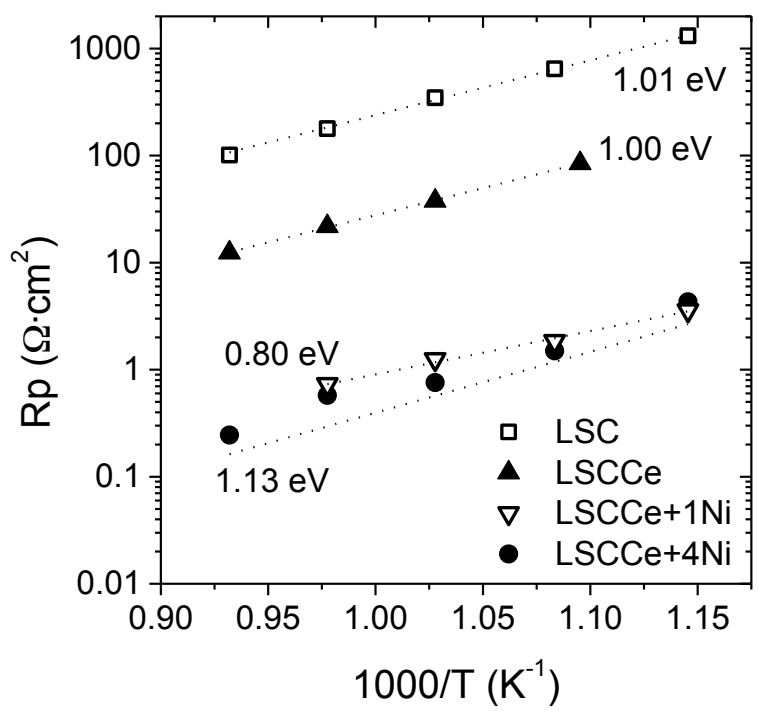

Figure 9.25. $\mathrm{Rp}$ as a function of the inverse temperature of the four anodes $\mathrm{La}_{0.85} \mathrm{Sr}_{0.15} \mathrm{CrO}_{3-\delta}$ (LSC), $\mathrm{La}_{0.75} \mathrm{Ce}_{0.1} \mathrm{Sr}_{0.15} \mathrm{Cr}^{\mathrm{O}} \mathrm{O}_{3-\delta}$ (LSCCe) and LSCCe infiltrated with $1 \mathrm{Ni}(\mathrm{LSCCe}+1 \mathrm{Ni})$ and with $5 \mathrm{Ni}(\mathrm{LSCCe}+5 \mathrm{Ni})$.

Electrochemical properties of the different anodes were analyzed by means of EIS measurements. Polarization resistances (Rp) extracted at different temperatures (Figure 9.25) for all the studied anodes allow comparing (1) the improvement caused by the enhancement of transport properties of the backbone chromite material, and (2) the further improvement of the LSCCe 
anode performance achieved through the infiltration and surface coating with $\mathrm{Ni}$ nanoparticles.

a)

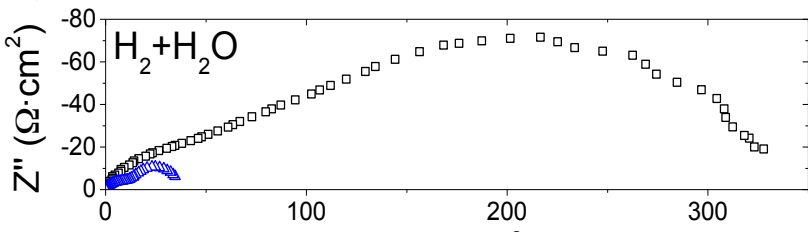

b)
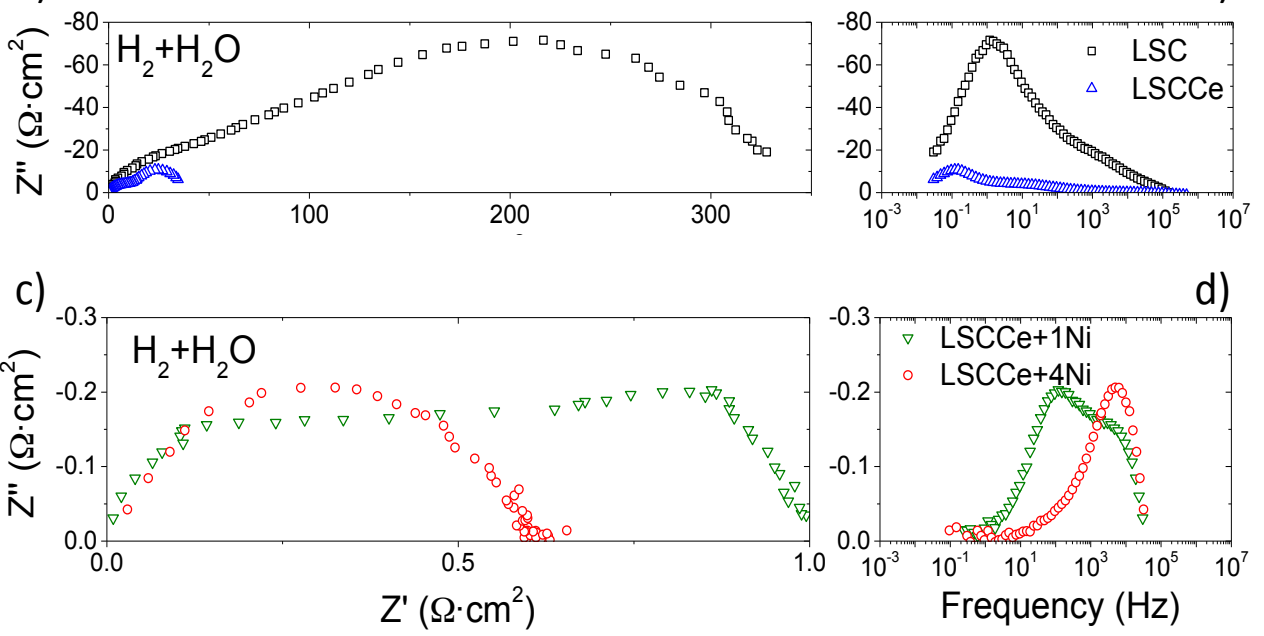

c)

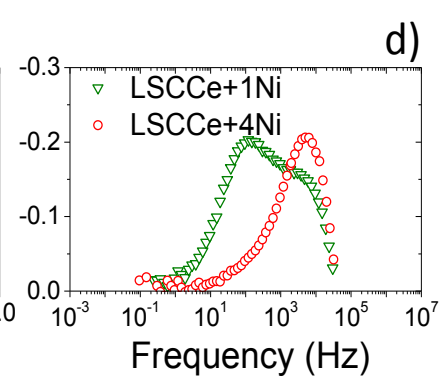

Figure 9.26. EIS spectra at $700{ }^{\circ} \mathrm{C}$ in $\mathrm{H}_{2}$ of LSC and LSCCe anodes, a) Nyquist and b) Bode plots and EIS spectra at $700{ }^{\circ} \mathrm{C}$ in $\mathrm{H}_{2}$ of LSCCe infiltrated with $1 \mathrm{Ni}$ and $5 \mathrm{Ni}, \mathrm{c}$ ) Nyquist and d) Bode plots.

The impedance spectra at $700{ }^{\circ} \mathrm{C}$ in wet $\mathrm{H}_{2}$ are plotted in Figure 9.26a-d. They can be analysed by adjusting to a double serial $\mathrm{R} \| \mathrm{CPE}$ elements (Figure 9.11). With the two arcs interpretation we are able to distinguish different processes occurring in different ranges of frequencies, low, medium and high. 


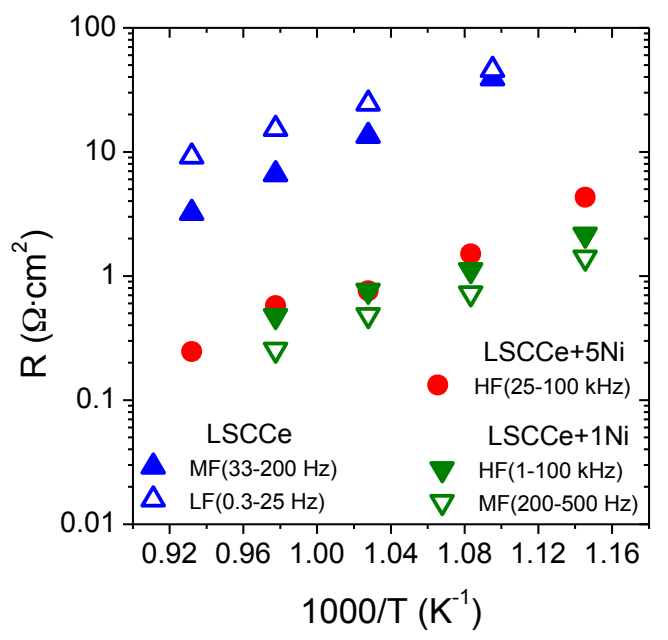

Figure 9.27. Modeled R contributions as a function of the inverse temperature of the LSCCe anode and when infiltrated with different amounts of $\mathrm{Ni}$ : $1 \mathrm{Ni}(\mathrm{LSCCe}+1 \mathrm{Ni})$ and with $5 \mathrm{Ni}(\mathrm{LSCCe}+5 \mathrm{Ni})$.

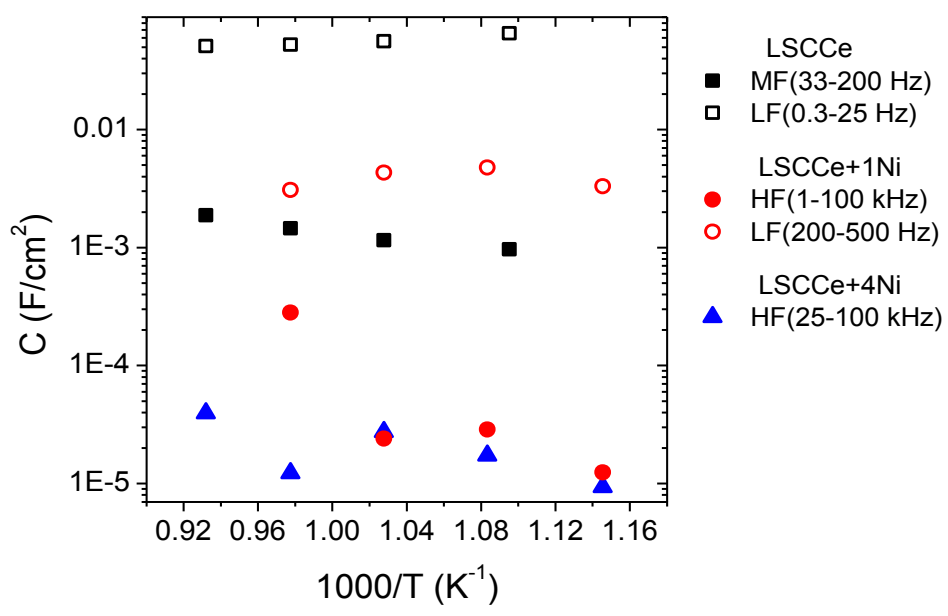

Figure 9.28. Associated capacitances as a function of the inverse temperature of the $\mathrm{LSCCe}, \mathrm{LSCCe}+1 \mathrm{Ni}$ and $\mathrm{LSCCe}+5 \mathrm{Ni}$ anodes.

Figure 9.27 presents the modeled resistances for the pristine LSCCe and the two infiltrated LSCCe electrodes ( $\mathrm{LSCCe}+1 \mathrm{Ni}$ and $\mathrm{LSCCe}+5 \mathrm{Ni}$ ) as a function of temperature $\left(600-800 \quad{ }^{\circ} \mathrm{C}\right)$; the corresponding associated 
capacitances are available in Figure 9.28. The LSCCe anode (Figure 9.24a and d) is principally limited by processes ascribed to low frequencies (LF, $0.3-25 \mathrm{~Hz})$ and this becomes more evident with decreasing temperatures. LSCCe anode shows another important contribution at medium frequencies (MF, 33-200 Hz). Due to the characteristic frequencies and associated capacitances, both contributions can be related to surface processes, being the MF one typically associated to surface exchange coupled with bulk ionic transport in mixed conducting electrodes. [36, 37]

With the introduction of a limited amount of $\mathrm{Ni}$ nanoparticles (LSCCe $+1 \mathrm{Ni}$, Figure $9.24 \mathrm{~b}$ and e) the LF contribution disappears, the MF associated processes shift to higher frequencies (MF, 200-500 Hz) and a new contribution becomes visible at high frequencies (HF, 1-100 kHz). This confirms the catalytic role of Ni particles, which reduce the surface associated resistances and confirm the surface nature of the abovementioned LF and MF processes. Additionally, the decrease of the LF and MF resistances allows the observation of other, less resistive processes, at higher frequencies. In summary, the reduction of the LF process can be directly linked with the improvement in surface kinetics due to the Ni dispersion on the LSCCe grains.

When the amount of infiltrated $\mathrm{Ni}$ is increased (LSCCe $+5 \mathrm{Ni}$, Figure 9.24c and 9.24f) the MF contribution disappears and exclusively the HF contribution $(\mathrm{HF}, 25-100 \mathrm{kHz})$ remains. The HF contribution is ascribed to the transport properties of the LSCCe material or to the LSCCe/LWO interfacial resistances, which remain unchanged upon $\mathrm{Ni}$ infiltration.

As a summary, a complete comparison between the different chromite based anodes on LWO electrolytes studied in this chapter can be seen in Figure 
9.29, where the Rp at $700{ }^{\circ} \mathrm{C}$ is plotted for LSC, LSCCe, LSCN10, LSCN, LSCCe $+1 \mathrm{Ni}$, LSCCe $+5 \mathrm{Ni}$ and LSCN $+5 \mathrm{Ni}$. The graph depicts the gradual decrease of the $\mathrm{Rp}$ from the $347 \Omega \cdot \mathrm{cm}^{2}$ obtained for LSC to 4.85 and $3.55 \Omega \cdot \mathrm{cm}^{2}$ when the LSC material is firstly substituted by 10 and $20 \% \mathrm{Ni}$, respectively and $37.81 \Omega \cdot \mathrm{cm}^{2}$ obtained for $10 \%$ Ce doped LSC. This anode improvement is increased with the Ni infiltrations on both LSCCe and LSCN anodes, being $\mathrm{LSCN}+5 \mathrm{Ni}$ anode the best electrode $\left(\mathrm{Rp}=0.47 \Omega \cdot \mathrm{cm}^{2}\right)$ with slightly better performance than LSCCe $+5 \mathrm{Ni}\left(\mathrm{Rp}=0.76 \Omega \cdot \mathrm{cm}^{2}\right)$.

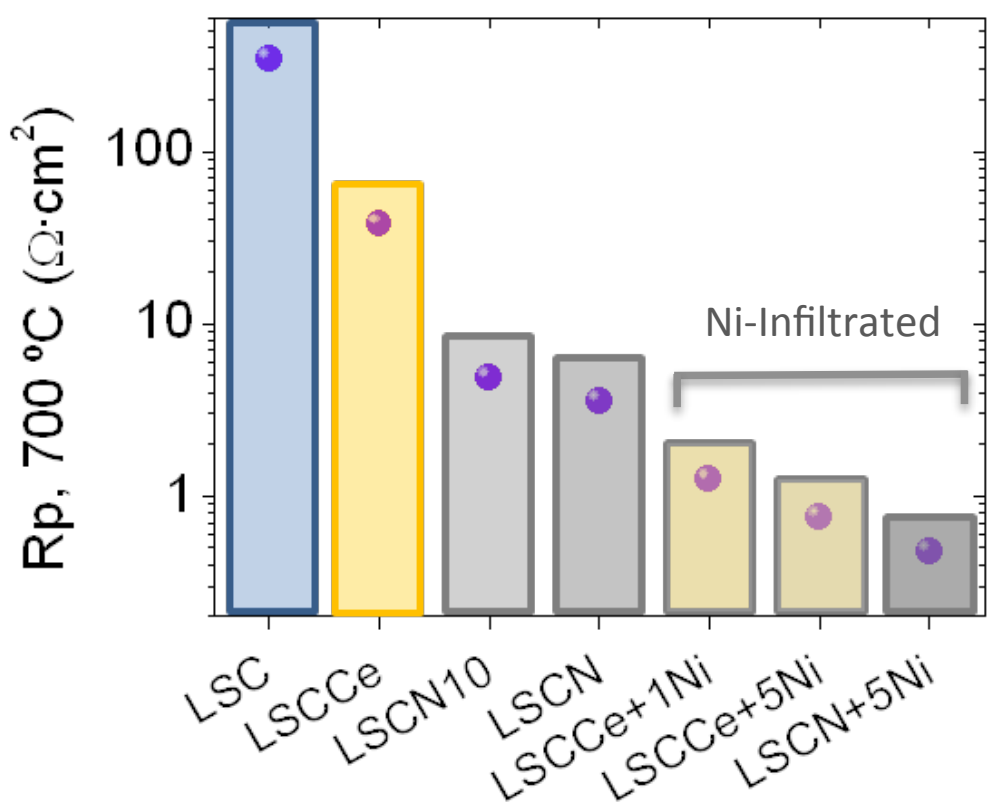

Figure 9.29. $\mathrm{Rp}$ at $700{ }^{\circ} \mathrm{C}$ in $\mathrm{H}_{2}+\mathrm{H}_{2} \mathrm{O}$ of different chromite based anodes: LSC, LSCCe, LSCN10, LSCN, LSCCe+1Ni, LSCCe+5Ni and LSCN+5Ni. 


\subsection{Conclusions}

In conclusion, this chapter presents the large potential of chromite-based anodes for LWO-based PC-SOFC. Aside from the chemical compatibility of LSC, LSCN10, LSCN and LSCCe with LWO, the protonic conductivity of reduced anodes combined with sufficient $p$-type electronic conductivity is illustrated. These properties are requisites for protonic anodes and make it possible to avoid the blending with other protonic or electronic materials, as it typically happens with protonic composite cathodes for PC-SOFC and Ni-based cermet anodes. Furthermore, the mixed conductivity allows enlarging the surface area, i.e., TPB, available for the $\mathrm{H}_{2}$ oxidation reactions and proton incorporation.

LSCN10 anode on LWO, in symmetrical cell configuration, demonstrates that under typical anode reducing conditions, structural $\mathrm{Ni}$ is segregated on the surface. Thus, the Ni nanoparticles become catalytically active for the $\mathrm{H}_{2}$ oxidation reaction improving the anode performance regarding to pure LSC, i.e., $2.68 \Omega \cdot \mathrm{cm}^{2}$ at $750{ }^{\circ} \mathrm{C}$ being principally limited below $850{ }^{\circ} \mathrm{C}$ by $\mathrm{LF}$ processes, ascribed to surface reactions. The surface properties of the chromite backbone structure (mixed $\mathrm{H}^{+} / \mathrm{e}^{-}$conducting) can be boosted either by higher Ni or Ce doping and specially by Ni infiltration. This results in the coating of the electrode surface with $\mathrm{Ni}$ nanoparticles and consequently in a strong improvement of the anode performance. Specifically, the infiltrated anode with the highest $\mathrm{Ni}$ loading $(\mathrm{LSCN}+5 \mathrm{~N})$ only presents $\mathrm{HF}$ associated resistance and Rp as low as $0.26 \Omega \cdot \mathrm{cm}^{2}$ at $750{ }^{\circ} \mathrm{C}$ in wet $\mathrm{H}_{2}$. 


\subsection{References}

[1] S. Tao, J.T.S. Irvine, A redox-stable efficient anode for solid-oxide fuel cells, Nat Mater, 2 (2003) 320-323.

[2] J. Sfeir, P.A. Buffat, P. Möckli, N. Xanthopoulos, R. Vasquez, H. Joerg Mathieu, J. Van herle, K. Ravindranathan Thampi, Lanthanum Chromite Based Catalysts for Oxidation of Methane Directly on SOFC Anodes, Journal of Catalysis, 202 (2001) 229244.

[3] E. Lay, G. Gauthier, L. Dessemond, Preliminary studies of the new Ce-doped La/Sr chromo-manganite series as potential SOFC anode or SOEC cathode materials, Solid State Ionics, 189 (2011) 91-99.

[4] H. Iwahara, Technological challenges in the application of proton conducting ceramics, Solid State Ionics, 77 (1995) 289-298.

[5] E. Fabbri, L. Bi, D. Pergolesi, E. Traversa, High-performance composite cathodes with tailored mixed conductivity for intermediate temperature solid oxide fuel cells using proton conducting electrolytes, Energy \& Environmental Science, 4 (2011) 49844993.

[6] A. Magraso, J.M. Polfus, C. Frontera, J. Canales-Vazquez, L.-E. Kalland, C.H. Hervoches, S. Erdal, R. Hancke, M.S. Islam, T. Norby, R. Haugsrud, Complete structural model for lanthanum tungstate: a chemically stable high temperature proton conductor by means of intrinsic defects, Journal of Materials Chemistry, 22 (2012) $1762-1764$.

[7] R. Haugsrud, Defects and transport properties in $\mathrm{Ln}_{6} \mathrm{WO}_{12}$ ( $\left.\mathrm{Ln}=\mathrm{La}, \mathrm{Nd}, \mathrm{Gd}, \mathrm{Er}\right)$, Solid State Ionics, 178 (2007) 555-560.

[8] R. Haugsrud, C. Kjølseth, Effects of protons and acceptor substitution on the electrical conductivity of $\mathrm{La}_{6} \mathrm{WO}_{12}$, Journal of Physics and Chemistry of Solids, 69 (2008) 1758-1765.

[9] C. Solis, S. Escolastico, R. Haugsrud, J.M. Serra, $\mathrm{La}_{5.5} \mathrm{WO}_{12-\delta}$ Characterization of Transport Properties under Oxidizing Conditions: A Conductivity Relaxation Study, The Journal of Physical Chemistry C, 115 (2011) 11124-11131.

[10] S.Escolástico, J. M. Serra, M. Ivanova, W. Meulenberg, J. Seeger, C. Solís Hydrogen permeation through $\mathrm{La}_{5.5} \mathrm{WO}_{12}$ membranes, in: 10th CMCee International Symposium on ceramic materials and components for energy and environmental applications, Dresden (Germany), 2012. 
[11] C. Solis, L. Navarrete, S. Roitsch, J.M. Serra, Electrochemical properties of composite fuel cell cathodes for $\mathrm{La}_{5.5} \mathrm{WO}_{12-\delta}$ proton conducting electrolytes, Journal of Materials Chemistry, 22 (2012) 16051-16059.

[12] E. Quarez, K.V. Kravchyk, O. Joubert, Compatibility of proton conducting $\mathrm{La}_{6} \mathrm{WO}_{12}$ electrolyte with standard cathode materials, Solid State Ionics, 216 (2012) 1924.

[13] F. Meschke, F.J. Dias, F. Tietz, Porous $\mathrm{Ni} / \mathrm{TiO}_{2}$ substrates for planar solid oxide fuel cell applications, Journal of Materials Science, 36 (2001) 5719-5728.

[14] A.M. Hussain, J.V.T. Høgh, T. Jacobsen, N. Bonanos, Nickel-ceria infiltrated Nbdoped $\mathrm{SrTiO}_{3}$ for low temperature SOFC anodes and analysis on gas diffusion impedance, International Journal of Hydrogen Energy, 37 (2012) 4309-4318.

[15] J.M. Serra, W.A. Meulenberg, Thin-Film Proton $\mathrm{BaZr}_{0.85} \mathrm{Y}_{0.15} \mathrm{O}_{3}$ Conducting Electrolytes: Toward an Intermediate-Temperature Solid Oxide Fuel Cell Alternative, Journal of the American Ceramic Society, 90 (2007) 2082-2089.

[16] S. Ricote, N. Bonanos, Enhanced sintering and conductivity study of cobalt or nickel doped solid solution of barium cerate and zirconate, Solid State Ionics, 181 (2010) 694-700.

[17] J. Seeger, M. Ivanova, W. Meulenberg, D. Stöver, S. Escolástico, J.M. Serra, S. Roitsch, Development of mixed proton-electron conducting materials based on $\mathrm{La}_{6} \mathrm{WO}_{12}$ for hydrogen separation, in: ECerS XII 12th conference of the European Ceramic Society, Stockholm (Sweden), 2011.

[18] A.L. Sauvet, J.T.S. Irvine, Catalytic activity for steam methane reforming and physical characterisation of $\mathrm{La}_{1-\mathrm{x}} \mathrm{Sr}_{\mathrm{x}} \mathrm{Cr}_{1-\mathrm{y}} \mathrm{Ni}_{\mathrm{y}} \mathrm{O}_{3-\delta}$, Solid State Ionics, 167 (2004) 1-8.

[19] W.Z. Zhu, S.C. Deevi, Development of interconnect materials for solid oxide fuel cells, Materials Science and Engineering: A, 348 (2003) 227-243.

[20] J. Sfeir, $\mathrm{LaCrO}_{3}$-based anodes: stability considerations, Journal of Power Sources, 118 (2003) 276-285.

[21] T. Caillot, G. Gauthier, P. Delichère, C. Cayron, F.J. Cadete Santos Aires, Evidence of anti-coking behavior of $\mathrm{La}_{0.8} \mathrm{Sr}_{0.2} \mathrm{Cr}_{0.98} \mathrm{Ru}_{0.02} \mathrm{O}_{3}$ as potential anode material for Solid Oxide Fuel Cells directly fed under methane, Journal of Catalysis, 290 (2012) 158-164. 
[22] A. Atkinson, S. Barnett, R.J. Gorte, J.T.S. Irvine, A.J. McEvoy, M. Mogensen, S.C. Singhal, J. Vohs, Advanced anodes for high-temperature fuel cells, Nat Mater, 3 (2004) $17-27$.

[23] S. Julsrud, B. Vigeland, in: N.H. ASA (Ed.), Norway, 2001.

[24] V.B. Vert, F.V. Melo, L. Navarrete, J.M. Serra, Redox stability and electrochemical study of nickel doped chromites as anodes for $\mathrm{H}_{2} / \mathrm{CH}_{4}$-fueled solid oxide fuel cells, Applied Catalysis B: Environmental, 115-116 (2012) 346-356.

[25] J. Sfeir, P.A. Buffat, P. Möckli, N. Xanthopoulos, R. Vasquez, H. Joerg Mathieu, J. Van herle, K. Ravindranathan Thampi, Lanthanum Chromite Based Catalysts for Oxidation of Methane Directly on SOFC Anodes, Journal of Catalysis, 202 (2001) 229244.

[26] W. Kobsiriphat, B.D. Madsen, Y. Wang, M. Shah, L.D. Marks, S.A. Barnett, Nickel- and Ruthenium-Doped Lanthanum Chromite Anodes: Effects of Nanoscale Metal Precipitation on Solid Oxide Fuel Cell Performance, Journal of The Electrochemical Society, 157 (2010) B279-B284.

[27] V.B. Vert, Electrodos avanzados para pilas de combustible de óxido sólido (SOFCs), in: Instituto de Tecnología Química (UPV-CSIC)-Departamento de Química, Universidad Politécnica de Valencia, Valencia, 2011, pp. 314.

[28] K.E.J. Eurenius, E. Ahlberg, C.S. Knee, Proton conductivity in $\mathrm{Sm}_{2} \mathrm{Sn}_{2} \mathrm{O}_{7}$ pyrochlores, Solid State Ionics, 181 (2010) 1577-1585.

[29] J.R. Macdonald, Impedance Spectroscopy: Emphasizing Solid Materials and Systems, New York, 1987.

[30] X. Dong, S. Ma, K. Huang, F. Chen, $\mathrm{La}_{0.9-\mathrm{x}} \mathrm{Ca}_{\mathrm{x}} \mathrm{Ce}_{0.1} \mathrm{CrO}_{3-\delta}$ as potential anode materials for solid oxide fuel cells, International Journal of Hydrogen Energy, 37 (2012) 10866-10873.

[31] A.L. Sauvet, J. Fouletier, Electrochemical properties of a new type of anode material $\mathrm{La}_{1-\mathrm{x}} \mathrm{Sr}_{\mathrm{x}} \mathrm{Cr}_{1-\mathrm{y}} \mathrm{Ru}_{\mathrm{y}} \mathrm{O}_{3-\delta}$ for $\mathrm{SOFC}$ under hydrogen and methane at intermediate temperatures, Electrochimica Acta, 47 (2001) 987-995.

[32] M.C. Verbraeken, B.A. Boukamp, D.H.A. Blank, P. Holtappels, U. Vogt, Electrochemical characterisation of supporting SOFC anodes, in: J.M.E. S.C. Singal (Ed.) Proc. 9th Intern. Symp. On Solid Oxide Fuel Cells (SOFC IX), The Electrochemical Society In., 2005, pp. 1218.

[33] S.P. Jiang, S.P.S. Badwal, An electrode kinetics study of $\mathrm{H}_{2}$ oxidation on $\mathrm{Ni} / \mathrm{Y}_{2} \mathrm{O}_{3^{-}}$ $\mathrm{ZrO}_{2}$ cermet electrode of the solid oxide fuel cell, Solid State Ionics, 123 (1999) 209224. 
[34] J.M. Serra, V.B. Vert, Compositional Improvement of $\mathrm{Ln}_{0.435} \mathrm{Ba}_{0.145} \mathrm{Sr}_{0.4} \mathrm{Fe}_{0.8} \mathrm{Co}_{0 .} 2 \mathrm{O}_{3-\delta}$ IT-SOFC Cathodes Performance by Multiple Lanthanide Substitution, Journal of The Electrochemical Society, 157 (2010) B1349-B1357.

[35] B.I. Lee, R.K. Gupta, C.M. Whang, Effects of solvent and chelating agent on synthesis of solid oxide fuel cell perovskite, $\mathrm{La}_{0.8} \mathrm{Sr}_{0.2} \mathrm{CrO}_{3-\delta}$, Materials Research Bulletin, 43 (2008) 207-221.

[36] S.B. Adler, J.A. Lane, B.C.H. Steele, Electrode Kinetics of Porous MixedConducting Oxygen Electrodes, Journal of The Electrochemical Society, 143 (1996) 3554-3564.

[37] E. Barsoukov, J.R.I. Macdonald, Impedance Spectroscopy Theory, Experiment, and Applications John Wiley \& Sons, 2005. 



\section{Chapter 10. Conclusions and remarks}

With the aim of finding new MIEC materials stable in the harsh working conditions found in the applications as ion transport membranes, SOFC and PC-SOFC electrodes, four classes of materials have been studied. The results presented in this thesis led to the following conclusions, which are divided by blocks.

\section{Fluorite structured $\mathrm{Ce}_{0.9} \mathrm{Ln}_{0.1} \mathrm{O}_{2-\delta}$ and $\mathrm{Ce}_{0.9} \mathrm{Ln}_{0.1} \mathrm{O}_{2-\delta}+2 \mathrm{~mol} \% \mathrm{Co}$}

- $\quad 10 \%$ of trivalent lanthanides are fully soluble in the fluorite lattice of ceria. Eu and $\mathrm{Yb}$ maintain their trivalent state in oxidising conditions. The trivalent lanthanides produce a linear distortion of the cell and the introduction of a fixed number of extrinsic oxygen vacancies.

- $\quad \operatorname{Pr}$ and $\mathrm{Tb}$ dopants are in a mixed tri- and tetravalent state. The ratio $\mathrm{Ln}^{3+} / \mathrm{Ln}^{4+}$ depends on temperature and oxygen partial pressure.

- The addition of $2 \mathrm{~mol} \%$ of $\mathrm{CoO}_{\mathrm{x}}$ succeed as sintering aid for all the doped cerias. It helps the grain growth and causes the densification improvement

- Raman spectroscopy results demonstrate that the introduction of lanthanides dopants produces a disorder due to the creation of oxygen vacancies. This disorder increases with the cell parameter.

- All the lanthanides improve the total conductivity with respect to pure ceria. Except of $\mathrm{Tb}$ and $\mathrm{Pr}$ doped ceria, all the compounds are predominantly ionic conductors, as deduced from the conductivity behaviour, which is independent on $p \mathrm{O}_{2}$.

- Conductivity of $\mathrm{Tb}$ and $\mathrm{Pr}$ doped ceria show a dependency on $\mathrm{pO}_{2}^{-1 / 6}$ at the most oxidizing conditions due to the formation of oxygen vacancies as $\mathrm{pO}_{2}$ reduces. The conductivity is still mainly ionic. 
- The addition of Co particularly improves the conductivity of Tb and Pr doped ceria due to the effect in the reducibility of the dopant (which occurs at low temperatures) and to the electronic conductivity promotion by either the creation of electronic paths through the grain boundary or the dissolution of part of the Co in the structure.

- Differences in conductivity of materials with the same concentration of ionic charge carriers are due to differences in the mobility of ions over the intrinsic oxygen vacancies created in the fluorite lattice.

- The highest ionic conductivity is obtained for the less distorted cells, i.e., $\mathrm{Gd}$ and $\mathrm{Nd}$, which represent an optimum balance between Coulomb interactions, steric effects and oxygen vacancy distribution.

- The lowest $E_{a}$ was found for materials with long cell parameters, up to a maximum.

\section{Co addition on $\mathrm{Ce}_{0.9} \mathrm{Pr}_{0.1} \mathrm{O}_{2-\delta}$}

- The $\mathrm{CoO}_{\mathrm{x}}$ causes the grain size enlargement by accelerating the densification rate and grain growth.

- Co does not dissolve in the ceria lattice.

- Co has a catalytic effect on the reduction of ceria, which shifts the bulk ceria reduction temperature in $\mathrm{H}_{2}$.

- The addition of Co improves the total conductivity of Pr- doped ceria, attributed to electronic pathways along the grain boundaries.

- The transport properties depend on the microstructure of the specimen. Higher sintering temperature produces larger grain size and the agglomeration of $\mathrm{CoO}_{x}$ in microdomains.

- Low temperature sintering conditions produces small particle size and the homogeneous spreading of the Co additive along the grain boundaries.

- The highest conductivity has been obtained for samples sintered at $1000{ }^{\circ} \mathrm{C}$, which are a trade off between grain size, Co distribution and densification. 


\section{Materials based on $\mathrm{Ce}_{1-\mathrm{x}} \mathrm{Tb}_{\mathrm{x}} \mathrm{O}_{2-\delta}$ and $\mathrm{Ce}_{1-\mathrm{x}} \mathrm{Tb}_{\mathrm{x}} \mathrm{O}_{2-\delta}+2 \mathrm{~mol} \% \mathrm{Co}$}

- The cell parameter depends on the concentration of dopant following the Vegard's rule.

- A constant ratio between $\mathrm{Tb}^{4+} / \mathrm{Tb}^{3+}$ is observed for all the concentrations at room temperature. The Co addition affects the oxidation state of $\mathrm{Tb}$ increasing the ratio $\mathrm{Tb}^{4+} / \mathrm{Tb}^{3+}$.

- Part of the $\mathrm{CoO}_{x}$ dissolves in the fluorite lattice. The sintering at very high temperatures may cause the segregation in the grain boundaries.

- The addition of Co increases the material reducibility, i.e., it lowers the reduction temperature.

- The addition of Co improves the densification of the specimens

- $\mathrm{Ce}_{1-\mathrm{x}} \mathrm{Tb}_{\mathrm{x}} \mathrm{O}_{2-\delta}$ shows predominantly ionic or mixed ionic-electronic conductivity depending on the $\mathrm{Tb}$ concentration, Co addition and temperature range.

- Tb doping introduces new electronic levels in the ceria band gap, which allow the small polaron hopping between $\mathrm{Tb}^{4+} / \mathrm{Tb}^{3+}$. Low $\mathrm{Tb}$ content $(10 \%)$ seem not to be enough for the hopping, while $50 \%$ Tb-doped ceria shows $p$-type electronic conductivity.

- High doping levels and low oxygen partial pressures may induce the oxygen vacancy association with the cations.

- The addition of Co further creates oxygen vacancies, so that improves the conductivity. The Co not incorporated in the structure may create electronic pathways along the grain boundaries.

- The characterization by XPS reveals the presence of $\mathrm{Tb}^{4+}, \mathrm{Tb}^{3+}$ and $\mathrm{Co}^{2+}$ in the surface and thus oxygen vacancies. $\mathrm{Ce}^{3+}$ is not observed as expected for oxidizing conditions (air).

- PIE experiments demonstrate the importance of oxygen vacancies and electrons on the surface exchange rate, $\Re_{0}$. It is found to increase profoundly with increasing $\mathrm{Tb}$ concentration. 
- $\Re_{\mathrm{o}}$ is limited by the dissociative adsorption step rather than the oxygen incorporation in the ceria lattice, which is two orders of magnitude higher.

- CT10 does not show any oxygen permeation flux, which is attributed to the mainly ionic conductivity since the low doping level still not allows the electron hopping.

- CT10Co shows an oxygen permeation flux up to $0.02 \mathrm{ml} \mathrm{cm}^{-2} \mathrm{~min}^{-1}$ at $1000{ }^{\circ} \mathrm{C}$. This is a consequence of the electronic pathways introduced by the Co with regard to CT10.

- CT20Co increases the oxygen permeation up to $0.08 \mathrm{ml} \mathrm{cm}^{-2} \mathrm{~min}^{-1}$ at $1000{ }^{\circ} \mathrm{C}$. The enhancement is ascribed to the higher oxygen vacancy concentration as well as the higher density of new electronic levels in the ceria bandgap and percolation pathways.

- CT20, CT50 and CT50Co membranes are not tested due to the impossibility to obtain dense specimens by pressing and firing.

- CTx and CTxCo families are stable in $\mathrm{CO}_{2}$ containing atmospheres, contrarily to the most widely used membrane material, the BSCF.

\section{Materials based on $\mathrm{Ce}_{0.8} \mathrm{Gd}_{0.1} \mathrm{X}_{0.1} \mathrm{O}_{2-\delta}(\mathrm{X}=\mathrm{Gd}, \mathrm{Cr}, \mathrm{Mg}, \mathrm{Bi}$ and $\mathrm{Ce})$}

- Gd doped ceria has high ionic conductivity. The co-doping with other metals aims to promote the electronic conductivity. $\mathrm{Gd}, \mathrm{Cr}, \mathrm{Mg}$, Bi and $\mathrm{Ce}$ as $\mathrm{X}$ dopant have been incorporated in the ceria structure.

- Electrical conductivity of LSM-Ce ${ }_{0.8} \mathrm{Gd}_{0.1} \mathrm{X}_{0.1} \mathrm{O}_{2-\delta}(\mathrm{X}=\mathrm{Gd}, \mathrm{Cr}, \mathrm{Mg}, \mathrm{Bi}$ and Ce) composite has been proved to be sufficient for SOFC cathode applications. Doped ceria contributes the ionic conduction improving the triple phase boundary area of a LSM cathode.

- These materials have been tested as LSM-CGXO composite cathodes in symmetrical cells. The polarization resistance decreases with increasing the conductivity of the ceria based material.

- Among the dopants, $\mathrm{Mg}$ seems to boost both the oxygen ion transport and the surface process for oxygen reduction. 
- However, the performance of these new co-doped ceria composite cathodes is not notably better than $\mathrm{Ce}_{0.8} \mathrm{Gd}_{0.2} \mathrm{O}_{1.9}$ material.

- EIS data may be fitted to an equivalent circuit comprising three ZARC elements in series with an inductance and a pure resistance.

- The use of $\mathrm{Ce}_{0.8} \mathrm{Gd}_{0.1} \mathrm{Mg}_{0.1} \mathrm{O}_{2-\delta}$ instead of $\mathrm{Ce}_{0.8} \mathrm{Gd}_{0.2} \mathrm{O}_{1.95}$ in the composite may be economically viable due to the lower price of the raw material.

\section{Materials based on doped-CaTiO ${ }_{3}$ perovskites}

- The perovskite stability predictions made by Goldschmidt and octahedral factor are not experimentally accomplished for all the doped $\mathrm{CaTiO}_{3}$.

- The stable perovskites have been electrochemically tested by DC-conductivity. All the dopants improve the total conductivity with respect to pure $\mathrm{CaTiO}_{3}$.

- The best transport properties are observed for Fe and Fe-Mg doping in the Bsite (named CTF and CTFM, respectively).

- DC conductivity up to $1000{ }^{\circ} \mathrm{C}$ demonstrated an increase of conductivity of CTFM over that of CTF upon $800{ }^{\circ} \mathrm{C}$, which has been ascribed to the mobility of associated oxygen vacancies released at high temperature. Below $800{ }^{\circ} \mathrm{C}$, the decrease in conductivity of CTFM is attributed to the oxygen vacancy association.

- CTF and CTFM present mainly ionic conductivity at high temperatures, while $p$-type electronic conductivity is ascertained in the low temperature range.

- XPS measurements demonstrate the presence of oxygen vacancies in the surface of CTFM, since $\mathrm{Ti}, \mathrm{Fe}$ and $\mathrm{Mg}$ in tri- and divalent oxidation states of are found.

- PIE experiments indicate that CTF and CTFM show a similar oxygen exchange rate, $\Re_{\mathrm{o}}$ up to $700{ }^{\circ} \mathrm{C}$. At this temperature, the transport properties of both materials are similar, so that the possible effect of the oxygen vacancy increase in CTFM is not quantifiable. 
- CTF and CTFM present a comparatively high $\Re_{\mathrm{o}}$ with regard to YSZ and CGO10, known as solid ionic conductors, and LSC20 perovskite. $\Re_{\mathrm{o}}$ is comparable to that of CGO20 and CT50. On the other hand, CTF and CTFM $\Re_{0}$ are lower than for BSCF.

- Using a catalytic layer over the membrane surface may allow overcoming the limits imposed by the oxygen surface exchange rate.

- CTFM membrane with a Pt catalytic layer delivers an oxygen permeation flux one order of magnitude higher than referenced CTF membranes, which is attributed to the temperature dependent increase of oxygen vacancies concentration and mobility.

- The CTFM membrane stability is tested under $\mathrm{CO}_{2}$ containing sweep gas, where it demonstrates to be stable.

- CTFM is stable to harsh environment conditions involving $1000 \mathrm{ppm}$ COS, 100 ppm $\mathrm{HCN}, 4 \% \mathrm{CO}_{2}, 46 \% \mathrm{CO}, 46 \% \mathrm{H}_{2}$ and $4 \% \mathrm{H}_{2} \mathrm{O}$ at 35 bar and up to $400{ }^{\circ} \mathrm{C}$.

- CTFM is not stable against a two weeks treatment on a continuous stream of $\mathrm{CO}_{2}$ containing $5 \% \mathrm{O}_{2}, 1 \%$ of $\mathrm{SO}_{2}$ and $2.5 \%$ of $\mathrm{H}_{2} \mathrm{O}$ at $800{ }^{\circ} \mathrm{C}$.

\section{Composite materials based on doped-ceria/ spinels}

- One-pot synthesis of fluorite/spinel composites has been demonstrated to be the most suitable method to obtain pure phases. One pot method produces narrow grain size distribution and homogeneous distribution of grains and phases.

- 60/40 spinel to fluorite phase volume ratio is the optimum for the creation of percolative ionic and electronic paths from side to side of the membrane.

- From the tested spinels, only $\mathrm{Fe}_{2} \mathrm{NiO}_{4}, \mathrm{Fe}_{2} \mathrm{CrO}_{4}$ and $\mathrm{Mn}_{1.5} \mathrm{Cu}_{1.5} \mathrm{O}_{4}$ are stable at high temperatures and to the contact with the fluorite. However, $\mathrm{Fe}_{2} \mathrm{CrO}_{4}$ and $\mathrm{Mn}_{1.5} \mathrm{Cu}_{1.5} \mathrm{O}_{4}$ are not able to deliver dense specimens.

- $\mathrm{Fe}_{2} \mathrm{NiO}_{4}(\mathrm{FNO})$ is the spinel of choice as it is capable to form dense composites without reacting with some of the fluorites. FNO only reacts with the $\mathrm{Ce}_{0.8} \mathrm{Gd}_{0.1} \mathrm{Pr}_{0.1} \mathrm{O}_{2-\delta}$. 
- Stable composites are achieved by mixing FNO and $\mathrm{Ce}_{0.8} \mathrm{Gd}_{0.2} \mathrm{O}_{1.9}$ (CGO), $\mathrm{Ce}_{0.8} \mathrm{~Tb}_{0.2} \mathrm{O}_{2-\delta}$ (CTO) and $\mathrm{Ce}_{0.8} \mathrm{~Tb}_{0.2} \mathrm{O}_{2-\delta}+2 \mathrm{~mol} \% \mathrm{Co}$ (CTOCo).

- At high temperatures, the FNO-CGO composite shows the highest conductivity, ascribed to the higher extrinsic ionic conductivity of CGO.

- At temperatures below $640{ }^{\circ} \mathrm{C}$, the conductivity of FNO-CTO overwhelms that of FNO-CGO, due to the improved electronic conductivity of the fluorite phase.

- The lowest conductivity is observed for FNO-CTOCo.

- Contrarily to single phase FNO, dense specimens are achieved by the combination with the fluorites, which are able to act also as sintering aid.

- A deeper study on FNO-CTO composite microstructure did not show evidence of impurities or reaction between phases up to the limits of the XRD and SEM devices.

- SEM analysis of FNO-CTO reveals a narrow grain size distribution and homogeneous allocation of grains. The existence of percolative paths from side to side of the dual-phase membranes necessary for the oxygen permeation is ascertained as well as a proper packing and bounding among the different grains in the membrane.

- The transport properties of FNO-CTO showed that the ambipolar transport involves (i) hopping of charge carriers, i.e., hopping of localized electrons between multivalent cations located in the octahedral $\mathrm{Fe}$ sublattice and (ii) the ionic transport in though the doped ceria lattice. Additionally, CTO presents significant $p$-type electronic conductivity although the ionic conductivity prevails.

- The oxygen permeation of FNO-CTO membrane has been measured and compared with the flux obtained by a FNO-CGO reference composite membrane, both using $\mathrm{CO}_{2}$ as sweeping gas. Both membranes are stable against $\mathrm{CO}_{2}$, but the oxygen permeation of FNO-CTO is twice the flux obtained by FNO-CGO.

- Higher $\mathrm{pO}_{2}$ gradients across the membranes lead to substantial increases in the oxygen flux. 
- FNO-CTO composite is stable against an environment containing wet $\mathrm{SO}_{2}$ and $\mathrm{CO}_{2}$, at $800{ }^{\circ} \mathrm{C}$ during 14 days. Contrarily, FNO-CGO and LSCF are not stable to the same treatment.

\section{Mixed protonic-electronic conductors based on lanthanum chromites}

- $\mathrm{La}_{0.87} \mathrm{Sr}_{0.13} \mathrm{CrO}_{3}$ (LSC) has been doped both in A and B site in order to increase the mixed protonic-electronic conductivity.

- LSC, $\mathrm{La}_{0.85} \mathrm{Sr}_{0.15} \mathrm{Cr}_{0.9} \mathrm{Ni}_{0.1} \mathrm{O}_{3}$ (LSCN10) and $\mathrm{La}_{0.85} \mathrm{Sr}_{0.15} \mathrm{Cr}_{0.8} \mathrm{Ni}_{0.2} \mathrm{O}_{3}$ (LSCN20) show $\mathrm{SrCrO}_{3}$ impurities that disappear in reducing conditions.

- Several dopants succeeded to form a pure perovskite at low temperatures, but they were not stable at the high temperatures required to obtain dense specimens, i.e., $\mathrm{La}_{0.85} \mathrm{Sr}_{0.15} \mathrm{Cr}_{0.9} \mathrm{Fe}_{0.1} \mathrm{O}_{3}$ (LSCrF), $\mathrm{La}_{0.85} \mathrm{Sr}_{0.15} \mathrm{Cr}_{0.9} \mathrm{Al}_{0.1} \mathrm{O}_{3}$ (LSCA), $\mathrm{La}_{0.85} \mathrm{Sr}_{0.15} \mathrm{Cr}_{0.9} \mathrm{Ti}_{0.1} \mathrm{O}_{3}(\mathrm{LSCT})$ and $\mathrm{La}_{0.85} \mathrm{Sr}_{0.15} \mathrm{Cr}_{0.9} \mathrm{Y}_{0.1} \mathrm{O}_{3}$ (LSCY).

- Other dopants only released a pure perovskite structure after sintering at high temperatures or in reducing conditions, i.e., $\mathrm{La}_{0.75} \mathrm{Sr}_{0.15} \mathrm{Ce}_{0.1} \mathrm{CrO}_{3}$ (LSCCe).

- The highest conductivities in wet $\mathrm{H}_{2}$ atmospheres were obtained for LSCCe and LSCY. LSCCe was chosen for further investigations.

- Densification of these materials is very difficult, so they have been focus on the application as anodes for PC-SOFC based on LWO electrolyte. Thus, the first stage of the study considered LSC, LSCN10, LSCN20 and LSCCe materials and the improvement of surface reactions by Ni infiltration.

- These materials do not react with the LWO electrolyte, contrarily to the widely used anode of $\mathrm{NiO}$.

- Structural Ni in LSCN10 and LSCN20 is able to segregate from the structure to the surface in reducing conditions. Thus, Ni nanoparticles become catalytically active for the $\mathrm{H}_{2}$ oxidation reaction. The more $\mathrm{Ni}$ in the structure the better performance of the anode. TEM images demonstrated the presence of the nanoparticles in the electrode surface.

- The improvement of LSCN10 and LSCN20 anodes with respect to the LSC is ascribed to the surface promotion by the Ni nanoparticles. They reduce the 
overall resistance, but they are still limited by low frequency resistance (LF), especially at low temperatures.

- The improvement of LSCCe anode with respect to the LSC is ascribed to the improvement in the mixed protonic electronic conductivity. It reduces the overall resistance, although the anode operation is still limited by the LF, i.e., surface processes.

- The infiltration of $\mathrm{Ni}$ nanoparticles allows decreasing the LF of the corresponding anodes without infiltration, which suggests that the surface reaction of $\mathrm{H}_{2}$ oxidation is catalyzed.

- The most concentrated infiltration (5Ni) completely eliminates the LF resistance in both anodes LSCN20 and LSCCe, and only high frequency (HF) processes remain.

- If the infiltration concentration decreases, as is the case of LSCCe+1Ni, the LF resistance disappears and the medium frequency (MF) resistance shifts to higher frequencies, combined with a new contribution at HF.

- The HF contribution is ascribed to the transport properties of the anode backbone and/or to the anode/electrolyte interfacial resistances, which remain unchanged upon Ni infiltration.

- The best anode performance is obtained for LSCNi20+5Ni. Polarization resistance is as low as $0.26 \Omega \cdot \mathrm{cm}^{2}$ at $750{ }^{\circ} \mathrm{C}$ in wet $\mathrm{H}_{2}$.

\subsection{Final remarks}

The key objectives of the research described in this thesis were to find new materials capable to transport oxygen ions or protons and electronic carriers that are stable in the working conditions to which they are submitted. Several materials emerged from this work have achieved their purpose. $\mathrm{Ce}_{1-\mathrm{x}} \mathrm{Tb}_{\mathrm{x}} \mathrm{O}_{2-\delta}$ and $\mathrm{Ce}_{1-\mathrm{x}} \mathrm{Tb}_{\mathrm{x}} \mathrm{O}_{2-\delta}+2 \mathrm{~mol} \% \mathrm{Co}$ are proposed as a family of materials 
that may provide competent oxygen permeation fluxes and be stable against $\mathrm{CO}_{2}$ containing atmospheres. Besides, the inclusion of this material as a component of a dual-phase membrane has increased the oxygen flux possibilities. The combination with a stable, Co and alkali-earth free spinel has delivered a promising membrane material for the integration in oxyfuel power plants. Furthermore, the studies about the behaviour of the materials and sintering properties may contribute to the next optimization of the microstructure and membrane architecture.

On the other hand, after a wide screening of doped $\mathrm{CaTiO}_{3}$ perovskites, CTFM has been shown as a promising material offering good oxygen permeation fluxes and keeping stable against $\mathrm{CO}_{2}$.

Finally, the study on lanthanum chromites as mixed protonic-electronic conductors allowed developing a new generation of anodes for LWO based PC-SOFC. Although deeper study on the transport properties of the doped materials and the optimization of the synthesis and sintering conditions is still required, milestone polarization resistance values have been reached with these anodes. 


\section{Scientific Contribution}

\section{Peer reviewed articles}

C. Solís, M. Balaguer, F. Bozza, N. Bonanos and J. M. Serra, Catalytic surface promotion of highly performance $\mathrm{La}_{0.85} \mathrm{Sr}_{0.15} \mathrm{Cr}_{0.8} \mathrm{Ni}_{0.2} \mathrm{O}_{3-\delta}$ anodes for $\mathrm{La}_{5.6} \mathrm{WO}_{11.4-\delta}$ based proton conducting fuel cells, Applied Catalysis $B$ (2013), to be submitted.

M. Balaguer, C. Solís, S. Roitsch, J. M. Serra, Engineering microstructure and redox properties in the mixed conductor $\mathrm{Ce}_{0.9} \mathrm{Pr}_{0.1} \mathrm{O}_{2-\delta}+\mathrm{Co} 2 \mathrm{~mol} \%$, Physical Chemistry Chemical Physics, (2013), to be submitted.

M. Balaguer, J. García-Fayos, C. Solís, J. M. Serra, Fast oxygen separation through $\mathrm{SO}_{2-}$ and $\mathrm{CO}_{2}$-stable dual-phase membranes, Chemistry of Materials, (2013), to be submitted.

C. Solís, L. Navarrete, M. Balaguer, J. M. Serra, Development and understanding of $\mathrm{La}_{0.85} \mathrm{Sr}_{0.15} \mathrm{Cr}_{1-\mathrm{x}} \mathrm{Ni}_{\mathrm{x}} \mathrm{O}_{3-\delta}$ anodes for $\mathrm{La}_{5.6} \mathrm{WO}_{11.4-\delta}$-based PC-SOFC, Journal of Power Sources, (2013), submitted.

M. Balaguer, C. Y. Yoo, H.J.M. Bouwmeester, J. M. Serra, Bulk transport and oxygen surface exchange of the mixed ionic-electronic conductor $\mathrm{Ce}_{1-\mathrm{x}} \mathrm{Tb}_{\mathrm{x}} \mathrm{O}_{2-\delta}$ $(\mathrm{x}=0.1, \quad 0.2, \quad 0.5) \quad$ Journal of Materials Chemistry A, (2013) DOI:10.1039/C3TA11610G. 
M. Balaguer, C. Solís, F. Bozza, N. Bonanos and J. M. Serra, High performance anodes with tailored catalytic properties for $\mathrm{La}_{5.6} \mathrm{WO}_{11.4-\delta}$ based proton conducting fuel cells, Journal of Materials Chemistry A, (2013), 1, 3004-3007.

M. Balaguer, V.B. Vert, L. Navarrete, J.M. Serra, SOFC composite cathodes based on LSM and co-doped cerias $\left(\mathrm{Ce}_{0.8} \mathrm{Gd}_{0.1} \mathrm{X}_{0.1} \mathrm{O}_{2-\mathrm{d}}, \mathrm{X}=\mathrm{Gd}, \mathrm{Cr}, \mathrm{Mg}, \mathrm{Bi}, \mathrm{Ce}\right)$, Journal of Power Sources, (2013) 223, 214-220.

C. Solís, V. B. Vert, M. Balaguer, S. Escolástico, S. Roitsch and J. M. Serra, Mixed proton-electronic chromite electrocatalysts as anode material for $\mathrm{La}_{5.6} \mathrm{WO}_{12-\delta}$ based protonic conducting SOFC, ChemSusChem, (2012), 21552158 .

M. Pilar Lobera, M. Balaguer, J. Garcia-Fayos, J.M. Serra, Rare Earth-doped Ceria Catalysts for ODHE Reaction in a Catalytic Modified MIEC Membrane Reactor, ChemCatChem, (2012), 4, 2102-2111.

M. Balaguer, C. Solís, J.M. Serra, Structural-Transport Properties Relationships on $\mathrm{Ce}_{1-\mathrm{x}} \mathrm{Ln}_{\mathrm{x}} \mathrm{O}_{2-\delta}+\mathrm{Co}$ System ( $\left.\mathrm{Ln}=\mathrm{Gd}, \mathrm{La}, \mathrm{Tb}, \mathrm{Pr}, \mathrm{Eu}, \mathrm{Er}, \mathrm{Yb}, \mathrm{Nd}\right)$ and Effect of Cobalt Addition, Journal of Physical Chemistry C, (2012), 116, 7975-7982.

M. Balaguer, C. Solís, J.M. Serra, Study of the transport properties of the mixed ionic electronic conductor $\mathrm{Ce}_{1-\mathrm{x}} \mathrm{Tb}_{\mathrm{x}} \mathrm{O}_{2-\delta}+\mathrm{Co}(\mathrm{x}=0.1,0.2)$ and evaluation as oxygen- transport membrane, Chemistry of Materials, (2011), 23, 2333-2343.

M. Balaguer, E. Matveeva, Quenching of porous silicon photoluminescence by molecular oxygen and dependence of this phenomenon on storing media and method of preparation of $\mathrm{pSi}$ photosensitizer, Journal of Nanoparticle Research, (2010), 12, 2907-2917. 
E. Pastor, M. Balaguer, L. Bychto, J. Salonen, V.P. Letho, E. Matveeva, V.S. Chirvony, Porous silicon for photosensitized formation of singlet oxygen in water and in simulated body fluid: two methods of modification by undecylenic acid, Journal of Nanoscience and Nanotechnology (2009), 9, 3455-3461.

M. Balaguer, E. Pastor, L. Bychto, P. Atienzar, M. A. Miranda, E. Matveeva, and V. S. Chirvony, Durability and photophysical properties of surfactantcovered porous silicon particles in aqueous suspensions, Phys Status Solidi A (2008), 205, 2585-2588.

L. Bychto, M. Balaguer, E. Pastor, V. S. Chirvony E. Matveeva, Influence of preparation and storage conditions on photoluminescence of porous silicon powder with embedded Si nanocrystals, Journal of Nanoparticle Research (2008), 10, 1241-1249.

E. Pastor, M. Balaguer, E. Matveeva, Nano suspension of porous silicon in water:two methods of material preparation and modification by surfactants, ECS Transactions (2007), 6, 63-70.

\section{Patent}

M. Balaguer, I. Moriña, M. A. Pérez, J.M. Serra, C.Solís, 'Composición para su uso en membranas permeables al oxígeno' Applicant: Instalaciones Inabensa S.A., Patent Number ES2392872A1.

\section{Oral presentations}

M. Balaguer, J. García-Fayos, C. Solís, J.M. Serra, Oxygen-Permeability through $\mathrm{CO}_{2}$-Tolerant $\mathrm{Fe}_{2} \mathrm{NiO}_{3}-\mathrm{Ce}_{0.8} \mathrm{~Tb}_{0.2} \mathrm{O}_{2-\delta}$ dual phase composite membrane, 
13th conference of the European Ceramic Society, ECERS XIII, Limoges (France), 2013.

M. Balaguer, Oxygen permeation membranes: fundamentals and applications, Invited talk in the Kepler Symposium on the 2nd International Conference on Materials for Energy, EnMat II, Karlsruhe (Germany), 2013.

M. Balaguer; C. Solís, S. Escolástico, J.M. Serra, Transport properties of $\mathrm{Ce}_{1-\mathrm{x}} \operatorname{Ln}_{\mathrm{x}} \mathrm{O}_{2-\delta}$ system and effect of $\mathrm{Co}$ addition. Application as oxygen permeation membrane, 12th conference of the European Ceramic Society Stockholm (Sweden), 2011.

M. Balaguer, C. Solís, S. Escolástico, J.M. Serra, Structural-transport properties relationships $\mathrm{Ce}_{1-\mathrm{x}} \operatorname{Ln}_{\mathrm{x}} \mathrm{O}_{2-\delta}$ system and effect of Co addition, Electroceramics XII, Trondheim (Norway), 2010.

M. Balaguer, E. Maveeva, Quenching of porous silicon photoluminescence by molecular oxygen. Dependence of this phenomenon on storing media and method of the preparation of pSi photosensitizer, 7th Conference on Porous Semiconductors Science and Technology, Valencia (Spain), 2010.

\section{Selected poster presentations}

M. Balaguer, C.Y. Yoo, H. J Bouwmeester, J.M. Serra, Oxygen Transport and surface Exchange Properties of $\mathrm{Ce}_{1-\mathrm{x}} \mathrm{Tb}_{\mathrm{x}} \mathrm{O}_{2-\delta}+\mathrm{Co} 2 \% \operatorname{mol}(\mathrm{x}=0.1,0.2,0.5)$, 12th International Conference on Inorganic Membranes, Enschede (The Netherlands), 2012. 
M. Balaguer, S. Escolástico, J.M. Serra, Oxygen permeation and transport properties of $\mathrm{CaTi}_{0.73} \mathrm{Fe}_{0.18} \mathrm{Mg}_{0.09} \mathrm{O}_{3-\delta}$, 12th International Conference on Inorganic Membranes, Enschede (The Netherlands), 2012

M. Balaguer, C. Solís, J.M. Serra, Influence of Co addition on the transport properties of $\mathrm{Ce}_{1-\mathrm{x}} \mathrm{Tb}_{\mathrm{x}} \mathrm{O}_{2-\delta}(\mathrm{x}=0.1,0.2)$ and evaluation as oxygen-transport membrane, 18th Conference on Solid State Ionics, Warsaw, (Poland) 2011.

M. Balaguer, C. Solís, J.M. Serra, Effect of sintering temperature on transport properties of $\mathrm{Ce}_{0.9} \mathrm{Pr}_{0.1} \mathrm{O}_{2-\delta}+\mathrm{Co} 2 \%$, 18th Conference on Solid State Ionics, Warsaw, (Poland) 2011. 


\section{List of Figures and Tables}

\section{List of figures}

Figure 1.1. a) Frenkel defect: cation in interstitial hole, and b) Schottky disorder: missing pair of ions.

Figure 1.2. a) Polaron particle: a charge inside its self-created lattice distortion [12] and b) ionic diffusion mechanisms (1) via vacancy, (2) via interstitial site.

Figure 1.3. Electron (e), and hole (h), mobilities in oxides. The lower limit for large polarons corresponds to a mean-free path equal to the interatomic distance. The upper limit for small polarons corresponds to one hop every lattice vibration period. Note the opposite trends with temperature: for small polarons and ions, thermal activation is needed, so higher temperatures raise the rate; for large polarons, phonon scattering reduces the mobility at higher temperatures. [13]

Figure 1.4. Kroger-Vink diagram for acceptor doped material. The acceptor concentration represents the ionized acceptor concentration. 31

Figure 1.5. Kröger-Vink diagram for donor doped material. The donor concentration represents the ionized donor concentration.

Figure 1.6. Oxygen permeation scheme for a dense membrane with catalytic layers for oxygen dissociation and recombination.

Figure 1.7. Schematic of an individual SOFC based on oxygen-ion conductors fed by $\mathrm{H}_{2}$

Figure 2.1. Scheme of the fabrication of the lanthanide doped ceria. The addition of Co by impregnation is indicated. The calcination temperature ensures the decomposition of the nitrates and carbonates from the precursors.

Figure 2.2. Main steps for rectangular probes fabrication. From left to rigth: initial powder, ball milling step, rinsing of the powder, pressing, high temperature calcination and final configuration of sintered samples. 
Figure 2.3. Main steps for membrane fabrication. From left to rigth: initial powder, ball milling step, rinsing and drying of the powder, uniaxial pressing, high temperature calcination and final configuration of sintered samples. Afterwards, a catalytic layer may be screen printed on the membrane surface.

Figure 2.4. Main steps for electrode fabrication. From left to rigth: initial powder, ball milling step, rinsing and drying of the powder, ceramic ink fabrication, screen printing of the electrode on the electrolyte surface and final electrode configuration after high temperature calcination. In addition, the electrode on the right is screen printed with a gold mesh for current collection. 66

Figure 2.5. Incident beam on a crystalline material and diffracted angle $(2 \theta)$, which is recorded.

Figure 2.6. Schematic diagram for grain size and crystallite of a crystalline powder sample. A particle or grain of a real crystalline powder generally consists of many crystallites, which can be considered as single crystals. 71

Figure 2.7. Four point configuration for DC conductivity measurements. The contacts are made with silver paste and wires.

Figure 2.8. a) Combinatorial DC conductivity setup, b) detail of the sample chamber containing the ten samples and c) from top to bottom, furnace controller, programmable current source (Keithley 2601) that and sixteen channel multimeter (Keithley 3706)... 80

Figure 2.9. Experimental setup permeation scheme. The gas inlet and outlet may be reversed. The membrane is pressed by a spring sited in the lid.

Figure 2.10. Equivalent circuits a) simple parallel resistance-capacitor (RC) circuit and its corresponding perfect semicircle response and b) double $\mathrm{RC}$ circuit, represented as a double arc.

Figure 3.1. Fluorite cubic structure of $\mathrm{CeO}_{2}$. Doping occurs by the Ln substitution of a Ce cation

Figure 3.2 Thermogravimetry analysis of $\mathrm{Ce}_{0.9} \mathrm{~Tb}_{0.1} \mathrm{O}_{2-\delta}$ and $\mathrm{Ce}_{0.9} \mathrm{Er}_{0.1} \mathrm{O}_{2-\delta}$, in air. The TG was performed up to $800{ }^{\circ} \mathrm{C}$, the calcination temperature of the as prepared materials.

Figure 3.3. XRD pattern of $\mathrm{Ce}_{0.9} \mathrm{Ln}_{0.1} \mathrm{O}_{2-\delta}$ and $\mathrm{Ce}_{0.9} \mathrm{Ln}_{0.1} \mathrm{O}_{2-\delta}+\mathrm{Co} 2 \mathrm{~mol} \%$ calcined at $800{ }^{\circ} \mathrm{C}$. 
Figure 3.4. Lattice parameter of doped ceria as a function of the trivalent ionic radius of the dopant cation (and tetravalent for $\mathrm{Pr}$ and $\mathrm{Tb}$ ) in eight-fold coordination. 98

Figure 3.5. SEM images of $\mathrm{Ce}_{0.9} \mathrm{Ln}_{0.1} \mathrm{O}_{2-\delta}$ and $\mathrm{Ce}_{0.9} \mathrm{Ln}_{0.1} \mathrm{O}_{2-\delta}+\mathrm{Co} 2 \mathrm{~mol} \%$ calcined at $1300{ }^{\circ} \mathrm{C}(\mathrm{Ln}=\mathrm{Tb}, \mathrm{Pr}, \mathrm{Eu}$ and $\mathrm{La})$

Figure 3.6. HT-XRD heating up in air (left side) and cooling down in $\mathrm{N}_{2}$ (right side) of $\mathrm{Ce}_{0.9} \mathrm{Ln}_{0.1} \mathrm{O}_{2-\delta}$. In the insets, the calculated cell parameters $\left(a_{700}{ }^{\circ} \mathrm{C}\right)$ at $700{ }^{\circ} \mathrm{C}$ in air (left side) and in $\mathrm{N}_{2}$ (right side) are plotted as a function of the trivalent ionic radii (and tetravalent for Pr and Tb). 102

Figure 3.7. a) Raman spectra of the $\mathrm{Ce}_{0.9} \mathrm{Ln}_{0.1} \mathrm{O}_{2-\delta}(\mathrm{Ln}=\mathrm{La}, \mathrm{Gd}, \mathrm{Eu}, \mathrm{Pr}, \mathrm{Tb}, \mathrm{Yb}, \mathrm{Er}$ ) (sintered at $1300{ }^{\circ} \mathrm{C}$ ) from $400-750 \mathrm{~cm}^{-1}$ and b) $\mathrm{F} 2 \mathrm{~g}$ Raman shift as a function of the cell parameter $\mathrm{Ce}_{0.9} \mathrm{Ln}_{0.1} \mathrm{O}_{2-\delta}$ and $\mathrm{Ce}_{0.9} \mathrm{Ln}_{0.1} \mathrm{O}_{2-\delta}+\mathrm{Co}$ calcined at $1300{ }^{\circ} \mathrm{C}$ and $800{ }^{\circ} \mathrm{C}$ (top) and after subtracting Grüneisen shifts (bottom). The inset shows a magnification of the band related to the oxygen vacancy formation. 104

Figure 3.8. Correlation length obtained from Raman line-shape fitting as a function of the cell parameter. 105

Figure 3.9. a) Arrhenius plot of the total conductivity in air of $\mathrm{CeO}_{2}$ and $\mathrm{Ce}_{0.9} \mathrm{Ln}_{0.1} \mathrm{O}_{2-\delta}$ $\left(\mathrm{Ln}=\mathrm{Tb}, \mathrm{Pr}, \mathrm{Eu}\right.$ and $\mathrm{Er}$ ), and b) $\log -\log$ plot of total conductivity at $700{ }^{\circ} \mathrm{C}$ of the Cofree and Co-containing $\mathrm{CeO}_{2}$ and $\mathrm{Ce}_{0.9} \mathrm{Ln}_{0.1} \mathrm{O}_{2-\delta}(\mathrm{Ln}=\mathrm{Tb}, \mathrm{Pr}, \mathrm{Eu}$ and $\mathrm{Er}$ ) as a function of $p \mathrm{O}_{2}$. 108

Figure 3.10. a) Arrhenius plot of the total conductivity in air of Co-free $\mathrm{Ce}_{0.9} \operatorname{Ln}_{0.1} \mathrm{O}_{2-\delta}$ $\mathrm{Ce}_{0.9} \mathrm{Ln}_{0.1} \mathrm{O}_{2-\delta}(\mathrm{Ln}=\mathrm{Yb}, \mathrm{Gd}, \mathrm{La}$ and $\mathrm{Nd})$ and b) log-log plot of the total conductivity versus $\mathrm{pO}_{2}$ at $700{ }^{\circ} \mathrm{C}$ of the $\mathrm{Co}$-free and Co-containing $\mathrm{CeO}_{2}$ and $\mathrm{Ce}_{0.9} \mathrm{Ln}_{0.1} \mathrm{O}_{2-\delta}$ $\mathrm{Ce}_{0.9} \mathrm{Ln}_{0.1} \mathrm{O}_{2-\delta}(\mathrm{Ln}=\mathrm{Yb}, \mathrm{Gd}, \mathrm{La}$ and $\mathrm{Nd})$. 108

Figure 3.11: $E_{a}$ (top) and total conductivity at $700{ }^{\circ} \mathrm{C}$ in air (bottom) of the different doped materials versus cell parameter measured at $700{ }^{\circ} \mathrm{C}$. Cell parameters at $700{ }^{\circ} \mathrm{C}$ in air have been calculated from HT-XRD or by using TEC values for similar dopants. $E_{a}$ solid lines have been fitted to Eq. 4 only taken into account samples with trivalent dopants while dashed fits include Pr and Tb. 111

Figure 4.1. a) XRD patterns and SEM images and b) temperature dependence of cell parameter of $\mathrm{CP}$ and $\mathrm{CPCo}$ sintered at $1300^{\circ} \mathrm{C}$. 125

Figure 4.2. a) TPR profile of $\mathrm{CeO}_{2}$ and $\mathrm{CP}$ and b) TPR profile of $\mathrm{CeO}_{2}+2 \mathrm{~mol} \% \mathrm{Co}$ and CPCo. All the samples are samples sintered at $1300{ }^{\circ} \mathrm{C}$. The flow gas is $10 \% \mathrm{H}_{2}$ in $\mathrm{Ar}$; heating ramp was $5{ }^{\circ} \mathrm{C} / \mathrm{min}$. 127 
Figure 4.3 SEM pictures of CPCo for different sintering temperature: 900, 1000, 1200, 1300 and $1450{ }^{\circ} \mathrm{C}$. The grain size grows with the temperature from $60 \mathrm{~nm}$ at $900{ }^{\circ} \mathrm{C}$ to 7 $\mu \mathrm{m}$ at $1450^{\circ} \mathrm{C}$. 129

Figure 4.4. Back scattering SEM of CPCo sintered at a) $1000^{\circ} \mathrm{C}$ and b) $1450{ }^{\circ} \mathrm{C}$. c) Less magnified image of CPCo at $1450^{\circ} \mathrm{C}$ and d) EDS Co mapping indicating Co rich zones. Arrows indicate the presence of the $\mathrm{CoO}_{\mathrm{x}}$ particles. 130

Figure 4.5. FIB-lamella TEM of CPCo sintered at $1000^{\circ} \mathrm{C}$ (left) and $1450{ }^{\circ} \mathrm{C}$ (right). 131

Figure 4.6. TEM-EDS on a $1000{ }^{\circ} \mathrm{C} \mathrm{CPCo} \mathrm{grain.} \mathrm{Co} \mathrm{appears} \mathrm{dispersed} \mathrm{along} \mathrm{the} \mathrm{grain}$ surface, while fine CP-CP grain interface is shown. 131

Figure 4.7. TEM-EDS on a $1450{ }^{\circ} \mathrm{C} \mathrm{CPCo}$ grain boundary. A fine $\mathrm{CP}-\mathrm{CP}$ grain interface is shown. Co was not detected either in the grain boundaries or in fringes and surfaces.

Figure 4.8. a) XRD patterns and b) TPD of oxygen in Ar for CPCo sintered at 1000, 1200 and $1450{ }^{\circ} \mathrm{C}$...

Figure 4.9. a) Total conductivity in air at $800{ }^{\circ} \mathrm{C}$ and $400{ }^{\circ} \mathrm{C}$ as a function of the sintering temperature and b) total conductivity of $\mathrm{CPCo}$ sintered at five different temperatures in air. For comparison, conductivity at $5 \times 10^{-5}$ atm for CPCo sintered at $1000^{\circ} \mathrm{C}$ is plotted... 134

Figure 4.10. a) Arrhenius plot of the total conductivity of CPCo sintered at 1000 and $1300{ }^{\circ} \mathrm{C}$ and $\mathrm{CP}$ sintered at $1300{ }^{\circ} \mathrm{C}$, in air. b) $\mathrm{pO}_{2}$ dependence of the total conductivity of CPCo sintered at different temperatures, $400{ }^{\circ} \mathrm{C}$ (open symbols) and $800{ }^{\circ} \mathrm{C}$ (solid symbols). 136

Figure 5.1. XRD patterns of CTx and CTxCo sintered at $1450{ }^{\circ} \mathrm{C}$ recorded at room temperature. 145

Figure 5.2. Scanning microscope pictures taken by backscattered electrons of CTx and CTxCo membrane cross sections, at $1200^{\circ} \mathrm{C}$.

Figure 5.3 Cell parameter dependence on the dopant amount for CTx and CTxCo and theoretical Vegard's slope for $\mathrm{Tb}^{3+}$ and $\mathrm{Tb}^{4+}$ total substitution..................................... 146

Figure 5.4. a) $\mathrm{H}_{2}$ TPR signal for CT10 and b) $\mathrm{H}_{2}$ TPR for CTxCo (x=10 and 20)..... 149 
Figure 5.5. Crystallite size dependence on the cell parameter for CT10, CT20, CT10Co and CT20Co after sintering at $1300{ }^{\circ} \mathrm{C}$

Figure 5.6. Normalized TPD measured in Ar of CTx and CTxCo. Inset: $\mathrm{CeO}_{2}$ and $\mathrm{CeO}_{2}+\mathrm{Co}$. The intensity (ion current) corresponds to $\mathrm{m} / z=32$.

Figure 5.7. (a) Oxygen non-stoichiometry $(\delta)$ determined by $\mathrm{TG}$ in air and (b) $\delta$ determined by TG in $\mathrm{N}_{2}$, for CTx and CTxCo. Temperature range include from 430 to $1000{ }^{\circ} \mathrm{C}$.

Figure 5.8. Kröger-Vink diagram for acceptor doped fluorite. Four $\mathrm{pO}_{2}$ regions delimit the main key defect reactions for a general fluorite. 153

Figure 5.9. Arrhenius plot of the total conductivity in air and in $\operatorname{Ar}\left(0.21\right.$ and $5 \cdot 10^{-5} \mathrm{~atm}$, respectively) for a) CTx and b) CTxCo

Figure 5.10. Total conductivity dependency on $\mathrm{Tb}$ dopant concentration in (a) air, $p O_{2}=0.21 \mathrm{~atm}$ and (b) Ar, $p O_{2}=2 \cdot 10^{-5} \mathrm{~atm}$. 159

Figure 5.11. Conductivity of CTx and CTxCo systems as a function of $\mathrm{pO}_{2}$ measured at (a) $400{ }^{\circ} \mathrm{C}$ and (b) $800{ }^{\circ} \mathrm{C}$ 160

Figure 5.12. UV-vis absorption spectra $h \nu F(R) 2$ versus photon energy of (a) CTx and (b) $\mathrm{CTxCo}$

Figure 5.13. a) Ce $3 d$ XPS spectra of CT20 and CT20Co quenched in air from $800{ }^{\circ} \mathrm{C}$ and b) Ce3d XPS separation of peaks of CT20 and CT20Co spectra. Pass energy was 30 $\mathrm{eV}$ 164

Figure 5.14. XPS of the Tb $3 d$ region of a) CT20 and b) CT20Co, quenched in air from $800{ }^{\circ} \mathrm{C}$, acquired with a pass energy of $30 \mathrm{eV}$.

Figure 5.15. XPS of the Co $2 \mathrm{~d}$ region of CT20 (a) and CT20Co (b), quenched in air from $800{ }^{\circ} \mathrm{C}$, acquired with a pass energy of $30 \mathrm{eV}$. The Co $2 \mathrm{~d}$ peak is shifted to higher binding energy than found in $\mathrm{CoO}(778 \mathrm{eV})$, suggesting Co lattice substitution 167

Figure 5.16. Arrhenius plot of the exchange rates of CT10, CT10Co, CT20. CT20Co, CT50, CT50Co

Figure 5.17. Surface exchange rate as a function of the oxygen nonstoichiometry at $700{ }^{\circ} \mathrm{C}$. 
Figure 5.18. Inverse temperature dependence of the rates of dissociative adsorption $(\mathfrak{R}$ a), oxygen incorporation $(\mathfrak{R} i)$ of the terbium doped studied series. 171

Figure 5.19. Oxygen permeation flux as a function of inverse temperature for CT10Co and CT20Co. The oxygen flux for similarly configuration BSCF and LSCF membranes is shown for comparison.

Figure 5.20. a) Ionic transport number and b) total, ionic and electronic conductivity of CT20Co measured across a $1.2 \mathrm{~mm}$ thick membrane under a nominal gradient of $p \mathrm{O}_{2}$ $0.21 \| 5 \times 10^{-5}$ atm (air/Ar). $J\left(\mathrm{O}_{2}\right)$ measured using chromatography analytical data. .... 174

Figure 5.21. Thermogravimetric analysis under $\mathrm{CO}_{2}$ flow of $\mathrm{CT} 10$ and $\mathrm{CT} 10 \mathrm{Co}$ in $\mathrm{CO}_{2}$, and BSCF.

Figure 6.1. Room temperature XRD patterns of the as-sintered doped cerias CG82, CGMg, CGCr, CGBi and CG91) at $800^{\circ} \mathrm{C}$. 187

Figure 6.2. Comparison of the initial particle size of a) CG82 (as-sintered) and b) LSM (as-received) and the resulting powders after calcination at $1150{ }^{\circ} \mathrm{C}$ : c) CG82 and d) LSM.

Figure 6.3. SEM images of several composite cathodes sintered at $1150{ }^{\circ} \mathrm{C}$ : a) CG82-LSM, b) CG91-LSM and c) CGBi-LSM. 190

Figure 6.4. a) Arrhenius plot in air and b) $\mathrm{pO}_{2}$ dependence, of the total conductivity of ceria-based materials

Figure 6.5. Arrhenius plot of the electrode polarization resistance of LSM-ceria-based composites and the single LSM electrodes. LSFC and LSM-YSZ are shown for comparison. 194

Figure 6.6. Polarization resistance as a function of conductivity at three different temperatures $\left(700,750\right.$ and $\left.800{ }^{\circ} \mathrm{C}\right)$ 195

Figure 6.7. Cross section SEM images of a) CGCr-LSM, b) CG82-LSM and c) CG91LSM electrodes on a dense CG82-Co electrolyte. The upper layer seen in a) corresponds to the gold current collecting mesh. 196

Figure 6.8. Electrochemical impedance spectra of symmetrical cells with different LSM-doped-ceria electrodes at $800{ }^{\circ} \mathrm{C}$ under air: a) Nyquist and b) imaginary 
impedance Bode plots. Logarithm of selected frequencies is provided on the Nyquist plot.

Figure 6.9. Resistances corresponding to the different processes obtained by fitting the spectra of symmetrical cells at $800{ }^{\circ} \mathrm{C}$ under air according to the equivalent circuit $\mathrm{LR}\left(\mathrm{R}_{\mathrm{A}} \mathrm{Q}_{\mathrm{A}}\right)\left(\mathrm{R}_{\mathrm{B}} \mathrm{Q}_{\mathrm{B}}\right)\left(\mathrm{R}_{\mathrm{C}} \mathrm{Q}_{\mathrm{C}}\right)$ shown in the inset. The corresponding logarithm of summit frequency is indicated on top of each bar.

Figure 7.1. $\mathrm{CaTiO}_{3}$ orthorhombic perovskite from the family $\mathrm{A}^{\mathrm{II}} \mathrm{B}^{\mathrm{IV}} \mathrm{O}_{3}$ 208

Figure 7.2. Comparative $\mathrm{pO}_{2}$ dependence of total electrical conductivity for pure and doped $\mathrm{CaTiO}_{3}$ perovskites.

Figure 7.3. a) Room temperature XRD pattern of orthorhombic CTFM calcined at 1200 ${ }^{\circ} \mathrm{C}$ and b) SEM picture of the formed membrane. 215

Figure 7.4. TG analysis under $5 \% \mathrm{CO}_{2} /$ air of a) CTFM and CTF and b) BSCF. Heating rate was $10^{\circ} \mathrm{C} \cdot \mathrm{min}^{-1}$. 215

Figure 7.5. a) DTG in air, b) TPD of oxygen, $\mathrm{m} / \mathrm{z}=32$, in $\mathrm{He}\left(\mathrm{pO}_{2}=10^{-5} \mathrm{~atm}\right)$, c) cell parameters extracted from HT-XRD in air and d) cell parameters extracted from HT-XRD in $\mathrm{N}_{2}$, for CTFM. Heating rate was $10^{\circ} \mathrm{C} \cdot \mathrm{min}^{-1}$

Figure 7.6. a) Arrhenius plot of the conductivity of CTFM, CTF and $\mathrm{CaTiO}_{3}$ and b) logarithm of the total conductivity versus $\mathrm{pO}_{2}$ of CTFM and CTF at 1000, 800 and $400{ }^{\circ} \mathrm{C}$.

Figure 7.7. Ca $2 \mathrm{p}$ spectrum of CTFM quenched from $800{ }^{\circ} \mathrm{C}$ in Ar. Pass energy was 30 $\mathrm{eV}$

Figure 7.8. Ti $2 \mathrm{p}$ spectrum of CTFM quenched from $800{ }^{\circ} \mathrm{C}$ in $\mathrm{Ar}$ and deconvolution of the spectrum. $\mathrm{Ti}^{4+}$ and $\mathrm{Ti}^{3+}$ components are highlighted. Pass energy was $30 \mathrm{eV} \ldots \ldots .220$

Figure 7.9. a) Fe 2p XPS spectrum and b) Mg 2s and Fe3s XPS spectra of CTFM quenched from $800{ }^{\circ} \mathrm{C}$ in Ar. Pass energy was $30 \mathrm{eV}$.

Figure 7.10. O1s XPS spectra of CTFM quenched from $800^{\circ} \mathrm{C}$. Pass energy was $30 \mathrm{eV}$.

Figure 7.11. Arrhenius plot of CTF and CTFM (a) the overall surface exchange rate $\mathfrak{R}_{0}$ and (b) partial $\mathfrak{R}_{\mathrm{a}}, \Re_{\mathrm{i}}$ and $\mathfrak{R}_{0}$. 
Figure 7.12. Comparative Arrhenius plot of the CTFM and CTF $\Re_{0}$ with literature data of widely used oxides measured by PIE as YSZ, CGO10, CGO20, CT50 or IEDP as LSC20 and BSCF. [30-32]

Figure 7.13. Oxygen permeation flow for CTFM membrane as a function of the temperature, with a sweep flow of $400 \mathrm{~mL} \cdot \mathrm{min}^{-1}$ of Ar. Synthetic air and pure oxygen at a flow rate of $100 \mathrm{~mL} \cdot \mathrm{min}^{-1}$ are used as feed gas. 226

Figure 7.14. a) Oxygen permeation flux for CTFM membrane as a function of the Ar sweep flow at three different temperatures using synthetic air as feed and b) using air and $\mathrm{O}_{2}$ as feed gas at $1000{ }^{\circ} \mathrm{C}$.

Figure 7.15. a) Oxygen permeation flow after 24 hours in $\mathrm{CO}_{2} / \mathrm{Ar}$ atmosphere. Oxygen flux using Ar as sweep gas is represented using open symbols, b) Oxygen permeation flux in Ar before and after of $\mathrm{CO}_{2}$ treatment. $\mathrm{Q}_{\mathrm{Ar}}=400 \mathrm{~mL} \cdot \mathrm{min}^{-1}$. 228

Figure 7.16. XRD patterns of as sintered CTFM powder and membranes after the stability treatments. Reference pattern and XRD of sample holder has been plotted for comparison.

Figure 8.1. Oxygen and electron transport scheme in a dual phase composite membrane composed of a) pure electronic and mixed ionic electronic conducting phases and b) pure electronic and ionic conducting phases.

Figure 8.2. XRD patterns of tested spinels: $\mathrm{Fe}_{2} \mathrm{NiO}_{4}, \mathrm{Cr}_{2} \mathrm{FeO}_{4}, \mathrm{Co}_{2} \mathrm{FeO}_{4}, \mathrm{Mn}_{2} \mathrm{CuO}_{4}$. Grey circles indicate impurity peaks. 242

Figure 8.3. a) XRD patterns of the composites prepared by $\mathrm{SS}$ and $\mathrm{OP}$, and the $\mathrm{Fe}_{2} \mathrm{NiO}_{4}$ and $\mathrm{Ce}_{0.8} \mathrm{Gd}_{0.2} \mathrm{O}_{1.9}$ single phases. b) Arrhenius plot of total electrical conductivity for the $50 / 50$ phase ratio composites prepared by SS and OP and for 60/40 phase ratio OP composite. c) SEM picture of 50/50 FNO-CGO prepared by PM and d) SEM picture of 50/50 FNO-CGO prepared by OP.

Figure 8.4. XRD patterns of FNO-CLnO synthesized by OP and sintered in air at $1400{ }^{\circ} \mathrm{C}$. Reference fluorite and spinel structure peaks are also indicated (ICDD 00-0431002 and 00-044-1485, respectively).

Figure 8.5. Arrhenius plot of the total electrical conductivity in air of FNO-CLnO composite series. 246

Figure 8.6. a) BS-SEM picture of the fractured cross-section of a FNO-CTO composite membrane sintered at $1400{ }^{\circ} \mathrm{C}$ in air; b) detail of the grain boundary between spinel 
(dark) and fluorite (light) phases; c) detail of two fluorite grains fringe; d) detail of two spinel grains fringe and; e) CGO-LSM-Pd catalytic layer on the composite.

Figure 8.7. a) Total electrical conductivity recorded in air as a function of temperature for the single phase CTO, FNO and for the composite, b) Arrhenius plot of the total electrical conductivity of the composite in air and Ar atmospheres and c) total electrical conductivity at $800{ }^{\circ} \mathrm{C}$ as a function of the $p O_{2}$

Figure 8.8. SEM image of $\mathrm{Fe}_{2} \mathrm{NiO}_{4}$ sample sintered at $1400{ }^{\circ} \mathrm{C}$ in air dwelling $10 \mathrm{~h}$. $\sim 90 \%$ relative density was achieved at these conditions. 250

Figure 8.9. a) Oxygen permeation flux as a function of temperature and sweep gas ( $\mathrm{Ar}$ or $\mathrm{CO}_{2}$ ) for FNO-CTO membrane and in Ar for single phase CTOCo; and b) flux stability with time on stream for $\mathrm{FNO}-\mathrm{CTO}$ membrane in $\mathrm{CO}_{2}$ sweeping at $900{ }^{\circ} \mathrm{C} . .253$

Figure 8.10. Oxygen flux as a function of reciprocal temperature for different $\mathrm{pO}_{2}$ gradients across the membrane, i.e., air- $\mathrm{Ar}, \mathrm{O}_{2}-\mathrm{Ar}$ and air- $10 \% \mathrm{CH}_{4} / \mathrm{Ar}$, on a $0.68 \mathrm{~mm}$ thick FNO-CTO membrane sintered at $1400{ }^{\circ} \mathrm{C}$ 255

Figure 8.11. XRD pattern of both sides of the FNO-CTO membranes after 7 days of permeation experiments in $\mathrm{Ar}, \mathrm{CO}_{2}, \mathrm{CH}_{4}$ sweeping.

Figure 8.12. Temperature dependency of oxygen permeation of FNO-CTO and FNOCGO composites. The membranes were fed by air and the sweep gas was $100 \% \mathrm{CO}_{2}$.

Figure 8.13. XRD pattern of FNO-CTO and FNO-CGO composites after 7 days of treatment under a continuous flux consisted of $1 \% \mathrm{SO}_{2}-5 \% \mathrm{O}_{2}$ in $\mathrm{CO}_{2}$, at $800{ }^{\circ} \mathrm{C} . \ldots .258$

Figure 8.14. XRD pattern of LSCF after 7 days of treatment under a continuous flux consisted of $1 \% \mathrm{SO}_{2}-5 \% \mathrm{O}_{2}$ in $\mathrm{CO}_{2}$, at $800{ }^{\circ} \mathrm{C}$, and comparison with pure perovskite phase. 259

Figure 9.1. XRD patterns of LSC calcined at $900{ }^{\circ} \mathrm{C}$, LSCN10 and LSCN at $1050{ }^{\circ} \mathrm{C}$ and $\mathrm{LSCrF}$ at $1000{ }^{\circ} \mathrm{C}$. The dotted lines represent the LSC reference pattern (ICSD 00032-1240) 270

Figure 9.2. XRD patterns of A-site doped LSC calcined at $1600{ }^{\circ} \mathrm{C}$ and corresponding SEM microstructure images of (top to bottom) SmSC, NSC and LSCCe. 271

Figure 9.3. XRD patterns and corresponding SEM images of B-site doped LSC pressed and calcined at $1600{ }^{\circ} \mathrm{C}$ : LSCY, LSCT, LSCA and LSCrF. The peak positions of $\mathrm{LaCrO}_{3}$ (LC, ICSD 00-024-1016) and LSC (ICSD 00-032-1240) perovskites are represented for comparison. 
Figure 9.4. XRD patterns of (from bottom to top): LSC, LWO and 50 vol.\% mixture at $\mathrm{RT}$, treated at $1400{ }^{\circ} \mathrm{C}$ in $\mathrm{H}_{2}$ for $48 \mathrm{~h}$ and treated at $1150{ }^{\circ} \mathrm{C}$ in air for $5 \mathrm{~h}$.

Figure 9.5: XRD patterns of (from bottom to top): LWO and $\mathrm{NiO} 50$ vol. $\%$ mixture at $\mathrm{RT}$, treated at $900{ }^{\circ} \mathrm{C}$ in $\mathrm{H}_{2}$ for $48 \mathrm{~h}$ and treated at $1150{ }^{\circ} \mathrm{C}$ in air for $5 \mathrm{~h}$; and LSCN10 and LWO 50 vol.\% mixture after calcination in air at $1150{ }^{\circ} \mathrm{C}$ for $5 \mathrm{~h}$. 276

Figure 9.6: a) SEM image and b) TEM image of a FIB-lamella of a LSCN10 anode on LWO electrolyte sintered at $1150^{\circ} \mathrm{C}$ 277

Figure 9.7: TEM images of as sintered LSCN10 on LWO electrolyte reveal the good bounding between electrolyte and porous electrode particles a) and LSCN10 powder after reduction at $900{ }^{\circ} \mathrm{C} \mathrm{b}$ ) 278

Figure 9.8: a) TPR analysis of $\mathrm{LaCrO}_{3-\delta}, \mathrm{LaCr}_{0.9} \mathrm{Ni}_{0.1} \mathrm{O}_{3-\delta}$ and $\mathrm{LSCN} 10$ materials and b) $\mathrm{R}_{\mathrm{p}}$ of LSCN10 as a function of time at $900{ }^{\circ} \mathrm{C}$ in $100 \% \mathrm{H}_{2}$.

Figure 9.9: Total conductivity of $\mathrm{La}_{0.85} \mathrm{Sr}_{0.15} \mathrm{Cr}_{0.9} \mathrm{Ni}_{0.1} \mathrm{O}_{3-\delta}$ in $5 \% \mathrm{H}_{2}, 5 \% \mathrm{D}_{2}, 5 \% \mathrm{H}_{2}+\mathrm{H}_{2} \mathrm{O}$ and $5 \% \mathrm{D}_{2}+\mathrm{D}_{2} \mathrm{O}$. 280

Figure 9.10: a) Nyquist and b) Bode plots of impedance spectra recorded in wet $(2.5 \%$ vol. $\left.\mathrm{H}_{2} \mathrm{O}\right) 100 \% \mathrm{H}_{2}$ for LSC at $700{ }^{\circ} \mathrm{C}$ and c) Nyquist and d) Bode plots of impedance spectra recorded in wet $\left(2.5 \%\right.$ vol. $\left.\mathrm{H}_{2} \mathrm{O}\right) 100 \% \mathrm{H}_{2}$ at different temperatures for LSCN10. 281

Figure 9.11. Equivalent circuit used for EIS impedance modeling. CPS are used instead of perfect capacitors due to the porous nature of the anode. 282

Figure 9.12. a) Modeled LF and MF resistances as a function of the temperature and b) as a function of the $\mathrm{pH}_{2}$.

Figure 9.13. XRD pattern in logarithm scale of LSCN10 anode after electrochemical test. The materials maintains its pure perovskite structure 284

Figure 9.14. a) XRD pattern and b) SEM image of LSCN powder sintered at $900{ }^{\circ} \mathrm{C} 285$

Figure 9.15. XRD patterns of LSC, LSCN and LSCN+5Ni symmetrical cells. The peaks corresponding to metallic $\mathrm{Au}$ from the current collector of the symmetrical cell and metallic $\mathrm{Ni}$ (infiltrated and structural) are indicated. 286 
Figure 9.16. Schematic diagram of the LSCN anode a) and its corresponding SEM image b) and scheme of the LSCN $+5 \mathrm{Ni}$ anode c) and its real SEM images d) and e). $5 \mathrm{M}$ water solution of $\mathrm{Ni}$ Nitrate was dropped on the surface of the LSCN electrode and allowed to penetrate into the pores for $30 \mathrm{~min}$. The infiltration step was repeated 4 times. Each infiltration step was followed by calcination at $300{ }^{\circ} \mathrm{C}$ to decompose the nitrates. 288

Figure 9.17. TEM image of $\mathrm{Ni}$ nanoparticles obtained by firing nitrate precursor at $800{ }^{\circ} \mathrm{C}$ in $\mathrm{H}_{2}$ 289

Figure 9.18. Rp as a function of inverse temperature for LSC, LSCN and $\mathrm{LSCN}+5 \mathrm{Ni}$ electrodes. 290

Figure 9.19. Impedance spectra recorded at $700{ }^{\circ} \mathrm{C}$ in wet hydrogen of LSC a) Nyquist and b) Bode plots and LSCN and LSCN+5Ni c) Nyquist and d) Bode plots. 291

Figure 9.20. Temperature dependency of modelled resistances a) and capacitances b) of LSC, LSCN and LSCN+5N samples. 292

Figure 9.21. XRD patterns of LSCCe as prepared powder and anode after Ni infiltration and reduction in wet $\mathrm{H}_{2}$ up to $800^{\circ} \mathrm{C}$. XRD positions of $\mathrm{LaCrO}_{3}, \mathrm{LWO}$ and $\mathrm{CeO}_{2}$ are indicated as reference. 294

Figure 9.22. TG analysis of the LSCCe in $\mathrm{Ar}(85 \%)-\mathrm{CO}_{2}(15 \%)$. The temperature was increased at a rate of $5^{\circ} \mathrm{C} / \mathrm{min}$.

Figure 9.23. a) LSCCe total conductivity in $\mathrm{H}_{2}+\mathrm{H}_{2} \mathrm{O}$ and $\mathrm{D}_{2}+\mathrm{D}_{2} \mathrm{O}$ and LSC in $\mathrm{H}_{2}+\mathrm{H}_{2} \mathrm{O}$, b) $\mathrm{pO}_{2}$ dependence of the total conductivity at $700^{\circ} \mathrm{C}$ of LSCCe in dry atmosphere and c) $\mathrm{pO}_{2}$ dependence of the total conductivity at $700{ }^{\circ} \mathrm{C}$ of LSCCe wet atmospheres.... 296

Figure 9.24. Schematic diagrams of the LSCCe a), LSCCe+1Ni b) and LSCCe+5Ni c) anodes and the corresponding SEM images d), e) and f). $1 \mathrm{M}$ and $5 \mathrm{M}$ water solution of $\mathrm{Ni}$ Nitrate (LSCe+1 $\mathrm{Ni}$ and $\mathrm{LSCe}+5 \mathrm{Ni}$, respectively) were dropped on the surface of the electrodes and allowed to penetrate into the electrodes pores for $30 \mathrm{~min}$. The infiltration step with the $5 \mathrm{M}$ solution was repeated 4 times. Each infiltration step was followed by calcination at $300{ }^{\circ} \mathrm{C}$ to decompose the nitrates. 297

Figure 9.25. $\mathrm{Rp}$ as a function of the inverse temperature of the four anodes $\mathrm{La}_{0.85} \mathrm{Sr}_{0.15} \mathrm{CrO}_{3-\delta}$ (LSC), $\mathrm{La}_{0.75} \mathrm{Ce}_{0.1} \mathrm{Sr}_{0.15} \mathrm{Cr} \mathrm{O}_{3-\delta}$ (LSCCe) and LSCCe infiltrated with $1 \mathrm{Ni}(\mathrm{LSCCe}+1 \mathrm{Ni})$ and with $5 \mathrm{Ni}(\mathrm{LSCCe}+5 \mathrm{Ni})$. 298

Figure 9.26. EIS spectra at $700{ }^{\circ} \mathrm{C}$ in $\mathrm{H}_{2}$ of LSC and LSCCe anodes, a) Nyquist and b) Bode plots and EIS spectra at $700{ }^{\circ} \mathrm{C}$ in $\mathrm{H}_{2}$ of LSCCe infiltrated with $1 \mathrm{Ni}$ and $5 \mathrm{Ni}, \mathrm{c}$ ) Nyquist and d) Bode plots. 
Figure 9.27. Modeled R contributions of the LSCCe anode and when infiltrated with different amounts of $\mathrm{Ni}: 1 \mathrm{Ni}(\mathrm{LSCCe}+1 \mathrm{Ni})$ and with $5 \mathrm{Ni}(\mathrm{LSCCe}+5 \mathrm{Ni})$. 300

Figure 9.28. Associated capacitances and relaxation times of the $\mathrm{LSCCe}, \mathrm{LSCCe}+1 \mathrm{Ni}$ and $\mathrm{LSCCe}+5 \mathrm{Ni}$ anodes. 300

Figure 9.29. Rp at $700{ }^{\circ} \mathrm{C}$ of different chromite based anodes: LSC, LSCCe, LSCN10, $\mathrm{LSCN}, \mathrm{LSCCe}+1 \mathrm{Ni}, \mathrm{LSCCe}+5 \mathrm{Ni}$ and $\mathrm{LSCN}+5 \mathrm{Ni}$.

\section{List of tables}

Table 1.1. Summary of electroneutrality equations in different regions .27

Table 1.2. Region dependent equation for defect species concentration in acceptor doped material.

Table 1.3. Region dependent equation for defect species concentration in donor doped material.

Table 2.1. Summary of the seven crystal systems and Bravais lattices. [6] .69

Table 3.1. Theoretical and experimental density of doped cerias. 101

Table 3.2. Thermal expansion coefficients calculated in Air and in $\mathrm{N}_{2}$ for different La, $\mathrm{Eu} \mathrm{Gd,} \mathrm{Pr} \mathrm{and} \mathrm{Tb}$ doped ceria. 103

Table 4.1 Thermal expansion coefficient of $\mathrm{Ce}_{0.9} \mathrm{Pr}_{0.1} \mathrm{O}_{2-\diamond}$ and CPCo $2 \%$ sintered at 1300 ${ }^{\circ} \mathrm{C}$.

Table 5.1. Apparent activation energies in the temperature ranges of $800-480{ }^{\circ} \mathrm{C}$ (high T) and $480-400{ }^{\circ} \mathrm{C}$ (low T) for CT10, CT10Co, CT20 and CT20Co and in the temperature range of $800-700{ }^{\circ} \mathrm{C}$ (high T) and $700-400{ }^{\circ} \mathrm{C}$ (low T) for CT50 and CT50Co. The conductivities at $800^{\circ} \mathrm{C}$ under air and $5 \times 10^{-5}$ atm is indicated. 
Table 5.2: Oxygen non-stoichiometry and surface exchange rate, at $700{ }^{\circ} \mathrm{C}$, and apparent activation energies for the surface exchange rate for the different compounds (Fig 14) 170

Table 6.1 Cerium-based compositions synthesized as cubic fluorite structure with the nomenclature used along the text, and the corresponding lattice parameter and crystallite size extracted from XRD patterns

Table 6.2. Fitting parameters obtained for each LSM-ceria-based composite at $800{ }^{\circ} \mathrm{C}$ under air. $\mathrm{R}$ is the resistance, $\mathrm{Q}_{0}$ and $n$ are the constant phase element parameters and $\omega$ the summit frequency of each ZARC element, respectively. Capital letters (A, B, C) indicate the process according to the proposed electrical equivalent circuit.

Table 7.1. Ionic radius of constituent ions $\mathrm{A}$ and $\mathrm{B}$, tolerance factor, octahedral factor $\left(r_{B} / r_{O}\right)$ and symmetry of different compounds $(\mathrm{O}=$ orthorrombic, $\mathrm{X}=$ not single phase).

Table 7.2. Cell volume and cell parameters of formed perovskite compounds (fitting errors in brackets)

Table 8.1. Spinel and fluorite structured compounds selected for dual-phase membranes

Table 9.1. Summary of doped lanthanum strontium chromites composition and short nomenclature. The main structural phase formation is indicated as well as the total conductivity in wet $\mathrm{H}_{2}$ atmosphere at $700{ }^{\circ} \mathrm{C}$, and the corresponding activation energy. 


\section{Appendix}

\section{Appendix 1}

Summary of the Kröger-Vink notation of a compound $\mathrm{MO}_{\mathrm{x}}$.

\begin{tabular}{|l|c|}
\hline Sites & Notation \\
\hline Interstitial oxygen ion & $\mathrm{O}_{\mathrm{i}}^{\prime \prime}$ \\
\hline Foreign acceptor metal, A on M site & $\mathrm{A}_{\mathrm{M}}^{\prime}$ \\
\hline Free electrons & $\mathrm{e}^{\prime}$ \\
\hline Occupied oxygen site & $\mathrm{O}_{\mathrm{O}}^{\mathrm{X}}$ \\
\hline Oxygen vacancy & $\mathrm{V}_{\mathrm{O}}^{\prime \prime}$ \\
\hline Foreign donor metal, D on M site & $\mathrm{D}_{\mathrm{M}}^{\prime}$ \\
\hline Free positive holes & $\mathrm{h}^{\prime}$ \\
\hline Vacancy in the metal & $\mathrm{V}_{\mathrm{Mi}}^{\prime \prime}$ \\
\hline
\end{tabular}




\section{Appendix 2}

Information of plane spacing for the seven Bravais crystal systems.

\begin{tabular}{|c|c|}
\hline Cubic & $\frac{1}{d^{2}}=\frac{h^{2}+k^{2}+l^{2}}{a^{2}}$ \\
\hline Tetragonal & $\frac{1}{d^{2}}=\frac{h^{2}+k^{2}}{a^{2}}+\frac{l^{2}}{c^{2}}$ \\
\hline Hexagonal & $\frac{1}{d^{2}}=\frac{4}{3}\left(\frac{h^{2}+h k+k^{2}}{a^{2}}\right)+\frac{l^{2}}{c^{2}}$ \\
\hline Trigonal & $\frac{1}{d^{2}}=\frac{\left(h^{2}+k^{2}+l^{2}\right) \sin ^{2} \alpha+2(h k+k l+h l)\left(\cos ^{2} \alpha-\cos \alpha\right)}{a^{2}\left(1-3 \cos ^{2} \alpha+2 \cos ^{3} \alpha\right)}$ \\
\hline Orthorhombic & $\frac{1}{d^{2}}=\frac{h^{2}}{a^{2}}+\frac{k^{2}}{b^{2}}+\frac{l^{2}}{c^{2}}$ \\
\hline Monoclinic & $\frac{1}{d^{2}}=\frac{1}{\sin ^{2} \beta}\left(\frac{h^{2}}{a^{2}}+\frac{k^{2} \sin ^{2} \beta}{b^{2}}+\frac{l^{2}}{c^{2}}-\frac{2 h l \cos \beta}{a c}\right)$ \\
\hline Triclinic & $\frac{1}{d^{2}}=\frac{1}{V^{2}}\left(S_{11} h^{2}+S_{22} k^{2}+S_{23} k^{2}+2 S_{12} h k+2 S_{23} k l+2 S_{13} h l\right)$ \\
\hline \multicolumn{2}{|c|}{ On the triclinic system, $V$ is the volume of a unit cell and the coefficients are given below. } \\
\hline & $S_{11}=b^{2} c^{2} \sin ^{2} \alpha, \quad S_{12}=a b c^{2}(\cos \alpha \cos \beta-\cos \gamma)$ \\
\hline & $S_{22}=a^{2} c^{2} \sin ^{2} \beta, \quad S_{23}=a^{2} b c(\cos \beta \cos \gamma-\cos \alpha)$, \\
\hline & $S_{33}=a^{2} b^{2} \sin ^{2} \gamma, \quad S_{13}=a b^{2} c(\cos \gamma \cos \alpha-\cos \beta)$ \\
\hline
\end{tabular}




\section{Appendix 3}

\begin{tabular}{|c|c|c|}
\hline Symbol & Definition & Units \\
\hline $\mathrm{A}_{\text {effective }}$ & Effective area of a membrane & $\mathrm{cm}^{2}$ \\
\hline$\Delta \mu_{\mathrm{O}_{2}}^{\text {tot }}$ & Total oxygen chemical potential difference & \\
\hline$\nabla \mu_{\mathrm{O}_{2}}$ & Oxygen chemical potential gradient & \\
\hline$a$ & Cell parameter of a crystal & Angstroms, $\AA$ \\
\hline $\mathrm{a}$ & Conductivity probe width & $\mathrm{cm}$ \\
\hline $\mathrm{b}$ & Conductivity probe thickness & $\mathrm{cm}$ \\
\hline$C$ & Capacitor & $\mathrm{F} / \mathrm{cm}^{2}$ \\
\hline $\mathrm{d}$ & $(h k l)$ interplanar space & $\mathrm{nm}$ \\
\hline$E_{a}$ & Activation Energy & $\mathrm{eV}$ \\
\hline$E_{\text {ass }}$ & Vacancy-dopant association energy & $\mathrm{eV}$ \\
\hline$E_{b}$ & Binding energy (characteristic of each element) & $\mathrm{eV}$ \\
\hline$E_{f}$ & Vacancy formation energy & $\mathrm{eV}$ \\
\hline$E_{g}$ & Bang gap energy & $\mathrm{eV}$ \\
\hline $\overrightarrow{E_{k}}$ & Kinetic energy of the electrons & $\mathrm{eV}$ \\
\hline$E_{m}$ & Migration barrier & $\mathrm{eV}$ \\
\hline$E_{w}$ & Work function of the spectrometer & $\mathrm{eV}$ \\
\hline$h, k, l$ & Miller indices defining a plane & \\
\hline$h v$ & Energy of the photons & $\mathrm{eV}$ \\
\hline$J_{O 2}$ & Permeation flux & $\mathrm{mL} \min ^{-1} \mathrm{~cm}^{-2}$ \\
\hline 1 & $\begin{array}{l}\text { Distance between electrodes in the } \\
\text { conductivity probe }\end{array}$ & $\mathrm{cm}$ \\
\hline$L$ & Correlation length & $\AA$ \\
\hline$N$ & Defect concentration & $\mathrm{cm}^{-3}$ \\
\hline NA & Avogadro's Number & $\mathrm{mol}^{-1}$ \\
\hline $\mathrm{Q}$ & Flow & $\mathrm{mL} \min ^{-1}$ \\
\hline$q$ & Wave vector & $2 \pi / a$ \\
\hline$R$ & Resistance & Ohm \\
\hline$t$ & Crystallite diameter & $\mathrm{nm}$ \\
\hline $\mathrm{Z}$ & Number of electrons in a reaction & \\
\hline$Z$ & System impedance & Ohm \\
\hline$\beta$ & Full width at half maximum intensity & $\mathrm{rad}$ \\
\hline$\Gamma$ & Linewidth of the Raman mode & $\mathrm{cm}^{-1}$ \\
\hline
\end{tabular}




\begin{tabular}{|l|l|l|}
\hline$\delta$ & Oxygen nonstoichiometry & \\
\hline$\Delta \omega$ & Raman frequency shift & $\mathrm{cm}^{-1}$ \\
\hline$\theta$ & Incidence angle of X-ray & ${ }^{\circ}$ \\
\hline$\lambda$ & Wavelength of X-rays & $\mathrm{nm}$ \\
\hline$\mu$ & Carrier mobility & $\mathrm{cm}^{2} V^{-1} \mathrm{~s}^{-1}$ \\
\hline$\sigma_{\mathrm{amb}}$ & Ambipolar conductivity & $\mathrm{S} \mathrm{cm}^{-1}$ \\
\hline$\sigma_{\mathrm{e}}$ & Electronic conductivity & $\mathrm{S} \mathrm{cm}^{-1}$ \\
\hline$\sigma_{\mathrm{i}}$ & Ionic conductivity & $\mathrm{S} \mathrm{cm}^{-1}$ \\
\hline$\omega$ & Frequency & $\mathrm{rad}^{-1}$ \\
\hline$\omega_{0}$ & Raman frequency & $\mathrm{cm}^{-1}$ \\
\hline $\mathrm{Q}_{0}$ & $\begin{array}{l}\text { Constant phase element parameters of ZARC } \\
\text { elements }\end{array}$ & $\mathrm{F}$ \\
\hline$\omega$ & Summit frequency ZARC elements & $\mathrm{Hz}$ \\
\hline
\end{tabular}




\section{Appendix 4}

\begin{tabular}{|l|l|}
\hline Acronyms & \\
\hline AC & Alternating Current \\
\hline AES & Auger Electrons \\
\hline AFC & Alkaline Fuel Cell \\
\hline ASR & Area Specific Resistivity \\
\hline ASU & Air Separation Unit \\
\hline AW & Atomic Weight \\
\hline BE & Binding energies \\
\hline BSE & Backscattered electrons \\
\hline CEC & Chemical Expansion Coefficient \\
\hline CMR & Catalytic Membrane Reactor \\
\hline CV & Volume of the unit Cell \\
\hline DC & Direct Current \\
\hline DTA & Differential Thermal Analysis \\
\hline ECR & Electrical Conductivity Relaxation \\
\hline EDS & Energy Dispersive x-ray Spectroscopy \\
\hline EDTA & Ethylenediaminetetraacetic acid \\
\hline EIS & Electrochemical Impedance Spectroscopy \\
\hline FE-SEM & Field Emission SEM \\
\hline FIB & Focused Ion Beam \\
\hline GB & Grain Boundary \\
\hline HT-SOFC & High Temperature SOFC \\
\hline HT-XRD & High Temperature XRD \\
\hline HTM & Hydrogen Transport Membrane \\
\hline IGCC & Integrated Gasification Combined Cycle \\
\hline IT-SOFC & Intermediate Temperature SOFC \\
\hline Ln & Lanthanide \\
\hline MCFC & Molten Carbonate Fuel Cell \\
\hline MFC & Mass Flow Controllers \\
\hline MIEC & Mixed Ionic Electronic Conductor \\
\hline & \\
\hline
\end{tabular}




\begin{tabular}{|l|l|}
\hline OCM & Oxidative Coupling of Methane \\
\hline OCV & Open Circuit Voltage \\
\hline OTM & Oxygen Transport Membrane \\
\hline PAFC & Phosphoric Acid Fuel Cell \\
\hline PC-SOFC & Proton Conducting Solid Oxide Fuel Cell \\
\hline PEMFC & Polymer Electrolyte Membrane \\
\hline PIE & Pulse Isotopic Exchange \\
\hline$p O_{2}$ & Oxygen partial pressure \\
\hline POM & Partial Oxidation of Methane \\
\hline$r_{c}$ & Ionic radius of the cerium $($ Ce \\
\hline$r_{d}$ & Ionic radius of the dopant \\
\hline SEM & Scanning Electron Microscopy \\
\hline SOFC & Solid Oxide Fuel Cell \\
\hline TCD & Thermal Conductivity Detector \\
\hline TEC & Thermal Expansion Coefficient \\
\hline TG & Thermogravimetry \\
\hline TPB & Triple Phase Boundary \\
\hline TPD & Temperature Programmed Desorption \\
\hline TPR & Temperature Programmed Reduction \\
\hline UV-vis & Ultra violet visible \\
\hline XPS & X-ray Photoelectron Spectroscopy \\
\hline XRD & X-Ray Diffraction \\
\hline & \\
\hline
\end{tabular}


Resumen / Resum / Summary 



\section{Resumen}

Los materiales conductores mixtos de electrones e iones (oxígeno o protones) son capaces de separar oxígeno o hidrógeno de los gases de combustión o de corrientes de reformado a alta temperatura. La selectividad de este proceso es del 100\%. Estos materiales, óxidos sólidos densos, pueden usarse en la producción de electricidad a partir de combustibles fósiles, así como formar parte de los procesos que forman parte del sistema de captura y almacenamiento de $\mathrm{CO}_{2}$. Las membranas de transporte de oxígeno (MTO) se pueden utilizar en las plantas energéticas con procesos de oxicombustión, así como en reactores catalíticos de membrana (RCM), mientras que las membranas de transporte de hidrógeno (MTH) se aplican en procesos de precombustión. Además, estos materiales encuentran aplicación en componentes de sistemas energéticos, como electrodos o electrolitos de pilas de combustible de óxido sólido, de ambas clases iónicas y protónicas (SOFC y PC-SOFC).

Los procesos mencionados implican condiciones de operación muy severas, como altas temperaturas y grandes gradientes de presión parcial de oxígeno $\left(\mathrm{pO}_{2}\right)$, probablemente combinadas con la presencia de $\mathrm{CO}_{2}$ y $\mathrm{SO}_{2}$. Los materiales más que mayor rendimiento de separación presentan y más ampliamente investigados en este campo son inestables en estas condiciones. Por tanto, existe la necesidad de encontrar nuevos materiales inorgánicos estables que proporcionen alta conductividad electrónica e iónica.

La presente tesis propone una búsqueda sistemática de nuevos conductores iónicos-electrónicos mixtos (MIEC, del inglés) con diferente estructura cristalina y/o diferente composición, variando la naturaleza de los elementos y la estequiometría del cristal. La investigación ha dado lugar a 
materiales capaces de transportar iones oxígeno, protones o cargas electrónicas y que son estables en las condiciones de operación.

La caracterización de una amplia serie de cerias $\left(\mathrm{CeO}_{2}\right)$ dopadas con lantánidos proporciona una comprensión general de las propiedades estructurales y de transporte, así como la relación entre ellas. Además, se estudia el efecto de la adición de cobalto a dicho sistema. Se ha completado el análisis con la optimización de las propiedades de transporte a partir de la microestructura. Todo esto permite hacer una clasificación inicial de los materiales basada en el comportamiento de transporte principal y permite adecuar la estructura y las condiciones de operación para obtener las propiedades deseadas para cada aplicación.

Algunos de los materiales extraídos de este estudio alcanzaron las expectativas. Las familias de materiales basadas en $\mathrm{Ce}_{1-\mathrm{x}} \mathrm{Tb}_{\mathrm{x}} \mathrm{O}_{2-\delta}$ y $\mathrm{Ce}_{1-\mathrm{x}} \mathrm{Tb}_{\mathrm{x}} \mathrm{O}_{2-\delta}$ +2 mol\% Co proporcionan flujos de oxígeno bajos pero competitivos, ya que son estables en atmósferas con $\mathrm{CO}_{2}$. Además, la inclusión de estos materiales en membranas de dos fases aumenta el flujo de oxígeno. La combinación con una espinela libre de cobalto y de metales alcalinotérreos como es el $\mathrm{Fe}_{2} \mathrm{NiO}_{4}$, ha dado lugar a un material prometedor en cuanto a flujo de oxígeno y estabilidad en $\mathrm{CO}_{2}$ y en $\mathrm{SO}_{2}$, que podría ser integrado en el proceso de oxicombustión.

Por otra parte, se ha añadido metales como codopantes en el sistema $\mathrm{Ce}_{0.9-\mathrm{x}} \mathrm{M}_{\mathrm{x}} \mathrm{Gd}_{0.1} \mathrm{O}_{1.95}$. Estos materiales, en combinación con la perovskita $\mathrm{La}_{1-\mathrm{x}} \mathrm{Sr}_{\mathrm{x}} \mathrm{MnO}_{3}$ usada comúnmente como cátodo de SOFC, han sido capaces de disminuir la resistencia de polarización del cátodo. La mejora es consecuencia de la introducción de conductividad iónica por parte de la ceria.

Las perovskitas dopadas basadas en $\mathrm{CaTiO}_{3}$ forman el segundo grupo de materiales investigados. La dificultad de obtener perovskitas estables y que 
presenten conducción mixta iónica y electrónica se ha hecho evidente. De entre los dopantes utilizados, el hierro y la combinación hierro-magnesio han sido los mejores candidatos. Ambos materiales presentan conductividad principalmente iónica a alta temperatura, mientras que a baja predomina la conductividad electrónica tipo $p$. $\mathrm{CaTi}_{0.73} \mathrm{Fe}_{0.18} \mathrm{Mg}_{0.09} \mathrm{O}_{3-\delta}$ se ha mostrado como un material competente en la fabricación de membranas de oxígeno, que proporciona flujos adecuados a la par que estabilidad en $\mathrm{CO}_{2}$.

Finalmente, la perovskita $\mathrm{La}_{0.87} \mathrm{Sr}_{0.13} \mathrm{CrO}_{3}$ (LSC) ha sido dopada con el objetivo de aumentar la conductividad mixta protónica electrónica. Este estudio ha llevado al desarrollo de una nueva generación de ánodos para PC-SOFC basadas en electrolitos de LWO. Las perovskitas dopadas con Ce en el sitio del $\mathrm{La}$ (LSCCe) y con Ni en el sitio del Cr (LSCN) son estables en condiciones de operación reductoras, así como en contacto con el electrolito. El uso de ambos materiales como ánodo disminuye la resistencia de polarización con respecto al LSC. El LSCCe está limitado por los procesos que ocurren a baja frecuencia (BF), relacionados con los procesos superficiales, y que son atenuados en el caso del LSCN debido a la formación de nanopartículas de Ni metálico en la superficie. La infiltración posterior con nanopartículas de Ni permite disminuir la resistencia a $\mathrm{BF}$ lo que sugiere que la reacción superficial de oxidación del $\mathrm{H}_{2}$ está siendo catalizada. La infiltración más concentrada en Ni (5Ni) elimina completamente la resistencia a $\mathrm{BF}$ en ambos ánodos, de forma que los procesos que ocurren a altas frecuencias son ahora limitantes. El ánodo constituido por LSCNi20+5Ni dio una resistencia de polarización de $0.26 \Omega \cdot \mathrm{cm}^{2}$ at $750{ }^{\circ} \mathrm{C}$ en $\mathrm{H}_{2}$ húmedo. 


\section{Resum}

Els materials conductors mixts d'electrons i ions (oxigen o protons) són capaços de separar oxigen o hidrogen dels gasos de combustió o de corrents de reformat a alta temperatura. La selectivitat d'aquest procés és del 100\%. Aquests materials, òxids sòlids densos, poden usar-se en la producció d'electricitat a partir de combustibles fòssils, així com formar part dels processos que formen part del sistema de captura i emmagatzematge de $\mathrm{CO}_{2}$. Les membranes de transport d'oxigen (MTO) es poden utilitzar en les plantes energètiques amb processos de oxicombustió, així com en reactors catalítics de membrana (RCM), mentre que les membranes de transport d'hidrogen (MTH) s'apliquen en processos de precombustió. A més, aquests materials troben aplicació en components de sistemes energètics, com a elèctrodes o electròlits de piles de combustible d'òxid sòlid, d'ambdues classes iòniques i protòniques (SOFC i PC-SOFC).

Els processos esmentats impliquen condicions d'operació molt severes, com ara altes temperatures i grans gradients de pressió parcial d'oxigen $\left(\mathrm{pO}_{2}\right)$, probablement combinades amb la presència de $\mathrm{CO}_{2} \mathrm{i} \mathrm{SO}_{2}$. Els materials que major rendiment de separació presenten i són més àmpliament investigats en aquest camp són inestables en aquestes condicions. Per tant, existeix la necessitat de trobar nous materials inorgànics estables que proporcionen alta conductivitat electrònica i iònica.

La present tesi proposa una cerca sistemàtica de nous conductors iònicselectrònics mixts (MIEC, de l'anglès) amb diferent estructura cristal·lina i/o diferent composició, variant la naturalesa dels elements i l'estequiometria del cristall. La recerca ha donat lloc a materials capaços de transportar ions oxigen, 
protons $\mathrm{o}$ càrregues electròniques $\mathrm{i}$ que són estables en les condicions d'operació.

La caracterització d'una àmplia sèrie de cerias $\left(\mathrm{CeO}_{2}\right)$ dopades amb lantànids proporciona una comprensió general de les propietats estructurals i de transport, així com la relació entre elles. A més, s'estudia l'efecte de l'addició de cobalt a aquest sistema. S'ha completat l'anàlisi amb l'optimització de les propietats de transport a partir de la microestructura. Tot açò permet fer una classificació inicial dels materials basada en el comportament de transport principal i permet adequar l'estructura i les condicions d'operació per a obtenir les propietats desitjades per a cada aplicació.

Alguns dels materials extrets d'aquest estudi van aconseguir les expectatives. Les famílies de materials basades en $\mathrm{Ce}_{1-\mathrm{x}} \mathrm{Tb}_{\mathrm{x}} \mathrm{O}_{2-\delta} \mathrm{i} \mathrm{Ce}_{1-\mathrm{x}} \mathrm{Tb}_{\mathrm{x}} \mathrm{O}_{2-\delta}$ +2 mol\% Co proporcionen fluxos d'oxigen baixos però competitius, ja que són estables en atmosferes amb $\mathrm{CO}_{2}$. A més, la inclusió d'aquests materials en membranes de dues fases augmenta el flux d'oxigen. La combinació amb una espinela lliure de cobalt i de metalls alcalinoterris com és el $\mathrm{Fe}_{2} \mathrm{NiO}_{4}$, ha donat lloc a un material prometedor quant a flux d'oxigen i estabilitat en $\mathrm{CO}_{2} \mathrm{i}$ en $\mathrm{SO}_{2}$, que podria ser integrat en el procés de oxicombustió.

D'altra banda, s'ha afegit metalls com codopants en el sistema $\mathrm{Ce}_{0.9-\mathrm{x}} \mathrm{M}_{\mathrm{x}} \mathrm{Gd}_{0.1} \mathrm{O}_{2-\delta}$. Aquests materials, en combinació amb la perovskita $\mathrm{La}_{1-\mathrm{x}} \mathrm{Sr}_{\mathrm{x}} \mathrm{MnO}_{3}$ usada comunament com a càtode de $\mathrm{SOFC}$, han sigut capaços de disminuir la resistència de polarització del càtode. La millora és conseqüència de la introducció de conductivitat iònica per part de la ceria. 
Les perovskites dopades basades en $\mathrm{CaTiO}_{3}$ formen el segon grup de materials investigats. La dificultat d'obtenir perovskites estables i que presenten conducció mixta iònica i electrònica s'ha fet evident. D'entre els dopants utilitzats, el ferro i la combinació ferro-magnesi han sigut els millors candidats. Tots dos materials presenten conductivitat principalment iònica a alta temperatura, mentre que a baixa predomina la conductivitat electrònica tipus $p$. $\mathrm{CaTi}_{0.73} \mathrm{Fe}_{0.18} \mathrm{Mg}_{0.09} \mathrm{O}_{3-\delta}$ s'ha mostrat com un material competent en la fabricació de membranes d'oxigen, que proporciona fluxos adequats a l'una que estabilitat en $\mathrm{CO} 2$.

Finalment, la perovskita $\mathrm{La}_{0.87} \mathrm{Sr}_{0.13} \mathrm{CrO}_{3}$ (LSC) ha sigut dopada amb l'objectiu d'augmentar la conductivitat mixta protònica electrònica. Aquest estudi ha portat al desenvolupament d'una nova generació d'ànodes per a PC-SOFC basades en electròlits de LWO. Les perovskites dopades amb Ce en el lloc del La (LSCCe) i amb Ni en el lloc del Cr (LSCN) són estables en condicions d'operació reductores, així com en contacte amb l'electròlit. L'ús de tots dos materials com a ànode disminueix la resistència de polarització pel que fa al LSC. El LSCCe està limitat pels processos que succeeixen a baixa freqüència $(\mathrm{BF})$, relacionats amb els processos superficials, i que són atenuats en el cas del LSCN a causa de la formació de nanopartícules de Ni metàl·lic en la superfície. La infiltració posterior amb nanopartícules de Ni permet disminuir la resistència a $\mathrm{BF}$, el que suggereix que la reacció superficial d'oxidació de l' $\mathrm{H}_{2}$ està sent catalitzada. La infiltració més concentrada en $\mathrm{Ni}(5 \mathrm{Ni})$ elimina completament la resistència a $\mathrm{BF}$ en tots dos ànodes, de manera que els processos que ocorren a altes freqüències són ara limitants. L'ànode constituït per LSCNi20+5Ni va donar una resistència de polarització de $0.26 \Omega \cdot \mathrm{cm} 2$ a $750{ }^{\circ} \mathrm{C}$ en $\mathrm{H}_{2}$ humit. 


\section{Summary}

Mixed ionic (oxygen ions or protons) and electronic conducting materials (MIEC) separate oxygen or hydrogen from flue gas or reforming streams at high temperature in a process $100 \%$ selective to the ion. These solid oxide materials may be used in the production of electricity from fossil fuels (coal or natural gas), taking part of the $\mathrm{CO}_{2}$ separation and storage system. Dense oxygen transport membranes (OTM) can be used in oxyfuel combustion plants or in catalytic membrane reactors (CMR), while hydrogen transport membranes (HTM) would be applied in precombustion plants. Furthermore, these materials may also be used in components for energy systems, as advanced electrodes or electrolytes for solid oxide fuel cells (SOFC) and proton conducting solid oxide fuel cells (PC-SOFC) working at high and moderate temperature.

The harsh working conditions stablished by the targeted processes include high temperatures and low $\mathrm{O}_{2}$ partial pressures $\left(p O_{2}\right)$, probably combined with $\mathrm{CO}_{2}$ and $\mathrm{SO}_{2}$ containing gases. The instability disadvantages presented by the most widely studied materials for these purposes make them impractical for application to gas separation. Thus, the need to discover new stable inorganic materials providing high electronic and ionic conductivity is still present.

This thesis presents a systematic search for new mixed ionic-electronic conductors. It includes different crystalline structures and/or composition of the crystal lattice, varying the nature of the elements and the stoichiometry of the crystal. The research has yielded new materials capable to transport oxygen ions or protons and electronic carriers that are stable in the working condition to which they are submitted. 
The characterization of a large series of lanthanide-doped ceria $\left(\mathrm{CeO}_{2}\right)$ provides an overall understanding of the structural and transport properties of these materials. Besides, the addition of cobalt oxide on this system is similarly studied. The analysis is completed by the optimization of the transport properties dependence on the microstructure. This allows a preliminary classification of the materials based on the main transport behavior and shows the possibility of tailoring both the structure and operating conditions to achieve desired properties for a number of applications, i.e., solid oxide fuel cells (SOFC) electrodes or oxygen separation membranes

Several materials emerged from this work have achieved their purpose. $\mathrm{Ce}_{1-\mathrm{x}} \mathrm{Tb}_{\mathrm{x}} \mathrm{O}_{2-\delta}$ and $\mathrm{Ce}_{1-\mathrm{x}} \mathrm{Tb}_{\mathrm{x}} \mathrm{O}_{2-\delta}+2 \mathrm{~mol} \% \mathrm{Co}$ are proposed as a family of materials that may provide competent oxygen permeation fluxes and stability against $\mathrm{CO}_{2}$ containing atmospheres. Besides, the inclusion of this material as a component of a dual-phase membrane increases the oxygen flux possibilities. The combination with a stable, Co and alkali-earth free spinel has delivered a promising membrane material for the integration in oxyfuel power plants.

On the other hand, metal dopants on the $\mathrm{Ce}_{0.9-\mathrm{x}} \mathrm{M}_{\mathrm{x}} \mathrm{Gd}_{0.1} \mathrm{O}_{2-\delta}$ structure have been added to lanthanide-doped ceria. They have been studied as SOFC cathode in combination with $\mathrm{La}_{1-\mathrm{x}} \mathrm{Sr}_{\mathrm{x}} \mathrm{MnO}_{3}$-perovskite. The results show that the ionic conductivity invested by the ceria allows decreasing the polarization resistance regarding to the single phase cathode.

The study of a second group of materials involves a wide screening of doped $\mathrm{CaTiO}_{3}$ perovskites. The difficulty of obtaining stable perovskites with high mixed ionic-electronic conductivity is evidenced. Iron and iron-magnesium doped $\mathrm{CaTiO}_{3}$ are the best candidates as they present mainly ionic conductivity at high temperatures, while $p$-type electronic conductivity is predominant in the 
low temperature range. $\mathrm{CaTi}_{0.73} \mathrm{Fe}_{0.18} \mathrm{Mg}_{0.09} \mathrm{O}_{3-\delta}$ is shown as a promising material offering good oxygen permeation fluxes and stability against $\mathrm{CO}_{2}$, although it reacts with $\mathrm{SO}_{2}$.

Finally, $\mathrm{La}_{0.87} \mathrm{Sr}_{0.13} \mathrm{CrO}_{3}$ (LSC) perovskites have been doped in order to increase the mixed protonic-electronic conductivity. The study has lead to the development of a new generation of anodes for LWO based PC-SOFC. Ce in the A-site (LSCCe) and Ni in the B-site (LSCN) are stable perovskites in the reducing anode working conditions and in contact to the LWO electrolyte. Both of them decrease the polarization resistance regarding to pure LSC. LSCCe is limited by low frequency processes (LF), which are alleviated in the case of LSCN due to the formation of metallic nanoparticles on the surface. The further infiltration of $\mathrm{Ni}$ nanoparticles allows decreasing the LF resistance, which suggests that the surface reaction of $\mathrm{H}_{2}$ oxidation is catalyzed. The most concentrated infiltration (5Ni) completely eliminates the LF resistance in both anodes and only high frequency (HF) processes remain. The best anode performance was obtained for LSCNi20 $+5 \mathrm{Ni}$. Polarization resistance was as low as $0.26 \Omega \cdot \mathrm{cm}^{2}$ at $750{ }^{\circ} \mathrm{C}$ in wet $\mathrm{H}_{2}$. 



\section{Acknowledgments/Agradecimientos}

¿Quién me iba a decir que esta iba a ser una de las partes más difíciles de escribir? Es que cuatro años, a pesar de pasar volando, dan para mucho. Sobre todo para ver que todo funciona mejor cuando se hace uniendo fuerzas y que 'tota pedra fa paret'. Empezaré por el principio, que no está mal para variar un poco...

Quería agradecer a mis directores de tesis la confianza depositada en mi trabajo. A José M. Gozálvez que me acercó al ITQ y me puso en contacto con Jose. Y a Jose M. Serra por hacerme parte de este equipo. He aprendido mucho en este tiempo gracias a su dedicación, paciencia y confianza, tanto en el trabajo de laboratorio como a la hora de escribir... y a esos "Te tengo que pasar un brownie".

El aporte financiero debo agradecérselo al Ministerio de Ciencia e Innovación tanto por la beca de formación del personal investigador (Proyecto ENE2008-06302, ENE2011-24761 y la beca BES-2009-015835), como por la dotación para la estancia breve realizada en la Universidad de Twente.

No todo ha sido fácil, pero he tenido la suerte de contar con unos compañeros excepcionales, muchos de ellos ahora amigos. Llegué a un laboratorio bastante reciente, con tres personas trabajando a tope. Por entonces no había tantos equipos, aunque sí los Sonia 1 y Sonia $2 \ldots$ ¿es así, Sonia? Es increíble que entre tantos buenos momentos haya salido tanto trabajo. O quizá es por eso. Ha sido estupendo trabajar juntas, las meriendas con Vicente y Francis, estar presente en ese "tú todo lo haces bien...", que consiguió sacarte 
los colores, y escribir la tesis a tu derecha, las dos con auriculares de protección antigases.

También estaba Vicente, ¿nos conocíamos de antes? Debimos ser hermanos en alguna vida anterior o en alguna paradoja espacio-temporal. Las conversaciones contigo siempre son fructíferas. Ya ves si lo son, descubrimos un nuevo elemento, el Molonio (de símbolo Molo), que en su estado excitado ya debe ser...molérrimo, you know.

Y con una sonrisa me recibió también Sandra, la "morena del pelo rizao'. Siempre me ponía al día con la vida moderna, tecnología, música, estrenos de cine... incluido ese "distrito 9" de marras... y además una campeona.

Después vino Cecilia, gran fichaje de la temporada. Una guaja tan rápida que a veces se desplaza al azul. Excelente compañera de trabajo, de fatiga iy de lío! Las veces que hemos oído ese "que miedo me dais". Siempre estás para lo que haga falta, en los buenos momentos y en los muy malos. Muchas gracias.

Fuera de los límites del laboratorio estaba Esther, o Sam Sagaz, Polvorilla... y también denominada Nancy actividades. Mira que tienes energía, y siempre dispuesta a echar un cable y a apuntarse a un buen plan. Me encanta haberte encontrado y nuestros viajes y excursiones y compras y... si es que funcionamos bien en grupo. Y también Aroa, con su buen hacer y paciencia para explicar las cosas (ese masas infernal...) y que se preocupa por nosotras en los veranos de soledad. Gracias, chicas.

Y poco después llegó María Fabuel, para mí la más vieja conocida. Y sobre todo amiga, no de ahora, sino desde el momento que hace ya (respira 
hondo, Conchín) 22 años, me dijo que tenía una bici como la mía...jesa gran 'Bolero $\mathrm{BH}$ '! Ha sido un placer trabajar contigo (incluso cuando me tatuaste con el portaminas).

El tiempo restante pasó volando. Llegó Laura, la persona más espontánea de Sarrión y sus bocadillos de aire. Eliner y Pilar. Cyril y Mischa, francés y alemán (el que mejor se lo monta del mundo) que llegaron en plena crisis, Merkozy. Pobres, los llevamos fritos. Llegaron Raquel y Julio, compañeros también de escuela y con los que he pasado grandes momentos de laboratorio. Fidel y Mateusz... ¡si es que así se puede!

También quiero acordarme del elenco de taller, Javi P., Paco, Rafa, Javi H., Juan, Jose, Óscar, Ma José, David, Javi Z. y Álvaro por su ayuda y paciencia. De las chicas de caracterización, Maribel, Amparo y Adelina y de José Luis. De Álex y Michael. De los compañeros de combinatoria, Francis, Eva y Juanjo y de Nano, Amparo y Laura. De Vicent y de su parte matutino: "esto no es nada para lo que va a venir".

I would also like to acknowledge the people that I met in the University of Twente, where I spent three months. Thanks to Henny Bouwmeester, who gave me the opportunity of performing PIE experiments under his supervision and to Louis Winnubst, who also made me feel hosted in the group. Of course to Chung-Yul Yoo, my guide and partner for the measurements. It has been a pleasure working with you. Thanks to Susanne and her priceless help to find a house and organize my stay in the university. Also to the office colleagues and many thanks to Ana, the girl of the group who became a friend. She host me also out from the lab and we had good dinners, barbecues, trips and parties... 
Federico, Enrique and Guillaume for your friendship, help, dinners, movies, cycling... I was really lucky to meet you.

Desde luego, igual de importante ha sido el apoyo fuera del laboratorio, un apoyo de fondo. ¿Qué agradecer a mi familia? Más bien la pregunta sería qué no agradecer, si me lo han dado todo. A mis padres su dedicación, su apoyo en todas mis decisiones y su comprensión (incluso cuando la lío un poco). Y a mi hermana, porque no recuerdo ni un solo día sin ti y porque me sigues sin protestar siempre que te necesito, aunque luego me llames 'cansaalmas'. Vosotros sois la base sobre la que he podido empezar. A mi abuela Paz, que me da los mejores consejos que puedo pedir. Y a los que me los dieron. A mis primas, que me alegran los fines de semana y me ayudan a desconectar. Y a todos los demás, que siempre tenéis algo bueno que aportar.

Y por último a Sergio, que llena todas las partes de mi vida. Esta tesis también es en parte tuya, te debo tantas horas... gracias por no fallarme nunca y por comprenderme y animarme. Gracias por tanto amor. TQM. 
"What makes the desert beautiful,' said the little prince, ' is that somewhere it hides a well..."

Antoine de Saint-Exupéry, The Little Prince 